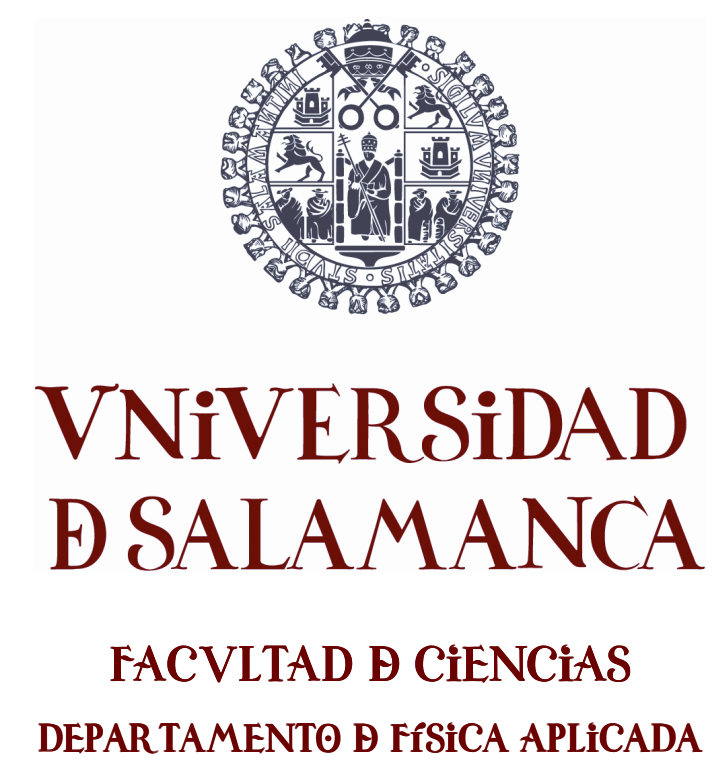

\title{
Modelado de estructuras Schottky y de transistores MOSFET con contactos de fuente y drenador metálicos para aplicaciones de alta frecuencia
}

\author{
Tesis Doctoral
}

Elena Pascual Corral 



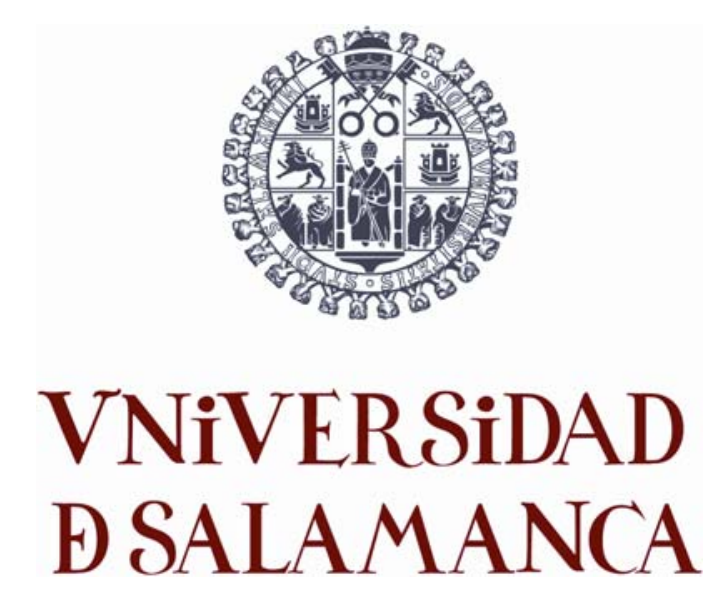

FACVLTAD B CIENCIAS

DEPARTAMENTO Đ FÍSİCA APLICADA

\begin{abstract}
Modelado de estructuras Schottky y de transistores MOSFET con contactos de fuente y drenador metálicos para aplicaciones de alta frecuencia
\end{abstract}

Memoria presentada por

Elena Pascual Corral para optar al grado de Doctor en Ciencias Físicas

Fdo. Elena Pascual Corral Salamanca, Mayo de 2010 

María Jesús Martín Martínez y Raúl Rengel Estévez, Profesores Titulares de Universidad del Área de Electrónica de la Universidad de Salamanca,

\section{AUTORIZAN,}

La presentación del trabajo de investigación que se recoge en la presente Memoria, realizada por $\mathrm{D}^{\mathrm{a}}$ Elena Pascual Corral para optar al Título de Doctor por la Universidad de Salamanca, y que ha sido desarrollado en su totalidad bajo su dirección en el Área de Electrónica del Departamento de Física Aplicada de la Universidad de Salamanca.

Salamanca, 1 de Mayo de 2010

María Jesús Martín Martínez

Profesor Titular de Universidad

Departamento de Física Aplicada

Universidad de Salamanca
Raúl Rengel Estévez

Profesor Titular de Universidad

Departamento de Física Aplicada

Universidad de Salamanca 



\section{Agradecimientos}

Antes de comenzar la Memoria de esta Tesis Doctoral, quisiera agradecer de forma sincera a aquellas personas que de un modo u otro contribuyeron al desarrollo de la misma:

A los directores de la presente Tesis, María Jesús Martín Martínez y Raúl Rengel Estévez, por su capacidad de guiarme a lo largo de esta ardua tarea, mostrándome su constante apoyo tanto científico como personal. Agradezco profundamente su disponibilidad, su gran capacidad de trabajo, su rigor científico y su incansable dedicación, suponiendo para mí un modelo a seguir tanto en el plano laboral como en el personal.

A todos los miembros del Área de Electrónica por el interés y la motivación que me han brindado a lo largo de estos años, en especial a Tomás González Sánchez, por su experiencia científica y sus consejos, que han supuesto una inestimable ayuda para el desarrollo de este trabajo, y a Daniel Pardo, por su interés y apoyo durante todo este tiempo.

Esta tesis doctoral ha sido realizada gracias al Proyecto Europeo METAMOS (IST016677), financiado por la Comisión Europea dentro del Sexto Programa Marco de la Unión Europea. Por ello, quisiera también agradecer a nuestros colegas del IEMN (Francia), Universidad católica de Lovaina (Bélgica), ITE (Polonia), NXT y ST Microelectronics participantes en de dicho proyecto, por su aportación de medidas experimentales y de nuevos puntos de vista, y en particular a Emmanuel Dubois, por su apoyo e intercambio de ideas, y a François Danneville por su colaboración científica.

Por otro lado, a mis amigos por entender que ha sido un camino largo y duro en el que, algunas veces, la fijación por lograr tus objetivos te hace olvidar la importancia del contacto humano. A mis padres y hermanos, por su apoyo incondicional y sus palabras de aliento, ayudándome a superar las etapas difíciles, recordándome lo importante que es disfrutar a cada momento. Y por último a David, por estar ahí, por comprenderme, por creer en mí cuando ni yo misma creía y por perdonar el continuo posponer de nuestros planes. 



\section{Índice}

\section{CAPÍTULO I}

Interfaz metal - semiconductor

I. 1. Unión metal-semiconductor en equilibrio 16

I. 1. A. Caso de banda plana 17

I. 1. B. Contacto óhmico 18

I. 1. C. Contacto rectificador 19

- Descripción cuantitativa básica del contacto rectificador en equilibrio 20

I. 2. Unión metal-semiconductor fuera de equilibrio 22

- Descripción cuantitativa básica del contacto rectificador fuera de equilibrio 23

I. 3. Transporte de Corriente a través del Contacto Schottky 24

I. 3. A. Emisión Termoiónica. [Bethe, 1942] 26

- Corriente de absorción, $J_{s \rightarrow m}$

- Corriente de inyección, $J_{m \rightarrow s} \quad 29$

I. 3. B. Teoría de Difusión. [Schottky, 1938] 29

I. 3. C. Teoría de Mott. [Mott, 1938] 30

I. 4. Efectos reales en la unión metal-semiconductor 31

I. 4. A. Efecto Schottky. [Schottky, 1914] 31 
I. 4. B. Corriente túnel 33

I. 4. C. Inyección de portadores minoritarios 36

I. 4. D. Estados superficiales o de interfaz. Estabilización del nivel de Fermi 36

I. 5. Caracterización de la altura de la barrera 39

I. 5. A. Modelos de determinación de la altura de la barrera 40

I. 5. B. Medida de la altura de la barrera 45

- Medida de corriente - voltaje 45

- Medida de capacidad-voltaje 46

- Medida fotoeléctrica 46

- Medida de energía de activación 47

I. 6. Fabricación de contactos Schottky.

- Limpieza del sustrato 48

- Formación del metal sobre el semiconductor 48

- Activación térmica 51

- Materiales empleados: Siliciuros 51

Bibliografía del Capítulo I

\section{CAPÍTULO II}

Método de Monte Carlo. Algoritmo de inyección/absorción en

$\begin{array}{ll}\text { barrera Schottky } & 57\end{array}$

II. 1. Métodos de estudio del transporte de carga 58

II. 2. Fundamentos del método Monte Carlo 62

II. 3. Estructura de la simulación Monte Carlo 63

II. 3. a. Definición del semiconductor 64

II. 3. b. Definición física del dispositivo 67

- Técnicas de realce estadístico 68 
II. 3. c. Condiciones iniciales 71

II. 3. d. Movimiento de los portadores 71

II. 3. e. Absorción de portadores por efecto túnel

II. 3. f. Cálculo espectroscópico 73

II. 3. g. Inyección de portadores por emisión termoiónica o efecto túnel 74

II. 3. h. Resolución de la ecuación de Poisson y cálculo del campo eléctrico 74

$\begin{array}{ll}\text { II. 3. i. Cálculo de corriente } & 78\end{array}$

II. 3. j. Consideración de resistencias en serie en los contactos 79

II. 3. $k$. Cálculo de los coeficientes de Transmisión 79

II. 3. l. Cálculo de perfiles longitudinales de magnitudes bidimensionales 80

II. 4. Modelo de inyección/absorción: Cálculo del coeficiente de transmisión a través de una barrera de potencial $\quad 80$

$\begin{array}{ll}\text { II. 4. a. Aproximación WKB } & 81\end{array}$

II. 4. b. Método de la matriz de transferencia de Airy (MTA) 87

II. 4. c. Comparación directa entre ambas aproximaciones 89

II. 5. Algoritmo de inyección/absorción: Implementación en el $\begin{array}{ll}\text { simulador Monte Carlo } & 91\end{array}$

II. 6. Modelo de inyección/absorción: Aplicación al caso de barreras sencillas 95

- Barrera triangular 95

a. Influencia de la anchura de la barrera 95

b. Influencia de la altura de la barrera 97

c. Influencia del voltaje aplicado 98

$\begin{array}{lr}\text { Bibliografía del Capítulo II } & 100\end{array}$

\section{CAPÍTULO III}

$\begin{array}{ll}\text { Diodos de barrera Schottky } & 107\end{array}$ 
III. 4. Diodo Schottky con semiconductor dopado tipo n

III. 4. a. Diodo Schottky (Si-n/ZrSi $i_{2}$ a $300 \mathrm{~K}$ de temperatura.

III. 4. a. 1. Comportamiento en directa

III. 4. a. 2. Comportamiento en inversa

o Función de distribución de velocidad, mecanismos de scattering

y ocupación de valles

- Ausencia de efecto túnel

- Estudio del efecto de las cargas imagen

- Estudio de la capacidad

III. 4. b. Estudio del efecto de la variación de la temperatura y de la altura de la barrera

III. 5. Diodo Schottky con semiconductor dopado tipo $p$

- Estudio del efecto de la variación de la temperatura.

\section{CAPÍTULO IV}

Diodos Back-to-Back: estudio del transporte de carga en estructuras fabricadas con barreras Schottky bajas 


\section{CAPÍTULO V}

V. 3. Proceso de fabricación de SB-CMOS y principales características de la arquitectura SB-MOS frente la convencional

V. 5. Simulación Monte Carlo de un transistor SB-MOSFET:

Características estáticas

V. 5. a. Características tensión-corriente

V. 5. b. Análisis del paso de corte a conducción en el dispositivo

V. 5. c. Regímenes de triodo y saturación

V. 5. d. Estudio de las características microscópicas del transporte de portadores en el canal 


\section{CAPÍTULO VI}

VI. 1. Caracterización de pequeña señal

VI. 2. Sensibilidad de las características dinámicas del SB-MOSFET con la variación de parámetros geométricos

VI. 2. a. Variación de la altura de barrera Schottky

VI. 2. b. Variación de la longitud de underlap

VI. 2. c. Variación de la longitud de puerta

VI. 3. Caracterización de ruido

VI. 3. a. Dependencia de las fuentes de ruido con la frecuencia

VI. 3. b. Dependencia de $S_{I D}$ con el voltaje aplicado

VI. 4. Efecto de la variación de parámetros geométricos sobre los resultados de ruido del SB-MOSFET

VI. 4. a. Variación de la altura de barrera

VI. 4. b. Variación de la longitud de underlap

VI. 4. c. Variación de la longitud de puerta 
APÉNDICE

Modelado del material de Silicio. Parámetros de la simulación

309

A. 1. Estructura de bandas del Silicio 309

A. 1. a. Banda de Conducción 310

A. 1. b. Banda de Valencia 311

A. 2. Mecanismos de scattering 312

A. 2. a. Electrones 312

$\begin{array}{ll}\text { A. 2. b. Huecos } & 315\end{array}$

A. 2. c. Probabilidades de scattering 316

A. 3. Modelado de Silicio material $\quad 317$

1. 3. a. Tiempo de permanencia en cada valle 317

A. 3. b. Velocidad de arrastre. Movilidad 318

$\begin{array}{ll}\text { Bibliografía del Apéndice } & 321\end{array}$

BIBLIOGRAFÍA 323 



\section{Introducción}

$\mathcal{F}_{\text {metal-óxido-semiconductor (MOSFET) de Silicio se han convertido en la piedra }}^{\text {nálimas décadas, las tecnologías basadas en el transistor de efecto de campo }}$ angular de los sistemas de telecomunicaciones y los ordenadores modernos [Khanna, 2004]. Una amplia mayoría de los chips contemporáneos utilizados en los productos

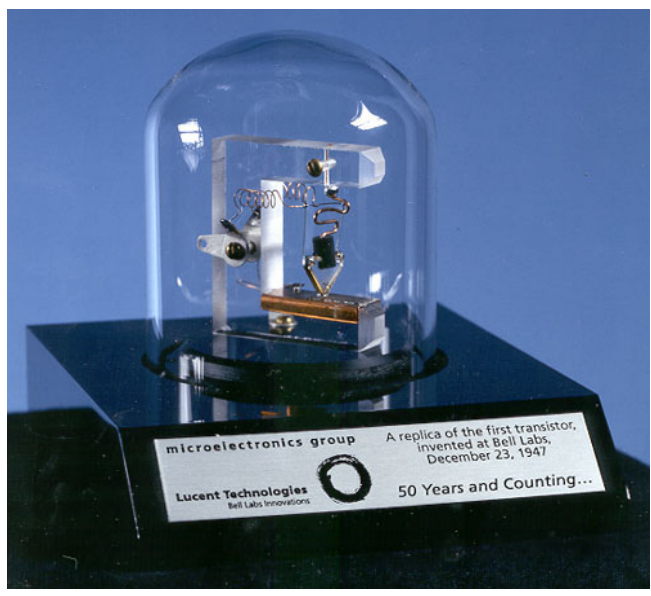

Figura 0.1. Réplica del primer transistor bipolar inventado en los laboratorios Bell de consumo masivo de comunicaciones, profesionales y de la industria electrónica en general tienen al transistor MOSFET como principal componente activo. Los impresionantes avances obtenidos han ido acompañados de una democratización de la tecnología que ha permeabilizado grandes capas de la sociedad. De este modo, hoy en día asistimos al nacimiento de una verdadera sociedad de la información en la que no es posible entender las relaciones

personales (y la forma de interactuar con nuestro propio entorno laboral y de ocio) sin avances tales como la telefonía móvil de última generación, los sistemas inalámbricos (Bluetooth, redes 
WLAN, etc.), los sistemas de posicionamiento global (GPS), los ordenadores personales con alta capacidad de procesamiento, Internet, etc. En consecuencia, el estudio del rendimiento de los transistores MOSFET y su perfeccionamiento, tanto para aplicaciones digitales como analógicas, su rapidez de operación y su comportamiento dinámico y de ruido a altas frecuencias (en el rango de radio frecuencias y microondas) se convierten en un sector estratégico de primer orden para el progreso tecnológico, económico y social del mundo en que vivimos [ITRS, 2007].

Desde sus orígenes, la industria de circuitos integrados ha seguido una estrategia de reducción continua del tamaño de los dispositivos MOSFET, denominada escalado, la cual ha permitido la consecución de los grandes avances tecnológicos obtenidos hasta la fecha [Moore, 1975], [Iwai, 1999]. Este ritmo de escalado ha conducido a una mayor densidad de empaquetamiento, permitiendo fabricar un mayor número de transistores en un único chip sin apenas impacto en el coste de fabricación del mismo [Dennard, 1974], [Taur, 2002]. Al mismo tiempo ha aumentado la funcionalidad de los sistemas al obtenerse una mayor velocidad de operación junto con un menor

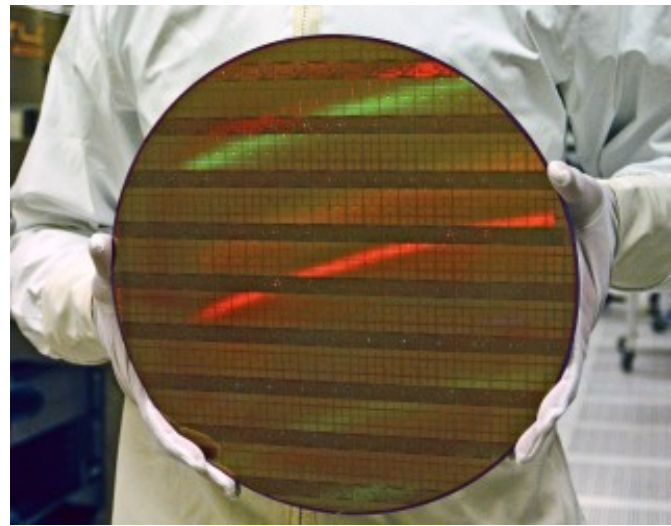

Figura 0.2. Oblea de $300 \mathrm{~mm}$ con chips de $45 \mathrm{~nm}$ fabricada por INTEL consumo, permitiendo simultáneamente a los dispositivos móviles alargar la vida de sus baterías y mejorar su fiabilidad para aplicaciones de circuitos integrados de ultra larga escala de integración (ULSI).

La predicción de las tendencias del escalado y su extensibilidad constituyen por tanto hoy en día un campo fundamental en la industria microelectrónica y en los emergentes sistemas nanoelectrónicos [Lundstrom, 2002], [Hutchby, 2002]. Como documento fundamental para guiar los esfuerzos de investigación y desarrollo necesarios para mejorar la tecnología, la industria semiconductora se ha dotado del llamado International Technology Roadmap for Semiconductors (ITRS) [ITRS, 2007], fruto del consenso del conjunto de principales actores involucrados en este proceso, tanto académicos como industriales. El resultado es una guía precisa de los requerimientos y especificaciones que deben presentar las futuras generaciones de 
dispositivos, así como un compendio de las posibles soluciones a analizar para solventar determinados problemas.

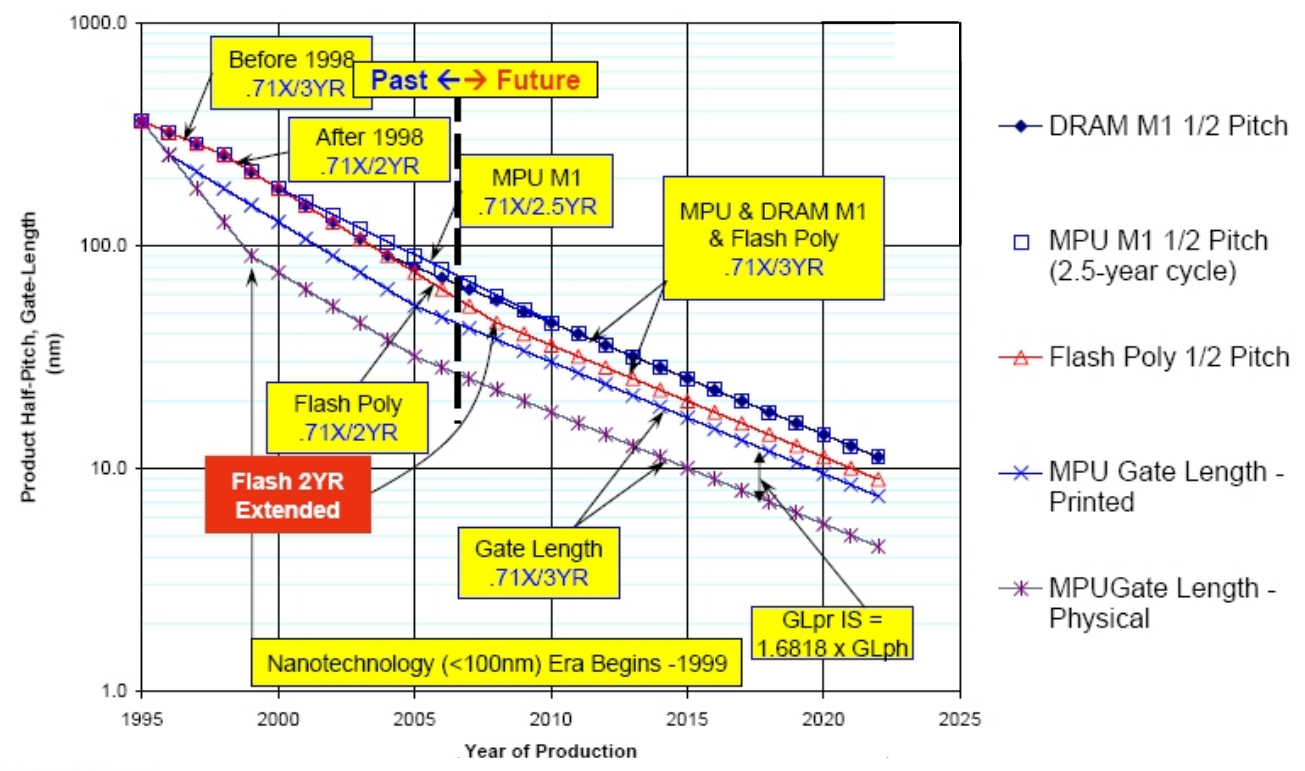

\section{TIT:}

Figura 0.3: Evolución del tamaño de la longitud de puerta y dimensiones características de los transistores constituyentes del MPU (microprocessor unit) y DRAM desde 1995 hasta el 2007, así como las predicciones hasta 2022; [ITRS, 2007].

Hasta la fecha, la evolución del escalado se ha basado fundamentalmente en el transistor MOSFET convencional [Wong, 2002], y en la misma topología básica (el MOS complementario o CMOS) junto con un número de materiales limitados: Silicio, dióxido de Silicio, Aluminio, y Nitruro de Silicio. Para ello han sido modificadas las dimensiones del dispositivo (incluyendo el espesor del óxido, la profundidad de las uniones, la longitud del canal y espaciado de aislamiento además de la concentración de dopantes del substrato y las tensiones de polarización) siguiendo unas reglas de escalado adecuadas. Sin embargo, en el momento actual nos encontramos próximos a un punto crítico en este proceso: hay motivos sobradamente fundamentados para creer que el escalado de los dispositivos en el futuro no será tan inmediato como ha sido en el pasado, puesto que ciertos límites están siendo alcanzados y para superarlos se requerirán cambios substanciales en las tecnologías, así como nuevos conceptos de dispositivo. El ITRS asume que la industria semiconductora ha entrado ya en la era del "escalado de dispositivos limitado por material" dado que los materiales que constituyen los dispositivos MOSFET convencionales (como Silicio, dióxido de Silicio, Silicio dopado y 
polisilicio) se han extendido hasta las limitaciones máximas de su rendimiento. Para el año 2010, la longitud de puerta impresa de los transistores constituyentes de la unidad de microprocesador (MPU) ha de ser de 34 nanómetros y la longitud de puerta física de tan solo 19 nanómetros (Véase Figura 0.3); como podemos comprobar, se ha entrado ya de facto en el dominio de la nanoelectrónica.

Como consecuencia del escalado de los transistores hasta semejantes dimensiones, aparecen una serie de efectos no deseables que pueden limitar notablemente el rendimiento de los dispositivos [Wong, 2002], [Frank, 1998], [Pikus, 1997]. Entre ellos, podemos destacar el llamado efecto de canal corto, que tiene como consecuencia la variación de parámetros del transistor tales como el voltaje umbral, junto con un aumento de la corriente de fugas subumbral, debido a la reducción de la barrera de fuente a substrato por parte del drenador. Otro efecto importante es la posible corriente a través del óxido de puerta, que limita la utilización del dióxido de Silicio como material aislante para espesores inferiores a los 2 nanómetros. Dada su gran importancia se están realizando enormes esfuerzos para identificar e implementar diferentes aislantes de puerta cuya constante dieléctrica sea mayor que la del dióxido de Silicio y cuyas características de estados interfaciales y distribución de trampas muestren un nivel de perfección análogo al de la interfaz $\mathrm{Si} / \mathrm{SiO}_{2}$.

Otro problema que transciende de la reducción de las dimensiones del dispositivo surge como consecuencia de los altos campos eléctricos en el interior, lo que provoca la aparición de los fenómenos de portadores calientes, que pueden degradar el comportamiento del dispositivo, afectando a la fiabilidad y estabilidad de las características.

Pero sin duda, uno de los mayores desafíos a los que se enfrenta esta arquitectura es el de reducir las resistencias parásitas externas al canal conductor o resistencias de acceso. La reducción de las dimensiones del dispositivo lleva consigo una reducción de la resistencia del canal intrínseco; sin embargo, esta reducción no se ve acompañada por una disminución equiparable de las resistencias de acceso. De este modo, la contribución relativa de las resistencias de acceso a la resistencia total del dispositivo aumenta, llegando incluso a ser totalmente dominante en comparación con la intrínseca. La resistencia de acceso se compone de distintas contribuciones en serie (véase Figura 0.4.a.) [Ng, 1986], [Kim, 2000]:

$$
R_{\text {serie }}=R_{\text {cont }}+R_{\text {sd }}+R_{\text {ext }}+R_{o v}
$$


(a)

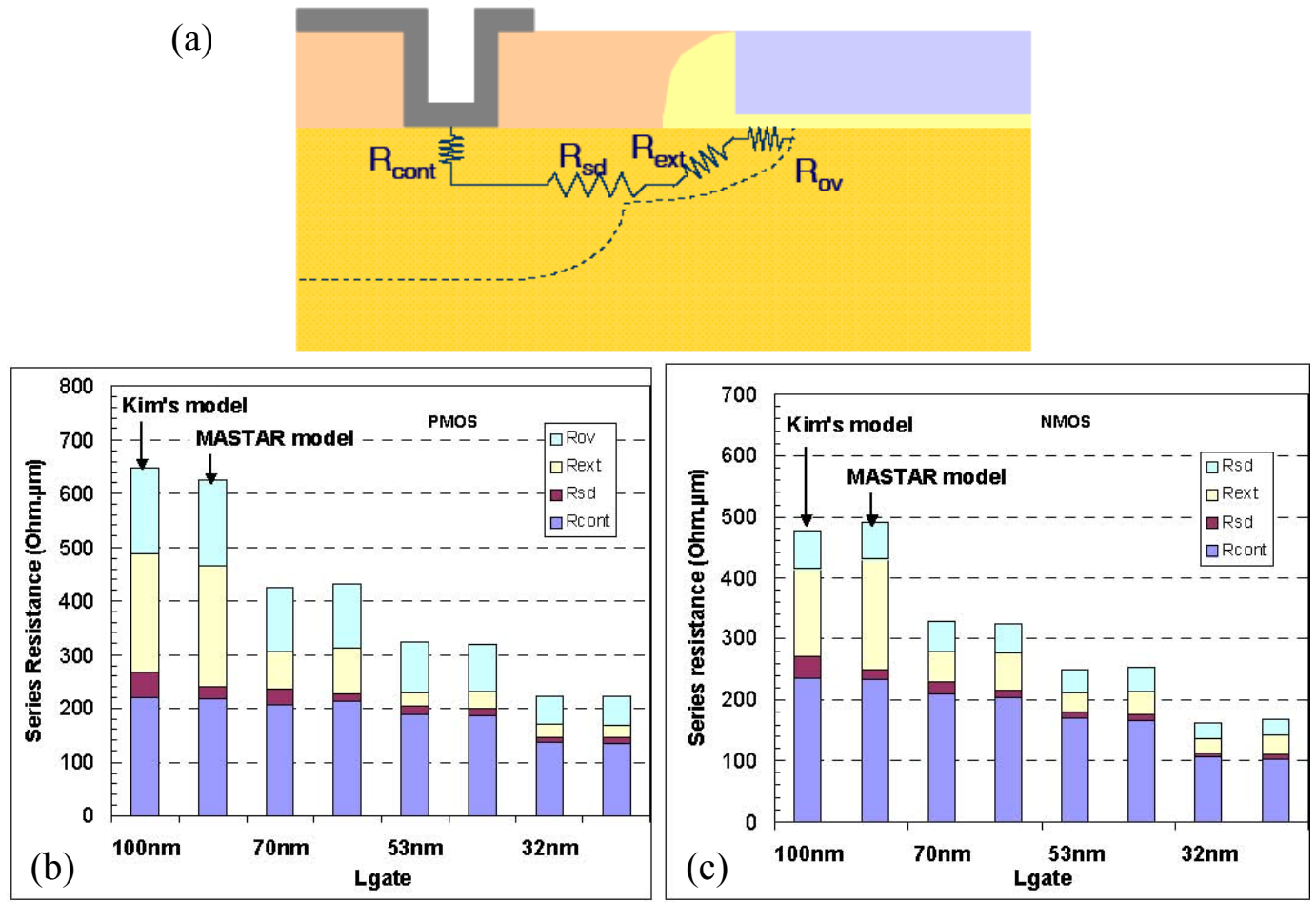

Figura 0.4.a. Diagrama esquemático de las componentes de la resistencia en serie externas al canal conductor. Gráficas del impacto de la resistencia en serie en la estructura .b. PMOS y.c. NMOS.

Donde $R_{\text {cont }}$ es la resistencia de los contactos, $R_{s d}$ la resistencia sheet (relacionada con la resistencia cuadrada de las regiones fuertemente dopadas [deep source/drain resistance]), $R_{\text {ext }}$ de extensión, atribuida al spacer de la puerta y $R_{o v}$ la resistencia que ofrece la región del overlap. Basándose en las conclusiones de los modelos analíticos de Kim y MASTAR [Kim, 2000], [Skotnicki, ITRS 2005], varios autores [Skotnicki, 2003], [Lenoble, 2006] destacan el papel dominante de la resistencia de los contactos $R_{\text {cont }}$ sobre la resistencia en serie total a medida que se reduce la longitud de puerta (véase la Figura 0.4.b. y .c.).

Los desafíos aquí mencionados se encuentran recogidos en el International Roadmap for Semiconductors [ITRS 2007] junto con otras muchas especificaciones a las que deben enfrentarse los diseñadores de dispositivos. En la Tabla 0.1 mostramos de manera esquemática los valores que tendrán que alcanzar diversos parámetros para cumplir las especificaciones del escalado, en particular destacamos los relacionados con los contactos y las resistencias de acceso. 


\begin{tabular}{|c|c|c|c|c|c|c|c|}
\hline \multicolumn{8}{|c|}{ ITRS '05: Focus on Source/Drain Architecture } \\
\hline year & - & 2004 & 2007 & 2010 & 2013 & 2016 & 2018 \\
\hline Technology node & & hp90 & hp65 & hp45 & hp32 & $\mathrm{hp22}$ & \\
\hline DRAM $1 / 2$ pitch & $\mathrm{nm}$ & 90 & 65 & 45 & 32 & 22 & 18 \\
\hline Physical gate length & $\mathrm{nm}$ & $37 / 53 / 65$ & $25 / 32 / 37$ & $18 / 22 / 25$ & $13 / 16 / 18$ & $09 / 11 / 2013$ & 7 \\
\hline Minimum logic Vdd (HP/LOP/LSTP) & $\mathrm{V}$ & $1.2 / 0.9 / 1.2$ & $1.1 / 0.8 / 1.1$ & $1 / 0.7 / 1$ & $0.9 / 0.6 / 0.9$ & $0.8 / 0.5 / 0.8$ & $0.7 / 0.5 / 0.8$ \\
\hline n-MOS current drive (HP/LOP/LSTP) & $\mathrm{mA} / \mu \mathrm{m}$ & $1.1 / 0.53 / 0.4$ & $1.5 / 0.57 / 0.51$ & $1.9 / 0.77 / 0.76$ & $2.05 / 0.78 / 0.88$ & $2.4 / 0.92 / 0.86$ & $2.19 / 0.95 / 0.99$ \\
\hline n-MOS leakage (HP/LOP/LSTP) & $\mathrm{nA} / \mu \mathrm{m}$ & $50 / 3 / 0.001$ & $70 / 5 / 0.025$ & $100000 / 7 / 0.06$ & $300000 / 10 / 0.08$ & $500000 / 30 / 0.1$ & $500000 / 30 / 0.1$ \\
\hline n-MOS intrinsic delay t (HP/LOP/LSTP) & ps & $0.95 / 1.76 / 2.6$ & $0.64 / 1.15 / 1.72$ & $0.39 / 0.73 / 0.98$ & $0.26 / 0.48 / 0.6$ & $0.15 / 0.26 / 0.43$ & $0.11 / 0.21 / 0.31$ \\
\hline Device Architecture & - & \multicolumn{2}{|c|}{ planar bulk } & FDSOI & \multicolumn{3}{|c|}{ FDSOI - multi-gate } \\
\hline Contact junction depth & $\mathrm{nm}$ & 40.7 & 27.5 & NA & NA & NA & NA \\
\hline Silicide thickness & $\mathrm{nm}$ & 20 & 17 & 13 & 19 & 13 & 9 \\
\hline Maximum silicon consumption by silicidation & $\mathrm{nm}$ & 20.4 & 13.8 & 10.8 & 15.6 & 10.8 & 7.7 \\
\hline Max contact specific resistivity & ohm.cm² & $1.6210^{-1}$ & $1.0510^{-1}$ & $6.0810^{-8}$ & $1.7110^{-8}$ & $8.6910^{-9}$ & $5.410^{-9}$ \\
\hline Contact silicide sheet resistance & ohm/sq & 7.9 & 9.6 & 12.3 & 8.5 & 12.3 & 17.2 \\
\hline Parasitic source+drain resistance & ohm. $\mu \mathrm{m}$ & 180 & $162 / 180 / 180$ & $135 / 160 / 180$ & $107 / 126 / 135$ & $79 / 98 / 107$ & $60 / 80 / 88$ \\
\hline Parasitic fringe/overlap capacitance & $\mathrm{fF} / \mu \mathrm{m}$ & 0.24 & $0.22 / 0.24 / 0.24$ & $0.18 / 0.22 / 0.24$ & $0.14 / 0.16 / 0.17$ & $0.1 / 0.13 / 0.14$ & $0.08 / 0.11 / 0.12$ \\
\hline Channel dopant concentration & $\mathrm{cm}^{-3}$ & $1.5-2.5 \times 10^{18}$ & $1.5-2.5 \times 10^{18}$ & NA & NA & NA & NA \\
\hline solution being pursued & & & \multicolumn{3}{|c|}{ no known solutions } & & \\
\hline
\end{tabular}

Tabla 0.1: Dificultades y requerimientos especificados en el ITRS del 2007 respecto a los obstáculos inherentes a la tecnología de los contactos. En color verde se destacan las tareas a las que se busca solución y en amarillo aquellas para las que aún no existe una solución fiable.

Sin embargo, existen ya una serie de potenciales soluciones a estos problemas (con diferentes grados de complejidad), que permitirían cumplimentar en gran parte las especificaciones del ITRS. Entre ellas, podemos destacar la ingeniería del canal, basada en el uso de canales graduales [Wong, 2002], regiones de pockets o halos, nuevos materiales en el canal como aleaciones de Silicio-Germanio o Silicio strained, etc. Otra posibilidad es el uso de sustratos alternativos tales como las obleas de Silicio sobre aislante o SOI, en sus siglas en inglés [Celler, 2003], que ya fueron ampliamente analizados en una Tesis Doctoral previa en nuestro grupo [Rengel, 2002].

Entre las soluciones más prometedoras y dentro del conjunto de dispositivos emergentes debemos destacar la posibilidad de reemplazar las islas de fuente y drenador del MOSFET convencional por contactos de tipo Schottky metálicos con valores de barrera bajos, formando de este modo los denominados MOSFET de barrera Schottky (SB-MOSFETs) [Dubois, 2002], [Larrieu, 2004], [Larson, 2006]. Este dispositivo se presenta como un excelente candidato, desde el punto de vista tecnológico, para reducir notablemente las resistencias de acceso al 
dispositivo; asimismo, por su compatibilidad con los equipos de fabricación actuales y sus prometedoras perspectivas, constituye el objeto principal de esta Tesis, que ha sido realizada en el marco de trabajo del proyecto Europeo METAMOS (IST-016677, VI programa Marco de la Unión Europea).

\section{MOSFET de barrera Schottky, ventajas de su utilización y modo de funcionamiento}

De entre las distintas soluciones propuestas al problema del escalado, nuestra investigación está centrada como decimos en los MOSFET de barrera Schottky. Vamos a examinar con más detalle las ventajas que ofrecen estos dispositivos en relación a sus equivalentes convencionales. En primer lugar, la sustitución de las regiones dopadas de fuente y drenador por metalizaciones implica una reducción y/o desaparición de algunas de las componentes de las resistencias parásitas de las estructuras convencionales que presentábamos en la Figura 0.4.a. Del mismo modo, los contactos de tipo Schottky permiten reducir notablemente el valor de las capacidades parásitas. Es importante destacar que los SBMOSFETs presentan una escalabilidad física inherente hasta valores de longitud de puerta de dimensiones por debajo de los $10 \mathrm{~nm}$ [Larson, 2006], la cual es debida a la baja resistencia del metal y a las uniones atómicamente abruptas que se forman en la interfaz de Silicio-Siliciuro (siendo el Siliciuro la aleación del Silicio con un metal). Además, su proceso de fabricación es paradójicamente más simple y requiere menos procesos fotolitográficos que en el caso de los transistores MOSFET convencionales (ya que mediante esta tecnología se puede prescindir de procesos tales como la implantación iónica profunda y los recocidos rápidos de alta temperatura). La tecnología SB-MOS es totalmente compatible con las instalaciones de fabricación actuales y con otros tipos de innovaciones tales como los substratos de tipo SOI e incluso la integración con otras soluciones (como dieléctricos de alta permitividad, sustratos de aleación, puertas metálicas, etc.) que sufren de cierta degradación a consecuencia de los procesos de altas temperaturas [Larson, 2006]. A más largo plazo, podría incluso incorporarse a dispositivos no basados en semiconductores convencionales, como sería el caso de los transistores de efecto de campo de nanotubos de carbono con metalizaciones Schottky [Jiménez, 2008]. 
En la Figura 0.5.a. podemos ver una representación esquemática del SB-MOSFET. Como vemos, la principal diferencia con un MOSFET convencional es la sustitución de las islas dopadas de fuente y drenador por metalizaciones; además, estos contactos no se encuentran solapados con la región del contacto de puerta, sino que están separados del canal una cierta distancia, denominada longitud de underlap. A pesar de sus similitudes topológicas con un MOSFET convencional, el comportamiento físico es muy distinto. A partir de los diagramas de bandas de la Figura 0.5 (b) y (c) vamos a analizar de manera sucinta el estado de corte y conducción de un SB-MOSFET, que serán estudiados de manera detallada en posteriores capítulos de esta memoria.
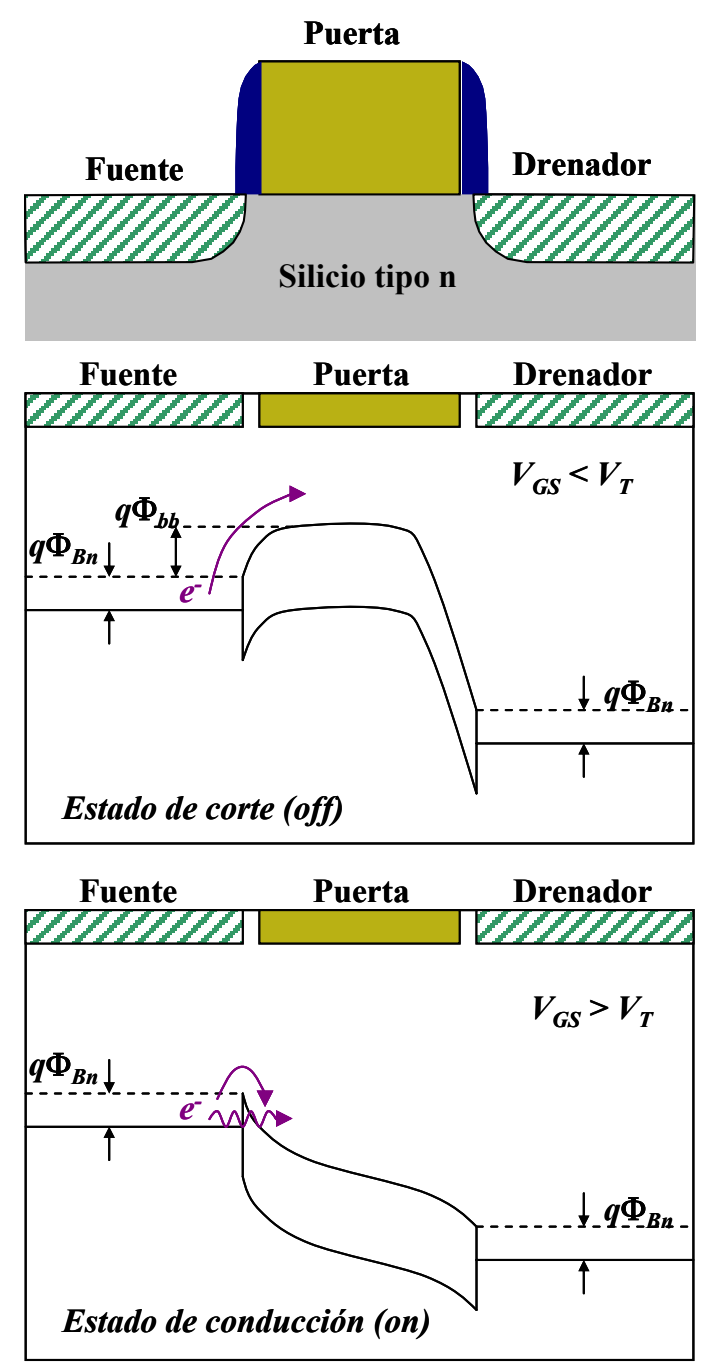

Figura 0.5.a. Representación esquemática de un MOSFET de barrera Schottky tipo n. Curvatura de bandas a lo largo del canal. En condiciones de $V_{D S}$ fijo mostramos dos situaciones diferentes: .b. Estado de corte y.c. estado de conducción. 
Para un voltaje entre fuente y drenador $\left(V_{D S}\right)$ fijo, es el voltaje de puerta $\left(V_{G S}\right)$ el que controla el paso entre el estado de corte (off) y conducción (on). Para un $V_{G S}$ inferior a la tensión umbral (Figura 0.5.b.), además de la barrera Schottky inherente a la unión de fuentesemiconductor $\left(q \Phi_{B n}\right)$ aparece una barrera adicional (body barrier, $q \Phi_{b b}$ ) creada como efecto del campo bajo la puerta. De este modo apenas hay corriente entre fuente y drenador (estado off), puesto que muy pocos electrones tienen energía suficiente para superar ambas barreras mediante emisión termoiónica. A medida que aumentamos $V_{G S}, q \Phi_{b b}$ disminuye hasta desaparecer; a partir de este momento, la barrera Schottky se hace más estrecha cuanto mayor es el voltaje de puerta. Esta situación se corresponde con el estado de conducción "on" (Figura 0.5.c.), en la que la contribución a la corriente del dispositivo es debida no sólo a los electrones que acceden al canal mediante emisión termoiónica, sino además también aquellos que atraviesan la barrera Schottky mediante efecto túnel, fenómeno también denominado como "emisión de campo". Los fenómenos túnel son muy importantes en el estado de conducción, por lo que en ocasiones se dice que el SB-MOSFET es un dispositivo de emisión de campo.

\section{Estructura de la presente Tesis Doctoral}

Para entender el comportamiento de este dispositivo es necesario establecer una base sólida acerca del transporte de electrones a través de los contactos de barrera Schottky en general, y analizar en profundidad el comportamiento de este tipo de transistores. Para esta tarea, el uso de simuladores se convierte en un requisito fundamental para determinar la viabilidad de los dispositivos como paso previo a su fabricación.

El objetivo de este trabajo es el ampliar el estudio de los dispositivos de la familia MOSFET de Silicio, abordando la investigación de estructuras alternativas de gran importancia para el futuro de la microelectrónica a corto y medio plazo, como son los transistores MOSFET de barrera Schottky, mediante el desarrollo y utilización de simuladores Monte Carlo. La estructura de la tesis es la siguiente:

En el primer capítulo llevaremos a cabo el estudio básico de los contactos Schottky de carácter general mediante las teorías que modelan el transporte de portadores a través de las barreras de potencial, además del análisis de diversos efectos reales a considerar en las uniones metal-semiconductor. Posteriormente revisaremos los distintos métodos de caracterización de la 
altura de la barrera y finalmente explicaremos sucintamente el proceso de fabricación de dichos contactos.

En el segundo capítulo presentamos los principales detalles de la herramienta de simulación empleada para el estudio de las diversas estructuras: el método Monte Carlo de dispositivos. Para poder abordar el estudio de transistores SB-MOSFET, ha sido necesario ampliar nuestro simulador incluyendo el transporte de portadores a través de barreras de potencial por emisión de campo o efecto túnel cuántico, tanto en inyección como en absorción. De entre las distintas posibilidades para evaluar la probabilidad de transmisión de los portadores a través de la barrera, las más comúnmente utilizadas son la aproximación de Airy y WKB; justificaremos la elección del segundo método para desarrollar un algoritmo de inyección/absorción a través de barreras Schottky y explicaremos además los pasos seguidos para llevar a cabo la implementación de este algoritmo en nuestro simulador Monte Carlo.

A continuación, en el tercer capítulo estudiaremos los diodos de barrera Schottky, dando en primer lugar unas pinceladas acerca de sus orígenes históricos y aplicaciones. Mostraremos los resultados de la simulación de diversos diodos, tanto para el caso de materiales dopados tipo $n$ como tipo $p$, que serán contrastados con datos experimentales extraídos de la literatura. De este modo podremos calibrar y verificar el funcionamiento del algoritmo de inyección/absorción que ha sido implementado.

En el cuarto capítulo se analizará el comportamiento de estructuras back-to-back; estas estructuras consisten en dos contactos Schottky situados sobre un sustrato de Silicio. Tales estructuras presentan una configuración del transporte en corriente muy cercana a la del SB-MOSFET (con excepción de la ausencia del terminal de puerta) [Dubois, 2004]. Realizaremos una comparación de las simulaciones numéricas Monte Carlo con medidas en dispositivos fabricados. Para ello contamos con la colaboración del Institut d'Electronique, Microelectronique et Nanotechnologies (I.E.M.N.) en Francia, la Universidad católica de Lovaina en Bélgica, ST Microelectronics y Philips Semiconductors, habiéndose desarrollado la colaboración en el marco del proyecto europeo METAMOS. Llevaremos a cabo un amplio análisis de la física del transporte en el dispositivo mediante el estudio de diversas magnitudes internas. 
Tanto el estudio de los diodos de barrera Schottky como de las estructuras back-to-back nos permitirán validar y garantizar la corrección del modelo de inyección/absorción implementado. Disponemos así de todas las herramientas para proceder al estudio de los transistores MOSFET de barrera Schottky. En el quinto capítulo comentaremos brevemente el desarrollo histórico de estos dispositivos y el interés actual por los mismos, así como los principales aspectos acerca de su fabricación y los modelos analíticos existentes para la determinación de su corriente. A continuación nos centraremos en el análisis de las características estáticas y de todo el conjunto de magnitudes internas proporcionadas por el simulador, con particular atención en los principios físicos y características y propiedades del transporte de carga en este tipo de transistores. Finalmente evaluaremos la influencia de la variación de diversos parámetros de diseño del SB-MOSFET sobre el transporte, como son la altura de la barrera, la longitud de underlap y la longitud de puerta.

Posteriormente, en el capítulo sexto efectuaremos un análisis del comportamiento a altas frecuencias de los SB-MOSFETs, incluyendo la respuesta dinámica y del ruido electrónico. Expondremos en primer lugar el circuito equivalente considerado para el estudio de alta frecuencia del dispositivo. A continuación presentaremos el análisis del comportamiento dinámico y de ruido del dispositivo en el dominio RF, calculando las principales figuras de mérito. En este estudio tendremos también en cuenta el efecto de la variación de la topología del dispositivo (altura de barrera, underlap y longitud de puerta).

Por último, pondremos de relieve las conclusiones más relevantes que se extraen del presente trabajo. Como complemento hemos añadido un apéndice en el que se evalúan los principales parámetros físicos considerados en nuestra simulación, así como resultados obtenidos para la simulación de material Silicio. Por otro lado, cabe mencionar que, con el fin de facilitar el acceso a la bibliografía utilizada en el presente trabajo, ésta ha sido recogida tras cada capítulo, además de proporcionar el compendio completo al final de la Tesis. 


\section{Bibliografía de la Introducción}

Celler G. K.and Cristoloveanu S., "Frontiers of silicon-on-insulator", J. Appl. Phys, 93, 4955 (2003)

Dennard R. H., Gaensslen F. H., Yu H. N., Rideout V. L., Bassous E., and LeBlanc A. R., "Design of Ion-Implanted MOSFETs with Very Small Physical Dimensions," IEEE J. Solid-State Circuits 9, 256 (1974)

Dubois E. and Larrieu G., "Low Schottky barrier source/drain for advanced MOS architecture: device design and material considerations", Solid-State Electronics, 46, 997 (2002)

Dubois E. and Larrieu G., "Measurement of low Schottky barrier heights applied to metallic source-drain metal-oxide-semiconductor field effect transistors" J. Appl. Phys., 91, 729 (2004)

Frank D. J., Taur Y.and Wong H. S., "Generalized scale length for two-dimensional effects in MOSFET's", IEEE Electron Device Lett. 19, 385 (1998)

Hutchby J. A., Bourianoff G. I., Zhirnov V. V. and Brewer J. E., "Extending the road beyond CMOS”, IEEE Circuits and Devices Magazine 18, 28 (2002)

ITRS "The International Technology Roadmap for Semiconductors (ITRS), Semiconductor Industry Association", Edition, Austin, TX, International Sematech (2007) (disponible en http://public.ITRS.net)

Iwai H., "Outlook of MOS devices into the next century", Microelectronics Engineering, 48, 7 (1999)

Jiménez D., "A current-voltage model for Schottky-barrier graphene-based transistors", Nanotechnology, 19, 345204 (2008)

Khanna V. K., "Physics of carrier-transport mechanisms and ultra-small scale phenomena for theoretical modelling of nanometer MOS transistors from diffusive to ballistic regimes of operation", Physics Reports, 67, 398 (2004) 
Kim S. D., Park C. -M., and Woo J. C. S., "Advanced Model and Analysis for Series Resistance in Sub-100nm CMOS Including Poly Depletion and Overlap Doping Gradient Effect", IEDM Tech. Dig., (2000)

Larrieu G. and Dubois E., "Schottky-Barrier Source/Drain MOSFETs on Ultrathin SOI Body With a Tungsten Metallic Midgap Gate”, IEEE Trans. Electron Dev., 25, 801 (2004)

Larson J. M. and Snyder J. P., "Overview and Status of Metal S/D Schottky-Barrier MOSFET Technology", IEEE Trans. Electron Dev. 53, 1048 (2006)

Lenoble D., "Advanced junction fabrication challenges at the $45 \mathrm{~nm}$ node", Semiconductor Fabtech 30th edition, 114 (2006)

Lundstrom M., "Is nanoelectronics the future of microelectronics?", Proceedings of the 2002 International Symposium on Low Power Electronics and Design, 172 (2002)

Moore G., "Progress in Digital Integrated Electronics," IEDM Tech. Digest, 11 (1975)

$\mathrm{Ng} \mathrm{K}$. K. and Lynch W. T., 'Analysis of the gate-voltage-dependent series resistance of MOSFET's', IEEE Trans. Electron Devices, 33, 965 (1986)

Pikus F. G., K. K. Likharev, "Nanoscale field effect transistors: an ultimate size analysis", Appl. Phys. Lett., 71, 3661 (1997)

Rengel R., "Análisis de los Fenómenos de Trasporte y Ruido Electrónico en Transistores MOSFET y SOI Submicrométricos”, Tesis Doctoral, Universidad de Salamanca (2002)

Skotnicki T., Pouydebasque A. and Villanueva D.; USJ Conf. Proceedings, (2003)

Skotnicki T. et al. http://www.itrs.net/Common/2005ITRS/Linked\%20Files/2005Files/PIDS/

MASTAR_Download.htm (2005)

Taur Y., "CMOS design near the limit of scaling", IBM Journal of Research and Development 46, 213 (2002)

Wong H.-S. P., "Beyond the conventional transistor", IBM Journal of Research and Development 46, 133 (2002) 



\section{Interfaz metal - semiconductor}

1 estudio de las uniones entre metal y semiconductor presenta una gran
relevancia, puesto que son un componente básico de cualquier dispositivo de estado sólido y en general de los circuitos integrados; además, para medir las propiedades eléctricas de un semiconductor, es necesario conectar el mismo a los aparatos de medida por medio de este tipo de contactos.

De la unión del metal y el semiconductor surgen dos posibles situaciones, dependiendo de las propiedades de los materiales utilizados. El contacto formado puede ser de tipo óhmico, permitiendo el paso de corriente del metal al semiconductor y viceversa, o de tipo rectificador, que principalmente permite el paso de corriente en sentido único, como ya veremos.

En este capítulo presentaremos las principales características y propiedades de estas uniones, centrándonos fundamentalmente en el contacto rectificador, también denominado contacto Schottky. Analizaremos además los distintos modelos analíticos que se utilizan comúnmente para representar la corriente que circula a través del contacto Schottky (posteriormente, en el Capítulo III presentaremos diversos modelos numéricos existentes en la literatura y basados en los modelos analíticos que aquí presentamos). A continuación analizaremos los efectos reales que surgen en dichas uniones apartándolas de su consideración 
de contacto ideal. El cálculo de la altura de la barrera de los contactos Schottky ha sido objeto de debate a lo largo de los últimos cuarenta años; presentaremos algunos de los modelos más importantes para la obtención de dicho parámetro, desde el cálculo sencillo partiendo de una consideración ideal del contacto a otros más completos, incluyendo algunos de los efectos reales que hemos mencionado previamente. También discutiremos sobre los métodos para la determinación experimental de la altura de la barrera Schottky, exponiendo las técnicas que se emplean con este fin, así como sus ventajas y aplicaciones. Por último, comentaremos brevemente el proceso de fabricación de los contactos Schottky.

\section{I.1. Unión metal-semiconductor en equilibrio}

Antes de afrontar los aspectos teóricos de los modelos analíticos que representan el transporte de corriente a través de los contactos metal-semiconductor, es conveniente definir ciertos parámetros básicos. En la Figura I.1 se muestra el diagrama de bandas de energía para un metal y un semiconductor antes de entrar en contacto. Consideraremos que los materiales son ideales, sin tener en cuenta la posible aparición de estados superficiales en la superficie del semiconductor en el proceso de fabricación o inducidos por el metal al realizarse el contacto.

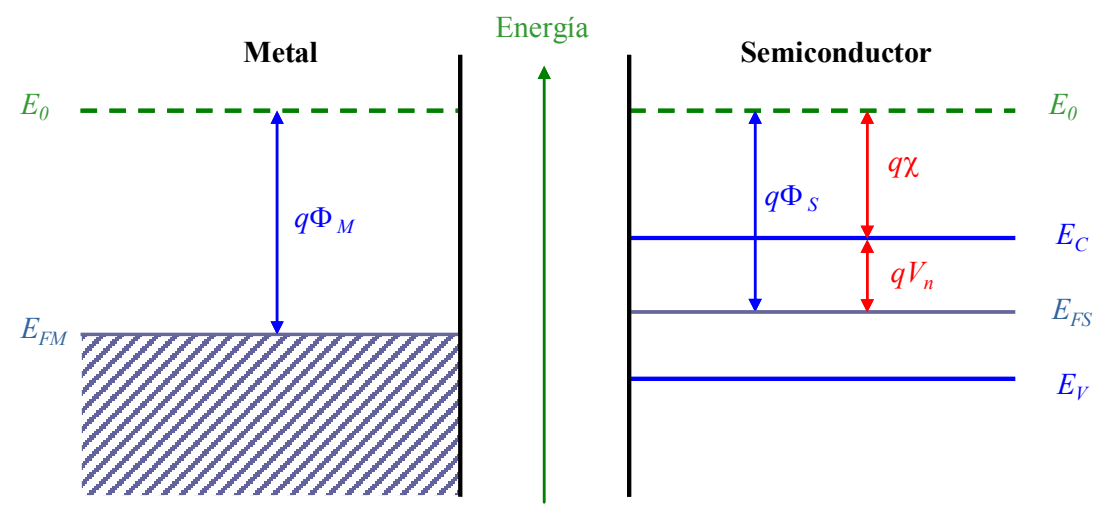

Figura I. 1. Diagrama de bandas de energía para un metal (izquierda) y un semiconductor (derecha) antes de entrar en contacto. Observamos que, en el caso del metal representamos únicamente el nivel de Fermi del metal $\left(E_{F M}\right)$ ya que la banda de conducción se encuentra muy poblada, mientras que en el semiconductor hemos representado el fondo de las bandas de valencia $\left(E_{V}\right)$ y conducción $\left(E_{C}\right)$, con el nivel de Fermi $\left(E_{F S}\right)$ entre ambas.

Se define para el semiconductor [Neudeck, 1993], [Albella, 1996] la afinidad electrónica $q \chi$ como la energía que necesita un electrón que se encuentra en el fondo de la 
banda de conducción para alcanzar la energía del electrón libre (nivel de energía del vacio $E_{0}$ ). La función de trabajo de un material, denotada por $q \Phi$ (es la diferencia de energía entre la del vacío $\left(E_{0}\right)$ y su nivel de Fermi $\left(E_{F}\right)$. En el caso del metal, $q \Phi_{M}$ depende del material considerado. Sin embargo, para el semiconductor $q \Phi_{S}$ varía en función del dopaje del mismo, puesto que el nivel de Fermi del semiconductor $\left(E_{F S}\right)$ está relacionado con el tipo de dopaje y la concentración de impurezas. De este modo, para un mismo semiconductor, $q \Phi_{S}$ será mayor cuando es dopado tipo $p$ que cuando es dopado tipo $n$. Finalmente $q V_{n}$ corresponde a la diferencia de energía entre el fondo de la banda de conducción $\left(E_{C}\right)$ y $E_{F S}$.

Cuando se realiza el contacto, en situación de equilibrio termodinámico y considerando una unión ideal, los niveles de Fermi de ambos materiales deben alinearse, lo que puede implicar la aparición de una barrera en la interfaz metal-semiconductor. En este caso, es la diferencia entre las funciones de trabajo del metal y del semiconductor la que condiciona la modificación de la estructura de bandas de energía en dicha interfaz y determinará las propiedades de la unión. A continuación analizaremos brevemente los diferentes diagramas de bandas de energía para los contactos, dependiendo de las características del semiconductor. Un estudio más detallado puede encontrarse en [Neudeck, 1993], [Sze, 2001]

\section{1. A. Caso de banda plana}

Tomamos como referencia la Figura I.1, particularizándola en el caso en el que las funciones de trabajo de metal y semiconductor (supuesto tipo $n$ ) fueran iguales. En esta situación, cuando consideramos ambos materiales aislados los niveles de Fermi se encuentran ya alineados, por lo que al entrar en contacto las bandas de valencia y conducción permanecen invariantes después de haberse realizado la unión: a esta situación se la denomina de "banda plana". De este modo, se alcanza el equilibrio termodinámico sin necesidad de efectuar intercambio neto de portadores entre el metal y el semiconductor. 


\section{1. B. Contacto óhmico}

Un contacto será de tipo óhmico cuando la función de trabajo del metal sea menor que la del semiconductor si éste es tipo $n\left(q \Phi_{M}<q \Phi_{S}\right)$, o mayor en caso de ser tipo $p\left(q \Phi_{M}>q \Phi_{S}\right)$ [Neudeck, 1993].

De este modo, considerando un semiconductor tipo $n$, al efectuarse el contacto se produce una transferencia de electrones del metal al semiconductor, alcanzándose el equilibrio cuando los niveles de Fermi de metal y semiconductor se igualan. Puesto que la función de trabajo del metal es inferior a la del semiconductor, la curvatura de la banda de conducción en el semiconductor será de tipo cóncavo tomando como referencia el nivel de Fermi (la banda de valencia se curva de manera simétrica a la de conducción) [Neudeck, 1993]. Se origina entonces un campo eléctrico en el interior del semiconductor (dirigido de metal a semiconductor) como consecuencia de las cargas acumuladas en la interfaz: una carga superficial positiva en el lado del metal, y en el semiconductor una carga negativa denominada usualmente capa de acumulación. Para el caso de semiconductor de tipo $p$, con $q \Phi_{M}>q \Phi_{S}$, tendríamos una situación análoga.

Como señalamos, en el semiconductor tendremos una zona de acumulación de portadores mayoritarios (electrones en el caso de semiconductor tipo $n$ ). Esto implica que la entrada o salida de un electrón del semiconductor es inmediatamente compensada con la salida o entrada de un hueco desde el metal. Debido al campo eléctrico creado, las bandas del semiconductor se curvan hacia abajo manteniéndose en todo caso el nivel de Fermi constante y la continuidad del nivel de vacío.

En el movimiento de los electrones desde el metal al semiconductor y en sentido inverso no existe barrera de potencial que se oponga al mismo; además la concentración de electrones a ambos lados de la unión con posibilidad de atravesarla es muy elevada, por lo que, de manera ideal, se considera que la resistencia asociada al contacto es despreciable. Se dice entonces que el contacto presenta un comportamiento óhmico. 


\section{1. C. Contacto rectificador}

La condición para que de la unión de un metal y un semiconductor tipo $n$ obtengamos un contacto de tipo rectificador es: $q \Phi_{M}>q \Phi_{S}$, mientras que en el caso de considerar un semiconductor tipo $p$ sería: $q \Phi_{M}<q \Phi_{S}$.

En la Figura I.2 se muestra la estructura de bandas para un metal y un semiconductor tipo $n$ ideal antes y después de producirse la unión entre ambos. Similarmente a lo que ocurre en una unión $p$-n [Neudeck, 1993], en la transición entre ambas situaciones se produce una difusión de electrones, provocando la creación de una región de carga espacial positiva (de anchura $W$ ) en el semiconductor, en la zona cercana a la unión, debida a la presencia de impurezas donadoras ionizadas. Esta región está prácticamente vacía de electrones libres por lo que se la conoce como región de vaciamiento. Como contrapartida, y para mantener la neutralidad de la unión, en el metal aparece una carga negativa, que estará localizada en la superficie ya que la densidad de estados del metal es mucho mayor que la del semiconductor. Por tanto, las cargas positivas y negativas quedan localizadas a ambos lados de la unión, originando con ello un campo eléctrico $\xi(x)$ dirigido del semiconductor al metal y que alcanza su máximo valor, $\xi_{m}$, en la interfaz de la unión.

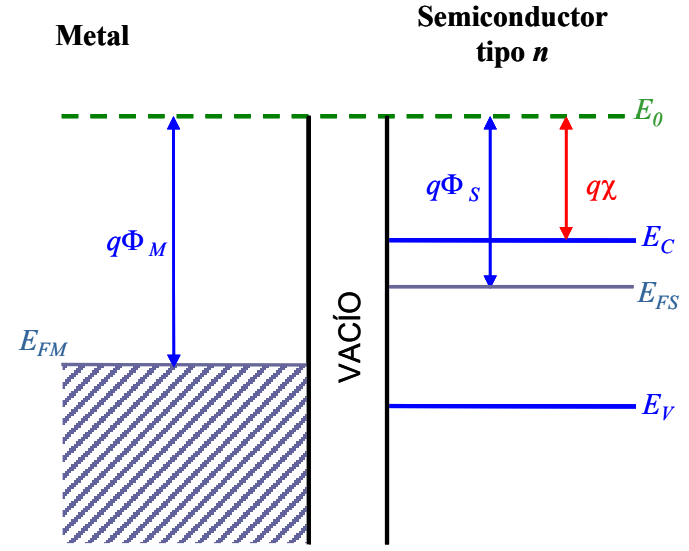

(a)

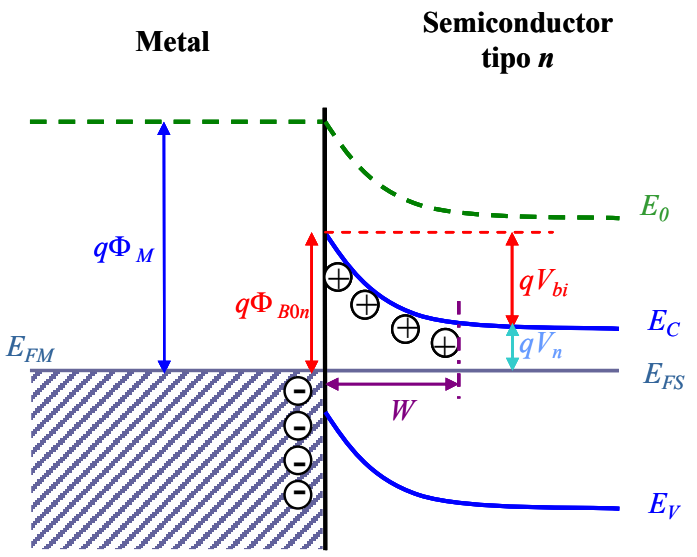

(b)

Figura I. 2. Contacto rectificador metal-semiconductor tipo n con $q \Phi_{M}>q \Phi_{S}$. a. Metal y semiconductor aislados .b. Bandas de energía después del contacto rectificador.

La presencia del campo eléctrico produce a su vez una variación del potencial en el interior del semiconductor y por tanto una curvatura de las bandas de energía en la región de carga espacial, teniendo en cuenta que la afinidad electrónica debe permanecer constante a lo 
largo del semiconductor y que el nivel de vacío debe ser continuo. La curvatura de las bandas en el semiconductor en la región cercana a la unión es contraria al caso I.B. En este caso la banda de conducción es convexa desde el punto de vista del nivel de Fermi (véase Figura I.2.b).

Finalmente, cuando la unión alcanza el equilibrio surgen sendas barreras de energía, $q \Phi_{B 0 n}$ y $q V_{b i}$, que se oponen al movimiento de los electrones desde el metal hacia el semiconductor tipo $n$ y en sentido opuesto, respectivamente. De ahora en adelante nos referiremos a estos conceptos como altura de la barrera Schottky, que se define como:

$$
q \Phi_{B 0 n}=q\left(\Phi_{M}-\chi\right)
$$

y potencial de contacto, cuyo valor se determina a partir de la siguiente diferencia:

$$
V_{b i}=\Phi_{B 0 n}-V_{n}
$$

En esta situación el flujo de corriente a través del dispositivo es no lineal, puesto que la corriente fluye con mayor facilidad en un sentido (del semiconductor al metal) pero no en el opuesto, ya que $q V_{b i}<q \Phi_{B 0 n}$, recibiendo así por este hecho el nombre de contacto rectificador. Esta situación se ve influenciada en gran medida por la tensión aplicada, efecto que estudiaremos en la Sección I.2.

\section{- Descripción cuantitativa básica del contacto rectificador en equilibrio}

(b)

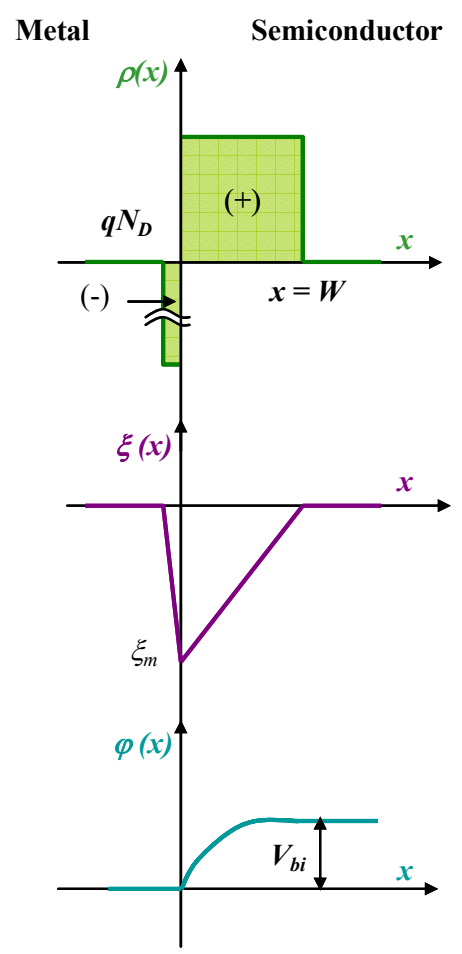

el caso de la unión rectificadora en condiciones de equilibrio.

Mediante la resolución de la ecuación de Poisson es posible calcular la dependencia con la

Figura I. 3 a. Diagrama de la distribución de carga bajo la hipótesis de vaciamiento total.b. Variación del campo eléctrico y.c. del potencial a lo largo de la unión. 
posición del campo eléctrico, $\xi(x)$, y del potencial, $\varphi(x)$, para lo cual es necesario conocer la densidad de carga. Como primera hipótesis, se suele considerar la aproximación de vaciamiento total, al igual que se hace en la unión $p^{+}-n$ abrupta (véase Figura I.3). Encontraremos más detalles de esta aproximación en [Albella, 1996] y [Pardo, 2007].

Considerando estas condiciones de contorno en la ecuación de Poisson y la densidad de carga dada por la aproximación de vaciamiento total se obtiene finalmente [Sze, 1981], [Neudeck, 1993], [Pardo, 2007] las expresiones para la anchura de vaciamiento $W$, el campo eléctrico $\xi(x)$ y el potencial $\varphi(x)$. Debe tenerse en cuenta también la contribución de la cola de la distribución de los portadores mayoritarios a través del factor $K_{B} T / q$ [Sze, 1981].

$$
\begin{aligned}
& W=\sqrt{\frac{2 \varepsilon_{s}}{q N_{D}}\left(V_{b i}-\frac{K_{B} T}{q}\right)} \\
& |\xi(x)|=\frac{q N_{D}}{\varepsilon_{s}}(W-x)=\xi_{m}-\frac{q N_{D}}{\varepsilon_{s}} x \\
& \varphi(x)=\frac{q N_{D}}{2 \varepsilon_{s}}(W-x)^{2}-V_{b i}+\frac{K_{B} T}{q}
\end{aligned}
$$

donde $\varepsilon_{s}$ es la permitividad del semiconductor, $K_{B}$ es la constante de Boltzmann, $T$ la temperatura absoluta en grados Kelvin y $\xi_{m}$ es el máximo valor del campo, que tiene lugar para $x=0$ :

$$
\xi_{m}=\xi(x=0)=\frac{2\left(V_{b i}-K_{B} T / q\right)}{W}
$$

De este modo, es posible calcular por tanto la carga almacenada por unidad de área en la zona de carga espacial:

$$
Q_{s c}=q N_{D} W=\sqrt{2 q \varepsilon_{s} N_{D}\left(V_{b i}-\frac{K_{B} T}{q}\right)}
$$

que debe corresponderse además (con el signo contrario) con la densidad de carga superficial en el metal, con lo que la carga total almacenada en el conjunto de la unión es nula.

Finalmente, debemos comentar que para el caso de uniones rectificadoras metalsemiconductor tipo $p\left(q \Phi_{M}<q \Phi_{S}\right)$ son válidas de manera general consideraciones similares a las descritas anteriormente para semiconductores tipo $n$, sin más que tener en cuenta que los portadores mayoritarios son los huecos [Neudeck, 1993]. En este caso, cuando ambos materiales 
están en contacto, se difunden los electrones desde el metal al semiconductor. Las impurezas aceptadoras son ionizadas, con lo cual aparece una zona de carga espacial en el semiconductor que restablece el equilibrio y permite el alineamiento de los niveles de Fermi. La unión es rectificadora, puesto que en principio sólo tendremos corriente desde el semiconductor al metal (situación análoga a la ya explicada para electrones), ya que a los huecos del metal se les presenta una barrera cuya altura viene dada por la expresión:

$$
q \Phi_{B 0 p}=E_{g}-q\left(\Phi_{M}-\chi\right)
$$

donde $E_{g}$ es el gap del semiconductor.

\section{2. Unión metal-semiconductor fuera de equilibrio}

Tomemos como ejemplo una unión rectificadora con semiconductor dopado tipo $\mathrm{n}$, en la cual se cumple que $q \Phi_{M}>q \Phi_{S}$ (diodo de barrera Schottky), como la descrita en el apartado anterior (Figura I.2). Si se aplica un voltaje $V_{a}$ desde el metal al semiconductor, este voltaje tendrá un efecto sobre el diagrama de bandas de energía [Neamen, 1992].

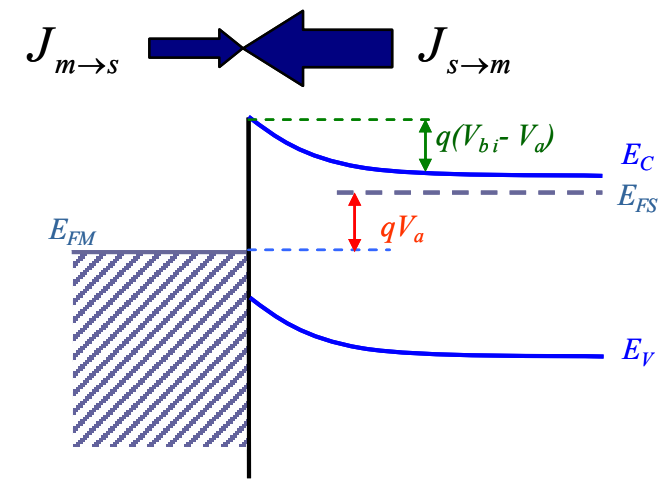

(a) $V_{a}>0$
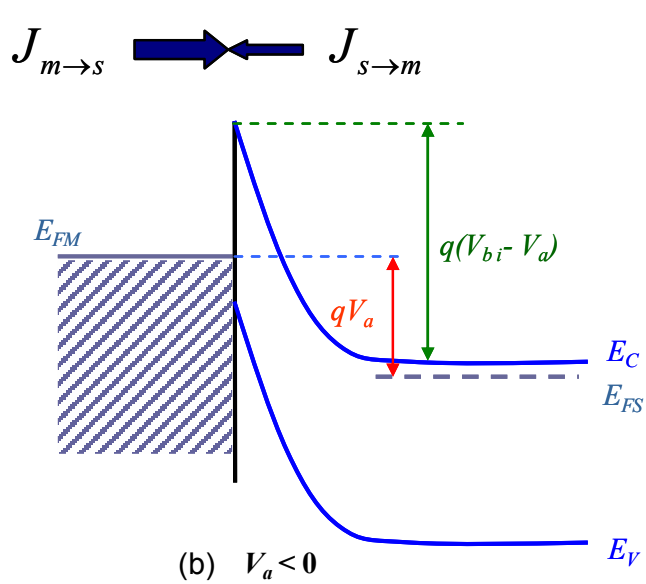

Figura I. 4. Diodo Schottky polarizado: .a. Polarización directa .b. Polarización inversa.

La unión se encuentra polarizada en directa cuando $V_{a}>0{ }^{1}$. En este caso, el potencial aplicado hace que el diagrama de bandas de energía se desplace hacia arriba del lado del semiconductor (o el del lado del metal hacia abajo). De este modo se reduce la altura de la

\footnotetext{
${ }^{1}$ Consideramos la polarización en directa como el voltaje positivo aplicado en el metal.
} 
barrera para los electrones que van desde el semiconductor hacia el metal, desde $q V_{b i}$ hasta $q\left(V_{b i}-V_{a}\right)$, como podemos ver en la Figura I.4.a, pudiendo éstos alcanzar el contacto con mayor facilidad (recordemos que los electrones son los portadores mayoritarios para la unión considerada). Para la situación de equilibrio la corriente del metal al semiconductor, $J_{m \rightarrow s}$, era compensada con la del semiconductor al metal, $J_{s \rightarrow m}$, teniendo por tanto una corriente neta nula. Sin embargo, en la situación que nos ocupa, la corriente $J_{s \rightarrow m}$ es mayor que la del sentido opuesto. Esta corriente aumenta con el voltaje aplicado, por lo que a partir de cierto $V_{a}$, la corriente $J_{m \rightarrow s}$ es despreciable frente a $J_{s \rightarrow m}$; nótese además que en esta región de polarizaciones $J_{m \rightarrow s}$ puede ser en principio considerada constante.

Cuando $V_{a}<0$, el diodo está polarizado en inversa, y el diagrama de bandas de energía se desplaza hacia abajo del lado del semiconductor, como vemos en la Figura I.4.b. Aquí la altura de la barrera para los electrones en el semiconductor ha aumentado en $\left|q V_{a}\right|$, por lo que la corriente $J_{s \rightarrow m}$ es inferior a la del sentido opuesto, $J_{m \rightarrow s}$, que en primera aproximación debe mantenerse como decimos constante pues la altura de la barrera vista desde el metal no ha variado (más adelante comprobaremos que $J_{m \rightarrow s}$ no permanece en realidad constante, debido al efecto túnel).

En el Capítulo III estudiaremos ambos regímenes de polarización en diodos de barrera Schottky, centrándonos principalmente en el de inversa, puesto que en los dispositivos MOSFET de barrera Schottky (objetivo final de este trabajo) el contacto rectificador de fuente, que regula la inyección de portadores en el canal, se encuentra bajo este régimen de polarización.

\section{Descripción cuantitativa básica del contacto rectificador fuera de equilibrio}

Podemos modelar el comportamiento de la unión rectificadora fuera de equilibrio calculando la expresión del potencial y demás magnitudes en la región de carga espacial de manera totalmente análoga a la situación en equilibrio. El único factor a tener en cuenta es que el valor del voltaje externo aplicado se superpone al de la altura de la barrera y así, donde aparecía $q V_{b i}$ en las Ecuaciones I.2 a la I.6, debemos ahora considerar $q\left(V_{b i}-V_{a}\right)$ para incluir el efecto del voltaje aplicado. De este modo se puede ver fácilmente (a partir de la Ecuación I.3) que la anchura de la región de carga espacial $(W)$ varía en función del voltaje aplicado. Cuando 
se polariza la unión en directa, $W$ disminuye y el campo eléctrico máximo es menos negativo. Mientras que cuando se polariza en inversa, $W$ aumenta y el campo eléctrico máximo es más negativo. La carga que se acumula en la región de carga espacial se obtiene de la Ecuación I.7:

$$
Q_{s c}=q N_{D} W=\sqrt{2 q \varepsilon_{s} N_{D}\left(V_{b i}-V_{a}-\frac{K_{B} T}{q}\right)}
$$

La capacidad asociada a la unión metal-semiconductor será en consecuencia:

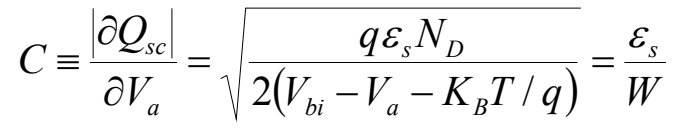

Es decir, corresponde a la capacidad de un condensador plano paralelo de permitividad igual a la del semiconductor y cuya separación entre placas equivale a la anchura de la zona de carga espacial.

\section{3. Transporte de Corriente a través del Contacto Schottky}

Una vez establecidos los conceptos básicos de la unión metal-semiconductor, nos encontramos en condiciones de estudiar el transporte de corriente de un modo más exhaustivo. Como comentábamos en la sección previa, de entre los distintos tipos de contacto metalsemiconductor nos centraremos únicamente en el contacto de tipo rectificador. Además realizaremos el análisis considerando de nuevo únicamente el caso del semiconductor dopado tipo $n$, puesto que el tratamiento del semiconductor dopado tipo $p$ es completamente análogo.

Como ya hemos explicado, en los contactos de tipo Schottky el transporte de corriente es debido principalmente a los portadores mayoritarios, en contraposición con las uniones $p-n$, donde son los minoritarios los que presentan la mayor contribución en condiciones de polarización en inversa [Sze, 1981]. Una consideración más detallada de las diferentes contribuciones a la corriente en el caso que nos ocupa debe tener en cuenta los procesos que especificamos a continuación y que se exponen gráficamente en la Figura I.5 [Rideout, 1978]:

1) Componentes de corriente termoiónica. En un contacto rectificador polarizado en directa, el fenómeno más significativo es el transporte de electrones del semiconductor al metal por encima de la barrera de potencial (en la Figura I.5, el proceso 1a). Este proceso es el de mayor importancia para los diodos Schottky con semiconductores de dopaje moderado (en el 
caso del Silicio, $N_{D} \leq 10^{17} \mathrm{~cm}^{-3}$ ). Cuando se polariza en inversa, predomina el transporte de electrones del metal al semiconductor (1b en la Figura I.5) como ya vimos en la anterior sección de este capítulo. El estudio de ambas corrientes se describirá con detalle en el siguiente apartado.

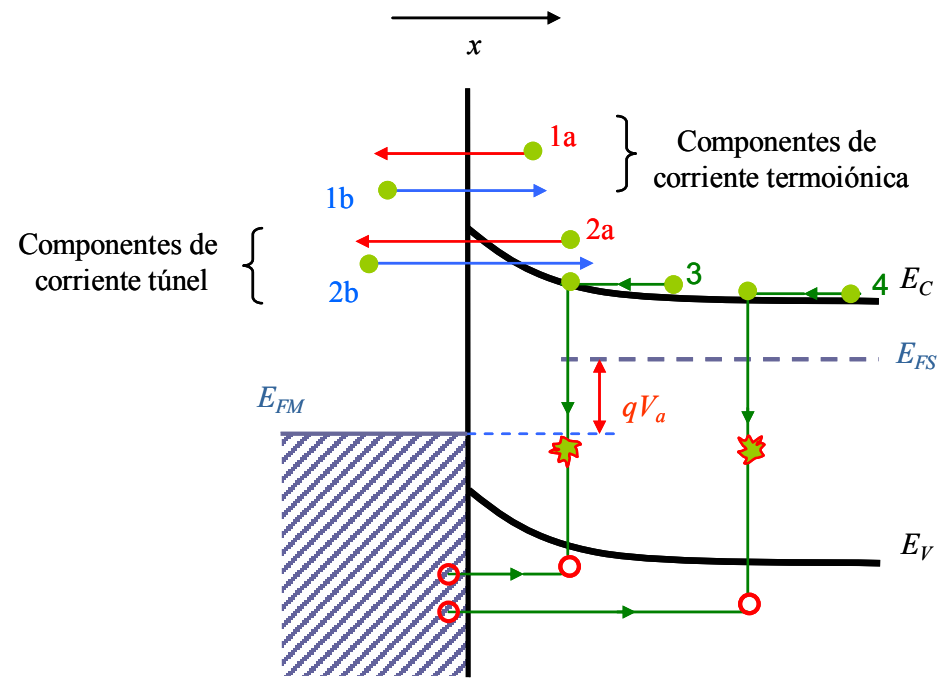

Figura I. 5. Procesos básicos de transporte de corriente bajo polarización directa $V_{a}$ en un contacto Schottky. En lo sucesivo consideraremos la inyección como el transporte de portadores desde el metal al semiconductor (flechas azules) y la absorción (flechas rojas) en el sentido opuesto (del semiconductor al metal).

2) Componentes de corriente túnel o de emisión de campo. En determinadas condiciones, también es importante la contribución a la corriente de procesos tales como el transporte por túnel cuántico directo a través de la barrera (en ambos sentidos; las contribuciones $2 \mathrm{a}$ y $2 \mathrm{~b}$ de la Figura I.5).

3) La recombinación en la zona de carga espacial (electrones del fondo de la banda de conducción que se recombinan con huecos del metal en la zona de carga espacial).

4) La inyección de huecos (portadores minoritarios) del metal al semiconductor, que equivale a una recombinación en la región neutra.

En esta tesis nos centraremos en el estudio de la corriente termoiónica sobre la barrera de potencial (1) y a través de ella mediante efecto túnel o emisión de campo (2), ya que dadas las características de los dispositivos que analizaremos y el voltaje al que serán sometidos, los procesos (3) y (4) son despreciables respecto al (1) y (2) [Crowell, 1966].

A continuación presentaremos un estudio analítico cuantitativo de la corriente a través del contacto Schottky, analizando los parámetros que influyen en la misma; consideraremos los 
principales modelos teóricos existentes. Este análisis nos servirá de base para el siguiente capítulo, en el que describiremos la implementación en nuestro simulador Monte Carlo de un algoritmo que evalúe de manera adecuada el transporte de corriente a través de barreras Schottky (Capítulo II); además, tanto en el Capítulo III, como en el V mencionaremos los distintos modelos numéricos para el estudio de diodos Schottky y de SB-MOSFETs, respectivamente, que aparecen en la bibliografía.

Para voltajes inferiores al de banda plana, el comportamiento de la corriente viene explicado por las teorías de emisión y difusión de portadores, mientras que, a polarizaciones superiores, dicho comportamiento se modela analíticamente a través de la teoría de Mott. Estas teorías serán presentadas con detalle en la presente sección.

El modelo de transporte de carga que desarrollamos a continuación será completado a través de la consideración de otros efectos que aparecen en situaciones no ideales, como veremos en la Sección I.4.

\section{3. A. Emisión Termoiónica. [Bethe, 1942]}

La teoría de emisión termoiónica fue enunciada por Bethe y está basada en las siguientes hipótesis:

1) La altura de la barrera $q \Phi_{B n}$ es mucho mayor que $K_{B} T$.

2) Suponemos equilibrio termodinámico en el plano de emisión ${ }^{2}$.

3) El equilibrio termodinámico no se ve afectado por la existencia de una corriente neta (condición de cuasi-equilibrio).

Bajo estas condiciones, tal y como ya veíamos en la Figura I.4, la densidad de corriente neta será el resultado de la suma de dos densidades de corriente, la que fluye del metal al semiconductor $\left(J_{m \rightarrow s}\right)$ y la que circula en sentido opuesto, $\left(J_{s \rightarrow m}\right)$, las cuales dependen únicamente de la altura de la barrera. Además puede suponerse que en la región de vaciamiento los portadores presentan un comportamiento balístico.

\footnotetext{
${ }^{2}$ El plano perpendicular a la dirección de incidencia $x$.
} 


\section{Corriente de absorción, $J_{s \rightarrow m}$}

La densidad de corriente $J_{s \rightarrow m}$ depende de $q\left(V_{b i}-V_{a}\right)$, barrera que se opone al paso de los portadores del semiconductor al metal y que a su vez depende del voltaje aplicado $V_{a}$ (véase Figura I.4). Para realizar el cálculo de la corriente, consideramos por simplicidad un semiconductor genérico cuya banda de conducción está formada por valles esféricos en los que el portador posee una masa efectiva $m^{*}$. La densidad de corriente puede determinarse a partir de la concentración de electrones con energía suficiente (en la dirección en la que se efectúa el transporte) como para superar la barrera [Sze, 1981]:

$$
J_{s \rightarrow m}=\int_{E_{F S}+q \Phi_{B n}}^{\infty} q v_{x} d n
$$

donde $v_{x}$ es la velocidad de los portadores en la dirección $x$. Dado que en este modelo únicamente consideramos la emisión termoiónica, el rango de energías a integrar es de $E_{F S}+q \Phi_{B n}$ a $\infty . d n$ es la densidad de electrones incremental en un intervalo de energía $d E$, que viene dada por:

$$
d n=n(E) f(E) d E
$$

siendo

$$
\begin{aligned}
& n(E)=\frac{4 \pi\left(2 m^{*}\right)^{3 / 2}}{h^{3}} \sqrt{E-E_{C}} \\
& f(E)=\exp \left(-\left(E-E_{F S}\right) / K_{B} T\right)
\end{aligned}
$$

$n(E)$ es la densidad de estados en energía en el caso particular que nos ocupa (superficies isoenergéticas esféricas). Considerando nuestro semiconductor como no degenerado, $f(E)$ es la función de distribución de Maxwell-Boltzmann.

Suponemos que toda la energía de los electrones en la banda de conducción es cinética:

$$
E-E_{C}=1 / 2 m * v^{2} \text { con } v^{2}=v_{x}^{2}+v_{y}^{2}+v_{z}^{2}
$$

Teniendo en cuenta todos estos conceptos, a partir de la Ecuación I.11 la densidad de corriente se podrá expresar de la siguiente forma:

$$
J_{s \rightarrow m}=\left(\frac{4 \pi q m^{*} K_{B}^{2}}{h^{3}}\right) T^{2} \exp \left(-\frac{q V_{n}}{K_{B} T}\right) \exp \left(-\frac{m^{*} v_{0 x}^{2}}{2 K_{B} T}\right)
$$


donde $h$ es la constante de Planck y $v_{0 x}$ es la velocidad mínima que deben tener los electrones para ser absorbidos termoiónicamente, es decir, el valor de la velocidad que hace que su energía cinética sea suficiente para superar la barrera de potencial:

$$
1 / 2 m * v_{0 x}^{2}=q\left(V_{b i}-V_{a}\right)
$$

De esta forma, podremos expresar la densidad de corriente en función de la altura de la barrera $q \Phi_{B 0 n}$ puesto que, por definición, ésta es la suma del potencial $V_{n}$ y $V_{b i}$ (ver Figura I.3.b):

$$
\begin{aligned}
& J_{s \rightarrow m}=A^{*} T^{2} \exp \left(-\frac{q \Phi_{B 0 n}}{K_{B} T}\right) \exp \left(\frac{q V_{a}}{K_{B} T}\right) \\
& \text { siendo } A^{*}=\frac{4 \pi q m * K_{B}^{2}}{h^{3}}
\end{aligned}
$$

la constante de Richardson efectiva, $A^{*}$, para la emisión termoiónica. Para los electrones en el vacío, el valor de la constante de Richardson, $A$, (con $m^{*}=m_{0}$ ) es $120 \mathrm{~A} \mathrm{~cm}^{-2} \mathrm{~K}^{-2}$ [Crowell, 1965], [Crowel, 1969b]. Si, tal y como hemos considerado, el semiconductor posee masa isótropa en el mínimo de la banda de conducción, la constante de Richardson efectiva se puede evaluar de manera directa:

$$
\frac{A^{*}}{A}=\frac{m^{*}}{m_{0}}
$$

donde $m^{*}$ es la masa efectiva del electrón y $m_{0}$ la masa del electrón libre. Sin embargo, esta relación varía cuando el semiconductor tiene múltiples valles elipsoidales, como es el caso del Silicio [Crowell, 1965], donde el valor de la constante de Richardson para cada valle es:

$$
\frac{A^{*}}{A}=\frac{1}{m_{0}}\left(l_{x}^{2} m_{y} * m_{z} *+l_{y}^{2} m_{z} * m_{x} *+l_{z}^{2} m_{x} * m_{y} *\right)^{1 / 2}
$$

siendo $l_{i},(i=x, y, z)$ los cosenos directores de la normal al plano de emisión referida a los ejes principales del elipsoide y $m_{i}{ }^{*}(i=x, y, z)$ las componentes del tensor masa efectiva. En este caso, para el Silicio con el campo aplicado orientado en la dirección $<100>$ el valor teórico de la constante de Richardson efectiva para electrones es de $246 \mathrm{~A} \mathrm{~cm}^{-2} \mathrm{~K}^{-2}$, mientras que para el caso de los huecos es $79.2 \mathrm{~A} \mathrm{~cm}^{-2} \mathrm{~K}^{-2}$ [Crowell, 1965]. 


\section{Corriente de inyección, $J_{m \rightarrow s}$}

Si examinamos la corriente que penetra al semiconductor desde el metal (que, como dijimos previamente, depende de la altura de la barrera $q \Phi_{B 0 n}$ ), llegamos a la conclusión de que $J_{m \rightarrow s}$ debe permanecer constante en todo el rango de voltajes aplicados, puesto que $q \Phi_{B 0 n}$ no varía con la polarización. Para calcular el valor de esta densidad de corriente partimos de la base de que en equilibrio termodinámico la corriente neta debe ser cero. Por tanto, sin más que tomar $V_{a}=0$ en la Ecuación I.18 obtenemos el valor de esta densidad de corriente:

$$
J_{m \rightarrow s}=-A^{*} T^{2} \exp \left(-\frac{q \Phi_{B 0 n}}{K_{B} T}\right)
$$

La densidad de corriente total será la suma de las Ecuaciones I. 18 y I. 22:

$$
\begin{aligned}
& J_{n}=A^{*} T^{2} \exp \left(-\frac{q \Phi_{B 0 n}}{K_{B} T}\right)\left[\exp \left(\frac{q V_{a}}{K_{B} T}\right)-1\right] \\
& =J_{S T}\left[\exp \left(\frac{q V_{a}}{K_{B} T}\right)-1\right]
\end{aligned}
$$

donde hemos introducido el concepto de densidad de corriente inversa de saturación:

$$
J_{S T}=A^{*} T^{2} \exp \left(-\frac{q \Phi_{B 0 n}}{K_{B} T}\right)
$$

\section{3. B. Teoría de Difusión. [Schottky, 1938]}

Para explicar el transporte por encima de la barrera de potencial, podemos considerar la teoría de difusión de Schottky como alternativa a la de emisión termoiónica. Las hipótesis en las que se basa son:

1) La altura de la barrera es mucho mayor que $K_{B} T$.

2) Considera los efectos de las colisiones de los electrones en la región de vaciamiento.

3) Supone además que la concentración de portadores al principio y al final de la zona de vaciamiento no varía con el flujo de corriente y la concentración de impurezas del semiconductor es no degenerada.

A partir de la expresión "clásica" para la densidad de corriente en función de los términos de arrastre y difusión, obtenemos: 


$$
J_{n}=q D_{n}\left[-\frac{q n(x)}{K_{B} T} \frac{\partial \varphi(x)}{\partial x}+\frac{\partial n}{\partial x}\right]
$$

donde $D_{n}$ es la constante de difusión y $\varphi(x)$ es el potencial para las barreras Schottky, dado por la Ecuación I.4. Partiendo del estado estacionario y con las condiciones de contorno adecuadas [Sze, 1981], llegamos a la siguiente expresión para la corriente:

$$
\begin{aligned}
J_{n} & \cong\left\{\frac{q^{2} D_{n} N_{C}}{K_{B} T}\left[\frac{q\left(V_{b i}-V_{a}\right) 2 N_{D}}{\varepsilon_{s}}\right]^{1 / 2} \exp \left(-\frac{q \Phi_{B 0 n}}{K_{B} T}\right)\right\}\left[\exp \left(\frac{q V_{a}}{K_{B} T}\right)-1\right] \\
& =J_{S D}\left(V_{a}\right)\left[\exp \left(\frac{q V_{a}}{K_{B} T}\right)-1\right]
\end{aligned}
$$

siendo $N_{C}$ es la densidad efectiva de estados de la banda de conducción.

Observamos cómo las expresiones que nos proporcionan las teorías de difusión y de emisión termoiónica son muy similares. Sin embargo, comparando las Ecuaciones I. 23 y I. 26, comprobamos que la densidad de corriente de saturación $J_{S T}$ en la teoría de emisión termoiónica es más sensible al cambio de la temperatura, mientras que $J_{S D}$, en la teoría de difusión varía más rápidamente con el voltaje.

Estas dos teorías fueron unificadas en 1966 por Crowell y Sze en un modelo que describiremos posteriormente.

\section{3. C. Teoría de Mott. [Mott, 1938]}

La expresión que nos proporcionan las teorías expuestas anteriormente es válida sólo para un cierto rango de tensiones aplicadas, esto es, siempre que $V_{a} \leq V_{b i}$. Para $V_{a}=V_{b i}$ nos encontramos en la condición de banda plana, y para voltajes superiores el comportamiento del dispositivo es explicado mediante la llamada Teoría de Mott.

Para este rango de voltajes aplicados, la barrera que el semiconductor ofrece a los electrones que intenten acceder al metal desaparece. El potencial en el semiconductor cae de manera lineal y surge por tanto un campo eléctrico constante que provoca una corriente de arrastre. La dependencia de la corriente total con el potencial deja de ser de tipo exponencial y 
pasa a ser prácticamente lineal puesto que el dispositivo se comporta de manera muy similar a una resistencia:

$$
J \cong \frac{V_{a}-V_{b i}}{S R}
$$

donde $S$ es la sección transversal del diodo y $R$ es la resistencia óhmica total equivalente de las distintas regiones del mismo.

\section{4. Efectos reales en la unión metal-semiconductor}

Hasta el momento, hemos descrito el comportamiento ideal del dispositivo y nos hemos centrado en los procesos de transporte de tipo termoiónico. Sin embargo, nuestro estudio de la unión metal-semiconductor rectificadora sería incompleto si no considerásemos efectos no ideales tales como el efecto de cargas imagen (que produce una reducción de la barrera, con el consiguiente aumento de corriente termoiónica) o un proceso de transporte de corriente distinto al descrito hasta ahora: la corriente túnel. Comentaremos también cuál es la contribución al transporte de los portadores minoritarios bajo ciertas condiciones de inyección, así como el efecto de la existencia de estados superficiales en la interfaz metal-semiconductor.

\section{4. A. Efecto Schottky. [Schottky, 1914]}

Cuando un electrón se encuentra a una distancia $x$ del metal, en este último se induce una carga positiva en su superficie que atrae al electrón. Esta fuerza de atracción es equivalente a la de atracción electrostática entre el electrón y una carga idéntica de signo positivo situada a una distancia $-x$ de la interfaz, que recibe el nombre de carga imagen. La fuerza imagen correspondiente será por tanto:

$$
F=-\frac{q^{2}}{4 \pi(2 x)^{2} \varepsilon_{s}}
$$

donde $\varepsilon_{S}$ es la permitividad del semiconductor. La energía potencial total $\left(q \varphi_{P}\right)$ para un electrón situado a una distancia $x$ de la interfaz metal-semiconductor se define como sigue 


$$
q \varphi_{P}(x)=\frac{q^{2}}{16 \pi \varepsilon_{s} x}+q \xi x
$$

Es decir, como el resultado de la combinación de la fuerza imagen y la ejercida por el campo eléctrico en la interfaz, $\xi$, (Figura I.6).

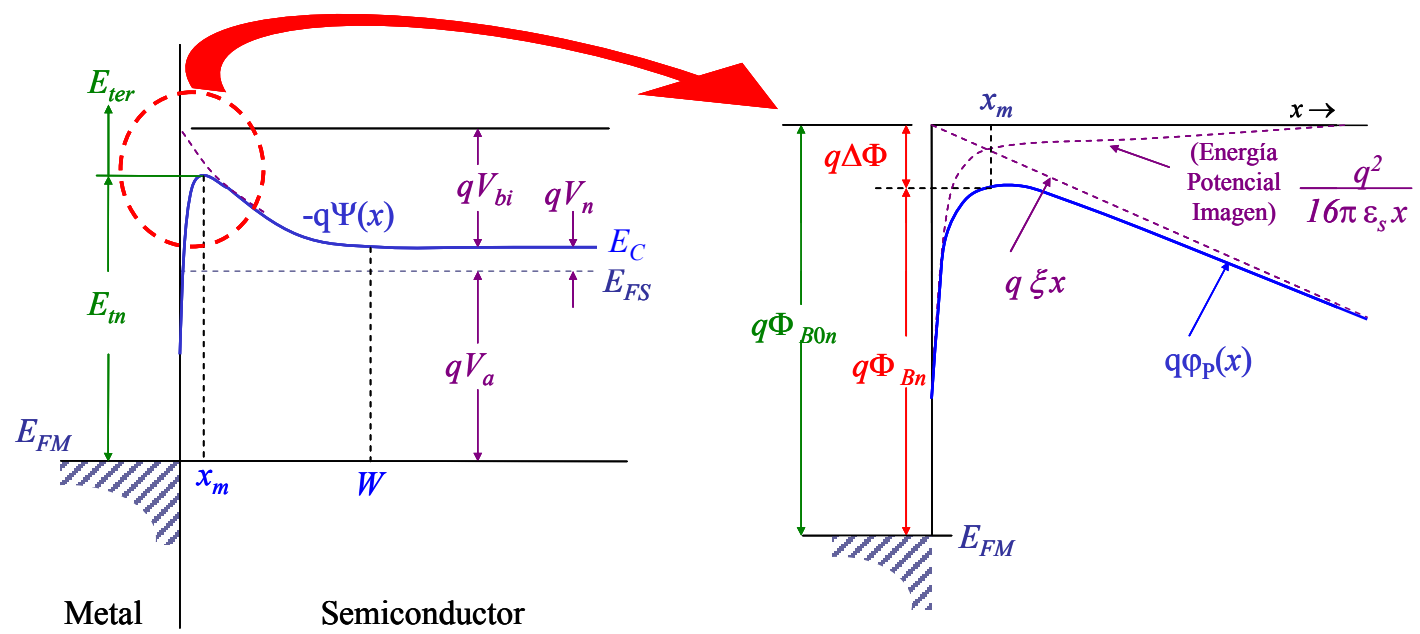

Figura I. 6. Distorsión de la barrera de potencial debido al efecto de la fuerza imagen.

A partir de esta expresión, podemos calcular el máximo valor de $\varphi_{P}$ y determinar su posición $\left(x_{m}\right)$, lo que nos permite obtener el valor de la disminución de la altura de la barrera, $\Delta \Phi$, indicada en la Figura I.6.

$$
\begin{aligned}
& x_{m}=\sqrt{\frac{q}{16 \pi \varepsilon_{s} \xi}} \\
& \Delta \Phi=\sqrt{\frac{q \xi}{4 \pi \varepsilon_{s}}}=2 \xi x_{m}
\end{aligned}
$$

Así, tal y como observamos en la Figura I.6, el valor de la altura de la barrera tras considerar el efecto Schottky, $q \Phi_{\mathrm{Bn}}$, que denominaremos a partir de ahora altura de barrera Schottky efectiva, será igual a la diferencia entre $q \Phi_{B 0 n} \mathrm{y} \Delta \Phi$.

Como cabe esperar, la reducción de la barrera tiene una importante repercusión en las teorías termoiónica y de difusión anteriormente expuestas. Teniendo en cuenta la similitud entre las expresiones que para la densidad de corriente se deducían de ambos modelos (Ecuaciones I. 23 y I. 26), es posible una síntesis de ambas, incluyendo además el efecto Schottky [Crowell, 1966], dentro de la llamada teoría de emisión termoiónica/difusión. 
En este caso, la densidad de corriente se expresa en función de una velocidad de recombinación $v_{R}$ asociada al máximo de energía potencial y una velocidad de difusión $v_{D}$ relacionada con el transporte de electrones desde el borde de la capa de vaciamiento $W$ al máximo de la energía potencial, situado en $x_{m}$ (ver Figura I.6). Las expresiones son [Sze, 1981]:

$$
J=\frac{q N_{C} v_{R}}{1+v_{R} / v_{D}} \exp \left(-\frac{q \Phi_{B n}}{K_{B} T}\right)\left[\exp \left(\frac{q V_{a}}{K_{B} T}\right)-1\right]
$$

donde las velocidades de difusión $v_{D}$ y recombinación $v_{R}$ vienen dadas por las expresiones:

$$
\begin{aligned}
v_{D}= & {\left[\int_{x_{m}}^{W} \frac{q}{\mu K_{B} T} \exp \left(-\frac{q}{K_{B} T}\left(\Phi_{B n}+\Psi\right)\right) d x\right]^{-1} } \\
v_{R}= & \frac{\int_{0}^{\infty} v_{x} \exp \left(-m^{*} v_{x}^{2} / K_{B} T\right) d v_{x}}{\int_{-\infty}^{\infty} \exp \left(-m^{*} v_{x}^{2} / 2 K_{B} T\right) d v_{x}}=\left(\frac{K_{B} T}{2 m^{*} \pi}\right)^{1 / 2}=\frac{A^{*} T^{2}}{q N_{C}}
\end{aligned}
$$

$\mu$ es la movilidad de los electrones, $v_{\mathrm{x}}$ es la componente de la velocidad en la dirección en la que se da la el transporte de los portadores y $A^{*}$ la constante de Richardson. $\Psi(x)$ representa, tal y como vemos en la Figura I.6, la barrera de potencial como superposición de los potenciales $\varphi(x)=\xi x$ y $\varphi_{P}(x)$ (Ecuaciones I.5 y I.29 respectivamente).

Examinando la expresión anterior para la corriente, podemos diferenciar las situaciones siguientes: si $v_{D} \gg v_{R}$, en el término que multiplica a las exponenciales tiene más importancia $v_{R}$, y la Ecuación I.32 quedaría reducida a la obtenida con la teoría de emisión termoiónica. En caso contrario, si $v_{D}<<v_{R}$, se obtiene la expresión propia de la teoría de difusión.

\section{4. B. Corriente túnel}

Como ya hemos mencionado con anterioridad, existe una contribución a la corriente debida a los electrones que son capaces de atravesar la barrera de potencial (el llamado efecto túnel o emisión de campo), dado que la probabilidad cuántica de que ocurra este fenómeno no es nula. La densidad de corriente que se obtiene mediante este proceso puede llegar a tener mucha importancia, sobre todo a voltajes aplicados en inversa altos, para los cuales se da un estrechamiento de la barrera mayor y la probabilidad de transporte por efecto túnel aumenta. 
Además, en el caso de semiconductores fuertemente dopados, el transporte por túnel puede convertirse en el proceso dominante, por lo que resulta imprescindible abordar su estudio si se quiere efectuar un correcto análisis del transporte en estos dispositivos.

Tomaremos como punto de partida la Ecuación I.11, pero en esta ocasión incluiremos no sólo la componente termoiónica, sino también la componente túnel [Chang, Sze 1970], además del efecto Schottky. La componente de la corriente $J_{s \rightarrow m}$ por efecto túnel será proporcional al coeficiente de transmisión cuántico $T_{C}$ multiplicado por la probabilidad de ocupación en el semiconductor y la de no ocupación en el metal:

$$
\begin{aligned}
J_{s \rightarrow m}= & \frac{A^{*} T}{K_{B}} \int_{q \Phi_{B n}}^{\infty} T_{C}\left(E_{t e r}\right) \exp \left(\frac{-q\left(V_{b i}+V_{n}+E_{t e r}-\Delta \Phi\right)}{K_{B} T}\right) d E_{t e r} \\
& +\frac{A^{*} T}{K_{B}} \int_{0}^{q \Phi_{B n}} T_{C}\left(E_{t n}\right) f_{s}\left(1-f_{m}\right) d E_{t n}
\end{aligned}
$$

$E_{\text {ter }}$ engloba los valores de energía de emisión termoiónica que pueden poseer los electrones mientras que $E_{t n}$ corresponde a sus posibles energías túnel (ver Figura I.6 ${ }^{3}$ ). Las probabilidades de ocupación de semiconductor y metal se expresan en términos de la función de distribución de Fermi-Dirac, $f_{s}$, para el semiconductor y $f_{m}$, en el caso del metal ${ }^{4}$. Así, el primer término del segundo miembro de la Ecuación I.35 representa la componente termoiónica de la corriente, que coincide con la expresión de la Ecuación I.18 si el coeficiente de transmisión $T_{C}\left(E_{t e r}\right)=1$. El segundo término es la componente túnel.

La corriente desde el metal al semiconductor se expresa de manera similar:

$$
\begin{aligned}
J_{m \rightarrow s}= & -\frac{A^{*} T}{K_{B}} \exp \left(\frac{-q \Phi_{B n}}{K_{B} T}\right) \int_{q \Phi_{B n}}^{\infty} T_{C}\left(E_{t e r}\right) \exp \left(-\frac{q E_{t e r}}{K_{B} T}\right) d E_{t e r} \\
& -\frac{A^{*} T}{K_{B}} \int_{0}^{q \Phi_{B n}} T_{C}\left(E_{t n}\right) f_{m}\left(1-f_{s}\right) d E_{t n}
\end{aligned}
$$

La corriente total será la suma de las expresiones I.35 y I.36.

Para incorporar el concepto de corriente túnel en las teorías desarrolladas previamente, es posible introducirlo como una condición de contorno más, describiendo así de manera más

\footnotetext{
${ }^{3}$ Consideramos que el origen de energías es el nivel de Fermi del metal

${ }^{4}$ Para la corriente termoiónica hemos supuesto que las energías son suficientemente altas como para que sea válida la aproximación de Maxwell-Boltzmann, con el fin de mostrar de una manera más precisa la relación con las ecuaciones anteriormente descritas.
} 
precisa el comportamiento del metal en la unión (como ya se ha visto en el caso de la velocidad de recombinación asociada a la emisión termoiónica). Para ello, se define una constante de Richardson efectiva, en la que se podrá tener en cuenta no sólo la transmisión por efecto túnel, sino también otros efectos, como el scattering con fonones ópticos y las reflexiones cuánticas [Crowell, 1965b], [Crowell, 1966]:

$$
A * *=\frac{f_{P} f_{Q}}{1+f_{P} f_{Q} v_{R} / v_{D}} A *
$$

siendo $f_{P}$ la probabilidad de scattering con fonones ópticos, y $f_{Q}$ la probabilidad de reflexiones y transmisiones cuánticas, ambas como función del campo eléctrico. En este caso, el valor que toma esta constante para Silicio tipo $n$ y tipo $p$ es [Andrews, 1970]:

$$
\begin{aligned}
& A^{* *} \approx 112 \mathrm{~A} \mathrm{~cm}^{-2} \mathrm{~K}^{-2} \quad \text { para electrones } \\
& A^{* *} \approx 32 \mathrm{~A} \mathrm{~cm}^{-2} \mathrm{~K}^{-2} \quad \text { para huecos }
\end{aligned}
$$

Así, la expresión para la densidad de corriente quedará:

$$
J=J_{S}\left(e^{q V / K_{B} T}-1\right)
$$

donde se ha definido la corriente de saturación, $J_{S}$, del siguiente modo:

$$
J_{S}=A^{* *} T^{2} \exp \left(-\frac{q \Phi_{B n}}{K_{B} T}\right)
$$

Llegamos por tanto a una expresión para la corriente completamente análoga a la clásica de partida, con la única salvedad de la corrección efectuada en la constante de Richardson. Puede por tanto considerarse a esta constante como un parámetro modificable que permite incorporar ciertos efectos o reproducir de manera global, en cuanto al cómputo de corriente, el comportamiento real de los dispositivos a analizar.

En este punto es preciso aclarar que, en nuestro caso, el tratamiento del efecto túnel en contactos Schottky se hace de manera completa e independiente partiendo de las Ecuaciones I.35 y I.36, a través de la adecuada determinación del coeficiente de transmisión cuántico en la simulación Monte Carlo. Para ello, calcularemos la solución de la Ecuación de Schrödinger mediante la aproximación WKB. El proceso completo será descrito en el Capítulo II. 


\section{4. C. Inyección de portadores minoritarios}

La inyección de portadores minoritarios desde el metal al semiconductor es despreciable cuando aplicamos tensiones en inversa o no muy elevadas en directa [Sze, 1981]; sin embargo, cuando el voltaje aplicado en directa es suficientemente alto, la inyección de portadores minoritarios comienza a tener importancia. Esto es debido a que en esta situación, el campo eléctrico causa una corriente de deriva de portadores significativa frente a la de difusión, por lo que la corriente de portadores minoritarios puede tener una contribución a la densidad de corriente total no despreciable. No obstante, dentro del rango de las polarizaciones estudiadas en esta Memoria, este tipo de procesos no tiene relevancia, por lo que no han sido considerados en las simulaciones.

\section{4. D. Estados superficiales o de interfaz. Estabilización del nivel de Fermi}

Hasta este momento, hemos supuesto que la interfaz entre metal y semiconductor es perfecta. Esto significa que, desde el punto de vista del semiconductor, éste se encuentra constituido por una cantidad infinita de distancias atómicas, y que todos los enlaces de Silicio

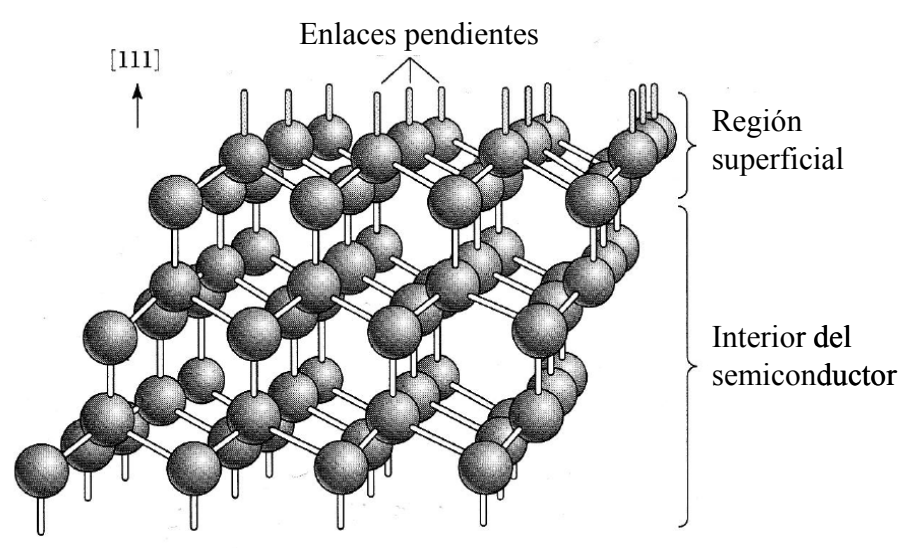

Figura I. 7. Diagrama esquemático de los enlaces en la superficie de un semiconductor. Los enlaces superficiales son anisotrópicos y son distintos de los del interior del material [Sze, 2001]. están completos de acuerdo con la estructura de diamante del mismo. Sin embargo no sucede así en los materiales reales: existe una discontinuidad abrupta de la estructura de la red en la superficie y no todos los enlaces pueden satisfacerse en esta región. De hecho ocurre que una fracción de los enlaces de los átomos de la superficie queda sin completar o incluso se completan con los átomos vecinos, produciendo una cierta asimetría en el enlace (ver Figura I.7). De este modo, los estados electrónicos de las últimas capas, donde se rompe la periodicidad del potencial eléctrico del cristal, no tienen la energía de 
los estados electrónicos del volumen. Puesto que estos estados energéticos se forman en la superficie entre el metal y el semiconductor, se denominan estados superficiales o de interfaz [Albella, 1996].

Existen también otros motivos para que surjan los estados superficiales, como la existencia de impurezas localizadas en la superficie de un semiconductor, sobre todo las de oxígeno, o defectos en la superficie (daños en el cristal). En realidad, aunque la superficie del semiconductor se considere limpia, siempre hay una pequeña concentración de impurezas en la misma; por esta y por otras razones podemos afirmar que no existe una interfaz ideal.

La propiedad más importante de los estados superficiales es que pueden atrapar cargas. Esto implica que la existencia de estos estados afectará a la curvatura de las bandas energéticas del semiconductor, y por tanto el valor de la altura de la barrera cambiará en comparación con la que se formaría sin la presencia de los estados superficiales (pueden aumentarla o disminuirla, dependiendo del estado de interfaz y del dopaje del semiconductor [Neudeck, 1993]).

Para estudiar el efecto de los estados superficiales sobre las bandas de energía en el semiconductor de una manera simplificada, analizamos el caso de un semiconductor tipo $n$ en ausencia de metal $^{5}$ con estados superficiales de tipo aceptador, tal y como se representa en la Figura I.8.a. Estos estados propician una transferencia de electrones, bien sea desde los niveles donadores del semiconductor o bien desde los niveles de la banda de conducción.

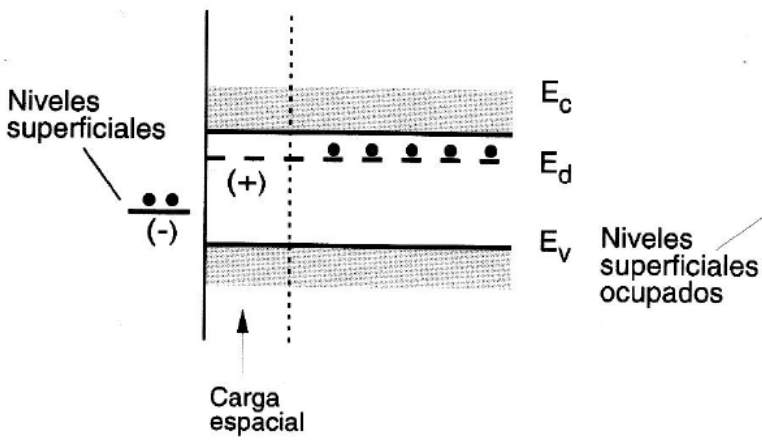

a)

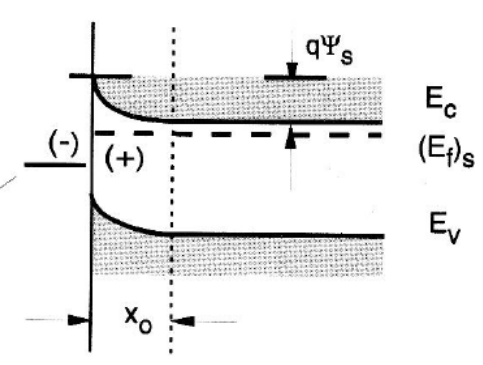

b)

Figura I. 8. Diagrama de bandas de energía de un semiconductor tipo n: .a. antes de iniciarse la transferencia de electrones desde los niveles donadores los estados superficiales aceptores y .b. después de establecerse el equilibrio, dando lugar a la curvatura de las bandas debido al proceso de estabilización del nivel de Fermi [Albella, 1996] (Fermi level pinning).

\footnotetext{
${ }^{5}$ En realidad los estados superficiales del semiconductor se verán alterados al estar en contacto con el metal, tal y como veremos en la siguiente sección.
} 
Como consecuencia, aparece una carga negativa en la superficie del semiconductor y una carga positiva en el interior del semiconductor (localizada en un espesor $x_{0}$ cerca de la superficie, debida a los niveles donadores ionizados). Por tanto, el sentido del campo eléctrico originado es negativo y así, las bandas del semiconductor se doblan como se indica en la Figura I.8.b. Al comparar las Figuras I.8.a y .b, observamos que, como consecuencia de los estados superficiales, el nivel de Fermi del semiconductor (situado cerca de los niveles donadores) se acerca al nivel de los estados superficiales. Si el número de estos es muy grande, el nivel de Fermi prácticamente coincide con el nivel de los estados superficiales. Cuando ambos niveles están a la misma altura ya no habrá más transferencia de electrones del interior del semiconductor a la superficie, con lo que el proceso de transferencia se detiene y las bandas ya no se curvan más. En esta situación se habla de estabilización del nivel de Fermi (Fermi level pinning) por los estados superficiales.

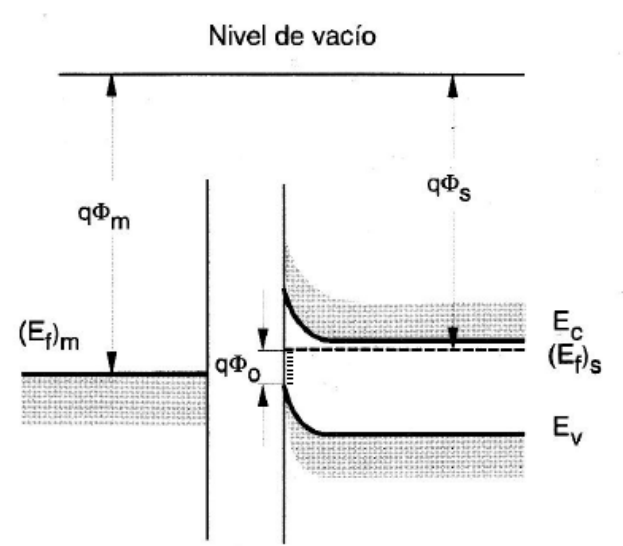

a)

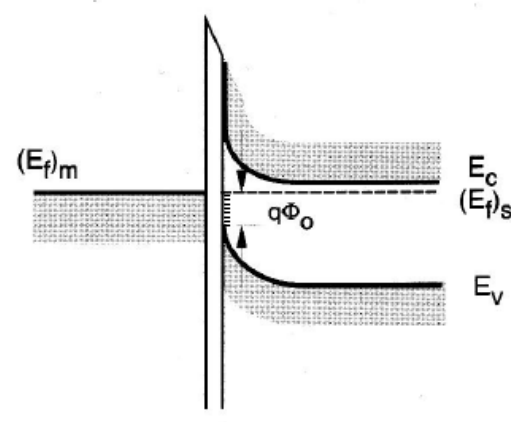

b)

Figura I. 9. Diagrama de bandas de energía de un metal y un semiconductor tipo $n$ con estados superficiales de tipo aceptor .a. antes y.b. después de entrar en contacto [Albella, 1996].

En la Figura I.9.a y .b presentamos el mismo semiconductor con superficie no ideal antes y después de entrar en contacto con un metal para formar una unión de tipo rectificador. $\mathrm{Al}$ poner ambos materiales en contacto, con el fin de equilibrar los niveles de Fermi, debe producirse una transferencia de carga. La carga negativa que ocupa los estados superficiales es la más susceptible de abandonar el semiconductor hacia el metal, por lo que la diferencia de densidad de carga en el metal con respecto al semiconductor al producirse la unión causará únicamente ligeras variaciones en la densidad de carga espacial [Albella, 1996]. De este modo, la curvatura de la banda no se ve apenas modificada al producirse la unión tal y como se puede 
observar comparando la Figura I.9.a y .b. Esta hipótesis muestra que cuando existen estados superficiales con una densidad suficientemente alta, el valor de $q \Phi_{B n}$ es prácticamente independiente de la función del trabajo del metal.

Denominamos $q \Phi_{0}$ (ver Figura I.9) al valor del nivel de energía de estos estados, que está relacionado con la neutralidad de carga de los estados superficiales: a temperatura nula, los estados superficiales se encuentran poblados desde el estado energético más bajo hasta el nivel de Fermi, tal y como sucede en la Figura I.9; sin embargo para temperaturas superiores no todos los estados superficiales se encuentran ocupados. De este modo, $q \Phi_{0}$ se define como la distancia del máximo de la banda de valencia en la interfaz al estado energético superficial más elevado que posea neutralidad de carga [Tung, 2001].

Por otra parte, la densidad de estos estados energéticos en la banda prohibida, $D_{S}$, no es uniforme, y además depende notablemente de las condiciones de deposición del metal. Por tanto, su importancia final dependerá de la técnica de preparación de la superficie considerada, pudiendo obtenerse resultados distintos en cada caso. Desde el punto de vista de la tecnología de fabricación, minimizar el efecto de los estados superficiales es una de las cuestiones más importantes hoy en día para técnicos e investigadores.

En nuestro caso el efecto de este tipo de estados podría incluirse en la simulación Monte Carlo mediante la consideración de trampas en la interfaz. Sin embargo, puesto que dicho efecto es finalmente sobre la altura de la barrera, sin propiciar cambios importantes en el fundamento del comportamiento de la unión Schottky, consideramos que en las interfaces de los contactos que estudiaremos en capítulos posteriores este efecto se encuentra incluido en el valor de la altura de la barrera medida experimentalmente.

\section{5. Caracterización de la altura de la barrera}

El estudio de la determinación de la altura de la barrera ha sido objeto de discusión a lo largo de los últimos 70 años. En esta sección describiremos en primer lugar los modelos teóricos más relevantes y posteriormente evaluaremos las distintas formas experimentales de determinación de la altura de la barrera. 


\section{5. A. Modelos de determinación de la altura de la barrera}

Recordemos de la Sección I.1.C que en una unión rectificadora metal-semiconductor tipo $n$, la altura de barrera que deben superar los electrones en el metal para acceder al semiconductor, $q \Phi_{B 0 n}$, es igual a la diferencia entre la función trabajo del metal $q \Phi_{M}$ y la afinidad electrónica del semiconductor $q \chi$ (Ecuación I.1). Fueron Mott [Mott, 1938] y posteriormente Schottky [Schottky, 1940] quienes propusieron primero esta regla simple para el cálculo de la altura de la barrera, a la cual denominaremos a partir de ahora como Relación de Mott-Schottky.

Sin embargo, cuando se mide la altura de la barrera por los métodos que indicaremos posteriormente, se observa que $q \Phi_{B 0 n}$ varía menos rápidamente con $q \Phi_{M}$ de lo que cabría esperar (véase la Figura I.10). Esto se debe a que la relación Mott - Schottky proporciona la altura de la barrera para contactos ideales, sin considerar diversos efectos reales como es el efecto Schottky y la aparición de estados superficiales, que ya analizamos previamente en la Sección I.4.

El pionero en considerar los estados superficiales como posible explicación para la desviación de los resultados experimentales de la relación de Mott - Schottky fue Bardeen [Bardeen, 1947]. Propuso que en el semiconductor, en la región cercana a la interfaz, además de la presencia de la zona de carga espacial también existirían estados superficiales con capacidad de absorber o ceder carga según su naturaleza. De este modo para alcanzar la neutralidad de carga de la

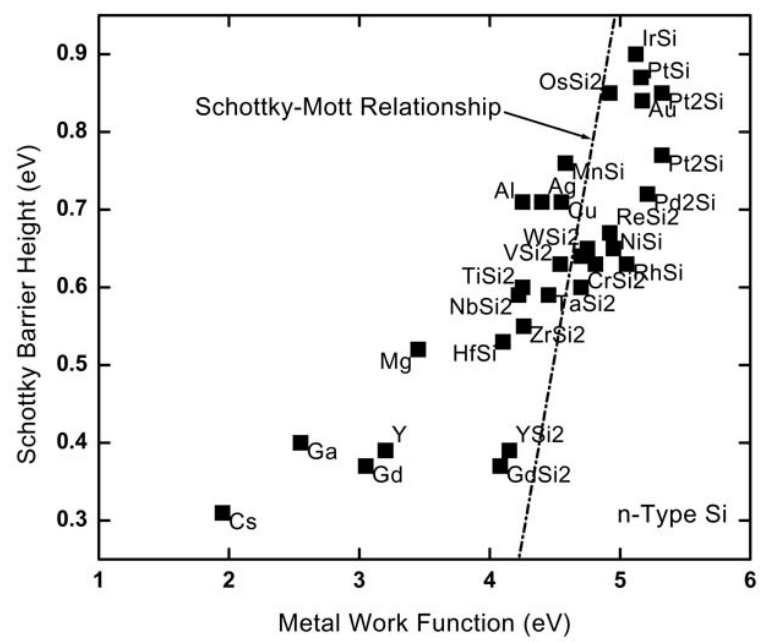

Figura I. 10. Variación de la altura de barrera del contacto para metales con distinta función de trabajo cuando el semiconductor es tipo n. La línea discontinua representa la Relación Mott-Schottky [Sze, 1981]. unión, la densidad de carga en el metal, $Q_{m}$, será igual pero de signo contrario a la del semiconductor $Q_{s}$, que se distribuye entre la zona de carga espacial $\left(Q_{s c}\right)$ y los estados superficiales $\left(Q_{s s}\right)$ :

$$
Q_{m}+Q_{s}=Q_{m}+Q_{s c}+Q_{s s}=0
$$


Tal y como ya vimos en la Sección I.4.D, la existencia de estos estados provoca una estabilización del nivel de Fermi (Fermi level pinning), con la correspondiente curvatura de las bandas de energía en el semiconductor incluso en ausencia del metal, explicando la cierta insensibilidad de $q \Phi_{B n}$ con la función de trabajo del metal empleado en la unión. La expresión para la altura de la barrera podemos extraerla a partir del diagrama de bandas de energía [Sze, 1981] (ver Figura I.9), que incluyendo el efecto de las cargas imagen es como sigue:

$$
q \Phi_{B n}=E_{g}-q \Phi_{0}-q \Delta \Phi
$$

Los primeros en aportar pruebas experimentales de la existencia de estos estados superficiales fueron Shockley y Pearson [Shockley, Pearson, 1948], Brattain y Bardeen [Brattain, Bardeen, 1953] y Morrison [Morrison, 1953]. Como consecuencia de este descubrimiento, se estimuló el desarrollo de la técnica de vacío ultra alto, requisito fundamental para obtener superficies en interfaces lo suficientemente limpias.

En 1965, Cowley y Sze [Cowley, Sze, 1965] cuantificaron el concepto de Bardeen de los estados superficiales en contactos metal - semiconductor demostrando la dependencia de la altura de la barrera con los estados superficiales y la función trabajo del metal. Plantearon el modelo de separación fija (fixed separation model), en el que presentaban una expresión de la altura de la barrera bajo dos hipótesis iniciales: (1) entre los dos materiales de la unión hay una capa interfacial de dimensiones atómicas que soporta una caída de potencial y permite el paso de electrones por efecto túnel a través de ella, y (2) los estados superficiales en la interfaz se consideran una característica de la superficie del semiconductor y son independientes del metal.
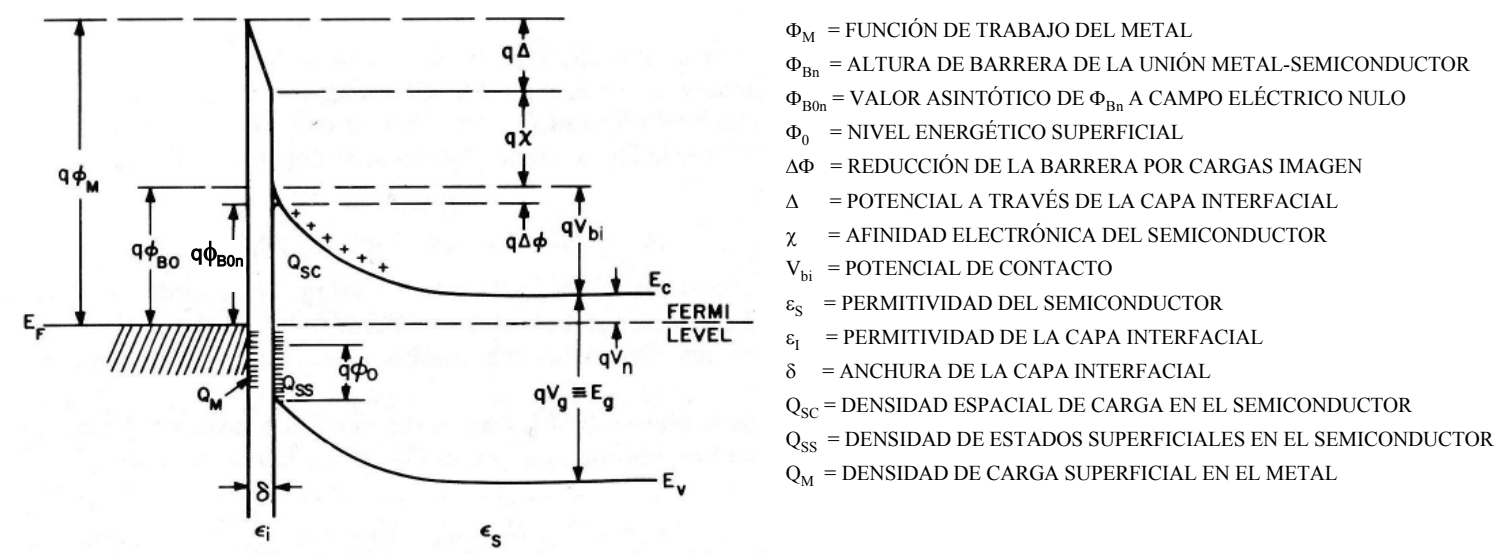

Figura I. 11. Diagrama de bandas de energía de un metal y un semiconductor tipo $n$ con estados superficiales de tipo aceptor con una capa interfacial de distancia atómica [Sze, 1981]. 
En la Figura I.11 presentamos el diagrama de bandas de energía así como la definición de las distintas variables que usaremos a continuación.

El valor de la densidad de carga de los estados superficiales se expresa como sigue:

$$
\begin{array}{lll}
Q_{s s}=-q D_{s}\left(E_{g}-q \Phi_{0}-q \Phi_{B n}-q \Delta \Phi\right) & {\left[\mathrm{C} / \mathrm{cm}^{2}\right]} & \text { I. } 42
\end{array}
$$

Donde $D_{s}$ es la densidad de los estados superficiales (medida en estados $/ \mathrm{cm}^{2} / \mathrm{eV}$ ), que permanece constante para energías comprendidas entre $q \Phi_{0}$ y el nivel de Fermi. Podemos observar que se incluye el efecto Schottky a través del término $q \Delta \Phi$.

Por otro lado, la ecuación de la densidad de carga de la zona de vaciamiento del semiconductor viene dada por la Ecuación I.6, expresada del siguiente modo al incluir el efecto Schottky:

$$
Q_{s c}=\sqrt{2 q \varepsilon_{s} N_{D}\left(\Phi_{B n}-V_{n}+\Delta \Phi-\frac{K_{B} T}{q}\right)}
$$

Además, el valor de esta carga $Q_{s c}$ está directamente relacionado con el potencial $\Delta$ que cae a lo largo de la capa interfacial. Este potencial podemos calcularlo de dos modos, en primer lugar aplicando la ley de Gauss en la superficie interfacial y mediante el diagrama de bandas de energía de la Figura I.11:

$$
\Delta=-\delta \frac{Q_{m}}{\varepsilon_{i}}=\Phi_{M}-\left(\chi+\Phi_{B n}+\Delta \Phi\right)
$$

Donde $\delta$ es la anchura de la capa interfacial. Despejando, por tanto de esta relación el valor de $Q_{m}$, y sustituyendo junto a $Q_{s s}$ y $Q_{s c}$ en la Ecuación I.40:

$$
\begin{aligned}
\left(\Phi_{M}-\chi\right)-\left(\Phi_{B n}+\Delta \Phi\right)= & \sqrt{\frac{2 q \varepsilon_{s} N_{D} \delta^{2}}{\varepsilon_{i}^{2}}\left(\Phi_{B n}-V_{n}+\Delta \Phi-\frac{K_{B} T}{q}\right)} \\
& -\delta \frac{q D_{s}}{\varepsilon_{i}}\left(E_{s}-q \Phi_{0}-q \Phi_{B n}-q \Delta \Phi\right)
\end{aligned}
$$

A partir de esta ecuación podemos obtener el valor de $q \Phi_{B n}$ sin más que despejar y considerando que $\varepsilon_{s} \approx 10 \varepsilon_{0}, \varepsilon_{i}=\varepsilon_{0} \mathrm{y} N_{D}<10^{18} \mathrm{~cm}^{-3}$, siendo así posible despreciar así algunos términos por su pequeño valor [Sze, 1981]. Por tanto, la expresión general de la altura de la barrera para el modelo de separación fija será:

$$
\Phi_{B n}=\gamma\left(\Phi_{m}-\chi\right)+(1-\gamma)\left(\frac{E_{g}}{q}-\Phi_{0}\right)-\Delta \Phi
$$

Donde el valor de $\gamma$ lo expresamos a continuación: 


$$
\gamma=\frac{\varepsilon_{i}}{\varepsilon_{i}+q^{2} \delta D_{s}}
$$

A partir de la Ecuación I.46 obtenemos la expresión de la altura de la barrera para los dos casos anteriores:

1) Cuando la densidad de los estados superficiales es muy elevada $\left(D_{s} \rightarrow \infty\right)$, entonces $\gamma \rightarrow 0$, así la expresión de $q \Phi_{B n}$ coincidirá con la Ecuación I.40 (incluyendo el efecto Schottky) para el modelo de estabilización del nivel de Fermi:

$$
q \Phi_{B n}=\left(E_{g}-q \Phi_{0}\right)-q \Delta \Phi
$$

2) Cuando la densidad de los estados superficiales es muy baja $\left(D_{s} \rightarrow 0\right)$, entonces $\gamma \rightarrow 1$, coincidiendo el valor de $q \Phi_{B n}$ con la relación de Mott - Schottky, teniendo en cuenta el efecto Schottky:

$$
q \Phi_{B n}=q\left(\Phi_{m}-\chi\right)-q \Delta \Phi
$$

Sin embargo, aunque el modelo de separación fija refuerce la explicación del modelo de estabilización del nivel de Fermi, el punto débil del mismo es la presencia de la capa interfacial. Sin esta capa interfacial, es decir: $\delta \rightarrow 0$, se recuperaría la relación de Mott - Schottky. Por otro lado, es difícil de entender porqué la distribución de los estados superficiales es independiente del metal, de hecho Heine [Heine, 1965] enunció que los estados energéticos de la banda prohibida del semiconductor se ven influenciados por la densidad de estados del metal, por lo que denominó a estos estados superficiales como MIGS, del inglés metal-induced gap states, o lo que es lo mismo: estados en la banda prohibida inducidos por el metal [Mönch, 2004].

El problema principal de esta teoría es que, en realidad el modelo de separación fija explica el fenómeno de estabilización del nivel de Fermi; sin embargo, la estructura real de la interfaz metal-semiconductor no es conocida. Existen diversas teorías para explicar qué sucede en la interfaz; entre ellas destaca la representación mediante de enlaces químicos. El modelo de polarización de enlaces desarrollado por Tung, [Tung, 2001] considera que la altura de barrera Schottky formada en la interfaz es el resultado de los enlaces químicos de la misma así como de los dipolos interfaciales correspondientes. Estos dipolos de la unión Schottky pueden analizarse utilizando técnicas de la física química, identificándolos con la polarización de los enlaces químicos: para ello, se considera a la interfaz metal - semiconductor como una molécula gigante. 
En la Figura I.12 mostramos el esquema de la interfaz que se utiliza en el modelo de polarización de enlaces, además del diagrama de bandas de energía correspondiente a la unión. Como podemos observar, se supone que la interfaz metal-semiconductor es atómicamente abrupta y se establece una densidad $N_{B}$ de enlaces uniforme.

El mayor inconveniente que presenta esta teoría es que, al asumir que la transferencia de carga se da únicamente en átomos que pertenecen a los planos atómicos de la interfaz, este método no podría utilizarse para materiales cuya densidad de estados superficiales fuera nula. Sin embargo, permitiría calcular los dipolos interfaciales de manera aproximada, ya que la mayor parte de los estados superficiales se forma en los planos atómicos inmediatos a la interfaz. En nuestro caso, tal y como decíamos en la Sección I.4.D, no tendremos en cuenta esta consideración del modelo de polarización de enlaces para la interfaz ni los estados energéticos prohibidos en la interfaz, sino que dichos efectos los tendremos en cuenta de manera intrínseca en el valor de la altura de barrera Schottky medida experimentalmente.
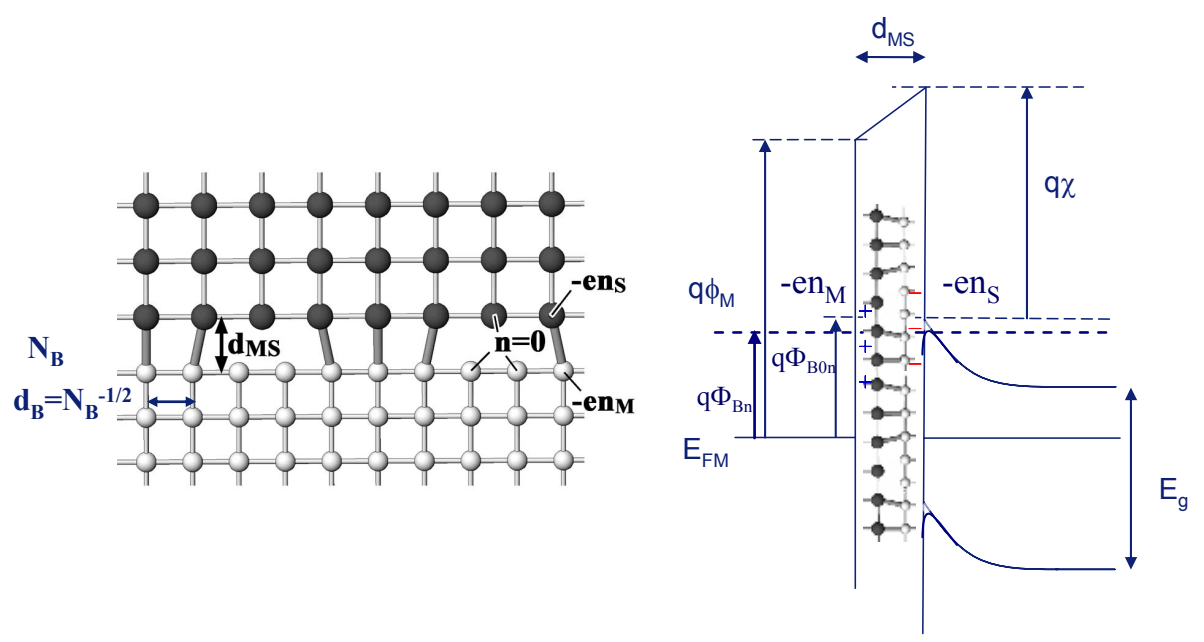

Figura I. 12. a. Vista en corte transversal de la interfaz metal - semiconductor utilizada en el modelo de polarización de enlaces. Las barras más gruesas representan los enlaces que constituyen la unión entre el semiconductor (mitad superior) y el metal (mitad inferior). Se considera que la transferencia de carga se da únicamente entre los átomos que forman parte de la unión [Tung, 2001]. - en $n_{S} y-e n_{M}$ es la carga neta de los átomos en el semiconductor y el metal respectivamente, $d_{M S}$ es la distancia entre los átomos del metal y semiconductor, $N_{B}$ es la densidad de enlaces $y d_{B}$ es la distancia entre los enlaces .b. Diagrama de bandas de energía de la unión. 


\section{5. B. Medida de la altura de la barrera}

A continuación describimos brevemente los distintos métodos existentes para la determinación experimental de la altura de barrera de los contactos Schottky.

\section{Medida de corriente - voltaje}

A partir de las Ecuaciones I.23, y I.24, incluyendo la reducción de la barrera $(\Delta \Phi)$ debida al efecto Schottky, obtenemos la siguiente expresión de la densidad de corriente para $V>3 K_{B} T$ :

$$
\begin{aligned}
& J_{n}=A^{*} T^{2} \exp \left(-\frac{q \Phi_{B 0 n}}{K_{B} T}\right) \exp \left(\frac{q\left(\Delta \Phi+V_{a}\right)}{K_{B} T}\right) \\
& \text { donde } J_{S}=A^{*} T^{2} \exp \left(-\frac{q \Phi_{B 0 n}}{K_{B} T}\right)
\end{aligned}
$$

En el régimen de directa, al representar el valor de la densidad de corriente frente al voltaje aplicado, del valor de corte de la recta con el eje de ordenadas, obtenemos el valor de la corriente de saturación $J_{S}$, y así, la altura de la barrera podrá ser determinada a través de la siguiente expresión:

$$
\Phi_{B 0 n}=\frac{K_{B} T}{q} \ln \left(\frac{A^{*} T^{2}}{J_{S}}\right)
$$

Este método es el más utilizado y ofrece muy buenos resultados cuando la corriente termoiónica es suficientemente pequeña para que la caída de voltaje debido a la resistencia del Silicio sea moderada. Sin embargo, presenta ciertas desventajas:

- En primer lugar, la superficie debe ser conocida, lo cual no es siempre fácil, dependiendo de la estructura bajo estudio.

- En el caso de polarización inversa en que la corriente túnel presente una importante contribución a la corriente total, este método no puede aplicarse ya que las expresiones anteriores no son válidas.

- Para contactos Schottky de altura de barrera baja en el que sustrato es poco dopado, la importancia de la resistencia en serie del Silicio es mayor frente a la resistencia del contacto Schottky, lo que implica que el método de extracción de la altura de la barrera no sea muy preciso. 


\section{Medida de capacidad - voltaje}

Este método está basado en la variación de carga en la superficie del metal y en el interior del semiconductor (de signo opuesto a la del metal) que se induce al someter al dispositivo a un pequeño incremento del voltaje respecto al que está trabajando. La relación entre $C$ y $V$ viene dada por la Ecuación I.10.

Para calcular la altura de la barrera Schottky, representaremos $1 / C^{2}$ frente a $V$ y sustituiremos el valor del corte de la recta con el eje de ordenadas, $V_{i}$, en la siguiente expresión [Sze, 1981]:

$$
\Phi_{B n}=V_{i}+V_{n}+\frac{K_{B} T}{q}-\Delta \Phi
$$

Este método es muy utilizado para la caracterización de semiconductores, sobre todo para la medida de la concentración de impurezas de los mismos [Albella, 1996]. Además, si el semiconductor bajo estudio presenta un nivel de impurezas donadoras superficial y otro más profundo, obtendremos la altura de barrera midiendo la curva $C-V$ a dos temperaturas distintas [Roberts, 1973]. Sin embargo, para alturas de barrera Schottky bajas, el método $C-V$ no es el más adecuado, ya que la linealidad de la recta $1 / C^{2}$ frente a $V$ puede verse afectada por la reducción de la altura de la barrera por efecto de las cargas imagen.

\section{Medida fotoeléctrica}

Cuando hacemos incidir una luz monocromática en la superficie de un metal, se genera fotocorriente a partir de la cual se pueden obtener datos sobre las interfaces semiconductormetal. Los experimentos de emisión fotoeléctrica se llevan a cabo en dos modos de operación diferentes:

- En primer lugar, la iluminación frontal, donde la luz $(h v)$ incide sobre la capa metálica desde el lado del aire, así que la mayoría de los electrones son excitados cerca de la interfaz aire - metal y viajan hacia la interfaz metal - semiconductor. Se generarán pares electrón - hueco en el semiconductor si el metal es suficientemente fino y además se cumple que $h v>q \Phi_{B n}$ y $h v>E_{g}$, siendo $v$ la frecuencia de la luz incidente y $h$ la constante de Planck.

- Por otro lado, la iluminación trasera, en la que la radiación incide en la cara del semiconductor, generará fotoelectrones siempre que $h v>q \Phi_{B n}$. Sin embargo, al cumplirse también que $h v>E_{g}$, la luz será absorbida en la superficie posterior del semiconductor y los 
pares de electrones y huecos tendrán muy poca probabilidad de llegar a la interfaz metal semiconductor.

La relación entre la fotocorriente por fotón absorbido $R$, y la energía del fotón, $h v$, viene dada por la teoría de Fowler, que bajo una serie de condiciones queda reducida a la siguiente expresión [Sze, 1981]:

$$
\sqrt{R} \approx h\left(v-v_{0}\right) \quad \text { para } h\left(v-v_{0}\right)>3 K_{B} T \quad \text { I. } 58
$$

Donde consideramos que la energía inicial del fotón, $h v_{0}$, es la altura de la barrera. Por tanto, al representar la raíz cuadrada de la fotocorriente en función de la energía del fotón, del corte con el eje de energías podremos calcular directamente el valor de la altura de la barrera Schottky.

La principal ventaja de este método es que permite determinar el valor de la reducción de la barrera $\Delta \Phi$ así como la dependencia de la altura de la barrera con la temperatura [Crowell, 1964]. Por otro lado, este método es eficaz únicamente para contactos de altura de barrera superiores a $0.4 \mathrm{~V}$ [Shalish, 2000] [Jiménez, 1995], dado que la detección de la fotocorriente que se obtiene para barreras bajas es muy débil (del orden de $\mu \mathrm{A}$ ).

\section{Medida de energía de activación}

Al contrario que el método $\mathrm{I}-\mathrm{V}$, éste puede ser utilizado perfectamente en el régimen de polarización inversa utilizando la siguiente expresión para la corriente en inversa, $I$, donde $S_{e}$ es el área eléctricamente activa [Leroux, 1999], [Zubrilov, 2002], [Dubois, 2004]:

$$
\ln \left(I / T^{2}\right)=\ln \left(S_{e} A^{*}\right)-q\left(\Phi_{B n}-V_{a}\right) / K_{B} T
$$

Se define la energía de activación como la diferencia $q\left(\Phi_{B n}-V_{a}\right)$. Considerando un $V_{a}$ en inversa fijo, podremos calcular el valor de la altura de la barrera Schottky a partir de la pendiente de la gráfica de $\ln \left(I / T^{2}\right)$ frente a $1 / T$. Por otro lado, el corte de la recta con el eje de ordenadas nos proporciona el producto del área eléctricamente activa por la constante de Richardson $\left(S_{e} A^{*}\right)$.

La representación $\ln \left(I / T^{2}\right)$ vs $1 / T$ recibe el nombre de diagrama de Arrhenius. Para determinar la altura de la barrera de las estructuras que analizaremos en este trabajo, es el método más adecuado [Dubois, 2004] ya que presenta una gran ventaja: no es necesario realizar ninguna suposición acerca del área eléctricamente activa. Esto es especialmente útil en el 
estudio de interfaces metal - semiconductor no caracterizadas previamente, puesto que no suele conocerse el verdadero valor del área de contacto. En capítulos posteriores estudiaremos este tipo de diagramas en mayor profundidad.

\section{6. Fabricación de contactos Schottky.}

El proceso de fabricación de un contacto metal-semiconductor se lleva a cabo depositando una fina capa de metal sobre las regiones del substrato (limpiado previamente) en las que se pretenden realizar los contactos [Rideout, 1978], [Larrieu, 2004]. En la actualidad, los materiales más empleados para la fabricación de este tipo de contactos son los Siliciuros (aleación de Silicio con un metal).

\section{Limpieza del sustrato}

En primer lugar se lleva a cabo la limpieza y preparación del sustrato (Si macizo, SOI...). Entre los diversos procesos de limpieza existentes destacans el de limpieza simple y el método RCA (Radio Corporation of America), que es el que se utiliza en el IEMN en la fabricación de los dispositivos que analizaremos en los siguientes capítulos.

Mediante el método de limpieza simple se eliminan los contaminantes orgánicos existentes en la superficie. Para ello, se cubre en primer lugar la superficie de la oblea con acetona. A continuación se frota minuciosamente la oblea con un hisopo y se enjuaga con IPA (alcohol isopropílico). Finalmente la muestra se seca con una pistola de $\mathrm{N}_{2}$.

Por otro lado el método de limpieza RCA es más completo que el anterior, ya que no sólo limpia la superficie de contaminantes orgánicos, sino también de contaminantes óxidos y metálicos mediante la aplicación de diversas soluciones.

\section{Formación del metal sobre el semiconductor}

De entre los diversos métodos para la formación de películas conductoras, describiremos los más empleados en la industria microelectrónica: evaporación, pulverización catódica y deposición química en fase de vapor [Sze, 2001], [Albella, 1996]. 
Evaporación térmica (Physical-Vapor Deposition -PVD-). Esta técnica consiste en el calentamiento de un material metálico por encima de su punto de fusión. Los átomos evaporados se condensan sobre el sustrato de Silicio formando una película. La evaporación se realiza en un aparato de vacío para evitar la oxidación del material.

Existen distintos tipos de evaporación, distinguiéndose entre sí por el procedimiento de calentamiento del material a evaporar. El más sencillo es la evaporación por filamento incandescente, en el que el metal a evaporar se coloca en el interior de filamentos de materiales con un punto de fusión muy alto (como tungsteno, molibdeno, etc.). Se hace circular una corriente por el filamento que produce calor por efecto Joule hasta que se alcanza la temperatura de fusión del metal. El metal en estado líquido libera un vapor de átomos con energía cinética dependiente de la temperatura. Los átomos metálicos viajan en línea recta desde el filamento a la muestra constituyéndose así la capa de metal sobre el semiconductor. Debe tenerse en cuenta que pueden aparecer problemas de contaminación del sustrato con partículas provenientes del filamento.
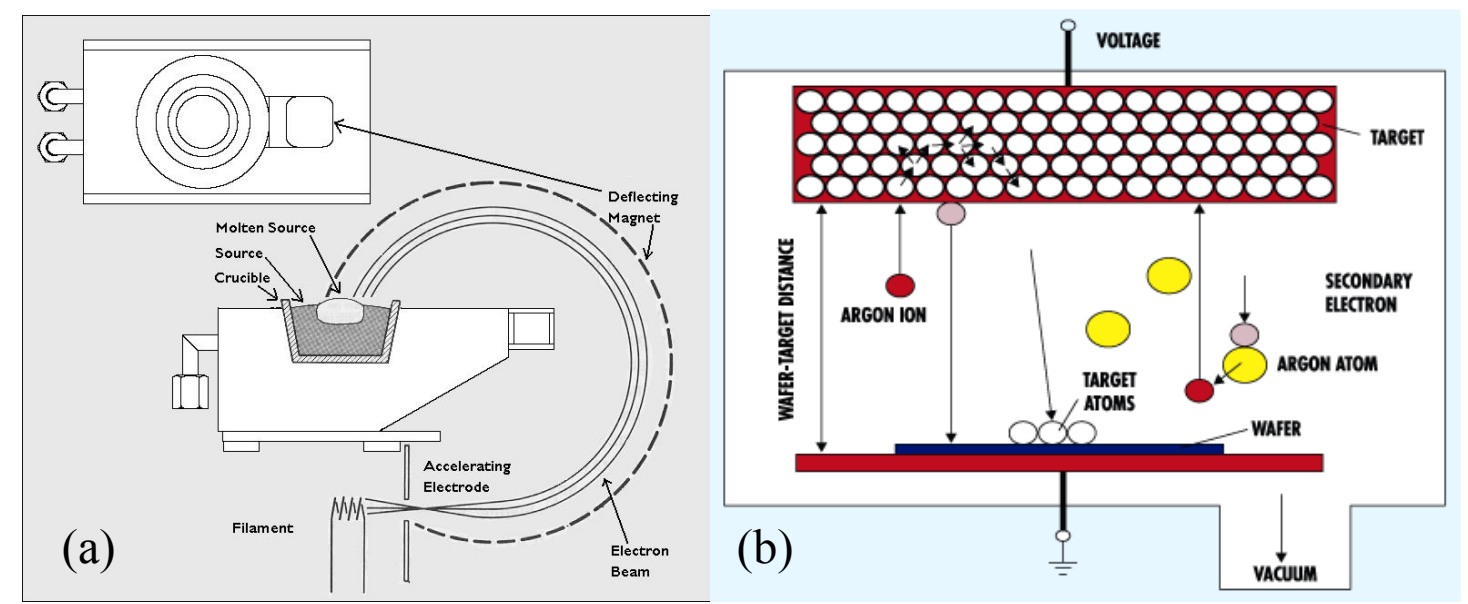

Figura I. 13. Esquema de un sistema de formación del contacto metálico por .a. bombardeo con cañón de electrones y.b. sputtering.

El método de bombardeo por cañón de electrones es muy utilizado en la industria microelectrónica. Según este método, los electrones producidos por un filamento incandescente (usualmente tungsteno) mediante emisión termoiónica son acelerados mediante un voltaje elevado hasta alcanzar unas energías de unos $10 \mathrm{KeV}$. El haz de electrones es enfocado contra el metal a evaporar, efectuando un giro de $270^{\circ}$ por efecto de un campo magnético; esto se realiza para proteger el filamento y prevenir así la contaminación (Ver Figura I.13.a). Puede suceder 
que, al golpear los electrones el metal, aparezcan rayos $\mathrm{X}$ que dañen los diversos componentes de la oblea; estos problemas son tenidos en cuenta en el paso de recocido.

Pulverización catódica (Sputtering). Este procedimiento es el que se utiliza en la fabricación de los dispositivos que estudiaremos en este trabajo en los capítulos posteriores. Está basado en el bombardeo de un cátodo compuesto por el metal a depositar, mediante un haz de iones. La generación de estos iones se consigue con la descarga eléctrica entre un ánodo y el propio cátodo. Durante el bombardeo del cátodo, los iones le transfieren su momento cinético y de este modo, los átomos de la superficie son expulsados para condensarse en el sustrato a recubrir. El sputtering se lleva a cabo en una cámara de vacío, que será evacuada antes de introducir el gas utilizado para la descarga. El gas elegido debe ser inerte para que no reaccione con el cátodo ni el substrato (típicamente $\mathrm{Ar}^{+}$, dado su bajo coste y su elevada masa). En la Figura I.13.b mostramos el esquema típico de un equipo de sputtering.

Esta técnica presenta una gran ventaja sobre la evaporación y es que no es necesario calentar el material a depositar a altas temperaturas, haciendo así que sea muy fácil trabajar con materiales de alto punto de fusión, como el tungsteno, el carbón, etc. Por otro lado, también permite una correcta deposición de las aleaciones (más difíciles de tratar mediante evaporación, ya que los componentes de la misma suelen tener distinto punto de evaporación).

Deposición química en fase de vapor (Chemical-Vapor Deposition CVD). Se lleva a cabo mediante el uso de compuestos volátiles, como haluros u organometálicos, que contengan el metal a depositar. Se hace reaccionar a estos compuestos con el sustrato sometiéndolos a temperaturas relativamente altas en el interior de una cámara. Esta técnica se suele emplear para la deposición de Tungsteno, Cobre o Aluminio.

En el caso de los contactos Schottky fabricados dentro del proyecto METAMOS que analizaremos en posteriores capítulos, se emplean Siliciuros, como el ErSi para la formación de los contactos metal - semiconductor tipo $n$ o el PtSi para contactos metal - semiconductor tipo p. El método empleado en la formación del Siliciuro sobre el semiconductor es el de la deposición por evaporación térmica (en el caso de las muestras tipo $n$ fabricadas en la Universidad de Lovaina) y mediante sputtering de $\operatorname{Ar}$ (en el de las muestras tipo $p$ fabricadas en el IEMN). 


\section{Activación térmica}

El último paso del proceso de formación del contacto es la activación térmica. Se someterá al material a fuentes intensas de calor en hornos de recocido, con el fin de conseguir la completa adherencia del metal al Silicio.

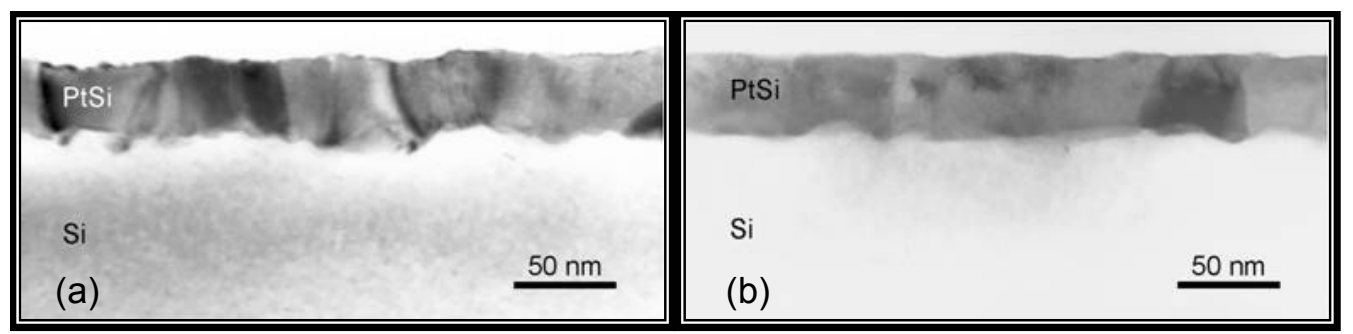

Figura I. 14. Diferencias en la interfaz de un contacto Siliciuro de platino-semiconductor: a. sin activación térmica, .b. con activación térmica (recocido). Como puede observarse, el recocido permite mejorar notablemente la calidad de la interfaz, consiguiéndose que sea más homogénea y con menos imperfecciones. (Imagen perteneciente al proyecto europeo METAMOS)

Este proceso también puede llevarse a cabo a mediante el sistema RTP clásico (rapid thermal processor), en el que se aumenta la temperatura de las obleas a valores elevados (por encima de los $1200^{\circ} \mathrm{C}$ ) en una escala de tiempo de pocos segundos. Estos procesos de calentamiento tan rápidos son llevados a cabo por lámparas de alta intensidad.

\section{Materiales empleados: Siliciuros}

El Aluminio fue el metal más utilizado al principio para contactos en semiconductores, debido a su alta conductividad eléctrica y a su facilidad para formar contactos óhmicos con el Silicio. Sin embargo, presenta varios inconvenientes, como es su bajo punto de fusión y la solubilidad del Silicio en el mismo. En la actualidad, para la fabricación de contactos tipo Schottky destaca el uso de materiales conocidos como Siliciuros metálicos, que pueden soportar procesos de temperatura más elevados que el Al. Esto es debido a que poseen una conductividad próxima a la de los metales y mayor estabilidad mecánica que estos de cara a la oxidación.

La formación de los Siliciuros metálicos se realiza siguiendo las mismas metodologías que expusimos anteriormente para la formación de un metal sobre el semiconductor: mediante deposición del metal puro sobre una capa de Si, evaporación simultánea del Silicio y el metal a partir de dos fuentes, deposición mediante sputtering o deposición química en fase de vapor. 
Después de la deposición del metal en el Silicio, se somete a la oblea a altas temperaturas para favorecer las reacciones químicas entre el metal y el Silicio necesarias para formar el Siliciuro. En dicha reacción metalúrgica, los Siliciuros ricos en metal se forman primero y continúan creciendo hasta que se ha consumido el metal completamente; en este momento, los Siliciuros con menor contenido de metal comienzan a surgir. En este proceso de formación del Siliciuro, se consume Silicio del substrato sobre el que se depositó el metal, por eso es importante que haya suficiente Silicio disponible para la reacción.

En el caso de la fabricación de contactos Schottky mediante el contacto del Siliciuro con $\mathrm{Si}$, estos materiales proporcionan las menores alturas de barrera, característica fundamental para la optimización de dispositivos basados en este tipo de contacto (como los diodos Schottky y los SB-MOSFETs), tal y como veremos en capítulos posteriores.

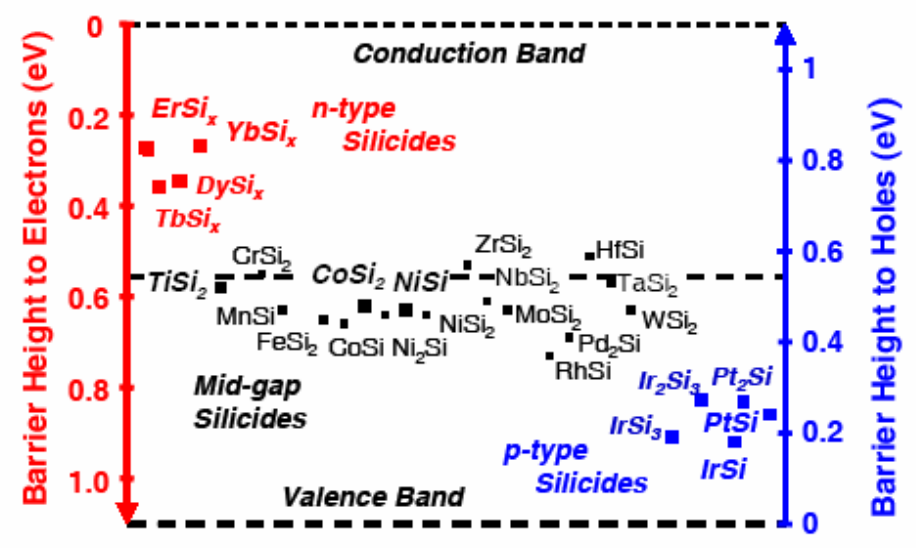

Figura I. 15. Alturas de barrera Schottky para distintos Siliciuros tipo n, $p$ y mid-gap.

Desde el punto de vista experimental se convierte así en fundamental la tarea de buscar el contacto Siliciuro/Silicio que proporcione una menor altura de barrera. En la Figura I.15 mostramos las alturas de barrera que se obtienen para contactos Schottky con distintos tipos de Siliciuros: En el caso de los contactos tipo $p$, la menor altura de barrera para huecos se obtiene usando el Siliciuro de Platino (PtSi) y es de $0.22 \mathrm{eV}$ aproximadamente [Dubois, 2004]. Por otro lado, para aquellos contactos tipo $n$ son los Siliciuros de Lantánidos o tierras raras los que proporcionan la menor altura de barrera; los más utilizados son el Siliciuro de Erbio $\left(\operatorname{ErSi}_{\mathrm{x}}\right.$, de $0.28 \mathrm{eV}$, ver [Kedzierski, 2000]) y el de Yterbio (YbSi, $0.27 \mathrm{eV}$, [Zhu, 2004]). En el presente trabajo, estudiaremos en primer lugar contactos Schottky con Siliciuro de Zirconio $\left(\mathrm{ZrSi}_{2}\right)$, de Rodio (RhSi) y de Platino (PtSi), los cuales presentan una altura de barrera mayor, pero que 
presentan las condiciones adecuadas para testar inicialmente el funcionamiento de nuestro simulador, como ya veremos en el Capítulo III. Por otro lado, los dispositivos back-to-back experimentales que analizaremos en el Capítulo IV o el SB-MOSFET en el Capítulo V, presentan alturas de barreras reducidas, de acuerdo con el estado-del-arte actual. 


\section{Bibliografía del Capítulo I}

Albella J. M. Y Martínez-Duartz J. M., "Fundamentos de electrónica física y microelectrónica", Addison-Wesley / Universidad Autónoma de Madrid (1996)

Andrews J. M. and Lepselter M. P, "Reverse current-voltage characteristics of metal-Silicide Schottky diodes", Solid State Electronics, 13, 1011 (1970)

Bardeen J., "Surface States and Rectification at a Metal Semi-Conductor Contact", Phys. Rev. 71, 717 (1947)

Bethe H. A., "Theory of the boundary layer of crystal rectifiers", Mass. Inst. Technol. Radiat. Lab. Rep. 43-12 (1942)

Brattain W.H., Bardeen J., Bell Systems Tech. J., 32, 1 (1953)

Chang C. Y. and Sze S. M. "Carrier transport across Metal-semiconductor barriers", Solid-State Electron, 13, 727 (1970)

Cowley A. M. and Sze S. M. "Surface States and Barrier Height of Metal-Semiconductor Systems", J. Appl. Phys., 36, 3212 (1965)

Crowell C. R., Sze S. M., Spitzer W. G., "Equality of the temperature dependence of the GoldSilicon surface barrier and the silicon energy gap in Au n-type Si diodes", Appl. Phys. Lett., 4, 91 (1964)

Crowell C. R., "The Richardson constant for thermionic emission in Schottky barrier diodes", Solid State Electronics, 8, 395 (1965)

Crowell C. R. and Sze S. M., "Electron-Optical-Phonon scattering in the emitter and collector barriers of semiconductor-metal-semiconductor structures", Solid-State Electron, 8, 979 (1965b)

Crowell C. R. and Sze S. M., "Current Transport in Metal-Semiconductor barriers", Solid-State Electron, 9, 1035 (1966)

Crowell C. R., "Richardson constant and tunneling effective mass for thermionic and thermionic-field emission in Schottky barrier diodes", Solid-State Electronics, 12, 55 (1969) 
Crowell C. R. and Rideout V. L., "Normalized Thermionic-fielg emission in metalsemiconductor (Schottky) barriers ", Solid-State Electronics, 12, 89 (1969b)

Dubois E. and Larrieu G., "Measurement of low Schottky barrier heights applied to metallic source-drain metal-oxide-semiconductor field effect transistors" J. Appl. Phys., 91, 729 (2004)

Heine V. "Theory of Surface States", Phys. Rev., 138, 1689 (1965)

Jiménez J. R., Xiao X., Sturm J.C., Pellegreni P.W., “Tunable, long-wavelength PtSi/SiGe/Si Schottky diode infrared detector", Applied Physics Letters, 67, 506 (1995)

Kedzierski J., Xuan P. et al., "A 20 nm gate-length ultra-thin body p-MOSFET with silicide source/drain”, Superlattices and Microstructures, 28, 445 (2000)

Larrieu G. "Elaboration et caractérisation de transistors MOS Schottky en regime nanométrique", Tesis Doctoral, Universidad de Ciencias y Tecnología de Lille (2004)

Leroux M., Grandjean N., Beaumont B., Nataf G., Semond F., Massies J., Gilbart P., "Temperature quenching of photoluminescence intensities in undoped and doped GaN" $J$. Appl. Phys. 86, 3721 (1999)

Mönch W., "Electronic properties of semiconductor interfaces", Springer, Germany (2004)

Morrison S. R., J. Phys. Chem. 57, 860 (1953)

Mott N. F., "Note on the contact between a metal and an insulator or semiconductor", Proc. Cambr. Phil. Soc. 34, 568 (1938)

Neudeck G. W., "El diodo PN de Unión", $2^{a}$ Edn. Addison-Wesley Iberoamericana, S. A., Delaware, USA (1993)

Neamen D. A., "Semiconductor Physics and devices", Richard Irwin (1992)

Pardo D. y Bailón L. A., "Elementos de electrónica", Secretariado de Publicaciones e Intercambio Editorial, Universidad de Valladolid (2007)

Rideout V. L., "A Review of the Theory, Technology and Applications of Metal-Semiconductor Rectifiers", Thin Solid Films, 48, 261 (1978)

Roberts G. I. and Crowell C. R. "Capacitive effects of $\mathrm{Au}$ and $\mathrm{Cu}$ impurity levels in Pt n-type Schottky barriers", Solid State Electronic., 16, 29 (1973)

Schottky W., Phys. Z., 15, 872 (1914)

Schottky W., "Halbleirtertheorie der Sperrschicht", Naturwissenschaften, 26,843 (1938)

Schottky, W., Physik. Zeitschr. 30, 839 (1940)

Shalish I., Kronik L., Segal G., Shapira Y., "Yellow luminescence and Fermi level pinning in GaN layers", Applied Physics Letters, 77, 987 (2000) 
Shockley, W. And Pearson G. L., Physic Rev. 74, 232 (1948)

Sze S. M., "Physics of Semiconductor Devices", $2^{\text {nd }}$ Edn. Wiley, New York (1981)

Sze S. M., "Semiconductor Devices. Physics and Technology", $2^{\text {nd }}$ Edn. John Wiley and Sons Inc., New York (2001)

Tung R. T., "Recent advances in Schottky barrier concepts", Material Science and Engineering $R, 35,1(2001)$

Zhu S. et al., "N-Type Schottky Barrier Source/Drain MOSFET Using Ytterbium Silicide" IEEE Elec. Dev. Letters, 25, 565 (2004)

Zubrilov A. S., Nikishin S. A., Kipshidze G. D., Kuryatkov V. V., Temkin H., Prokofyeva T. I., Holtz M. "Optical properties of GaN grown on $\mathrm{Si}$ (111) by gas source molecular beam epitaxy with ammonia”, J. Appl. Phys. 91, 1209 (2002) 


\section{Método de Monte Carlo. Algoritmo de inyección/absorción en barrera Schottky}

as simulaciones realizadas a lo largo de este trabajo para el análisis de las distintas
estructuras bajo estudio, han sido llevadas a cabo mediante un simulador propio (desarrollado en el área de Electrónica de la Universidad de Salamanca, dentro del grupo de investigación de dispositivos de Silicio), basado en el Método Monte Carlo de dispositivos [González, 1994], [Martín, 1996b], [Rengel, 2002]. En este capítulo presentaremos las principales características de este método, comúnmente reconocido como uno de los más adecuados para el estudio de los dispositivos electrónicos debido a su carácter microscópico. Nos centraremos principalmente en las modificaciones que han sido implementadas en el simulador para abordar de manera adecuada el análisis de los dispositivos con contactos de barrera Schottky, objetivo principal de esta Memoria.

A la hora de realizar un estudio del transporte de carga en semiconductores, podemos llevarlo a cabo mediante varios modelos. Comenzaremos en la primera sección presentando cuáles son estos modelos y mostrando tanto sus ventajas como sus principales inconvenientes. Posteriormente, en la segunda sección, describiremos los fundamentos del método de Monte Carlo, mientras que en la sección siguiente describiremos la estructura de la simulación, así como su aplicación al estudio de dispositivos electrónicos, prestando especial atención a los procesos de importancia que tendrán lugar en los contactos Schottky. En la cuarta sección 
presentaremos distintas aproximaciones para el cálculo de la probabilidad de transmisión cuántica a través de la barrera Schottky y justificaremos de entre todas ellas nuestra elección de la aproximación WKB. Finalmente, explicaremos con detalle cómo se ha realizado la implementación del algoritmo de inyección/absorción basado en esta aproximación en nuestro simulador Monte Carlo de dispositivos.

\section{II.1. Métodos de estudio del transporte de carga}

Para el estudio de un dispositivo nos valemos de ciertas magnitudes macroscópicas que describen el comportamiento de los portadores; dichas magnitudes son el resultado de una serie de procesos microscópicos que se dan en el interior del dispositivo. La ecuación que describe estos procesos (y por tanto el transporte de carga en el interior del semiconductor) es la ecuación de transporte de Boltzmann [Snowden, 1986]. Dado que la dimensión en la dirección del transporte de los dispositivos simulados a lo largo de esta Memoria es muy superior a la longitud de onda de De Broglie del portador, podemos considerar que la aproximación semiclásica es válida. Esto es, supondremos que el portador está totalmente localizado (conocemos su posición y su momento en todo instante de tiempo) a cambio de considerar al portador como una partícula con masa dependiente de la energía (aproximación de masa efectiva) y una carga positiva o negativa dependiendo de su posición en el sistema de bandas de energía ${ }^{1}$.

Por tanto, el objetivo primero para poder hacer una descripción del transporte de carga es obtener la solución de la ecuación de Boltzmann, lo cual es una labor extremadamente complicada en la mayoría de los casos. Por este motivo se recurre a diversos modelos numéricos, que realizan aproximaciones más o menos restrictivas según su complejidad. Entre ellos destacaremos los siguientes: el modelo de deriva-difusión, el modelo hidrodinámico y el método de Monte Carlo.

El modelo de deriva-difusión [Snowden, 1986] es de tipo macroscópico, y entre sus principales hipótesis destaca el hecho de que considera a los portadores en equilibrio térmico permanente con la red, y además supone que el ajuste de la velocidad con el campo aplicado es

\footnotetext{
${ }^{1}$ Esta consideración afecta al movimiento de los portadores dentro del semiconductor, y es independiente del tratamiento de los fenómenos cuánticos involucrados en el transporte por efecto túnel, que serán descritos con más detalle en el siguiente capítulo.
} 
instantáneo. El resultado es un modelo muy sencillo, que necesita de pocos recursos de cálculo y con gran fiabilidad en muchos casos. Sin embargo, los inconvenientes surgen a medida que las condiciones de la simulación contradicen las hipótesis básicas de partida. Por ejemplo, no permite estudiar los efectos asociados a portadores calientes [Lugli, 1993], ya que como hemos mencionado considera en todo momento que los portadores se encuentran en equilibrio térmico con la red. Debido a que se exige una respuesta instantánea de la velocidad de los portadores frente al campo eléctrico total, este modelo es incapaz de reflejar los efectos de sobrevelocidad que puedan aparecer en el estudio del trasporte de los portadores; por tanto, no es adecuado para el análisis de dispositivos cuyas dimensiones sean comparables a la distancia sobre la cual aparecen los fenómenos de no equilibrio portador-red [Brennan et al. 1983], [Brennan y Hess, 1984].

El modelo hidrodinámico [Constant, 1980], también de carácter macroscópico, aporta grandes mejoras sobre el modelo de deriva-difusión a costa de una mayor complejidad y aumento del tiempo de cálculo. En este modelo se emplea la ecuación de Boltzmann de manera completa, considerando la dependencia de los parámetros tanto con el momento como con la energía. Las ventajas de este modelo sobre el de deriva-difusión son, entre otras, que permite describir fenómenos no estacionarios (por ejemplo la sobrevelocidad), por tanto, es adecuado para el estudio de transitorios en los dispositivos y de fenómenos de portadores calientes [Lugli, 1993]. Estas características hacen que el método sea muy adecuado y fiable para el estudio de numerosas magnitudes y para abordar el análisis de dispositivos submicrométricos; sin embargo, presenta limitaciones importantes, como por ejemplo la necesidad de que le proporcionen la dependencia de numerosos parámetros con la energía. Para este fin suelen emplearse resultados obtenidos con modelos más complejos, como es el caso de las simulaciones Monte Carlo, de las que sólo puede emplear los valores estacionarios.

Un problema común a ambos modelos es que no proporcionan la función de distribución instantánea de la velocidad de los portadores o la corriente instantánea en los terminales, con lo que su aplicación para el estudio de ruido en dispositivos es limitada. Para ello, se suele emplear el método del campo de impedancia [Shockley et al., 1966], [Van Vliet, 1975], [van de Roer 1994] que, a pesar de que en numerosas condiciones ofrece resultados satisfactorios, parte de una consideración macroscópica del sistema. 
El método de Monte Carlo [Kurosawa, 1966], [Kurosawa, 1966b] surge como un modelo de resolución de la ecuación de Boltzmann en cada instante, basándose en una descripción microscópica del movimiento de los portadores en el interior del dispositivo. Presenta, por tanto, múltiples ventajas frente a los demás modelos [Jacoboni 1989], [González, 1994], [Martín, 1996b], entre las cuales destacamos:

- Reproduce de manera natural el movimiento de los portadores dentro de la red cristalina del semiconductor, considerando los diferentes mecanismos de dispersión o scattering que tienen lugar dentro del mismo.

- Tiene en cuenta los efectos que surgen en dispositivos de dimensiones reducidas, tales como portadores calientes o fenómenos no estacionarios.

- Permite el estudio de las características tensión-corriente al proporcionar de manera instantánea el valor de la corriente entre los terminales del dispositivo. Además proporciona valores medios y funciones de distribución de magnitudes de interés, tales como velocidad y energía de los portadores, concentración, perfiles de potencial y campo eléctrico, etc.

- Proporciona el valor medio de magnitudes microscópicas como el tiempo de tránsito, el número de mecanismos de scattering, el tiempo medio entre mecanismos de scattering, el recorrido libre medio, la longitud recorrida longitudinal y trasversal y la total, así como las funciones de distribución de las mismas.

- Presenta la posibilidad de considerar determinados fenómenos cuánticos (como es el estudio del trasporte de portadores a través de barreras de potencial) mediante su acoplamiento con diversos métodos de resolución de la ecuación de Schrödinger, así como la inclusión de los efectos de la cuantización espacial mediante el método del potencial efectivo, por mencionar dos ejemplos.

La gran potencialidad del método puede también comprobarse por el hecho de que además permite analizar el comportamiento a alta frecuencia de los dispositivos, tanto su respuesta dinámica de como el ruido electrónico, ya que la propia naturaleza de la simulación reproduce los fenómenos causantes del mismo. Sin embargo, presenta el principal inconveniente de un alto consumo de recursos de computación, problema que en cierta manera se va solventando con los constantes avances en la velocidad y potencia de los medios informáticos.

Dependiendo del sistema físico que se vaya a estudiar, el nivel de complejidad para el método Monte Carlo puede variar. Cuando se quiere efectuar el estudio del transporte de carga en semiconductores homogéneos en condiciones estacionarias, el modelo más sencillo y 
adecuado es el Monte Carlo de partícula única [Jacoboni y Reggiani, 1983]. Basándose en el principio de ergodicidad, simula el movimiento de un único portador durante un tiempo lo suficientemente largo; de ese modo permite obtener magnitudes como velocidad, energía o movilidad de los portadores, así como sus funciones de distribución en función del campo eléctrico. Si se desea estudiar fenómenos no estacionarios y procesos de difusión en semiconductores homogéneos, el método más adecuado es el Monte Carlo multipartícula (o ensemble Monte Carlo) [Jacoboni y Reggiani 1983, Jacoboni y Lugli 1989]; basado en la simulación de un conjunto de partículas en el material semiconductor.

En el caso de los dispositivos, debemos modificar el Monte Carlo multipartícula para considerar dos propiedades importantes de los mismos: la primera es la restricción espacial al movimiento de los portadores, por lo que se deben establecer las condiciones de contorno adecuadas para las partículas que alcanzan las fronteras del dispositivo. La segunda propiedad a tener en cuenta es que el cálculo del potencial eléctrico debe realizarse autoconsistentemente con la distribución espacial de las partículas a través de la resolución de la ecuación de Poisson con las condiciones de contorno apropiadas. Por tanto, es necesario acoplar un simulador multipartícula con la resolución autoconsistente de la ecuación de Poisson, obteniendo el llamado Monte Carlo de dispositivos [Moglestue, 1986]. Mediante este método pueden simularse las geometrías complejas que corresponden a los dispositivos en una, dos o tres dimensiones, según los requerimientos del problema en concreto.

Cabe destacar que para el estudio de las estructuras de Silicio que presentamos en este trabajo ha sido necesario adaptar el simulador Monte Carlo específicamente a la física de estos dispositivos; con este fin, hemos desarrollado un algoritmo de inyección/absorción que permite tratar los efectos de transporte a través de barreras de potencial mediante túnel cuántico directo. Tras el análisis de barreras de potencial para comprobar el correcto funcionamiento del algoritmo, afrontaremos el estudio de diversas estructuras: En primer lugar, analizaremos dispositivos en los que puede considerarse como aproximación que el transporte se lleva a cabo en la dirección longitudinal [como es el caso de los diodos Schottky o los diodos back-to-back (diodos con dos contactos Schottky enfrentados en sus extremos)], para ello nos valdremos el simulador Monte Carlo unidimensional (a partir de este momento denominaremos al modelo MC1D por comodidad), obteniendo resultados igualmente precisos que si escogiéramos uno bio tridimensional, con la gran ventaja de una notable reducción del tiempo de cálculo. Finalmente, estudiaremos estructuras en las que no puede simplificarse el trasporte como 
unidimensional, pero sí considerar que es de tipo bidimensional (SB-MOSFET), haciéndose imprescindible el uso del simulador Monte Carlo bidimensional (MC2D). Como veremos en la Sección II.5, el algoritmo de inyección/absorción está basado en la aproximación WKB. Inicialmente ha sido implementado en el simulador MC1D, y con la experiencia adquirida en el estudio de los diodos, posteriormente realizamos la implementación en el MC2D para el análisis de los dispositivos SB-MOSFET. Sin embargo, debido a las características inherentes del caso bidimensional, el tratamiento de los procesos túnel en contactos Schottky 2D presenta una gran complejidad. Por ejemplo, el perfil de potencial 2D no se correspondería al de una unión Schottky en condiciones de polarización convencional debido al efecto modulador inducido por el contacto de puerta; ello exigió un importante trabajo en la fase de depuración, haciéndonos considerar un rango más amplio de condiciones y aumentando notablemente el número de líneas de código, sirviendo de hecho para mejorar el algoritmo inicial.

Existen en la literatura otros simuladores avanzados que incorporan fenómenos de carácter estadístico, como es el presentado por [García-Loureiro, 2005], [Aldegunde, 2006], que les permite estudiar el efecto de la fluctuación de diversos parámetros (influencia del polisilicio de puerta, posición de dopantes, dieléctricos de alta permitividad, etc.) en MOSFETs avanzados mediante un simulador tridimensional de elementos finitos. Este mismo grupo de investigación también ha presentado un modelo Monte Carlo tridimensional incorporando elementos finitos tetraédricos para el estudio de MOSFETs convencionales de tres puertas [Aldegunde, 2008].

Por otro lado, encontramos modelos Monte Carlo que resuelven de manera autoconsistente las ecuaciones unidimensionales de Poisson y de Schrödinger, empleados en el estudio de transistores SOI convencionales de doble puerta [Gámiz, 2004], [Sampedro, 2005] o bidimensionales para el estudio de efectos cuánticos en transistores multipuerta [Godoy, 2007].

\section{II.2. Fundamentos del método Monte Carlo}

El movimiento de los portadores en el interior de un semiconductor se encuentra sometido a la acción de un campo eléctrico aplicado y a las interacciones con la red cristalina circundante [Yang, 1988], la cual transfiere al mismo una masa efectiva, además de alterar su movimiento mediante colisiones o mecanismos de scattering [Nag 1980, Madelung 1981, Ridley 1983]. Muchos parámetros, como la frecuencia de estos mecanismos, están gobernados por distribuciones de probabilidad complejas, difíciles de manejar analítica y numéricamente. 
Como consecuencia, el método de Monte Carlo precisa de la generación de números aleatorios con distribuciones de probabilidad adecuadas. La función aleatoria más conveniente y sencilla, es la distribución uniforme entre 0 y 1 , que cuenta además con la ventaja de estar disponible fácilmente en cualquier compilador informático. Podremos así obtener la solución de la ecuación de Boltzmann en cada instante de tiempo sin necesidad de hacer las restricciones propias de los modelos macroscópicos.

A partir de la distribución uniforme puede obtenerse cualquier otra. Sean $p(r)$ y $q(\phi)$ las densidades de probabilidad de dos distribuciones, $p(r)$ correspondiente a una distribución uniforme con $r$ entre 0 y 1 (implementada de manera pseudoaleatoria en los compiladores informáticos) mientras que $q(\phi)$ es la densidad de probabilidad de una distribución de probabilidad arbitraria. Si $p(r)$ y $q(\phi)$ están normalizadas podemos escribir [Boardman, 1980]:

$$
\int_{0}^{\phi} q\left(\phi^{\prime}\right) d \phi^{\prime}=\int_{0}^{r} p\left(r^{\prime}\right) d r^{\prime}
$$

En una distribución uniforme $p(r)=1$ por lo que la ecuación anterior pasa a ser:

$$
r=\int_{0}^{\phi} q\left(\phi^{\prime}\right) d \phi^{\prime}
$$

En el caso en que sea posible evaluar de forma analítica esta integral, obtendremos el valor aleatorio de $\phi$ sin más que despejarla en función de $r$. En el caso contrario, pueden aplicarse ciertas técnicas para poder realizar la inversión que proporciona $\phi$.

Este procedimiento es la base del método de Monte Carlo y debe aplicarse a cada mecanismo que actúe de manera aleatoria en el sistema bajo estudio. A pesar de que es necesario un conocimiento previo de las probabilidades de aparición de cada mecanismo aleatorio, posteriormente no se impone ninguna condición sobre la naturaleza del problema, por lo que es aplicado en numerosos campos de la ciencia.

\section{II.3. Estructura de la simulación Monte Carlo}

En esta sección estudiaremos de manera sucinta la estructura del simulador Monte Carlo bipolar de dispositivos que hemos empleado en la presente Memoria (para un mayor detalle nos remitimos a las anteriores tesis desarrolladas en el área, dentro del grupo de Silicio [Martín, 
1996b], [Rengel, 2002]). Para ello, nos basaremos en el diagrama de flujo del simulador bidimensional (las diferencias con el simulador unidimensional serán mencionadas en aquellas secciones que sea necesario), analizando los distintos niveles del mismo y comentando sus principales características.

\section{II.3.a. Definición del semiconductor}

Como se observa en la Figura II.1, la simulación comienza con la definición física de los materiales semiconductores considerados en el dispositivo en cuestión. Los parámetros físicos correspondientes al Silicio utilizados en las simulaciones se encuentran detallados en el Apéndice. Es necesario destacar que una vez descrita la estructura de bandas del semiconductor y los mecanismos de scattering, será posible proceder a la simulación propiamente dicha del dispositivo sin necesidad de hipótesis adicionales, característica en la cual radica la gran potencia del método como herramienta de simulación. En el Apéndice encontraremos una discusión detalla acerca del modelo de bandas de energía y de los diferentes mecanismos de scattering de electrones y huecos, sus funciones de probabilidad, así como los diferentes parámetros empleados en las mismas. Este modelo físico para el Silicio ha sido validado mediante la comparación con medidas experimentales encontradas en la literatura para diferentes magnitudes (véase también el Apéndice).

Bandas de energía. El modelo necesita construir las trayectorias semiclásicas de la partícula considerada en el espacio fásico. Éstas estarán condicionadas tanto por la estructura de bandas como por los mecanismos de scattering presentes en el cristal.

La aproximación empleada para la estructura de bandas consiste en una descripción de los valles X y L de la banda de conducción del Silicio como bandas elipsoidales no parabólicas [Cohen, 1988], [Tomizawa, 1993], mientras que para la banda de valencia se consideran subbandas de huecos ligeros y pesados [Kane, 1965], [Madelung, 1981] como bandas esféricas no parabólicas [Martín, 1996b] (véanse más detalles en el Apéndice). 


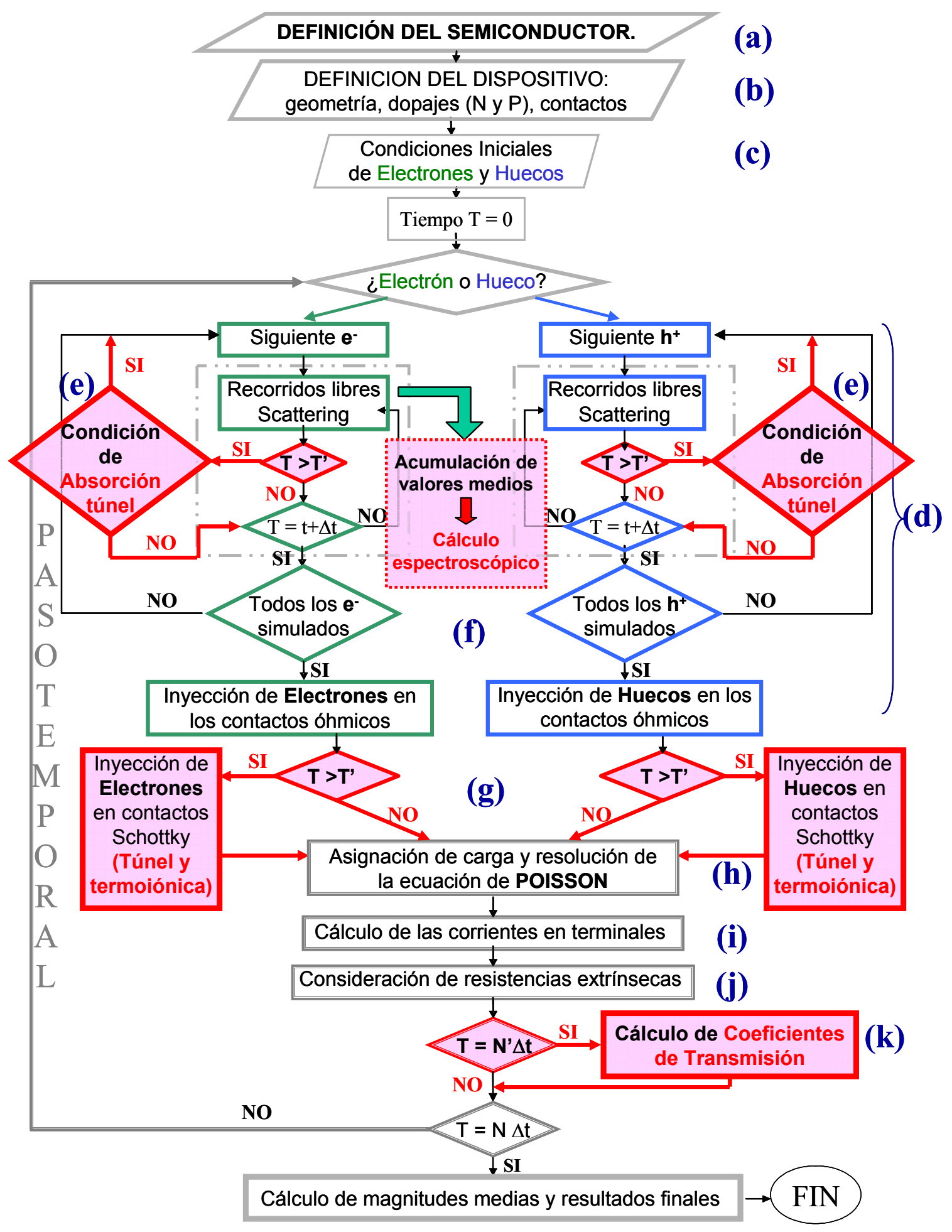

Figura II.1. Diagrama de flujo de la simulación Monte Carlo de dispositivos 
Es importante destacar el hecho de que, en el espacio real, la simulación tiene carácter unidimensional en el caso del simulador MC1D y bidimensional en el MC2D, mientras que en el espacio fásico es tridimensional. Además, es posible simular tanto electrones como huecos, lo que nos permitirá efectuar el modelado tanto de diodos tipo $p$ como de diodos tipo $n$. Por otro lado, dado que a pesar de que el transporte en transistores de tipo MOSFET es de carácter unipolar, para poder efectuar una simulación lo más completa y precisa posible (que tenga por ejemplo en cuenta los efectos dinámicos asociados al substrato o fenómenos de ionización por impacto) es necesario simular ambos tipos de portadores [Rengel et al. 2001].

Mecanismos de scattering. En un cristal real, los portadores ven modificado su movimiento debido a la interacción con la red cristalina, a través de colisiones o mecanismos de scattering con las imperfecciones de la misma o mediante el efecto de las vibraciones de los átomos del cristal. Se produce así una tendencia a la relajación de la energía y del momento de los portadores, que se mantienen por tanto en los extremos de la banda de conducción y de valencia, lo que permite considerar como válida la aproximación que hemos tenido en cuenta para las bandas de energía.

Los mecanismos de scattering considerados en este trabajo para electrones son: interacción con impurezas ionizadas, mecanismos intravalle con fonones acústicos (inelástico), y mecanismos intervalle con fonones ópticos (entre valles equivalentes y no equivalentes). Para los huecos se ha tenido en cuenta la interacción con impurezas ionizadas, el scattering con fonones acústicos y el scattering con fonones ópticos. Además de estos mecanismos, hemos considerado el scattering de superficie y los fenómenos de ionización por impacto [Sze, 1981], [Jacoboni y Lugli, 1989], [Tsividis, 1999], aunque no sea un mecanismo de scattering propiamente dicho. La definición de los mecanismos así como sus probabilidades están reflejadas en el Apéndice.

Sería posible tener en cuenta otros mecanismos de scattering además de los considerados, como las colisiones portador-portador ${ }^{2}$ o las interacciones con defectos del cristal, etc. sin embargo dichas interacciones no serán de importancia en las estructuras simuladas en la presente Memoria, por lo que no han sido consideradas en el modelo.

\footnotetext{
${ }^{2}$ Cuando la concentración de portadores en el semiconductor es muy elevada (superior a $1.5 \cdot 10^{25} \mathrm{~m}^{-3}$ en el Silicio), la probabilidad de que el estado energético que va a ocupar el electrón tras la colisión esté lleno no es despreciable. El principio de exclusión de Pauli impide que se ocupen estados energéticos previamente poblados.
} 


\section{II.3.b. Definición física del dispositivo}

El segundo paso de la simulación engloba la definición del dispositivo, para lo que deben determinarse de manera precisa las dimensiones, dopaje de las regiones (tipo $n$ o tipo $p$ ), posición de los contactos, etc. A continuación se realiza una discretización espacial del dispositivo que permita resolver adecuadamente la ecuación de Poisson. Se definirá pues un mallado en el que el tamaño de las celdas debe ser inferior a la longitud de Debye del material ${ }^{3}$, $\lambda_{D}$, de modo que permita efectuar el correcto seguimiento de las variaciones espaciales del campo eléctrico [Jacoboni y Lugli, 1989].

El campo eléctrico debe, además, actualizarse mediante la resolución de la ecuación de Poisson en los intervalos temporales adecuados. El paso temporal ha de seleccionarse de modo que su valor máximo quede limitado por el inverso de la frecuencia de las oscilaciones del plasma $^{3}, \omega_{p}$, para considerar únicamente aquéllas que se producen de manera natural [Hockney, 1988] y también por el tiempo de relajación dieléctrico ${ }^{3}, \tau_{d}$, para evitar inestabilidades asociadas a redistribuciones de carga. Elegiremos el paso temporal $\Delta t$ que cumpla que $\omega_{p} \Delta t<2$ y $\Delta t<\tau_{d}$, tomando la mayor $\omega_{p}$ de todos los materiales del dispositivo y el menor $\tau_{d}$. Además, se fijarán otros parámetros importantes, como son la temperatura, la tensión aplicada en los contactos, etc.

Llegados a este punto, debemos señalar el hecho de que en el MC1D y MC2D no se simulan todos los portadores que existirían físicamente en el dispositivo, puesto que tal situación sería inabordable desde el punto de vista computacional. Es por ello que cada partícula simulada representa a un número determinado de portadores (electrones o huecos) "reales"; esto es lo que se denomina equivalente partícula-electrón $(E P E)$ o equivalente partícula-hueco $(E P H)$ según sea el caso ${ }^{4}$. Por tanto, también debe determinarse el número de partículas a considerar, así como el EPE y EPH, que deben ser elegidos llegando a un compromiso entre precisión de los resultados y reducción en el tiempo de cálculo.

\footnotetext{
${ }^{3}$ En el Apéndice podemos encontrar la definición de estos parámetros $\left(\lambda_{D}, \omega_{p} \mathrm{y} \tau_{d}\right)$ así como una tabla de valores para los materiales simulados en la Memoria.

${ }^{4}$ En el caso de dispositivos reales, para un correcto análisis de los mismos este parámetro debe corresponder al inverso de la dimensión no simulada.
} 


\section{Técnicas de realce estadístico}

Cuando nos enfrentamos a casos en los que la concentración de portadores varía en muchos órdenes de magnitud a lo largo del dispositivo y/o se desea analizar estados de alta energía, cuyos niveles de ocupación son poco probables [Hockney, 1988], [Lugli, 1993], el tiempo de CPU requerido por el método Monte Carlo para realizar la simulación aumenta demasiado. Este es el caso de los diodos Schottky en las condiciones que nos interesan en el presente trabajo, donde les someteremos a un gran rango de voltajes, tanto en directa como en inversa. En particular, estamos interesados (como ya veremos en los posteriores capítulos de resultados) en el rango de polarización en inversa donde, debido a la débil población de partículas equivalentes que existe en la región del contacto metal-semiconductor comparado con las regiones de alto dopaje del dispositivo, existirían en principio grandes fluctuaciones en el cálculo de las magnitudes físicas de interés (velocidad, energía...).

Para solucionar este problema en nuestro simulador Monte Carlo unidimensional, emplearemos técnicas de realce estadístico mediante un algoritmo de asignación de peso variable a las partículas simuladas. De este modo, dependiendo de la región del dispositivo en que estén localizadas, equivaldrían a un número distinto de electrones/huecos para poder mantener el número de partículas equivalentes aproximadamente igual en cada malla del dispositivo. O lo que es lo mismo, el peso de la partícula o valor de EPE sigue las variaciones del perfil de concentración, reduciendo así considerablemente las fluctuaciones estadísticas antes mencionadas y el tiempo de cálculo de CPU del método Monte Carlo de modo que puede mantenerse el número de partículas simuladas por malla aproximadamente constante en todo el dispositivo [Martín et al, 1994], [Martín, 1996b].

El esquema seguido para la implementación del algoritmo es el siguiente:

- Inicialmente se realiza una división espacial del dispositivo en distintas zonas $\left(S_{i}, i=1\right.$, $2 \ldots n)$, de modo que cada una de estas $n$ regiones tiene asignado un valor de equivalente partícula-electrón $E P E_{i}$ (Ver Figura II.2). Es conveniente que los límites de estas regiones coincidan con fronteras de las mallas utilizadas para la resolución de la ecuación de Poisson.

- La simulación se inicia asignando a cada malla $j$ un valor de $E P E_{j}$ que es determinado por la ecuación:

$$
E P E_{j}=\frac{\Delta x_{j} D O P_{j}}{N P_{j}}
$$


donde $\Delta x_{j}$ es la anchura de las mallas, $D O P_{j}$ la densidad de impurezas ionizadas y $N P_{j}$ el número de partículas de la malla $j$. Inicialmente se toman valores similares de $N P_{j}$ en cada celda, se comparan los valores de EPE de las mallas adyacentes para así determinar las fronteras de las regiones $S_{i}$ y los factores multiplicativos ${ }^{5}\left(N_{i}\right)$.

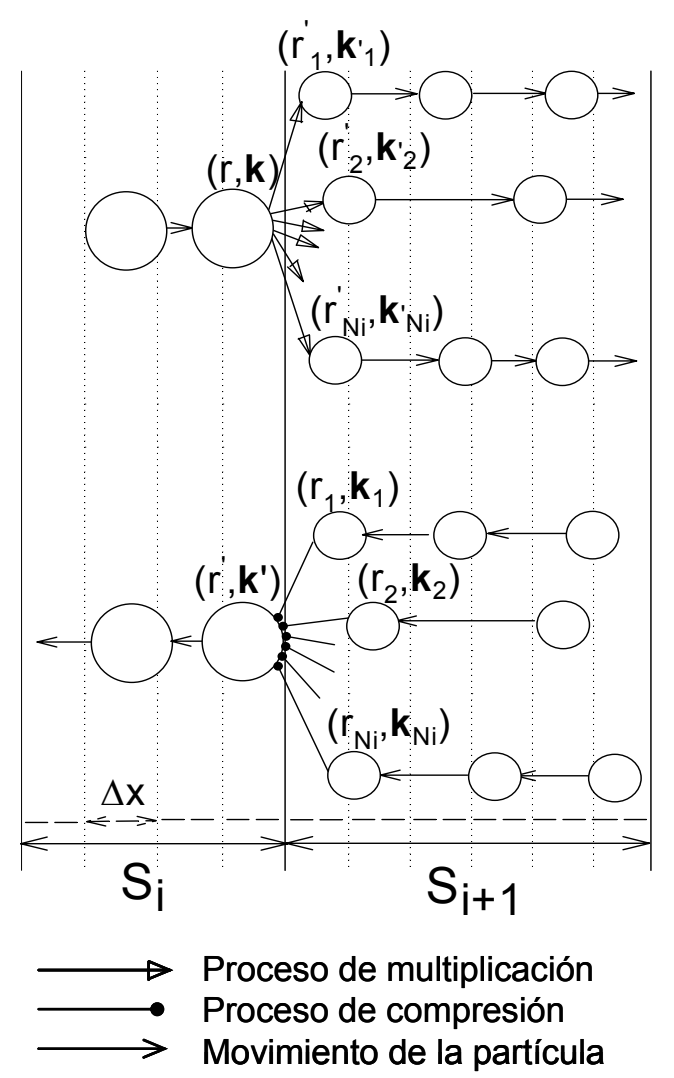

Figura II.2. Posibles procesos de multiplicación-compresión. Las líneas continuas indican los límites de las regiones $S_{i}$ de distinto EPE y las líneas discontinuas representan los límites de las mallas de la resolución de la ecuación de Poisson.

- Se realiza una simulación Monte Carlo con estos valores de $E P E$ durante un número de iteraciones adecuado (1-5ps) para poder realizar una estimación del perfil de la densidad de portadores libres y así reajustar los valores de EPE de cada malla según la Ecuación II.3, sustituyendo el dopaje de cada malla por la concentración de portadores. A partir de estos nuevos datos se reajustan también las fronteras de las regiones y demás parámetros

\footnotetext{
${ }^{5}$ En el paso de una región a la siguiente se produce un proceso de multiplicación o división de la partícula correspondiente, el factor de multiplicación $N_{i}$ viene dado por el cociente entre el $E P E$ de la región final y la inicial, de modo que se generan $N_{i}$ partículas a partir de la partícula "madre", que desaparece, cumpliendo las leyes de conservación de energía y momento.
} 
mencionados. (El procedimiento es análogo para los huecos en caso de simular semiconductores tipo $p$ ).

-Finalmente, obtenemos un valor del perfil de $E P E$ adecuado (ver Figura II.3.a) que permite mantener, como hemos comentado anteriormente, un número aproximadamente constante de partículas por malla a lo largo de todo el dispositivo.
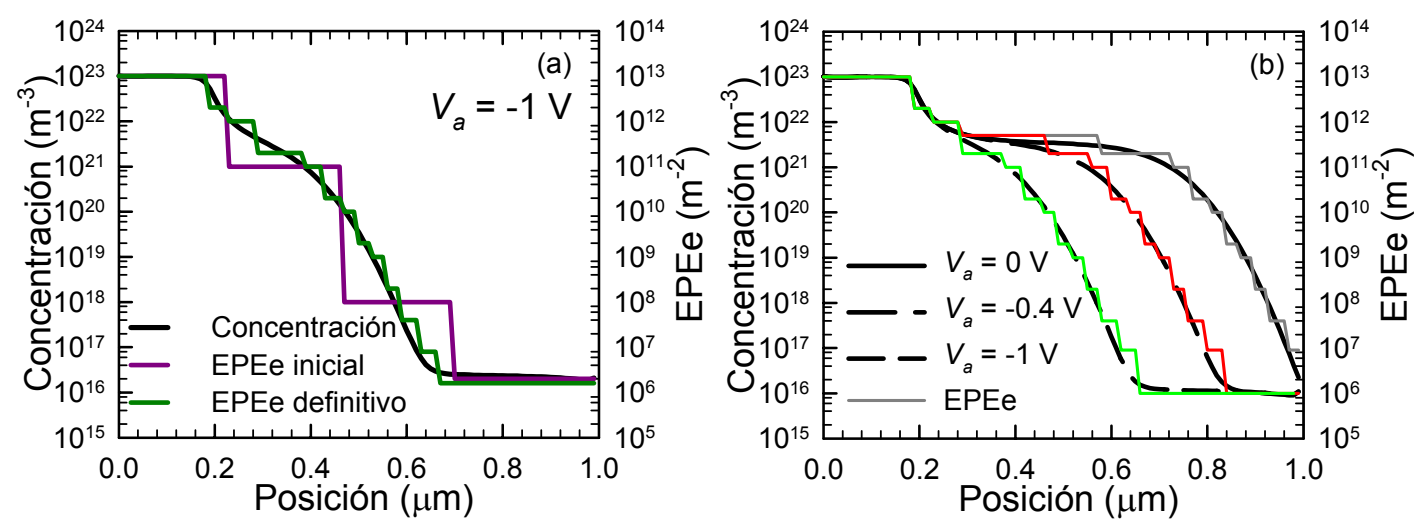

Figura II.3.a. Diferentes perfiles de EPE utilizados en la simulación de un diodo Schottky con polarización en inversa de -1 V. Se observan los distintos reajustes del perfil de EPE hasta alcanzar el perfil satisfactorio más cercano al perfil de densidad de portadores .b. Concentración de portadores en un diodo Schottky sometido a tres polarizaciones en inversa $(0 \mathrm{~V},-0.4 \mathrm{Vy}-1 \mathrm{~V})$ con sus perfiles de EPE correspondientes.

El resultado de la asignación variable de los valores de los $E P E$ y las fronteras de las diferentes regiones de expansión/compresión en un diodo Schottky como los que estudiaremos en el Capítulo III $\left[n^{+}\left(0.2 \mu \mathrm{m}, 10^{17} \mathrm{~cm}^{-3}\right)-n\left(0.8 \mu \mathrm{m}, 3.5 \cdot 10^{15} \mathrm{~cm}^{-3}\right)\right.$-Siliciuro] se muestra en la Figura II.3. En la Figura II.3.b, mostramos la variación de la concentración de portadores en el mismo diodo sometido a tres polarizaciones, en equilibrio y en inversa, $(0 \mathrm{~V},-0.4 \mathrm{~V} \mathrm{y}-1 \mathrm{~V})$; como podemos observar en dicha gráfica, el perfil del EPE debe variar teniendo en cuenta la variación de la concentración.

La aplicación de esta técnica de realce es fundamental en las estructuras simuladas en primer lugar en el presente trabajo, es decir, en los diodos Schottky y diodos back-to-back, debido a los notables gradientes de concentración que se presentan la zona de carga espacial de la unión semiconductor-Siliciuro. De este modo se evitan las fluctuaciones en la distribución espacial de la concentración a pesar de que ésta varía en más de 10 órdenes de magnitud a lo largo de la estructura. Además, el hecho de utilizar el método de realce hace que el número de partículas en las mallas próximas al contacto sea elevado, pudiendo así estimar con gran precisión las diferentes componentes de la corriente. Sin embargo, en el caso de las estructuras 
SB-MOSFET esta técnica no es necesaria puesto que, como veremos en el capítulo correspondiente, la variación entre los distintos niveles de concentración no es tan agresiva como en el caso que acabamos de mencionar.

\section{II.3.c. Condiciones iniciales}

Teniendo en cuenta que el tiempo de simulación debe ser suficientemente elevado, las condiciones iniciales impuestas al portador no tendrán influencia en los resultados finales, que vendrán determinados exclusivamente por las condiciones de polarización aplicadas en los terminales del dispositivo. Sin embargo, una buena elección de estas condiciones permitirá un tiempo de simulación menor para obtener resultados fiables. Para ello, los portadores se inicializan con energía en torno a la térmica de la red, $3 / 2 K_{B} T$, y las componentes del vector de onda se determinan de manera aleatoria, así como la posición dentro de la malla correspondiente $^{6}$. Para evitar efectos no deseados de la elección de las condiciones iniciales, no se considerará la contribución de la primera parte de la simulación (transitorio hasta el estado estacionario) sobre los resultados.

\section{II.3.d. Movimiento de los portadores}

Como hemos comentado anteriormente, el movimiento de los portadores en el dispositivo consta de dos partes bien diferenciadas. Cada portador (electrón o hueco) se simula durante un intervalo de tiempo $\Delta t$, durante el cual puede experimentar una serie de recorridos libres, en los que el movimiento del portador se supone balístico y su velocidad y posición vienen determinadas por la acción del campo eléctrico a través de las ecuaciones del movimiento semiclásicas [Martín, 1996b]. Cada cierto tiempo, estos recorridos se ven interrumpidos por los mecanismos de scattering, que suponemos instantáneos, que pueden modificar la trayectoria y la energía de los portadores. Una vez que ha transcurrido el intervalo temporal $\Delta t$, se pasa a simular el movimiento del siguiente portador, y cuando se han simulado todos los portadores, se actualiza el valor del campo eléctrico mediante la resolución de Poisson.

\footnotetext{
${ }^{6}$ La ubicación espacial inicial se determina de tal modo que asegure la neutralidad del dispositivo al inicio de la simulación [González, 1994].
} 
Tras ello, se comienza de nuevo a simular el movimiento de cada portador, tal y como se refleja en el diagrama de flujo de la Figura II.1.

La duración del recorrido libre se determina aleatoriamente de acuerdo con la probabilidad (dependiente de la energía) de que tenga lugar algún proceso de scattering. Por tanto, la estructura que se sigue es la siguiente: primero se determina la duración del recorrido libre, se efectúa el mismo y posteriormente se escoge el mecanismo de scattering que sufrirá el portador. Tras el mecanismo de scattering, se determina el estado final (nuevos valores de momento y energía de manera adecuada según sea el tipo de portador) para a continuación comenzar el siguiente recorrido libre. Una descripción más detallada puede encontrarse en [Martín, 1996b].

\section{II.3.e. Absorción de portadores por efecto túnel}

En una primera caracterización sencilla del contacto Schottky, se considera únicamente la absorción termoiónica del semiconductor al metal [Martín, 1993]. Sin embargo, para realizar un modelado preciso y adecuado de estos contactos, debemos tener en cuenta también efectos tales como la emisión termoiónica del metal al semiconductor y el efecto de túnel cuántico (o efecto de campo) en ambos sentidos. Tales procesos, junto con la metodología empleada para ser incorporados en el modelo, serán explicados con detalle en la Sección II.5; sin embargo, para dar una primera idea acerca de su implementación en el modelo, a continuación los describimos brevemente en el proceso de evolución del diagrama de flujo de nuestro simulador Monte Carlo (Figura II.1)

Como podemos observar en la Figura II.1 y ya hemos mencionado en el apartado anterior, simulamos el movimiento de los portadores durante intervalos de tiempo $\Delta t$. Para considerar la absorción por efecto túnel, únicamente una vez superado un tiempo predeterminado T' examinamos las condiciones impuestas en nuestro modelo: en primer lugar debe calcularse la probabilidad de transmisión a través de la barrera de potencial para un rango de energías suficientemente amplio. Los portadores que en el semiconductor son susceptibles de ser absorbidos por efecto túnel serán aquellos que no tengan energía suficiente para rebasar la altura de la barrera Schottky (en caso contrario serán absorbidos por emisión termoiónica). 
Cuando tales portadores llegan al punto de retroceso ${ }^{7}$ se evalúa la componente del vector de onda paralela al camino túnel; en caso de que sea nula se emplea una técnica de rechazo que tiene en cuenta la probabilidad de absorción. El portador en cuestión que supere la condición impuesta abandonará el dispositivo dejando de simularse su movimiento, en caso contrario será reflejado. Condiciones similares para el tratamiento del efecto túnel pueden encontrarse en [Matsuzawa, 2000], [Sun et al., 2003], [Shen et al., 2004].

\section{II.3.f. Cálculo espectroscópico}

Para analizar los procesos microscópicos relacionados con el transporte de electrones y dado el carácter de nuestro simulador Monte Carlo, es posible obtener del mismo magnitudes internas tales como el recorrido libre medio o el tiempo entre mecanismos de scattering. Para ello hemos considerado en nuestro simulador MC2D una región de conteo limitada por superficies de entrada y de salida que pueden definirse al comienzo de la simulación. En el caso del SB-MOSFET analizado en los Capítulos V y VI, la región de conteo coincide con la zona bajo el contacto de puerta con el fin de considerar en igualdad de condiciones aquellos portadores que han entrado o salido del dispositivo mediante emisión termoiónica o efecto túnel.

Cada electrón que entra en la región de conteo es etiquetado adecuadamente y se sigue su avance, registrando todos los parámetros significativos relacionados con su movimiento, esto es, no sólo los mecanismos de scattering sino también los tiempos de vuelo libre, el recorrido libre medio, etc. Este completo análisis del movimiento de cada partícula individual permite determinar para cada electrón que cruza el canal diferentes magnitudes de distintos parámetros claves para el estudio de dispositivos de dimensiones reducidas, como son el tiempo de tránsito, el número de mecanismos de scattering, el tiempo medio entre dichos mecanismos... Por ello es posible, realizando un estudio estadístico adecuado, el obtener no sólo los valores medios de estos parámetros sino también las funciones de distribución de los mismos.

\footnotetext{
${ }^{7}$ El punto de retroceso para un portador que se acerca a una barrera de potencial con una determinada energía es la posición en el en el que dicha energía es igual al valor del potencial.
} 
Además, también se ha incorporado en nuestro modelo MC2D la posibilidad de "desconectar" los mecanismos de scattering en las diferentes regiones que componen el dispositivo, permitiéndonos por tanto simular el dispositivo en un modo puramente balístico y así compararlo con el comportamiento de la estructura real, estudiando así las diferencias entre ambos. En este aspecto, el método Monte Carlo posee grandes ventajas frente a otros modelos tradicionales, como el deriva-difusión o el hidrodinámico, ya que realiza una correcta descripción del transporte balístico o cuasibalístico, incluyendo de forma directa los procesos microscópicos aleatorios causantes del ruido. Se han llevado a cabo en nuestro área diversos estudios de dispositivos balísticos, evidenciando la capacidad del método Monte Carlo para este tipo de aplicaciones [González, 2004], [Vasallo, 2004], [Mateos, 2005].

\section{II.3.g. Inyección de portadores por emisión termoiónica o efecto túnel}

Tal y como vimos en el Capítulo I, aquellos portadores que en el metal tienen energía suficiente para superar la barrera Schottky, serán inyectados termoiónicamente. Por otro lado, aquellas partículas con energía inferior, podrán ser inyectadas mediante efecto túnel o emisión de campo en cada intervalo temporal una vez superado un tiempo T'. Para ello y al igual que en el caso de la absorción túnel, debemos tener en cuenta la probabilidad de transmisión cuántica a través de la barrera. Se considerará la posibilidad de inyección en el semiconductor mediante una técnica de rechazo teniendo en cuenta el valor de la carga a inyectar (como ya veremos en la Sección II.5). El portador se inyectará en la malla adecuada con la componente del vector de onda paralela al camino túnel nula mientras que las otras dos componentes tienen (en suma) el total correspondiente a la energía térmica ${ }^{8}$ [Shen, 2004].

\section{II.3.h. Resolución de la ecuación de Poisson y cálculo del campo eléctrico}

Como ya hemos comentado previamente, en el presente trabajo abordaremos el estudio de diodos Schottky y back-to-back mediante el simulador MC1D, mientras que para el análisis de MOSFET de barrera Schottky es necesario llevar a cabo una simulación bidimensional en el

\footnotetext{
${ }^{8}$ En el caso de la simulación MC1D, y dado que consideramos un $E P E$ variable, al inyectar la partícula en una malla deberá tenerse en cuenta el EPE correspondiente a la misma.
} 
espacio real. La resolución de la ecuación de Poisson bipolar en este caso es notablemente más costosa en términos de tiempo de cálculo y ocupación de memoria. A continuación describimos sucintamente los pasos necesarios para la resolución de la ecuación de Poisson y el posterior cálculo del campo eléctrico en dos dimensiones. Para un análisis más detallado nos remitimos a las anteriores tesis realizadas en el área [González, 1994], [Martín, 1996b], [Rengel, 2002]. Efectuando el estudio del problema bidimensional, la reducción al caso unidimensional es sencilla.

A la hora de afrontar la resolución de la ecuación de Poisson, se plantean una serie de ecuaciones diferenciales para cuya solución se suele emplear como técnica más habitual el método de diferencias finitas [Selberherr 1984], consistente en la discretización de las ecuaciones en cada nodo del mallado rectangular en el que se ha dividido el dispositivo. El mallado que hemos considerado se compone de $\mathrm{N}$ columnas y $\mathrm{M}$ filas, de tamaño no uniforme para adaptarse a las singularidades de cada dispositivo simulado (concentración de portadores, gradiente de potencial local, etc.). Por tanto constará de $(\mathrm{N}+1) \times(\mathrm{M}+1)$ nodos, de modo que cada malla está rodeada de 4 nodos (cada uno en uno de sus vértices) ${ }^{9}$. El proceso de resolución consta de los siguientes pasos:

1) Asignación de densidad de carga efectiva a cada nodo. Este es un aspecto importante del modelo, ya que la resolución de la ecuación de Poisson se realiza en cada nodo y la densidad de carga se obtiene por malla. Como ya hemos comentado anteriormente, la densidad de carga tiene un carácter bipolar dada la naturaleza de nuestro simulador (que nos permite simular de manera simultánea electrones y huecos).

De entre las distintas técnicas existentes en la literatura [Jacoboni y Lugli, 1989], [Tomizawa, 1993], en nuestro caso, para proceder a la asignación de carga en cada nodo, realizamos una media ponderada de la densidad de carga media $\rho$ dividida por la permitividad del material $\left(\varepsilon_{0} \varepsilon_{r}\right)$ en las cuatro mallas que rodean a dicho nodo (Figura II.3) [González 1994, Martín 1996b, Rengel 2002]. De este modo, obtenemos el segundo miembro de la ecuación de Poisson (ver Ecuación II.4).

2) Resolución numérica de la ecuación de Poisson. Tras la asignación de la carga a cada nodo, el siguiente paso consiste en la discretización de la ecuación de Poisson para proceder después a la resolución del sistema resultante. La ecuación de Poisson en 2D puede

\footnotetext{
${ }^{9}$ En el caso 1D, se define un mallado (que puede ser no-uniforme) en la geometría de los dispositivos que consta de $\mathrm{N}$ mallas de longitud variable y $\mathrm{N}+1$ nodos.
} 
representarse de la siguiente forma (considerando nulo el término asociado a la derivada espacial de la permitividad del material) [Moglestue, 1993]:

$$
\frac{\partial^{2} \varphi}{\partial x^{2}}+\frac{\partial^{2} \varphi}{\partial y^{2}}=-\frac{\rho}{\varepsilon_{0} \varepsilon_{r}}
$$

Aplicando diferencias finitas, y teniendo en cuenta el segundo miembro de la ecuación de Poisson calculado en el paso anterior, obtenemos una expresión para la Ecuación II.1 que podemos aplicar a cada uno de los nodos de la red para determinar así el valor del potencial $\varphi$ en cada nodo.

3) Cálculo del campo eléctrico. A partir de los valores de potencial obtenidos, el campo eléctrico en cada malla vendrá dado por:

$$
\begin{aligned}
& E_{x}^{n, m}=-\nabla_{x} \varphi=-\frac{1}{2}\left(\frac{\varphi_{i+1, j+1}-\varphi_{i, j+1}}{\Delta x_{n}}+\frac{\varphi_{i+1, j}-\varphi_{i, j}}{\Delta x_{n}}\right) \\
& E_{y}^{n, m}=-\nabla_{y} \varphi=-\frac{1}{2}\left(\frac{\varphi_{i, j+1}-\varphi_{i, j}}{\Delta y_{m}}+\frac{\varphi_{i+1, j+1}-\varphi_{i+1, j}}{\Delta y_{m}}\right)
\end{aligned}
$$

Donde $\mathrm{i}, \mathrm{j}$ son los índices relativos a cada nodo del mallado.

4) Condiciones de contorno. Estas forman parte de la simulación tanto en la resolución de la ecuación de Poisson como en la dinámica de las partículas.

- En primer lugar, en la resolución de la ecuación de Poisson se consideran dos tipos de condiciones de contorno [Hockney y Eastwood, 1988; Jacoboni y Lugli, 1989]:

- Se imponen condiciones de Dirichlet en los electrodos, esto es, potencial fijo.

- En el contorno del dispositivo, cuando existe interfaz entre el semiconductor y un dieléctrico, se impone la condición de Neumann ${ }^{10}$, que implica la continuidad de la componente normal del vector desplazamiento.

Para afrontar la simulación de transistores de tipo MOSFET, debemos considerar regiones en el dispositivo de material dieléctrico (tanto para el óxido de puerta como para el óxido enterrado en los dispositivos de tipo SOI, como es el caso del SB-MOSFET que analizaremos en los Capítulo V y VI).

En un óxido real existen cargas tanto en su interior como en la superficie óxidosemiconductor [Sze 1983, Ghandhi 1994], pudiendo considerarse las cargas en el volumen de

\footnotetext{
${ }^{10}$ En nuestro caso particular de las estructuras unidimensionales que simulamos no poseen interfaz entre el semiconductor y el vacío, por lo que no se aplica esta condición de contorno.
} 
manera equivalente como una carga superficial adicional [Pierret 1994]. La condición de contorno asociada a las superficies semiconductor-dieléctrico es considerada en nuestro simulador como sigue [Mateos 1999, Rengel 2002]:

$$
\varepsilon_{1} \xi_{1}-\varepsilon_{2} \xi_{2}=\sigma_{12}
$$

Siendo $\sigma_{12}$ la densidad de carga superficial (que tomaría el valor 0 en caso de no considerar carga de superficie o en las superficies entre dos materiales dieléctricos), $\xi$ el campo eléctrico en la dirección perpendicular a la superficie y $\varepsilon$ la permitividad, correspondiendo 1 al semiconductor y 2 al óxido. Todas estas condiciones de contorno son consideradas en el conjunto del sistema de ecuaciones para la resolución de la ecuación de Poisson.

- Las condiciones de contorno para la dinámica de los portadores se aplican consistentemente con las consideradas en el cálculo del campo eléctrico.

- En el caso de que existiera una frontera semiconductor / dieléctrico (es decir, cuando la partícula tiene posibilidad de sufrir scattering superficial con la misma) en la que se aplican las condiciones de Neumann, consideramos que los portadores sufren dos tipos de reflexiones: reflexión especular y difusiva en el simulador bidimensional, mientras que en el caso unidimensional sólo está considerada la especular. En la reflexión especular la componente del momento perpendicular a la superficie cambia de signo en los límites del dispositivo, mientras que en la reflexión difusiva las componentes del momento conservan el mismo módulo que antes de la reflexión, pero el ángulo de salida es independiente del de entrada (se realiza un sorteo aleatorio para el cálculo de dicho ángulo). El modo de determinar si la reflexión será especular o difusiva se realiza mediante el parámetro de Fuchs [Fuchs, 1937]: se sortea un número aleatorio, y en caso de ser superior a dicho parámetro se considera de tipo especular, si es inferior es difusiva.

- Los contactos óhmicos, donde se aplican las condiciones de Dirichlet, son considerados como una región del dispositivo que permanece en equilibrio térmico incluso en el caso de que fluya una corriente térmica a través de ella. Para simular tal comportamiento se impone que todos los portadores que llegan al electrodo son absorbidos por él, y se inyectan en la malla adyacente al contacto los portadores necesarios en cada $\Delta t$ para mantener la neutralidad de carga y el equilibrio térmico en la región adyacente al electrodo. Existen 
diferentes métodos de inyección de portadores: en nuestro caso, hemos optado por una distribución hemi-Maxwelliana ponderada en velocidad [González y Pardo 1996].

- En los contactos de tipo Schottky, en los que también se aplican condiciones de Dirichlet para el potencial, el modelo más simple consiste en considerar el contacto metálico como una frontera perfectamente absorbente, es decir, que toda partícula capaz de superarla abandona el dispositivo mediante absorción termoiónica, sin inyectar ningún portador al interior del mismo. Sin embargo, en nuestro modelo, tal y como ya hemos comentado en anteriores apartados, hemos realizado una visión más realista de este tipo de contactos mediante la consideración adicional de fenómenos tales como el efecto túnel y la emisión termoiónica en ambos sentidos (de inyección y absorción). Además se consideran efectos reales como la disminución de la altura de la barrera efectiva por el efecto de las cargas imagen.

\section{II.3.i. Cálculo de corriente}

El cálculo de la corriente es fundamental a la hora de afrontar el análisis de un dispositivo, siendo por tanto necesario disponer de un valor preciso de la misma en los terminales para cada intervalo temporal de que consta la simulación. Existen varias posibilidades para evaluar la corriente, como contabilizar el número de partículas neto ${ }^{11}$ que sale o entra de los terminales. Sin embargo, cuando nuestro objetivo es realizar un estudio del ruido en el dispositivo, es necesario utilizar modelos que reduzcan al máximo el ruido matemático, como es el caso del Teorema de Ramo-Shockley [Shockley, 1938], [Ramo, 1939], [Kim et al., 1991], que nos permite obtener mayor precisión en el valor instantáneo de la corriente, ya que tiene en cuenta no sólo las partículas que entran o salen del dispositivo, sino también las del interior.

El procedimiento para el cálculo de la corriente a partir de este teorema consiste en la asignación de carga a cada uno de los electrodos del sistema, para posteriormente calcular la

\footnotetext{
${ }^{11}$ Con su carga y pesos respectivos, recordando que en el caso del simulador MC1D, el valor del EPE depende de la malla
} 
corriente a partir de las derivadas temporales de dicha carga [Rengel, 2002]. La expresión final para el cálculo de la corriente en el caso de una dimensión:

$$
i_{j}(t)=-\sum_{i=1}^{N} q_{i} \vec{v}_{i}(t) \nabla f_{j}\left(\vec{r}_{i}\right)
$$

Donde $q_{i}$ y $\vec{v}_{i}$ son la carga y la velocidad de la partícula $i$-ésima, $f_{j}(r)$ es un factor geométrico independiente de la densidad de carga (que corresponde al potencial eléctrico en la posición $r$ cuando el electrodo j-ésimo se encuentra a tensión 1 y los demás a tierra), considerando $N$ partículas y $j=1,2$ electrodos.

La implementación de este teorema en nuestro simulador Monte Carlo, nos proporciona como decíamos el valor instantáneo de la corriente, pudiendo así realizar una adecuada caracterización de los dispositivos SB-MOSFET tanto de pequeña señal como de ruido, tal y como veremos en el Capítulo VI de esta tesis.

\section{II.3.j. Consideración de resistencias en serie en los contactos}

En el caso de las estructuras MOSFET, a la hora de afrontar el estudio de dispositivos fabricados, en ocasiones puede ser necesario incluir el efecto de una resistencia macroscópica en serie con la parte del dispositivo simulada de modo microscópico, que vamos a denominar dispositivo intrínseco. En un dispositivo real, existe una resistencia en serie en los accesos del dispositivo, asociada a la resistencia de las conexiones, del contacto óhmico y la resistencia distribuida de la oblea [Sze 1981, Liou et al. 1998, Tsividis 1999]. Por tanto, este es un efecto que debe ser tenido en cuenta si se desea realizar una comparación efectiva de los resultados de la simulación con medidas experimentales.

\section{II.3.k. Cálculo de los coeficientes de Transmisión}

Para poder considerar adecuadamente el efecto túnel en sus dos vertientes (inyección y absorción), es necesario determinar la probabilidad cuántica de transmisión de las partículas a través de la barrera Schottky. Para la evaluación de dicha probabilidad hemos considerado diferentes aproximaciones (en particular la aproximación WKB y el método de la matriz de 
transferencia de Airy), que serán objeto de un estudio detallado en la próxima sección (II.4). Como ya veremos, necesitamos el conocer la variación de la barrera de potencial a lo largo de la estructura, por lo que los cálculos de estas probabilidades comienzan tras un tiempo $T^{\text {' (ver }}$ Figura II.1) lo suficientemente grande para obtener un perfil de potencial estable. Este cálculo se actualiza cada $N^{\prime}$ iteraciones.

\section{II.3.l. Cálculo de perfiles longitudinales de magnitudes bidimensionales}

Finalmente, es importante destacar que la simulación MC2D nos permite calcular el valor de las distintas magnitudes asociadas a los portadores, como su concentración $\bar{n}(x, y)$, velocidad $\bar{v}(x, y)$, etc, siendo $x$ (dirección longitudinal) e $y$ (dirección transversal) las coordenadas de la malla correspondiente. En el caso de dispositivos MOSFET, el estudio de estas cantidades proporciona una gran cantidad de información sobre el transporte de portadores; sin embargo, en ciertas circunstancias, el análisis de esta información en transistores MOSFET puede hacerse de un modo más sencillo si consideramos los perfiles longitudinales de estas magnitudes a lo largo del canal, apropiadamente pesados por la concentración local en el eje vertical. Así, para una magnitud $\bar{A}(x, y)$, donde la barra superior denota media temporal, determinaremos estos perfiles longitudinales como sigue [Lusakowski, 2007]:

$$
\begin{aligned}
\left\langle A_{x}\right\rangle= & \left(\iint d x d y \bar{A}(x, y) \bar{n}(x, y)\right) / n_{S} \\
& \text { Donde } n_{S}=\iint d x d y \bar{n}(x, y) \text { es la concentración media de portadores en el canal. Las }
\end{aligned}
$$
integrales se calculan en toda la región activa del transistor.

\section{II.4. Modelo de inyección/absorción: Cálculo del coeficiente de transmisión a través de una barrera de potencial}

Como hemos visto en el Capítulo I, los procesos de transporte a través de la barrera Schottky más importantes son la inyección termoiónica (del metal al semiconductor), la absorción termoiónica (del semiconductor al metal) y el transporte túnel en ambos sentidos. En particular, en el régimen de polarización en inversa, la inyección mediante túnel cuántico tiene 
especial importancia si se desea efectuar una caracterización precisa del comportamiento de los diodos Schottky. Por tanto, es necesaria la implementación en nuestro simulador Monte Carlo de un algoritmo que modele adecuadamente tales procesos. Se hace así imprescindible resolver la ecuación de Schrödinger para determinar la probabilidad de transmisión a través de la barrera Schottky.

En 1926 Schrödinger presentó una teoría que describía la dependencia espacio-temporal de los sistemas mecánico-cuánticos, jugando un papel análogo al de la segunda ley de Newton para la mecánica clásica [Schrödinger, 1926], [Schrödinger, 1926b]. De este modo, en el caso de una partícula de masa $m$ moviéndose en una dimensión sujeta al potencial $V(x)$ independiente del tiempo, la ecuación de Schrödinger se expresa como sigue:

$$
-\frac{\hbar^{2}}{2 m} \frac{d^{2} \psi(x)}{d x^{2}}+V(x) \psi(x)=E \psi(x)
$$

donde $\Psi(x)$ es la función de onda que describe el movimiento de la partícula, $V(x)$ es la energía potencial y $E$ la energía. La importancia de esta ecuación reside en su aplicación a diversos fenómenos, como el túnel a través de una barrera de potencial, la determinación de las autoenergías y autofunciones en pozos de potencial, etc. Sin embargo, su resolución es extremadamente compleja pues implica tratar con ecuaciones diferenciales que no tienen solución analítica ni tan si quiera en una dimensión, excepto en casos especiales muy simples.

Presentamos a continuación dos métodos comúnmente usados en la literatura para afrontar la evaluación de la ecuación de Schrödinger: la aproximación de Wentzel-KramersBrillouin (WKB) [ATLAS, 1999], [Matsuzawa, 2000] y [Sun, 2003] y el método de la matriz de transferencia de Airy (MTA) [Lui, 1986], [Brennan, 1987], [Vattania, 1996], [Winstead, 2000], [Vega, 2006], [Vega, 2006b].

\section{II.4.a. Aproximación WKB}

En 1926 G. Wentzel, H. A. Kramers y L. Brillouin publicaron de forma simultánea e independiente un método que se basaba en aproximar la solución de la ecuación de Schrödinger por una combinación de funciones de onda sinusoidales, solventando así en gran medida los diversos problemas que se planteaban al enfrentarse a dicha ecuación [Wentzel, 1926], 
[Kramers, 1926], [Brillouin, 1926]. La aproximación se denominó WKB a partir de las iniciales de sus autores.

Las hipótesis en que se basa la aproximación $\mathrm{WKB}^{12}$ son:

1. El potencial $V(x)$ del problema en cuestión debe variar suavemente en comparación con la longitud de onda de de Broglie

$$
\lambda(x)=\frac{h}{p(x)}
$$

Siendo $h$ la constante de Planck y $p(x)$ el momento clásico del electrón en la posición $x$ :

$$
p(x)=[2 m|E-V(x)|]^{\frac{1}{2}}
$$

En el límite clásico $\lambda$ tiende a 0 , por lo que la variación "lenta" del potencial implica que, desde el punto de vista de la mecánica cuántica, nos encontramos en una situación cuasiclásica.

2. La diferencia entre energía y energía potencial, $E-V(x)$, es suficientemente grande.

Ambas condiciones se ven reflejadas en la siguiente ecuación, que puede emplearse como criterio de validez del método [Bransden, 1986]:

$$
\left|\frac{\hbar m d V(x) / d x}{[2 m(E-V(x))]^{3 / 2}}\right|<<1
$$

A partir de estas suposiciones se puede hacer un desarrollo completo del modelo, tal y como veremos a continuación. Aunque a primera vista estas hipótesis pueden resultar restrictivas, la aproximación WKB es ampliamente utilizada, no sólo en contactos Schottky ([Matsuzawa, 2000], [Sun, 2003]), sino también en heteroestructuras de doble barrera [Martínez, 2001] o de tipo escalón [Havart, 1998], entre otras. Esta aproximación es también implementada en simuladores comerciales como el ATLAS Silvaco [ATLAS, 1999], ya que presenta resultados satisfactorios en la comparación frente a las medidas experimentales y es además un método computacionalmente muy eficiente.

Para el cálculo de la densidad de corriente total en la simulación de un contacto Schottky es necesario determinar el valor de las cuatro contribuciones de la corriente: inyección túnel y termoiónica además de la absorción túnel y termoiónica. Como ya vimos en la Sección I.IV, las densidades de corriente se expresan en función del coeficiente de transmisión

\footnotetext{
${ }^{12}$ Presentamos aquí el desarrollo unidimensional del problema, el planteamiento en dos dimensiones se haría de manera análoga para la variable $y$.
} 
(Ecuaciones I.35 y I.36). Estas ecuaciones se pueden formular de manera más compacta, tal y como lo hacen varios autores ([Sun, 2003], [Huang, 1998], etc.). En el caso de la inyección:

$$
J_{m \rightarrow s}=\frac{A^{*} T}{K_{B}} \int_{0}^{\infty} T_{C m s}(E) f_{m}(E)\left[1-f_{s}(E)\right] d E
$$

donde $A^{*}$ es la constante de Richardson efectiva, $T$ la temperatura, $K_{B}$ la constante de Boltzmann, $T c_{m s}$ es el coeficiente de transmisión del metal al semiconductor. La probabilidad de ocupación del metal y la de desocupación del semiconductor se expresan en términos de la función de distribución de Fermi-Dirac, $f_{m}$ y $f_{s}$. Mientras que en el caso de la densidad de corriente de absorción:

$$
J_{s \rightarrow m}=\frac{A^{*} T}{K_{B}} \int_{0}^{\infty} T_{C s m}(E) f_{s}(E)\left[1-f_{m}(E)\right] d E
$$

El coeficiente de transmisión ( $T_{C}$ ms $y T_{C s m}$ ) se define como el ratio de la intensidad de la densidad de corriente transmitida y la incidente y determina la probabilidad de transmisión a través de la barrera.

$$
T_{C \mathrm{~ms} / \mathrm{sm}}=\frac{J_{\text {transmitida }}}{J_{\text {incidente }}}
$$

donde la densidad de probabilidad de corriente transmitida e incidente se obtienen del siguiente modo:

$$
j(x)=\frac{\hbar}{2 m i}\left[\psi^{*}(x) \frac{d \psi(x)}{d x}-\psi(x) \frac{d \psi^{*}(x)}{d x}\right]
$$

Siendo $\Psi^{*}(x)$ la conjugada de la función de onda $\Psi(x)$.

Debemos, por tanto, calcular dichas densidades de corriente, para lo que es necesario resolver la ecuación de Schrödinger. Con este objetivo, y a fin de realizar el desarrollo teórico de la aproximación de WKB de manera global, consideramos una barrera genérica como la representada en la Figura II.4 y un flujo de partículas que incide por la izquierda con energía $E$.

Los puntos de retroceso $\left(x_{1}\right.$ y $\left.x_{2}\right)$ en los que la energía incidente iguala al potencial de la barrera, determinan tres regiones (véase la Figura II.4), en cada una de las cuales calcularemos la solución para la ecuación de Schrödinger siguiendo los pasos marcados a continuación. 


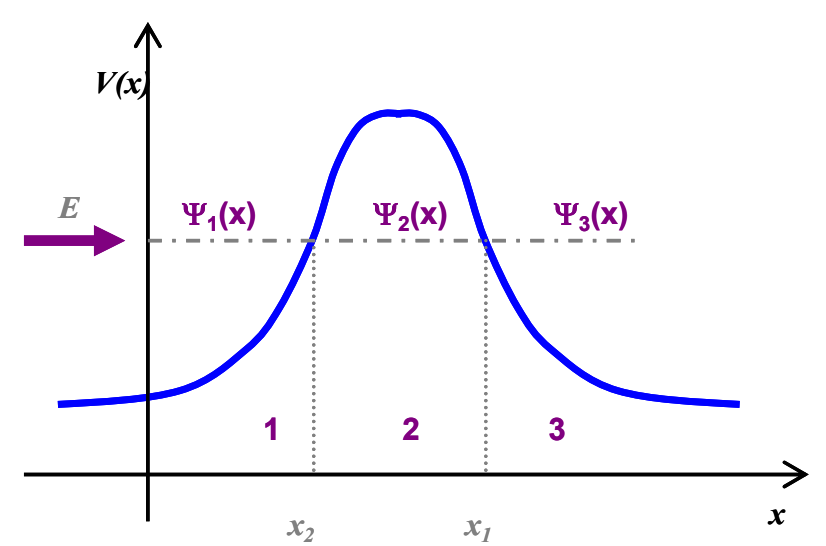

Figura II.4. Barrera de potencial genérica $V(x)$, donde $E$ es la energía de las partículas incidentes, $x_{1}$ y $x_{2}$ son los puntos de retroceso y $\Psi_{1}(x), \Psi_{2}(x)$ y $\Psi_{3}(x)$ son las funciones de onda solución de la ecuación de Schrödinger para cada región.

A partir de la solución de la ecuación de Schrödinger para el caso particular de potenciales constantes podemos considerar que bajo las hipótesis descritas anteriormente, la función de onda puede expresarse como:

$$
\psi(x)=A \exp \left(\frac{i}{\hbar} S(x)\right)
$$

donde $S(x)$ es una función proporcional al momento de la partícula que varía con la posición. De este modo la ecuación de Schrödinger se enuncia de la siguiente manera:

$$
-\frac{i \hbar}{2 m} \frac{d^{2} S(x)}{d x^{2}}+\frac{1}{2 m}\left[\frac{d S(x)}{d x}\right]^{2}+V(x)=E
$$

De manera aproximada, si el potencial es constante, $V(x)=V_{0}$, entonces $S(x)$ toma el siguiente valor: $S(x)= \pm p_{0} x^{13}$, de modo que el primer sumando de la Ecuación II.18 desaparecería. Observamos además que este sumando es proporcional a $\hbar$, luego en el límite semi-clásico $(\hbar \rightarrow 0)$ se despreciaría. Podemos expandir por tanto $S(x)$ en serie de potencias del siguiente modo:

$$
S(x)=S_{0}(x)+\hbar S_{1}(x)+\frac{\hbar^{2}}{2} S_{2}(x)+\ldots
$$

Sustituyendo el valor de $S(x)$ en la Ecuación II.18 (una vez despreciado el primer sumando) obtenemos un sistema de ecuaciones sin más que igualar a 0 cada uno de los

\footnotetext{
${ }^{13}$ El valor de $p_{0}$ se determina a partir de la Ecuación II.11 considerando el potencial constante, es decir $V(x)=V_{0}$.
} 
coeficientes de las distintas potencias de $\hbar$ separadamente. Resolviendo el sistema encontramos los valores de $S_{0}(x), S_{1}(x), S_{2}(x)$, etc.

De acuerdo con las hipótesis consideradas, tenemos en cuenta únicamente los dos primeros términos de la serie, pudiendo despreciar por tanto los de mayor orden. De este modo se obtienen las siguientes funciones de onda, soluciones de la ecuación de Schrödinger, tanto para las regiones clásicamente permitidas ( $E>V(x)$, Ecuación II.20), como para las prohibidas $(E<V(x)$, Ecuación I.21):

$$
\begin{array}{ll}
\psi(x)=\widetilde{A}[p(x)]^{-\frac{1}{2}} \exp \left[ \pm \frac{i}{\hbar} \int p\left(x^{\prime}\right) d x^{\prime}\right] & E>V(x) \\
\psi(x)=\widetilde{B}|p(x)|^{-\frac{1}{2}} \exp \left[ \pm \frac{1}{\hbar} \int\left|p\left(x^{\prime}\right)\right| d x^{\prime}\right] & E<V(x)
\end{array}
$$

Donde el momento $p(x)$ es imaginario puro en el caso de $E<V(x)$ (ver la Ecuación II.11) y $\widetilde{A}$ y $\widetilde{B}$ son constantes arbitrarias. En ambas regiones energéticas $(E>V(x)$ y $E<V(x)$ ), la solución WKB general será una combinación lineal de las soluciones positiva y negativa. Para las energías superiores al potencial de la barrera tendremos:

$$
\psi(x)=[p(x)]^{-\frac{1}{2}}\left\{A \exp \left[+\frac{i}{\hbar} \int p\left(x^{\prime}\right) d x^{\prime}\right]+B \exp \left[-\frac{i}{\hbar} \int p\left(x^{\prime}\right) d x^{\prime}\right]\right\} \quad E>V(x) \text { II.22 }
$$

Con $A$ y $B$, constantes arbitrarias. La exponencial positiva corresponde al paquete de ondas moviéndose en sentido positivo y la exponencial negativa en sentido opuesto.

Para la región energética clásicamente prohibida la función de onda general será:

$$
\psi(x)=|p(x)|^{-\frac{1}{2}}\left\{C \exp \left[+\frac{1}{\hbar} \int\left|p\left(x^{\prime}\right)\right| d x^{\prime}\right]+D \exp \left[-\frac{1}{\hbar} \int\left|p\left(x^{\prime}\right)\right| d x^{\prime}\right]\right\} \quad E<V(x) \text { II.23 }
$$

Con $C$ y $D$, constantes arbitrarias.

Estas soluciones WKB son totalmente válidas cuando se cumple el criterio expuesto en la Ecuación II.12, por tanto en las proximidades de un punto de retroceso en el que $E=V$, la aproximación WKB no puede utilizarse. Sin embargo, teniendo en cuenta que la función de onda debe ser continua para todo valor de $x$, podemos obtener una fórmula de conexión entre los dos tipos de soluciones WKB (Ecuaciones II.22 y I.23) en torno al punto de retroceso. 
Como decíamos anteriormente, se supone un flujo de partículas que incide desde la izquierda con energía $E$. Algunas partículas se reflejan en la región 1 ( $x<x_{2}$ en Figura II.4), el resto son transmitidas y emergen en la región $3\left(x>x_{1}\right)$. En esta zona la energía es mayor que la barrera de potencial y el paquete de ondas se desplaza únicamente hacia la derecha, por lo que utilizamos la Ecuación II.22 particularizada para esta situación:

$$
\psi_{3}(x)=A[p(x)]^{-\frac{1}{2}} \exp \left[\frac{i}{\hbar} \int_{x_{1}}^{x} p\left(x^{\prime}\right) d x^{\prime}\right] \quad x>x_{1}
$$

La solución de la ecuación de Schrödinger en la región 2 se obtiene a partir de la Ecuación II.23 (la energía es inferior al valor del potencial) y aplicando una fórmula de conexión ${ }^{14}$ en el punto de retroceso $x_{1}$ para mantener la continuidad de la función de onda en el paso de la región 2 a la 3.

$$
\psi_{2}(x)=-i A|p(x)|^{-\frac{1}{2}} \exp [\Lambda] \exp \left[-\frac{1}{\hbar} \int_{x_{2}}^{x_{1}}\left|p\left(x^{\prime}\right)\right| d x^{\prime}\right] \quad x_{2}<x<x_{1}
$$

donde se ha definido el parámetro $\Lambda$ del siguiente modo:

$$
\Lambda=\frac{1}{\hbar} \int_{x_{2}}^{x_{1}}|p(x)| d x=\frac{1}{\hbar} \int_{x_{2}}^{x_{1}}\{2 m[V(x)-E]\}^{\frac{1}{2}} d x
$$

A partir de esta solución y de la consideración de la continuidad de la función de onda en el punto de retroceso $x_{2}$ llegamos a la siguiente expresión para la función de onda en la región 1:

$$
\begin{aligned}
& \psi_{1}(x)=-i A[p(x)]^{-\frac{1}{2}} e^{\Lambda}\left\{\exp \left[\frac{i}{\hbar} \int_{x}^{x_{2}} p\left(x^{\prime}\right) d x^{\prime}\right]\right. \\
& \left.+\exp \left[-\frac{i}{\hbar} \int_{x}^{x_{2}} p\left(x^{\prime}\right) d x^{\prime}\right]\right\} \quad x<x_{2}
\end{aligned}
$$

En esta solución, la primera exponencial representa al paquete de ondas moviéndose hacia la derecha y la segunda, moviéndose hacia la izquierda.

\footnotetext{
${ }^{14}$ Considerando una variación prácticamente lineal del potencial en las cercanías del punto de retroceso, la función de onda solución de la ecuación de Schrödinger es la función de Airy [Bransden, 1986], que finalmente puede expresarse como vemos en la Ecuación II.24 y siguientes, en los distintos puntos de retroceso. Es a esta metodología de resolución a lo que se le considera como fórmula de conexión.
} 
Una vez que hemos determinado las soluciones de la función de onda para cada región, ya podemos proceder al cálculo de las densidades de probabilidad de corriente, cuya expresión general dimos en la Ecuación II.16.

El ratio entre la densidad de corriente transmitida (calculada a partir de la función de onda transmitida, es decir, $\Psi_{3}(x)$ ) y la incidente (a partir de la función de onda incidente, es decir, el primer sumando de la Ecuación II.27) nos proporciona el valor del coeficiente de transmisión de la región 1 a la 3 (Ecuación II.15):

$$
T_{C 1 \rightarrow 3}=\exp (-2 \Lambda)=\exp \left[-\frac{2}{\hbar} \int_{x_{2}}^{x_{1}}\{2 m[V(x)-E]]^{\frac{1}{2}} d x\right]
$$

Este coeficiente de transmisión proporcionado por la aproximación WKB es, como ya hemos visto anteriormente, la clave para calcular la corriente inyectada (que, particularizando, es $\left.T_{C m s}\right)$ y absorbida $\left(T_{C s m}\right)$, tanto por efecto túnel $\left(E<q \Phi_{B}\right)$ como por emisión termoiónica $\left(E>q \Phi_{B}\right)$. Además, es muy adecuado para implementarlo en el simulador Monte Carlo puesto que la relativa sencillez de esta expresión comparada con la proporcionada por otros métodos agiliza los ya de por sí pesados cálculos que debe efectuar el simulador.

\section{II.4.b. Método de la matriz de transferencia de Airy (MTA)}

A continuación mostramos sucintamente los pasos a seguir en el cálculo del coeficiente de transmisión mediante el método MTA: encontraremos una descripción más detallada de los mismos en [Lui, 1986], [Brennan, 1987].

Consideramos nuevamente una barrera de potencial arbitraria, como la mostrada en la Figura II.4; se divide la misma en un número de regiones y se realiza la interpolación del potencial entre cada uno de los nodos resultantes. Tras aplicar la condición de continuidad de las funciones de onda y sus derivadas en cada interfaz, la función de onda incidente y transmitida se expresan del siguiente modo, en función de los coeficientes $A, B, C$ y $D$ :

$$
\begin{aligned}
& \psi_{\text {incidente }}(x)=A \exp \left[i k_{m} x\right]+B \exp \left[-i k_{m} x\right] \\
& \psi_{\text {transmitida }}(x)=C \exp \left[i k_{s c}(x-L)\right]+D \exp \left[-i k_{s c}(x-L)\right] \\
& \left(\begin{array}{l}
A \\
B
\end{array}\right)=\left(\begin{array}{cc}
1 & 1 \\
i k_{s c} & -i k_{s c}
\end{array}\right)^{-1} S(0, L)\left(\begin{array}{cc}
1 & 1 \\
i k_{m} & -i k_{m}
\end{array}\right)\left(\begin{array}{l}
C \\
D
\end{array}\right)
\end{aligned}
$$


donde $L$ es la anchura total de la barrera y $S(0, L)$ es la matriz de transferencia de Airy de la barrera, la cual se obtiene a partir de la multiplicación de las matrices de Airy ${ }^{15}$ dadas por las condiciones de contorno en los nodos anteriormente considerados [Lui, 1986], [Brennan, 1987]. A partir de las expresiones resultantes, el coeficiente de transmisión puede ser evaluado como el ratio entre la corriente transmitida e incidente (Ecuación II. 15), obteniendo así la siguiente expresión [Winstead, 2000], [Rengel, 2007]:

$$
T_{C}\left(E_{\text {inc }}\right)=\frac{\left|\psi_{\text {transmitida }}\right|^{2}}{\left|\psi_{\text {incidente }}\right|^{2}} \cdot \frac{m_{m}^{*} k_{s c}}{m_{s c}^{*} k_{m}}
$$

$m_{m}^{*} \mathrm{y}{ }^{*}{ }_{s c}$ representan la masa efectiva en el metal y en el semiconductor y $k_{m}$ y $k_{s c}$ son los correspondientes vectores de onda. En nuestro caso, aunque es posible considerar también la dependencia del coeficiente $T_{C}$ con la componente del vector de onda en el metal paralela al movimiento de la partícula, no hemos encontrado una dependencia significativa con este parámetro, lo cual coincide con los resultados expuestos en [Winstead, 2000].

Finalmente, el coeficiente de transmisión puede expresarse en términos de los coeficientes A, B, C y D de la matriz $S(0, L)$ del siguiente modo, considerando la misma masa efectiva en el metal y en el semiconductor $\left(0.26 m_{0}\right)$ por simplicidad [Rengel, 2007]:

$$
T_{C}\left(E_{i n c}\right)=\frac{4 k_{m} / k_{s c}}{\left[\left(A+\frac{k_{s c}}{k_{m}}\right)^{2}+\left(\frac{C}{k_{m}}-k_{s c} B\right)^{2}\right]}
$$

Una vez determinado el valor del coeficiente de transmisión, la densidad de corriente inyectada desde el metal al semiconductor se obtiene como en el caso anterior, a partir de la Ecuación II.13.

El método de la matriz de transferencia de Airy proporciona una solución exacta al potencial lineal, sin mostrar las limitaciones de la aproximación WKB. Además, también tiene en cuenta las reflexiones cuánticas en el rango de energía termoiónica (y también para las energías túnel una vez que la partícula ha sobrepasado el punto de retroceso $x_{2}$-ver Figura II.4). Por otro lado, es mucho más complicado de desarrollar que la aproximación WKB puesto que presenta dificultades importantes como la determinación del origen de energías en el metal, siendo además más costoso desde el punto de vista computacional (debe tenerse en cuenta que

\footnotetext{
${ }^{15}$ Las matrices de Airy son un conjunto de matrices 2 x 2 que se componen de las funciones de Airy y sus derivadas.
} 
hay que llevar a cabo numerosas multiplicaciones de las matrices de Airy y sus derivadas, entre otros cálculos).

\section{II.4.c. Comparación directa entre ambas aproximaciones}

Hemos desarrollado un algoritmo que determina el coeficiente de transmisión a través de una barrera de potencial mediante ambos métodos, el WKB y el MTA. Esto nos ha permitido contrastar, por primera vez en la literatura, las diferencias obtenidas entre ambas soluciones de la ecuación de Schrödinger al considerar una barrera de potencial de tipo Schottky, estos resultados han sido publicados en [Rengel, 2007].

Para ello se ha tenido en cuenta el perfil de una barrera correspondiente a un contacto Schottky de altura igual a $0.2 \mathrm{eV}$ sobre Silicio tipo $n\left(10^{16} \mathrm{~cm}^{-3}\right)$; este perfil de potencial ha sido proporcionado por una simulación Monte Carlo. El algoritmo calcula tanto el coeficiente de transmisión como la carga inyectada mediante ambas aproximaciones (WKB y MTA).
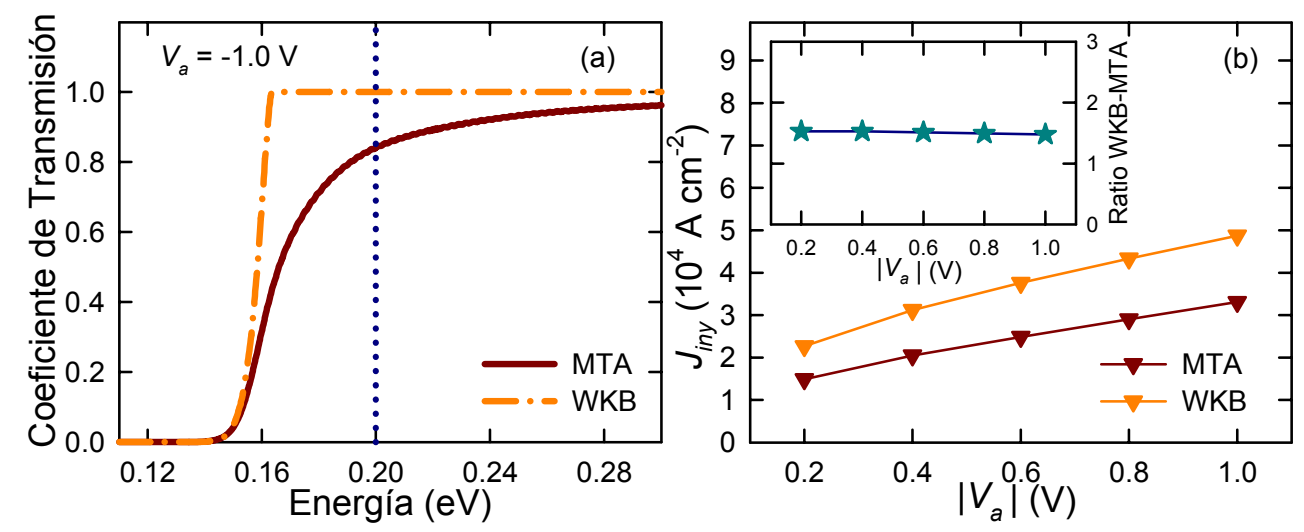

Figura II.5.a. Coeficiente de transmisión cuántico en función de la energía incidente para un voltaje en inversa aplicado igual a -1 V: resultados WKB y MTA .b. Corriente inyectada en función del voltaje en inversa aplicado para ambas aproximaciones; el recuadro interior de esta figura muestra el ratio entre los resultados MTA y WKB.

Las simulaciones muestran discrepancias significativas en la dependencia con la energía y los valores del coeficiente de transmisión, como puede observarse en la Figura II.5. En el caso de la aproximación WKB, para energías incidentes superiores al valor de la altura de barrera efectiva, se obtiene que el valor de $T_{C}$ es igual a la unidad: esto significa que todos los portadores en el metal con energías superiores a $q \Phi_{B n}$ pueden entrar en el dispositivo. Sin 
embargo, esto no es completamente exacto desde el punto de vista físico: debido a la existencia de la barrera, e incluso en el rango de energías termoiónicas existe la posibilidad de que las partículas sean reflejadas y no penetren en el dispositivo, como puede observarse claramente en el perfil del coeficiente de transmisión proporcionado por la aproximación MTA. Es más, para las energías inferiores a la altura de barrera efectiva, la probabilidad real de atravesar dicha barrera por efecto túnel es también más baja que la que predice el método WKB.

Sin embargo, la cuestión principal desde el punto de vista del modelado es la determinación de la corriente total a inyectar, mostrada en la Figura II.5.b en función del voltaje en inversa aplicado. Por otro lado, en la Figura II.6 mostramos la dependencia de la corriente inyectada con la energía para dos valores de voltaje en inversa aplicado. Como podemos observar en ambas figuras, la aproximación WKB sobreestima la corriente total, aunque el comportamiento frente a la energía es similar, presentando el valor máximo de corriente inyectada en la región energética próxima al valor de la altura de la barrera Schottky. Sin embargo, si examinamos el ratio entre la corriente proporcionada mediante WKB y MTA, se obtiene un valor prácticamente constante (recuadro interior de la Figura II.5.b). Este resultado es particularmente significativo, puesto que indica que la aproximación WKB puede predecir razonablemente el valor de la corriente total mediante una consideración adecuada de los parámetros del modelo (por ejemplo de la constante de Richardson). Por tanto, podemos afirmar que el método WKB puede predecir de manera razonable el valor de la corriente a través de la barrera (en función del voltaje en inversa aplicado) en el caso de una unión Schottky. A la vista de estos resultados, hemos considerado la aproximación WKB para su implementación en el simulador Monte Carlo.

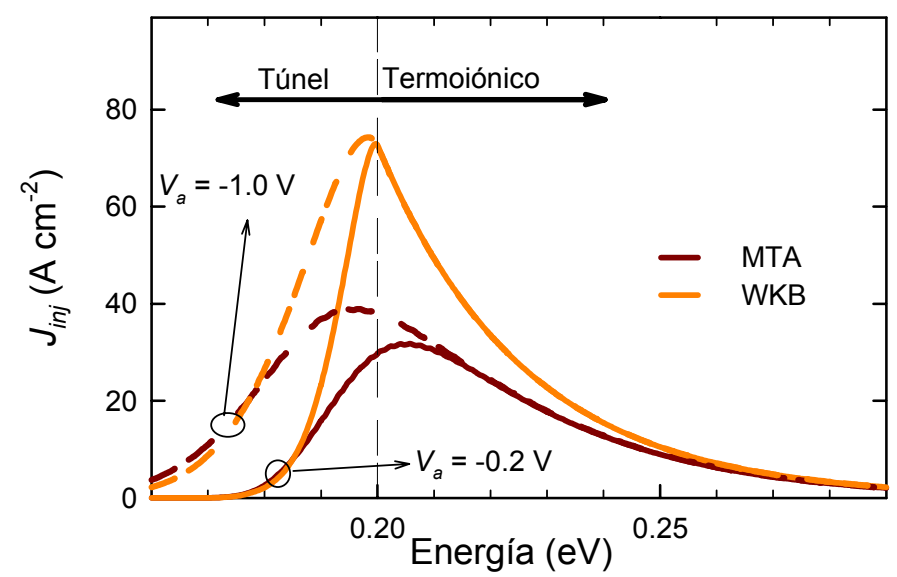

Figura II.6. Corriente inyectada en función de la energía para dos valores de voltaje en inversa aplicado $(\mathrm{Va}=-0.2 \mathrm{Vy}-1.0 \mathrm{~V})$. Mostramos los resultados para ambas aproximaciones (MTA y WKB). 


\section{II.5. Algoritmo de inyección/absorción: Implementación en el simulador Monte Carlo}

A continuación presentamos los pasos seguidos para la implementación del algoritmo de inyección/absorción basado en la aproximación WKB en el simulador Monte Carlo bidimensional. La reducción del problema bidimensional al unidimensional es obvio, por lo que no lo mostramos en el presente trabajo, sin embargo, podemos encontrar detalles acerca de la implementación en el simulador Monte Carlo 1D en [Pascual, 2007].

En primer lugar, se evalúan todas las condiciones de contorno en las mallas del dispositivo simulado, identificando aquellas celdas que representen fronteras metal/semiconductor ${ }^{16}$. Estas celdas son susceptibles de inyectar o absorber portadores y se les asigna convenientemente un número de situación $(l)$. Con motivo de optimizar tiempo de CPU, hemos considerado una región que denominamos de influencia, la cual se extiende desde cada celda numerada que pertenezca a un contacto Schottky hacia el interior del dispositivo en la dirección perpendicular. Es importante señalar que esta distancia debe elegirse lo suficientemente grande como para no despreciar ninguna posible contribución a la inyección de portadores en el interior del dispositivo (ni absorción desde el semiconductor al metal), evitando al mismo tiempo la consideración innecesaria de zonas en las que no puede tener lugar el transporte túnel por encontrarse demasiado alejadas del contacto.

Acto seguido, se lleva a cabo una discretización $(j)$ de las energías incidentes $\left(E_{j, l}\right)$ en cada una de las celdas numeradas correspondientes a contactos Schottky. El coeficiente de transmisión $T_{C j, l}$ es evaluado para cada uno de los intervalos de energía siguiendo la expresión de la Ecuación II. 28, proporcionada por la aproximación WKB, para el caso particular de un contacto vertical (los contactos horizontales seguirán la misma metodología, cambiando la dimensión $x$ por la $y$ ):

$$
T_{C j, l}=\exp \left[-\frac{2}{\hbar} \int_{x_{2}}^{x_{1}}\left\{2 m\left[V_{l}(x)-E_{j, l}\right]\right\}^{\frac{1}{2}} d x\right]
$$

El potencial de barrera Schottky $V_{l}(x)$ se determina a partir del potencial bidimensional proporcionado por el simulador Monte Carlo y corregido teniendo en cuenta el efecto de las cargas imagen, cuya implementación será detallada al final de esta sección. Debemos recordar

\footnotetext{
${ }^{16}$ En nuestro caso, como ya hemos comentado en el capítulo anterior, el contacto es de tipo Siliciuro/Silicio.
} 
que el potencial proporcionado por el simulador está auto-consistentemente acoplado a la resolución de la ecuación de Poisson en cada paso temporal. El coeficiente de transmisión se evalúa para todas las regiones de influencia existentes, teniendo en cuenta la interpolación del potencial Monte Carlo en los nodos adyacentes a cada una de las superficies de inyección / absorción consideradas. Los puntos de retroceso $\left(\begin{array}{lll}x_{1} & \text { y } & x_{2}\end{array}\right)$ correspondientes a cada energía incidente deben ser determinados cuidadosamente para evaluar la integral numérica.

La carga a inyectar en cada intervalo de energía para el número de regiones de influencia establecido se calcula como sigue [Sun, 2003]:

$$
Q_{j, l}=\frac{A^{*} T \Delta t}{K_{B}} T_{C j, l} f_{m}\left(E_{j, l}\right)\left[1-f_{m}\left(E_{j, l}\right)\right] \Delta E_{j, l}
$$

A partir de esta cantidad, el número de partículas a ser inyectadas en cada paso temporal se determina del siguiente modo:

$$
N_{l}=\sum_{j=1}^{n_{E}} \frac{Q_{j, l}}{E P E} \Delta d
$$

Donde $n_{E}$ es el número total de intervalos de energía incidente considerados (para todas las regiones de influencia) y $E P E$ es el peso de la partícula equivalente; también tenemos en cuenta la anchura $\Delta d$ de la superficie de la celda paralela al contacto. Una vez calculado el número total de partículas a inyectar en las distintas regiones de influencia, determinamos la zona energética de inyección para cada partícula de acuerdo con la distribución de la carga inyectada valiéndonos de la siguiente expresión:

$$
\sum_{k=1}^{j} \frac{Q_{k, l}}{E P E} \Delta d<r N_{l}<\sum_{k=1}^{j+1} \frac{Q_{k+1, l}}{E P E} \Delta d
$$

Las partículas termoiónicas (aquellas cuya energía incidente es superior a la altura de la barrera) son depositadas en la interfaz del contacto Schottky (la posición exacta en la superficie de la celda es seleccionada mediante un número aleatorio) con un vector de onda perpendicular a la superficie y orientado hacia el interior del semiconductor igual a:

$$
k_{\perp} \mid=\sqrt{\frac{2 m_{\perp}^{*}}{\hbar}\left(\frac{1}{2} K_{B} T+E_{j, l}-q \Phi_{B}\right)}
$$

Nótese que la energía térmica $\left(3 / 2 K_{B} T\right)$, se reparte entre las tres direcciones del espacio. Si la superficie del contacto Schottky de la región de influencia $l$ fuera vertical, esta cantidad sería igual a $k_{\perp}$, con signo positivo o negativo dependiendo de la orientación del contacto. La 
componente paralela al vector de onda, $k_{\|}$, se elige con orientación aleatoria en el plano paralelo al contacto; además el valor de su módulo se corresponde con a una energía igual a $K_{B} T$.

La posición en el interior del dispositivo para aquellas partículas que son inyectadas mediante el efecto túnel viene definida por el punto de retroceso correspondiente a su energía incidente. Su valor inicial de $k_{\perp}$ es cero; $k_{\|}$es elegido de manera análoga al caso termoiónico [Shen, 2004].

El procedimiento a seguir con las partículas absorbidas desde el semiconductor al metal es distinto al de las inyectadas. En primer lugar consideramos las mismas áreas de influencia que en los procesos de inyección. En el caso termoiónico, todas aquellas partículas que alcanzan el contacto con energía suficiente para sobrepasar la barrera son absorbidas y abandonan la estructura simulada. Por otro lado, evaluamos del siguiente modo a las partículas que en el interior del semiconductor pueden ser absorbidas mediante efecto túnel: examinamos su componente perpendicular del vector de onda; si en cualquier momento de un recorrido libre es igual a cero, empleamos una técnica de rechazo ${ }^{17}$ para determinar si son absorbidas o no. Esta técnica de rechazo tiene en cuenta el coeficiente de transmisión correspondiente al valor del potencial $V_{l}(x)$ en ese punto (cuando $k_{\perp}=0$ ) multiplicado por la probabilidad de que el correspondiente estado de energía en el metal esté desocupado [Matsuzawa, 2000].

Mediante el procedimiento expuesto anteriormente, obtenemos cuatro contribuciones distintas a la corriente total (inyección túnel y termoiónica, absorción túnel y termoiónica) a partir del conteo del número de partículas inyectadas o absorbidas correspondientes a cada proceso.

Como decíamos al principio de la sección, los pasos seguidos son similares a los de la implementación del algoritmo en el simulador Monte Carlo 1D [Pascual, 2007], sin embargo la complejidad del problema bidimensional es mucho mayor.

Por otro lado, además de considerar la emisión termoiónica y el efecto túnel, hemos incluido el efecto de reducción de la barrera Schottky debido a las cargas imagen [Sze, 1981]. Para ello consideramos la influencia de la fuerza imagen de atracción entre el electrón y el semiconductor de manera continua mediante la modificación del perfil de potencial de Poisson.

\footnotetext{
${ }^{17}$ La técnica de rechazo está basada en la generación de números aleatorios uniformemente distribuidos entre 0 y 1 a partir de una función de probabilidad [Jacoboni, 1989]
} 
Teniendo en cuenta este hecho, el perfil de la banda de conducción proporcionado por el simulador Monte Carlo se modifica localmente como sigue para el cálculo del coeficiente de transmisión:

$$
V_{l}^{\prime}(x)=V_{l}(x)-\frac{q^{2}}{16 \pi \varepsilon_{0} x}
$$

En la Figura II. 7 mostramos un ejemplo de la reducción de la barrera por el efecto de las cargas imagen. La línea negra representa la banda de energía Monte Carlo $\left(V_{l}(x)\right)$ para un contacto Schottky con $q \Phi_{B}=0.5 \mathrm{eV}$. El efecto de las cargas imagen está representado por la línea roja, mientras que la composición de ambas energías $\left(V_{l}{ }^{\prime}(x)\right)$ está dibujado en verde. En este ejemplo en particular, la reducción de la altura de la barrera es de $0.0655 \mathrm{eV}$ y el máximo (véase, en el capítulo anterior, la Ecuación I.30) está situado a $0.933 \mathrm{~nm}$ del contacto Schottky. Como podemos observar, el resultado es un perfil de potencial suave, el cual se corresponde con la física del problema. Este perfil corregido por el efecto de las cargas imagen es el que se emplea en la Ecuación II.33 para determinar el coeficiente de transmisión de cada celda correspondiente a una interfaz Schottky.

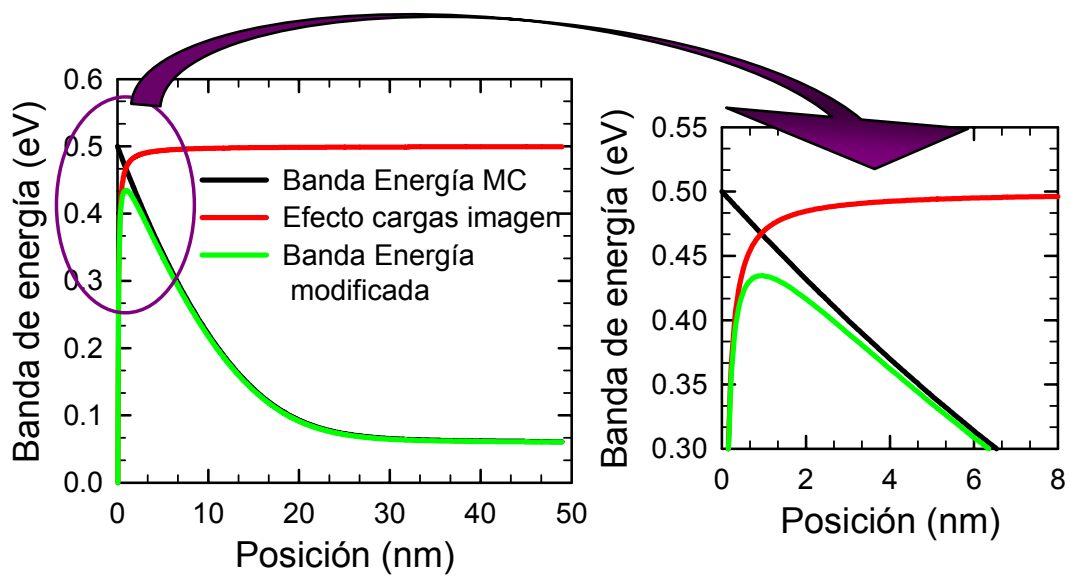

Figura II.7. Diagrama de energías de un contacto Schottky con $q \Phi_{B}=0.5 \mathrm{eV}$. Aparecen representados los perfiles de la banda de energía Monte Carlo, del efecto de las cargas imagen y la nueva banda de energía. A la derecha mostramos una ampliación de la banda en la región cercana al contacto. 


\section{II.6. Modelo de inyección/absorción: Aplicación al caso de barreras sencillas}

Como paso previo a la implementación del algoritmo de inyección/absorción en el simulador Monte Carlo y antes de considerar barreras realistas correspondientes a contactos Schottky, desarrollamos un algoritmo numérico para la determinación del coeficiente de transmisión de modo que pudiéramos comprobar el correcto funcionamiento de la aproximación WKB. De este modo, hemos considerado diferentes barreras arbitrarias de geometría sencilla, concentrándonos más específicamente en el caso de barreras triangulares por su mayor semejanza al perfil ideal, en las cercanías del contacto, de una barrera Schottky de altura relativamente elevada (como la presentada en la Figura II.7). A continuación mostramos algunos de los resultados obtenidos.

\section{Barrera triangular}

Como primera aproximación sencilla de la barrera Schottky que nos interesa, tomamos la barrera triangular y calculamos el coeficiente de transmisión a través de la misma así como la carga inyectada teórica correspondiente. En este caso particular, la barrera es una recta definida por dos puntos, $\left(0, q \Phi_{B n}\right)$ y $\left(d, E_{C}\right)$ donde $q \Phi_{B n}$ es la altura de la barrera y $d$ es la anchura de la misma. El valor del nivel de Fermi del semiconductor se calcula a partir de la concentración de impurezas en el material y es fijado como origen de energías. Analizaremos la influencia de distintos parámetros, como son la altura, la anchura de la barrera y la tensión aplicada.

\section{a) Influencia de la anchura de la barrera}

Para realizar este estudio efectuamos varias simulaciones en las que inicialmente procedemos a fijar la altura de la barrera a $q \Phi_{B n}=0.8 \mathrm{eV}$ y variamos su anchura (Ver Figura II.8). Comprobamos así el efecto de esta variación sobre el coeficiente de transmisión y la carga inyectada. 


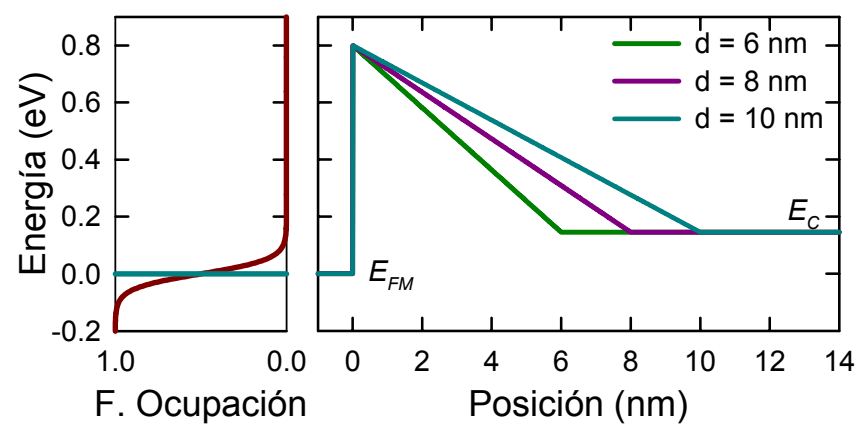

Figura II.8. Barrera triangular de $0.8 \mathrm{eV}$ de altura fija y distintas anchuras de $6 \mathrm{~nm}, 8 \mathrm{~nm}$ y $10 \mathrm{~nm}$. También se muestra en la figura la variación de la función de ocupación de los electrones en el metal con la energía (a la izquierda).

El coeficiente de transmisión correspondiente (Figura II.9) toma valor uno para energías de la región termoiónica, tal y como indica la aproximación WKB, lo que implica que todas las partículas con energía incidente superior a $0.8 \mathrm{eV}$ son inyectadas en el semiconductor. Esto se ve reflejado en el comportamiento exponencial de la carga inyectada para la región de energías termoiónicas (Figura II.9.b), que sigue al perfil de la función de distribución de Fermi-Dirac en el metal.
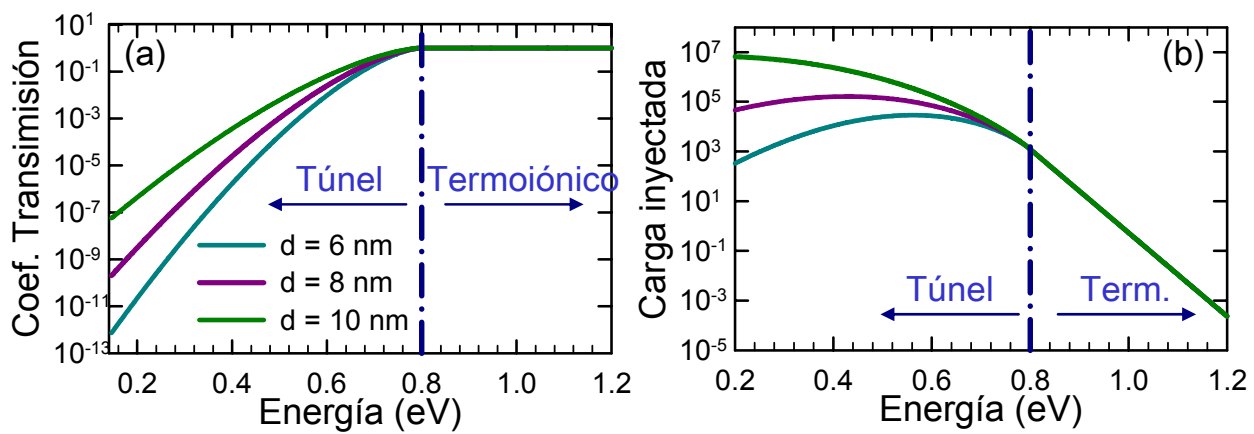

Figura II.9.a. Coeficiente de transmisión correspondiente a cada barrera .b. Carga inyectada a través de las mismas.

Para las energías incidentes inferiores a la altura de la barrera (relativas al efecto túnel), el coeficiente de transmisión disminuye al aumentar la anchura de la barrera, consecuencia de que el camino túnel es cada vez mayor para la misma energía (Figura II.8). Del mismo modo, la carga inyectada mediante el efecto túnel disminuye con la anchura de la barrera. Además, el máximo de la carga túnel se sitúa a energías inferiores al disminuir este parámetro; esto es debido al efecto conjunto del aumento del valor del coeficiente de transmisión y la función de 
ocupación, que toma el valor más elevado para la energía correspondiente al fondo de la banda de conducción, mientras que a energías superiores su valor es prácticamente cero (Figura II.8).

\section{b) Influencia de la altura de la barrera}

En este apartado estudiamos el efecto de la variación de la altura de la barrera sobre el coeficiente de transmisión y la carga inyectada. En esta ocasión hemos realizado una serie de simulaciones en las cuales fijamos la anchura de la barrera triangular a $4 \mathrm{~nm}$ y variamos el valor de su altura, como se observa en la Figura II.10.

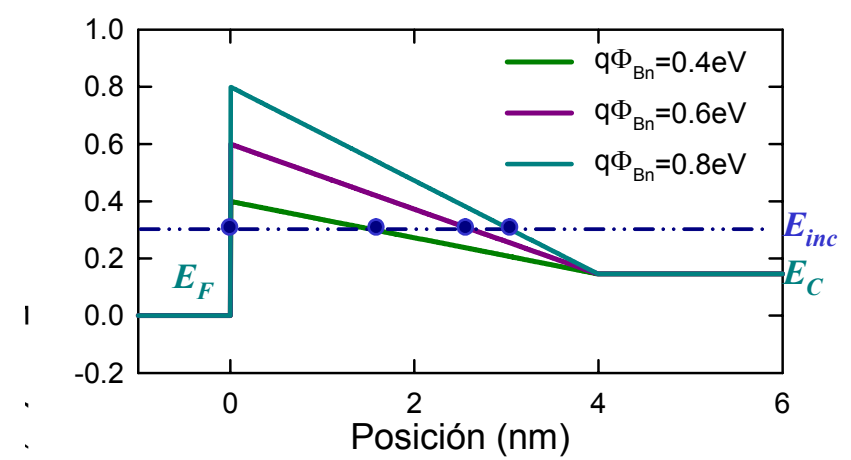

Figura II.10. Barrera de $4 \mathrm{~nm}$ de anchura fija y distintas alturas, desde 0.4 a $0.8 \mathrm{eV}$. También mostramos cómo para una misma energía incidente el camino túnel es mayor según aumenta $q \Phi_{B n}$

De nuevo comprobamos cómo el coeficiente de transmisión toma el valor uno para energías superiores a la altura de la barrera (Figura II.11.a), con el correspondiente comportamiento exponencial de la carga inyectada para estos valores de energía (Figura II.11.b).

En cuanto a la región energética túnel, observamos (Figura II.11.a) que al reducir la altura de la barrera la probabilidad de que una partícula con igual energía incidente la atraviese aumenta. Esto se debe a que al disminuir su altura, la diferencia entre la barrera de potencial y la energía considerada también se ve reducida, obteniéndose así un coeficiente de transmisión de mayor valor (ver Ecuación II.28). Además, el camino túnel se estrecha para el mismo valor de energía (al disminuir la altura de la barrera, ver Figura II.10), como consecuencia, la carga inyectada mediante el túnel cuántico aumenta puesto que la probabilidad de transmisión es ahora más elevada para energías con mayor nivel de ocupación en el metal. 


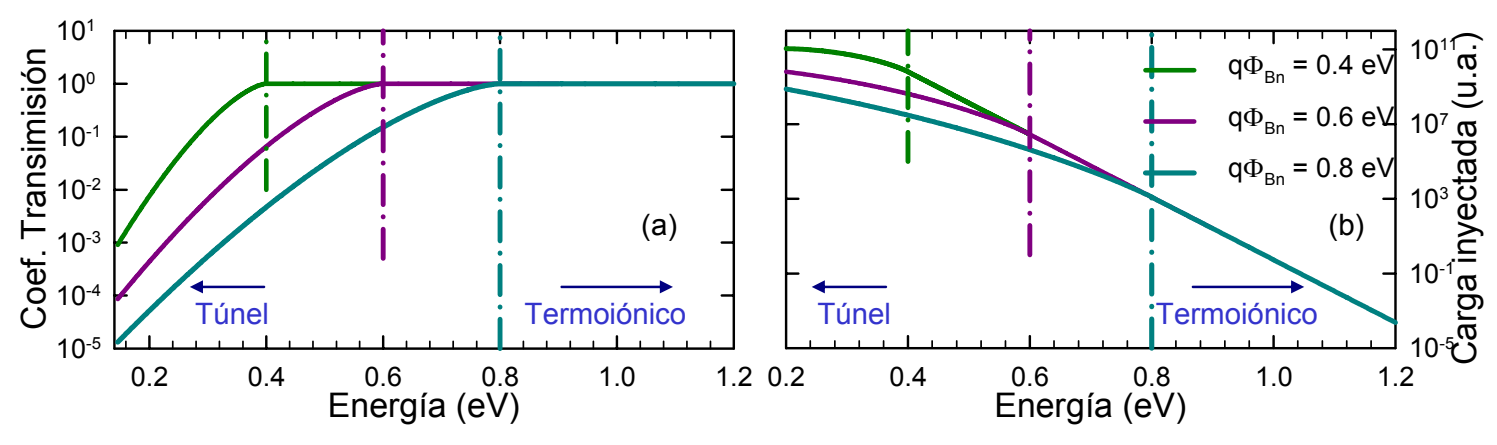

Figura II.11.a. Coeficientes de transmisión correspondiente a cada barrera .b. Carga inyectada a través de las distintas barreras.

\section{c) Influencia del voltaje aplicado}

Examinamos aquí de forma breve el efecto de la variación de la tensión aplicada sobre el coeficiente de transmisión y la carga inyectada. Sometemos una barrera triangular de $0.8 \mathrm{eV}$ de altura y $10 \mathrm{~nm}$ de anchura a distintos voltajes, de $0.4 \mathrm{~V}$ en directa, $0 \mathrm{~V}$ y $-0.4 \mathrm{~V}$ en inversa. La representación esquemática de la barrera en las distintas situaciones se muestra a continuación (Figura III.12).

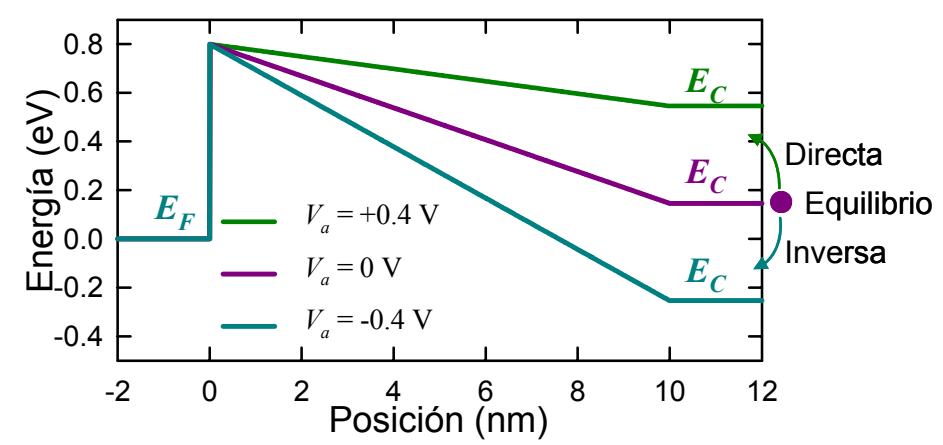

Figura II.12. Barrera triangular a distintas polarizaciones, de $0 \mathrm{~V}, 0.4 \mathrm{~V}$ en directa y $-0.4 \mathrm{~V}$ en inversa

La altura de la barrera para los electrones en el metal permanece invariante con la tensión aplicada, sin embargo el fondo de la banda de conducción en el semiconductor asciende para tensiones en directa y desciende en el régimen de inversa. Por tanto para tensiones aplicadas en directa la probabilidad de transmisión desde el metal al semiconductor es inferior que para polarizaciones en inversa, como podemos observar en la Figura II.13.a. 

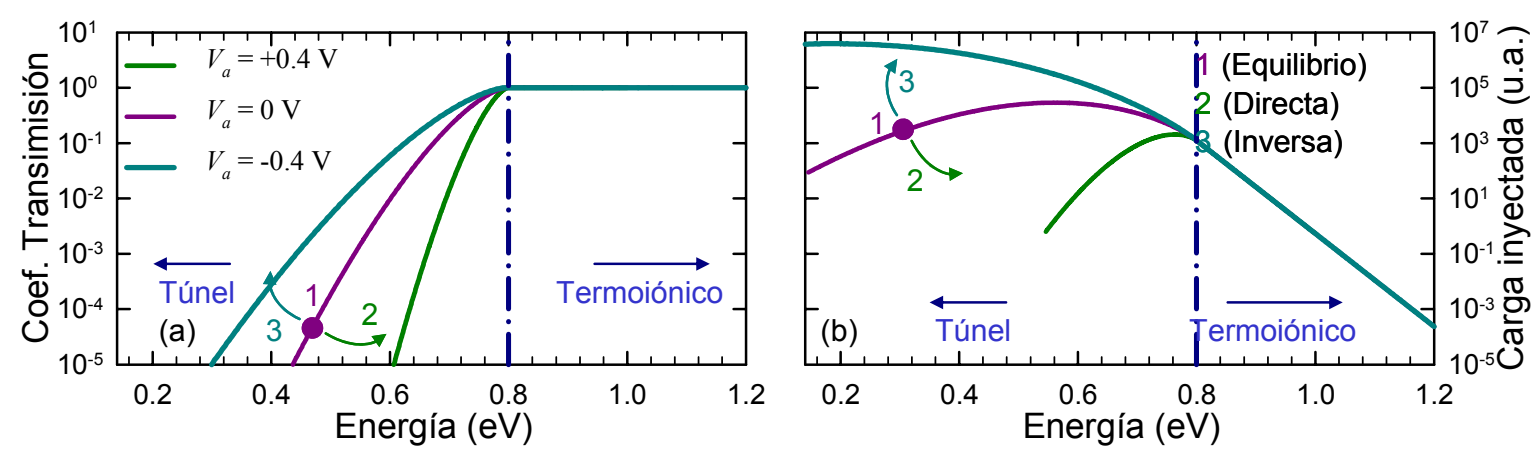

Figura II.13.a. Coeficiente de transmisión a través de la barrera para las distintas tensiones aplicadas

.b. Correspondiente carga inyectada.

La carga inyectada (Figura II.13.b.) por emisión termoiónica sigue el comportamiento exponencial típico mientras que la inyectada a través del efecto túnel aumenta considerablemente según aplicamos tensiones en inversa, al hacerse la barrera más estrecha para una determinada energía de inyección dada.

Para completar el estudio del modelo de inyección implementado, hemos contrastado nuestro coeficiente de transmisión con los resultados encontrados en la literatura para el caso de diversas barreras de potencial obteniendo resultados altamente satisfactorios. Una vez testado el algoritmo en los casos de barrera lineal y parabólica, se procedió a su implementación en el simulador Monte Carlo unidimensional, lo que nos ha permitido abordar el estudio del transporte túnel en el caso de diodos de barrera Schottky y diodos back-to-back, como veremos en los Capítulos III y IV. Posteriormente, realizamos la implementación en el simulador bidimensional con el fin de estudiar transistores SB-MOSFET (Capítulos V y VI). 


\section{Bibliografía del Capítulo II}

Aldegunde M., García-Loureiro A. J., Kalna K., Asenov A., "Study of fluctuations in advanced MOSFETs using a 3D finite element parallel simulator" J. Comput. Electron., 5, 311, (2006)

Aldegunde M., García-Loureiro A. J., Martínez A., Kalna K., "3D Monte Carlo Simulation of Tri-Gate MOSFETs Using Tetrahedral Finite Elements" IEEE, International Conference on Simulation of Semiconductor Processes and Devices, 2008. SISPAD. (2008)

ATLAS "New Thermionic Emission and Tunneling Models in ATLAS", The Simulation Standard, 10, no. 8, pp. 6-7 (1999).

Boardman A. D., "Computer Simulations of Hot Electron Behaviour in Semiconductor Using Monte Carlo Methods", en Physics Programs, Boardman A. D., Wiley, New York, 315 (1980)

Bransden B. H. and Joachain C. J., "Introduction to quantum mechanics", Longman Scientific \& Technical (1986)

Brennan K. F., Hess K., Tang J.Y. y Lafrate G. J., "Transient Electronic Transport in InP Ander the Condition of High-Energy Electron Injection", IEEE Trans. Electron. Devices, ED30, 1750, (1983)

Brennan K. F. and Summers C. J., "Theory of resonant tunneling in a variably spaced multiquantum well structure: An Airy function approach," J. Appl. Phys., 61, 614 (1987)

Brennan K. y Hess K., "High field transport in GaAs InP and InAs", Solid-State Electron. 27, 347 (1984)

Brillouin L., Comptes Rendus 183, 24 (1926)

Cohen M. L. y Bergstresser T. K., "Band Structure and Pseudopotential Form Factors for Fourteen Semiconductors of the Diamond and Zinc-Blende Structures", Phys. Rev. 141, 789, (1966)

Cohen M. L. y Chelikowsky J. R., "Electronic Structure and Optical Properties of Semiconductors", Berlin, Springer-Verlag, (1988) 
Constant E., "Modelling of Sub-Micron Devices", Solid State Devices, Carroll J. E., IOP Conf. Series, 57, 141, (1980)

Fuchs K. and Wills H. H., Proceedings of the Cambridge Philosophical Society, 100-108 (1937)

Gámiz F., Roldán J. B., Godoy A., Carceller J. E., Cartujo P., "Double gate silicon on insulator transistors. A Monte Carlo study”, Solid-State Electronics, 48, 937 (2004)

García-Loureiro A. J., Kalna K., Asenov A., "Intrinsic fluctuations induced by a high-kappa gate dielectric in sub-100 nm Si MOSFETs" ICNF: Int. Conf. Noise and Fluctuations, 3 (2005)

Ghandhi S. K., VLSI Fabrication Principles, Wiley, New York (1994)

Godoy A., Ruiz-Gallardo A., Sampedro C., Gámiz F., "Quantum-mechanical effects in multiple-gate MOSFETs", J. Comput. Electron., 6,145 (2007)

González T., "Análisis del Ruido Electrónico en Materiales y Dispositivos Semiconductores Unipolares mediante el Método de Monte Carlo", Tesis Doctoral, Universidad de Salamanca (1994)

González T. y Pardo D., "Physical Models of Ohmic Contacts for Monte Carlo Device Simulation”, Solid-State Electron. 39, 555 (1996)

González T., Vasallo B. G., Pardo D. and Mateos J., "Room temperature nonlinear transport in ballistic nanodevices", Semiconductor Science and Technology, 19, S125-S127 (2004)

Havart R., Lheurette E., "Step-like heterostructure barrier varactor" IEEE Transactions on Electron Devices, 45, Num. 11 (1998)

Hockney R. W. y Eastwood J. W., "Semiconductor device simulation" en Computer simulation using particles, IOP, Bristol (1988)

Huang C. K., Zhang W. E. And Yang C. H. "Two - dimensional numerical simulation of Schottky Barrier MOSFET with channel length to $10 \mathrm{~nm}$ " IEEE Transactions on Electron Devices, 45, 4 (1998)

Jacoboni C. y Reggiani L., "The Monte Carlo Method for the Solution of Charge Transport in Semiconductors with Applications to Covalent Materials", Rev. Mod. Phys., 55, 645 (1983)

Jacoboni C. y Lugli P. "The Monte Carlo Method for Semiconductor Device Simulation", Viena: Springer (1989)

Kalna K., Asenov A., "Role of Multiple Delta Doping in PHEMTs scaled to sub-100 nm Dimensions" Solid-State Electronics, 48, 1223 (2004) 
Kane O., "Energy Band Structure in p-type Germanium and Silicon", J. Phys. Chem. Solids, 1, $82(1965)$

Kim H., Min H. S., Tang T. W. y Park Y. J., "An extended proof of the Ramo-Shockley theorem", Solid-State Electron. 34, 1251 (1991)

Kramers H. A., Zeits. F. Phys., 39, 828 (1926)

Kurosawa T., "Monte Carlo calculation of hot electron problems", J. Phys. Soc. Japan Suppl. S 21, 424 (1966)

Kurosawa T., "Proceedings of the International Conference on the Physics of Semiconductors", Kyoto, J. Phys. Soc. Japan Suppl. A 49 345, (1966b)

Liou J. J., Ortiz-Conde A., García-Sánchez F., “Analysis and design of MOSFETs- Modelling, Simulation and Parameter extraction", Kluwer, Norwer MA, (1998)

Lugli P., "Monte Carlo Models and Simulations", Compound Semiconductor Device Modelling, Ed. C. M. Snowden y R. E. Miles, Londres: Springer-Verlag (1993)

Lui W. and Fukuma M., "Exact solution of the Schrödinger equation across an arbitrary onedimensional piece-linear potential barrier”, J. Appl. Phys. 60, Num. 5 (1986)

Lusakowski J., Martín M. J., Rengel R. et al, "Quasiballistic transport in nonometer Si metaloxide-semiconductor field-effect transistors: Experimental and Monte Carlo analysis", Journal of Applied Physics, 101, 114511(1-6) (2007)

Madelung O., "Introduction to Solid-State Theory", Springer-Verlag, Berlin (1981)

Martín M. J., González T., Velázquez J. E. y Pardo D., "Simulation of electron transport in silicon: impact-ionization processes", Semicond. Sci. Technol. 8, 1291 (1993)

Martín M. J., González T., Pardo D. y Velázquez J. E., "Monte Carlo Analysis of a Schottky Diode with an Automatic Space-Variable Charge Algorithm”, Semicond. Sci. Technol. 9, 1316 (1994)

Martín M. J., “Análisis del Transporte de Carga y de los Fenómenos de Ruido Electrónico en Estructuras Si/Si1-xGex bipolares", Tesis Doctoral, Universidad de Salamanca (1996b)

Martínez J. C. And Polatdemir E., "Semiclassical approach to tunneling throug double barrier structure" Quant-ph/0111103 (2001)

Mateos J, González T., Pardo D., Hoel V. and Cappy A., "Effect of the T-gate on the performance of recessed HEMTs. A Monte Carlo analysis" Semicond. Sci. Technol. 14, 864 (1999) 
Mateos J., Vasallo B. G., Pardo D. and González T., "Operation and high-frequency performance of nanoscale unipolar rectifying diodes”, Applied Physics Letters 86, 212103 [1-3] (2005)

Matsuzawa K., Uchida K. and Nishiyama A., "Monte Carlo simulation of sub-0.1mm devices with Schottky contact model" IEICE Trans. Electron., E83-C, 1212 (2000)

Moglestue C., "A Self-Consistent Monte Carlo Particle Model to Analyze Semiconductor Microcomponents of Any Geometry”, IEEE Trans. Computer-Aided Design, 5, 326 (1986)

Moglestue C., "Monte Carlo Simulation of Semiconductor Devices", Boston: Artech House (1993)

Nag B. R., "Electron Transport in Compound Semiconductors", Springer-Verlag, Berlin (1980)

Pascual E., Rengel R., Martín M. J., "Microscopic modelling of reverse biased Schottky diodes: influence of non-equilibrium transport phenomena", Semicond. Sci. Technol., 22, 1003 (2007)

Pierret R. F., "Dispositivos de efecto de campo", Wilmington, DE: Addison-Wesley Iberoamericana, (1994)

Ramo S., "Currents Induced by Electron Motion", Proc. IRE 27, 584 (1939)

Rengel R., Mateos J., Pardo D., González T. and Martín M. J., "Monte Carlo analysis of dynamic and noise performance of submicron MOSFETs at RF and microwave frequencies" Semicond. Sci. Technol., 16939 (2001)

Rengel R., “Análisis de los Fenómenos de Trasporte y Ruido Electrónico en Transistores MOSFET y SOI Submicrométricos", Tesis Doctoral, Universidad de Salamanca (2002)

Rengel R., Pascual E., Martín M. J., "Injected Current and Quantum Transmission Coefficient in Low Schottky Barriers: WKB and Airy Approaches", IEEE Elec. Dev. Letters, 28, 171 (2007)

Ridley K. K., "Quantum Processes in Semiconductors”, Oxford: Clarendon Press (1983)

Sampedro C., Gámiz F., Godoy A., Prunnila M., Ahopelto J., "A comprehensive study of carrier velocity modulation in DGSOI transistors", Solid-State Electronics, 49, 1504 (2005)

Schrödinger E., "Quantization as a problem of proper values. Part I", Annalen der Physik, Leipzig. 79, 361 (1926)

Schrödinger E., "An undulatory theory of the mechanics of atoms and molecules", Phys. Rev. 28, 1049 (1926b) 
Selberherr, S., "Analysis and Simulation of Semiconductor Devices", Springer-Verlag, Wien (1984)

Shen M., Saikin S, Cheng M., "Monte Carlo Modelling of Spin Injection Through a Schottky Barrier and Spin Transport in a Semiconductor Quantum Well", Journal of Applied Physics, 96, 4319 (2004)

Shockley W., "Current to conductors induced by a moving point charge", J. Appl. Phys., 9, 635 (1938)

Shockley W., Copeland J. A. y James P., "Quantum Theory of Atoms, Molecules and the Solid State", ed. P. O. Löwdin, New York: Academic Press, (1966)

Snowden C. M., "Introduction to Semiconductor Device Modelling", Singapur: World Scientific (1986)

Sun L., Liu X. Y., Du G. y Han R. Q., "Monte Carlo Simulation of Schottky Contact with Direct Tunnelling Model”, Semiconductor Science and Technology, 18, 576 (2003)

Sze S. M., "Physics of Semiconductor Devices", $2^{\text {nd }}$ Edn. Wiley, New York (1981)

Sze S. M., VLSI Technology, New York, McGraw-Hill (1983)

Tomizawa K., "Numerical Simulation of Submicron Semiconductor Devices", Artech House, Boston (1993)

Tsividis Y., "Operation and modelling of the MOS transistor" $2^{\text {nd }}$ Edition, McGraw-Hill Boston (1999)

Van de Roer T. G., "Microwave electronic devices", Chapman and Hall, London (1994)

Van Vliet K., Friedmann A. et al., "Noise in Single Injections Diodes. I. Survey of Methods", J. Appl. Phys., 46, 1804 (1975)

Vasallo B. G., González T., Pardo D. and Mateos J., "Monte Carlo analysis of four-terminal ballistic rectifiers", Nanotechnology, 15, S250-S253 (2004)

Vatannia S. and Gildenblat G., "Airy's functions implementation of the transfer-matrix method for resonant tunneling in variably spaced finite superlattices," IEEE J. Quantum Electron., 32, 1093 (1996)

Vega R. A., "On the modeling and design of Schottky field-effect transistors," IEEE Trans. Electron Devices, 53, 4, 866 (2006)

Vega R. A., "Comparison study of tunneling models for Schottky field effect transistors and the effect of Schottky barrier lowering," IEEE Trans. Electron Devices, 53, 1593 (2006b)

Wentzel G., "Una generailzacione delle condizioni quantiche al fini della meccanica ondulatoria", Zeitschr. F. Phys. 38, 518 (1926) 
Winstead B., Ravaioli U., "Simulation of Schottky Barrier MOSFET’s with a Coupled Quantum Injection/Monte Carlo Technique”, IEEE Trans. on Elect. Dev., 47, 1241 (2000)

Yang E. S., "Microelectronic Devices", McGraw-Hill, Singapore (1988) 



\section{Diodos de barrera Schottky}

$\mathcal{L}_{\text {Schottky, presentado en primer lugar la evolución histórica de estos dispositivos, }}^{\text {nestulo hemos desarrollado un estudio completo de los diodos de barrera }}$ así como sus principales propiedades y aplicaciones. Una vez analizado el modelo de inyección/absorción a través de barreras Schottky en el Capítulo II, el siguiente paso es la inclusión del mismo en nuestro simulador Monte Carlo unidimensional. Para comprobar la fiabilidad de este simulador, llevaremos a cabo el modelado de diversas estructuras experimentales [Andrews, 1970], de las que disponemos de los datos necesarios para abordar su estudio (alturas de barrera, dopajes...) así como medidas experimentales de corriente tanto para diodos tipo $n$ como de tipo $p$. Cabe comentar que, en el caso de los diodos tipo $n$, las alturas de barrera son elevadas (entre 0.50 y $0.90 \mathrm{~V}$ ). Estas condiciones son particularmente complicadas de modelar, por lo que podremos testar nuestro modelo en las condiciones más adversas posibles desde el punto de vista de la simulación.

Analizaremos en profundidad la variación de diversos parámetros, tales como la variación de la temperatura y de la altura de la barrera. El simulador también nos permitirá mostrar la relevancia del efecto túnel en este tipo de dispositivos; para ello, compararemos los resultados obtenidos con el caso de considerar únicamente la emisión termoiónica (inyección y 
absorción). Evaluamos además las consecuencias de la reducción de la altura de la barrera debido al efecto de las cargas imagen.

Además de contrastar con las medidas experimentales las características corrientetensión obtenidas a través del método de Monte Carlo, también mostraremos los valores predichos por los modelos analíticos que describíamos en el Capítulo I y comentaremos sus limitaciones. Estudiaremos el comportamiento del diodo en régimen de tensiones en directa y en inversa, aunque dedicaremos un mayor esfuerzo al análisis en inversa puesto que en este régimen los fenómenos de transporte túnel son más relevantes. Por otra parte, la unión metalsemiconductor de fuente de los transistores MOSFET de barrera Schottky (SB-MOSFET) es la que regula el acceso de portadores al canal, encontrándose polarizada en inversa. Dado que por tanto la corriente en estos dispositivos (que estudiaremos en los Capítulos V y VI) estará determinada por el comportamiento de un contacto Schottky en inversa, es necesario asegurar completamente la fiabilidad de nuestro modelo en este régimen de operación.

\section{1. Evolución histórica del diodo Schottky}

La conexión de los dispositivos electrónicos de estado sólido con el exterior (otros dispositivos, elementos electromecánicos, etc.)

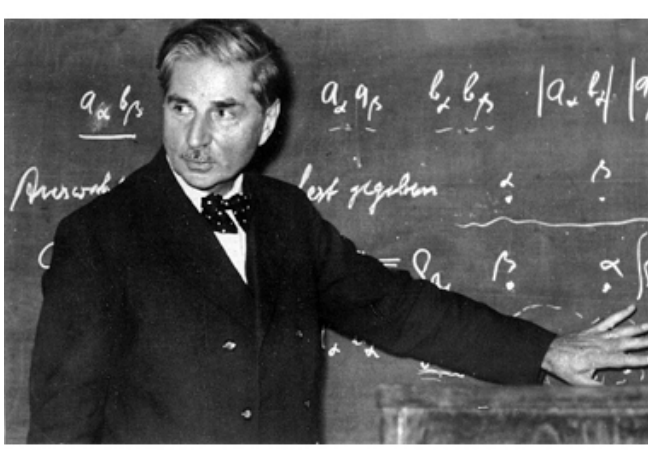

Figura III.1. Walter H. Schottky (1886-1976), inventor del diodo que lleva su nombre. ha sido desde los inicios de la Electrónica una cuestión fundamental. Comúnmente, la solución adoptada ha sido emplear estructuras metalsemiconductor, principalmente uniones de tipo óhmico ${ }^{1}$, ya que este tipo de contactos ofrecen una baja resistividad, y permiten el flujo de corriente en ambos sentidos. Sin embargo, en determinadas circunstancias el comportamiento de este tipo de uniones puede tener un carácter rectificador ${ }^{2}$, siendo el exponente más claro de

\footnotetext{
${ }^{1}$ Como ya hemos comentado en el Capítulo I, la unión de tipo óhmico es aquella en la que entre metal y semiconductor la resistencia de contacto es despreciable comparada con la que presenta el propio semiconductor, y por tanto no produciría caída de voltaje en la interfaz cuando la corriente circula en cualquiera de los dos sentidos.

${ }^{2}$ La rectificación es el procedimiento que permite convertir una corriente alterna en una señal unidireccional de tipo pulsado.
} 
esta característica el denominado diodo Schottky. En los contactos rectificadores bajo condiciones ideales, la corriente es debida principalmente a los portadores mayoritarios en el sentido semiconductor a metal, pero no en el sentido contrario, lo que da lugar a una serie de propiedades que pueden ser aprovechadas en diversas aplicaciones.

Desde el momento en que se efectuó la primera descripción de las propiedades rectificadoras de los contactos metal-semiconductor (atribuida a Ferdinand Braun hacia finales de 1874) hasta nuestros días, se han presentado diversas teorías y modelos sobre el comportamiento de este tipo de contactos [Mönch, 2004]. Sin embargo, en sus comienzos y a pesar de su importancia por el interés industrial de sus aplicaciones, el análisis teórico del contacto metal-semiconductor se desarrollaba con bastante lentitud en comparación con su evolución tecnológica. Debido a la baja pureza de los semiconductores, el estudio teórico de estos contactos se realizaba inicialmente mediante la adaptación de los modelos desarrollados para la interfaz metal-vacío ${ }^{3}$. Un ejemplo de esta situación es el hecho de que W. Schottky postulase ya en 1914 el efecto de las cargas imagen (o "image force lowering") de la barrera del contacto metal-vacío [Schottky, 1914], y que dicho efecto no fuese comprobado en los rectificadores metal-semiconductor hasta cincuenta años después [Sze, 1964]. De igual manera, en 1921 Richardson explicó el proceso de emisión termoiónica de electrones desde cátodos de metal al vacío [Richardson, 1921], pero fue necesario esperar hasta la década de 1940 para demostrar dicho proceso en contactos rectificadores metal-semiconductor [Bethe, 1942].

Entre los principales avances teóricos conseguidos a principios y mediados del siglo $\mathrm{XX}$, podemos destacar el desarrollo de modelos que explicaban el comportamiento rectificador basándose en la difusión de portadores sobre la barrera de potencial [Schottky y Spenke, 1939] o en la teoría de emisión termoiónica [Bethe, 1942]. Es importante mencionar la unificación de estas dos teorías en el modelo de emisión termoiónica-difusión realizado por [Crowell y Sze, 1966] (Véase el Capítulo I).

Sin embargo, no es hasta bien entrada la década de los sesenta del siglo pasado cuando podemos señalar el inicio de la "época dorada" de los diodos de barrera Schottky. ¿Por qué no antes? Pues sencillamente porque, como señalábamos anteriormente, el modelado de estos dispositivos estaba sujeto a

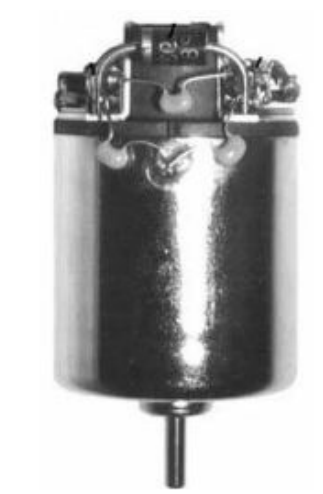

Figura III.2. Imagen de un diodo de barrera Schottky.

\footnotetext{
${ }^{3}$ No fue hasta alrededor de 1940 que se demostró la similitud entre la conducción en el diodo de tubo de vacío y el rectificador metal-semiconductor, gracias al trabajo realizado por Schottky y Bethe.
} 
la previa experimentación en contactos metal-vacío, por lo que en las aplicaciones comerciales se utilizaban otros dispositivos cuyo comportamiento era más fácil de predecir y de entender, como las uniones $p$ - $n$ rectificadoras o los tubos de vacío. Durante los años sesenta, el interés en los diodos de barrera Schottky aumentó considerablemente debido, en parte, a la invención y desarrollo del proceso tecnológico planar (relacionado en un primer momento con los transistores de efecto de campo FETs), el cual arrojó una nueva luz sobre la física de los contactos y las técnicas de fabricación de los mismos. De este modo se favoreció el desarrollo de los dispositivos rectificadores metal-semiconductor de estado sólido. A este notable impulso del que hablamos también contribuyó el descubrimiento de la formación de aleaciones metálicas de Silicio (Siliciuros) a bajas temperaturas, ya que, utilizando el Siliciuro en el contacto con el semiconductor se evita la influencia de la contaminación superficial. Gracias a ello aparecieron nuevas combinaciones de metal-semiconductor [Crowell, 1969], [Andrews, 1970], [Rideout, 1978], que contribuyeron al despegue definitivo de las aplicaciones basadas en este tipo de estructuras.

Mencionaremos a continuación algunos de los trabajos sobre diodos Schottky con los que hemos trabajado en esta Memoria.

En primer lugar, destacamos las siguientes investigaciones: [Crowell, 1965], [Crowell, 1966], [Crowell, 1968], [Andrews, 1970], [Rideout, 1978] y [Sze, 1981]. En estos trabajos se lleva a cabo un análisis teórico detallado sobre el transporte de corriente en contactos Schottky, sirviendo de referencia en múltiples ocasiones para estudios posteriores de otros autores. En el caso de [Andrews, 1970] muestra también resultados experimentales de características I-V de diversos diodos comparados con los modelos de corriente que expone en su análisis teórico inicial; en el presente capítulo utilizaremos estas medidas experimentales como referencia para contrastar con nuestros resultados Monte Carlo.

Por otro lado, con el fin de conseguir una reducción de la altura de barrera Schottky efectiva, surgen estudios de diodos Schottky con una capa de dopantes junto a la unión metalsemiconductor. Shenai et al. presentan en 1985 un modelo para caracterizar la corriente a través de estos dispositivos. Calculan la corriente a través del contacto teniendo en cuenta la aproximación WKB, además calculan la dependencia con el campo eléctrico de la profundidad de penetración de la carga de la capa de dopantes en el semiconductor. Simulan un diodo Schottky de Al/GaAs (tipo $n$ ) y comparan los resultados obtenidos con medidas experimentales del dispositivo. En este trabajo se centran exclusivamente en comparar las características I-V 
simuladas y experimentales, obteniendo buenos resultados; también estudian la dependencia de la capacidad con el voltaje aplicado. Sin embargo, no profundizan en el transporte de portadores a través del dispositivo. [Shenai, 1985], [Shenai, 1988].

Señalamos a continuación el estudio desarrollado en 1996 por Martín et al. En este trabajo se analizan diodos de barrera Schottky tipo $n$ en directa mediante un simulador Monte Carlo unidimensional que fue adaptado al cálculo de este tipo de dispositivos mediante la inclusión de un algoritmo de carga variable. En este trabajo se muestra la característica tensióncorriente del dispositivo además de distintas magnitudes internas como concentración, potencial, la función de distribución y la velocidad de recombinación de los portadores en la región próxima a la interfaz Schottky. Es importante mencionar que en este caso sólo se consideran los procesos de absorción termoiónica, puesto que en el régimen de polarización estudiado es la contribución más importante a la corriente total, pudiéndose considerar despreciables a las demás contribuciones [Martín, 1996]. En la primera parte de la presente Memoria hemos partido de este simulador, sin embargo, para analizar los diodos en el régimen de polarización inversa, ha sido necesario mejorar el simulador Monte Carlo implementando el algoritmo de inyección/absorción tal y como hemos explicado en el Capítulo II. De este modo se consideran no sólo la absorción termoiónica, sino también la absorción túnel, además de los procesos de inyección termoiónica y túnel, siendo imprescindible la consideración de todos estos procesos para el estudio de estos dispositivos, como veremos a lo largo de este capítulo.

Posteriormente, otros autores han afrontado también el estudio de diodos Schottky tipo $n$ mediante un simulador Monte Carlo; sin embargo focalizan sus esfuerzos en el régimen de inversa [Matsuzawa, 1999], [Matsuzawa, 2000], [Sun, 2003]. Para ello acoplan un algoritmo que proporciona la solución de la ecuación de Schrödinger mediante la aproximación WKB. Sin embargo estos trabajos se centran sobre todo en el estudio de la característica I-V del diodo Schottky. En [Matsuzawa, 1999] estudian además una estructura $n^{+}-n-n^{+}$, en la que también analizan la velocidad de dispersión del dispositivo (con el fin de demostrar que la dependencia de la constante de difusión debe ser tenida en cuenta en el modelo hidrodinámico). En [Sun, 2003], también muestran la densidad de portadores a lo largo del diodo Schottky para distintas polarizaciones, demostrando que el algoritmo de expansión/comprensión presentado en [Martín, 1996b] es totalmente necesario para el estudio de estos dispositivos.

Por otro lado, podemos también mencionar el trabajo presentado por [Shen, 2004], en el que utiliza un simulador Monte Carlo para modelar el flujo de spin a través de uniones de metal ferromagnético/semiconductor y su transporte en un pozo cuántico. Incluye tanto la emisión 
termoiónica como la inyección túnel. Estudia el flujo del spin y la polarización de la corriente de spin a lo largo del dispositivo. Sin embargo, es importante mencionar que la fabricación experimental de dispositivos con una fuente de spin lo suficientemente eficiente es una tarea difícil hoy por hoy.

Destacamos además los trabajos de [Dubois, 2004], [Jang, 2005] y [Jian, 2007] sobre extracción de los valores de la altura de barrera en contactos Schottky. En el primero de ellos se realiza mediante el diagrama de Arrhenius o método de energía de activación, en el segundo mediante el método de capacidad-voltaje y en el tercero mediante el método de admitancia, que puede considerarse como una variante del método de corriente-voltaje (Capítulo I.5.b). Este segundo método se basa en la medida de la admitancia para $0 \mathrm{~V}$ y a un voltaje en directa alto; sin embargo es sólo válido para barreras altas $(>0.4 \mathrm{eV})$ y moderadamente bajas $(0.2-0.4 \mathrm{eV})$. En nuestro caso utilizaremos el diagrama de Arrhenius, como ya comentamos en el capítulo anterior.

Mención aparte merece el excelente comportamiento de los diodos Schottky formados en materiales III-V, como es el Arseniuro de Galio (GaAs), a muy alta frecuencia [González, 1997], [Pérez, 2005].

\section{2. Diodos de barrera Schottky, propiedades y aplicaciones}

En algunos aspectos, el diodo de barrera Schottky se asemeja a una unión $p$ - $n$ donde una de las regiones semiconductoras es reemplazada por un metal o Siliciuro; sin embargo el diodo Schottky posee notables ventajas frente a las uniones $p-n$ convencionales [Neamen, 1992], [Neudeck, 1993]:

- Presentan una tensión umbral (para polarizaciones en directa) muy baja.

- Son capaces de mantener corrientes muy elevadas con voltajes relativamente bajos, inferiores a los que corresponden a un diodo de unión $p-n$.

- Sólo los portadores mayoritarios tienen un papel significativo en el transporte de corriente, lo cual implica que sean despreciables las recombinaciones entre electrones y huecos que ralenticen su comportamiento, como sucede en las uniones $p-n$ convencionales. 
- La capacidad de vaciamiento es independiente de la frecuencia hasta frecuencias elevadas y, en comparación con la unión $p$ - $n$, apenas tiene capacidad de difusión ${ }^{4}$. Por tanto, al polarizar en directa la capacidad total es inferior, pudiendo así funcionar a frecuencias mucho mayores que la unión $p-n$.

- Las velocidades de conmutación del diodo Schottky son muy elevadas debido a que la acumulación de portadores minoritarios en el semiconductor es prácticamente nula, por lo que los tiempos de conexión y desconexión del diodo están limitados únicamente por el movimiento de los portadores mayoritarios.

La desventaja más clara del diodo Schottky, en comparación con los dispositivos bipolares, es la corriente inversa de pérdidas en el caso de voltajes elevados y temperaturas de operación altas. Por ejemplo, para un diodo Schottky rectificador a $100 \mathrm{~V}$, la corriente de pérdidas es del orden de $100 \mathrm{~mA}$. En estas condiciones, la potencia disipada en inversa es de unos $10 \mathrm{~W}$. Sin embargo, hoy en día estos problemas están siendo paliados gracias al diseño de nuevos rectificadores Schottky a $100 \mathrm{~V}$ de altas temperaturas, los cuales tienen menor corriente en inversa (en torno a un factor 10 con respecto a los diseños precedentes) por lo que la potencia disipada sería de sólo $1 \mathrm{~W}$ en las mismas condiciones. Además esta disminución de la corriente de pérdidas favorece el aumento de la temperatura de operación máxima permitida acercándose a las especificaciones proporcionadas por diodos de unión $p-n$ para este tipo de aplicaciones.

Las ventajas anteriormente descritas de estos diodos hacen que se encuentren en una gran variedad de aplicaciones [Neudeck, 1993], [Albella, 1996]:

- Son de gran utilidad en circuitos de alta velocidad en los que se necesiten grandes velocidades de conmutación, donde se emplean como interruptores rápidos, ofreciendo además la posibilidad de integración en áreas muy pequeñas.

- Como limitadores de tensión o diodos "clamp" evitando así que los circuitos sean dañados (por ejemplo, como protección de descarga de células solares conectadas a baterías de plomo ácido).

- El hecho de que sólo intervengan portadores mayoritarios, sin posibilidad de recombinación, permite que el área del dispositivo sea más pequeña, siendo útiles en conversores de potencia en modo conmutador (switch-mode power converter) que operan en frecuencias próximas a $1 \mathrm{MHz}$ [Neudeck, 1993].

\footnotetext{
${ }^{4}$ Esto se debe, principalmente al almacenamiento pequeño o nulo de portadores minoritarios en el semiconductor.
} 
- Debido a su buen comportamiento en frecuencia, los diodos Schottky constituyen un elemento importante en detectores RF y mezcladores, que usualmente operan por encima de 5 GHz [Albella, 1996], [Shiktorov, 2004], [Pérez, 2005].

- Por último, es necesario destacar la utilización de este tipo de uniones metal-semiconductor en la región de puerta en los MESFET o de fuente/drenador en MOSFETs denominados MOSFET de barrera Schottky (SB-MOSFET) [Larson, 2006], [Sun, 2003]. En el caso de dispositivos de Silicio, como ya hemos comentado anteriormente, este último es de especial interés para permitir extender la escalabilidad de las tecnologías MOS de Silicio, y constituye el núcleo principal del presente trabajo.

Como señalamos en la introducción, nuestro objetivo principal es adquirir un grado de conocimiento exhaustivo acerca de los SB-MOSFET; de este modo, se hace indispensable un análisis previo de la física del transporte en los diodos de barrera Schottky de Silicio.

\section{III.3. Estructuras simuladas}

En la Figura III.3 mostramos la representación esquemática de los dispositivos simulados en este capítulo, cuya definición ha sido realizada a partir de la descripción experimental de las mismas [Andrews, 1970]. Como vemos, se han modelado dos tipos de estructuras unidimensionales, dependiendo de si estudiamos el transporte de electrones o de huecos: $n^{+}$-n-Siliciuro en el primero de los casos y $p^{+}-p$-Siliciuro, en el segundo.

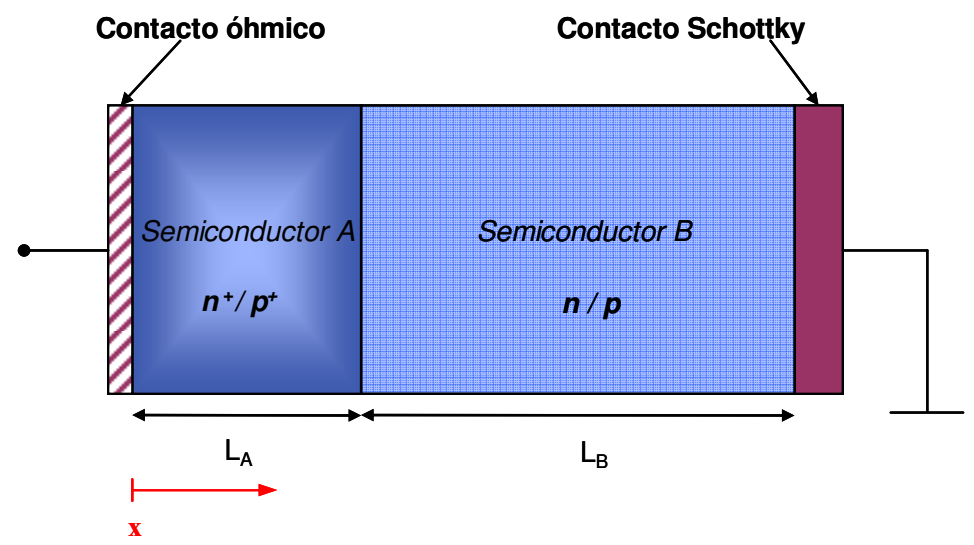

Figura III.3. Esquema del diodo de barrera Schottky analizado. 
A la izquierda del dispositivo se ha situado el contacto óhmico y a la derecha el contacto Schottky con los distintos Siliciuros metálicos, Siliciuro de Zirconio $\left(\mathrm{ZrSi}_{2}\right)$, Siliciuro de Rodio (RhSi) y Siliciuro de Platino (PtSi), formados sobre Silicio dopado tipo $n$ (o $p$ en el caso de huecos), presentando así alturas de barrera distintas. Las características específicas de cada una de las estructuras simuladas se muestran en la Tabla III.1.

\begin{tabular}{|c|c|c||c|c|c|c||}
\hline Estructura & $\begin{array}{c}\text { Dopaje A } \\
\left(\mathbf{c m}^{-3}\right)\end{array}$ & $\begin{array}{c}\mathbf{L}_{\mathbf{A}} \\
(\boldsymbol{\mu m})\end{array}$ & $\begin{array}{c}\text { Dopaje B } \\
\mathbf{( c m}^{-3} \mathbf{)}\end{array}$ & $\begin{array}{c}\mathbf{L}_{\mathbf{B}} \\
(\boldsymbol{\mu m})\end{array}$ & $\begin{array}{c}\mathbf{q} \mathbf{V}_{\mathbf{b i}} \\
(\mathbf{e V})\end{array}$ & $\begin{array}{c}\mathbf{q} \mathbf{\Phi}_{\mathbf{B n} / \mathbf{p}} \\
(\mathbf{e V})\end{array}$ \\
\hline $\mathrm{Si}-\mathrm{n} / \mathrm{ZrSi}_{2}$ & $10^{17}$ & 0.2 & $3.5 \cdot 10^{15}$ & 0.8 & 0.3178 & 0.55 \\
\hline $\mathrm{Si}-\mathrm{n} / \mathrm{RhSi}$ & $10^{17}$ & 0.2 & $3.5 \cdot 10^{15}$ & 0.8 & 0.4478 & 0.68 \\
\hline $\mathrm{Si}-\mathrm{n} / \mathrm{PtSi}$ & $10^{17}$ & 0.2 & $3.2 \cdot 10^{15}$ & 0.8 & 0.6380 & 0.87 \\
\hline $\mathrm{Si}-\mathrm{p} / \mathrm{RhSi}$ & $10^{17}$ & 0.2 & $9.0 \cdot 10^{16}$ & 0.8 & 0.1909 & 0.33 \\
\hline
\end{tabular}

Tabla III.1. Características de las distintas estructuras simuladas (tipo de Siliciuro, dopaje y longitud de los semiconductores A y B, potencial de contacto y altura de la barrera Schottky)

\begin{tabular}{|c|c|c|c|}
\hline \hline Dopaje Si $\left(\mathbf{m}^{-3}\right)$ & $\lambda_{\boldsymbol{D}}(\mathbf{n m})$ & $\boldsymbol{\omega}_{\boldsymbol{p}}\left(\mathbf{s}^{-1}\right)$ & $\tau_{\boldsymbol{d}}(\mathbf{s})$ \\
\hline \hline $\mathrm{N}_{\mathrm{D}}=10^{23}$ & 12.9 & $1.04 \cdot 10^{13}$ & $8.09 \cdot 10^{-14}$ \\
\hline $\mathrm{N}_{\mathrm{D}}=3.5 \cdot 10^{21}$ & 69.1 & $1.95 \cdot 10^{12}$ & $1.71 \cdot 10^{-12}$ \\
\hline $\mathrm{N}_{\mathrm{D}}=3.2 \cdot 10^{21}$ & 72.2 & $1.86 \cdot 10^{12}$ & $1.86 \cdot 10^{-12}$ \\
\hline $\mathrm{N}_{\mathrm{A}}=10^{23}$ & 12.9 & $7.48 \cdot 10^{12}$ & $1.62 \cdot 10^{-13}$ \\
\hline $\mathrm{N}_{\mathrm{A}}=9 \cdot 10^{22}$ & 13.6 & $7.09 \cdot 10^{12}$ & $1.71 \cdot 10^{-13}$ \\
\hline
\end{tabular}

Tabla III.2. Valores de la longitud de Debye, de la frecuencia del plasma y del tiempo de relajación dieléctrico de Silicio con distintas inmpurificaciones. Estos valores sirven como base para el criterio de elección del tamaño de las celdas del mallado del dispositivo y del paso temporal de la simulación.

Disponemos de medidas experimentales para tres temperaturas distintas, 300, 279 y $343 \mathrm{~K}$, lo que nos permitirá comprobar el efecto de este parámetro sobre el transporte de carga. Para asegurar la correcta resolución de la ecuación de Poisson (ver Capítulo II), la estructura se divide en celdas de $10 \mathrm{~nm}$ de longitud, y el paso temporal elegido es de $1 \mathrm{fs}$, siguiendo los criterios presentados en el Capítulo II, Sección 3.b y considerando los valores de longitud de 
Debye, frecuencia de oscilación del plasma y tiempo de relajación del dieléctrico presentados en la Tabla III.2. El tiempo total de la simulación varía entre 1.5 y 3 ns dependiendo del punto de operación y de la precisión requerida.

\begin{tabular}{|c|c||c||c|c||}
\hline \multirow{2}{*}{ Estructura } & $\boldsymbol{V}_{\boldsymbol{a}}(\mathbf{V})$ & $\begin{array}{c}\text { EPE (malla 1) } \\
\left(\mathbf{m}^{-\mathbf{2}} \mathbf{)}\right.\end{array}$ & $\begin{array}{c}\text { EPE (malla n) } \\
\left(\mathbf{m}^{-2}\right)\end{array}$ & Cociente \\
\hline \hline \multirow{2}{*}{$\mathrm{Si}-\mathrm{n} / \mathrm{ZrSi}_{2}$} & -0.001 & $1.0 \cdot 10^{13}$ & $8.0 \cdot 10^{6}$ & $1.25 \cdot 10^{6}$ \\
\cline { 2 - 5 } & -1 & $1.0 \cdot 10^{13}$ & $1.6 \cdot 10^{6}$ & $6.25 \cdot 10^{6}$ \\
\hline \multirow{3}{*}{$\mathrm{Si}-\mathrm{n} / \mathrm{RhSi}$} & -0.001 & $1.0 \cdot 10^{13}$ & $1.0 \cdot 10^{5}$ & $1.00 \cdot 10^{8}$ \\
\cline { 2 - 5 } & -1 & $1.0 \cdot 10^{13}$ & $5.0 \cdot 10^{3}$ & $2.00 \cdot 10^{9}$ \\
\hline \multirow{2}{*}{$\mathrm{Si}-\mathrm{n} / \mathrm{PtSi}$} & -0.005 & $1.0 \cdot 10^{13}$ & $1.0 \cdot 10^{2}$ & $1.00 \cdot 10^{11}$ \\
\cline { 2 - 5 } $\mathrm{Si}-\mathrm{p} / \mathrm{RhSi}$ & -1 & $1.0 \cdot 10^{13}$ & $1.0 \cdot 10^{1}$ & $1.00 \cdot 10^{12}$ \\
\cline { 2 - 5 } & -0.003 & $1.0 \cdot 10^{13}$ & $2.0 \cdot 10^{10}$ & $5.00 \cdot 10^{2}$ \\
\hline
\end{tabular}

Tabla III.3. Valores del EPE en la primera y última malla de la correspondiente estructura para distintas polarizaciones en inversa aplicadas. La última columna muestra el cociente entre estos dos valores de EPE.

El EPE se ajusta al perfil de la concentración para cada simulación en particular y su valor varía en varios órdenes de magnitud a lo largo del dispositivo. Este hecho se ve reflejado en la Tabla III.3, en la que mostramos el valor del EPE para la primera y la última malla de las diversas estructuras. De este modo se consigue que el número de partículas sea aproximadamente constante para todas las celdas, mejorando notablemente la precisión de la simulación. El número de partículas con que se inicia la simulación es aproximadamente 100 partículas por malla.

Observamos que en el caso de las estructuras con mayor altura de barrera, la variación del perfil de EPE a lo largo del dispositivo debe ser más grande; además, el cociente entre los valores de EPE también es mayor a medida que aumenta el voltaje en inversa aplicado. 


\section{III.4. Diodo Schottky con semiconductor dopado tipo $n$}

El diagrama de bandas en equilibrio para este tipo de estructuras es el mostrado en la Figura III.4. Como ya vimos en el Capítulo I, Sección 1, el nivel de Fermi debe permanecer constante en cualquier sistema en equilibrio termodinámico, por lo que la unión de las tres regiones implica el desplazamiento de las bandas de energía dando lugar finalmente a la situación que se muestra en la figura.

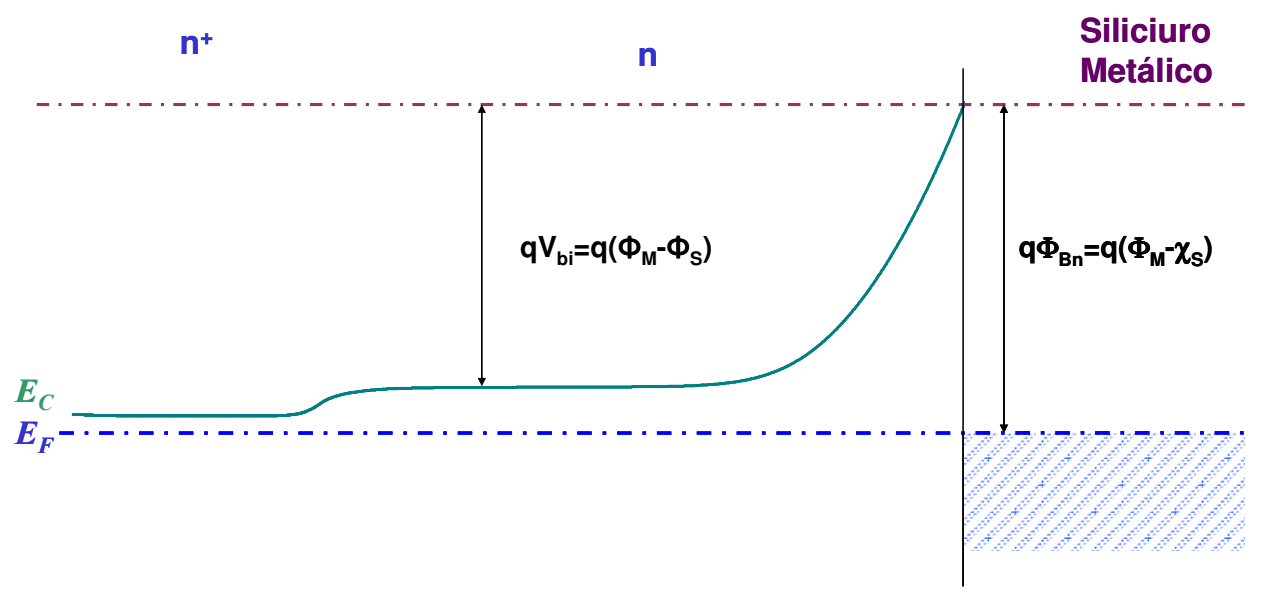

Figura III.4. Diagrama de bandas de la estructura $n^{+}-n$-Siliciuro en equilibrio.

Vamos a comenzar analizando exhaustivamente la estructura en la que el Siliciuro empleado es $\mathrm{ZrSi}_{2}$. De este modo validaremos el comportamiento del modelo de inyección/absorción de partículas implementado en el simulador Monte Carlo unidimensional mediante la comparación de la característica corriente-tensión obtenida con los modelos teóricos estudiados en el Capítulo I, Sección 3 y las medidas experimentales.

\section{III.4.a. Diodo Schottky (Si-n/ZrSi $\left.i_{2}\right)$ a $300 K$ de temperatura.}

En la presente sección realizaremos el estudio de esta estructura en el régimen de directa y, con mayor exhaustividad, en inversa. En primer lugar, los resultados mostrados no incluyen el efecto de las cargas imagen analizado en el Capítulo I, Sección 4.a, el cual será discutido con posterioridad. Calibraremos inicialmente el modelo de inyección/absorción para poder realizar en segundo término el análisis de la física del mismo y la influencia de diversos parámetros en el transporte de la corriente. 


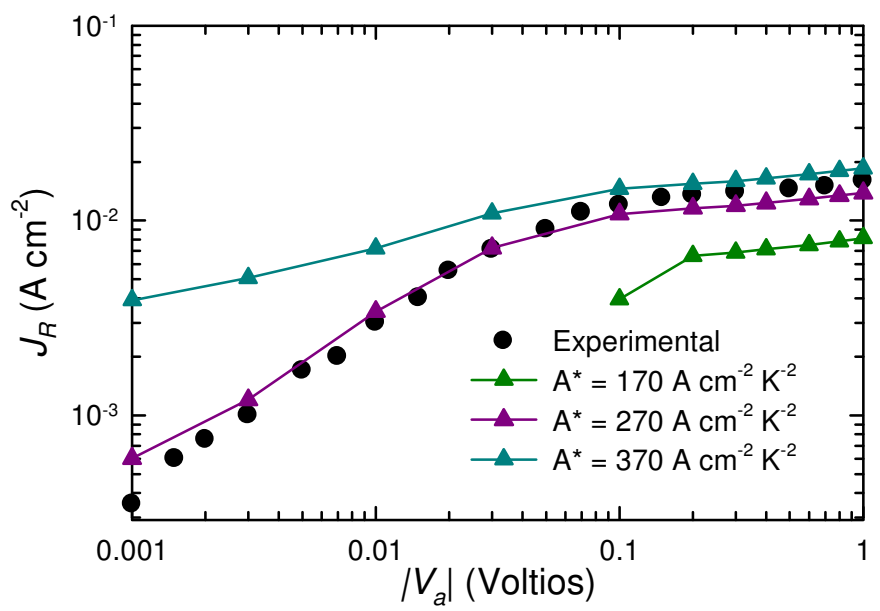

Figura III.5. Características J-V en inversa de la estructura $\mathrm{Si}-n / \mathrm{ZrSi}_{2}$ a $300 \mathrm{~K}$ de temperatura. Los círculos son los resultados experimentales [Andrews, 1970] y los triángulos son los resultados numéricos de la simulación Monte Carlo con constante de Richardson $A^{*}=170 \mathrm{~A} \mathrm{~cm}^{-2} \mathrm{~K}^{-2}, \mathrm{~A}^{*}=270 \mathrm{~A} \mathrm{~cm}^{-2} \mathrm{~K}^{-2}, \mathrm{~A}^{*}=370 \mathrm{~A} \mathrm{~cm}^{-2} \mathrm{~K}^{-2}$.

A la hora de reproducir los resultados experimentales para $300 \mathrm{~K}$, consideraremos como parámetro de ajuste del modelo la constante de Richardson de acuerdo con las conclusiones obtenidas a partir de la comparación entre la aproximación WKB y de la matriz de transferencia de Airy (Capítulo II, Sección 4). Para determinar el valor de dicho parámetro en nuestro modelo, realizamos diversas pruebas con diferentes valores de $\mathrm{A}^{*}$ en el rango de polarización inversa y comparamos los resultados obtenidos con las medidas experimentales. En la Figura III.5 se muestra la característica corriente-tensión de la estructura que estamos analizando con algunos de los valores de la constante de Richardson que han sido considerados: $170 \mathrm{~A} \mathrm{~cm}^{-2} \mathrm{~K}^{-2}$, $270 \mathrm{~A} \mathrm{~cm}^{-2} \mathrm{~K}^{-2}, 370 \mathrm{~A} \mathrm{~cm}^{-2} \mathrm{~K}^{-2}$.

En ausencia de polarización, la corriente debe ser nula (la corriente inyectada y la absorbida son iguales). Como podemos observar, el valor de $270 \mathrm{~A} \mathrm{~cm}^{-2} \mathbf{K}^{-2}$ proporciona una correspondencia adecuada de la característica $\mathbf{J}-\mathbf{V}$, incluyendo el ajuste de la corriente a $0 \mathrm{~V}$, de modo que los resultados Monte Carlo reproducen correctamente los datos experimentales.

La constante de Richardson ha sido ajustada en el régimen de polarización de inversa puesto que las medidas experimentales de las que disponemos pertenecen a este rango. A continuación, comprobaremos la validez del valor elegido ya no sólo en inversa sino también en el régimen de polarización directa. En la Figura III.6 se muestra la característica corriente- 
tensión obtenida mediante el método Monte Carlo para tensiones aplicadas en directa e inversa, junto con los resultados que proporcionan los distintos modelos teóricos:

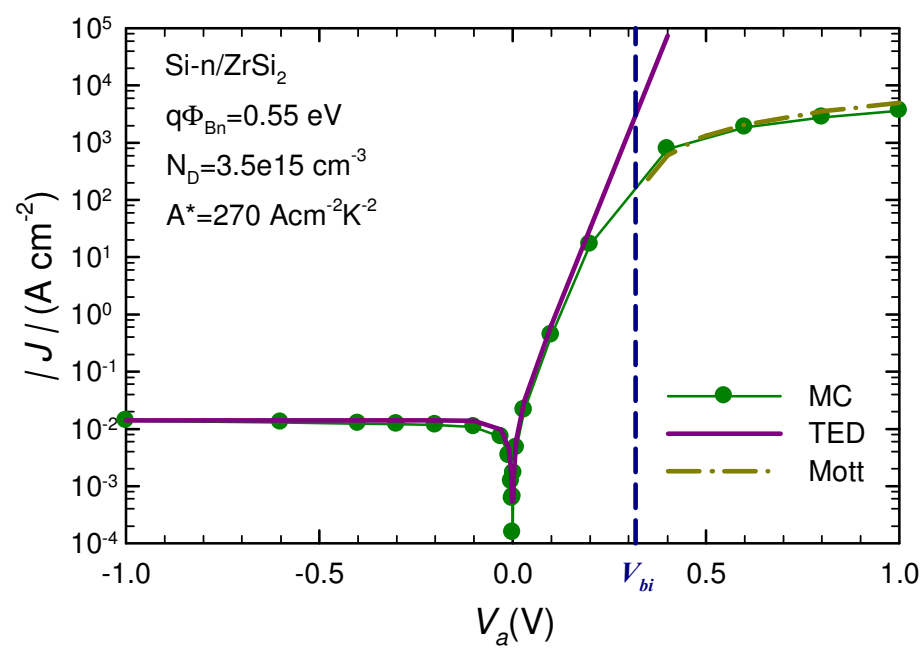

Figura III.6. Característica J-V de la estructura $\mathrm{Si}-\mathrm{n} / \mathrm{ZrSi}_{2}$ en polarización directa e inversa obtenida mediante el método Monte Carlo unidimensional incluyendo el algoritmo de inyección/absorción con $\mathrm{A}^{*}=270 \mathrm{Acm}^{-2} \mathrm{~K}^{-2}$. Nótese que la densidad de corriente está en módulo, puesto que en directa y en inversa tienen distinto signo. La figura muestra también los resultados obtenidos mediante las teorías de Mott y Emisión Termoiónica-Difusión.

- $\quad$ Para polarizaciones en directa superiores a $V_{b i}$ compararemos con la teoría de Mott (cuya expresión de la corriente, recordemos, viene dada por la Ecuación I.26, [Mott, 1938]). La corriente en esta región se expresa en función de las resistencias en condiciones óhmicas del dispositivo, calculadas mediante la siguiente expresión:

$$
R=\frac{1}{q N_{D} \mu_{0}} \frac{L}{A}
$$

donde $L$ es la longitud de la región neutra en la que se calcula la resistencia, $A$ es la sección transversal del diodo y $\mu_{0}$ es la movilidad de los portadores (véase Apéndice). En el caso de la estructura simulada se obtiene que $R_{n+}+R_{n} \square 1.3762 \cdot 10^{-8} \Omega \square \mathrm{m}^{-2}$.

- Para voltajes inferiores a $V_{b i}$ (tanto en directa como en inversa) comprobamos los resultados derivados de la teoría de Emisión Termoiónica-Difusión (Ecuación I.23) [Bethe, 1942].

Observamos una primera región $\left(V_{a}<V_{b i}\right)$ en la que la dependencia de la corriente con respecto al voltaje exhibe un comportamiento exponencial como el descrito por la teoría de emisión termoiónica. Debemos destacar que en la región en inversa el efecto predominante 
hasta $-1 \mathrm{~V}$ es la inyección termoiónica, de ahí el aparente buen acuerdo en principio de la predicción teórica con los resultados Monte Carlo. Analizaremos en profundidad esta región de la característica $\mathrm{J}-\mathrm{V}$ en posteriores secciones. Para voltajes superiores a $V_{b i}$ la corriente muestra una dependencia lineal, tal y como afirma la teoría de Mott. En la región de altas tensiones en directa el comportamiento de la característica difiere ligeramente de los valores indicados por la curva teórica; esta discrepancia está asociada al aumento de la resistencia en serie por la aparición de portadores calientes en la región $n$ del dispositivo próxima al contacto óhmico [González, 1994].

Para una mejor comprensión del comportamiento de la densidad de corriente mostramos a continuación, en la Figura III.7, la contribución de las diferentes componentes de corriente en función del voltaje aplicado.
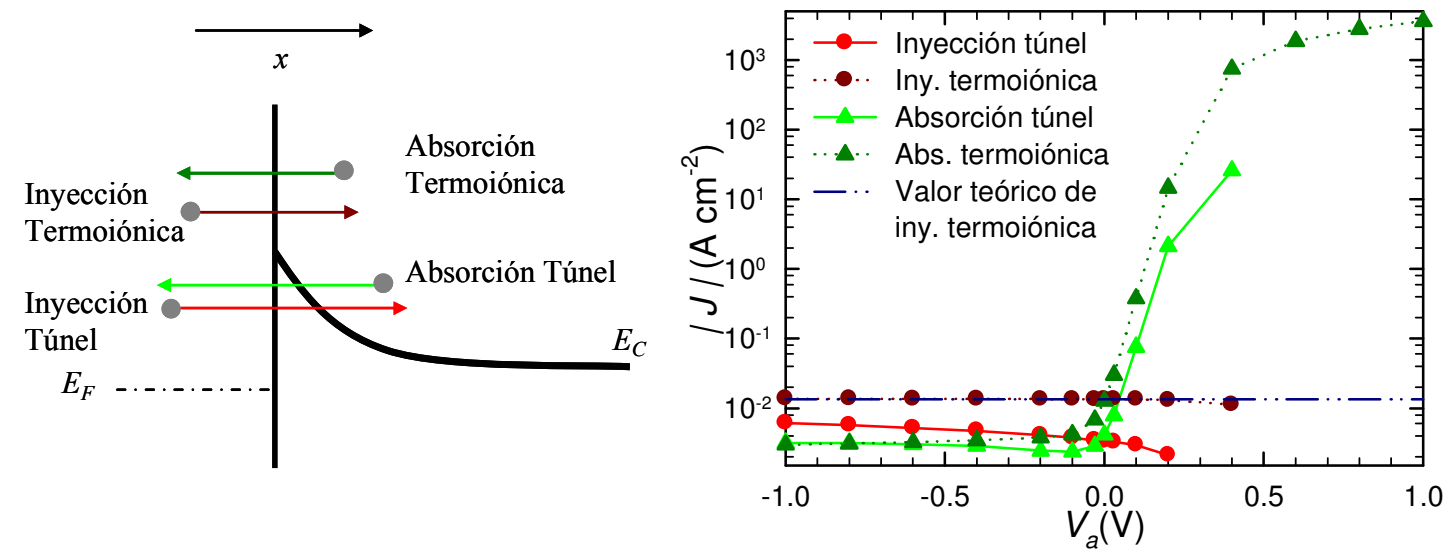

Figura III.7. Componentes de la corriente en función del voltaje aplicado.

\section{Para tensiones aplicadas en directa superiores a $V_{b i}$ no hay posibilidad de} absorción ni inyección por efecto túnel, como comprobaremos en la siguiente sección mediante el estudio del perfil de potencial en esta región. De este modo la componente con mayor contribución a la corriente total es la de absorción termoiónica, puesto que la inyección termoiónica, que permanece constante para todo el rango de tensiones, es despreciable frente a ella. Para polarizaciones inferiores a banda plana en el régimen de directa hay posibilidad de transmisión por túnel, cobrando importancia la absorción túnel, que junto con la termoiónica representan el mayor porcentaje de la corriente total. Sin embargo, en el régimen de voltajes en inversa, las componentes de absorción disminuyen, predominando la inyección desde el metal al semiconductor. Cuanto mayor es la tensión en inversa aplicada, más importancia 
cobra la inyección debida al efecto de túnel cuántico. El método de Monte Carlo nos ofrece no sólo la característica corriente-tensión que acabamos de analizar, sino también una serie de magnitudes que nos proporcionan información de interés a cerca del transporte de carga en el interior del dispositivo. Veamos a continuación con más detalle las principales magnitudes internas del dispositivo en ambos regímenes de operación.

\section{III.4.a.1. Comportamiento en directa}

En este rango de polarizaciones se distinguen claramente dos regiones donde el dispositivo se comporta de manera distinta, esto es, para tensiones superiores e inferiores a la de banda plana (recordemos que para esta estructura $V_{b i}=$ $0.32 \mathrm{~V})$.

Examinando el perfil de la banda de conducción (Figura III.8.a), observamos que para débiles tensiones aplicadas $\left(V_{a}=0 \mathrm{~V}\right.$ ó $\left.V_{a}=0.2 \mathrm{~V}\right)$, cerca del contacto rectificador (posición $\mathrm{x}=1 \mu \mathrm{m})$ aparece la barrera característica del mismo; esto se refleja en el campo eléctrico positivo que se opone al paso de los electrones desde el semiconductor al metal, mientras que en el resto de la zona $n$ el campo se mantiene con valores próximos a cero (Figura III.8.b). En cuanto a la concentración a lo largo del dispositivo, es claramente apreciable el vaciamiento de portadores como efecto de ese campo eléctrico (Figura III.8.c).
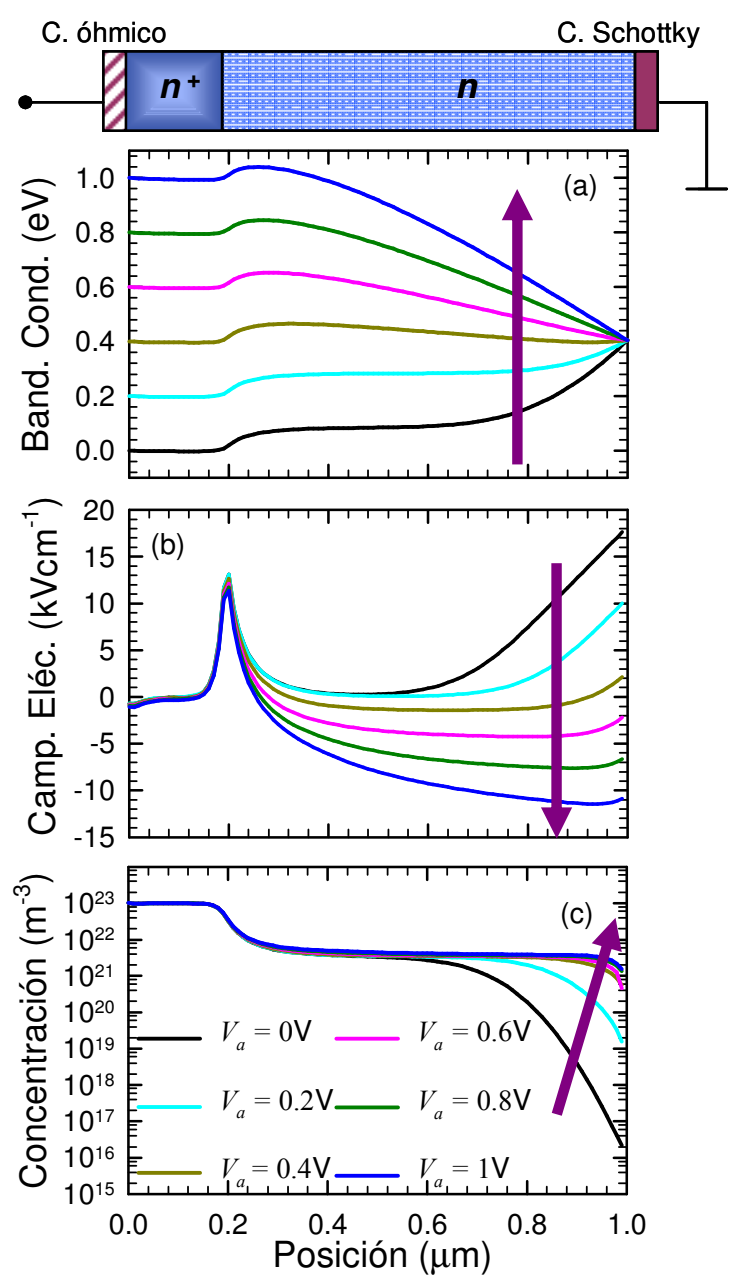

Figura III.8. Diodo de contacto $\mathrm{Si}-n / \mathrm{ZrSi}_{2}$. Dependencia con la posición de las siguientes magnitudes bajo polarización en directa: . a. Banda de conducción .b. Campo eléctrico .c. Concentración.

A medida que aumentamos la polarización en directa, la barrera del semiconductor al metal va disminuyendo, el perfil de potencial se suaviza y en consecuencia se reduce el campo 
eléctrico en esa zona, aumentando así la concentración de portadores en las cercanías del contacto rectificador.

A partir del momento en el cual el voltaje aplicado supera al de banda plana observamos una caída lineal del potencial en la zona $n$ y la desaparición de la barrera semiconductor-metal. Por ello, surge en la región un campo eléctrico negativo de arrastre que favorece el movimiento de los portadores hacia el contacto Schottky, mientras que la concentración permanece prácticamente constante a lo largo de toda la región $n$. Otra repercusión de la desaparición de la barrera Schottky es que, lógicamente, la transmisión por túnel cuántico a través de ella no es posible, como ya vimos anteriormente en la Figura III.7.
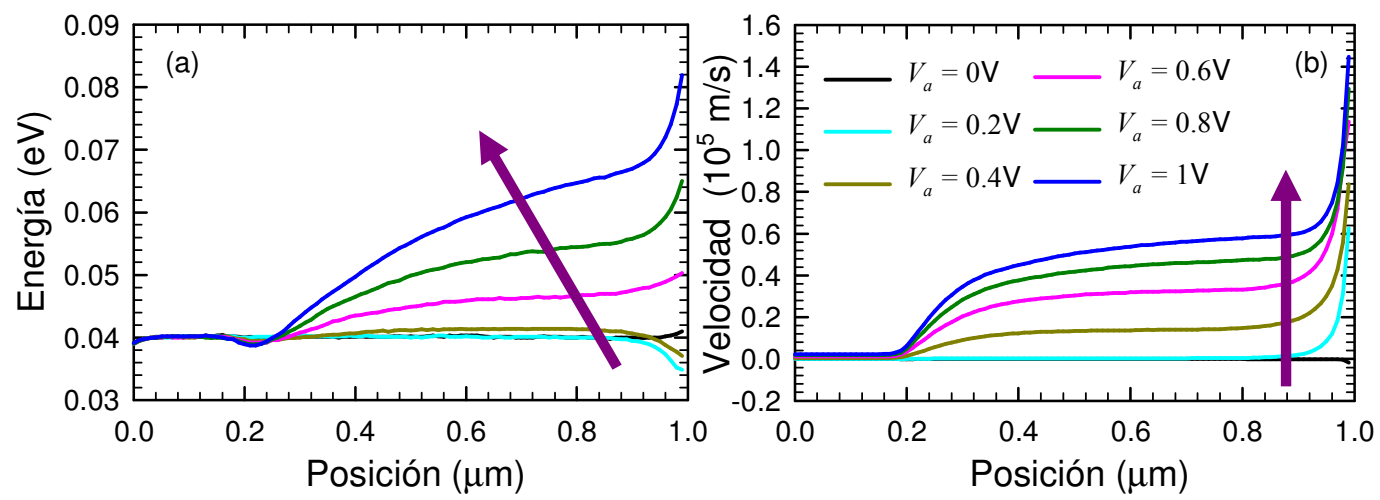

Figura III.9. Diodo de contacto $\mathrm{Si-n} / \mathrm{ZrSi}_{2}$. Perfiles de las siguientes magnitudes bajo polarización en directa:

.a. Energía y.b. velocidad.

Analizando la velocidad y la energía medias (Figura III.9), encontramos que para voltajes inferiores a banda plana los portadores se encuentran prácticamente en equilibrio térmico a lo largo de la estructura, correspondiéndose con valores de velocidad muy reducidos (exceptuando la región más cercana al contacto Schottky, a partir de $0.9 \mu \mathrm{m}$ hasta $1 \mu \mathrm{m}$, donde la velocidad en valor medio es elevada como podemos ver en la Figura III.9.b para $V_{a}=0.2 \mathrm{~V}$ ). Cabe destacar que incluso, en las proximidades del contacto Schottky, se produce un enfriamiento de los portadores [González, 1994], [Stratton, 1962]. Por el contrario, cuando aumentamos la polarización en directa hasta la desaparición de la barrera, debido a la presencia del campo eléctrico de arrastre que comentábamos previamente, los portadores de la región $n$ ganan energía progresivamente a lo largo de la estructura. De manera análoga, se produce un aumento significativo de la velocidad de los electrones para estas polarizaciones $\left(V_{a}>V_{b i}\right)$ a medida que pasan de la unión $n^{+}-n$ hacia el contacto. Observamos que tenemos una subida 
apreciable de la velocidad en las cercanías de la frontera; este efecto es debido al carácter rectificador de este contacto para las polarizaciones en directa aplicadas (la inyección de portadores es despreciable frente a la absorción). En el caso de altas tensiones aplicadas, la velocidad alcanza valores muy elevados (en torno a $1.4 \cdot 10^{5} \mathrm{~m} \mathrm{~s}^{-1}$ ) para regiones cuyo campo eléctrico local ronda los $10 \mathrm{kV} \mathrm{cm}^{-1}$, mientras que para Silicio material la saturación de la velocidad de arrastre en el caso de electrones toma valores de $10^{5} \mathrm{~m} \mathrm{~s}^{-1}$, aproximadamente, para campos eléctricos aplicados superiores a $50 \mathrm{kV} \mathrm{cm}^{-1}$ [Martín, 1992]. Esta diferencia entre lo que ocurre en Silicio material y en las últimas mallas próximas al contacto Schottky puede explicarse principalmente porque en este último únicamente hay portadores con velocidad positiva.

\section{III.4.a.2. Comportamiento en inversa}

En esta sección estudiaremos con detalle el comportamiento del dispositivo sometido a polarización inversa. A continuación examinaremos el perfil de las diferentes magnitudes que nos proporciona el método Monte Carlo, del mismo modo que en el caso de polarización en directa, para obtener una mejor comprensión de la física del diodo.

A medida que aumentamos la polarización en inversa, la barrera vista por los electrones del semiconductor al metal se hace más alta, y por tanto la probabilidad de absorción de portadores por parte del metal es muy pequeña. Sin embargo la barrera que deben superar los electrones desde el metal al semiconductor permanece constante en altura y es cada vez más estrecha (Figura III.10.a). Al aumentar la altura de la barrera

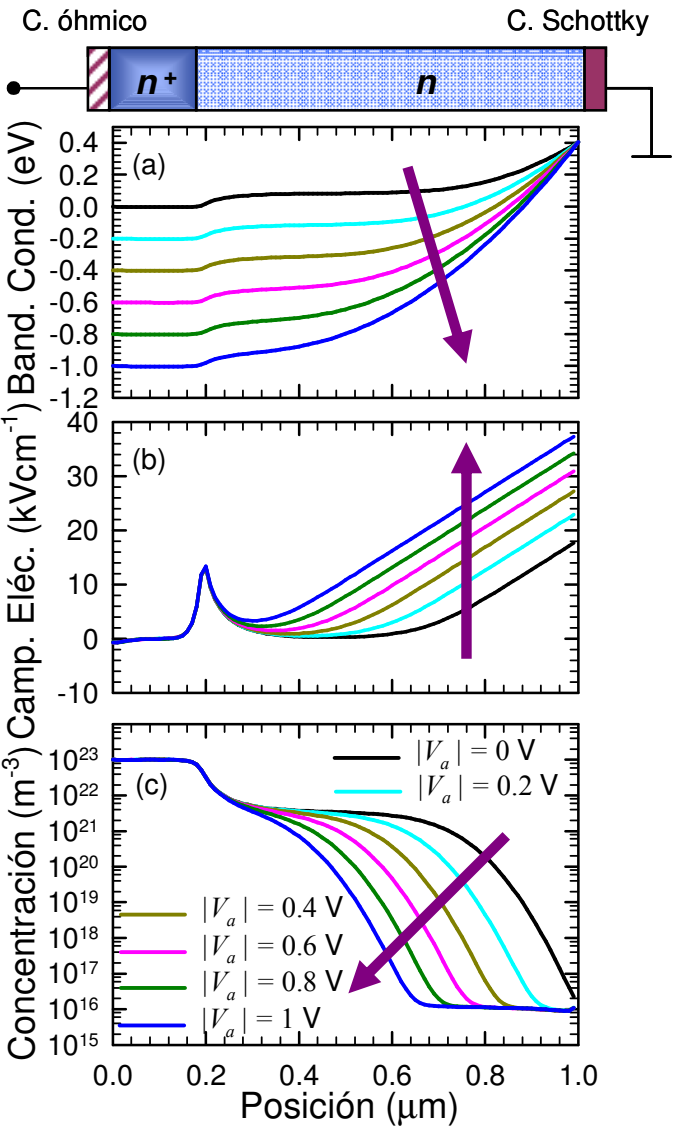

Figura III.10. Diodo de contacto $\mathrm{Si}-\mathrm{n} / \mathrm{ZrSi} \mathrm{S}_{2}$. Perfiles de las siguientes magnitudes bajo polarización inversa: .a. Banda de conducción .b. Campo eléctrico .c. Concentración. 
de semiconductor a metal, observamos en la región $n$ la presencia de un campo positivo cada vez más elevado que se opone al paso de los electrones al metal, y cuyo valor máximo se encuentra en el límite del contacto Schottky (Figura III.10.b). El perfil de concentración muestra cómo la zona de vaciamiento es mayor a medida que incrementamos la polarización en inversa, debido al campo eléctrico que "repele" a los electrones libres e impide que se acerquen al contacto Schottky.
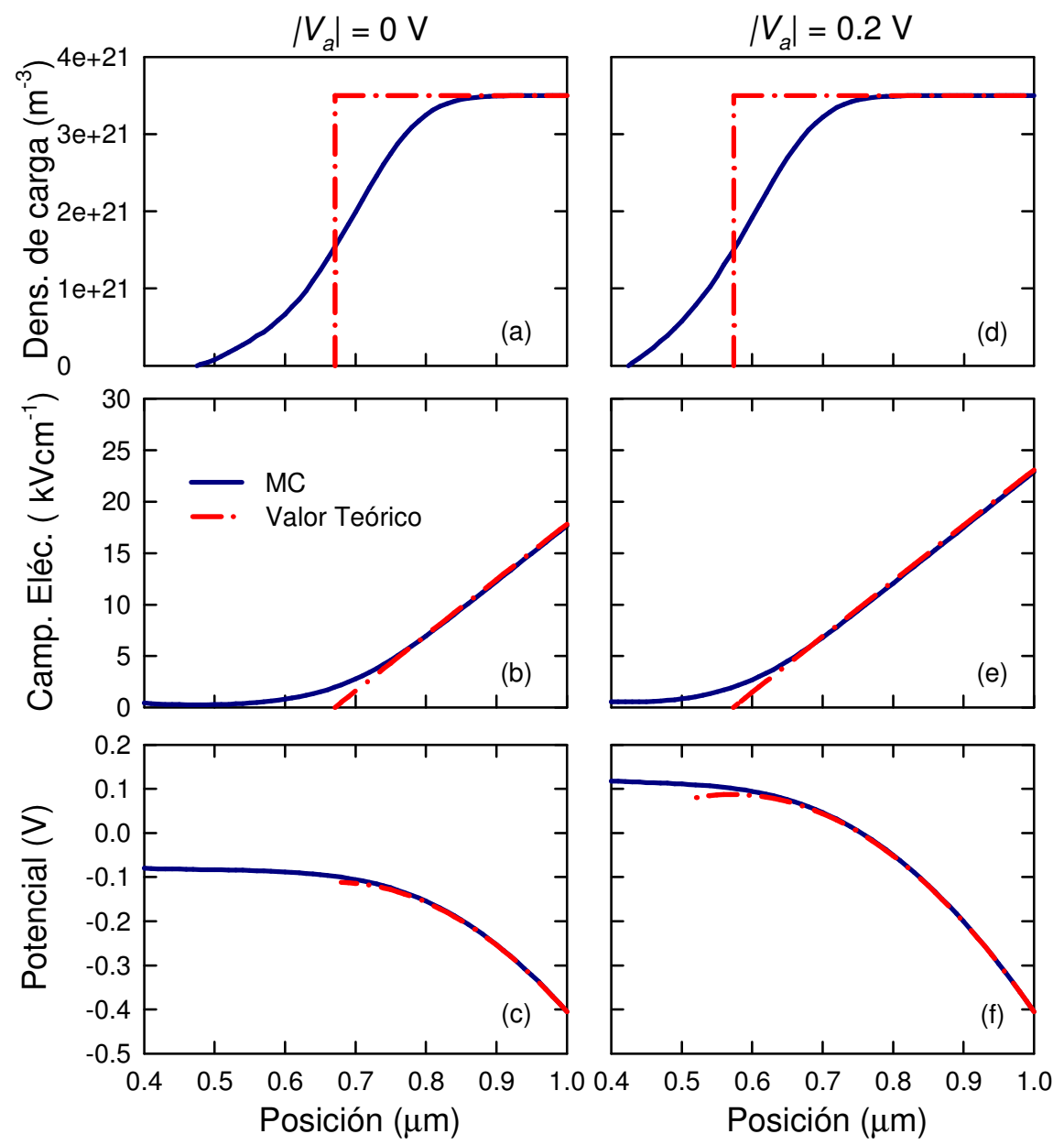

Figura III.11. Densidad de carga, campo eléctrico y potencial para $V_{a}=0$ Vy $0.2 \mathrm{~V}$ en inversa, donde se compara el valor teórico con el proporcionado por el simulador Monte Carlo.

Llegados a este punto, es interesante comparar los resultados obtenidos con el método de Monte Carlo para estas magnitudes y los predichos mediante la aproximación de vaciamiento total para obtener las expresiones de la anchura de vaciamiento $W$, del campo eléctrico $\xi$ y del potencial $\varphi \square$ en función de la posición (Ecuaciones I.3 a I.5). 
En la Figura III.11 mostramos los valores obtenidos mediante este modelo teórico (líneas púrpura) y mediante el simulador Monte Carlo (líneas azules, se corresponden con las mostradas en la Figura III.10, representando únicamente la zona cercana al contacto Schottky) para la situación de equilibrio y pala el voltaje en inversa aplicado $\left|V_{a}\right|=0.2 \mathrm{~V}$. El valor teórico de la anchura de vaciamiento aumenta con la polarización inversa aplicada, siendo igual a $0.33 \mu \mathrm{m}$ para $0 \mathrm{~V}$ (ver Figura III.11.a) y $0.57 \mu \mathrm{m}$ para $0.2 \mathrm{~V}$ (Figura III.11.d). En el caso de la simulación Monte Carlo la zona de carga espacial presenta una variación suave con la posición tal y como debe darse en una estructura real, en lugar de la transición abrupta propia de la aproximación de vaciamiento.

El campo eléctrico (Figuras III.11.b y e) varía de manera prácticamente lineal con la posición debido a la densidad de carga constante en la región de vaciamiento. Sin embargo, en el límite de esta zona no presenta comportamiento lineal, puesto que, como decíamos anteriormente, la variación de la carga no es abrupta. En todo caso, podemos destacar el buen acuerdo general con los resultados Monte Carlo para el campo eléctrico y el potencial, lo que implica que la hipótesis de vaciamiento total es razonable para describir a grandes rasgos el comportamiento de estos dispositivos.

A continuación procedemos a analizar la inyección de los portadores desde el metal al semiconductor. Para ello, el modelo de inyección/absorción implementado en nuestro simulador Monte Carlo nos permite examinar la carga inyectada para cada valor de energía incidente, así como el valor del coeficiente de transmisión. Como se comprueba en la Figura III.12.a, tenemos una distinción clara entre dos regiones energéticas:
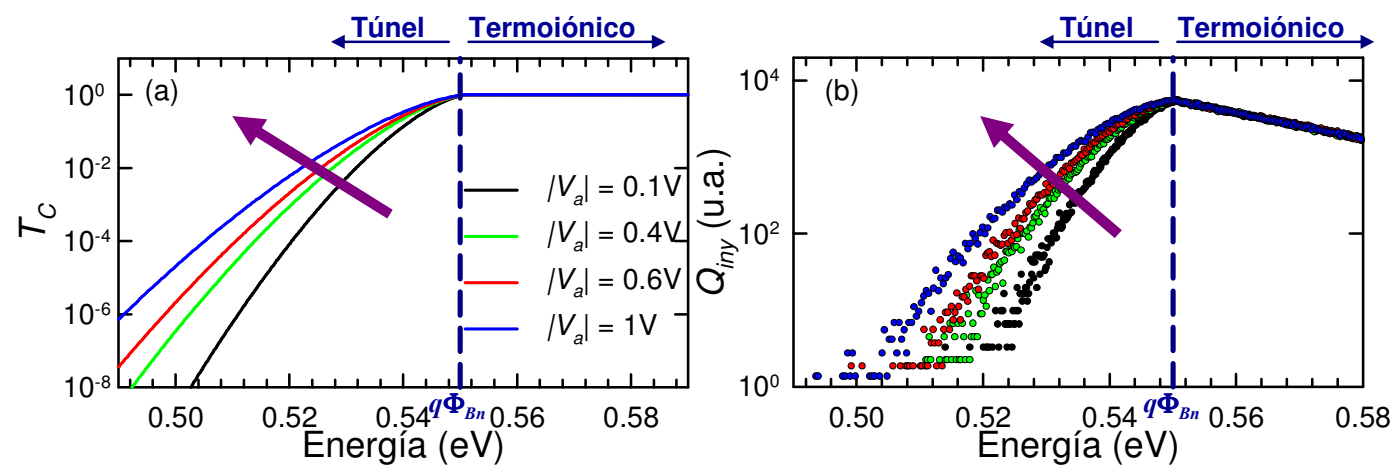

Figura III.12. Considerando la inyección de metal a semiconductor: a. Coeficiente de transmisión del metal al semiconductor .b. Carga real inyectada, ambas magnitudes expresadas en función de la energía, para distintos valores de tensión en inversa aplicada. 
- $\quad$ Para energías superiores a la altura de la barrera (en la estructura que actualmente nos ocupa recordemos que la altura de la barrera Schottky es $0.55 \mathrm{eV}$, véase Tabla III.1) el coeficiente de transmisión que asigna la aproximación WKB es la unidad, es decir, todos los portadores con energía superior a la barrera son inyectados al metal, sin considerar posibles reflexiones cuánticas. Esto implica la dependencia exponencial de la carga inyectada para esos valores de energía, asociada a la cola de la función de distribución de Fermi - Dirac en el metal, que se puede asimilar a la de Maxwell - Boltzmann para estos valores de energía.

- En el rango de energías inferiores a la altura de la barrera, observamos que el coeficiente de transmisión toma valores mayores a medida que aumenta la polarización debido al estrechamiento de la barrera de potencial, como ya veíamos en la Figura III.10.a; y por tanto la carga inyectada también aumenta con el voltaje aplicado.

En la Figura III.12.b puede comprobarse que el máximo de carga inyectada tiene lugar para el valor de energía de $0.55 \mathrm{eV}$, altura máxima que alcanza la barrera (es importante destacar que esta gráfica muestra valores reales de carga inyectada proporcionados por el simulador Monte Carlo).

El comportamiento de estas magnitudes, así como la variación de la barrera de potencial con la tensión aplicada, nos dan información suficiente para poder comprender cómo contribuye cada componente de corriente a la total.

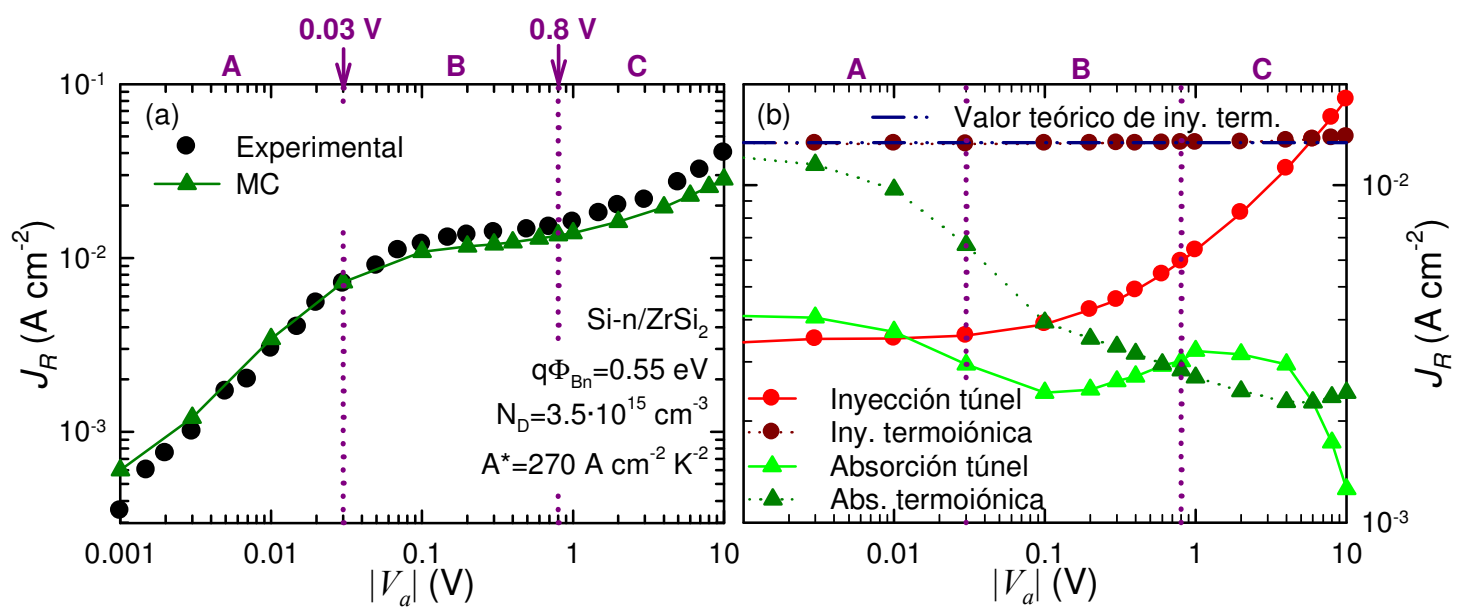

Figura III.13.a. Características $J-V$ en inversa de la estructura $\mathrm{Si-n} / \mathrm{ZrSi}_{2}$ a $300 \mathrm{~K}$. Los círculos son los resultados experimentales [Andrews, 1970] y los triángulos son los resultados numéricos de la simulación Monte Carlo con constante de Richardson $A^{*}=270 \mathrm{Acm}^{-2} \mathrm{~K}^{-2}$. b. Componentes de la corriente total.

En primer lugar, analizamos la característica corriente-tensión en inversa proporcionada por nuestro simulador en comparación con medidas experimentales (Figura III.13.a). Como 
observamos en esta figura, en general tenemos un buen ajuste de los resultados de la simulación con los experimentales. Para un análisis más preciso de la característica, podemos distinguir en ella tres regiones de comportamiento en función del voltaje.

En la región A (hasta el valor aproximado en módulo de $0.03 \mathrm{~V}$, es decir, tensiones débiles en inversa) la corriente total aumenta considerablemente debido a la progresiva disminución de la corriente de absorción termoiónica ${ }^{5}$ frente a la de inyección termoiónica, que permanece constante (Figura III.13.b), puesto que la barrera desde el metal es igualmente constante $\left(\Phi_{B n}=\right.$ cte.). El valor obtenido con el simulado Monte Carlo para esta corriente de inyección coincide con el calculado teóricamente:

$$
J_{\text {Term }}=A^{*} T^{2} \exp \left(-\frac{q \Phi_{B n}}{K_{B} T}\right)=134 A K^{-2} m^{-2}
$$

Las componentes de inyección y absorción túnel son notablemente inferiores a las termoiónicas en este rango de tensiones aplicadas, dado que los valores de anchura de la barrera son todavía elevados.

En la región B (para $V_{a}$ entre $0.03 \mathrm{~V}$ y $0.8 \mathrm{~V}$, tensiones medias en inversa) la corriente total presenta un comportamiento prácticamente constante con el voltaje. Esto es debido a que la principal contribución en este rango procede de la inyección termoiónica, puesto que la absorción termoiónica ha disminuido considerablemente, como también lo ha hecho la absorción túnel (Figura III.13.b). Destaquemos el progresivo incremento de la componente de inyección túnel, sin embargo aún despreciable en comparación con la inyección termoiónica.

Finalmente, en la región $\mathbf{C}$ (para tensiones en inversa elevadas) la corriente total experimenta un aumento (Figura III.13.a), el cual no se podría explicar sin considerar la inyección túnel, como veremos más adelante. Esta componente supone la contribución más importante a la corriente total en este rango de tensiones aplicadas (y por supuesto, a superiores). Observamos en la característica corriente total-tensión que en esta región los resultados numéricos tienen el mismo comportamiento que los experimentales pero su valor es inferior, lo cual es debido a que en esta ocasión no se está considerando la reducción efectiva de la barrera por el efecto de la carga imagen, como veremos más adelante.

La componente de inyección túnel aumenta con la tensión en inversa puesto que la barrera que tienen que atravesar los portadores por efecto túnel es cada vez más estrecha, lo que

\footnotetext{
${ }^{5}$ La disminución de la corriente de absorción termoiónica con la tensión en inversa aplicada es debida a la dificultad, cada vez mayor, de los electrones de sobrepasar la barrera del semiconductor al metal.
} 
aumenta la probabilidad de transmisión como veíamos en la Figura III.11.a. A polarizaciones negativas superiores en módulo a $8 \mathrm{~V}$, esta componente es la más importante.

En relación al comportamiento decreciente de las corrientes de absorción, vemos que la termoiónica es superior a la túnel en un primer rango de polarizaciones, sin embargo después pasa la absorción túnel a ser superior, debido a que la anchura de la barrera a atravesar comienza a ser menor para estos voltajes y por tanto aumenta la probabilidad de que se dé este fenómeno.

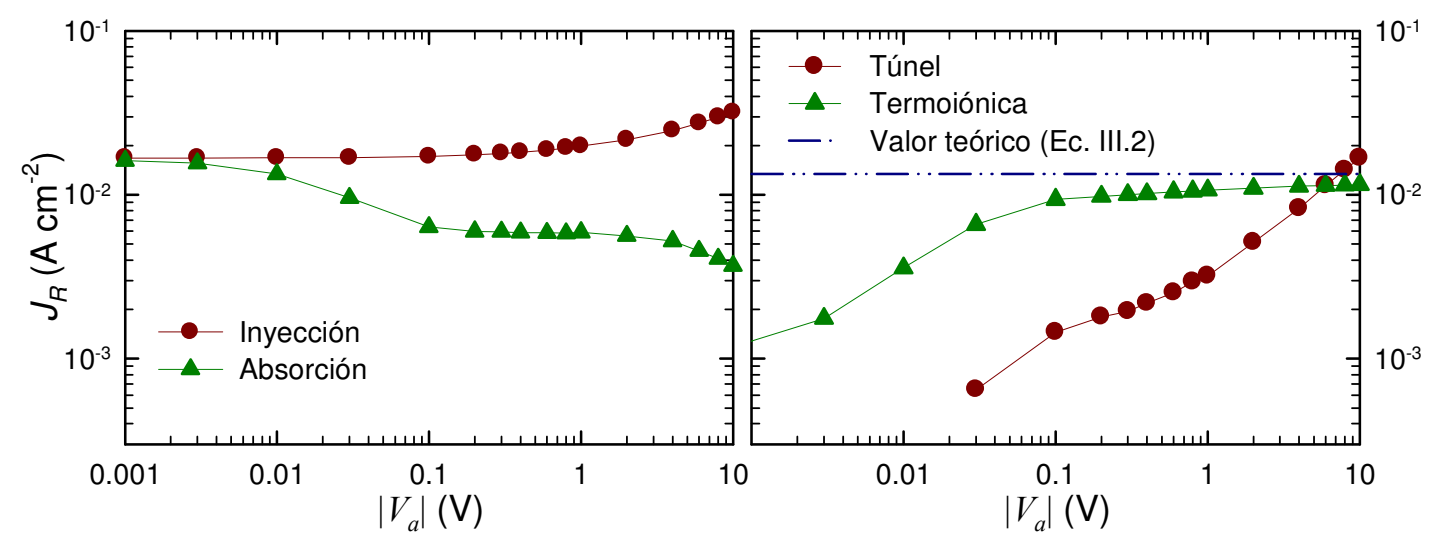

Figura III.14.a. Contribución de la corriente de inyección y de absorción para polarizaciones en inversa del contacto $\mathrm{Si}-n / \mathrm{ZrSi}_{2}$.b. Comportamiento de las componentes termoiónica total y túnel total, donde además se muestra el valor teórico de la componente de inyección termoiónica (ver Ecuación III.2).

La adición de las componentes termoiónica y túnel (Figura III.14.a) nos permite observar más claramente las diferencias entre inyección y absorción. En la región A, para los voltajes más cercanos a cero, éstas muestran valor similar; sin embargo, la absorción presenta una clara tendencia a disminuir con la tensión en inversa aplicada. En la región $\mathbf{B}$ ambas componentes toman un valor prácticamente constante, para luego aumentar su diferencia en la región $\mathbf{C}$, donde la inyección predomina considerablemente en la corriente total. La Figura III.14.b nos muestra el comportamiento de las corrientes termoiónica y de túnel calculadas mediante la diferencia entre sus componentes de inyección y absorción. Observamos cómo la corriente termoiónica aumenta en un primer rango de tensiones en inversa (debido a la anteriormente comentada reducción de su componente de absorción) y a tensiones mayores permanece constante correspondiendo prácticamente al valor teórico de la Ecuación III.2. Sin embargo, la componente túnel presenta un crecimiento importante y continuo con el voltaje en inversa, fundamentado en el fuerte incremento de la inyección túnel.

En la Figura III.15 mostramos los perfiles de energía y velocidad medias en función de la posición para diferentes valores de tensión en inversa aplicada. Al aumentar el voltaje en 
inversa, en la región cercana al contacto la energía de los portadores es cada vez más elevada, así como su velocidad (en valor absoluto). La población existente en esa región proviene en su mayor parte de los portadores inyectados en el semiconductor desde el metal. El hecho de que la velocidad tome valores negativos es consistente con que el flujo de carga se realice de derecha a izquierda. Los electrones son inyectados en el semiconductor desde el metal con energías cercanas a la térmica. Inmediatamente y como consecuencia del elevado campo eléctrico positivo existente en la zona de vaciamiento de la región $n$, son arrastrados hacia la región $n^{+} \mathrm{y}$ ganan rápidamente energía y velocidad. Sin embargo, dado que el campo eléctrico se va reduciendo a medida que los electrones se desplazan hacia la izquierda, también disminuye su energía y su velocidad de modo que una vez superada la región de vaciamiento se llega a una situación de equilibrio térmico, tomando la energía en promedio un valor de $3 / 2 K_{B} T$ y siendo la velocidad longitudinal muy reducida.
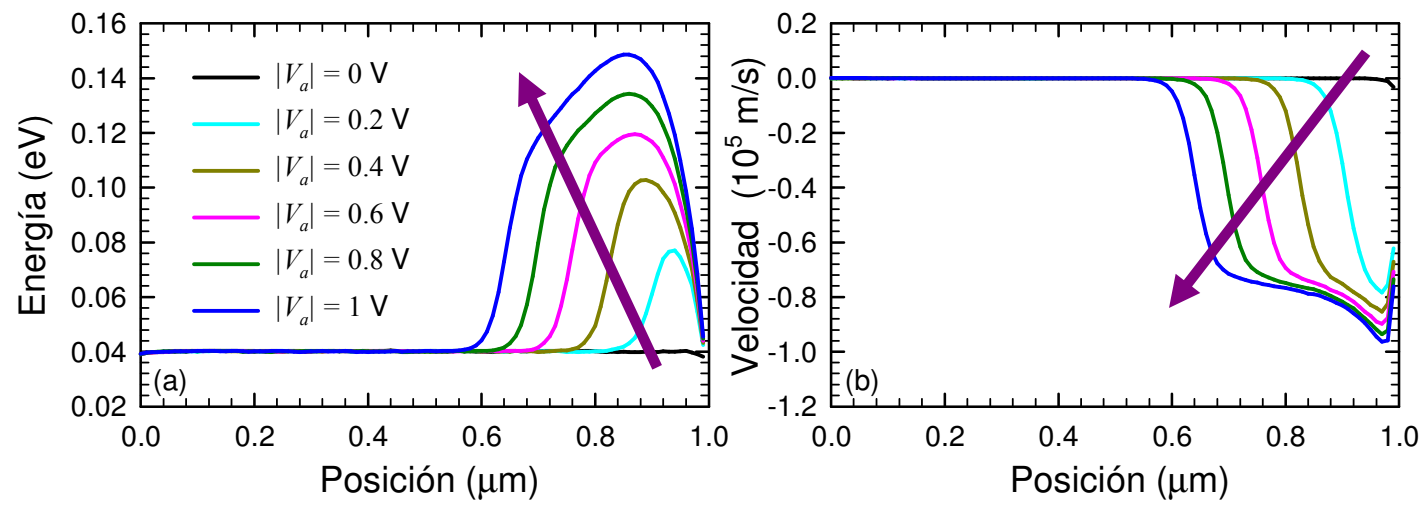

Figura III.15. Diodo de contacto Si-n/ZrSi ${ }_{2}$. Perfiles de las siguientes magnitudes bajo polarización en inversa:

$$
\text { .a. Energía .b. Velocidad. }
$$

Función de distribución de velocidad, mecanismos de scattering y ocupación de valles

El análisis que acabamos de realizar, aunque justifica el comportamiento de la velocidad y la energía, no describe con suficiente detalle la física del transporte en esta región. Llegados a este punto podemos aprovechar toda la información aportada por nuestro simulador para ofrecer una visión más amplia del transporte bajo estas condiciones de polarización en inversa; en concreto examinaremos a continuación las condiciones que se presentan para el voltaje en inversa aplicado de $1 \mathrm{~V}$ (en módulo). En esta situación las componentes de la corriente de 
inyección (tanto túnel como termoiónica) son mucho mayores que las de absorción (ver Figura III.14.a).

Los electrones inyectados por procesos termoiónicos poseen, considerando las condiciones iniciales de la simulación [ver Capítulo II, Sección 5], una componente $x$ del momento hacia el interior del dispositivo, mientras que para aquellos que acceden al semiconductor por procesos túnel, dicha componente toma inicialmente valor nulo.

En la Figura III.16 presentamos la densidad de ocupación de los valles X del Silicio y la densidad de mecanismos de scattering. En posiciones muy próximas al contacto Schottky $(\mathrm{x}=1 \mu \mathrm{m})$ observamos (véase Figura III.16.a) que existe un fuerte fenómeno no estacionario asociado a la interacción con fonones intervalle (Figura III.16.b) que provoca que, a pesar de que los portadores han sido inyectados en el semiconductor con igual probabilidad de ocupación en cada tipo de valle X (al $33 \%$ ), exista una redistribución entre los mismos, haciendo que el valle longitudinal (X1) presente una fuerte sobreocupación. Todos estos efectos se explican por el elevado campo eléctrico junto al contacto (Figura III.10.b).
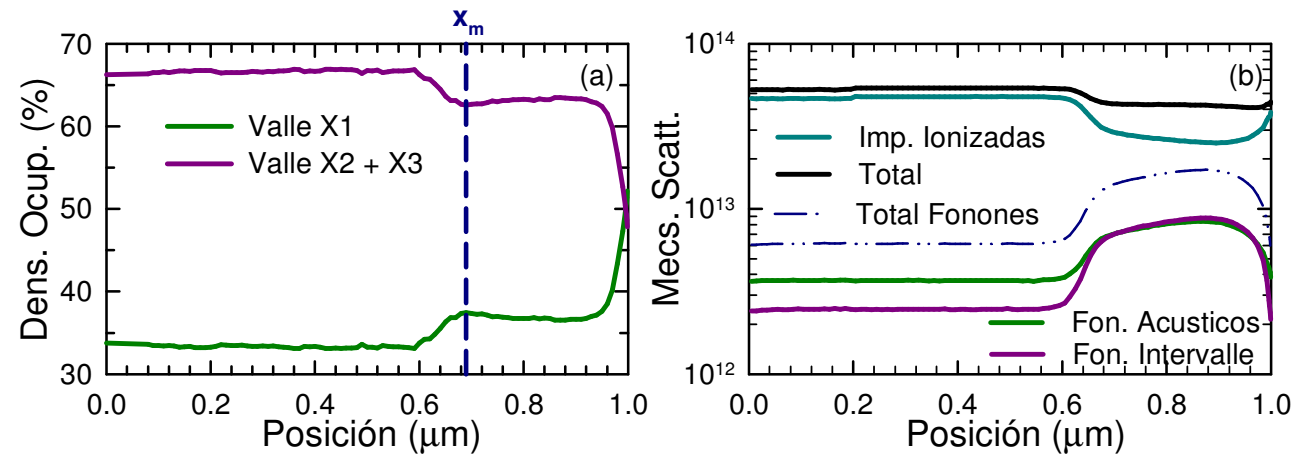

Figura III.16.a. Densidad de ocupación de los valles $X$ del Silicio. $x_{m}$ representa la posición del máximo de ocupación relativo de valle X1 .b. Densidad de mecanismos de scattering con impurezas, con fonones acústicos y ópticos.

A medida que nos adentramos en el interior del dispositivo (desplazamiento hacia la izquierda), el fuerte aumento de energía de los portadores ocasiona que, en correspondencia, aumenten también los mecanismos de scattering con fonones (Figura III.16.b). Este hecho produce una reducción de la ocupación del valle X1 y un aumento de la ocupación de los valles transversales. Asimismo, examinando la función de distribución de velocidad en la región próxima al contacto Schottky (Figura III.17) observamos que la existencia de estos mecanismos provoca que algunos de los portadores se den la vuelta acercándose al contacto Schottky, lo que 
explica la pequeña cola positiva de la función de distribución de velocidad (Figura III.17.e, en la posición más cercana al contacto).

El hecho de que un portador se encuentre en un valle longitudinal (pesado, con masa $m_{l}=0.91 m_{0}$ en la dirección del campo aplicado) o en uno transversal (ligero, con masa $m_{t}=$ $0.19 \mathrm{~m}_{0}$ ) afecta de manera significativa a su velocidad; examinando las funciones de distribución a medida que nos alejamos del contacto (Figuras III.17.d, c y b), observamos la existencia de dos máximos en la función de distribución total, cada uno de ellos asociado a portadores moviéndose en valles longitudinales (máximo de menor velocidad) y en valles transversales (máximo situado a velocidades más elevadas). A $25 \mathrm{~nm}$ de la interfaz con el contacto Schottky (Figura III.17.b), el máximo correspondiente a los portadores en valles longitudinales aparece para valores de velocidad de $-1.7 \cdot 10^{5} \mathrm{~m} \mathrm{~s}^{-1}$, mientras que para los valles transversales ocurre a $-4.0 \cdot 10^{5} \mathrm{~m} \mathrm{~s}^{-1}$. A partir del modelo semiclásico [Martín, 1996b] podemos realizar un cálculo analítico aproximado del valor de velocidad que poseerían los portadores que son inyectados desde el metal en el caso de que no sufrieran ningún mecanismo de scattering (esto es, la velocidad de los denominados portadores balísticos). Para el caso de los electrones del valle longitudinal el valor correspondiente es de $1.95 \cdot 10^{5} \mathrm{~m} \mathrm{~s}^{-1}$ y para el valle transversal es $4.4 \cdot 10^{5} \mathrm{~m} \mathrm{~s}^{-1}$. Estos valores son muy próximos a los obtenidos en la función de distribución, lo que nos indica el carácter cuasi-balístico del transporte de algunos portadores, que pueden ser fácilmente identificados en la función de distribución de velocidad.

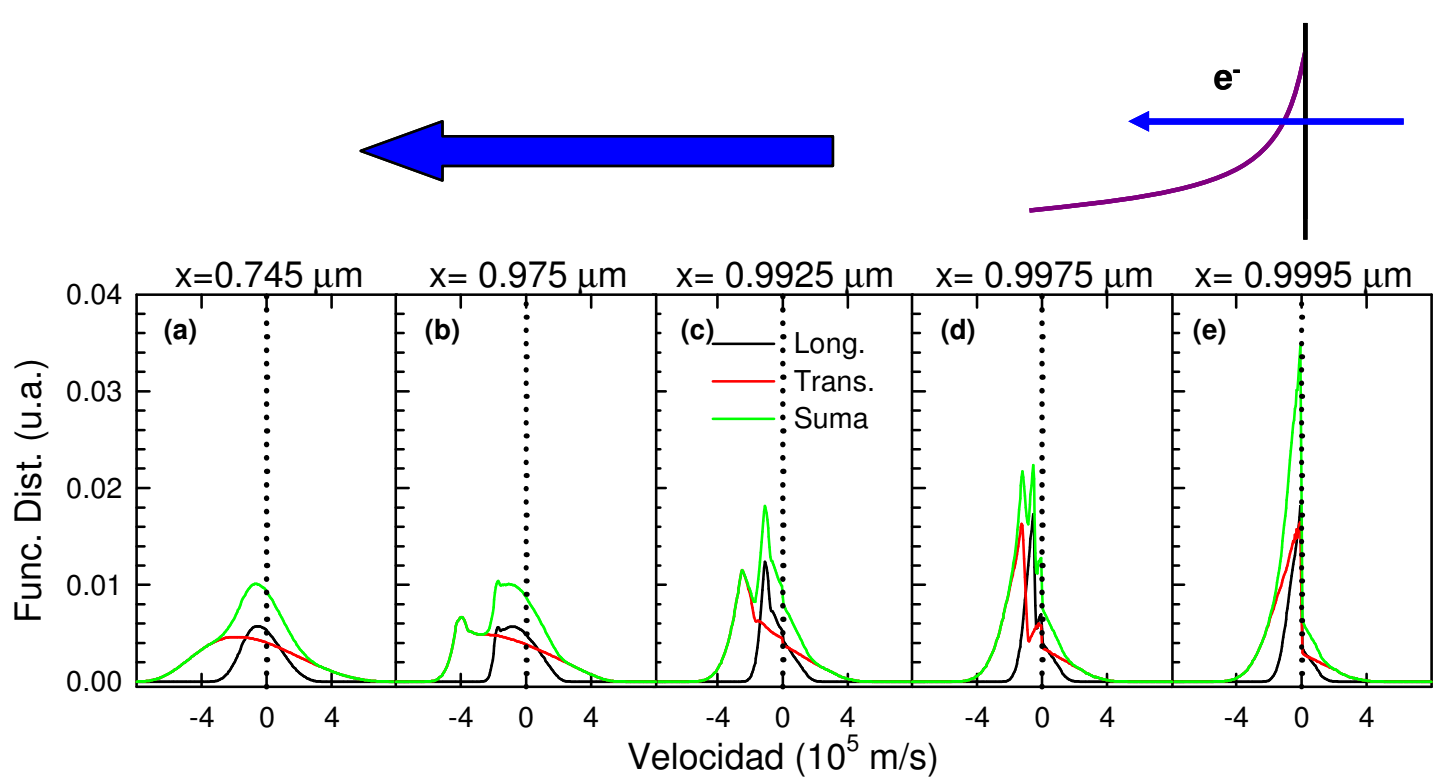

Figura III.17.a-e. Funciones de distribución de velocidad de los portadores en el valle longitudinal y en los valles transversales y la suma de ambas contribuciones en diferentes regiones del dispositivo para un voltaje en inversa aplicado de $1 \mathrm{~V}$. 
Llegados a este punto, debemos prestar especial atención de nuevo al perfil de densidad de mecanismos de scattering. Como puede apreciarse en la Figura III.16.b, la densidad de mecanismos de scattering con impurezas ionizadas es mayor que con fonones acústicos y ópticos a lo largo de todo el dispositivo; sin embargo, la naturaleza anisótropa de los mecanismos de scattering por impurezas hace que su efecto, de manera individual, sea menor que el de los mecanismos con fonones, de carácter isótropo. Como ya hemos comentado con anterioridad, inicialmente existe un fuerte aumento de las interacciones con fonones a medida que nos alejamos del contacto, así como una reducción de densidad de mecanismos de scattering con impurezas debido al fuerte incremento de la energía de los portadores. Estos valores de la densidad de mecanismos de scattering son reflejo de la variación de las probabilidades de las mismas con la energía (véase el Apéndice, Figura 2). Se da por tanto una zona del dispositivo en la cual existe un fuerte arrastre de los portadores por el campo elevado en conjunción con una fuerte acción termalizadora de los mecanismos de scattering. Este hecho se ve reflejado en la función de distribución de velocidad de la Figura III.17.d hacia la III.17.a, donde se observa una clara tendencia a aumentar de las componentes de velocidad positivas (o de valores negativos reducidos), aproximándose el perfil de la función de distribución a una maxwelliana completa. El efecto termalizador de los fonones se acaba imponiendo, de manera que observamos, para posiciones inferiores a $0.6 \mu \mathrm{m}$ una situación en equilibrio correspondiente a la mencionada en el apartado anterior, en la cual los tres valles se encuentran prácticamente equipoblados, tal y como sucede para campos reducidos.

Una vez examinado el comportamiento para un punto de polarización, vamos a comprobar cómo afecta la variación de la tensión en inversa aplicada a estos perfiles de ocupación de valles y mecanismos de scattering que acabamos de analizar. En la Figura III.18 mostramos ambas magnitudes para $\left|V_{a}\right|=0.4 \mathrm{~V},\left|V_{a}\right|=0.6 \mathrm{~V},\left|V_{a}\right|=1 \mathrm{~V} \mathrm{y}\left|V_{a}\right|=2 \mathrm{~V}$. A medida que aumenta la polarización, la zona de vaciamiento se hace mayor (ver Figura III.10.c), así como la región en la que los portadores alcanzan energías elevadas (Figura III.15.a). Consecuentemente, la región en la cual los mecanismos de scattering con fonones tienen una mayor presencia también se hace mayor, como puede observarse en las Figuras III.18.a-d, y la posición del máximo relativo de la ocupación del valle X1 se desplaza hacia el interior del dispositivo (Figura III.18.e-h). 


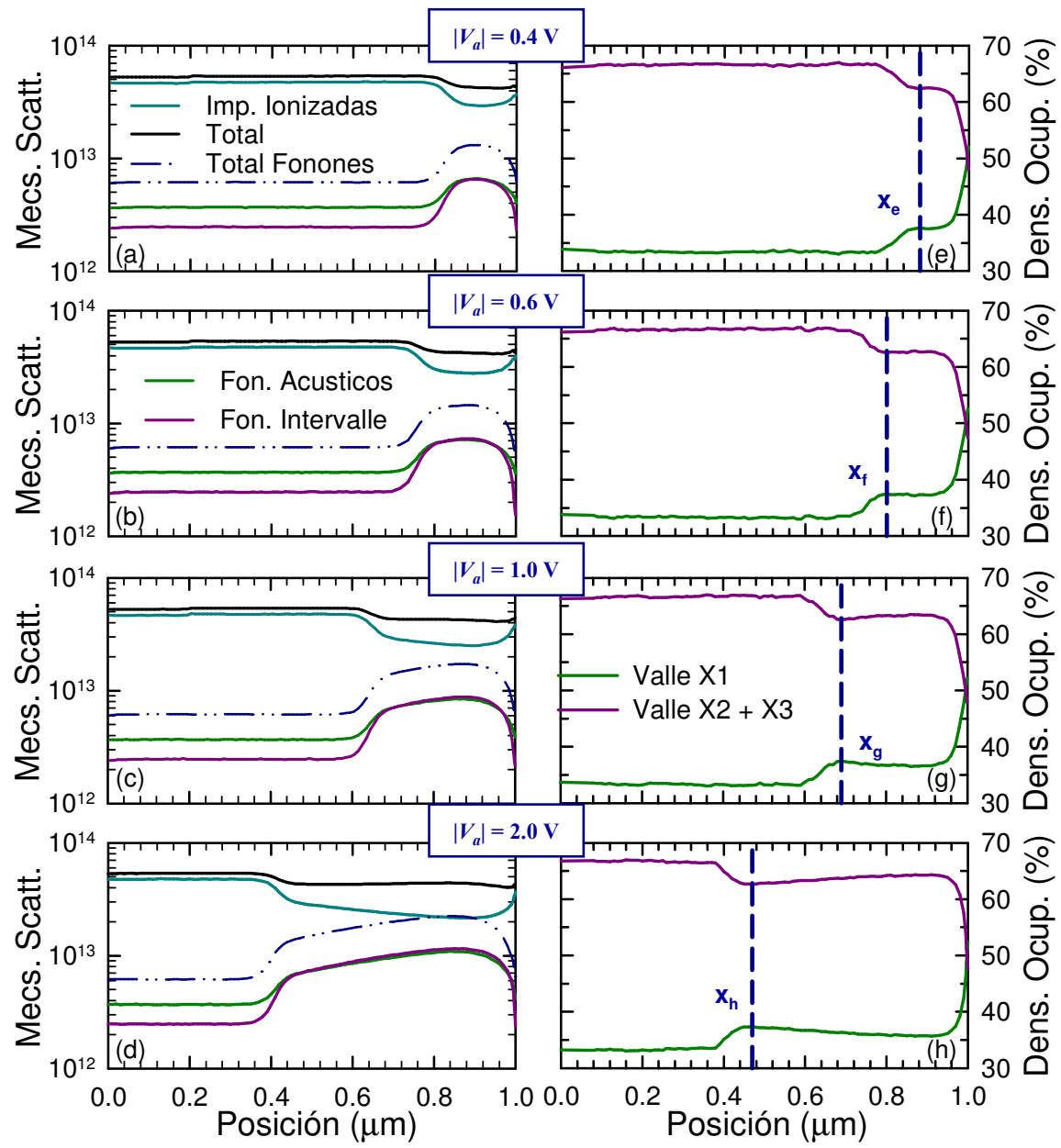

Figura III.18. Densidad de mecanismos de scattering con impurezas, con fonones acústicos y ópticas y la suma de todas ellas en función de la posición para los voltajes en inversa aplicados: .a. $0.4 \mathrm{~V}$, .b. $0.6 \mathrm{~V}$, .c. $1 \mathrm{Vy}$.d. $2 \mathrm{~V}$ .e. Densidad de ocupación de los valles X del Silicio para 0.4 Vy.f. 0.6 V, .g. 1 Vy.h. 2 V. La posición del máximo de ocupación relativo de valle X1 viene indicada por $x_{e}, x_{f}, x_{g} y x_{h}$.

Debemos señalar que todos los resultados mostrados en densidad de ocupación corresponden a los valles $\mathrm{X}$, ya que la ocupación asociada a los valles $\mathrm{L}$ del Silicio es meramente testimonial (nula para tensiones negativas aplicadas inferiores en módulo a $3 \mathrm{~V}$, aumentando para tensiones superiores. Por ejemplo para $\left|V_{a}\right|=4 \mathrm{~V}$ dicha densidad alcanza un máximo de $0.001 \%$ y para $\left|V_{a}\right|=10 \mathrm{~V}$ es aproximadamente del $0.04 \%$ ). 


\section{Ausencia de efecto túnel}

Con el fin de demostrar la importancia de la inclusión de los efectos del transporte por túnel cuántico, es interesante examinar la corriente obtenida si se elimina en el simulador Monte Carlo la posibilidad de que existan este tipo de procesos, es decir, si consideramos únicamente los procesos termoiónicos.

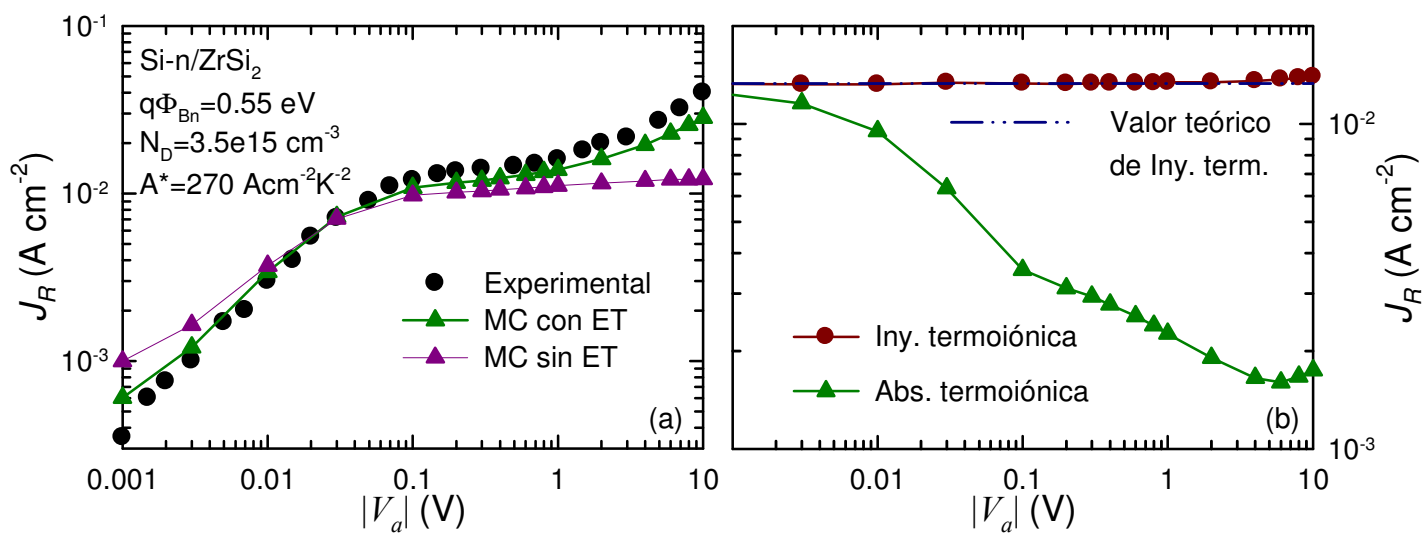

Figura III.19.a. Características J-V del contacto Si-n/ZrSi ${ }_{2}$ (Ver Figura III.13). La línea de triángulos púrpura es el resultado de la simulación sin la consideración del efecto túnel .b. Componentes de corriente para la simulación Monte Carlo sin efecto túnel.

En el caso de no incluir el transporte túnel, la discrepancia de la característica corriente tensión con las medidas experimentales es notable, particularmente a tensiones negativas superiores en módulo a 0.1V (Ver Figura III.19.a). En el rango de débiles tensiones aplicadas, no observamos tanta diferencia; este hecho es debido a que, (recuérdense las Figuras III.13.b y III.14.b), la contribución a la corriente total de la componente túnel es despreciable frente a la termoiónica hasta los $0.1 \mathrm{~V}$, donde la inyección túnel comienza a cobrar importancia. Para tensiones superiores a tal valor comprobamos que al no incluir el efecto túnel la corriente es muy inferior y prácticamente constante.

La Figura III.19.b muestra las componentes de corriente obtenidas en la simulación sin efecto túnel. A tensiones en inversa superiores a $0.1 \mathrm{~V}$ la absorción disminuye, haciéndose despreciable frente a la inyección. Es por esto que vemos cómo en la característica corrientetensión, sin considerar el efecto túnel, el valor de la corriente total permanece prácticamente constante en ese rango de polarizaciones. 
El hecho de considerar efecto túnel tiene además una repercusión importante en el perfil de concentración para altas tensiones aplicadas negativas (superiores en módulo a $1 \mathrm{~V}$ ). Tal y como ya hemos visto, en esta región de voltajes la barrera es muy estrecha lo que provoca un notable aumento de la inyección túnel de partículas desde el metal, convirtiéndose en la contribución más importante a la corriente total. Por este motivo es en esta zona en especial donde se encuentra la mayor discrepancia entre los perfiles de concentración para la simulación con y sin efecto túnel. Mostramos como ejemplo la variación de la densidad de portadores con

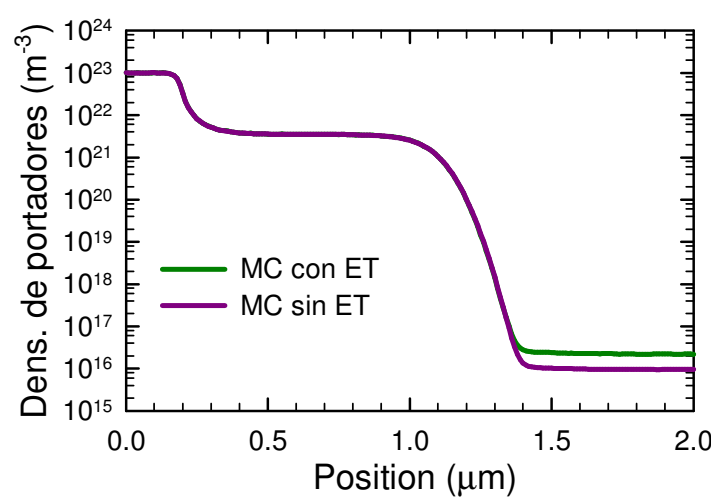

Figura III.20. Perfil de concentración de la estructura $\mathrm{Si}$-n/ZrSi $i_{2}$ para polarización aplicada de $-10 \mathrm{~V}$ en inversa, con y sin efecto túnel. la posición para un voltaje en inversa aplicado de $10 \mathrm{~V}$.

Al considerar el efecto túnel, el número de partículas inyectadas a través del contacto es mucho mayor que si no lo tenemos en cuenta, siempre considerando el mismo perfil de EPE. Éstas incrementan el valor de la concentración en la región cercana al contacto, tal y como se observa en la Figura III.20.

Por tanto, podemos concluir que la inclusión del efecto túnel cuántico en los cálculos es de vital importancia para reproducir los resultados experimentales, sobre todo para las tensiones más elevadas en inversa, donde su peso es más importante.

\section{Estudio del efecto de las cargas imagen}

Como ya mencionamos en el Capítulo I, Sección 4.a, existe un efecto no ideal de reducción de la barrera (denominado también barrier lowering, BL) por efecto de las cargas imagen. Recordemos que dicho efecto consiste en la disminución efectiva (de manera proporcional a la raíz cuadrada del campo del eléctrico aplicado [Ecuación I.31]) de la altura de la barrera que ven los portadores. Nosotros lo implementamos mediante el procedimiento detallado en la Sección 5 del Capítulo II. El valor de la altura de la barrera es un factor muy importante en el cálculo de la carga a inyectar, por tanto es de esperar que afecte de manera significativa en el transporte de corriente.

En la gráfica de la Figura III.21.a se comparan los resultados obtenidos con y sin el efecto de las cargas imagen. Obtenemos un ajuste muy bueno para tensiones en inversa entre 
$0.003 \mathrm{~V}$ y $1 \mathrm{~V}$, que es el rango de polarizaciones que consideramos de mayor interés: sin embargo, a voltajes negativos más elevados los valores obtenidos incluyendo el efecto de reducción de la barrera presentan un mayor aumento de la corriente que los datos experimentales. Un aspecto importante a destacar es que la constante de Richardson empleada en las simulaciones con $\mathrm{BL}$ es de $200 \mathrm{~A} \mathrm{~cm}^{-2} \mathrm{~K}^{-2}$, sensiblemente inferior a la necesaria para el caso de no considerar la reducción de la barrera.

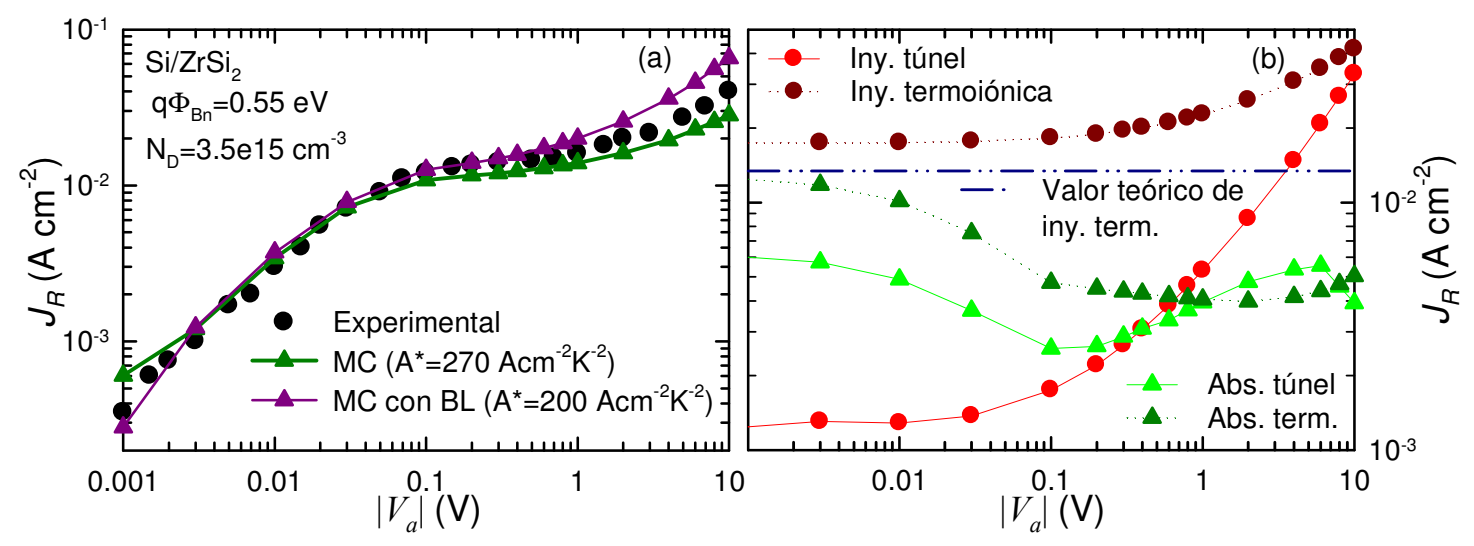

Figura III.21.a. Característica corriente-tensión del la estructura Si-n/ZrSi $i_{2}$ La línea de triángulos púrpura muestra los resultados incluyendo el efecto de cargas imagen con $A^{*=200 ~} \mathrm{~A} \mathrm{~cm}^{-2} \mathrm{~K}^{-2}$ mientras que la de triángulos verdes corresponde a los que se mostraban en la Figura III. 13 con $\mathrm{A}^{*}=270 \mathrm{~A} \mathrm{~cm}^{-2} \mathrm{~K}^{-2}$ y sin efecto de cargas imagen .b. Componentes de corriente para la simulación con BL. El valor teórico para la corriente de inyección termoiónica mostrado es para dicha componente sin incluir BL (Ecuación III.2).

Las componentes de corriente también se ven modificadas al considerar el efecto de las cargas imagen. En primer lugar, la corriente de inyección termoiónica no es constante y aumenta con el voltaje aplicado; esto se debe a que según aumenta el voltaje aplicado también lo hace el campo en las inmediaciones de la barrera, por lo que la reducción efectiva de la barrera es también mayor. Como vemos en la Figura III.21.b (en comparación con la característica que presentamos en Figura III.13.b, sin BL), la inclusión de este efecto favorece la inyección termoiónica en detrimento de la túnel para el rango de polarizaciones negativas de $0 \mathrm{a}$ $1 \mathrm{~V}$ (en módulo). Sin embargo a tensiones en inversa más elevadas, la componente túnel es superior incluyendo el efecto de cargas imagen que sin dicha consideración.

Este comportamiento se explica fácilmente a partir de los resultados mostrados en la Figura III.22, donde representamos la carga inyectada en función de la energía incidente para tres polarizaciones distintas con y sin el efecto de las cargas imagen. Como vemos, la carga inyectada termoiónicamente aumenta gracias a la reducción de la barrera efectiva. Esto implica 
una reducción de la carga inyectada por túnel, que es más acusada en el caso de débiles tensiones (por ejemplo, $V_{a}=0 \mathrm{~V}$ ). Sin embargo, para $1 \mathrm{~V}$ en inversa aplicado, la cantidad de carga inyectada comienza a igualarse para ambos casos (carga inyectada túnel y termoiónica) hasta ser superior la carga inyectada por túnel con la inclusión de dicho efecto, tal y como se refleja en el caso de voltaje negativo de $10 \mathrm{~V}$, teniendo su consecuencia directa en los valores de las corrientes inyectadas mostradas en la gráfica III.18.b. Se produce por tanto un compromiso entre dos efectos: la reducción de la altura de la barrera, que favorece a la componente termoiónica, y su estrechamiento, que favorece el efecto túnel [Pascual, 2007b].

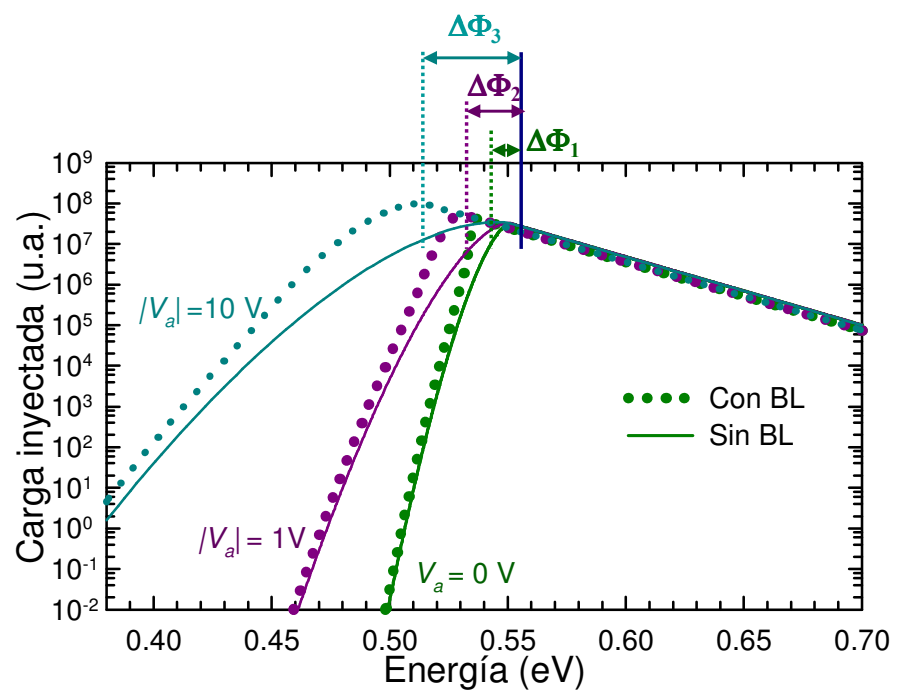

Figura III.22. Carga inyectada en función de la energía para tres polarizaciones distintas incluyendo efecto de cargas imagen (líneas discontinuas) y sin él (líneas continuas). El valor de la reducción de la barrera para cada voltaje es $\Delta \Phi_{1}=0.014 \mathrm{eV}$ para $V_{a}=0 \mathrm{~V}, \Delta \Phi_{2}=0.021 \mathrm{eV}$ para $\left|V_{a}\right|=1 \mathrm{~V}, \Delta \Phi_{3}=0.036 \mathrm{eV} \mathrm{para}\left|V_{a}\right|=10 \mathrm{~V}(\mathrm{Ver}$ Ecuación I.31)

\section{Estudio de la capacidad}

Como ya hemos mencionado con anterioridad (Capítulo I, Sección 2), el diodo Schottky presenta una capacidad variable con la tensión aplicada, cuyo origen físico está asociado a la zona de vaciamiento próxima al contacto rectificador. El valor de la capacidad es inversamente proporcional a la anchura de dicha zona de vaciamiento ( $W$, Ecuación I.10), y su comportamiento puede asimilarse al de un condensador plano paralelo con permitividad igual a la del semiconductor y separación entre placas $W$.

En nuestro caso, además de emplear esta expresión teórica, podemos evaluar de manera directa mediante el método de Monte Carlo el valor de la capacidad de la unión metal- 
semiconductor, determinando la variación de la carga almacenada en el conjunto del dispositivo para incrementos pequeños de la tensión aplicada (de $0.05 \mathrm{~V}$ ) sobre un punto de polarización estacionario.

En la Figura III.23.a representamos el incremento de la carga almacenada en la zona de vaciamiento en función de la posición para distintas polarizaciones. Como puede observarse, el incremento de carga presenta un máximo que se aleja del contacto Schottky a medida que aumentamos la polarización. Este máximo se corresponde con el inicio de la zona de vaciamiento que, como sabemos, tiende a alejarse del contacto cuanto mayor es el voltaje en inversa aplicado, $V_{a}$, (ver Figura III.10.c). Por otra parte, el área asociada a las diferentes curvas (que corresponde al incremento de carga total) tiende a disminuir según se aumenta el voltaje en inversa, lo cual es indicativo de una progresiva menor sensibilidad del dispositivo ante pequeñas variaciones de voltaje.
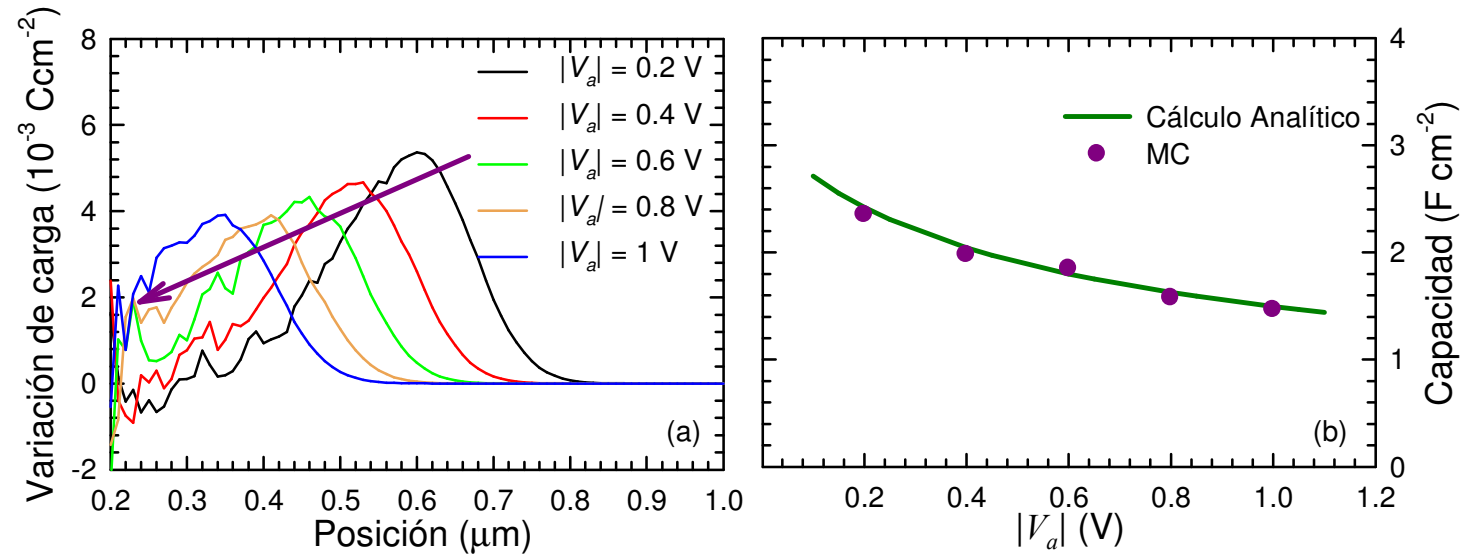

Figura III.23.a. Variación de la carga para incrementos de voltaje de $0.05 \mathrm{~V}$ en función de la posición para la estructura Si-n/ZrSi $i_{2}$.b. Capacidad de dicha estructura. Cálculo teórico (línea) y resultados Monte Carlo (símbolos).

En la Figura III.23.b, mostramos el valor de la capacidad de la estructura calculada de manera analítica a través de la Ecuación I.9, y la comparación con los resultados obtenidos mediante el método de Monte Carlo. Podemos apreciar el excelente acuerdo existente entre ambos cálculos. Debemos señalar que el cálculo Monte Carlo realizado corresponde a una situación cuasiestacionaria de baja frecuencia. Como puede apreciarse en la figura, a medida que aumenta la tensión aplicada en inversa, el valor de la capacidad tiende a disminuir. Esta disminución está asociada a la reducción de la variación de carga con $V_{a}$ previamente comentada [Pascual, 2007]; desde el punto de vista del formalismo asociado a un condensador, 
el aumento de la anchura equivalente entre placas es el responsable de los valores progresivamente menores de la capacidad de la unión rectificadora.

\section{III.4.b. Estudio del efecto de la variación de la temperatura y de la altura de la} barrera

Como ya hemos mencionado, uno de los parámetros de interés a considerar en nuestro estudio es la variación de la temperatura de operación, ya que el análisis de la dependencia de la corriente con la temperatura es empleado con frecuencia en las medidas experimentales para determinar la altura de la barrera de los contactos fabricados. Por otra parte, la altura de la barrera es otro parámetro fundamental en el diseño de dispositivos Schottky por su gran influencia en el transporte de corriente. Sobre este último parámetro ya se han comentado algunos detalles brevemente en el Capítulo II, Sección 6 para el caso de una barrera triangular. En esta sección vamos a estudiar con más detalle el efecto de ambas magnitudes (temperatura y altura de barrera) en el comportamiento del diodo Schottky de tipo $n$.

Inicialmente hemos realizado simulaciones para tres valores de temperatura distintos: $279 \mathrm{~K}, 300 \mathrm{~K}$ y $343 \mathrm{~K}$, para las cuales disponemos de medidas experimentales para tensiones aplicadas en un rango de inversa. También hemos considerado tres estructuras con diferentes Siliciuros, $\mathrm{ZrSi}_{2}$ (que corresponde a los resultados que acabamos de presentar), $\mathrm{RhSi}$ y PtSi, en los cuales la altura de la barrera Schottky toma valores mayores (véase Tabla III.1). Con el fin de mantener idénticas condiciones de simulación a las ya empleadas para el $\mathrm{ZrSi}_{2}$, para las estructuras de $\mathrm{RhSi}$ y PtSi hemos considerado los mismos valores de la constante de Richardson, $\mathrm{A}^{*}=200 \mathrm{~A} \mathrm{~cm}^{-2} \mathrm{~K}^{-2}$, incluyendo el efecto de cargas imagen en todos los casos.

A medida que se incrementa la altura de la barrera, el campo eléctrico aumenta y provoca un mayor vaciamiento de los portadores en las inmediaciones del contacto. Esto, desde el punto de vista de la simulación Monte Carlo, supone unas condiciones mucho más exigentes pues requiere emplear un gradiente de valores de EPEe más elevado (de la zona $n^{+}$hasta el contacto), como puede comprobarse en la Tabla III.3. Nos encontramos así en unas condiciones aún más adversas para el cálculo de la corriente, haciéndose de nuevo necesaria la utilización del realce estadístico para la simulación [Martín, 1996], [Sun, 2003]. 


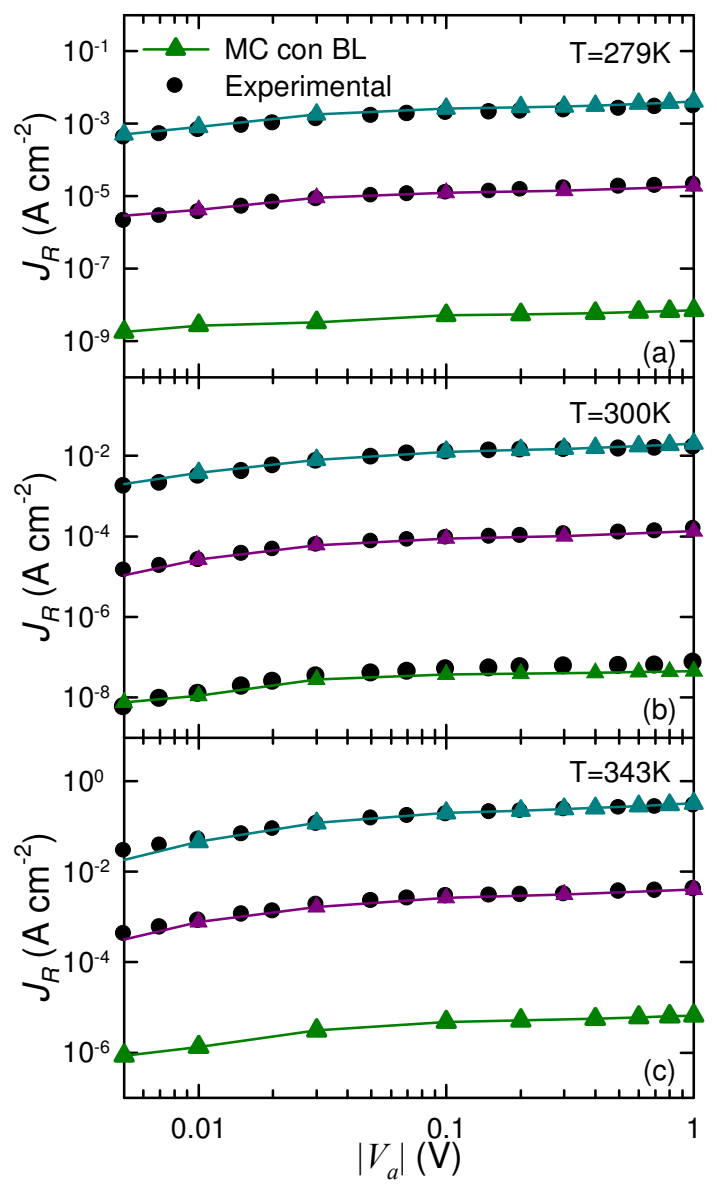

Figura III.24. Características corriente-tensión de las estructuras de Si-n/ZrSi, Si-n/RhSi y Si-n/PtSi para voltajes en inversa aplicados a distintas temperaturas: a. 279 K, .b. $300 \mathrm{~K}$ y.c. $343 \mathrm{~K}$. Los círculos muestran las medidas experimentales y las líneas con triángulos los resultados del método Monte Carlo. En todas las simulaciones se ha considerado una constante de Richardson de $200 \mathrm{~A} \mathrm{~cm}^{-2} \mathrm{~K}^{-2}$ y el efecto de las cargas imagen.

En la Figura III.24 mostramos las características corriente-tensión para las tres alturas de barrera y las tres temperaturas consideradas. En primer lugar debemos destacar el excelente acuerdo obtenido de los resultados de la simulación Monte Carlo con las medidas experimentales. Además, apoyándonos en esta gran concordancia, también presentamos los resultados Monte Carlo para 279 K y $343 \mathrm{~K}$, aunque en el caso de la estructura PtSi disponemos de medidas experimentales únicamente para $300 \mathrm{~K}$, pudiendo así evaluar dichas características corriente-tensión. Con respecto a la variación de la altura de la barrera, según ésta es mayor, la corriente disminuye notablemente (recordemos que la estructura con mayor altura de barrera Schottky es la de Siliciuro de Platino, y como puede observarse en cada una de las gráficas, es la que menores valores de corriente presenta). Esto se debe a que según aumenta la altura de la 
barrera las energías incidentes necesarias para efectuar con éxito la inyección son mayores, correspondiendo a una zona de menor ocupación en el metal; en consecuencia, el número de portadores inyectados es menor, lo que a su vez reduce la corriente. El hecho de haberse empleado en todos los casos los mismos valores de la constante de Richardson confirma la validez del modelo presentado en esta Memoria.

En cuanto a la contribución de las componentes de corriente (no mostradas en las gráficas) en cada una de las características simuladas, su comportamiento es totalmente análogo al mostrado en la Figura III.13.b para la barrera más baja de $\mathrm{ZrSi}_{2}$, con la particularidad de que en el caso de los contactos de RhSi y PtSi las dos componentes de absorción sufren una disminución al aumentar la tensión más acusada, debido a la mayor altura de barrera que deben superar los portadores en semiconductor para alcanzar el metal en comparación con el diodo de $\mathrm{ZrSi}_{2}$ previamente estudiado.

Vamos a continuación a estudiar con más detalle el efecto de variar la temperatura de operación del dispositivo. En primer lugar debemos señalar que al variar la temperatura se produce un cambio del valor del nivel de Fermi del semiconductor y por tanto se modifica el valor del potencial de contacto $V_{b i}$ (ver Tabla III.4). Recordemos además que la corriente inyectada depende de forma explícita con la temperatura (Ecuaciones I.30 y I.35) y también de manera implícita a través de las funciones de ocupación y del coeficiente de transmisión.

\begin{tabular}{|c|c|c|c||}
\hline $\mathbf{T}(\mathbf{K})$ & $E_{F}\left(\boldsymbol{n}^{+}\right)$ & $E_{F}(n)$ & $V_{b i}$ \\
\hline \hline $\mathbf{1 5 3}$ & 0.0611 & 0.1053 & 0.4447 \\
\hline \hline $\mathbf{1 9 1}$ & 0.0817 & 0.1370 & 0.4130 \\
\hline $\mathbf{2 2 9}$ & 0.1030 & 0.1690 & 0.3810 \\
\hline $\mathbf{2 7 9}$ & 0.1330 & 0.2137 & 0.3363 \\
\hline $\mathbf{3 0 0}$ & 0.1461 & 0.2321 & 0.3179 \\
\hline $\mathbf{3 4 3}$ & 0.1720 & 0.2720 & 0.2780 \\
\hline
\end{tabular}

Tabla III.4. Variación del nivel de Fermi de la región $n^{+}$y de la $n$ así como el potencial de contacto $V_{\text {bi }}$ para distintos valores de la temperatura en la estructura $\mathrm{Si}-\mathrm{n} / \mathrm{ZrSi}{ }_{2}$.

Para analizar la dependencia de la corriente con la temperatura de manera más precisa, hemos sometido a la estructura $\mathrm{Si}-\mathrm{n} / \mathrm{ZrSi}_{2}$ a un rango más amplio de variación de temperaturas (desde 153 hasta $343 \mathrm{~K}$ ) en condiciones de inversa para las tensiones de $0.1 \mathrm{~V}$ y $1 \mathrm{~V}$ en módulo. 
En la Figura III.25 mostramos la variación de la función de distribución de Fermi en el metal con la temperatura, el coeficiente de transmisión y la carga inyectada en el diodo para una tensión en inversa de 1 V. Como podemos observar (Figura III.25.a), al aumentar la temperatura la función de ocupación presenta una variación más suave, con lo que para idénticas energías por encima del nivel de Fermi del metal (las involucradas principalmente en la inyección), la función de ocupación presenta un valor más alto a medida que aumenta la temperatura. Sin embargo, el coeficiente de transmisión (Figura III.25.b) en el rango de energías inferiores a la altura de la barrera (correspondiente a procesos túnel) tiende a disminuir ligeramente. Aún así, esta reducción no es suficiente para compensar el notable aumento de portadores susceptibles de ser inyectados, con lo que el efecto final es el importante incremento de la carga inyectada que se observa en la Figura III.25.c.
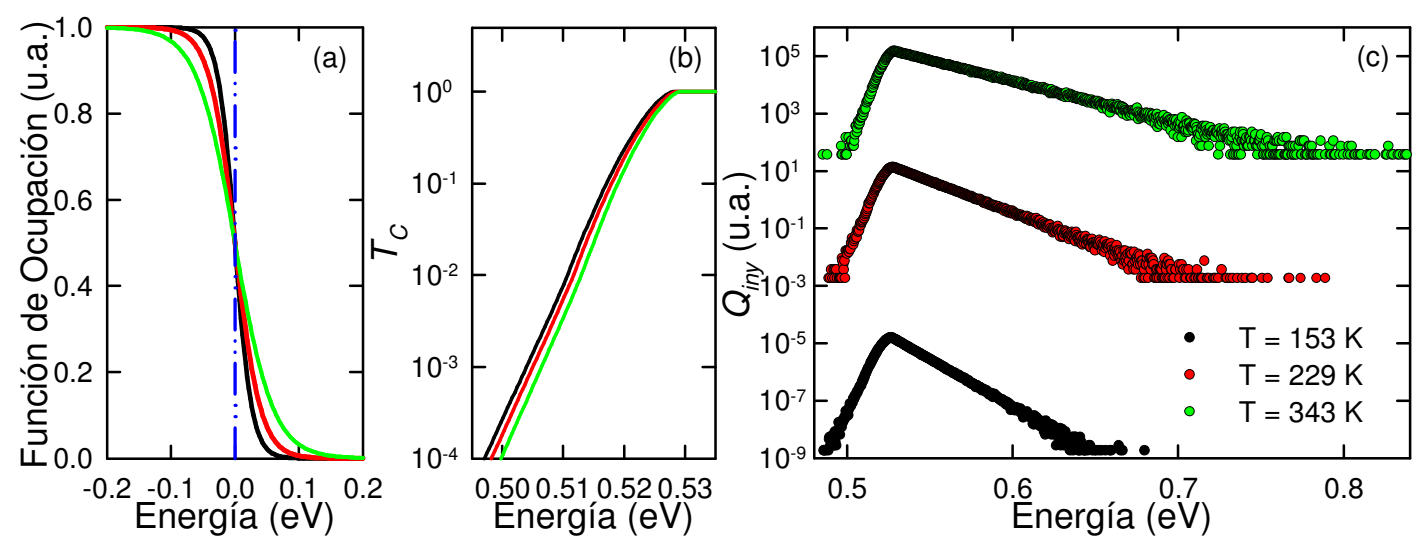

Figura III.25. Variación con la temperatura de la función de ocupación de los electrones en el metal a $153 \mathrm{~K}, 229 \mathrm{~K}$ y 343 K para tensión en inversa aplicada de 1 V para la estructura $\mathrm{Si}-\mathrm{n} / \mathrm{ZrSi}_{2}$.

Entre los métodos experimentales para la determinación de la altura de las barreras fabricadas destaca el llamado método de la energía de activación o diagrama de Arrhenius [Dubois, 2004], consistente en la representación del logaritmo neperiano de la corriente (dividida por la temperatura al cuadrado) frente al inverso de la temperatura. En la Figura III.26 mostramos el diagrama de Arrhenius con los resultados Monte Carlo obtenidos para dos polarizaciones negativas, $0.1 \mathrm{~V}$ (Figura III.26.a) y $1 \mathrm{~V}$ (Figura III.26.b), para el contacto de $\mathrm{ZrSi}_{2}$ (altura de barrera $0.55 \mathrm{eV}$, con barrier lowering esta altura es aproximadamente de $0.53 \mathrm{eV})$. 
Junto a los resultados de la simulación hemos incluido los valores proporcionados por la Ecuación I.23. Dividiendo ambos miembros de la ecuación por el cuadrado de la temperatura y tomando logaritmos neperianos obtenemos:

$\ln \left(\left|\frac{J}{T^{2}}\right|\right)=\ln A^{*}-\frac{q \Phi_{B n}}{K_{B} T}+\ln \left[\exp \left(\frac{q V_{a}}{K_{B} T}\right)-1\right]$
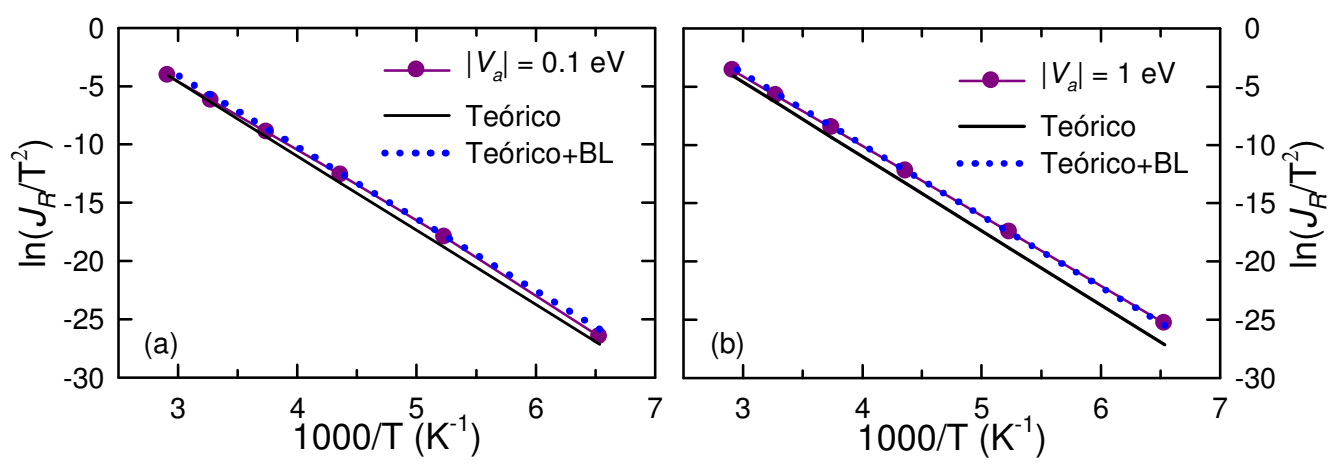

Figura III.26. Diagrama de Arrhenius. Variación de la corriente con la temperatura para la estructura Si-n/ZrSi ${ }_{2}$

sometida a una tensión en inversa de .a. $0.1 \mathrm{Vy}$.b. $1 \mathrm{~V}$ junto con el valor teórico con y sin barrier lowering.

Para valores de tensión en inversa apreciables esta expresión corresponde a una recta en el diagrama de Arrhenius, cuya pendiente proporciona la altura de la barrera. Los valores obtenidos mediante la Ecuación III.3 se muestran en las gráficas (líneas continuas negras). Como puede observarse, el ajuste con los resultados Monte Carlo es relativamente razonable para la polarización inferior. Sin embargo, a polarizaciones mayores (Figura III.26.b) la discrepancia es significativa, especialmente a temperaturas bajas. El origen de este desacuerdo se debe a que la Ecuación III.3 no incluye el efecto del barrier lowering, que es más acusado a baja temperatura y polarización elevada. Incorporándolo al modelo analítico obtendríamos:

$$
\ln \left(\left|\frac{J}{T^{2}}\right|\right)=\ln A^{*}-\frac{q \Phi_{B n}}{K_{B} T}+\frac{q \Delta \Phi}{K_{B} T}+\ln \left[\exp \left(\frac{q V_{a}}{K_{B} T}\right)-1\right]
$$

Los resultados correspondientes se muestran en las gráficas (líneas punteadas azules). Como puede comprobarse, el ajuste del modelo teórico con los resultados Monte Carlo es muy bueno, por lo que podemos concluir que en este caso (barreras relativamente elevadas y tensiones en inversa aplicadas de hasta $1 \mathrm{~V}$ ), modelos sencillos para el cálculo de la corriente, como los presentados en el Capítulo I, pueden proporcionar una estimación razonable de la 
altura de la barrera, a pesar de no ser adecuados para un análisis detallado del transporte a través de la misma.

El modelo de Monte Carlo nos permite extender el uso del diagrama de Arrhenius al estudio de la dependencia con la temperatura de las cuatro componentes de corriente (Figura III.27).

Comprobamos que a medida que aumentamos la polarización en inversa, la componente de corriente por inyección túnel aumenta significativamente, como ya confirmamos con anterioridad (véase Figura III.13), alcanzando valores cercanos (aunque aún inferiores) a los de la componente de inyección termoiónica para la polarización negativa de $1 \mathrm{~V}$. Además, para todas las polarizaciones en inversa, la inyección túnel se ve enormemente favorecida por la disminución de la temperatura como consecuencia del estrechamiento de la barrera que se produce al variar la posición de la banda de conducción y mantenerse constante la altura de la barrera.
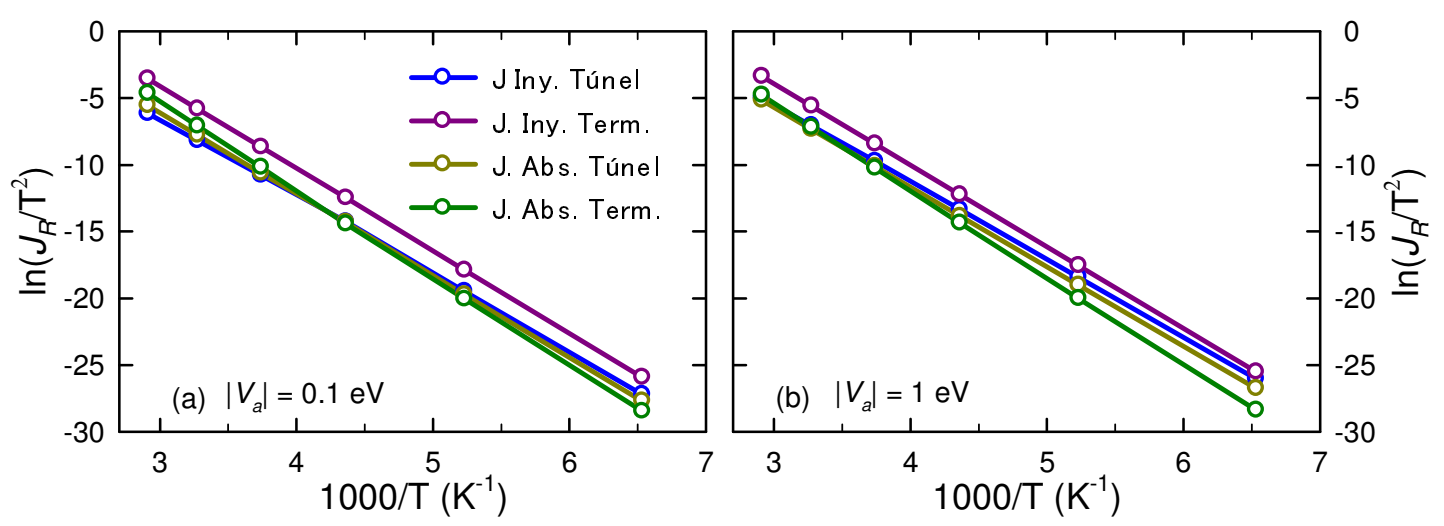

Figura III.27. Diagrama de Arrhenius. Variación de las componentes con la temperatura para la estructura $\mathrm{Si-n/ZrSi} i_{2}$ sometida a una tensión en inversa de a. $0.1 \mathrm{Vy}$.b. $1 \mathrm{~V}$.

Debemos destacar también el importante aumento con la temperatura de la componente de absorción termoiónica, relacionado directamente con el menor potencial $V_{b i}$ (ver Tabla III.4), lo que favorece el tránsito de portadores del semiconductor al metal.

Como conclusión principal del estudio de los diodos de tipo n, podemos afirmar que el método de Monte Carlo reproduce fielmente las medidas experimentales con una constante de 
Richardson de $200 \mathrm{~A} \mathrm{~cm}^{-2} \mathrm{~K}^{-2}$, incluyendo el efecto de reducción de la barrera debido a las cargas imagen.

Hemos realizado el análisis del transporte de los electrones en el interior del dispositivo en profundidad, comprobando el carácter cuasibalístico de los mismos en las mallas próximas al contacto Schottky. Observamos que a medida que aumenta la altura de la barrera, la densidad de corriente disminuye. Por otra parte, el aumento de la temperatura favorece notablemente el aumento de la corriente. El estudio de la dependencia con la temperatura ha sido objeto de un análisis más minucioso a través de la información provista por el diagrama de Arrhenius, donde hemos comprobado el buen ajuste de los resultados obtenidos con el modelo teórico presentado.

\section{III.5. Diodo Schottky con semiconductor dopado tipo p}

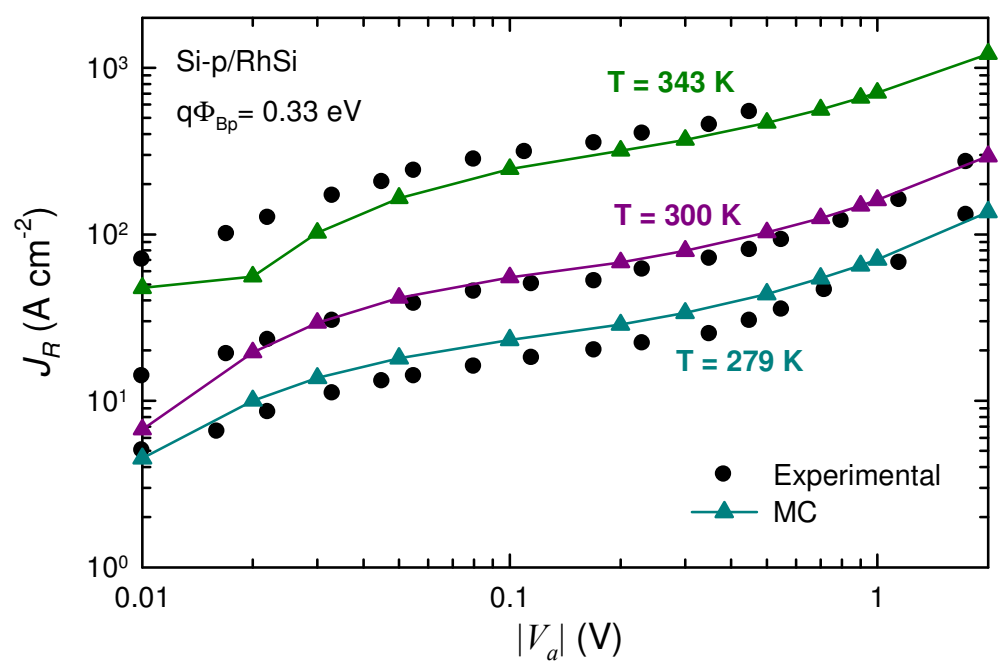

Figura III.28. Características corriente-tensión en inversa de la estructura Si-p/RhSi para tres temperaturas: 279K, $300 K$ y $343 \mathrm{~K}$. Los círculos negros muestran los resultados experimentales y los triángulos los resultados que ofrece nuestro simulador Monte Carlo con $A^{*}=60 \mathrm{Acm}^{-2} \mathrm{~K}^{-2}$, incluyendo Barrier Lowering o efecto de cargas imagen.

Una vez estudiado el comportamiento de los diodos tipo $n$, donde los electrones son los portadores involucrados en el transporte de carga, y aprovechando el carácter bipolar del simulador Monte Carlo unidimensional, hemos completado nuestro estudio de los diodos Schottky investigando el transporte de huecos en diodos tipo $p$. Hemos analizado una estructura con contacto rectificador de Siliciuro de Rodio $(\mathrm{Si}-\mathrm{p} / \mathrm{RhSi})$ con dopaje de la zona $p$ de valor $9 \cdot 10^{16} \mathrm{~cm}^{-3}$, más elevado que en el caso de los tipo $n$, y altura de barrera de $0.33 \mathrm{eV}$ 
aproximadamente, valores correspondientes a los dispositivos experimentales para los que disponemos de medidas [Andrews, 1970]. El hecho de que el semiconductor esté más dopado implicará, como comprobaremos, que la barrera sea notablemente más estrecha que en los dispositivos estudiados hasta ahora. Además la altura de barrera es la más baja de todas las analizadas en el caso de los diodos Schottky.

Es importante destacar el hecho de que al estar considerando huecos el valor de la constante de Richardson debe ser distinto al de electrones [Sze, 1981], [Crowell, 1965]. Para su determinación en este caso hemos seguido el mismo método empleado en el caso del diodo tipo $n$ que fue descrito en la Sección 2.A.1 del presente capítulo. Así, hemos estimado el valor de $\mathbf{A}^{*}$ (incluyendo el barrier lowering) en $60 \mathbf{A ~ c m}^{-2} \mathbf{K}^{-2}$ para el caso de huecos. En la Figura
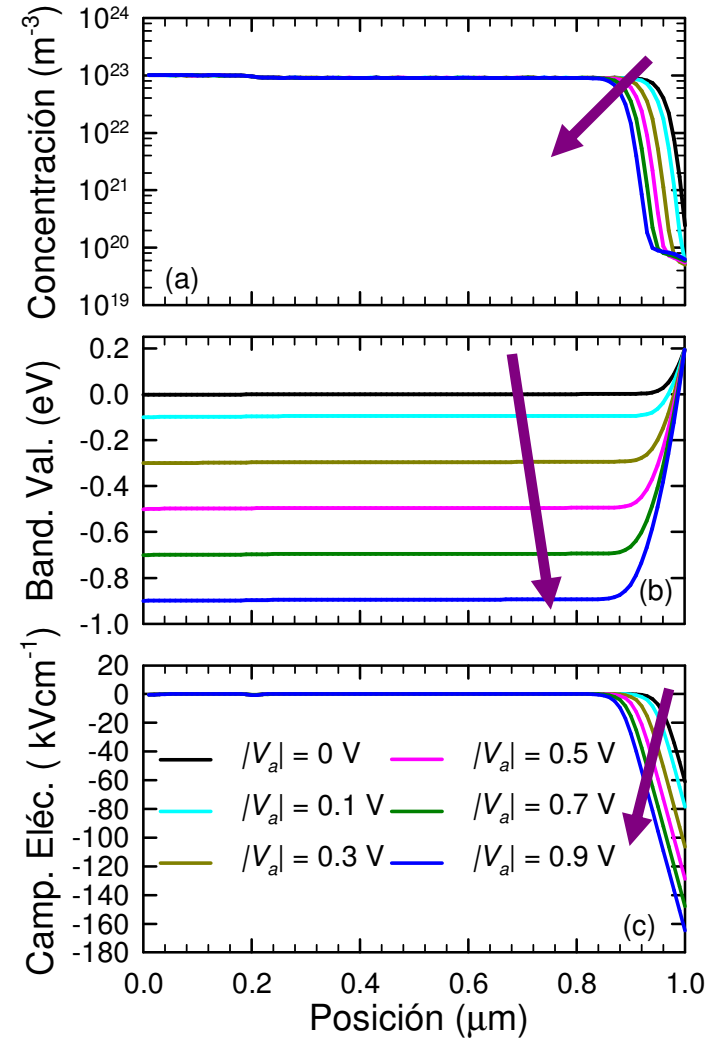

Figura III.29. Diodo de contacto Si-p/RhSi para temperatura de 300 K. Perfiles de las siguientes magnitudes bajo diferentes valores de polarización en inversa: .a. Concentración .b. Banda de valencia .c. Campo Eléctrico.
III.28 mostramos la característica corriente-tensión para diversas temperaturas. Como puede observarse, existe muy buen acuerdo entre los resultados que ofrece el método Monte Carlo para la corriente total y las medidas experimentales

disponibles.Antes de efectuar un análisis detallado de la dependencia con la temperatura, vamos a examinar el perfil de concentración, de potencial y de campo eléctrico para la temperatura de 300 K (Figura III.29).

Tal y como nos muestra la Figura III.29.a, la concentración permanece prácticamente constante a lo largo de todo el dispositivo, debido a la escasa diferencia entre los dopajes de la región $p^{+}$y $p$ (véase Tabla III.1), encontrándonos lógicamente con una zona de vaciamiento $W$ de menor anchura que en las situaciones anteriores. 
Recordemos que la anchura, $W$, de esta región (Ecuación I.2 para diodos tipo $n$, la expresión es análoga para los tipo $p$ ) es inversamente proporcional a la raíz cuadrada de la concentración de impurezas aceptadoras. Como consecuencia de este efecto, el espesor de la barrera de potencial de partida es menor que en los casos anteriores para diodos tipo $n$, de menor dopaje (Figura III.29.b), produciéndose además un estrechamiento adicional por efecto de la polarización en inversa aplicada. La región de campos elevados se encuentra también localizada en una zona muy cercana al contacto (Figura III.29.c), alcanzando valores mayores que para el resto de diodos simulados, debido a la rápida variación del perfil del potencial en esa zona.

Para una mejor comprensión del comportamiento de la corriente total en función de la tensión en inversa aplicada mostramos en la Figura III.30 la variación de las cuatro componentes de corriente (incluido el efecto de cargas imagen), para la temperatura de $300 \mathrm{~K}$ (para $279 \mathrm{~K}$ y $343 \mathrm{~K}$ las tendencias observadas son totalmente análogas).

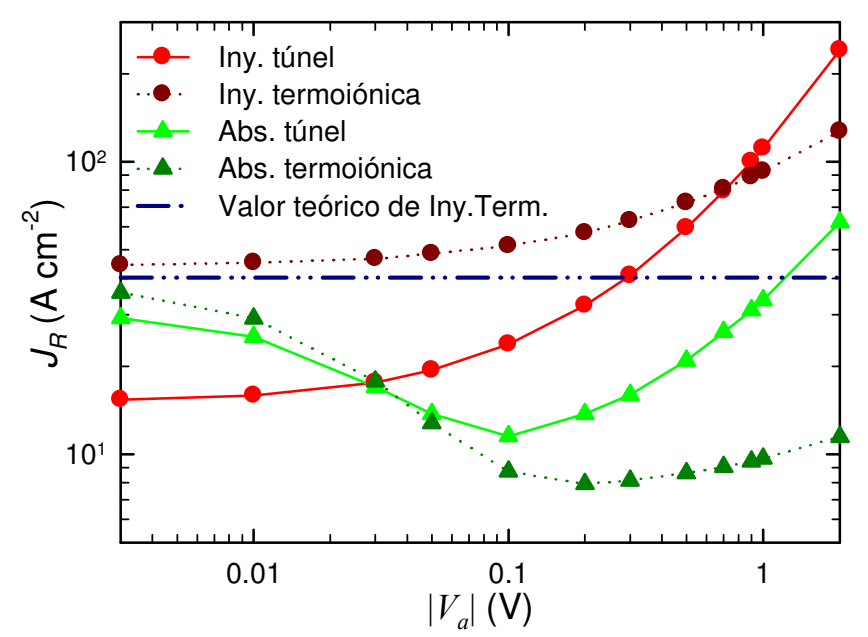

Figura III.30. Componentes de corriente para $300 \mathrm{~K}$ de temperatura para la estructura Si-p/RhSi.

Debemos destacar que la corriente de inyección túnel es muy elevada (en comparación con las de las estructuras tipo $n$ estudiadas) incluso a débiles tensiones en inversa, ya que este proceso se ve favorecido tanto por el alto dopaje (y la consiguiente menor anchura) como por la menor altura de la barrera [Sze, 1981]. Para materiales muy dopados, el efecto túnel es por tanto especialmente relevante. También vemos en la Figura III.30 cómo las dos componentes de absorción son también más elevadas en este caso. El aumento de las componentes de absorción con $V_{a}$ para tensiones superiores a $0.1 \mathrm{~V}$ en inversa es debido a un proceso autoconsistente: a 
causa del elevado número de portadores inyectados, existen más partículas en la región cercana al contacto susceptibles de ser absorbidas, por lo que estas componentes se ven también favorecidas.
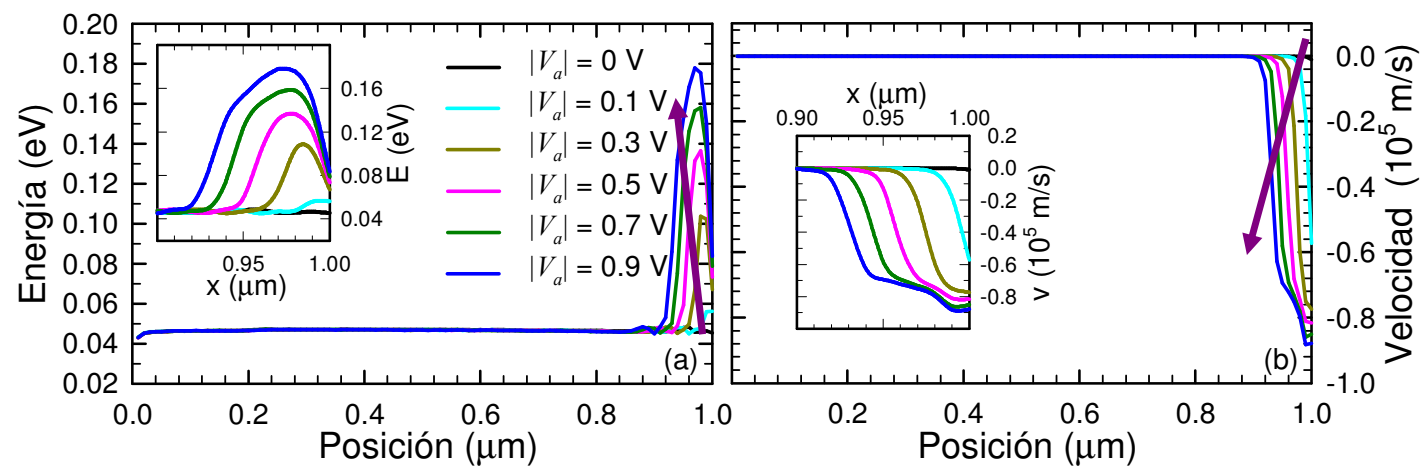

Figura III.31. Diodo de contacto Si-p/RhSi. Perfiles de las siguientes magnitudes bajo diferentes valores de polarización en inversa: .a. Energía .b. Velocidad. En los recuadros interiores correspondientes a cada figura se muestra una ampliación de la región de interés, es decir, la cercana al contacto Schottky.

Los perfiles de energía y velocidad nos proporcionan también información interesante (Figura III.31). Observamos cómo a medida que aumenta la tensión en inversa aplicada, la energía en la región cercana al contacto (véase el recuadro interior de la Figura III.31.a) también toma valores mayores, mientras que en el resto del dispositivo la energía es muy próxima a la térmica. Con la velocidad sucede algo similar, los portadores alcanzan grandes velocidades en valor absoluto en la región cercana al contacto y en el resto del dispositivo su velocidad es reducida. La velocidad es negativa ya que el campo también lo es, arrastrando a los huecos de derecha a izquierda. En general, la situación es, de forma cualitativa, análoga al caso de los electrones (Figura III.15. a y b). Para ambas magnitudes las regiones de valores elevados se encuentran de nuevo muy localizadas en las proximidades del contacto.

En cuanto a la ocupación de las subbandas de valencia de huecos ligeros y pesados, tal y como vemos en la Figura III.32, permanecen prácticamente constantes a lo largo de todo el dispositivo y no es hasta la región más cercana al contacto donde sufren modificaciones, debido a que se alcanzan valores de campo eléctrico muy elevados, como comprobamos en la Figura III.29.c.

La subbanda de huecos pesados posee mayor probabilidad de ocupación que la de ligeros, en torno a un $79 \%$ para la primera y un $21 \%$ para la segunda. Esta situación es 
totalmente consecuente con la variación de la densidad de ocupación de los valles con el campo eléctrico en Silicio material tipo $p$ (véase Agpéndice, Figura 5). Sin embargo, en la zona de campos mayores (región próxima al contacto) la subbanda de pesados alcanza un máximo del $88 \%$ aproximadamente y la de ligeros un mínimo del $12 \%$. Sendos máximos y mínimos se alejan del contacto Schottky a medida que aumenta la polarización aplicada. La aparición del máximo y mínimo en las bandas es debida a fenómenos de no equilibrio en el diodo consecuencia directa del campo elevado que se encuentran los portadores al ser inyectados en el interior del dispositivo (Figura III.29.c), y que ya hemos comentado en profundidad en el caso de los diodos tipo $n$.

Estudio del efecto de la variación de la temperatura. Diagrama de Arrhenius.

Como ya hicimos en el caso de electrones, dado su interés desde el punto de vista de la caracterización experimental de los contactos, vamos a analizar en profundidad la dependencia de la densidad de corriente con la temperatura, con el valor añadido de que en el presente caso la menor altura y anchura de la

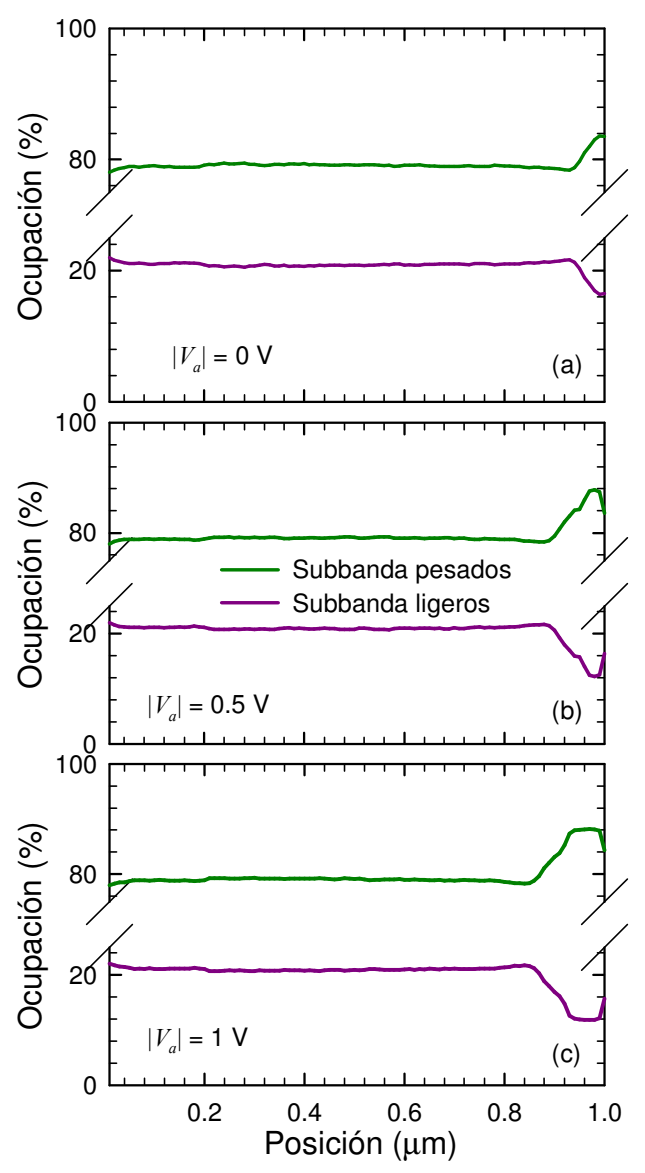

Figura III.32. Ocupación de las subbandas de valencia de la estructura RhSi/Si-p en función de la posición para distintas polarizaciones aplicadas en inversa. barrera favorecerá la influencia de ciertos efectos en el comportamiento de la corriente frente a la temperatura.

La Figura III.33 muestra los resultados Monte Carlo en el diagrama de Arrhenius para las tensiones de inversa $V_{a}=0.1 \mathrm{~V}$ (símbolos púrpura) y $V_{a}=1 \mathrm{~V}$ (símbolos verdes) junto a los resultados analíticos proporcionados por las Ecuaciones III.2 y III.3. 


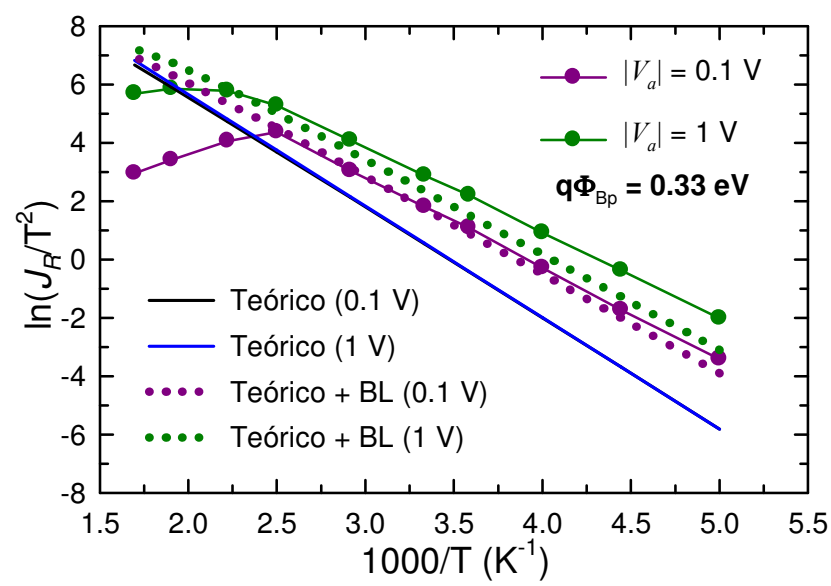

Figura III.33. Diagrama de Arrhenius. Dependencia de la densidad de corriente con la temperatura para la estructura Si-p/RhSi sometida a polarización en inversa de 0.1 Vy $1 \mathrm{~V}$. También se muestra la variación de la expresión teórica con y sin barrier lowering (Ecuaciones III.2 y III. 3 respectivamente).

A medida que aumenta la polarización en inversa aplicada también lo hace la densidad de corriente, como podemos ver en la figura correspondiente a los resultados Monte Carlo (Figura III.28). Al comparar dichos valores con el modelo teórico sin considerar la reducción de la barrera (líneas continuas) encontramos gran discrepancia (mayor que en el caso de los electrones, véase la Figura III.26). Sin embargo, a diferencia de lo que observamos en el caso de electrones, cuando incluimos el efecto del barrier lowering (líneas punteadas) la predicción teórica sigue sin ser acertada, sobre todo en el caso de mayor tensión en inversa aplicada (1 V).

Podemos distinguir dos regiones de temperaturas bien diferenciadas. Una primera zona (por debajo de $400 \mathrm{~K}$ para $V_{a}=0.1 \mathrm{~V}$ y $450 \mathrm{~K}$ para $V_{a}=1 \mathrm{~V}$, en la gráfica valores superiores a $2.50 \mathrm{~K}^{-1}$ y $2.22 \mathrm{~K}^{-1}$ respectivamente), donde a pesar de las discrepancias cuantitativas ya mencionadas, los resultados Monte Carlo y las predicciones teóricas presentan la misma tendencia cualitativa (reducción exponencial de $\mathrm{J} / \mathrm{T}^{2}$ con el inverso de $\mathrm{T}$ ). Una segunda zona, a temperaturas elevadas, donde los resultados MC muestran un comportamiento aparentemente anómalo en comparación con los modelos de la Ecuaciones III.2 y III.3, ya que se advierte una reducción de $\mathrm{J} / \mathrm{T}^{2}$ a medida que se aumenta $\mathrm{T}$, presentando el diagrama de Arrhenius un máximo para $400 \mathrm{~K}(0.1 \mathrm{~V})$ y $525 \mathrm{~K}(1 \mathrm{~V})$ aproximadamente, es decir, observándose un desplazamiento del máximo a temperaturas superiores a medida que aumentamos la polarización en inversa [Pascual, 2007]. Dicho comportamiento (presencia de un máximo y reducción de $\mathrm{J} / \mathrm{T}^{2}$ con $\mathrm{T}$ ) ha sido observado de manera experimental para valores de altura de 
barrera bajos por parte de otros autores [Dubois, 2004]. Para dar una explicación a este fenómeno, debemos recurrir al análisis de las magnitudes internas del dispositivo.

\begin{tabular}{|c||c||c|c||}
\hline $\mathbf{T}(\mathbf{K})$ & $E_{F}\left(p^{+}\right)$ & $E_{F}(p)$ & $V_{b i}$ \\
\hline \hline $\mathbf{2 0 0}$ & 0.0080 & 0.0082 & 0.2497 \\
\hline \hline $\mathbf{2 2 5}$ & 0.0094 & 0.0096 & 0.2361 \\
\hline \hline $\mathbf{2 5 0}$ & 0.1075 & 0.1101 & 0.2225 \\
\hline \hline $\mathbf{2 7 9}$ & 0.1240 & 0.1260 & 0.2040 \\
\hline $\mathbf{3 0 0}$ & 0.1368 & 0.1391 & 0.1930 \\
\hline $\mathbf{3 4 3}$ & 0.1620 & 0.1650 & 0.1680 \\
\hline $\mathbf{4 0 0}$ & 0.1969 & 0.2010 & 0.1331 \\
\hline $\mathbf{4 5 0}$ & 0.2284 & 0.2320 & 0.1019 \\
\hline \hline $\mathbf{5 2 5}$ & 0.2774 & 0.2815 & 0.0526 \\
\hline \hline $\mathbf{5 9 0}$ & 0.3194 & 0.3255 & 0.0106 \\
\hline
\end{tabular}

Tabla III.5. Variación del nivel de Fermi de la región $p^{+} y$ de la $p$ asi como el potencial de contacto $V_{b i}$ con la temperatura para la estructura Si-p/RhSi.

En la Figura III.34.a mostramos los valores del perfil de la banda de valencia para diferentes temperaturas para una tensión en inversa de $1 \mathrm{~V}$. Al aumentar la temperatura, el nivel de Fermi en el semiconductor se aleja de la banda de valencia y el potencial de contacto $V_{b i}$ disminuye (Tabla III.5). Para valores de temperatura bajos, existe una barrera Schottky bien definida, cuya resistencia es notablemente superior a la correspondiente al Silicio "masivo", es decir, la zona $p$ neutra. En este caso, el comportamiento de la estructura se encuentra gobernado principalmente por el contacto Schottky, lo cual se ve reflejado en el hecho de que el diagrama de Arrhenius sigue una dependencia exponencial bien definida (más adelante discutiremos las causas de las discrepancias con los modelos teóricos en ese régimen). Sin embargo, al aumentar la temperatura, la resistencia del contacto Schottky $\left(R_{S c h}\right)$ tiende a disminuir al reducirse la anchura de la zona de vaciamiento y el potencial $V_{b i}$, mientras que la resistencia en serie del Silicio aumenta [Sze, 1981]. Debido a la variación del nivel de Fermi (ver Tabla III.5), observamos una disminución notable de la zona de vaciamiento (Figura III.34.b), aumentando la concentración (en la región cercana al contacto) con la temperatura. De este modo, el efecto rectificador del contacto tiende a desaparecer, de manera que el comportamiento del 
dispositivo vendrá determinado principalmente por la resistencia en serie del sustrato de Silicio, como puede apreciarse claramente en la Figura III.34.a, donde para temperaturas elevadas la tensión aplicada cae de manera homogénea en todo el dispositivo y la concentración es prácticamente uniforme (Figura III.34.b). Para valores de temperatura superiores a los mostrados, el nivel de Fermi superaría a la altura de la barrera, produciéndose incluso una acumulación de portadores en las inmediaciones del contacto.

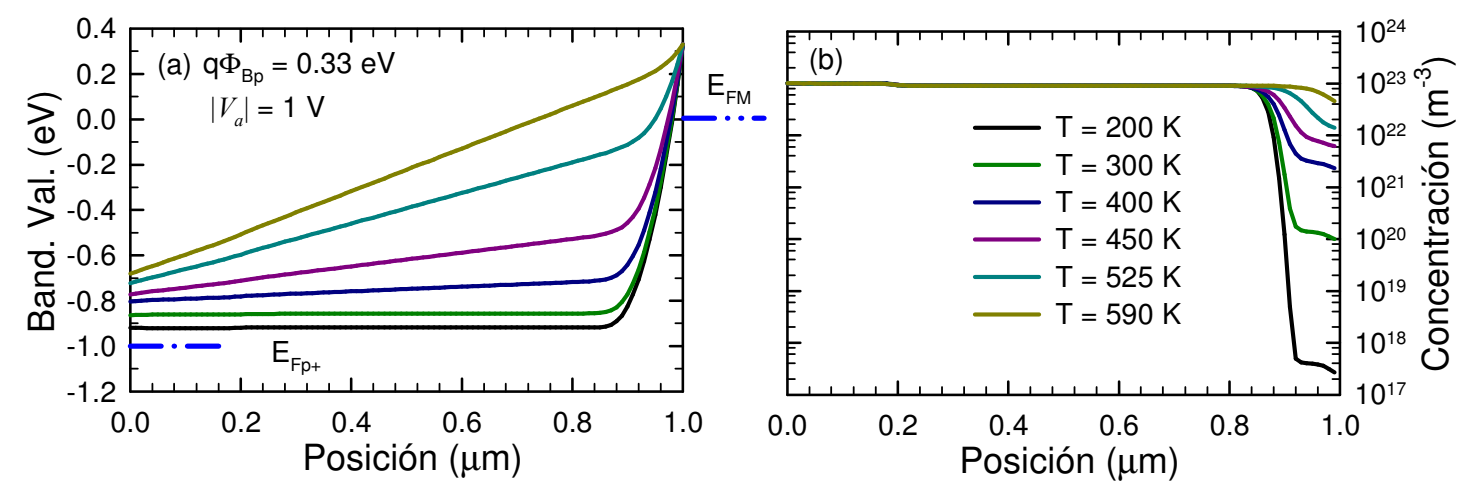

Figura III.34. Dependencia de la banda de valencia con la temperatura en la estructura Si-p/RhSi sometida a polarización en inversa de $1 \mathrm{~V}$.

A la vista del comportamiento del diagrama de Arrhenius y de los fenómenos que acabamos de describir, resulta interesante determinar cuál es la variación de las componentes de corriente en función de la temperatura. En la Figura III.35 mostramos los resultados obtenidos para $0.1 \mathrm{~V}$ y $1 \mathrm{~V}$ en inversa.

En la región de bajas temperaturas las componentes de inyección, tanto túnel como termoiónica, presentan la mayor contribución a la densidad de corriente total; nótese que la inyección túnel en el caso de $1 \mathrm{~V}$ en inversa aplicado es incluso mayor que la termoiónica en esta región de temperaturas, Figura III.35.b. Esto explica la discrepancia observada en el diagrama de Arrhenius (Figura III.33) en relación al valor proporcionado por los modelos teóricos, esencialmente termoiónicos y que por tanto no son capaces de describir de manera adecuada el incremento de la corriente proporcionado por el efecto túnel. Tanto la Ecuación III.3 como la III.4 predicen valores inferiores de $\mathrm{J} / \mathrm{T}^{2}$ en ese rango de temperaturas, ya que desprecian una componente de corriente cuya importancia queda reflejada en la Figura III.35. Queda por tanto patente la necesidad absoluta de incorporar los fenómenos de transporte túnel para describir adecuadamente el comportamiento de los diodos, especialmente en el caso de alturas bajas o moderadas. 

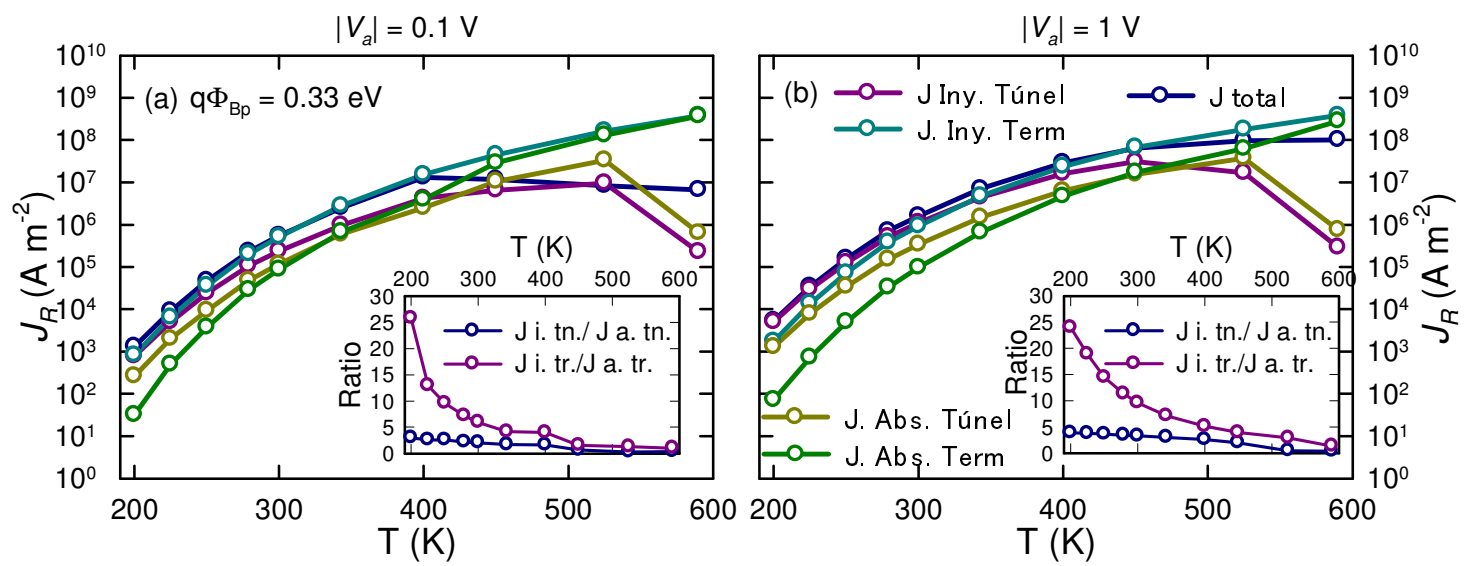

Figura III.35. Dependencia de la densidad de corriente y sus distintas componentes con la temperatura en la estructura Si-p/RhSi sometida a polarización en inversa de .a. 0.1 Vy .b. 1 V. En el recuadro interior de cada figura se muestra el cociente entre la densidad de corriente de inyección túnel con respecto a la de absorción túnel, así como el cociente entre la componente de inyección termoiónica respecto a la de absorción termoiónica.

A medida que aumentamos la temperatura para una misma energía incidente la barrera se hace más ancha (Figura III.34.a), por lo que la contribución a la corriente mediante el efecto túnel (tanto de inyección como de absorción) es cada vez menor, mientras que las componentes termoiónicas se ven en cambio favorecidas. De hecho, para valores de temperatura elevados (para los cuales la barrera Schottky tiende a desaparecer y el comportamiento se acerca al resistivo) las componentes túnel sufren una caída brusca, asociada al hecho de que el perfil de la banda (que ya no corresponde al de una barrera Schottky bien definida) impide en la práctica este tipo de procesos. La componente de absorción termoiónica es la que sufre un aumento comparativo mayor con la temperatura, como consecuencia de la variación de la banda de valencia, que hace que el paso de huecos del semiconductor al metal sea más favorable al ser menor $V_{b i}$ (Tabla III.5). En los recuadros interiores de las Figuras III.35.a y b podemos comprobar cómo a medida que la temperatura es mayor las componentes de absorción se equiparan a las de inyección, haciéndose este efecto más notable en el caso de las termoiónicas, cuya diferencia a temperaturas bajas es más elevada.

Podemos concluir, a la vista de los resultados mostrados, que la discrepancia de la predicción teórica con los resultados Monte Carlo en la región de débiles temperaturas (Figura III.34) es debida al efecto de la componente de inyección túnel, no considerada en el modelo teórico. En el caso de temperaturas elevadas la diferencia entre el comportamiento teórico y el de los resultados Monte Carlo es debida al mayor peso de la resistencia del sustrato de Silicio en 
comparación con la del contacto, que pierde las características inherentes a un contacto Schottky en el momento en que el nivel de Fermi supera la altura de la barrera.

Cabe preguntarse si, a la vista de las conclusiones obtenidas, este efecto anómalo en el diagrama de Arrhenius se vería acentuado al reducir aún más la altura de la barrera. En la Figura III.36 mostramos el diagrama de Arrhenius correspondiente a una estructura con el mismo dopaje que la anterior, pero con altura de barrera $q \Phi_{B p}=0.20 \mathrm{eV}$.

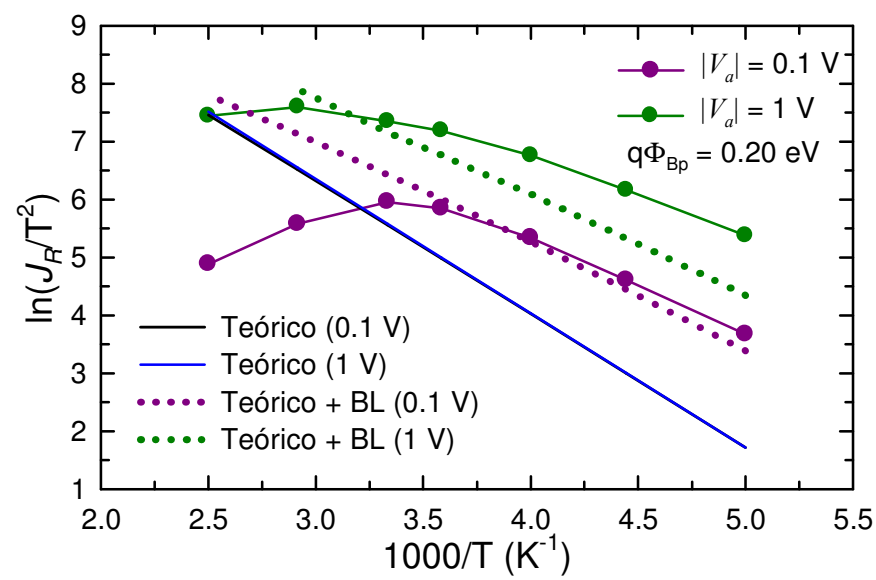

Figura III.36. Diagrama de Arrhenius. Dependencia de la densidad de corriente con la temperatura para la estructura con $q \Phi_{B p}=0.20 \mathrm{eV}$ sometida a polarización en inversa de $0.1 \mathrm{~V}$ y $1 \mathrm{~V}$ junto a los modelos teóricos con y sin barrier lowering (Ecuaciones III.2 y III.3 respectivamente).

Como podemos observar, además del aumento de corriente debido a la menor altura de barrera, el máximo en el diagrama de Arrhenius se desplaza hacia la derecha en comparación con la Figura III.33, es decir, tiene lugar para temperaturas inferiores. Observamos además que la predicción teórica se aparta en mayor medida de los resultados Monte Carlo que en los casos anteriores, debido a la mayor contribución de la componente de inyección túnel a la densidad de corriente total, favorecida por las características de la barrera Schottky en cuestión.

En conclusión, debemos subrayar que cuanto menor es la altura de la barrera mayor es la componente de inyección túnel en la región de bajas temperaturas, provocando mayor divergencia del modelo teórico (incluso incluyendo la reducción de la barrera por cargas imagen en el mismo). Además, el máximo de corriente en el diagrama de Arrhenius aparece a temperaturas menores a medida que se reducen tanto la polarización inversa aplicada como la altura de la barrera, ya que la resistencia asociada al contacto Schottky es menor. Por tanto, podemos concluir que en el caso de valores de altura de barrera bajos (como son las 
consideradas en el caso de los transistores SB-MOSFETs) la temperatura de operación puede afectar de manera significativa al comportamiento rectificador del contacto, por lo que el diseño de las características del mismo (altura de barrera, dopaje del semiconductor) debe efectuarse con sumo cuidado. Sin embargo, debemos señalar que (como veremos en el Capítulo V) en el caso de los SB-MOSFET este efecto se verá paliado por la modulación adicional del perfil de la barrera producto de la tensión de puerta aplicada, por lo que su utilización será perfectamente factible con el fin de lograr las figuras de mérito descritas en el ITRS, en particular en relación a la reducción de las resistencias asociadas a los contactos de fuente y drenador. 


\section{Bibliografía del Capítulo III}

Albella J. M. Y Martínez-Duartz J. M., "Fundamentos de electrónica física y microelectrónica”, Addison-Wesley / Universidad Autónoma de Madrid (1996)

Andrews J. M. and Lepselter M. P, "Reverse Current-Voltage Characteristics of Metal-Silicide Schottky Diodes", Solid State Electronics, 13, 1011 (1970)

Bethe H. A., "Theory of the boundary layer of crystal rectifiers", Mass. Inst. Technol. Radiat. Lab. Rep. 43-12 (1942)

Crowell C. R., "The Richardson constant for thermionic emission in Schottky barrier diodes", Solid State Electron. 8, 385 (1965)

Crowell C. R. y Sze S. M., "Current Transport in Metal-Semiconductor barriers", Solid-State Electron, 9, 1035 (1966)

Crowell C. R., "Richardson constant and tunneling effective mass for thermionic and thermionic-field emission in Schottky barrier diodes", Solid-State Electronics, 12, 55 (1969)

Dubois E. And Larrieu G., "Measurement of low Schottky barrier heights applied to metallic source/drain metal-oxide-semiconductor field effect transistors", J. Appl. Phys., 96, 729 (2004)

González T., "Análisis del ruido electrónico en materiales y dispositivos semiconductoras unipolares mediante el método de Monte Carlo", Tesis Doctoral, Universidad de Salamanca (1994)

González T., Pardo D., Varani L. and Reggiani L., "Microscopic analysis of electron noise in GaAs Schottky-barrier diodes", J. Appl. Phys., 82, 2349 (1997)

Jang M. et al., "Characterization of Erbium-Silicided Schottky Diode Junction” IEEE Elec. Dev. Letters, 26, 354 (2005)

Jian Y. L., "Schottky contact barrier height extraction by admittance measurement" J. Appl. Phys., 053705, 101 (2007) 
Larson J. M. and Snyder Y. P., "Overview and Status of Metal S/D Schottky- Barrier MOSFET Technology", IEEE Trans. Electron Dev., 53, 1048 (2006)

Martín M. J., "Estudio del transporte de carga en silicio mediante un método de Monte Carlo", Grado de Salamanca (1992)

Martín M. J., González T., Pardo D. y Velázquez J. E., "Monte Carlo Analysis of a Schottky Diode with an Automatic Space-Variable Charge Algorithm", Semicond. Sci. Technol. 11, 380 (1996)

Martín M. J., "Análisis del transporte de carga y de los fenómenos de ruido electrónico en estructuras $\mathrm{Si} / \mathrm{Si}_{1-\mathrm{x}} \mathrm{Ge}_{\mathrm{x}}$ bipolares", Tesis Doctoral, Universidad de Salamanca (1996b)

Matsuzawa, K. Uchida, K. Nishiyama, A. "Monte Carlo simulation of $50 \mathrm{~nm}$ devices with Schottky contact model" International Conference on Simulation of Semiconductor Processes and Devices, SISPAD '99, 35 (1999)

Matsuzawa K., Uchida K. and Nishiyama A., "Monte Carlo simulation of sub-0.1mm devices with Schottky contact model" IEICE Trans. Electron., E83-C, 1212 (2000)

Mott N. F., "Note on the contact between a metal and an insulator or semiconductor", Proc. Cambr. Phil. Soc. 34, 568 (1938)

Mönch W., "Electronic properties of semiconductor interfaces", Springer (2004)

Neamen D. A., "Semiconductor Physics and devices", Richard Irwin (1992)

Neudeck G. W., "El diodo PN de Unión", $2^{a}$ Edn. Addison-Wesley Iberoamericana, S. A., Delaware, USA (1993)

Pascual E., Rengel R., Martín M. J., "Microscopic modelling of reverse biased Schottky diodes: influence of non-equilibrium transport phenomena", Semicond. Sci. Technol., 22, 1003 (2007)

Pascual E., Rengel R., Martín M. J., "Monte Carlo analysis of quantum tunnelling and thermionic transport in a reverse biased Schottky diode", 2007 Spanish Conference on Electron Devices, IEEE Catalog 07EX1644, 108 (2007b)

Pérez S. and González T., "Current noise spectra of Schottky barrier diodes with electron traps in the active layer", J. Appl. Phys., 073708, 97 (2005)

Schottky W., Phys. Z., 15, 872 (1914)

Schottky W. and Spenke E., Wiss. Veroff. Siemens-Werken, 18, 225 (1939)

Shen M., Saikin S, Cheng M., "Monte Carlo Modelling of Spin Injection Through a Schottky Barrier and Spin Transport in a Semiconductor Quantum Well", Journal of Applied Physics, 96, 4319 (2004) 
Shenai K. et al., "Modeling and Characterization of Dopant Redistlributions in Metal and Silicide Contact" IEEE Trans. on Elec. Devices, ED-32, 793 (1985)

Shenai K. et al., "Current Transport Mechanisms in Atomically Abrupt Metal-Semiconductor Interfaces" IEEE Trans. on Elec. Devices, 35, 468 (1988)

Shiktorov P., Starikov E., Gruzinskis V., Perez S., González T., Reggiani L., Varani L. and Vaissiere J. C., "Monte Carlo simulation of Schottky diodes operating under TeraHertz cyclostationary conditions", IEEE Electron Dev. Lett., 25, 1, (2004)

Stratton R. "Difussion of hot and cold electrons in semiconductor barriers", Phys. Rev., 2002, (1962)

Sun L., Liu X. Y., Du G. y Han R. Q., "Monte Carlo Simulation of Schottky Contact with Direct Tunnelling Model”, Semiconductor Science and Technology, 18, 576 (2003)

Sze S. M., Crowell C. R. and Kahng D., "Photoelectric Determination of the Image Force Dielectric Constant for Hot Electrons in Schottky Barriers”, J. Appl. Phys., 35, 2534 (1964)

Sze S. M., Physics of Semiconductor Devices, $2^{\text {nd }}$ edn., Wiley, New York (1981)

Richardson O. W., "The Emission of Electricity from Hot Bodies", Longmans Green, Harlow, Essex (1921)

Rideout V. L., "A Review of the Theory, Technology and Applications of Metal-Semiconductor Rectifiers", Thin Solid Films, 48, 261 (1978) 


\section{Diodos Back-to-Back: estudio del transporte de carga en estructuras fabricadas con barreras Schottky bajas}

n el capítulo anterior, centrado en el estudio del diodo Schottky, hemos
conseguido un doble objetivo. Por un lado se ha comprobado la validez del modelo de inyección implementado en nuestro simulador Monte Carlo unidimensional mediante la comparación de nuestros resultados con datos experimentales (tanto diodos tipo $n$ como tipo $p$ ). Por otra parte, se ha realizado un exhaustivo análisis de la unión Schottky. Todo este trabajo nos sirve de base para el desarrollo del presente capítulo, en el que afrontamos el modelado de estructuras back-to-back fabricadas dentro del proyecto europeo METAMOS; en el caso de las estructuras tipo $n$ los datos experimentales nos han sido proporcionados por el grupo perteneciente a la Universidad Católica de Lovaina (Bélgica), mientras que el grupo del IEMN de Lille (Francia) nos ha facilitado los resultados para los diodos tipo $p$. Este tipo de dispositivos consisten en un sustrato de Silicio con dos contactos Schottky (en lugar de uno óhmico y uno Schottky, como en el caso del diodo estudiado hasta el momento). La principal peculiaridad de las estructuras simuladas es el reducido valor de la altura de las barreras Schottky (de $0.145 \mathrm{eV}$ en el caso tipo $p$ y de $0.325 \mathrm{eV}$ y $0.35 \mathrm{eV}$ en los tipo $n$ ), correspondientes al estado del arte más avanzado en la actualidad respecto a la búsqueda de nuevos materiales para los contactos. 
En este capítulo presentaremos inicialmente las principales propiedades de los diodos back-to-back; posteriormente realizaremos una comparativa de los resultados Monte Carlo con las medidas experimentales proporcionadas, además del estudio de las características del transporte de carga en el dispositivo.

\section{IV.1. Funcionamiento del diodo back-to-back}

Experimentalmente, se buscan valores de altura de barrera bajos con el fin de obtener una mejor respuesta en corriente en los dispositivos basados en contactos de barrera Schottky, particularmente en el caso de los transistores SB-MOSFET, de modo que puedan competir con los niveles de corriente proporcionados por los MOSFET convencionales. Para ello, en el caso de estructuras tipo $n$, se utilizan contactos Schottky basados en Siliciuros de tierras raras, como por ejemplo, Siliciuro de Erbio (ErSi) e Yterbio (YbSi), y Siliciuro de Platino (PtSi) para las tipo $p$, ya que son estos materiales con los que se consiguen las alturas de barrera más pequeñas [Duboz, 1989].

(a)

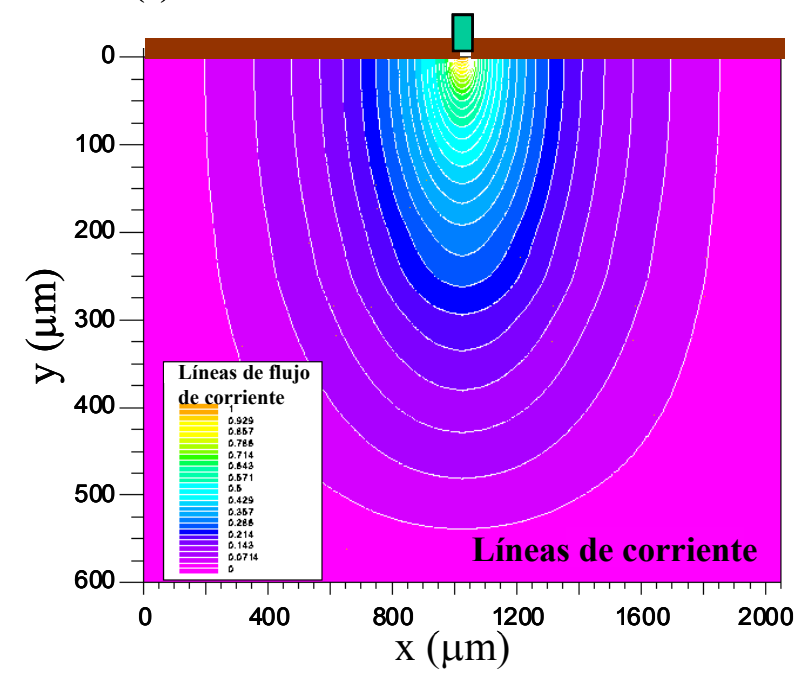

(b)

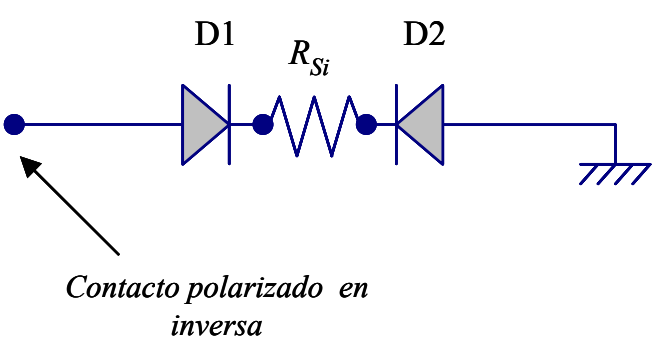

Figura IV.1.a. Esquema del flujo de corriente en una estructura back-to-back fabricada dentro del proyecto METAMOS con contactos de ErSi y sustrato de Si tipo $n\left(10^{-15} \mathrm{~cm}^{-2}\right)$.b. Esquema $1 D$ simplificado de los diodos backto-back

Sin embargo, medir la corriente y caracterizar la altura de la barrera de los contactos se convierte en una tarea complicada precisamente cuando las barreras Schottky formadas son 
bajas. Por este motivo se plantea la fabricación de diodos back-to-back como paso previo a los SB-MOSFET, para así estudiar de manera apropiada la barrera que forma el contacto metalsemiconductor [Dubois, 2004]. Los diodos backto-back deben reunir una serie de características, como es la utilización de un sustrato de Silicio poco dopado, al igual que ocurre en el canal de un SB-MOSFET. En la Figura IV.1.a mostramos un ejemplo de una estructura back-to-back, en la que se han representado las líneas de corriente entre ambos contactos Schottky; además, en la Figura IV.2 podemos examinar una fotografía de un

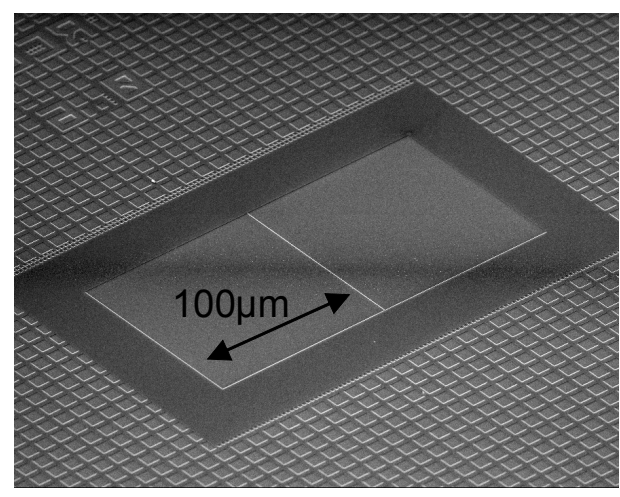

Figura IV.2. Fotografía SEM de un diodo Schottky back-to-back tipo n. El contacto Schottky está basado en ErSi. La formación de este Siliciuro se ha realizado mediante un grabado con iones de Ar a $150 \mathrm{eV}$ durante 90 sg, seguida de una deposición de una capa de Er de $20 \mathrm{~nm}$ de espesor. diodo back-to-back fabricado dentro del proyecto

METAMOS. Como podemos comprobar en ambas figuras, el montaje experimental corresponde a dos contactos de tipo "top" crecidos sobre la superficie del sustrato de Silicio. Sin embargo, desde el punto de vista del transporte, podemos considerar que éste tiene lugar fundamentalmente en un sentido unidimensional, por lo que simularemos el diodo con los contactos Schottky a ambos extremos del sustrato (facilitando así el estudio del dispositivo con nuestro simulador Monte Carlo 1D). La estructura puede ser representada por la combinación en serie de dos diodos Schottky back-to-back con una sección de Silicio resistivo entre ellos, tal y como se muestra en la Figura IV.1.b [Dubois, 2004]. Al aplicar voltaje, la corriente total se determina mediante la corriente proporcionada por el correspondiente contacto Schottky en condiciones de polarización inversa.

La longitud del camino recorrido en las estructuras back-to-back reales (usualmente superiores a los $100 \mu \mathrm{m}$, véase Figura IV.1.a) implicarían unos tiempos de CPU inabordables desde el punto de vista de la simulación Monte Carlo. Por este motivo, la resistencia del Silicio ha sido parcialmente incorporada como un elemento extrínseco de la simulación, pudiendo así tenerse en cuenta una longitud del substrato de Silicio realista manteniendo al mismo tiempo condiciones aceptables en la simulación en cuanto al uso de los recursos de CPU. La determinación de dicha resistencia extrínseca se realiza a partir de las características I-V experimentales que nos han sido proporcionadas dentro del proyecto METAMOS. A la hora de comparar los resultados de la simulación con las estructuras experimentales, es necesario 
determinar una superficie equivalente para obtener la corriente total a partir de la densidad de corriente obtenida en una estructura 1D; dicha superficie equivalente se obtiene por comparación entre los resultados Monte Carlo y la medida de la corriente experimental a bajas temperaturas (cuando la resistencia Schottky es dominante) [Pascual, 2008].

Para la caracterización del contacto Schottky en la estructura back-to-back se usa el método de la energía de activación. Como ya vimos en el Capítulo I, éste es el método más adecuado porque no se necesita ninguna suposición acerca del área eléctricamente activa [Sze, 1981], ya que en las interfaces metal-semiconductor el área geométrica no tiene por qué coincidir necesariamente con la superficie de inyección. Conocido el valor de la corriente $I$ (obtenida en la unión Schottky inversamente polarizada), podemos extraer experimentalmente el valor de la altura de barrera Schottky mediante el diagrama de Arrhenius (véase Ecuación I.59).

Debe tenerse en cuenta que este valor de altura de barrera sólo podrá calcularse en el rango de temperaturas en que la resistencia del contacto Schottky $\left(R_{S c h}\right)$ sea dominante sobre la resistencia de Silicio $\left(R_{S i}\right)$, es decir, a bajas temperaturas, donde el voltaje aplicado total coincide con la caída de potencial a través de la unión inversamente polarizada (diodo D1 en Figura IV.1.b). Al aumentar $T$, la caída de potencial en la resistencia del Silicio es mayor, dejando así (progresivamente) de estar regido el comportamiento del dispositivo por la ley Schottky.

Mediante este procedimiento se ha determinado que las alturas de barrera más bajas para los Siliciuros usualmente utilizados en la fabricación de SB-MOSFETs son, en el caso de estructuras tipo $n$, de $0.28 \mathrm{eV}$ para el Siliciuro de erbio [Kedzierski, 2000] y $0.27 \mathrm{eV}$ para el de yterbio [Zhu, 2004]; para las estructuras tipo $p$, el Siliciuro de platino presenta una altura de barrera de $0.22 \mathrm{eV}$ [Dubois, 2004].

Sin embargo, publicaciones recientes han señalado la necesidad de tender hacia alturas de barreras inferiores a $0.10 \mathrm{eV}$ para poder así competir plenamente con los MOSFETs convencionales que constan de fuente y drenador altamente dopados, ya que la corriente de conducción en los SB-MOSFETs se ve significativamente disminuida para alturas de barrera grandes. Con el fin de conseguir barreras efectivas más bajas, se ha propuesto la técnica de segregación de dopantes (SD) [Kinoshita, 2004], [Larrieu, 2007]. Analizaremos los detalles de esta técnica en la Sección VI.4.

Tanto para el caso de las estructuras back-to-back sin segregación de dopantes como para las que llevan integrada esta capa $\mathrm{SD}$, es necesario realizar un modelado preciso del transporte de portadores a través de la barrera. Los procesos más importantes que se dan en este 
tipo de contactos, como ya es sabido por el estudio del diodo Schottky en el Capítulo III, son los de inyección termoiónica y túnel así como de absorción termoiónica y túnel.

\section{IV.2. Diodos back-to-back tipo n: resultados}

El diodo back-to-back tipo $n$ que hemos analizado mediante el simulador MC1D está basado en una estructura experimental con las siguientes características: está constituido por dos contactos Schottky de ErSi fabricados sobre un sustrato de Silicio poco dopado $\left(10^{15} \mathrm{~cm}^{-3}\right)$. El proceso de fabricación de dicha estructura comienza con la evaporación de la oblea de Si en $U H V$ tras enmascarar la muestra. Posteriormente, se cubre el Erbio con una fina capa protectora de Titanio; después de la deposición, el Siliciuro de Er es crecido mediante RTA a $450{ }^{\circ} \mathrm{C}$ durante dos minutos en $\mathrm{N}_{2}$. Finalmente, se elimina la capa de Ti mediante una mezcla de peróxido sulfúrico. La longitud de cada contacto es de $100 \mu \mathrm{m}$, con una separación entre ambos de $0.5 \mu \mathrm{m}$. La superficie equivalente de la estructura simulada es $8.5 \cdot 10^{3} \mu \mathrm{m}^{2}$ y la altura de barrera es $0.35 \mathrm{eV}$, extraída mediante el método presentado en [Dubois, 2004]. La resistencia extrínseca del Silicio considerada en la simulación Monte Carlo es de $580 \Omega$ a $300 \mathrm{~K}$.
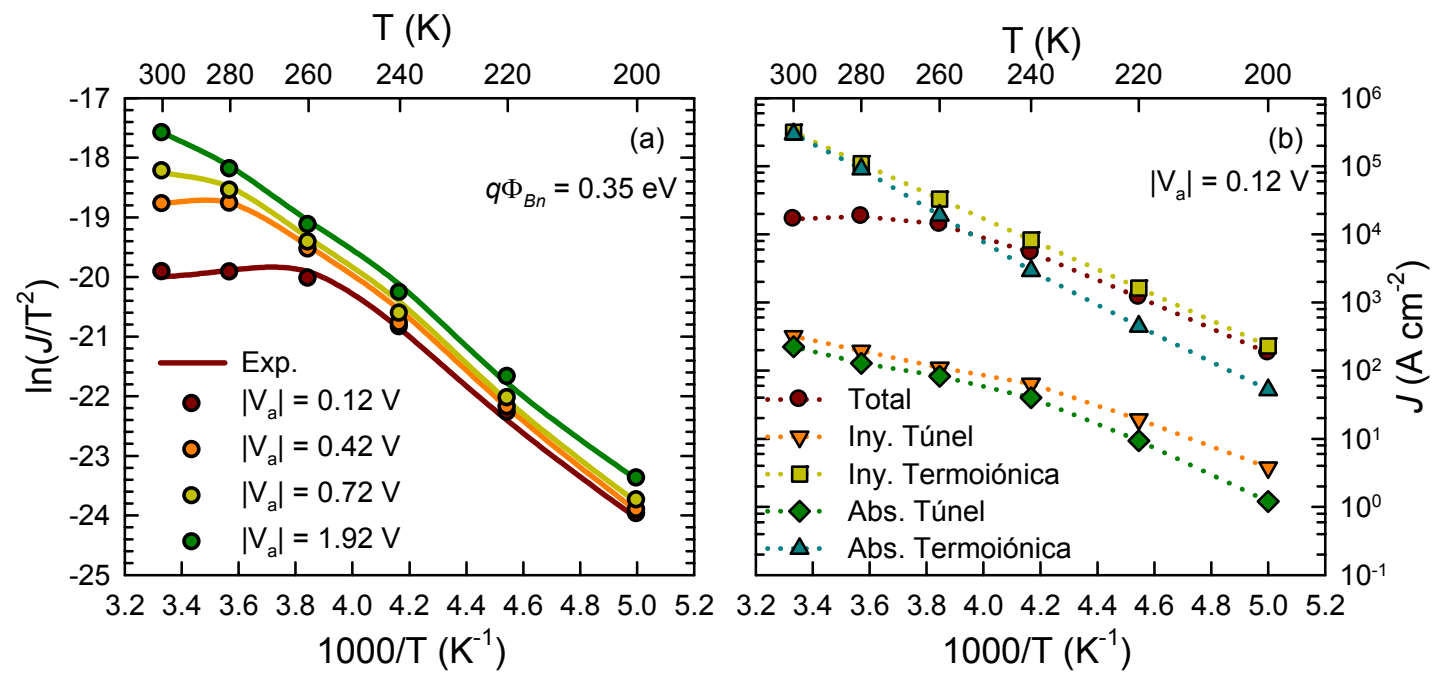

Figura IV.3.a. Diagrama de Arrhenius para el diodo back-to-back tipo n analizado: las lineas corresponden a los datos experimentales y los símbolos a los resultados Monte Carlo para diferentes valores de la tensión en inversa aplicada .b. Componentes de corriente obtenidas mediante la simulación Monte Carlo para la tensión en inversa

$$
\left|V_{a}\right|=0.12 \mathrm{~V}
$$


La Figura IV.3 a muestra el diagrama de Arrhenius de la estructura analizada para el caso de electrones sometida a diversos valores de voltaje aplicado en inversa. Las líneas continuas representan las medidas experimentales y los símbolos los resultados Monte Carlo. Como se puede verse en dicha figura, se obtiene un excelente acuerdo entre los datos experimentales y la simulación Monte Carlo (confirmando definitivamente la fiabilidad de nuestro modelo). Además, la simulación ratifica el valor de la altura de barrera Schottky extraído de manera experimental. Por otro lado, a partir de esta gráfica es posible analizar en detalle la transición entre los dos distintos regímenes observados en el diagrama de Arrhenius, más marcado para el caso de bajos voltajes aplicados.

En la Figura IV.3.b mostramos las componentes de corriente para un voltaje aplicado de $0.12 \mathrm{~V}$ (en inversa). Como puede observarse, a bajas temperaturas la contribución dominante es el proceso de inyección termoiónica; la absorción termoiónica es también significativa, pero mucho menor que su homóloga de inyección. Por otro lado, la contribución de la inyección túnel es menor, y sin embargo superior a de la absorción túnel. Según aumenta la temperatura (valores superiores a $260 \mathrm{~K}$ en el diagrama de Arrhenius), tanto la inyección como la absorción aumentan, con un incremento superior del fenómeno de absorción: de este modo, ambos mecanismos tienden a compensarse y así la corriente total muestra un máximo a unos $280 \mathrm{~K}$ y la subsiguiente reducción a mayor temperatura.

La explicación de este comportamiento puede obtenerse a partir del análisis del perfil de la banda de conducción (Figura IV.4.a). En la región de bajas temperaturas, como consecuencia de la existencia de la barrera Schottky, aparece una curvatura significativa de la banda de conducción. Esta curvatura implica un elevado valor del campo eléctrico en esa zona, y en consecuencia, según son inyectados los portadores en el contacto Schottky en inversa, se ven rápidamente acelerados, alejándose de la unión y apareciendo así una ancha región de vaciamiento (Figura IV.4.b y .c). Esta región es altamente resistiva y en ella se da la mayor parte de la caída de potencial de un contacto a otro, lo que explica que el comportamiento de la estructura esté correctamente descrito mediante la teoría de emisión termoiónica (el diagrama de Arrhenius -Figura IV.3.a- presenta en este rango de temperaturas la típica dependencia exponencial en función de $\mathrm{T}^{-1}$ ) [Sze, 1981]. 

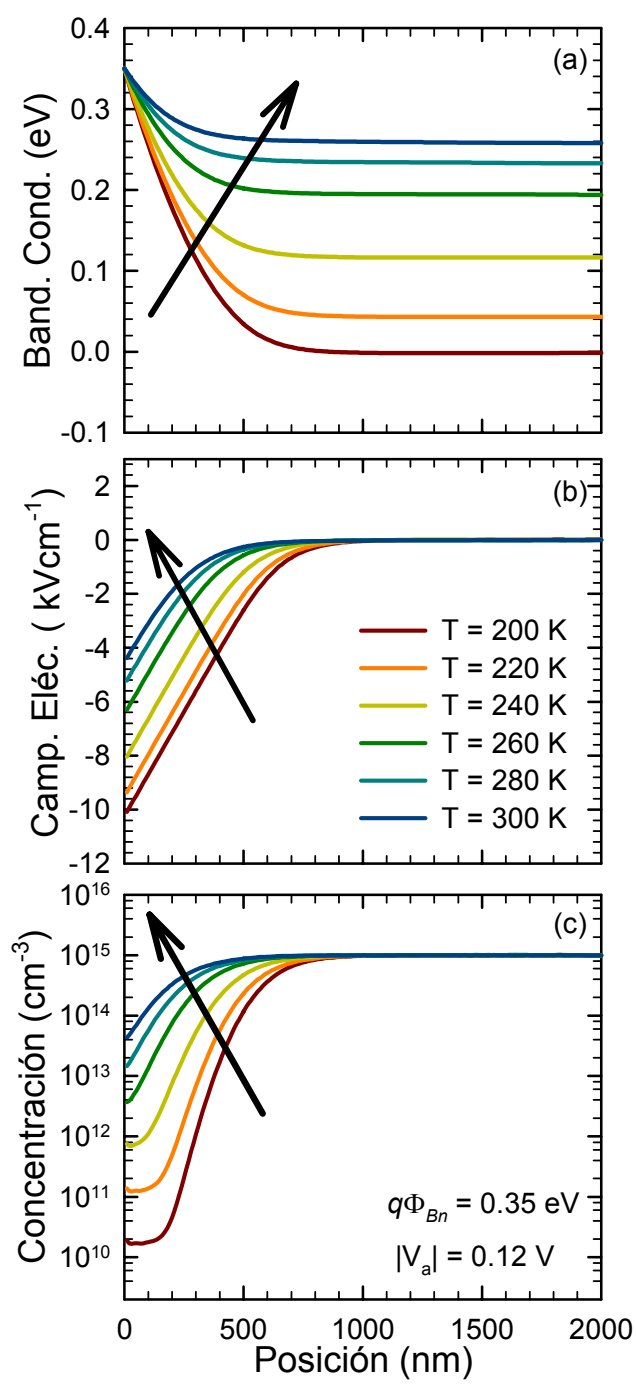

Figura IV.4. Perfil del campo eléctrico y de la densidad de portadores para las primeras 2 um desde la unión Schottky inversamente polarizada a varias temperaturas, desde $200 \mathrm{~K}$ a $300 \mathrm{~K}$.

Por tanto, en este régimen de "bajas" temperaturas el comportamiento del dispositivo sigue la denominada ley Schottky, siendo la resistencia del contacto Schottky superior a la del sustrato de Silicio. La resistencia de este contacto (resistencia Schottky, $R_{S c h}$ ) puede expresarse del siguiente modo bajo condiciones de débil polarización en una aproximación a primer orden [Sze, 1981]:

$$
R_{S c h}=\left(\frac{\partial I}{\partial V_{a}}\right)^{-1}=\frac{K_{B}}{S q A^{*} T} \exp \left(\frac{q \Phi_{B n}}{K_{B} T}\right)
$$


Mientras que la resistencia del Silicio $\left(R_{S i}\right)$ presenta una dependencia con la temperatura del siguiente tipo, siendo $\alpha$ un parámetro de valor que toma valores entre 1 y 1.5 [Dubois, 2004]:

$$
R_{S i}(T)=R_{S i}(300) \cdot(T / 300)^{\alpha}
$$

Según elevamos la temperatura, el dispositivo deja de obedecer la ley Schottky: la diferencia entre el nivel de Fermi y la banda de conducción en el semiconductor aumenta, reduciéndose la curvatura de la banda de conducción asociada a la unión Schottky. El campo eléctrico que los portadores inyectados se encuentran es inferior, por lo que se aceleran en menor medida; así, la región de vaciamiento es más estrecha y su correspondiente resistencia equivalente disminuye. Además, la separación entre el nivel de Fermi y la banda de conducción anteriormente comentada implica un aumento de la resistencia del Silicio. Por tanto, para altas temperaturas hay una caída de potencial significativa en el semiconductor, y $R_{S i}$ pasa a ser dominante en comparación con $R_{S c h}$ [Dubois, 2004]. Como consecuencia de este hecho, tiene lugar el máximo del diagrama de Arrhenius (Figura IV.3.a). El fenómeno anteriormente descrito es análogo al que ocurre en un diodo Schottky convencional al variar la temperatura y que ha sido explicado con detalle en el capítulo anterior.

A continuación vamos a examinar con más detalle cómo tiene lugar del transporte de portadores a través del diodo; para ello, estudiamos en primer lugar los perfiles de energía y velocidad medias de los electrones al alejarse de la interfaz (Figura IV.5.a y .b). En la región de vaciamiento (altamente resistiva, recordemos) los portadores inyectados desde el Siliciuro hacia el semiconductor adquieren elevados valores de energía y velocidad consecuencia del gran campo eléctrico existente, alcanzando así un máximo. Esta subida inicial del valor de la energía induce a un importante aumento de la probabilidad de sufrir un mecanismo de scattering de tipo isótropo con fonones (Figura IV.5.c). Sin embargo, según aumenta la temperatura, el valor máximo de velocidad y energía como se reduce siendo incluso despreciable para las temperaturas más altas. Para las temperaturas inferiores, donde la presencia del máximo de energía es aún notable, dicho scattering con fonones produce un efecto de relajación significativo sobre el momento y la energía. Simultáneamente, la velocidad media y la energía aumentan por el efecto del campo eléctrico, como decíamos con anterioridad. Ambos efectos tienden a compensarse mutuamente, pero finalmente la influencia del scattering con fonones tiende a imponerse a unos cientos de nanómetros de la interfaz, ya superada la región de carga 
espacial (donde además el campo eléctrico es menor, Figura IV.4.b) llegando así a la situación de cuasi-equilibrio, que tiene lugar más cerca del contacto Schottky según $T$ es mayor.

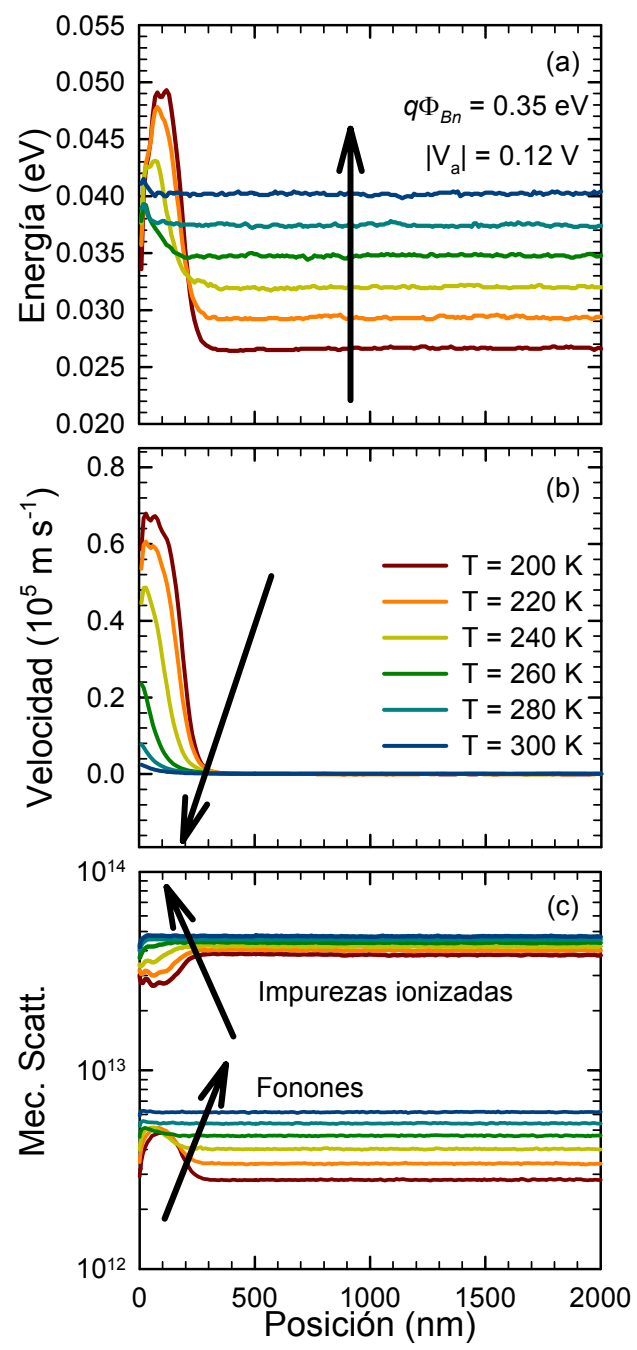

Figura IV.5.a. Perfiles de Energía, .b. velocidad y .c. densidad de mecanismos de scattering en función de la posición para los primeros 2 um desde la unión Schottky en inversa a diferentes temperaturas, desde $200 \mathrm{Ka} 300 \mathrm{~K}$ $y\left|V_{a}\right|=0.12 \mathrm{~V}$.

A través del análisis de la función de distribución de velocidad (FDV) podremos profundizar aún más en el comportamiento de los electrones que son inyectados en el contacto Schottky en inversa. En la Figura IV.8, mostramos los resultados de la FDV para $\left|V_{a}\right|=0.12 \mathrm{~V}$ a $200 \mathrm{~K}$ (parte superior) y a $300 \mathrm{~K}$ (parte inferior) en diversas posiciones distintas en el dispositivo: a $0.5 \mathrm{~nm}$ del contacto polarizado en inversa, a $3.5 \mathrm{~nm}$, a $15 \mathrm{~nm}$ y a $255 \mathrm{~nm}$. Para este valor de voltaje en inversa aplicado, los procesos de inyección túnel y termoiónica son más importantes frente a sus homólogos de absorción a bajas temperaturas, como ya veíamos en la 
Figura IV.5.b; esto se refleja en que la FDV se corresponda con una hemi-Maxwelliana positiva en la región más cercana al contacto (Figura IV.6, T $=200 \mathrm{~K}$ en $\mathrm{x}=0.5 \mathrm{~nm}$ ). Sin embargo, la importancia de los procesos de absorción aumenta con la temperatura (Figura IV.3.b), contribuyendo así con una considerable cola negativa en la función de distribución (Figura IV.6, $\mathrm{T}=300 \mathrm{~K}$ en $\mathrm{x}=0.5 \mathrm{~nm})$.

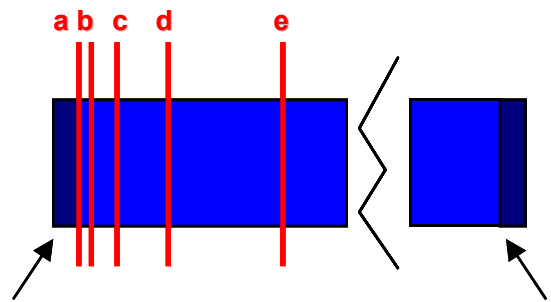

Contacto Schottky polarizado en inversa
Contacto Schottky polarizado en directa
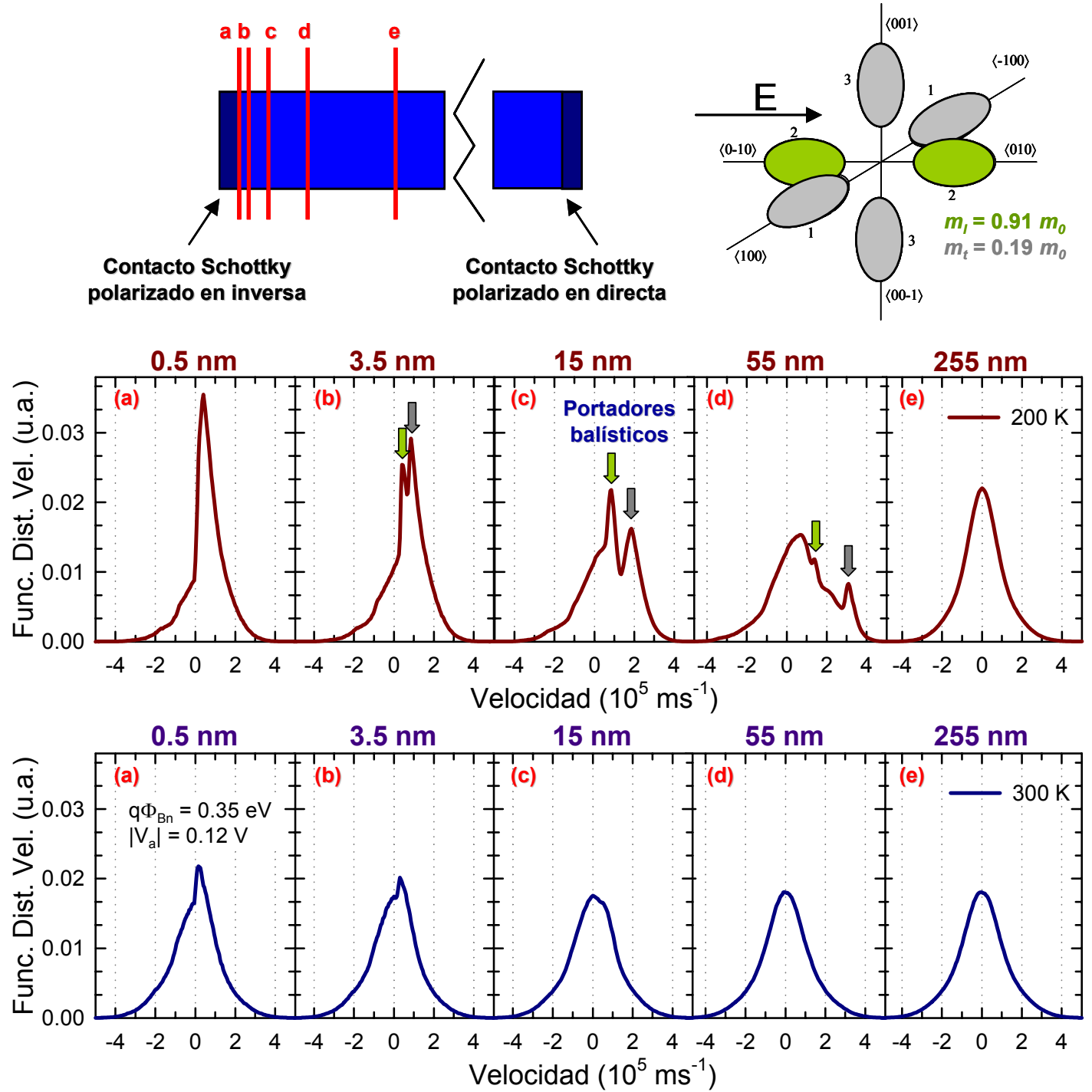

Figura IV.6. En primer lugar mostramos un esquema del dispositivo en el que marcamos las posiciones en que presentamos las funciones de distribución en velocidad. Al lado mostramos la dirección del campo eléctrico aplicado $y$ las superficies isoenergéticas de los valles $X$ de la banda de conducción: los valles longitudinales de verde y los transversales de gris. Finalmente representamos la función de distribución de velocidad de los portadores en varias posiciones para la tensión en inversa $\left|V_{a}\right|=0.12 \mathrm{~V}$ a diferentes temperaturas: $200 \mathrm{~K}$ en la parte superior y $300 \mathrm{~K}$ en 
A medida que los portadores son inyectados en el dispositivo a través del contacto Schottky, son rápidamente acelerados adquiriendo elevados valores de velocidad, como ya se ha expuesto en el Capítulo III. Sin embargo, la acción del campo eléctrico varía dependiendo de en cual de los seis mínimos elipsoidales de la banda de conducción estén situados los portadores (Figura IV). Los portadores en los valles longitudinales presentan una masa efectiva elevada y consecuentemente adquieren una velocidad menor; por el contrario, aquellos portadores situados en los valles transversales son más ligeros y alcanzan una mayor velocidad. Esto se traduce en la aparición de dos máximos en la función de distribución a medida que los portadores se mueven desde el contacto hacia la derecha [Figura IV.6, de (b) a (d)]. Cada uno de estos máximos están asociados a los electrones cuasi-balísticos en los valles longitudinales (el máximo situado a velocidades inferiores, señalado con una flecha verde) y en los transversales (máximo a velocidades superiores, con una flecha gris). Al alejarnos del contacto los portadores están prácticamente termalizados, efecto que se ve reflejado en la FDV, donde observamos que los máximos de velocidad tienden a suavizarse incluso para bajas temperaturas, aproximándose finalmente la función de distribución a una Maxwelliana completa (Figura IV.6.e, a $200 \mathrm{~K}$ ). Si aumentamos la temperatura (ver Figura IV.6 a $300 \mathrm{~K}$ ) la presencia de estos máximos no es tan clara; esto es debido a que la región de carga espacial es muy estrecha, llegando así rápidamente a una situación prácticamente difusiva.

Para analizar con más detalle el fenómeno de la cuasi-balisticidad de los portadores en la región más cercana a la unión, evaluamos el número de mecanismos de scattering con fonones de la red cristalina (Figura IV.7). Podemos ver cómo a $60 \mathrm{~nm}$ del contacto Schottky inversamente polarizado en media, los electrones sufren un total de unos 2 mecanismos de scattering a $200 \mathrm{~K}$, mientras que a $300 \mathrm{~K}$ el número de mecanismos aumenta considerablemente hasta 200. Por otro

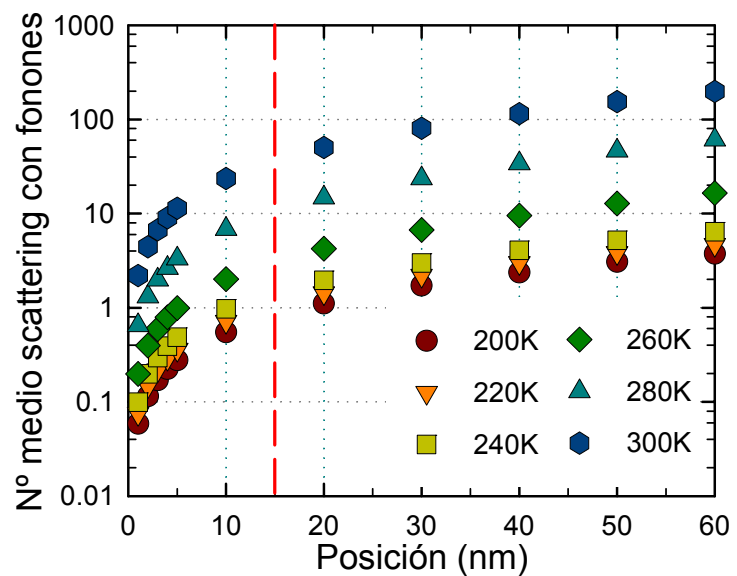

Figura IV.7. Número medio de mecanismos de scattering con fonones en función de la posición para varias temperaturas desde $200 \mathrm{~K}$ hasta $300 \mathrm{~K}$ para $\left|V_{a}\right|=0.12 \mathrm{~V}$ lado, en los primeros $15 \mathrm{~nm}$ desde el contacto, el número de mecanismos es inferior a uno para temperaturas inferiores a $260 \mathrm{~K}$, y aumenta de manera lineal a medida que se mueven los 
portadores hacia el interior del dispositivo. Para el mismo valor de posición, lógicamente el número de scatterings con fonones aumenta con la temperatura [Jacoboni y Lugli, 1989]. Podemos afirmar que en la región de carga espacial, hasta unas pocas decenas de $\mathrm{nm}$ del contacto Schottky, el transporte de carga inyectada desde el Siliciuro hacia el semiconductor presenta características fuertemente balísticas a bajas temperaturas.

\section{IV.3. Resultados y discusión para diodos back-to-back tipo p}

Hemos realizado también un estudio detallado de diodos back-to-back tipo $p$, análogo al presentado en la sección anterior para el tipo $n$. En este caso, y dado las similitudes, vamos a centrarnos principalmente en destacar las diferencias más notables con respecto a la estructura tipo $n$, derivadas (además de las características inherentes al dopado tipo $p$ ) del hecho de que en este caso el diodo analizado presenta una altura de barrera inferior a su homólogo tipo $n$ $\left(q \Phi_{B}=0.145 \mathrm{eV}\right)$.

Las características de la estructura back-to-back tipo $p$ fabricada que simularemos en esta sección son: contactos de PtSi de $1000 \mu \mathrm{m}$ separados por un gap de Si de $5 \mu \mathrm{m}$ (a partir del recocido de $20 \mathrm{~nm}$ de Pt en RTA $300{ }^{\circ} \mathrm{C} / 2 \mathrm{~min}$ ). La altura de la barrera considerada en la simulación es $0.145 \mathrm{eV}$, y el dopaje del sustrato de $\mathrm{Si}$ es $5 \cdot 10^{15} \mathrm{~cm}^{-3}$. La superficie "equivalente" del dispositivo unidimensional simulado es de $3 \cdot 10^{3} \mu \mathrm{m}^{2}$ y la longitud es $1 \mu \mathrm{m}$. El valor de la resistencia extrínseca del Silicio a tener en cuenta en la simulación Monte Carlo es $35 \Omega$ a $300 \mathrm{~K}$.

En la Figura IV.8.a mostramos el diagrama de Arrhenius para distintos voltajes aplicados (las líneas representan los datos experimentales y los símbolos los resultados MC). Debemos destacar nuevamente el excelente acuerdo entre las medidas y la simulación, que es capaz de reproducir con total fidelidad las medidas experimentales. 

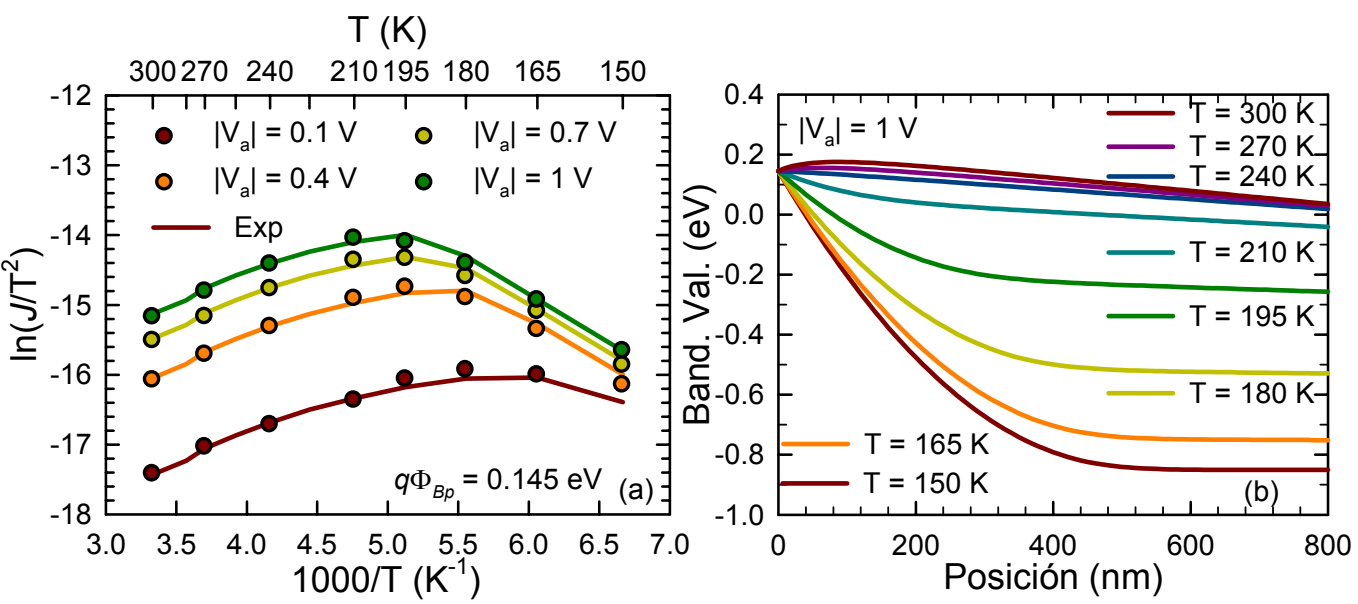

Figura IV.8.a. Diagrama de Arrhenius de la estructura back-to-back tipo p analizada: las lineas se corresponden con los datos experimentales y los símbolos con los resultados Monte Carlo.b. Perfil de la banda de conducción de la estructura simulada a diferentes temperaturas, desde $150 \mathrm{~K}$ a $300 \mathrm{~K}$ para la tensión en inversa $\left|V_{a}\right|=1 \mathrm{~V}$.

A partir de las Ecuaciones IV.1 y IV.2 podemos afirmar que cuando la altura de barrera Schottky $q \Phi_{B n}$ disminuye y aumenta la temperatura $T, R_{S c h}$ tiene una menor influencia en el comportamiento en corriente del dispositivo, imponiéndose así la resistencia del Silicio. Podemos corroborar esta afirmación al analizar la Figura IV.8.a, donde observamos que el reducido valor de la altura de la barrera Schottky provoca un desplazamiento del máximo del diagrama de Arrhenius hacia temperaturas sensiblemente más bajas que en las descritas para el diodo tipo $n$, convirtiéndose (para débil voltaje aplicado) la resistencia del Silicio en el efecto dominante para la mayor parte del rango de temperaturas considerado. Esto se traduce en algunas diferencias a la hora de estudiar la transición entre los dos regímenes de temperatura observados en el diagrama de Arrhenius.

Analizaremos el comportamiento del dispositivo en cada uno de los regímenes de temperatura para la tensión en inversa $\left|V_{a}\right|=1$ V. La Figura IV.8.b muestra el perfil de la banda de valencia proporcionada por la simulación Monte Carlo. A bajas temperaturas la situación es similar a la previamente comentada, con una curvatura de la banda de valencia claramente definida y asociada a la existencia de la región de vaciamiento, tal y como se puede observar en la Figura IV.9.a. Como consecuencia del elevado campo eléctrico en esta región, aparecen importantes fenómenos de no equilibrio: sobrevelocidad de los portadores en la región cercana al contacto Schottky (Figura IV.9.b), así como un gran aumento de su energía (ver el recuadro 
interior de la Figura IV.9.b). A medida que los portadores se mueven hacia el interior del dispositivo se alcanza una situación de cuasi-equilibrio.
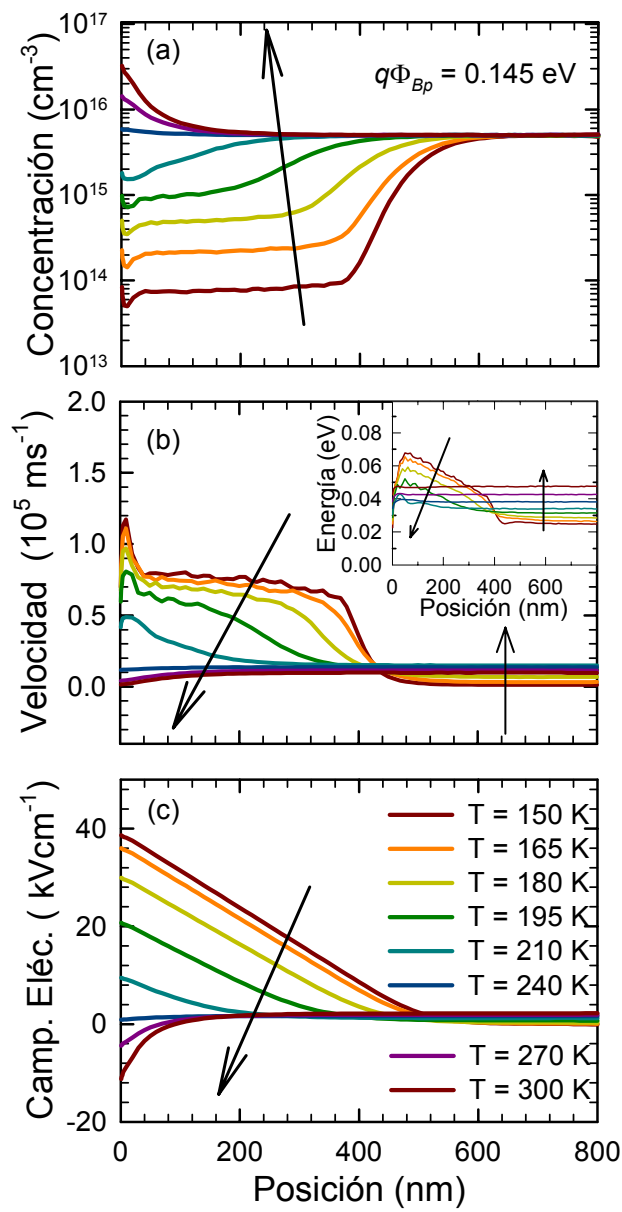

Figura IV.9. Perfiles de .a. concentración de portadores, .b. velocidad y .c. campo eléctrico para diversas temperaturas $\left(\left|V_{a}\right|=1 \mathrm{~V}\right)$.

Cuando la temperatura aumenta, el valor del nivel de Fermi del semiconductor excede el valor de la altura de la barrera Schottky (esto sucede para temperaturas superiores a $210 \mathrm{~K}$ ). En esta situación el dispositivo es muy resistivo y se observa una ligera curvatura de la banda de valencia en el sentido opuesto del que ocurre a bajas temperaturas, provocando una acumulación de huecos en la región cercana al contacto (Figura IV.9.a). Esta curvatura de la banda de valencia se ve reflejada en un campo eléctrico negativo que favorece el movimiento de los portadores hacia el contacto Schottky, presentando los mismos una energía próxima a la térmica a lo largo de todo el dispositivo. En definitiva, la unión metal-semiconductor en el rango de 
temperaturas más elevadas se comporta, en líneas generales, como un contacto óhmico en lugar de rectificador.

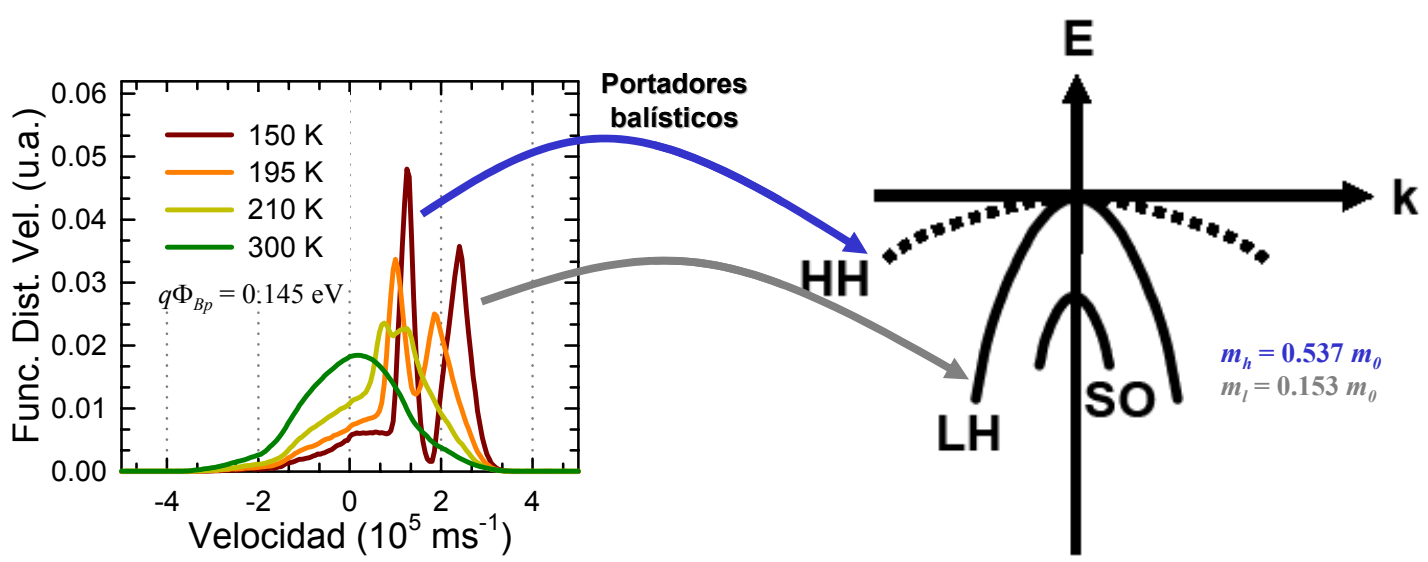

Figura IV.10. Función de distribución de velocidad de los portadores a $7.5 \mathrm{~nm}$ del contacto para $\left|V_{a}\right|=1 \mathrm{Va}$ diferentes temperaturas, $150 \mathrm{~K}, 195 \mathrm{~K}, 210 \mathrm{~K}$ y $300 \mathrm{~K}$. A la derecha de la figura encontramos la representación energética de las subbandas de huecos ligeros y pesados de la Banda de Valencia del Silicio.

Del estudio del transporte microscópico realizado en el diodo back-to-back tipo $p$ debemos destacar la aparición, nuevamente, de máximos en la FDV (ver Figura IV.10) asociados en esta ocasión a los huecos balísticos situados en tanto en las subbandas de huecos pesados como en la de ligeros (mostradas a la derecha de la Figura IV.10). Con el aumento de la resistencia del Silicio en comparación con la resistencia Schottky (a medida que la temperatura es mayor), disminuye el peso de los portadores balísticos; por tanto, el número de portadores que presentan un transporte de tipo difusivo es más elevado. Esto puede verse reflejado en la Figura IV.10, en la que observamos como a $7.5 \mathrm{~nm}$ del contacto Schottky inversamente polarizado, los máximos relativos debido a portadores balísticos (muy pronunciados a $\mathrm{T}=150 \mathrm{~K}$ ) se suavizan al aumentar la temperatura dando lugar a una maxwelliana, típica del transporte difusivo para $\mathrm{T}=300 \mathrm{~K}$, tal y como hemos querido indicar con las flechas más gruesas en la Figura IV.10.

\section{4. Contacto Schottky con capa de segregación de dopantes}

Como destacábamos al principio del capítulo, el principal objetivo de la fabricación de los diodos back-to-back es la caracterización de las alturas de barrera Schottky de los contactos que luego se utilizarán en los SB-MOSFET, buscando además las menores alturas de barrera 
posibles. Algunos autores han demostrado, mediante el estudio de las características I-V, un importante aumento de la corriente en contactos Schottky empleando una capa de segregación de dopantes [Knoch, 2005], [Zhang, 2006], [Tsui, 2007]. Sin embargo, el efecto de incluir este tipo de finas capas de dopantes adyacentes al contacto no ha sido investigado desde un punto de vista microscópico. Por tanto, el objetivo de este apartado es analizar, en estructuras back-toback, el efecto sobre el transporte de portadores de la inclusión de esta capa de dopantes, comparándolo con el caso en que no se considere esta solución.

La técnica de la segregación de dopantes en una unión Schottky consiste en la incorporación de una capa altamente dopada junto a la interfaz Siliciuro-Silicio. De este modo se modifica el perfil de potencial dentro del semiconductor en las proximidades del contacto favoreciéndose así la inyección de portadores. En la Figura IV.11 podemos observar el cambio de curvatura de la banda junto al contacto Schottky provocado al introducir la capa de dopantes. Como se observa, el resultado de la misma es el estrechamiento notable de la barrera, reduciéndose así el camino túnel que deben recorrer las partículas desde el metal al semiconductor.

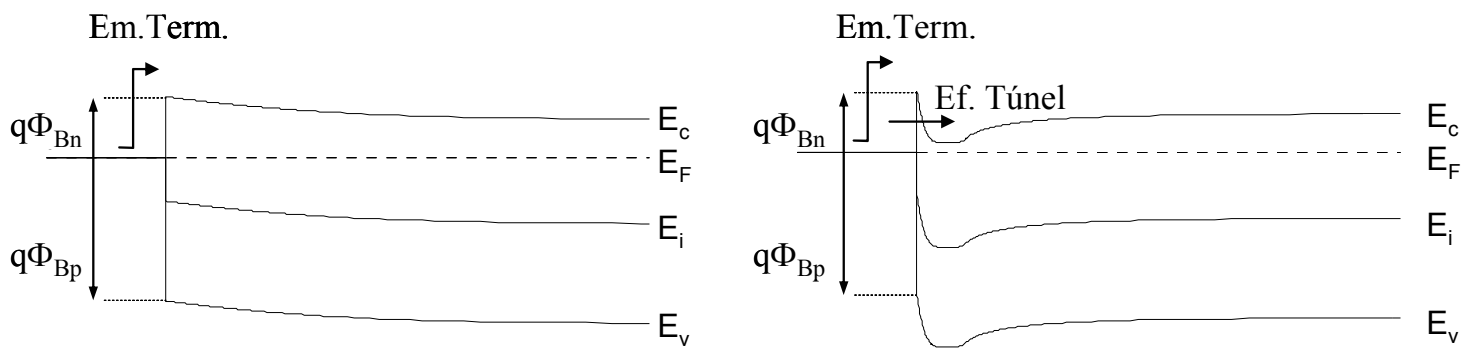

Figura IV.11.a. Estructura de bandas en equilibrio de un contacto Schottky en un sustrato base con baja concentración tipo $n$.b. La misma representación de estructura de bandas cuando se sitúa una fina capa de segregación de dopantes tipo n junto a la interfaz Schottky.

Existen diversos métodos para llevar a cabo la introducción de la capa de segregación de dopantes [Chen, 1996], [Larrieu, 2007]. La primera posibilidad es realizar una implantación poco profunda en el Si seguida de una deposición de metal, para llevar a cabo finalmente un recocido que permita la fabricación del Siliciuro (implant before silicide, IBS). De acuerdo con esta técnica, la energía de implantación debe ser controlada con precisión para confinar la mayor parte de los dopantes sobre una capa de Silicio superficial que será totalmente consumida en el proceso de fabricación del Siliciuro. La acumulación de los dopantes debe tener lugar en la interfaz Silicuro/Silicio debido a la poca solubilidad de los dopantes en la fase de Siliciuro. Una 
segunda técnica es la implantación a través del metal (implantation-trough-metal, ITM) para evitar la implantación en el Silicio y así prevenir problemas relacionados con la generación de defectos. En este caso, la segregación de dopantes también tiene lugar debido a las temperaturas alcanzadas en la reacción de generación del Siliciuro. Finalmente, la tercera posibilidad es la implantación a través del Siliciuro (implantation-trough-silicide, ITS), en la que se realiza una acumulación de dopantes mediante un paso de activación a baja temperatura tras la fabricación del Siliciuro.

La ventaja asociada a las dos últimas técnicas es que se mejora el efecto de segregación dado que los dopantes quedan confinados en la capa adyacente al Siliciuro sin generación de defectos [Larrieu, 2007]. En el caso de transistores SB-MOSFET tipo $p$, se ha comprobado que la integración de contactos Schottky de fuente y drenador constituidos por un valor de altura de barrera bajo de Siliciuro (como el PtSi) más una segregación de Boro (B), da lugar a una mejora del $50 \%$ de la corriente de conducción [Larrieu, 2007]. De manera alternativa, pueden introducirse impurezas tipo $n$ para producir en la banda la curvatura contraria, favoreciendo la inyección de electrones. Siguiendo esta estrategia, en el caso particular de las estructuras fabricadas en el proyecto METAMOS con las que hemos trabajado, se procede a la

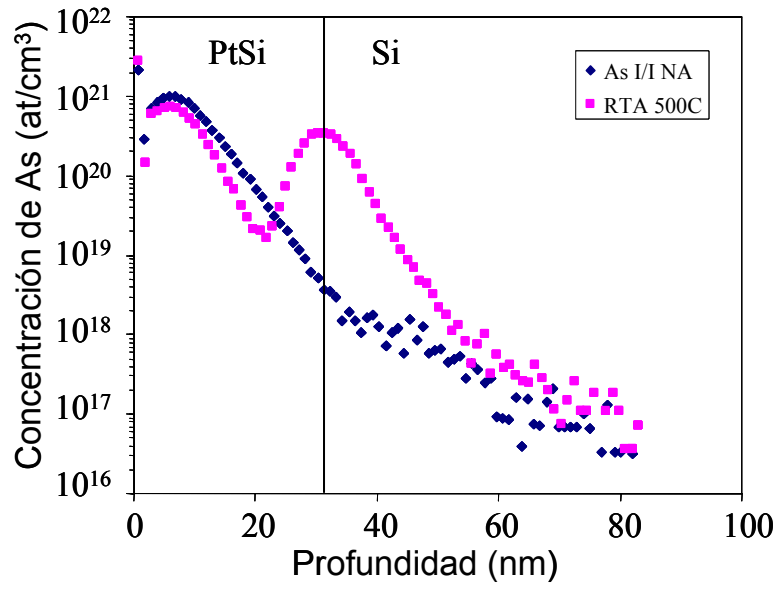

Figura IV.12. Perfiles de concentración de Arsénico obtenidos mediante espectroscopia de masas de iones secundarios (SIMS) según son implantados en una capa de PtSi de $32 \mathrm{~nm}$ de espesor (símbolos azules)y después del recocido tras la implantación (símbolos rosas). La acumulación elevada de As (donadores) indica que se espera que surja la curvatura en la banda de energía en la región localizada junto a la interfaz $\mathrm{PtSi} / \mathrm{Si}$ tal y como se muestra en la Figura IV.2.b implantación de $\mathrm{As}^{+}$a $25 \mathrm{keV}$ con una concentración de $10^{15} \mathrm{~cm}^{-2}$ en una capa del Siliciuro en cuestión, que en el caso particular mostrado en la Figura IV.12 es PtSi de $32 \mathrm{~nm}$ de espesor. Dicha figura muestra el perfil de concentración de As resultante tras el proceso químico obtenido mediante una espectrometría de masas de iones secundarios (SIMS). La espectrometría se lleva a cabo tanto en el momento de la implantación como en la situación final (tras el paso de recocido posterior al proceso de fabricación del Siliciuro). En este caso la duración del tiempo de recocido es de 5 minutos y

la temperatura que se alcanza es de $500{ }^{\circ} \mathrm{C}$. Como podemos observar en la Figura IV.12, en la 
interfaz PtSi/Si hay un importante depósito de Arsénico, similar al caso de la segregación de Boro en contactos tipo $p$ [Larrieu, 2007]. Este sistema sirve como referencia para el caso que estudiaremos en esta sección, relativo a la segregación de dopantes para favorecer la inyección de electrones.

Hemos tomado como punto de partida un diodo back-to-back con un sustrato de Silicio tipo $n$ con dopaje (denominado dopaje "base") de $2 \cdot 10^{15} \mathrm{~cm}^{-3}$ y altura de barrera de $0.325 \mathrm{eV}$ (con contactos constituidos por Siliciuro de Yterbio). De ahora en adelante llamaremos a esta estructura NSD ("no segregación de dopantes"). Simulamos la misma estructura añadiendo una capa SD adyacente al contacto Schottky con anchura de $20 \mathrm{~nm}$ (a partir de ahora estructura SD, "segregación de dopantes"). Asumimos que los dopantes en el Silicio están activados en las proximidades de la interfaz. Aunque la Figura IV.12 muestra que la concentración de dopantes de As puede alcanzar valores superiores a $10^{20} \mathrm{~cm}^{-3}$, debe tenerse en cuenta que el análisis SIMS proporciona información química pero no revela el estado de activación de los dopantes. En realidad, la densidad de electrones en equilibrio, relacionada directamente con la concentración de dopantes activados, sigue una ley de activación térmica proporcional a $\exp \left(-0.47 \mathrm{eV} \mathrm{K} \mathrm{K}_{\mathrm{B}} \mathrm{T}^{-1}\right)$ [Derdour, 1991]. Además, debe tenerse en cuenta que la segregación de As se produce generalmente a temperaturas entre 500 y $700{ }^{\circ} \mathrm{C}$ [Zhang, 2007], por lo que es razonable asumir que la concentración de dopantes activos es del orden de $10^{18} \mathrm{~cm}^{-3}$. La longitud total de estos dispositivos es de $2.72 \mu \mathrm{m}$, y con una superficie equivalente del contacto igual a $4 \cdot 10^{4} \mu \mathrm{m}$. La resistencia extrínseca del Silicio es de $164 \Omega$ a $300 \mathrm{~K}$.

En primer lugar mostramos en la Figura IV.13 la comparativa de las simulaciones Monte Carlo con medidas experimentales realizadas en una estructura de Siliciuro de Yterbio, a la izquierda sin dopantes y a la derecha con la capa de segregación de dopantes, incluida mediante la técnica IBS. Destacamos la excelente correspondencia de los resultados Monte Carlo con las medidas experimentales en el régimen de altas temperaturas en el caso de la estructura sin capa de segregación de dopantes (Figura IV.13.a). Para temperaturas inferiores a $235 \mathrm{~K}$ no disponemos de datos de medidas experimentales puesto que han surgido diversos problemas en la determinación de la corriente experimental relacionados con la aparición de defectos en la superficie, ya que la técnica de fabricación de contactos Schottky tipo $n$ está menos perfeccionada que la de contactos tipo $p$. 


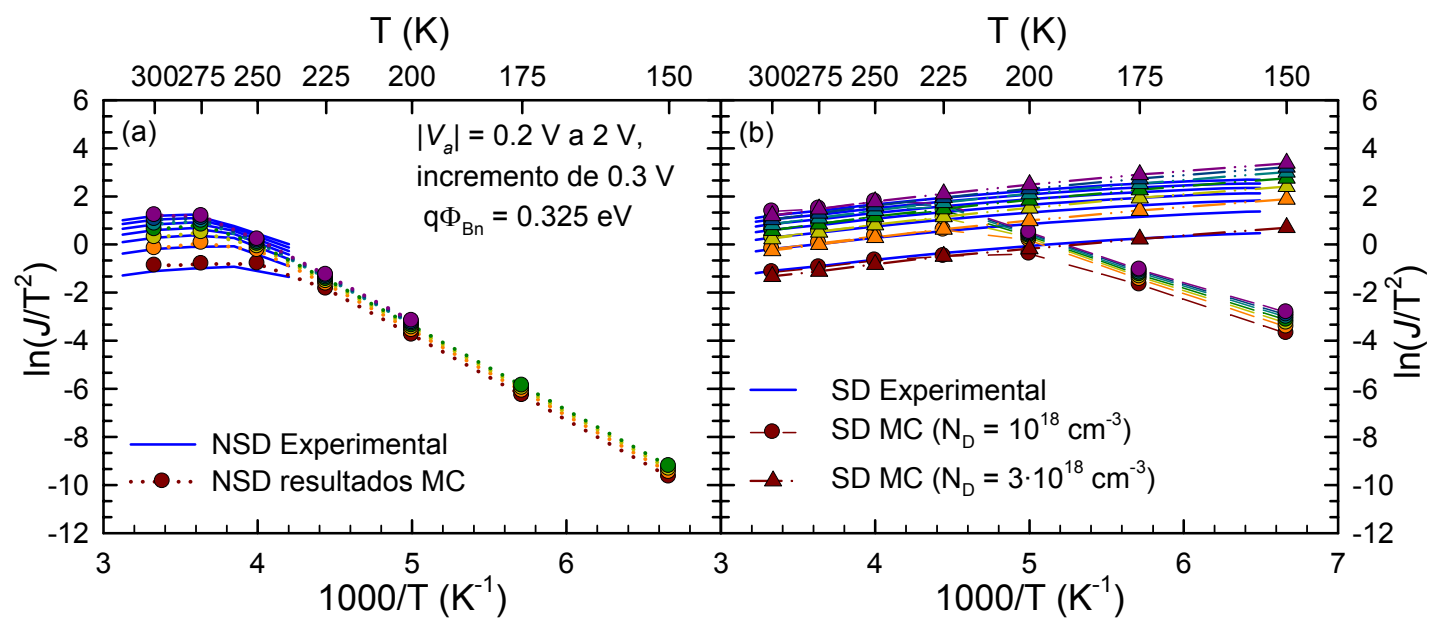

Figura IV.13.a. Diagrama de Arrhenius de la estructura sin dopantes NSD. Las lineas representan las medidas experimentales y los símbolos son resultados Monte Carlo .b. Diagrama de Arrhenius de la estructura con capa de dopantes, SD. En este caso, las líneas son las medidas experimentales y los círculos representan los resultados Monte Carlo con una capa $S D$ de dopaje de $10^{18} \mathrm{~cm}^{-3}$, los triángulos se corresponden con la simulación de una estructura de capa SD con dopaje de $3 \cdot 10^{18} \mathrm{~cm}^{-3}$.

Con respecto a la estructura con capa de segregación de dopantes (Figura IV.13.b), además de las medidas experimentales, mostramos los resultados Monte Carlo para dos valores de concentración de la capa SD, $3 \cdot 10^{18} \mathrm{~cm}^{-3}$ y $10^{18} \mathrm{~cm}^{-3}$. Como podemos ver, el comportamiento de la estructura es totalmente resistivo en todo el rango de temperaturas analizado, existiendo además una buena coincidencia con los resultados experimentales para la estructura Monte Carlo con SD de $3 \cdot 10^{18} \mathrm{~cm}^{-3}$. Para el caso de SD de dopaje $10^{18} \mathrm{~cm}^{-3}$, el valor de corriente no coincide con el experimental a bajas temperaturas. Sin embargo, desde el punto de vista de nuestro análisis se trata de una estructura más interesante, pues en este caso la corriente sigue la dependencia de la ley Schottky a bajas temperaturas. Por ello, a partir de este momento nos centraremos en el estudio de las estructuras NSD y SD con dopaje $10^{18} \mathrm{~cm}^{-3}$, dejando a un lado la estructura con dopaje de la capa SD igual a $3 \cdot 10^{18} \mathrm{~cm}^{-3}$, ya que este caso no tendía sentido extraer una altura de barrera equivalente sin capa de segregación de dopantes.

La Figura IV.14 muestra con más detalle, a través del diagrama de Arrhenius, la comparación de la corriente obtenida en la simulación Monte Carlo de la estructura NSD (sin la capa de segregación de dopantes) y la SD (la misma estructura incluyendo dicha capa junto al contacto Schottky inversamente polarizado) para varios voltajes en inversa aplicados. Para 
ambos diodos se observa la aparición del máximo que separa los dos regímenes de temperatura anteriormente comentados. Para la estructura NSD, este máximo está localizado en torno a $275 \mathrm{~K}$, mientras que para el diodo SD se sitúa a temperaturas inferiores (225 K aproximadamente), como puede observarse en la Figura IV.14. Además, la presencia de la capa de segregación de dopantes produce un importante incremento de la corriente en la estructura $\mathrm{SD}$, particularmente en el rango de bajas temperaturas. Para comprobar el efecto neto en la

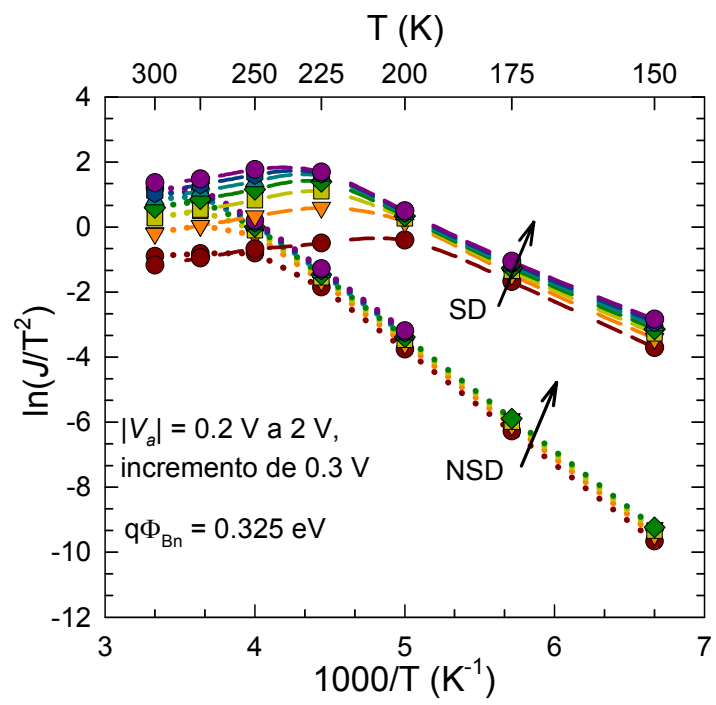

Figura IV.14. Diagrama de Arrhenius para las estructuras tipo n NSD y SD analizadas para diferentes voltajes en inversa aplicados (desde $\left|V_{a}\right|=0.2 \mathrm{Va} 2 \mathrm{~V}$, con un incremento de $0.3 \mathrm{~V}$ ). corriente de la capa de segregación de dopantes hemos llevado a cabo diversas simulaciones Monte Carlo sin capa de dopantes y reduciendo progresivamente la altura de la barrera Schottky. Así, hemos encontrado que para una altura de barrera de $0.254 \mathrm{eV}$ y sin capa de segregación de dopantes (a la que llamaremos estructura NSD2) se obtiene la misma corriente que en la estructura $\mathrm{SD}$ con barrera de $0.325 \mathrm{eV}^{1}$. Por tanto, puede deducirse que el efecto de la capa SD sobre la corriente sería el de una reducción de la altura de barrera Schottky efectiva en la estructura NSD. Sin embargo, aunque las corrientes para las estructuras NSD2 y SD coinciden, su origen no es el mismo [Pascual, 2009].

\begin{tabular}{|c|c|c|c|c|}
\hline \multirow{2}{*}{ Estructuras } & \multirow{2}{*}{$\begin{array}{c}\text { Dopaje } \\
\text { base }\left(\mathrm{cm}^{-3}\right)\end{array}$} & \multicolumn{2}{|c|}{ Capa de Seg. Dopantes } & \multirow{2}{*}{$\begin{array}{c}q \Phi_{B n} \\
(e V)\end{array}$} \\
\hline & & Dopaje $\left(\mathrm{cm}^{-3}\right)$ & Anchura (nm) & \\
\hline$N S D$ & $2 \cdot 10^{15}$ & - & - & 0.325 \\
\hline$S D$ & $2 \cdot 10^{15}$ & $10^{18}$ & 20 & 0.325 \\
\hline NSD2 & $2 \cdot 10^{15}$ & - & - & 0.254 \\
\hline
\end{tabular}

Tabla IV.1. Características principales de las distintas estructuras simuladas.

\footnotetext{
${ }^{1}$ En la Tabla VI.1 presentamos un resumen de las principales características de las estructuras simuladas en esta sección.
} 
A continuación evaluamos las componentes de la corriente total de las estructuras simuladas NSD y SD (ver Figura IV.15.a. y .b.). Dado que la contribución de las componentes de corriente a la total en el caso de la estructura NSD2 así como el comportamiento de las diversas magnitudes internas es muy similar al de la estructura NSD, no mostramos los resultados correspondientes a dicha estructura en las gráficas; si se quiere examinar en detalle el comportamiento de NSD2 nos remitimos a la referencia [Pascual, 2009].

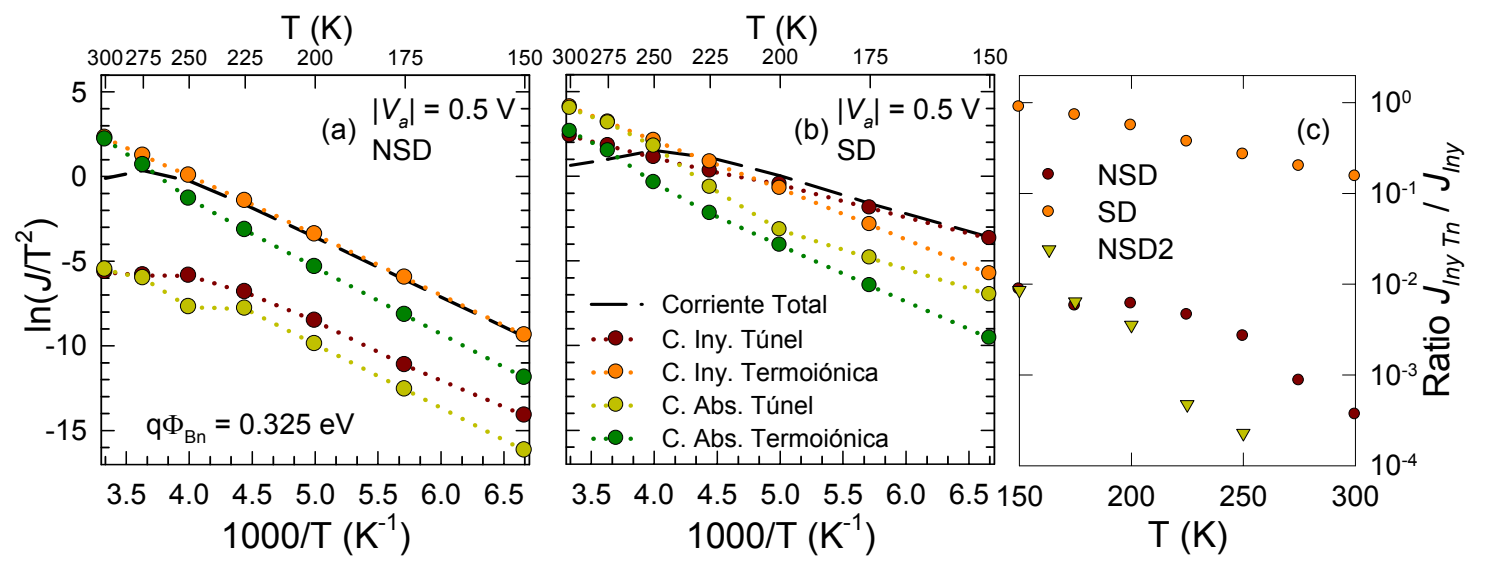

Figura IV.15. Diagrama de Arrhenius de las estructuras back-to-back analizadas mostrando las cuatro componentes de corriente: .a. NSD y .b. SD para la tensión negativa $\left|V_{a}\right|=0.5 \mathrm{~V}$. El comportamiento de las componentes de corriente de NSD2 es muy similar al de NSD. Las lineas discontinuas negras se corresponden con la corriente total mientras que las otras cuatro se corresponden con las distintas contribuciones a la corriente total .c. Ratio entre corriente de inyección túnel y corriente total inyectada para las tres estructuras simuladas (NSD, SD y NSD2).

En ausencia de la capa de segregación de dopantes (NSD y NSD2), la mayor contribución a la corriente total es debida a la inyección termoiónica en el régimen de bajas temperaturas. Para explicar este comportamiento examinamos la banda de conducción de las estructuras NSD y SD en los primeros $400 \mathrm{~nm}$ junto al contacto Schottky (ver Figura IV.16.a. y b.). Como podemos ver, sin capa de segregación de dopantes el camino túnel es muy ancho y la barrera que deben superar las partículas en el semiconductor hacia el metal es además muy grande (véase la Figura IV.16.a para la estructura NSD, como hemos dicho anteriormente, el comportamiento de NSD2 es muy similar). Sin embargo, cuando se introduce la capa de segregación de dopantes, observamos que la curvatura de la banda que se produce en los primeros nanómetros de la estructura SD junto al contacto Schottky inversamente polarizado da lugar a un importante estrechamiento de la barrera (Figura IV.16.b), volviéndose prácticamente transparente para los portadores que intentan atravesar la misma 
mediante efecto túnel. De este modo los fenómenos de inyección túnel se ven así enormemente favorecidos sobre los demás, como comprobamos en la Figura IV.15.c, en la que mostramos el ratio entre la corriente de inyección túnel y la corriente total inyectada [Pascual, 2009].

Como ya analizamos en la Sección IV.2 en el caso de la estructura sin capa de segregación de dopantes, en el régimen de altas temperaturas la contribución más importante a la corriente total es debida a las componentes de inyección y absorción termoiónicas (ver Figura IV.15.a). Sin embargo, al incluir la capa de segregación de dopantes son la corriente inyectada termoiónica y la corriente absorbida túnel las que contribuyen a la corriente total en mayor medida (Figura IV.15.b); nótese además que aunque la importancia de la componente de inyección túnel disminuye con respecto al régimen de bajas temperaturas, sigue presentando una mayor influencia sobre la corriente total que en las estructuras sin capa SD (Figura IV.15.c).
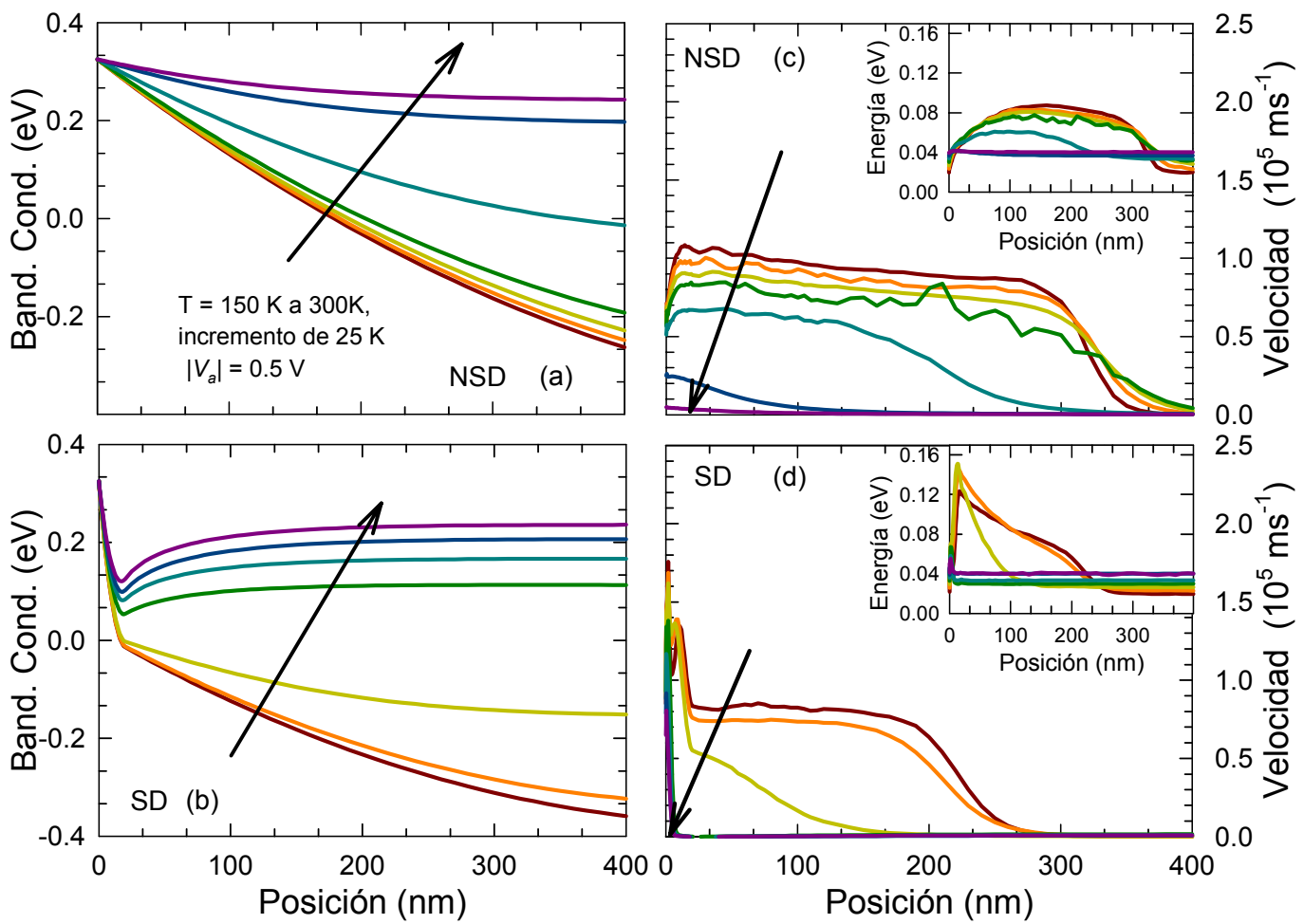

Figura IV.16. Perfil de la banda de conducción de los diodos .a. NSD y .b. SD para $\left|V_{a}\right|=0.5$ V para diferentes temperaturas entre 150 y $300 \mathrm{~K}$.c-d. Perfil de velocidad y de energía (recuadros interiores) en función de la posición de las estructuras back-to-back NSy, SD respectivamente, para $\left|V_{a}\right|=0.5 \mathrm{~V}$ y a varias temperaturas (de $150 \mathrm{~K}$ a $300 \mathrm{~K}$ en incrementos de $25 \mathrm{~K}$ ). 
Completamos el estudio de la variación de las magnitudes internas en los primeros $400 \mathrm{~nm}$ de las estructuras NSD y SD presentando el perfil de la velocidad media de los portadores en la Figura IV.16.c-d., el campo eléctrico y la concentración de portadores en la Figura IV.17. Dado que ya se ha llevado a cabo el análisis de dichas magnitudes para la estructura sin capa de segregación de dopantes en la Sección IV.2, nos centraremos en la estructura SD, permitiéndonos comprender así los efectos de introducir dicha capa junto al contacto Schottky.
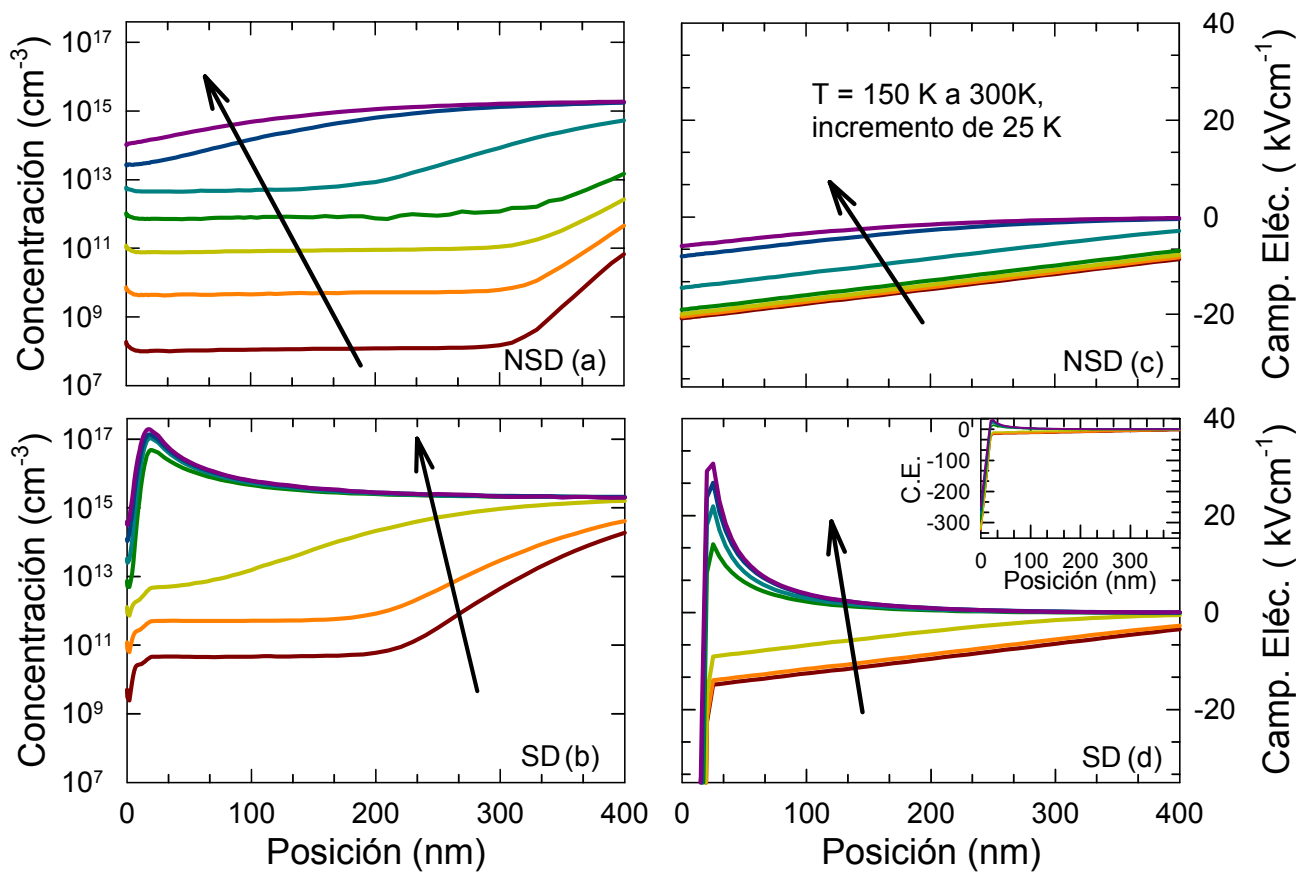

Figura IV.17.a-b. Concentración de portadores y.c-d. campo eléctrico en función de la posición de las estructuras back-to-back NSD y SD respectivamente, para $\left|V_{a}\right|=0.5 \mathrm{~V}$ y a varias temperaturas (de $150 \mathrm{Ka} 300 \mathrm{~K}$ en incrementos de $25 \mathrm{~K}$ ).

Cuando se tiene en cuenta la capa de segregación de dopantes junto al contacto Schottky, la región de carga espacial es más pequeña (Figura IV.17.b y .a. respectivamente). Además, cuando los portadores son inyectados en el interior del dispositivo SD, se encuentran con un campo eléctrico extremadamente elevado en los primeros $20 \mathrm{~nm}$ del dispositivo, consecuencia del elevado dopaje en esa zona (alrededor de $-320 \mathrm{kV} \mathrm{cm}^{-1}$, ver el recuadro interior de la Figura IV.17.d), por lo que son mucho más acelerados que en la estructura NSD. También debemos destacar el cambio de pendiente que presenta el campo eléctrico en el dispositivo con capa SD, el cual es debido al cambio de dopaje entre la región SD y el sustrato. 
Debido al elevado campo eléctrico junto al contacto, los portadores adquirieren un máximo de velocidad y de energía en los primeros $20 \mathrm{~nm}$ muy elevado (por ejemplo, para $\mathrm{T}=150 \mathrm{~K}$, el valor del máximo de velocidad es $1.76 \cdot 10^{5} \mathrm{~m} \mathrm{~s}^{-1}$ y el de energía es $0.15 \mathrm{eV}$, véase Figura IV.16.d).

Analizamos a continuación con más detalle el efecto del campo eléctrico sobre la velocidad de los portadores a través de la función de distribución de velocidad (FDV). Realizaremos este estudio en el régimen de bajas temperaturas para $\mathrm{T}=175 \mathrm{~K}$ (Figura IV.19) y en el de temperaturas elevadas, para $\mathrm{T}=225 \mathrm{~K}$ (Figura IV.20). Nuevamente nos remitimos a la Sección IV.2 para el análisis de la estructura NSD, puesto que el comportamiento es totalmente análogo al diodo back-to-back que analizábamos en la misma (también encontramos el análisis detallado tanto de NSD como de NSD2 en [Pascual, 2009]).

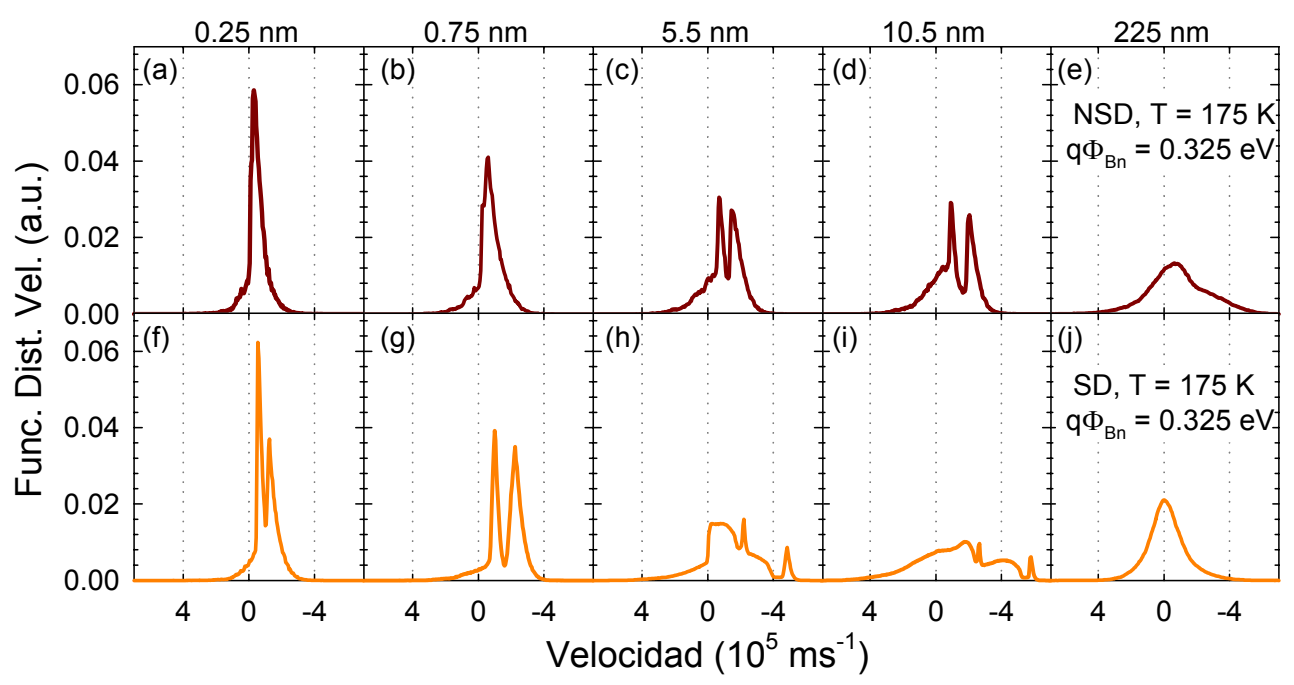

Figura IV.19. Función de distribución de velocidad para las estructuras: .a-e. NSD y.f-j. SD para T=175 K, a $0.25 \mathrm{~nm}, 0.75 \mathrm{~nm}, 5.5 \mathrm{~nm}, 10.5 \mathrm{~nm}$ y $225 \mathrm{~nm}$ del contacto Schottky inversamente polarizado.

Para el diodo con la capa SD los máximos asociados al transporte balístico están presentes incluso a $0.25 \mathrm{~nm}$ del contacto y son desplazados rápidamente a velocidades mayores (compárense las Figuras IV.19.c y IV.19.h, a $5.5 \mathrm{~nm}$ del contacto). Podemos estudiar la balisticidad de este dispositivo a través del número de scattering con fonones sufridos por un electrón en función de la posición (Figura IV.21.b); como vemos, este número es inferior a uno en los primeros nanómetros en las inmediaciones del contacto en inversa. Sin embargo, debido al rápido aumento de la energía en los primeros nanómetros del dispositivo, la acción de los 
mecanismos de scattering es más intensa en la estructura SD que en aquellas en las que no hay capa de dopantes (ver Figura IV.21). Además, aún en el régimen de bajas temperaturas, cuando los electrones han superado la capa SD se encuentran con el cambio de pendiente en el campo eléctrico anteriormente descrito (ver el recuadro interior de la Figura IV.17.d), lo que nos indica que los portadores aún son acelerados pero no de una manera tan extrema como antes. Confirmamos este hecho observando el perfil de velocidad (Figura IV.16.c. y .d.): para la misma posición que en el caso de la estructura NSD los portadores presentan una velocidad inferior (por ejemplo a $70 \mathrm{~nm}$ de la interfaz y para $\mathrm{T}=150 \mathrm{~K}$, la velocidad de los portadores en el diodo NSD es de $10^{5} \mathrm{~m} \mathrm{~s}^{-1}$ y de $0.85 \cdot 10^{5} \mathrm{~m} \mathrm{~s}^{-1}$ en el diodo SD).

El número de mecanismos de scattering aumenta rápidamente según se alejan los portadores del contacto (Figura IV.21.b); de este modo, aquellos electrones que inicialmente eran de tipo balístico presentan ahora un transporte difusivo debido al efecto de los mecanismos de scattering, como se puede ver en la FDV a $225 \mathrm{~nm}$ para la estructura SD (Figura IV.19.j).

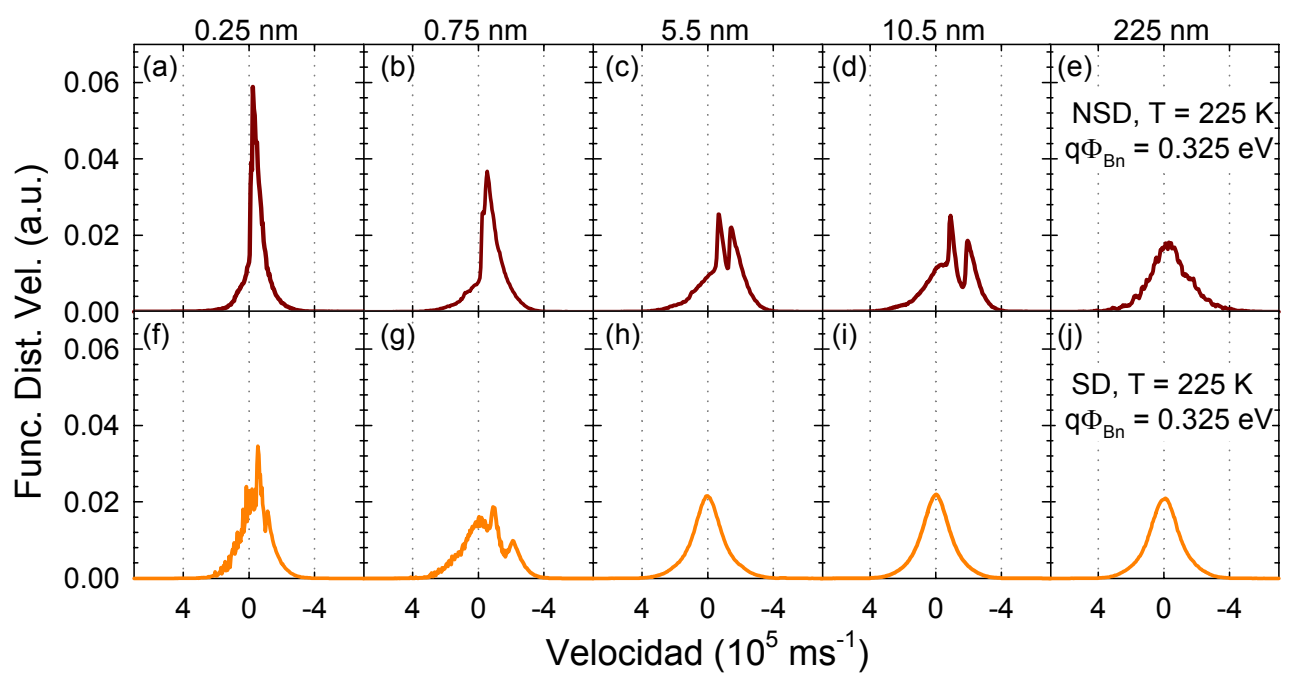

Figura IV.20. Función de distribución de velocidad para las estructuras: .a-e. NSD y.f-j. SD para T=225 K, a $0.25 \mathrm{~nm}, 0.75 \mathrm{~nm}, 5.5 \mathrm{~nm}, 10.5 \mathrm{~nm}$ y $225 \mathrm{~nm}$ del contacto Schottky inversamente polarizado.

En el rango de temperaturas elevadas (véase Figura IV.20, a $225 \mathrm{~K}$ ), para el diodo back-to-back SD (Figura IV.20.h) así como para el NSD2 (no mostrado en las gráficas, véase [Pascual, 2009]) la resistencia del Silicio es superior a la resistencia de la barrera Schottky, mientras que el comportamiento en corriente del diodo NSD aún sigue regido por la ley Schottky para este valor de temperatura (ver Figura IV.14). El comportamiento de la estructura 
NSD es por tanto similar al que nos encontramos para $175 \mathrm{~K}$, aunque con un valor inferior del campo eléctrico, que deriva en unos máximos en la FDV menos prominentes (ver Figura IV.20). Sin embargo, para la estructura SD, la banda de conducción presenta una curvatura bien formada (Figura IV.16.b), con la correspondiente acumulación de portadores en la región junto al contacto (ver Figura IV.17.b). La curvatura de la banda da lugar a una reducción drástica del campo, llegando a cambiar de signo y presentando un máximo a valores positivos, como se puede observar en la Figura IV.17.d a temperaturas superiores a $200 \mathrm{~K}$. Este perfil del campo eléctrico provoca que durante los primeros nanómetros desde la interfaz los electrones sean fuertemente acelerados hacia el interior del dispositivo a través de una barrera de potencial casi transparente. Sin embargo, tan pronto el campo eléctrico cambia de signo, los electrones se ven decelerados, tal y como se ve en el perfil de velocidad (Figura IV.16.d). Cuando los portadores se han alejado lo suficiente de la interfaz, el campo eléctrico tiende a cero, llegando a una situación cuasi-difusiva. En el caso de la estructura NSD2, puesto que la altura de barrera Schottky es inferior, la resistencia del Silicio supera a la Schottky a temperaturas inferiores que en la NSD, presentando antes el comportamiento resistivo [Pascual, 2009].

Llegados a este punto, es interesante comentar las diferencias en el transporte a través de las estructuras SD y NSD2 mediante las funciones de distribución, ya que la corriente a través de ambas estructuras es prácticamente la misma. Mientras que, en la estructura SD, para altas temperaturas los máximos de la FDV relativos a los portadores en los valles longitudinales y transversales aparecen cerca de la unión Schottky, en el diodo NSD2 se llega rápidamente a una situación difusiva ya en los primeros nanómetros del dispositivo (no mostrados en las gráficas). Podemos así afirmar que el transporte balístico se ve favorecido con la inclusión de la capa de segregación de dopantes, incluso a altas temperaturas. Sin embargo, cuando aumenta la temperatura también lo hace la probabilidad de scattering con fonones, provocando una fuerte relajación del momento. Este hecho, combinado con los valores positivos del campo eléctrico en los primeros nanómetros a partir de la interfaz, provoca que los portadores muestren un comportamiento difusivo en una región más cercana al contacto que para temperaturas inferiores. En comparación con el diodo NSD, la estructura que incluye la capa de segregación de dopantes muestra una FDV típicamente difusiva a una menor distancia del contacto (a $5.5 \mathrm{~nm}$, Figura IV.20.h). 

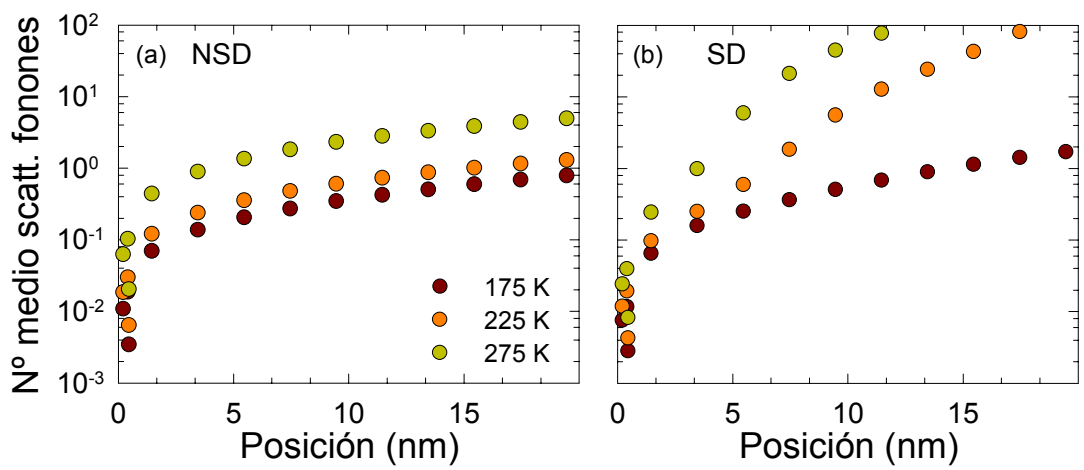

Figura IV.21. Número medio de mecanismos de scattering para $V_{a}=-0.5$ Vy a varias temperaturas $(175 \mathrm{~K}, 225 \mathrm{Ky}$ $300 \mathrm{~K})$ para las estructuras NSD y SD.

A partir de este análisis podemos considerar que, en general, la segregación de dopantes es una técnica adecuada para aumentar la corriente de inyección, promover el trasporte balístico y conseguir barreras efectivas más bajas en estructuras como el SBMOSFET. Debemos mencionar que en los siguientes capítulos, en los que nos centraremos en el análisis de los transistores SB-MOSFET, no hemos tenido en cuenta de manera explícita la inclusión de la capa de dopantes puesto que al tener los contactos de fuente y drenador separados una cierta distancia del contacto de puerta (underlap), la influencia de la capa SD sobre el transporte en el canal conductor debe ser poco relevante, siendo determinante únicamente a la hora de establecer el número de portadores inyectados. Es decir, nos interesa fundamentalmente la altura de la barrera Schottky equivalente que dichos portadores tuvieron que atravesar y por ello puede efectuarse la simulación considerando por simplicidad un contacto Schottky de tipo estándar, sin capa adyacente. 


\section{Bibliografía del Capítulo IV}

Chen B. S. and Chen M. C. "Formation of cobalt silicided shallow junction using implant into/through silicide technology and low temperature furnace annealing", IEEE Trans. Electron Devices, 43, 258 (1996)

Derdour A M, Nobili D and Solmi S, "High-Temperature Equilibrium Carrier Density of Arsenic-Doped Silicon"J. Electrochem. Soc. 138857 (1991)

Dubois E. And Larrieu G., "Measurement of low Schottky barrier heights applied to metallic source/drain metal-oxide-semiconductor field effect transistors”, J. Appl. Phys., 96, 729 (2004)

Duboz J. Y., Badoz P.A., Arnaud F. and Chroboczek J.A., "Electronic transport properties of epitaxial erbium silicide/silicon heterostructures". Appl. Phys. Lett. 55, 84 (1989)

Jacoboni C. y Lugli P. "The Monte Carlo Method for Semiconductor Device Simulation", Viena: Springer (1989)

Kedzierski J., Xuan P. et al., "A 20 nm gate-length ultra-thin body p-MOSFET with silicide source/drain", Superlattices and Microstructures, 28, 445 (2000)

Kinoshita A et al., "Solution for High-Performance Schottky-Source/Drain MOSFETs: Schottky Barrier Height Engineering with Dopant Segregation Technique", VLSI Symp. Technical Digest, 168 (2004)

Knoch J et al., "Effective Schottky barrier lowering in silicon-on-insulator Schottky-barrier metal-oxide-semiconductor field-effect transistors using dopant segregation" Appl. Phys. Lett., 87, 263505 (2005)

Larrieu G. et al., "Low Temperature Implementation of Dopant-Segregated Band-edge Metallic S/D junctions in Thin-Body SOI p-MOSFETs", International Electron Device Meeting, IEDM'07 (Washington), 147 (2007)

Pascual E., Rengel R., Reckinger N., Tang X., Bayot V., Dubois E., Martín M. J., “A Monte Carlo investigation of carrier transport in fabricated back-to-back Schottky diodes: 
Influence of direct quantum tunnelling and temperature" Physica Status Solidi (c), 5, 119 (2008)

Pascual E., Martín M. J., Rengel R., Larrieu G. and Dubois E., "Enhanced carrier injection in Schottky contacts using dopant segregation: a Monte Carlo research", Semicond. Sci. Technol., 24, 025022 [1-6] (2009)

Sze S. M., "Physics of Semiconductor Devices", $2^{\text {nd }}$ Edn. Wiley, New York (1981)

Tsui B-Y and Lu C-P, "Current Transport Mechanisms of Schottky Barrier and Modified Schottky Barrier MOSFETs" Conf. Proc. ESSDERC 07, 307 (2007)

Zhang M., Knoch J., Zhao Q.T., Breuer U., Mantl S. "Impact of dopant segregation on fully depleted Schottky-barrier SOI-MOSFETs" Solid State Electronics., 50, 594 (2006)

Zhang Z., Qiu Z., Liu R., Östling M. and Zhang S. L. "Schottky-barrier height tuning by means of ion implantation into preformed silicide films followed by drive-in anneal" Solid State Electronics., 28, 565 (2007)

Zhu S. et al., "N-Type Schottky Barrier Source/Drain MOSFET Using Ytterbium Silicide" IEEE Elec. Dev. Letters, 25, 565 (2004) 



\section{El Transistor SB-MOSFET}

$\mathcal{L}_{\text {la que las regiones de fuente y drenador están constituidas por metalizaciones, ha }}^{\text {n lonos, la arquitectura MOSFET de barrera Schottky (SB-MOS), en }}$ sido el objeto de un creciente interés como alternativa a las estructuras MOS de Silicio convencionales. Este interés es debido a sus particulares propiedades, como por ejemplo, una menor resistencia de acceso, junto con interfaces fuerte/drenador extremadamente abruptas, permitiendo así mayor escalabilidad y menores efectos de canal corto [Larson, 2006]. Es también importante la eliminación de la acción bipolar parásita propia de dispositivos ultraescalados [Sugino, 1982]. Los SB-MOSFETs ofrecen además numerosas ventajas relacionadas con el proceso de fabricación y sus costes en comparación con la arquitectura CMOS convencional.

En el presente capítulo realizaremos un estudio detallado de este tipo de estructuras bajo régimen de operación estacionario. En primer lugar, llevaremos a cabo una revisión histórica de la evolución del SB-MOSFET para posteriormente analizar el interés de estas estructuras en la actualidad. Describiremos someramente el proceso de fabricación de este tipo de dispositivos y también presentaremos los diversos modelos para el cálculo de la corriente de drenador en el SB-MOSFET que existen en la literatura. Para conocer el modo de operación y las 
características del transporte en este tipo de transistores hemos llevado a cabo un análisis minucioso mediante nuestro simulador Monte Carlo bidimensional; los principales detalles están expuestos en las secciones siguientes, donde evaluamos las características en condiciones estacionarias del SB-MOSFET. Mediante el análisis de las magnitudes internas proporcionadas por el simulador, explicaremos el modo de funcionamiento del dispositivo y describiremos el transporte a través del mismo en los regímenes de triodo y saturación. Finalmente, evaluaremos el efecto que tiene sobre el transporte la variación de diversos parámetros de la estructura, tales como la altura de la barrera Schottky, la longitud de underlap y la longitud de puerta.

\section{V.1. Orígenes históricos}

La idea de reemplazar las islas altamente dopadas de fuente y drenador de un MOSFET convencional por metalizaciones Schottky fue propuesta por primera vez en 1966 por Y. Nishi, que obtuvo la patente algunos años después [Nishi, 1970]. A continuación, M. P. Lepselter y S.
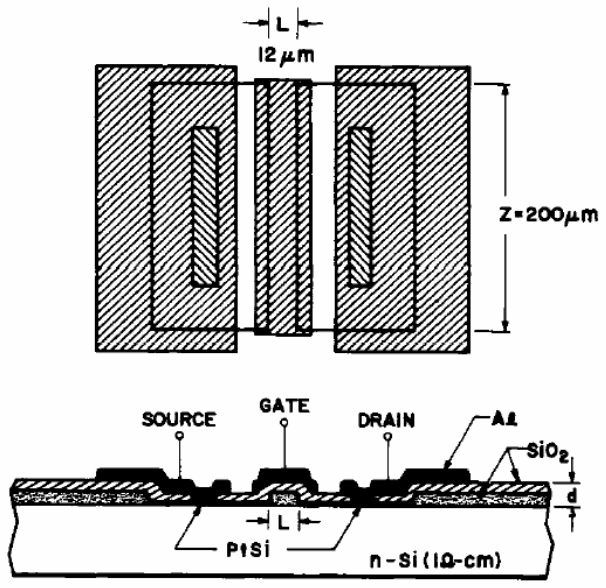

Figura V.1. Figura donde se muestra la geometría del dispositivo y la sección transversal de un MOSFET con contactos Schottky en fuente y drenador [Lepselter, 1968]. M. Sze fabricaron este tipo de estructuras y en 1968 realizaron la primera publicación [Lepselter, 1968], en la que presentaron una estructura $p$ MOS donde los contactos de fuente y drenador estaban compuestos por Siliciuro de Platino (PtSi). Sin embargo el rendimiento del dispositivo era muy bajo, con una corriente a temperatura ambiente diez veces inferior a la del MOSFET convencional.

En general, debido a la escasez de recursos técnicos, los dispositivos experimentales que fueron fabricados en la década de los 70 y 80 no presentaron muy buen rendimiento en términos de corriente comparación con la tecnología convencional; sin embargo, los estudios teóricos publicados en esta época eran muy relevantes. Se estudiaron las diversas ventajas del SB-MOSFET frente al MOSFET convencional, como es la minimización de los efectos de canal corto y la baja resistencia en serie de las regiones de fuente y drenador [Lepselter, 1968]. Pero el problema 
fundamental era como decimos encontrar el modo de solventar el inconveniente de la baja corriente. Ya en 1981, Koeneke et al. demostraron de manera experimental que la distancia entre los contactos de fuente y drenador respecto al electrodo de puerta tiene un fuerte efecto sobre la corriente, por tanto, reduciendo la misma obtuvieron una mejoría en el rendimiento del dispositivo [Koeneke, 1981].

Pero la principal manera de conseguir una mayor corriente es disponer de alturas de barrera Schottky lo menores posibles. Casi todos los metales pueden formar Siliciuros (aleaciones de Silicio y metal) y la mayoría de ellos constituyen alturas de barrera Schottky bajas en Silicio tipo $p$ y altas en tipo $n^{1}$. Este hecho facilitó un mayor desarrollo del estudio de los SB-pMOSFETs [Lepselter, 1968], [Koeneke, 1982]. El principal impulso para la tecnología SB-MOSFET tipo $n$ se produjo en 1981, cuando Tu et al. y Norde et al. [Tu, 1981], [Norde, 1981] demostraron que los Siliciuros de tierras raras producen alturas de barrera Schottky bajas para Silicio tipo $n$ y altas para tipo $p$, como por ejemplo el Erbio, con altura de $0.28 \mathrm{eV}$ en tipo $n$ y $0.73 \mathrm{eV}$ en tipo $p$ [Duboz, 1989]. De este modo, la primera investigación experimental sobre dispositivos SB-nMOS se publicó en 1984 a cargo de Mochizuki y Wise utilizando Tantalio para los contactos Schottky [Mochizuki, 1984].

Por otro lado, Sugino et al. y Swirhun et al. en 1983 fueron los primeros en poner de relieve otra característica importante de la tecnología SB-MOS: al reemplazar las regiones dopadas de fuente y drenador por uniones Schottky se eliminaba la inyección de portadores minoritarios en el sustrato desde la fuente, evitándose así problemas como la acción bipolar parásita. Analizaron una estructura CMOS constituida por un dispositivo SB- $p$ MOS y un $n$ MOS convencional, comprobando la inmunidad del dispositivo al fenómeno de latchup [Sugino, 1982], [Sugino, 1983], [Swirhun, 1985].

Como decíamos anteriormente, los estudios presentados en esta época presentaban gran interés teórico; sin embargo, la fabricación de dispositivos no producía resultados óptimos en comparación con las estructuras convencionales. Ya en 1994 Tucker et al. [Tucker, 1994], [Tucker, 1994b] y Snyder [Snyder, 1996] pusieron de relieve las ventajas del escalado de la arquitectura SB-MOS; la tecnología de los procesos de fabricación de los dispositivos había avanzado, pudiéndose así realizar estudios más precisos. Desde entonces, el uso del SB-

\footnotetext{
${ }^{1}$ Por ejemplo, el Siliciuro de Platino presenta una altura de barrera de $0.22 \mathrm{eV}$ en Silicio tipo $p$ y 0.84 en Silicio tipo $n$ [Maex, 1995]
} 
MOSFET como alternativa al MOSFET convencional ha ido cobrando más fuerza; sin embargo, es necesario aún optimizar los procesos de fabricación y el diseño de los dispositivos, además de buscar materiales adecuados que den lugar a la formación de alturas de barrera lo suficientemente bajas para hacer a esta tecnología competitiva con la convencional.

\section{V.2. Interés del SB-MOSFET, requerimientos y situación actual}

Como ya destacamos en la introducción de este trabajo, la arquitectura SB-MOSFET está llamada a ocupar un importante puesto en la tecnología de semiconductores. Se plantea como posible alternativa a algunos de los problemas que surgen al reducir el tamaño de los dispositivos: las resistencias parásitas de fuente y drenador y las capacidades parásitas. En la Figura V.2 mostramos los objetivos propuestos por el ITRS para estas magnitudes en un plazo de 10 años. Como podemos observar, en el camino planificado para la miniaturización de los transistores, se requiere el decrecimiento exponencial de las resistencias y capacidades parásitas. Sin embargo, en el caso de continuar con la arquitectura MOSFET convencional (regiones de alto dopaje de fuente y drenador), surgen dificultades para cumplir los requerimientos del ITRS debido al aumento de las resistencias y capacidades parásitas con respecto a la parte intrínseca del transistor a medida que la longitud de puerta del transistor se reduce. Por ello, los SB-MOSFETs, que de manera natural tienen resistencias parásitas reducidas, suponen una excelente opción para cumplimentar estas especificaciones.

A la hora de comparar el nivel de rendimiento del MOSFET convencional con el SB-MOSFET para un determinado nodo tecnológico ${ }^{2}$, cabe destacar el trabajo desarrollado por

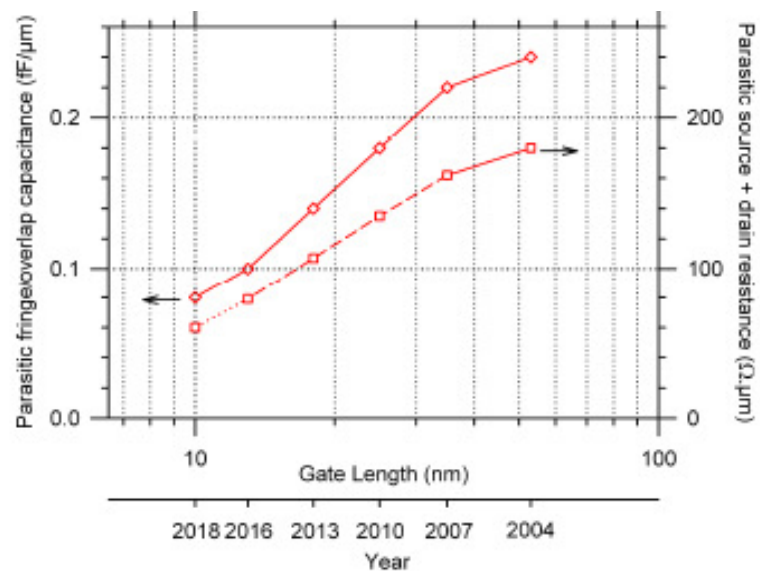

Figura V.2. Capacidades parásitas y resistencias parásitas de fuente y drenados en función de la longitud de puerta publicadas en [ITRS, 2006]. Las líneas continuas indican que existe solución para la reducción de la longitud de puerta, mientras que las discontinuas o de puntos advierten que no habría solución viable de continuar con la tecnología actual

\footnotetext{
${ }^{2}$ Los requerimientos para los sucesivos nodos tecnológicos están establecidos en el ITRS.
} 
Connelly et al. [Connelly, 2003], [Connelly, 2003b] con respecto a las aplicaciones digitales de estos dispositivos. Estos autores llevaron a cabo la simulación de circuitos de cadenas NAND con CMOS de doble puerta de $25 \mathrm{~nm}$, por un lado utilizando transistores con regiones de fuente y drenador metálicas y por otro empleando dispositivos convencionales con estas regiones altamente dopadas, y comparando diversas magnitudes, como el tiempo de retardo o la característica de transferencia. La finalidad de este tipo de estudio es asegurar la capacidad de integración del SB-MOSFET en una aplicación práctica. De acuerdo con estos trabajos es posible llegar a una serie de conclusiones: las regiones de fuente y drenador en una estructura de tipo Schottky deben estar separadas una distancia underlap del contacto de puerta, contrariamente al solapamiento de la longitud de overlap propia de los MOSFETs convencionales. Al aumentar el valor de la altura de barrera Schottky de los contactos, la longitud de underlap más apropiada es menor. Además, las simulaciones muestran que el rendimiento de la estructura con fuente y drenador metálicos con altura de barrera de $0.1 \mathrm{eV}$ es comparable a la tecnología de fuente y drenador dopados con resistividad de $3 \Omega \mu \mathrm{m}^{2}$ en los $\operatorname{contactos}^{3}$. Si la altura de barrera Schottky formada por el metal fuera de $0 \mathrm{eV}$, la arquitectura SB- CMOS sería un 50\% más rápida que la convencional. Por tanto, en este contexto, puede entenderse fácilmente la necesidad de contactos Schottky de altura de barrera muy baja, ya que es la clave para superar en rendimiento a los dispositivos convencionales.

Por otro lado, también es importante mencionar que a medida que la altura de la barrera es menor, se consigue que la corriente de conducción del SB-MOSFET tenga un valor razonable para aplicaciones prácticas en circuitos integrados; sin embargo el ratio entre la corriente de drenador en estado de corte $\left(I_{o f f}\right)$ y conducción $\left(I_{o n}\right)$ se reduce, lo cual no es conveniente. La principal contribución al aumento de la $I_{\text {off }}$ con la reducción de la barrera es debida a la inyección termoiónica de portadores con energías superiores a la barrera Schottky en el electrodo de fuente, que es esencialmente independiente del voltaje aplicado y aumenta con la disminución de la altura de la barrera. Se debe por tanto buscar un compromiso entre el aumento de la $I_{o n}$ y la disminución de la $I_{\text {off }}$ a la hora de seleccionar la altura de la barrera Schottky.

Existen diversas soluciones para mejorar el ratio de la corriente $I_{o n} / I_{\text {off. }}$ En primer lugar, se debe intentar reducir el valor de la $I_{\text {off }}$, la cual se ve afectada en gran medida por el

\footnotetext{
${ }^{3}$ El valor aquí proporcionado para la resistividad de los contactos de fuente y drenador dopados es el que se establece como valor requerido para el nodo tecnológico de 2015 según el [ITRS, 01].
} 
denominado efecto de punch-through, es decir, que existe una corriente de fuga en la parte más profunda del substrato la cual apenas se ve afectada por el voltaje del contacto de puerta. Para contrarrestar este fenómeno, diversos grupos proponen la implementación del diseño de fuente y drenador metálicos sobre un substrato fino de Silicio-Sobre-Aislante (Silicon-on-Insulator, SOI) [Snyder, 1995], [Wang, 1999], [Winstead, 2000], [Calvet, 2002], [Frizte, 2004]. De este modo también es posible mejorar la pendiente subumbral (subthreshold swing SS) [Saitoh, 1999], [Kedzierski, 2000]. El ratio de corriente $I_{o n} / I_{\text {off }}$ también mejora si aumentamos la corriente en conducción con técnicas como la que ya presentamos en el caso de un diodo back-to-back (Capítulo IV), basados en la introducción de una capa de segregación de dopantes en la proximidad del electrodo.

En el caso de estructuras SB-MOSFETs de tipo $p$, se han obtenido excelentes valores de $I_{o n}: 425 \mu \mathrm{A} / \mu \mathrm{m}$ para $-2 \mathrm{~V}$ de voltaje aplicado en la puerta $\left(V_{G S}\right)$ y en el drenador $\left(V_{D S}\right)$, junto con un valor de $I_{\text {off }}$ de $368 \mathrm{nA} / \mu \mathrm{m}$ para longitudes de puerta de $40 \mathrm{~nm}$ [Larrieu, 2005]. Mediante la inclusión de una capa de segregación de dopantes, técnica propuesta por Kinoshita [Kinoshita, 2004], se ha demostrado la optimización de la corriente de conducción $I_{o n}$ en un $50 \%$ con respecto a las estructuras sin dicha capa dopada [Larrieu, 2007]. En la Figura V.3 mostramos el estado del arte actual del ratio $I_{o n} / I_{\text {off }}$ para este tipo de estructuras.

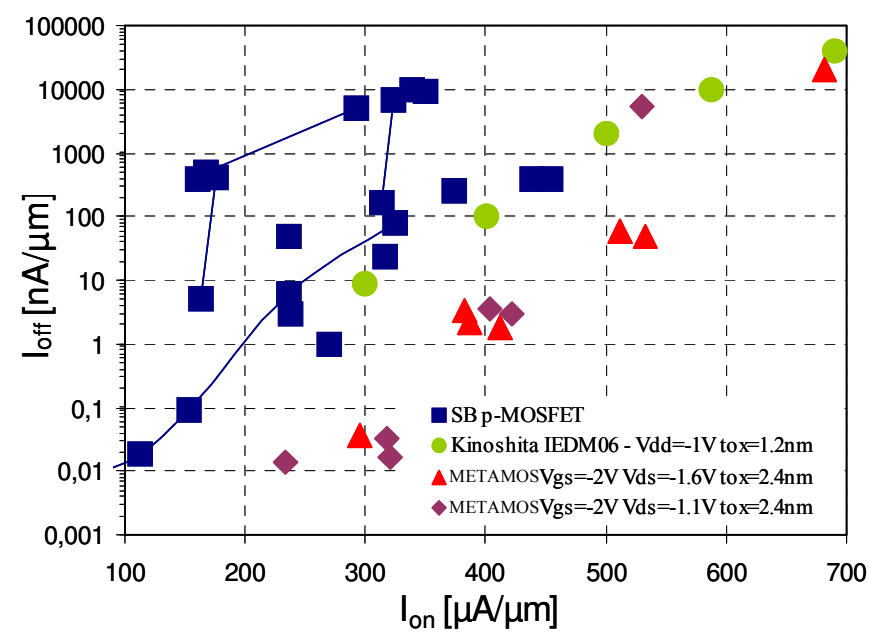

Figura V.3. Ratio $I_{o n} / I_{\text {off }}$ de SB-MOSFETs existentes en literatura. Comparación del estado del arte con resultados recientes presentados por Spinnaker [Fritze, 2004], [Larson, 2004b], [Larson, 2006] y los fabricados dentro del proyecto METAMOS [Dubois, 2004], [Larrieu, 2004], [Larrieu, 2005].

Con respecto a los SB- $n$ MOSFETs nos encontramos en la literatura con SB-MOSFETs como el presentado por M. Jang et al., (de $50 \mathrm{~nm}$ de longitud de puerta) con valores de $I_{\text {off }}$ inferiores a 
$10^{-4} \mu \mathrm{A} / \mu \mathrm{m}$ y corriente on de $120 \mu \mathrm{A} / \mu \mathrm{m}\left(V_{G S}=3 \mathrm{~V}, V_{D S}=1 \mathrm{~V}\right)$ [Jang, 2004]. Más recientemente, han sido publicados resultados para estructuras de longitudes de puerta de $5 \mu \mathrm{m}$ [Tsui, 2007] y $0.17 \mu \mathrm{m}$ [Wong, 2008], que presentan valores de corriente de conducción de aproximadamente $5 \mu \mathrm{A}\left(V_{G S}=2.5 \mathrm{~V}, V_{D S}=1 \mathrm{~V}\right)$ y $100 \mu \mathrm{nA} / \mu \mathrm{m}\left(V_{G S}=V_{D S}=1.2 \mathrm{~V}\right)$ respectivamente, ambos con inclusión de capa de segregación de dopantes. Sin embargo, optimizando la técnica experimental de la segregación de dopantes mediante LSA (laser spike annealing), Awano et al. han presentado un SB-MOSFET tipo $n$ con valores de corriente de conducción record: $I_{o n}=1310 \mu \mathrm{A} / \mu \mathrm{m}$ frente a $I_{\text {off }}=100 \mathrm{nA} / \mu \mathrm{m}$ (longitud de puerta de $40 \mathrm{~nm}$, $\left.V_{G S}=V_{D S}=1 \mathrm{~V}\right)$ [Awano, 2008].

\section{V.3. Proceso de fabricación de SB-CMOS y principales características de la arquitectura SB-MOS frente la convencional}

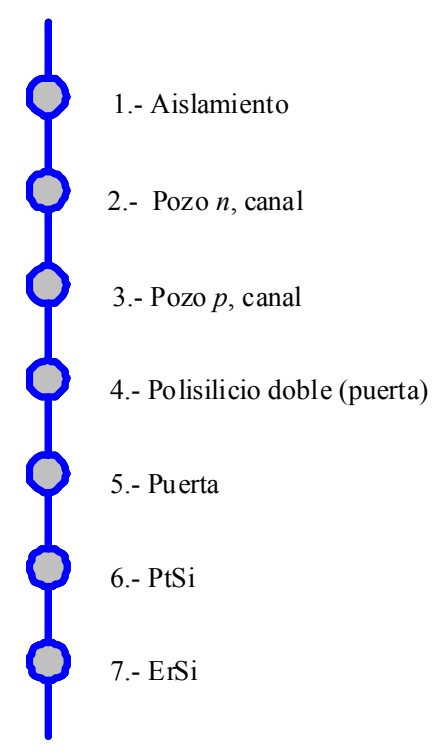

Figura V.4. Proceso de fabricación seguido para un dispositivo CMOS de barrera Schottky.
La tecnología necesaria para el proceso de fabricación de estructuras SB-CMOS es totalmente compatible con la tecnología CMOS de Silicio. A continuación presentamos un posible ejemplo de los pasos a seguir para fabricar un transistor MOSFET de barrera Schottky (Figura V.4) integrado en una estructura SB-CMOS como la presentada en la Figura V.5 [Larson, 2004].

En primer lugar se realiza el aislamiento de la oblea mediante técnicas como LOCOS (LOCal Oxidation of Silicon, oxidación local de Silicio). En los pasos 2 y 3 se realizan implantaciones estándar de los pozos de aislamiento y de los canales de dopaje. A continuación, en el paso 4, se hace uso de una única máscara de doble dopaje de polisilicio para realizar las puertas tipo $n$ y $p$ de los transistores $n$ MOS y $p$ MOS respectivamente; también es posible utilizar metal para el material de puerta. En el siguiente paso se lleva a cabo una litografía estándar y tecnología de grabado para la puerta. Finalmente, 
cuando se utilizan Siliciuros complementarios como en este ejemplo, es necesario emplear una máscara doble de Siliciuro para formar las regiones de fuente y drenador de los dispositivos $p$ MOS y $n$ MOS: PtSi para el primero y ErSi para el segundo (pasos 6 y 7). Es importante mencionar que la máxima temperatura de recocido requerida para la reacción de Siliciuración es inferior a $600^{\circ} \mathrm{C}$.

En el proceso descrito se utilizan alrededor de tres máscaras menos que en el caso del CMOS convencional, reduciéndose así notablemente los costes de fabricación.

Por otro lado, el hecho de que la temperatura máxima del proceso sea inferior a los $600{ }^{\circ} \mathrm{C}$ es una ventaja muy importante que facilita la integración de diversos materiales que realcen las características de los dispositivos CMOS, tales como aislantes de alta permitividad dieléctrica para la puerta, puertas metálicas o Silicio strained para el canal. Las propiedades de dichos materiales tienden a degradarse si se emplean temperaturas elevadas en el proceso de fabricación, por lo que su uso en conjunción con metalizaciones Schottky para los contactos de fuente y drenador parece natural.

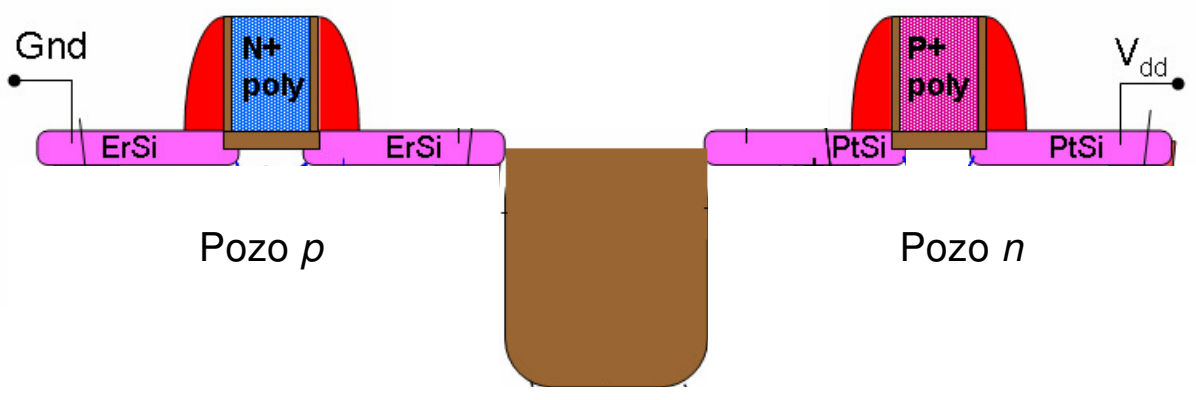

Figura IV.5. Representación esquemática de una estructura SB-CMOS [Larson, 2004b]

Las regiones de fuente y drenador en el SB-MOSFET, al contrario de las convencionales, constituyen una interfaz atómicamente abrupta con el sustrato y el canal, lo cual favorece el escalado del dispositivo en el régimen inferior a los $10 \mathrm{~nm}$. Además presenta excelentes valores de resistencia sheet con el canal (PMOS: $6 \Omega / \mathrm{sq}$; NMOS: $60 \Omega / \mathrm{sq}$ ) y resistencia de contacto (PMOS: $1.6 \cdot 10^{-9} \Omega \mathrm{cm}^{2}$; NMOS: $8 \cdot 10^{-9} \Omega \mathrm{cm}^{2}$, ambos valores son inferiores a los previstos por el ITRS para el 2016, ver Tabla 0.1 en el capítulo de Introducción) [Larson, 2004]. Se eliminan además, con respecto a las regiones de fuente y drenador dopados del MOSFET convencional, problemas de latchup y efecto bipolar parásito [Sugino, 1983], como ya comentábamos en la primera sección de este capítulo. 
Otro factor diferenciador de la arquitectura SB-MOS es que la puerta no se encuentra superpuesta a las regiones de fuente y drenador, sino que se encuentran separadas una cierta longitud de underlap, en contraposición con el overlap (solapamiento) típico de los MOSFET convencionales.

Por otro lado, mientras que en un MOSFET convencional el dopaje del canal es elevado, en el SB-MOSFET, se encuentra muy poco dopado. Esto se debe a que (además de un mejor comportamiento inherente ante el efecto de canal corto) gracias a la barrera Schottky, que actúa de manera similar a un pocket o halo, es posible controlar así la pérdida de corriente subumbral. De este modo podemos eliminar las implantaciones de halos o pockets de la estructura del dispositivo y del proceso de fabricación [Larson, 2004].

Con respecto al transporte de portadores en el interior del canal, en un MOS convencional es de tipo superficial, viéndose fuertemente afectado por el scattering de superficie del óxido de puerta. Esto implica una reducción notable de la movilidad efectiva en el canal. Sin embargo, en los dispositivos SB-MOS el movimiento los portadores de fuente a drenador se realiza también en el volumen del canal y no exclusivamente bajo la puerta. Este factor explica que el efecto del scattering de superficie sea despreciable cuando el dispositivo se encuentra en el estado on, tal y como afirman Winstead et al. [Winstead, 2000]; sin embargo esta afirmación no puede extenderse a todo el rango de voltajes aplicados en drenador, como veremos en la sección de resultados. Se puede afirmar por tanto que, junto con una elección de dopaje bajo del canal, la arquitectura SB-MOS mejora la movilidad de los portadores en el interior de los dispositivos [Larson, 2004].

\section{V.4. Estado del arte del modelado de transistores SB-MOSFET}

En la literatura, aunque encontramos diferentes modelos relacionados con el estudio del SB-MOSFET, la mayoría de ellos no van más allá del análisis de la corriente frente al voltaje de puerta y drenador. A continuación comentaremos de manera sucinta los principales detalles de estos modelos, así como las referencias bibliográficas más importantes que han tratado el estudio del SB-MOSFET en los últimos años.

En primer lugar analizaremos aquellos trabajos en los que se emplean también simuladores Monte Carlo para el modelado de SB-MOSFETs. Existen muy pocas referencias en 
este campo, debido presumiblemente a la dificultad que entraña el desarrollo de este modelo, estando en su mayoría centradas en el estudio de las curvas I-V. El trabajo más relevante es el presentado por Winstead y Ravaioli [Winstead, 2000] de la Universidad de Chicago. En este artículo, los autores desarrollan una simulación Monte Carlo de las características estáticas de un transistor SB-MOSFET tipo $p$, usando la aproximación de la matriz de transferencia de Airy para determinar el coeficiente de transmisión cuántico. El análisis está principalmente limitado a la presentación de las características I-V y los perfiles de corriente de inyección a través de la unión Schottky, proporcionando sin embargo escasa información acerca de los procesos responsables del transporte en dichos dispositivos.

Otro artículo importante es el presentado por Sun y sus colaboradores [Sun, 2003], de la Universidad de Pekín. En este trabajo estudian en primer lugar un diodo Schottky, como ya vimos en el Capítulo III. Además muestran algunos resultados para SB-MOSFETs tipo $n$ de barreras Schottky elevadas (superiores a $0.40 \mathrm{eV}$ ), pero centrándose en las características de transferencia y de salida del dispositivo. Del mismo grupo de investigación encontramos además otros trabajos relacionados con los transistores SB-MOSFET: [Du, 2004], [Xia, 2005], [Sun, 2006], [Zeng, 2009] y [Zeng, 2010]. El primero de los artículos consiste en un escueto informe sobre los resultados de la simulación Monte Carlo de un SB-MOSFET de doble puerta (tipo $n$ ). En este caso, además de mostrar la característica de transferencia, presentan la distribución bidimensional de diversas magnitudes internas (potencial, densidad de electrones y velocidad). También representan algunas de estas magnitudes de manera lineal a lo largo del canal (en el punto medio del eje vertical del canal); sin embargo es importante destacar que en nuestro caso, las magnitudes 1D que mostraremos en este capítulo, y para dar una mayor agilidad a la presentación de los resultados, están obtenidos mediante las magnitudes 2D (x,y) pero pesadas por el valor de la concentración a lo largo del eje vertical de modo que se obtiene un adecuado valor de las mismas a lo largo del canal (ver Capítulo II, Sección II.3.i). Por otro lado, el siguiente artículo que aquí mencionamos ([Xia, 2005]) describe la metodología seguida para realizar un análisis de las características dinámicas de los SB-MOSFETs mediante el simulador Monte Carlo. Presentan la variación de diversas magnitudes con la tensión aplicada en la puerta (la frecuencia de corte, la transconductancia, las capacidades y los tiempos de tránsito y de retardo). Debe tenerse en cuenta que en este trabajo, la determinación de algunas de estas magnitudes, como por ejemplo el tiempo de tránsito, se realiza integrando el cociente de la distancia recorrida por los portadores en el eje longitudinal y la componente longitudinal de la velocidad. Sin embargo, en la presente Memoria aprovecharemos toda la potencialidad del 
simulador Monte Carlo evaluando el movimiento bidimensional de cada portador que entra en la región de conteo (como ya explicamos en el Capítulo II, Sección II.3.f). Finalmente Zeng analiza el transporte en un SB-MOSFET ambipolar en [Zeng, 2009], estudiando los cuatro modos de operación posibles; los resultados que presenta son diversas características tensióncorriente, la distribución del potencial a lo largo del canal y la concentración de portadores en el mismo; destaca la importancia del efecto túnel en este tipo de transistores. En [Zeng, 2010] se ha simulado un SB-MOSFET con capa de segregación de dopantes; con el fin de optimizar estos dispositivos, se estudia la dependencia de la altura de la barrera Schottky efectiva con la anchura de esta capa; también muestra características tensión-corriente, la velocidad de los portadores en el canal, y el valor de la corriente y de la velocidad en las regiones de fuente y drenador en función de la anchura de la capa de segregación de dopantes.

Por otro lado, también encontramos en la literatura diversos trabajos en que simulan las estructuras SB-MOSFET mediante otros métodos numéricos. En primer lugar mencionaremos el trabajo de Huang et al., de la Universidad de Maryland, College Park [Huang, 1998]. Estos autores realizan simulaciones bidimensionales basadas en un bucle autoconsistente para resolver la ecuación de Poisson y determinar la corriente a través del dispositivo (partiendo de un potencial inicial hipotético y calculando la carga a partir del mismo). Los fenómenos de emisión termoiónica y efecto túnel son incorporados como una condición de contorno en el cálculo de la corriente (utilizan la aproximación WKB para el cálculo de la probabilidad de transmisión). Los resultados se centran en la discusión de perfiles de la banda de conducción para diversos voltajes de puerta y en las características de salida y transferencia.

Otros trabajos han afrontado el estudio de los dispositivos SB-MOSFET mediante un simulador basado en la resolución de la ecuación de Poisson acoplada de manera autoconsistente con la ecuación de Schrödinger (usando para ello el formalismo de las funciones de Green detallado en [Datta, 2000] y [Knoch, 2002]). En el caso de Guo et al., de la Universidad de Purdue (West Lafayette, USA ) [Guo, 2002] resuelven la ecuación de Poisson en dos dimensiones y llevan a cabo el análisis de un SB-MOSFET de doble puerta tipo $n$. Muestran la característica de salida así como el perfil longitudinal de la banda de conducción para diversos valores de altura de barrera y comparan estos resultados con los de un MOSFET convencional de igual geometría. En este trabajo no se consideran efectos tales como la reducción de la barrera por las cargas imagen ni el scattering con fonones. 
Encontramos además otros trabajos que utilizan un simulador similar al anterior, aunque con la diferencia de que resuelven la ecuación de Poisson de manera unidimensional; hablamos del trabajo elaborado por Knoch et al., del Centro de Sistemas Nanoelectrónicos para la Tecnología de la Información, en Jülich (Alemania). Mediante este simulador han llevado a cabo el estudio de diversos dispositivos. En primer lugar destacamos el artículo [Knoch, 2002], en el que simulan un SOI SB-MOSFET y estudian la influencia de la anchura del canal en la corriente del dispositivo; muestran además resultados para la banda de energía y la transconductancia variando este parámetro. También han llevado a cabo el estudio del impacto de la técnica de segregación de dopantes en SB-MOSFETs en [Knoch, 2005] y [Zhang, 2006]. En estos artículos se centran principalmente en las características de salida y transferencia de dichos dispositivos, además de en el cálculo de la barrera Schottky efectiva. Por último, mencionamos el estudio de un SB-MOSFET tipo SOI, en el que realizan un análisis similar al de [Knoch, 2002], es decir, sobre el efecto de la anchura del canal (mostrando curvas I-V y la variación de la transconductancia con dicho parámetro).

Es también interesante mencionar la investigación presentada por Kedzierki et al. Han desarrollado un algoritmo numérico sencillo para el cálculo de las corrientes basado en un modelo de transmisión simplificado y ajustado con datos experimentales, en el que se calcula la corriente a partir de la altura de barrera Schottky efectiva; tiene en cuenta la reducción de la altura de la barrera por el efecto de las cargas imagen y la influencia del efecto túnel [Kedzierski, 2000], [Kedzierski, 2000b]. Los resultados que presentan en este trabajo son principalmente características de salida y transferencia para estructuras tipo $n$ y tipo $p$ con distintas longitudes de puerta.

Recientemente ha sido presentado por Su-Zhen et al. [Su-Zhen, 2008] un modelo cuántico compacto del transporte de corriente en estas estructuras. Considerando el potencial en el canal como un pozo rectangular infinito y una barrera infinita en la interfaz Silicio/óxido a lo largo del eje vertical, calcula las autoenergías teniendo en cuenta la corrección a primer orden impuesta por la teoría de perturbaciones independiente del tiempo. El coeficiente de transmisión es calculado a través de la aproximación WKB. En este trabajo analizan un SB-MOSFET de doble puerta, siendo los principales resultados presentados los siguientes: representan diferentes niveles de energía en función de la anchura del canal junto con resultados de un simulador comercial, mostrando que los efectos cuánticos son despreciables para anchuras de canal 
superiores a 10nm. Además, presentan la variación de la densidad de carga media en función del voltaje en puerta aplicado y de las características de transferencia para distintas anchuras del canal de Silicio y diferentes valores de altura de barrera.

Otros autores llevan a cabo la simulación de transistores SB-MOSFET utilizando condiciones de contorno sencillas. Por ejemplo, Jang et al., del Semiconductor and Basic Research Laboratory y la Universidad de Seúl, en Korea [Jang, 2004] han desarrollando un método numérico basado en la continuidad de la corriente túnel en la barrera Schottky y la corriente en el canal. Mediante la continuidad de la corriente calculan la caída de potencial en el dispositivo y además obtienen las características corriente-voltaje (salida y transferencia) para transistores SB-MOSFETs tipo $n$ con distintas alturas de barrera.

Por otro lado, Vega del Instituto de Tecnología de Rochester en Nueva York, [Vega, 2006], [Vega, 2006b] presenta un método numérico unidimensional para el estudio de SB-MOSFETs. Se basa en el modelo de bandas de Lui et al. [Lui, 1986] junto con la aproximación de la matriz de transferencia de Airy para la resolución de la ecuación de Schrödinger [Brennan, 1987]. Los cálculos del modelo se han sido efectuados mediante MATLAB 7. El estudio que presenta de estructuras SB-MOSFETs tipo $p$ y $n$ se centra en las características de transferencia de los dispositivos, estudiando el efecto de la variación de la temperatura y la altura de la barrera [Vega, 2006]. Además lleva a cabo una comparación de las características I-V proporcionadas mediante el método de Airy y la aproximación WKB [Vega, 2006b]. Sin embargo, es importante señalar que en estos artículos sólo se considera una matriz de transferencia, lo que hace que el modelo no sea adecuado para evaluar el coeficiente de transmisión en barreras no lineales; en segundo lugar, la anchura de la barrera es determinada mediante el punto de retroceso (donde la energía es igual al potencial), despreciando así las reflexiones cuánticas de la región donde la energía es superior al potencial. Además, realiza una definición errónea del vector de onda, que combinado con una derivada incompleta de las funciones de Airy da lugar a un coeficiente de transmisión de un orden de magnitud correcto aunque inexacto por definición.

Fuera del ámbito de los transistores de Silicio, Jiménez et al. [Jiménez, 2006], [Jiménez, 2008] han desarrollado modelos de corriente para transistores FET de barrera Schottky con nanotubos de carbono y nanohilos de grafeno. En estos trabajos se muestra la 
característica tensión-corriente para dispositivos prototipo, mostrando un gran acuerdo con un modelo de corriente basado en las funciones de Green.

Dentro del campo de los simuladores comerciales, el más empleado es el DESSIS, una herramienta TCAD. El software ISE TCAD es un paquete de herramientas de litografía, dispositivos y simulación de circuitos del Integrated System Engineering -ISE-. DESSIS es la herramienta para la simulación multidimensional de dispositivos. El tratamiento de las corrientes en los contactos de fuente y drenador implementado está basado en el modelo túnel de [Ieong, 1998] que utiliza la aproximación WKB. Entre otros, los siguientes autores utilizan esta herramienta de simulación: [Matsuzawa, 2000], [Connelly, 2003], [Saha, 2005], [Xiong, 2005], [Sun, 2006], [Ding-Yu, 2007], [Vega, 2008] y [Valentin, 2008].

Matsuzawa et al., pese haber desarrollado un simulador Monte Carlo para el estudio de diodos Schottky (como vimos en el Capítulo III) realizan el estudio del transistor SB-MOSFET tipo $n$ con un simulador comercial. Llevan a acabo únicamente una comparativa a nivel de corriente con un MOSFET convencional.

Connelly et al., de "Acorn Technologies", Palo Alto (USA) simulan circuitos de cadenas NAND con CMOS de doble puerta, con regiones de fuente y drenador metálicas y por otro con estas regiones altamente dopadas. Los principales resultados de este artículo ya fueron expuestos en la Sección V.2.

Sun et al. y Ding-Yu et al. realizan un estudio de estructuras SB-MOSFET novedosas, aunque en esta ocasión no utilizan el simulador Monte Carlo, sino el DESSIS. En el primero de los artículos se presenta un transistor SB-MOSFET asimétrico (de canal $n$ ), con alturas de barrera Schottky en los contactos de fuente y drenador distintas, mientras que en el segundo trabajo el Siliciuro de los contactos de fuente y drenador se diseña sobre metal enterrado (de este modo se forman dos barreras Schottky distintas entre las capas de Siliciuro y el canal de Silicio, en la parte superior y en la inferior del canal). En ambos artículos realizan la comparativa con un SB-MOSFET convencional, pero únicamente a nivel de características tensión-corriente.

Saha et al., del Departamento de Electrónica \& ECE en Kharagpur (India) presentan un estudio de las características dinámicas de un SB-MOSFET tipo $n$. Los resultados principales mostrados en este trabajo son (además de las características I-V) la frecuencia de corte, el tiempo de retardo y la figura mínima de ruido en función de la longitud de puerta.

Xiong et al., de la Universidad de California presentan los resultados de la simulación de un SB-MOSFET de doble puerta tipo $n$. Estudian la variación de las características de 
transferencia con la altura de la barrera (y el ratio $I_{o n} / I_{o f f}$ ). Analizan también la influencia de la anchura del canal sobre la corriente de los dispositivos. Comparan estos resultados para transistores con canal de Silicio y de Germanio, dando mejores resultados los primeros.

Vega ha presentado, además del trabajo anteriormente descrito, un estudio comparativo entre un transistor SB-MOSFET con capa de segregación de dopantes y un MOSFET convencional de contactos de fuente y drenador elevados (ambos de doble puerta), nuevamente centrado principalmente en las corrientes de los dispositivos. En esta ocasión utiliza un simulador comercial (TCAD), que emplea la aproximación WKB para el estudio del efecto túnel.

A continuación comentaremos la investigación publicada en [Valentin, 2008], la cual ha sido desarrollada también dentro del proyecto METAMOS. Estos autores han llevado a cabo un estudio de las características dinámicas de un transistor SB-MOSFET tipo $p$ de topología similar al que nosotros estudiamos en este trabajo. Algunos de los principales resultados mostrados son la variación de diversas magnitudes con el voltaje en puerta aplicado, como la frecuencia de corte, la transconductancia y las capacidades. Además evalúan la influencia de la altura de la barrera sobre dichas magnitudes. Como veremos posteriormente en este capítulo, este simulador no considera la corriente de absorción túnel; sin embargo, y dado que este estudio se realiza con el dispositivo en saturación, esta deficiencia no supone graves consecuencias. De hecho, los resultados obtenidos para frecuencia de corte, transconductancia y demás magnitudes son cualitativamente similares a los que obtenemos mediante la simulación Monte Carlo como comentaremos en secciones posteriores.

Otro tipo de simulador comercial frecuentemente empleado es el MEDICI. Sheng-Pin Yeh et al. de la Universidad de Taiwan [Yeh, 2009] lo utilizan para estudiar el ruido en transistores SOI SB-MOSFETs de canal tipo $n$, comparando los resultados con el de un MOSFET convencional. Además de las características I-V de ambos dispositivos y su banda de conducción, muestra la corriente de ruido normalizada $\left(S_{I d} / I_{d}^{2}\right)$ en función de la frecuencia y de la corriente de drenador.

Los trabajos aquí mencionados configuran principalmente el estado actual de la simulación de los transistores SB-MOSFET. Como vemos, se trata de una temática aún poco desarrollada, centrada fundamentalmente en el estudio de las características tensión-corriente y de puntualmente algunos otros parámetros de interés. En general, los modelos numéricos existentes en la literatura no ofrecen información detallada acerca de la física interna ni del 
origen del comportamiento de las características estáticas. Es aquí donde el trabajo presentado en esta Memoria cobra particular relevancia, al ser la primera vez en la que se explotan las potencialidades del método de Monte Carlo para el estudio detallado de los transistores SB-MOSFET, no sólo desde el punto de vista estático, sino también del comportamiento dinámico y del ruido electrónico a alta frecuencia, como veremos en el Capítulo VI.

\section{V.5. Simulación Monte Carlo de un transistor SB-MOSFET: Características estáticas}

En la presente sección, analizaremos los resultados obtenidos de la simulación de un transistor SB-MOSFET de canal $n$ en modo de acumulación fabricado sobre sustrato SOI. En la Figura V.6 presentamos la topología del dispositivo simulado, que está basado en las estructuras fabricadas dentro del proyecto METAMOS [Valentin, 2008]. Este proyecto está financiado por el sexto programa Marco de la Comisión Europea, en el que los miembros integrantes son grupos pertenecientes al CNRS (Francia), la Universidad de Salamanca (España), la Université Catholique de Louvain (Bélgica), el I.T.E. (Polonia) y ST Microelectronics y Philips Semiconductors. Los objetivos principales del proyecto son la fabricación, caracterización y optimización de MOSFETs de barrera Schottky de modo que puedan ser una alternativa real para resolver los problemas críticos asociados a la arquitectura convencional de fuente y drenador dopados.

Como podemos ver en la Figura V.6, en cuanto a la dimensión vertical, se trata de una estructura metal-óxido-semiconductor sobre sustrato SOI, mientras que en horizontal se corresponde con el esquema ya analizado en el Capítulo IV para los diodos back-to-back. Por tanto, la topología es similar a la de un SOI-MOSFET convencional en el que los contactos óhmicos y las islas dopadas de fuente y drenador hubieran sido reemplazados por uniones Schottky. Sin embargo, tal y como hemos expuesto en la introducción de este capítulo, en este caso las regiones Schottky de fuente y drenador no se encuentran solapadas con la puerta, sino separadas una distancia (underlap) que denominaremos $L_{u n}$. En la Tabla V.1 detallamos las principales características de la geometría del dispositivo, como la altura de la barrera, la longitud de underlap y la longitud de puerta. En posteriores secciones evaluaremos el efecto de la variación de algunos de estos parámetros sobre el transporte de carga en el dispositivo. 


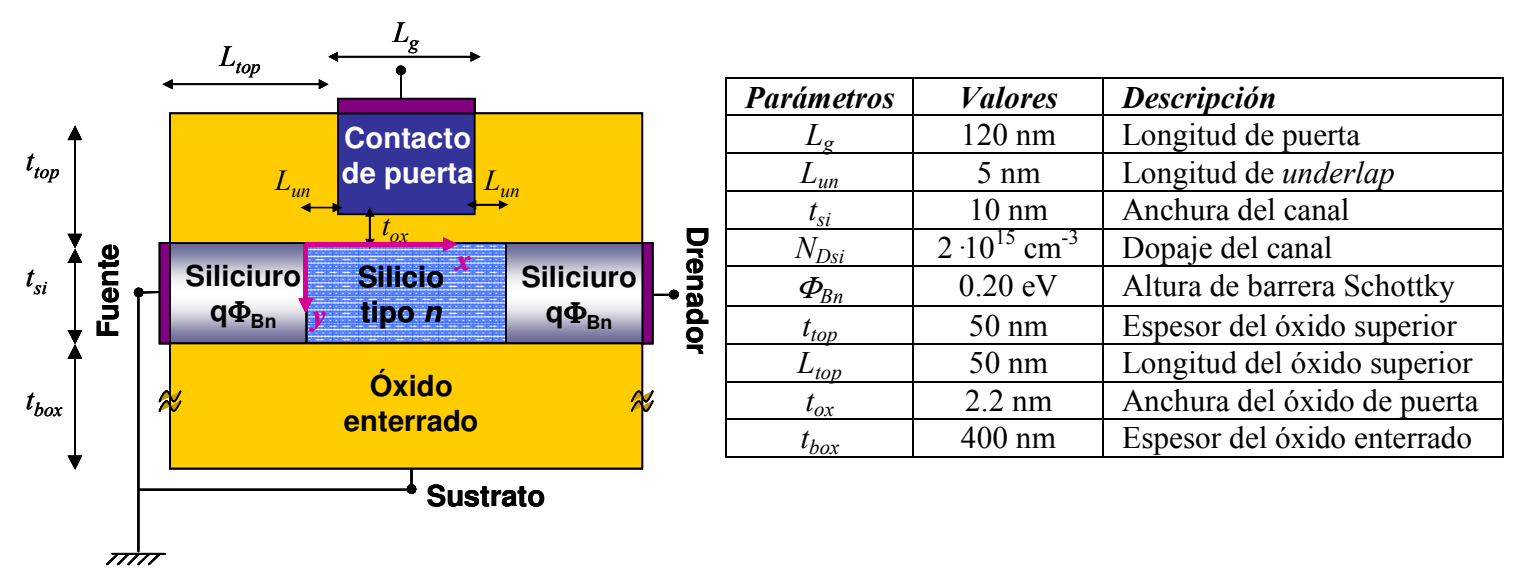

Figura V.6. Geometría del transistor SB-MOSFET.

Tabla V.1. Principales parámetros considerados en la estructura simulada.

En el interior de la Figura V.6 podemos observar mediante los ejes coordenados cómo la dimensión $x$ corresponde con la dirección paralela al canal (que a su vez coincide con la dirección cristalográfica $<100>$ ). Situamos su origen de coordenadas en el contacto Schottky entre la fuente y el substrato, con sentido positivo hacia el drenador. La dirección y es perpendicular al canal, con origen en la interfaz óxido-semiconductor (sentido positivo hacia el contacto de substrato).

Con el fin de resolver la ecuación de Poisson del modo más adecuado, los parámetros de la simulación Monte Carlo bidimensional han sido convenientemente escogidos y son los siguientes: el paso temporal elegido es de $1 \mathrm{fs}$ y el tamaño del mallado en el canal es de $0.5 \mathrm{~nm}$ en la dirección vertical y de $1 \mathrm{~nm}$ en la dirección horizontal; en el óxido de puerta hemos considerado un tamaño inferior del mallado, mientras que en el óxido enterrado aumentamos el tamaño de las mallas de manera gradual hacia el substrato con el fin de reducir el tiempo de CPU. Dado que el perfil de concentración de portadores muestra una gran variación con la polarización, con el fin de buscar una resolución estadística similar para todas las polarizaciones, se ha variado el EPE de modo que el número medio de partículas de la simulación sea 50000 aproximadamente; por ejemplo en el caso de la característica de transferencia de $V_{D S}=2 \mathrm{~V}$ el EPE varía desde 1 cuando $V_{G S}=0 \mathrm{~V}$ a $7 \cdot 10^{4}$ cuando $V_{G S}=2 \mathrm{~V}$. Todos nuestros cálculos han sido realizados para una temperatura de $300 \mathrm{~K}$. 


\section{V.5.a Características tensión-corriente}

En la Figura V.7.a presentamos la característica de transferencia de la estructura simulada para un voltaje aplicado entre las islas de fuente y drenador $\left(V_{D S}\right)$ igual a $2 \mathrm{~V}$. Podemos calcular de manera gráfica el potencial umbral $\left(V_{T}\right)$ del dispositivo a través de la característica de transferencia. Para ello debemos tener en cuenta la relación entre la corriente de saturación y el voltaje en puerta aplicado en un MOSFET [Rengel, 2002], [Ong, 1984]. El valor del voltaje umbral, $V_{T}$, obtenido para la estructura simulada es de $0.7 \mathrm{~V}$. La función trabajo del metal de puerta, $\Phi_{M}$, puede calcularse a partir del voltaje umbral [Sze, 1981], [Pierret, 1994]; en nuestro caso el valor de $\Phi_{M}$ para el metal considerado en nuestras simulaciones sería $4.9 \mathrm{eV}$ aproximadamente.

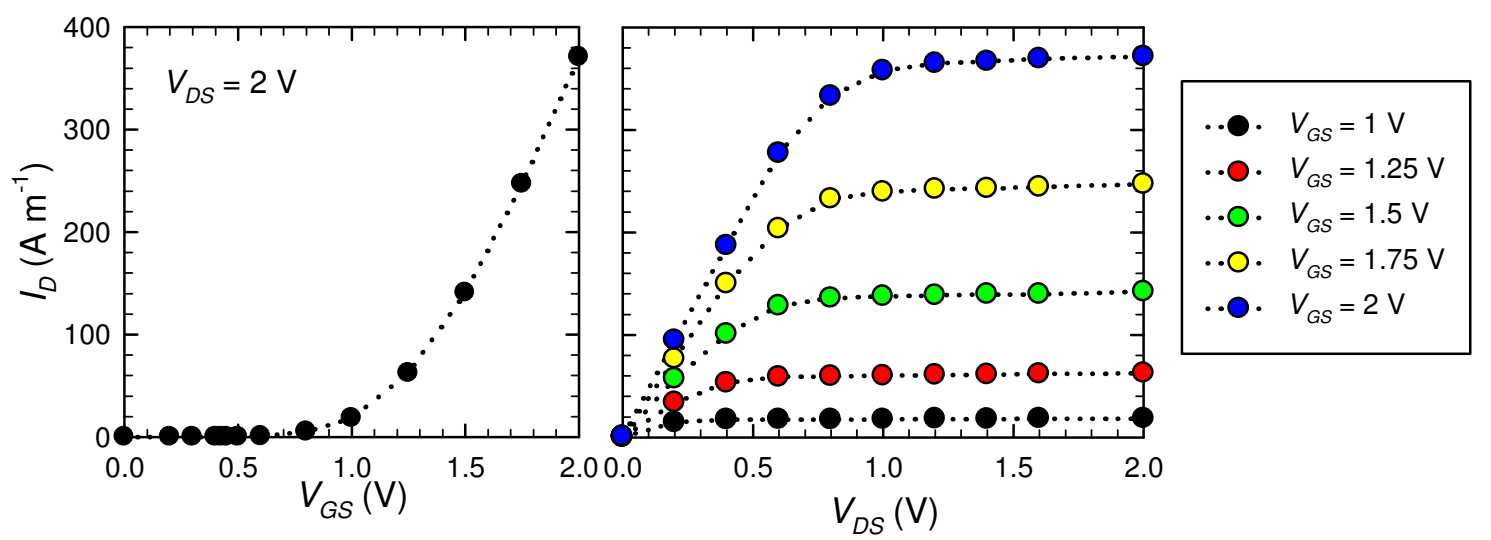

Figura V.7.a. Característica de transferencia para $V_{D S}=2 V y$.b. característica de salida para varios valores de $V_{G S}$.

La Figura V.7.b muestra las características de salida para diversos valores de voltaje en puerta aplicados $\left(V_{G S}\right)$, permitiéndonos observar una saturación de la corriente a partir de un cierto valor de $V_{D S}$ de modo análogo a como sucede en un MOSFET convencional.

En la V.8.a presentamos la comparación de las características de salida obtenidas mediante nuestro simulador Monte Carlo bidimensional (símbolos) y el simulador Atlas/Silvaco (líneas continuas). Los resultados del simulador ATLAS nos han sido facilitados a través de nuestra participación en el proyecto europeo METAMOS por nuestros compañeros pertenecientes al IEMN. 


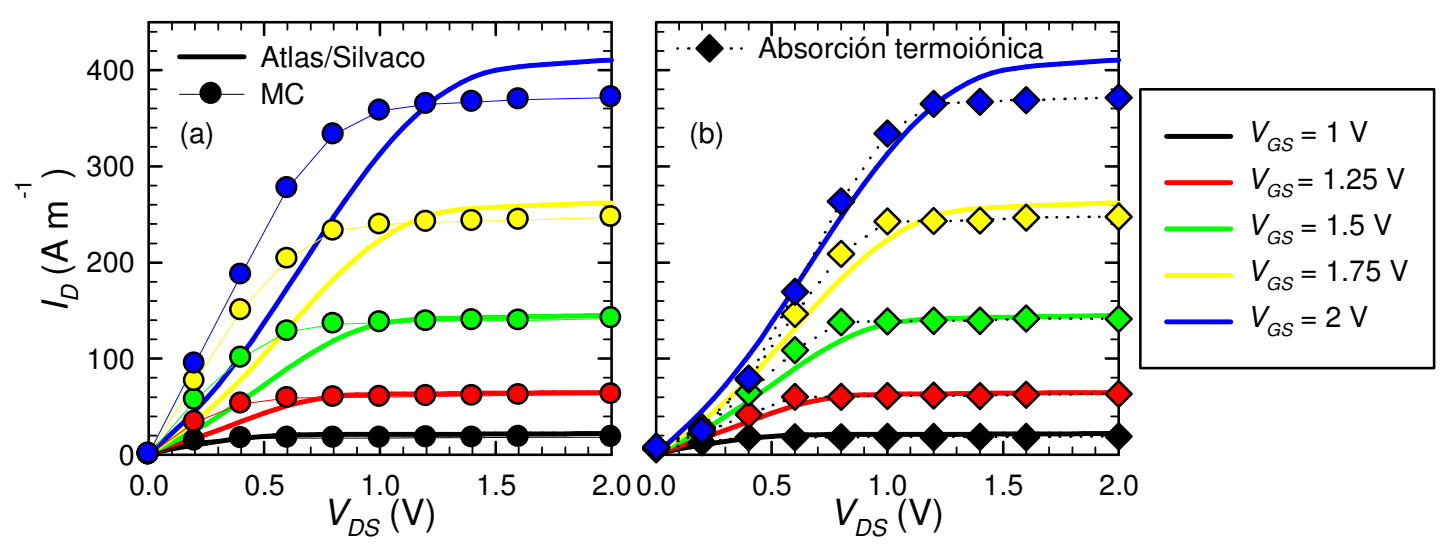

Figura V.8. Características de salida. Las lineas continuas corresponden a los resultados del simulador Atlas/Silvaco, mientras que los símbolos representan los resultados Monte Carlo .a. Los círculos simbolizan la corriente total mientras que .b. los rombos, la componente de absorción termoiónica.

Como podemos observar, las discrepancias más importantes entre ambos resultados están localizadas en el régimen de triodo. Dado que el simulador MC nos proporciona de manera independiente la contribución de las distintas componentes de corriente a la corriente de drenador total, podremos así determinar el origen de estas diferencias. En la Figura V.8.b presentamos la comparación de los resultados de Silvaco para la corriente total de drenador junto con los resultados Monte Carlo en los que mostramos únicamente la componente de absorción termoiónica. Mediante esta comparación vemos que dicha componente de corriente Monte Carlo presenta el mismo comportamiento de no linealidad en el régimen de triodo. Por tanto, las diferencias entre los resultados de ambos simuladores en condiciones de bajo $V_{D S}$ aplicado pueden atribuirse al hecho de que el simulador Silvaco no considera la componente de absorción túnel. Ésta presenta una gran importancia en estas condiciones de polarización, como veremos en la Sección V.5.c.

\section{V.5.b Análisis del paso de corte a conducción en el dispositivo}

En el presente apartado analizaremos el modo de funcionamiento del dispositivo en su paso de corte a conducción siguiendo la característica de transferencia para voltaje aplicado en drenador constante. Hemos seleccionado el valor de $V_{D S}=2 \mathrm{~V}$ (Figura V.7.a). 
(a) $V_{G S}=0 \mathrm{~V} / / V_{D S}=2 \mathrm{~V}$

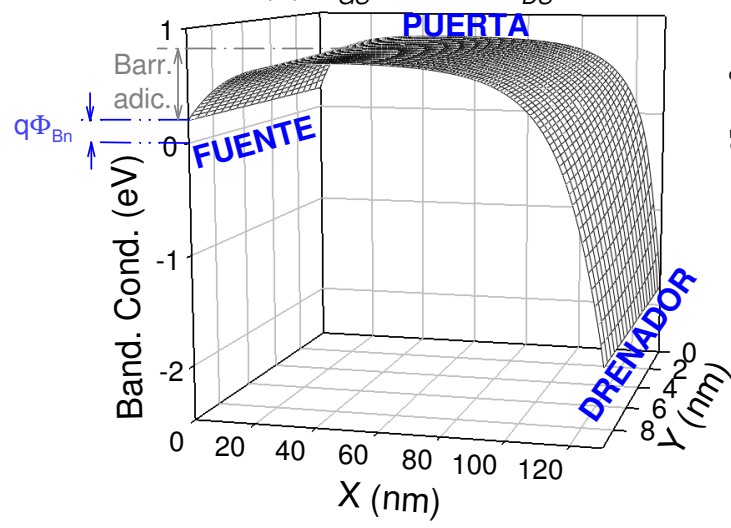

(c) $V_{G S}=0.7 \mathrm{~V} / / V_{D S}=2 \mathrm{~V}$

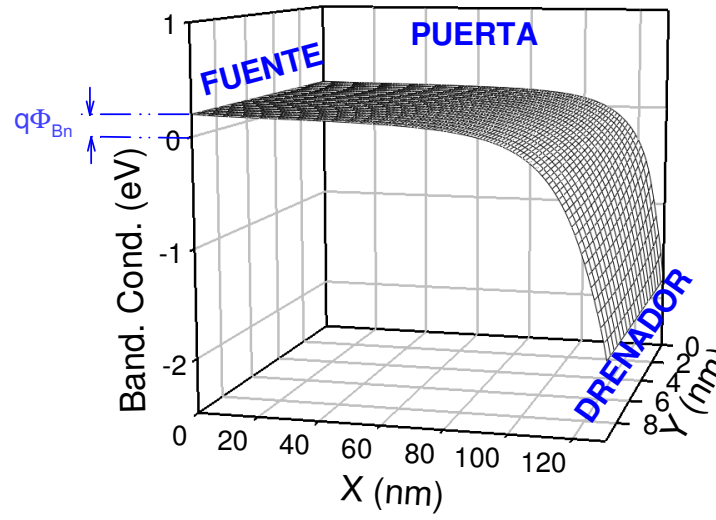

(e) $V_{G S}=2 \mathrm{~V} / / V_{D S}=2 \mathrm{~V}$

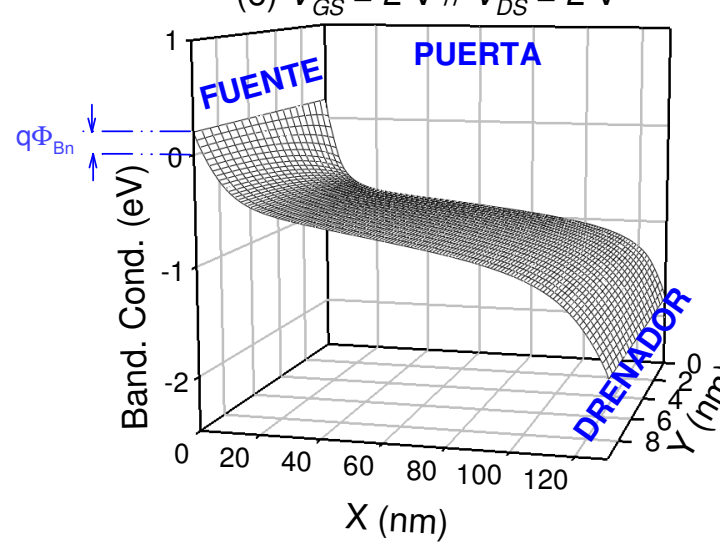

(b) $V_{G S}=0 \mathrm{~V} / / V_{D S}=2 \mathrm{~V}$

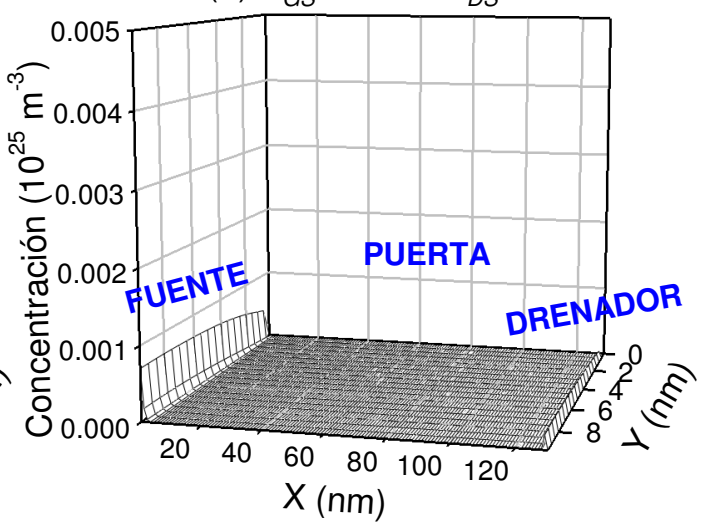

(d) $V_{G S}=0.7 \mathrm{~V} / / V_{D S}=2 \mathrm{~V}$

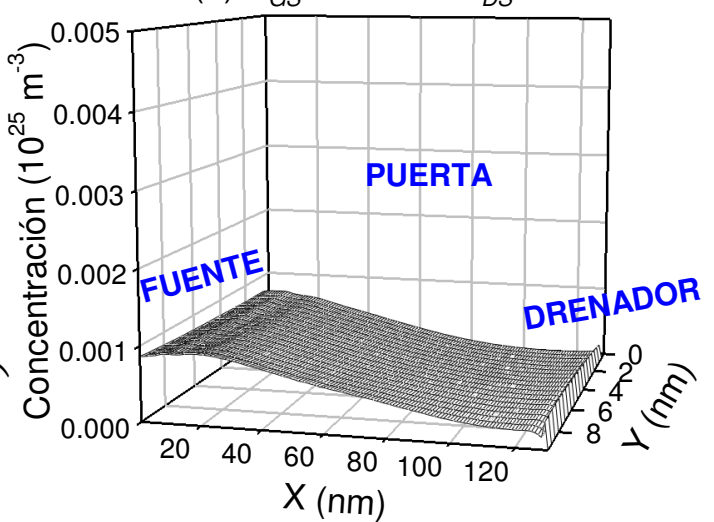

(f) $V_{G S}=2 \mathrm{~V} / / V_{D S}=2 \mathrm{~V}$

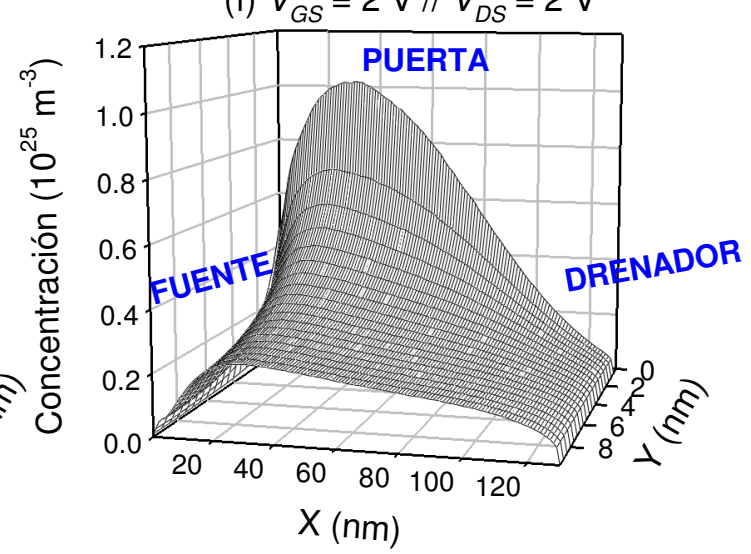

Figura V.9. Banda de conducción y concentración de portadores en el canal para $V_{D S}=2$ Vy varios valores de $V_{G S}(0 \mathrm{~V}, 0.7 \mathrm{Vy} 2 \mathrm{~V})$

En la Figura V.9, representamos los resultados bidimensionales correspondientes a la banda de conducción y la concentración de electrones en el canal para tres valores de voltaje en puerta aplicados. Se observa en el caso en que $V_{G S}=0 \mathrm{~V}$ (Figura V.9.a) que además de la 
barrera Schottky $(0.20 \mathrm{eV})$, los electrones deben superar de manera termoiónica una barrera adicional para ser inyectados en el canal. Muy pocos son los portadores capaces de superar la suma de ambas barreras, encontrándose el dispositivo en estado de corte (off). Por ello, tal y como se refleja en la Figura V.9.b, la concentración de portadores es prácticamente nula en todo el canal, salvo en la región próxima a la fuente, donde está teniendo lugar un proceso continuo de inyección y absorción de portadores.

Cuando aumentamos el potencial aplicado en la puerta, esta barrera adicional se ve reducida, desapareciendo por completo para $V_{G S} \square V_{T}$ (véase la Figura V.9.c, en la que mostramos la banda de conducción para $V_{G S}=0.7 \mathrm{~V}$ ), lo que permite el paso de los electrones al interior del dispositivo mediante emisión termoiónica y marca la transición del estado de corte a conducción (on) [Dubois, 2004], [Pascual, 2009b]. Como consecuencia, la concentración de portadores junto a la fuente es mayor y además, comienza a poblarse el canal (Figura V.9.d).

Si seguimos aumentando el valor de $V_{G S}$, la banda de conducción presenta una curvatura contraria a la del estado de corte (en la Figura V.9.e mostramos como ejemplo $V_{G S}=2 \mathrm{~V}$ ); de este modo se posibilita la inyección de portadores mediante emisión de campo (efecto túnel). Como podemos en la Figura V.9.f, la concentración de portadores se encuentra distribuida en volumen en todo el canal, aunque de manera más importante en la región próxima a la puerta, donde presenta un máximo a $20 \mathrm{~nm}$ de la fuente (nótese que hay un cambio en la escala del eje de concentración con respecto a las Figuras V.9.b y d).

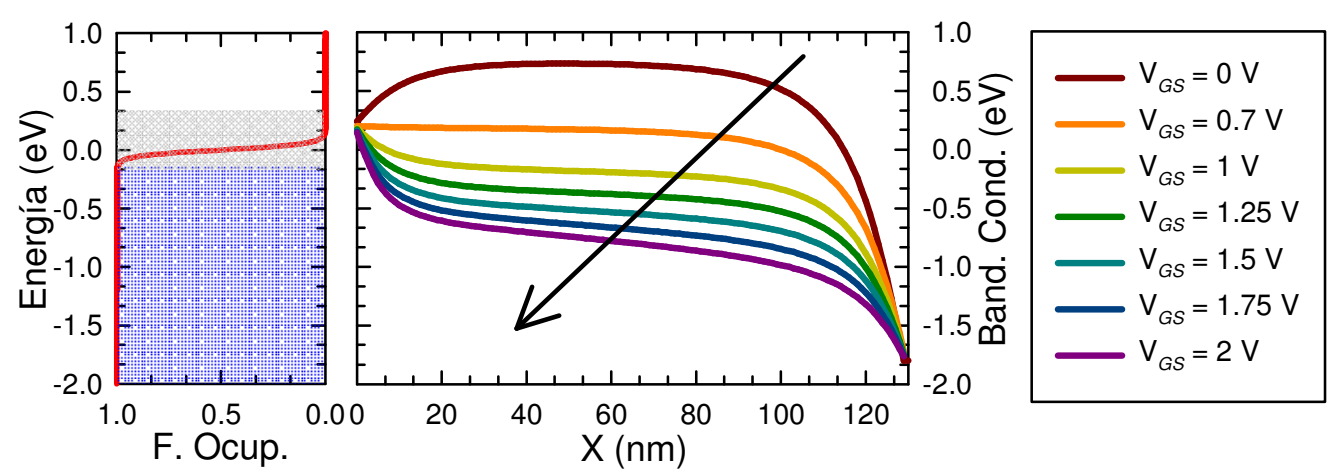

Figura V.10.a. Función de ocupación del metal y b. banda de conducción en el canal para $V_{D S}=2 \mathrm{Vy}$ varios valores de $V_{G S}$. En lo sucesivo, el sentido de las flechas en las figuras indica el aumento de la tensión aplicada.

El estudio de la variación de las magnitudes bidimensionales con las condiciones de polarización hace la tarea más complicada y muy laboriosa. Por ello, y para examinar de una manera más detallada las características internas del transporte en el paso de off a on del SB- 
MOSFET (y por tanto en función de $V_{G S}$ ) es conveniente obtener los perfiles unidimensionales de las diferentes magnitudes en una dimensión - eje $x$ - a partir de los datos bidimensionales proporcionados por el simulador, promediando dichos datos por el perfil de concentración a lo largo del canal, tal y como fue explicado en el Capítulo II, Sección 3.1. En la Figura V.10 mostramos el perfil de la banda de conducción a lo largo del canal para diversos valores de voltaje de puerta y $V_{D S}=2 \mathrm{~V}$. Mostramos a su izquierda, y para facilitar la discusión, la función de ocupación del metal de acuerdo con la distribución de Fermi-Dirac. En estado off $\left(V_{G S}<V_{T}\right)$, como afirmamos con anterioridad, muy pocos portadores tienen la energía suficiente para superar la barrera de potencial existente. Además, es importante destacar que en este estado la mayor parte del potencial cae en la región del canal próxima al drenador (Figura V.10.b).
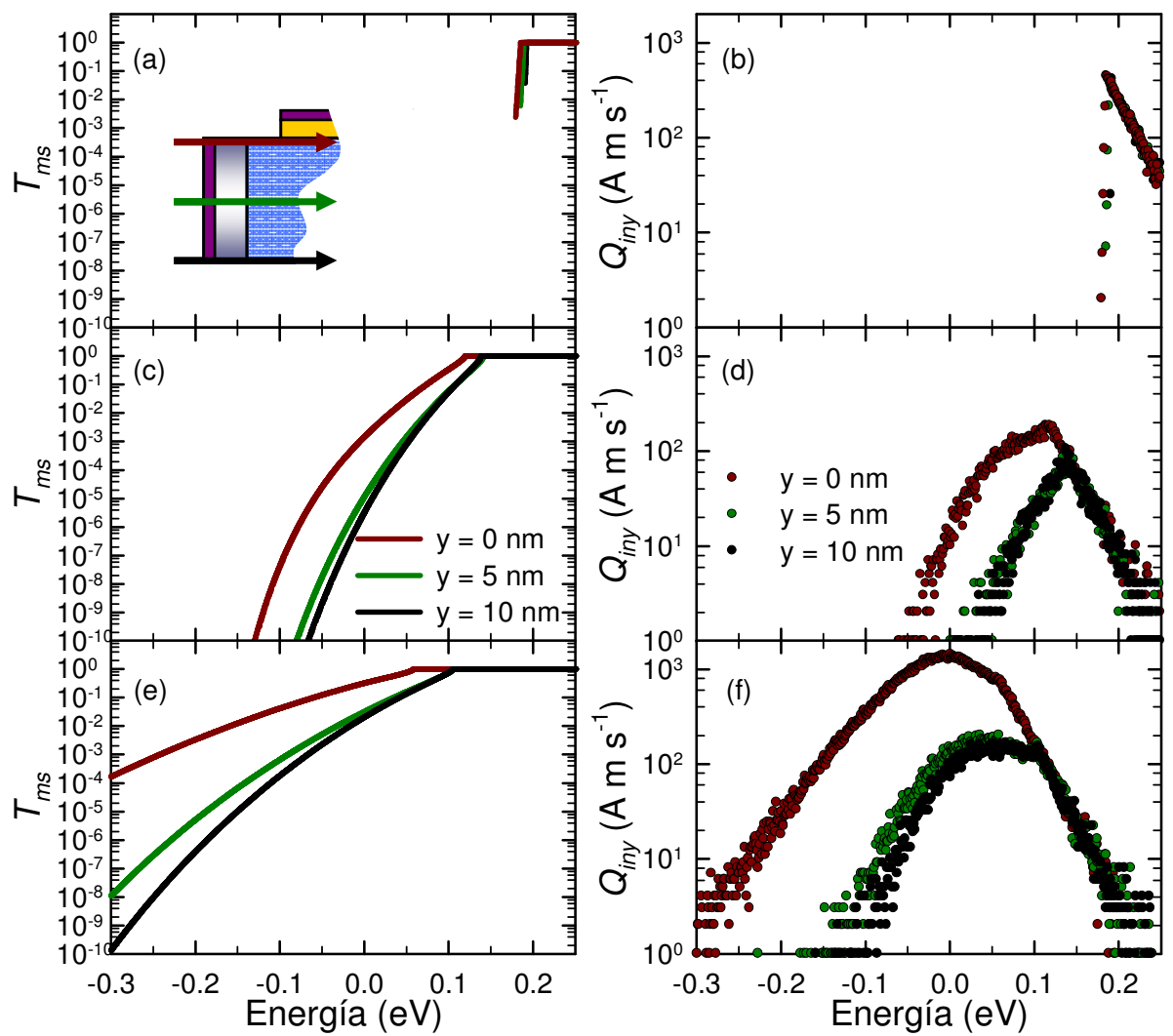

Figura V.11. Coeficiente de transmisión y carga real inyectada a través del contacto de fuente para $V_{D S}=2 \mathrm{Vy}$ varios valores de voltaje aplicado en la puerta: . a-b. $V_{G S}=0.7 \mathrm{~V}, \boldsymbol{c} \boldsymbol{c}-\boldsymbol{d} . V_{G S}=1.25 \mathrm{~V}$, e e-f. $V_{G S}=2 \mathrm{~V}$. Todas están calculadas en tres posiciones a lo largo del eje vertical del canal, a $0 \mathrm{~nm}, 5 \mathrm{~nm}$ y $10 \mathrm{~nm}$ del óxido de puerta para comprobar el efecto modulador de la puerta. 
En la Figura V.11 representamos el coeficiente de transmisión del metal al semiconductor $\left(T_{C m s}\right)$ y la carga real inyectada $\left(Q_{i n y}\right)$ (calculadas mediante la evaluación de las Ecuaciones II.33 y II.34 respectivamente) en función de la energía incidente para tres posiciones del contacto de fuente (junto al óxido de puerta - $0 \mathrm{~nm}$-, en el punto medio del contacto en la dirección transversal - $5 \mathrm{~nm}$ - y junto al óxido enterrado - $10 \mathrm{~nm}$-, tal y como podemos observar en el interior de la Figura V.11.a) para $V_{D S}=2 \mathrm{~V}$ y tres valores de $V_{G S}: 0.7 \mathrm{~V}, 1.25 \mathrm{~V}$ y $2 \mathrm{~V}$.

Para $V_{G S}=0.7 \mathrm{~V}$ (Figura V.11.a), la probabilidad de transmisión más significativa se encuentra localizada en el rango de energías termoiónicas, ya que el camino túnel es muy ancho para este voltaje en puerta aplicado (véase Figura V.10), y así, prácticamente todas las partículas inyectadas por el contacto de fuente son termoiónicas (Figura V.11.b). Debido al reducido valor del campo eléctrico junto al contacto de fuente para esta polarización, como veremos a continuación, la reducción de la altura de la barrera Schottky debido al efecto de las cargas imagen no es muy apreciable ( $\mathrm{q} \Delta \Phi_{\mathrm{Bn}} \square 11 \mathrm{meV}$ ).

Para voltajes aplicados en la puerta superiores al voltaje umbral, (en condiciones de saturación), debemos destacar cómo el potencial cae en la región cercana a ambos contactos, no sólo en el contacto de drenador (como sucedía a voltajes inferiores). A medida que el voltaje aplicado en la puerta aumenta, la probabilidad de emisión de campo se ve también incrementada debido a la reducción de la anchura del camino túnel (Figura V.10). Podemos certificar este hecho observando la transición del coeficiente de transmisión de $V_{G S}=1.25 \mathrm{~V}$ a $2 \mathrm{~V}$ (Figura V.11.c y e) y más claramente en las gráficas de la carga inyectada correspondientes, donde el máximo de la carga inyectada túnel es muy superior para el caso de voltaje aplicado en la puerta aplicado de $2 \mathrm{~V}$. La modulación del perfil de la banda de conducción inducida por la puerta (tal y como puede observarse en la Figura V.9.e), explica que la probabilidad túnel sea mayor a 0 $\mathrm{nm}$ del óxido de puerta que a medida que nos acercamos hacia el óxido enterrado, o lo que es lo mismo, el camino túnel es más estrecho en la región más cercana a la puerta. Por otro lado, la reducción de la barrera por efecto Schottky es mayor también bajo la puerta, debido a que el campo eléctrico junto al contacto de fuente presenta su valor máximo en esa región, disminuyendo a medida que nos acercamos al óxido enterrado. Este efecto de reducción de la barrera efectiva debida al efecto de las cargas imagen es además mayor cuanto mayor sea la tensión de puerta aplicada.

Para voltajes de puerta superiores a los mostrados en esta gráfica, el dispositivo entra en el régimen de operación de triodo. Este modo de operación será analizado con detalle en la sección siguiente. 

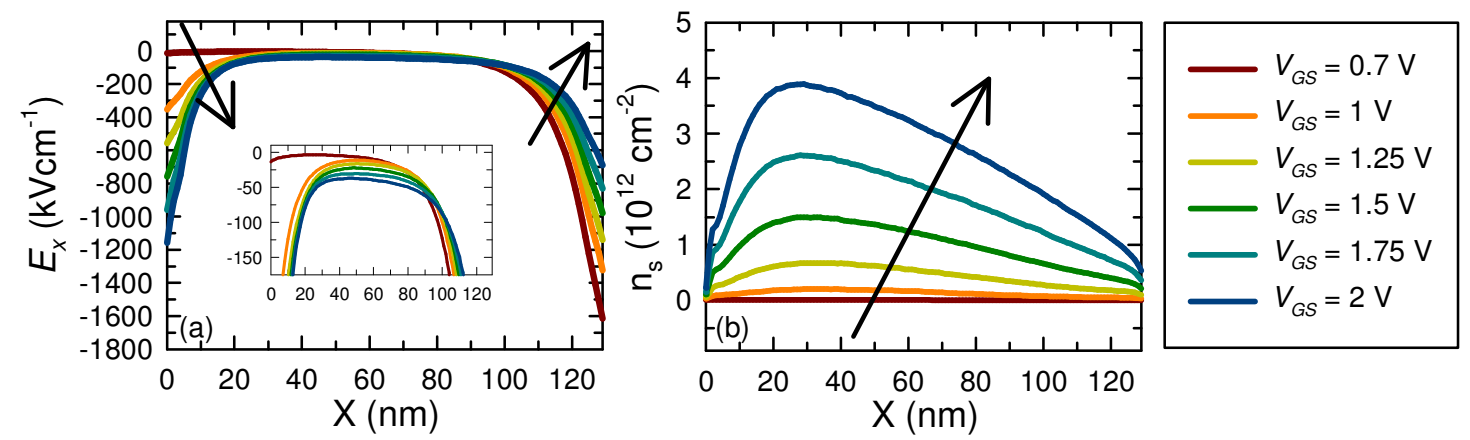

Figura V.12.a. Campo eléctrico y.b. concentración de portadores en el canal $t^{4}$ para $V_{D S}=2$ Vy varios valores de $V_{G S}$. En el recuadro interior de la Figura .a. mostramos una ampliación campo eléctrico con respecto al eje y.

La variación de la curvatura de la banda con el voltaje aplicado en la puerta está directamente relacionada con el campo eléctrico, mostrado en la Figura V.12.a. Como podemos ver, el campo eléctrico aumenta con $V_{G S}$ en la región cercana a la fuente, arrastrando a los portadores inyectados (mayoritariamente mediante efecto túnel) hacia el interior del canal. Además, en la zona cercana al drenador, el campo eléctrico que expulsa a los portadores del dispositivo decrece con $V_{G S}$ ya que la caída de tensión en esa región disminuye (Figura V.10). Debemos destacar que el valor del campo eléctrico en el canal es muy bajo en comparación con los elevados valores que se alcanzan junto a los contactos; sin embargo, este campo no es despreciable, como podemos ver en el recuadro interior de la Figura V.12.a (alrededor de $-10 \mathrm{kV} \mathrm{cm}^{-1}$ cuando $V_{G S}$ es igual a $1 \mathrm{~V}$, o de $-40 \mathrm{kV} \mathrm{cm}^{-1}$ cuando $V_{G S}$ es igual a $2 \mathrm{~V}$ ).

Es interesante estudiar también el perfil de la concentración de portadores, que tal y como vemos en la Figura V.12.b, aumenta notablemente a medida que aumenta el valor de $V_{G S}$. Como observamos, en este régimen de saturación la capa de acumulación de portadores aumenta rápidamente al principio del canal y presenta un valor máximo en la región cercana a la fuente, con una caída aproximadamente lineal a lo largo del canal, hasta alcanzar su valor mínimo junto al drenador, lo que supone una situación notablemente distinta respecto a la distribución de portadores de carga en un dispositivo MOS convencional. Recuérdese cómo para un MOSFET convencional operando en régimen de saturación, la capa de inversión junto al drenador desaparece, produciéndose el fenómeno denominado de estrangulamiento del canal o pinch-off, lo cual no ocurre en el SB-MOSFET.

\footnotetext{
${ }^{4}$ La concentración media de portadores en el canal, que se define como: $n_{S}=\int d y \bar{n}(x, y)$, como ya definimos en el Capítulo II, Sección 3.1. A partir de ahora la denominaremos, por comodidad, concentración de portadores.
} 

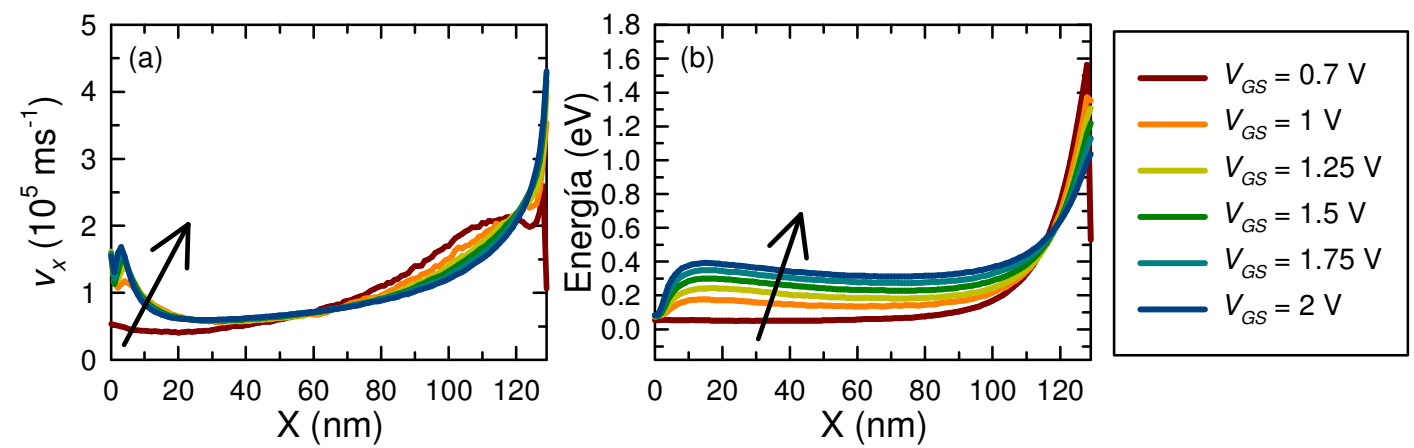

Figura V.13.a. Velocidad y b. energía de portadores en el canal para $V_{D S}=2 \mathrm{~V} y$ varios valores de $V_{G S}$

En la Figura V.13 exponemos el perfil de la velocidad y energía de los portadores a lo largo del canal. A medida que el campo eléctrico aumenta con $V_{G S}$, en la zona cercana al contacto de fuente los portadores inyectados son rápidamente acelerados, presentando un máximo local de velocidad y de energía localizado en los primeros diez nanómetros. Téngase en cuenta que este máximo de velocidad presenta un valor claramente superior a la velocidad de saturación del Silicio material (cercana a $10^{5} \mathrm{~m} \mathrm{~s}^{-1}$ ). De hecho, en la mayor parte del canal se observan valores de velocidad cercanos o superiores a la velocidad de saturación en Silicio, así como valores notables de energía. Junto al drenador, la velocidad y energía de los portadores sufren un drástico aumento debido al elevadísimo campo eléctrico que expulsa a los mismos fuera del dispositivo, como ya comentamos anteriormente (Figura V.11.a). Puede observarse que llegan a alcanzarse valores de energía y velocidad muy elevados (con energías incluso superiores a $1 \mathrm{eV}$ y velocidades por encima de $4 \cdot 10^{5} \mathrm{~ms}^{-1}$ para $V_{G S}=2 \mathrm{~V}$ ). El hecho de que se obtengan valores de velocidad superiores a la velocidad de saturación es común en los transistores MOSFET, dando lugar al fenómeno de sobrevelocidad u overshoot [Laux, 1988]. Sin embargo, el perfil de velocidad a lo largo del canal en un MOSFET convencional es notablemente distinto al caso del SB-MOSFET aquí descrito, estando aquel caracterizado por valores reducidos de velocidad cerca de la fuente y una caída brusca de velocidad desde el máximo próximo al drenador, antes de alcanzar el contacto [Rengel, 2001]. Por otro lado, los portadores altamente energéticos (a los que se les denomina portadores calientes o hot carriers) pueden tener una gran importancia en numerosas magnitudes, especialmente en las asociadas al ruido electrónico; sin embargo, también hay que destacar que la región del dispositivo en que se alcanzan esas energías elevadas es muy pequeña en comparación con el tamaño total de la puerta del transistor. Es importante también hacer notar que en este caso los portadores alcanzan 
el drenador con energías muy elevadas, a diferencia de lo que ocurre en un MOSFET convencional, en el que llegan totalmente termalizados a la metalización gracias a la presencia de la isla fuertemente dopada de drenador [Rengel, 2001].
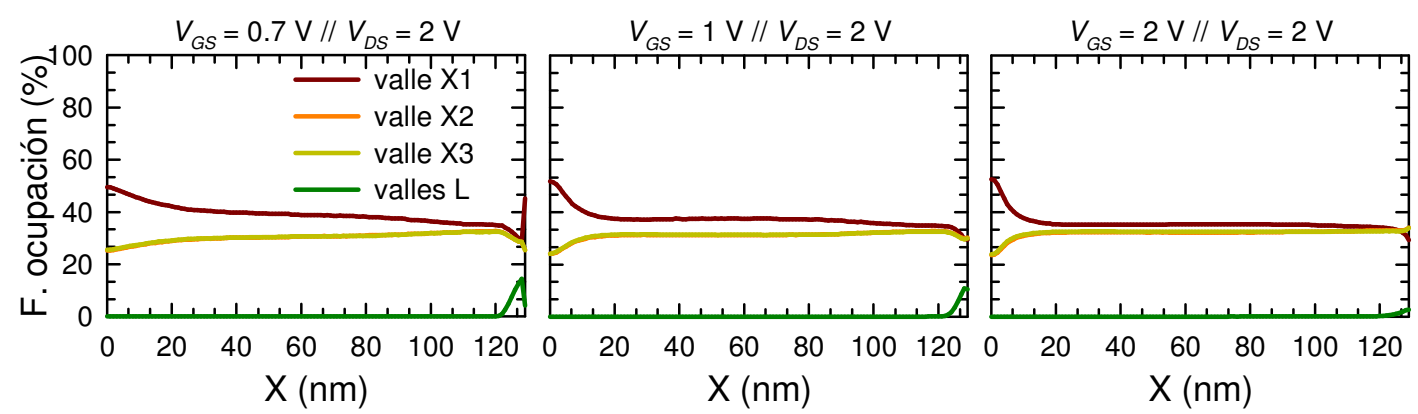

Figura V.14 Función de ocupación de los valles $X$ para $V_{D S}=2$ Vy varios valores de $V_{G S}$.

Como se explica en el Apéndice, nuestro modelo de banda de conducción del Silicio está formado por seis valles elipsoidales correspondientes al mínimo absoluto de la banda de conducción localizado en las direcciones cristalinas [100] (denominados valles X) y ocho correspondientes a los mínimos relativos en las direcciones [111] (valles L). En la Figura V.14, analizamos la ocupación electrónica de los valles para $V_{D S}=2 \mathrm{~V}$ y varios valores de $V_{G S}(0.6 \mathrm{~V}$, 1 V y 2 V) a lo largo del canal. Cuando los portadores son inyectados en el canal, se aceleran rápidamente por efecto del campo eléctrico en la dirección longitudinal. Como consecuencia, la probabilidad de ocupación del valle longitudinal (X1, donde los portadores presentan una masa más elevada, de $0.91 \mathrm{~m}_{0}$, frente al campo eléctrico longitudinal) es mayor que la de los valles transversales (X2 y X3, que presentan una masa transversal de $0.19 \mathrm{~m}_{0}$ frente al campo eléctrico). A medida que la energía de los portadores se reduce a lo largo del canal (aunque siguen siendo aún muy energéticos) la probabilidad de ocupación del valle longitudinal cae al $40 \%$ aproximadamente. En las proximidades del drenador, el campo alcanza valores muy elevados (Figura V.12.a), y la población de los tres valles tiende a equilibrarse, tal y como sucede en Silicio material, a causa del importante aumento de las transiciones intervalle. Además, según los portadores alcanzan energías elevadas, algunos comienzan a ocupar los valles superiores de la banda de conducción (L).

A medida que el voltaje aplicado en puerta aumenta, el máximo de ocupación del valle $\mathrm{X} 1$ junto al contacto de fuente es mayor (debido a que el campo eléctrico en la dirección $\mathrm{x}$ aumenta con $V_{G S}$ ), mientras que en la región del drenador la ocupación de los valles L se ve disminuida (recordemos que el campo eléctrico disminuye con $V_{G S}$ en esta región, Figura V.12.a). En el régimen de bajos voltajes de puerta aplicados, la mayor probabilidad de 
ocupación de los valles L provoca una disminución progresiva de la velocidad en la región cercana al drenador (véase en Figura V.13.a para $V_{G S}=0.6 \mathrm{~V}$ ). Sin embargo, respecto a la energía total, ésta sigue siendo elevada, pues aunque los portadores pierden energía cinética al comenzar a poblar los valles superiores, ganan energía potencial.

\section{V.5.c Regímenes de triodo y saturación}

Una vez que hemos analizado el funcionamiento del dispositivo en condiciones de $V_{D S}$ constante, es interesante realizar el estudio bajo voltaje aplicado en puerta constante ( $V_{G S}$ igual a 2 V), siguiendo la característica de salida (Figura V.7.b). En la Figura V.15 mostramos la variación con $V_{D S}$ del perfil de la banda de conducción. Podemos distinguir entre el régimen de triodo (para $V_{D S}$ entre $0 \mathrm{~V}$ y $1.2 \mathrm{~V}$ ) y de saturación (para $V_{D S}$ superiores a $1.2 \mathrm{~V}$ ) mediante la curvatura de la banda en la región cercana al contacto de drenador.
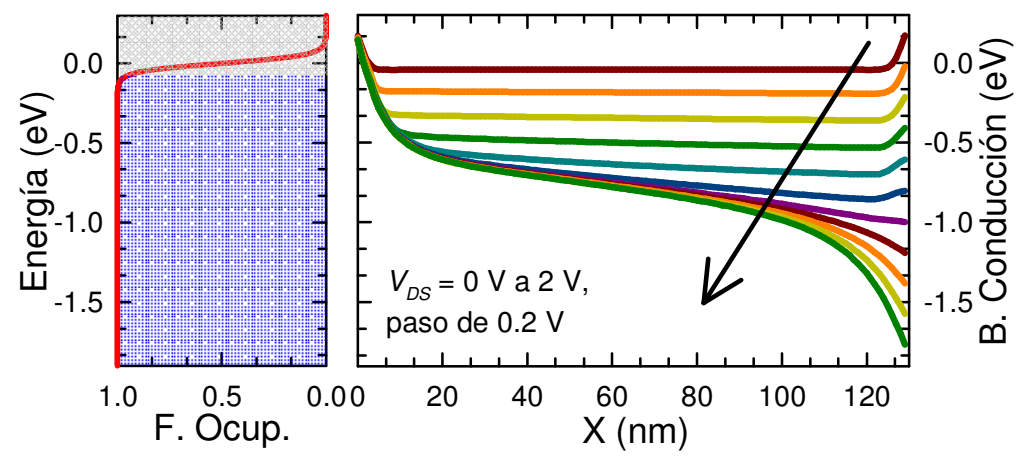

Figura V.15.a. Función de ocupación del metal y b. banda de conducción en el canal para $V_{G S}=2$ Vy varios valores de $V_{D S}$.

En el caso de polarización de drenador nula observamos una total simetría del dispositivo; la curvatura de la banda junto a ambos contactos está inducida por el voltaje en puerta aplicado. Cuando se aplica una tensión $V_{D S}$ la simetría desaparece, observándose en primer lugar en condiciones de triodo que la mayor parte del potencial cae en la región cercana a la fuente, provocando una curvatura de la banda más pronunciada en esa región. Si examinamos las componentes de corriente del contacto de fuente, mostradas en la Figura V.16.a, vemos que la emisión de campo aumenta con $V_{D S}$, debido a que la probabilidad de inyección túnel se extiende a regiones energéticas más bajas en el metal, donde hay un mayor 
número de electrones capaces de ser inyectados en el dispositivo (véase la función de ocupación en el metal, Figura V.15.a). La componente de inyección túnel es la más importante en la corriente total en la fuente, seguida por la inyección termoiónica, mientras que las componentes de absorción son prácticamente despreciables.

Por otro lado, en el contacto de drenador, la absorción túnel predomina sobre las demás para bajos $V_{D S}$, presentando un aumento con el voltaje para $V_{D S}<0.5 \mathrm{~V}$; sin embargo, para voltajes de drenador mayores que $0.5 \mathrm{~V}$, esta componente comienza a disminuir debido a que la curvatura de la banda junto al drenador se suaviza, ensanchándose así el camino túnel. Así, la absorción termoiónica pasa a ser particularmente relevante para $V_{D S}>0.5 \mathrm{~V}$, superando al resto de componentes en su contribución a la corriente total de drenador.

Para tensiones de drenador superiores, ya en la región de saturación $\left(V_{D S}>1.2 \mathrm{~V}\right)$, la tensión adicional cae prácticamente en su totalidad en la región del canal más próxima al drenador, como podemos ver en la Figura V.15, mientras que para el resto del canal el perfil de la banda de conducción apenas se ve modificado. El máximo valor de la curvatura de la banda en la región cercana a la fuente, constante con $V_{D S}$ en la región de saturación y asociado a la tensión aplicada en la puerta, es el responsable de la saturación de las componentes de corriente (Figura V.16.a), con una clara superioridad de la inyección túnel sobre las demás contribuciones a la corriente total. Comparando el comportamiento de este dispositivo con el del MOSFET convencional, este fenómeno de límite de inyección en la fuente sería análogo al proceso de estrangulación del canal o pinch-off.

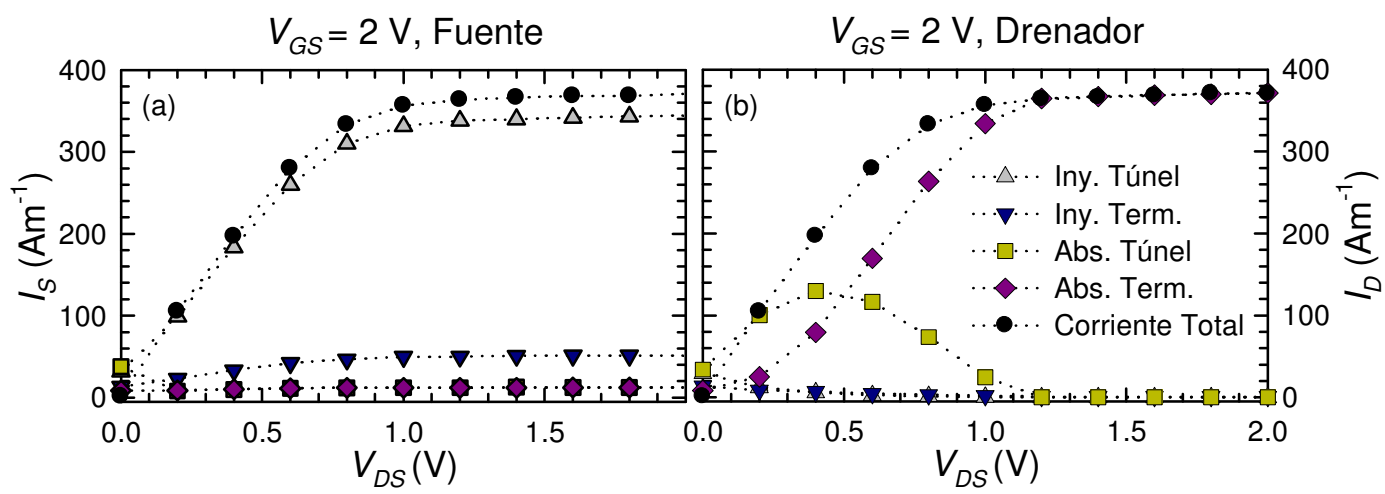

Figura V.16. Componentes de corriente de fuente y drenador en función de $V_{D S}$ para $V_{G S}=2 \mathrm{~V}$.

Para estas polarizaciones de $V_{D S}$ elevado, en el drenador ambas componentes túnel son nulas y la absorción termoiónica representa la principal contribución a la corriente total (Figura V.16.b). Cabe destacar que para $V_{D S}$ igual a $1.2 \mathrm{~V}$, el perfil de la banda de conducción está en 
condiciones de banda plana en la región del drenador, lo que supone la transición de la región de triodo a la de saturación (en triodo dicha curvatura es en el mismo sentido que en la región de fuente, permitiendo la inyección túnel en el drenador, mientras que en saturación, la curvatura es contraria, por lo que la probabilidad túnel es nula -Figura V.15-).

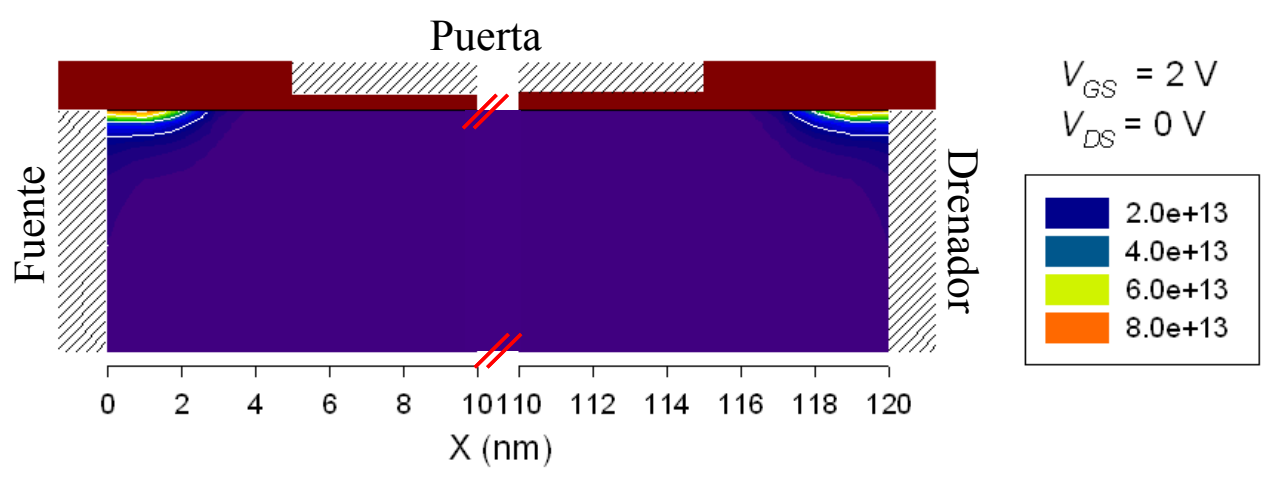

Figura V.17. Mapa de inyección total de los contactos de fuente y drenador para $V_{G S}=2 \mathrm{Vy} V_{D S}=0 \mathrm{~V}$.

Teniendo en cuenta lo estudiado anteriormente, nos interesa particularmente el estudio de la inyección a través de los contactos (fundamentalmente en el de fuente, dado que los fenómenos de inyección túnel son la clave en el funcionamiento de este dispositivo). Nuestro simulador, junto con el modelo WKB implementado, nos proporciona la carga inyectada total a través de los contactos Schottky. Podemos analizar por tanto su dependencia con el voltaje de drenador y su carácter bidimensional. En la Figura V.17 mostramos el mapa de inyección en la zona cercana a los contactos de fuente y drenador cuando $V_{D S}$ es igual a cero y $V_{G S}$ igual a $2 \mathrm{~V}$. En este caso, podemos observar un comportamiento simétrico en los contactos, lo cual corresponde con la previamente mencionada simetría del potencial a $V_{D S}=0 \mathrm{~V}$ (Figura V.15). Como podemos ver, la mayor parte de la inyección de carga tiene lugar en la parte superior de ambos contactos, donde el voltaje aplicado en la puerta tiene un mayor efecto sobre la banda de conducción, dando lugar a un estrechamiento del camino túnel más pronunciado en esa zona. Estos resultados están en consonancia con lo encontrado por otros autores [Winstead, 2000]. Una vez que los portadores son inyectados, se dispersan hacia abajo y al interior del dispositivo. 


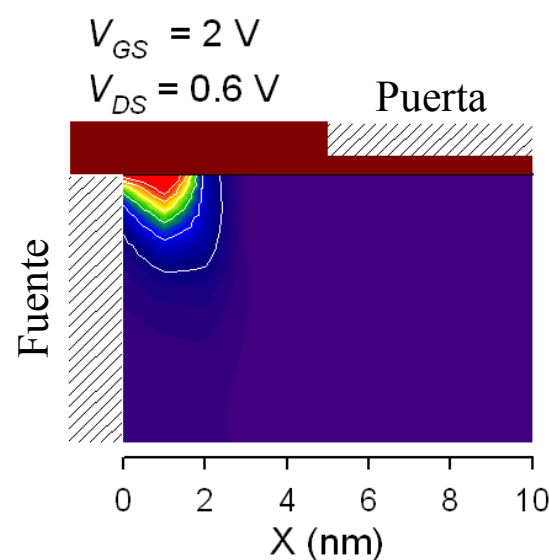

(a)
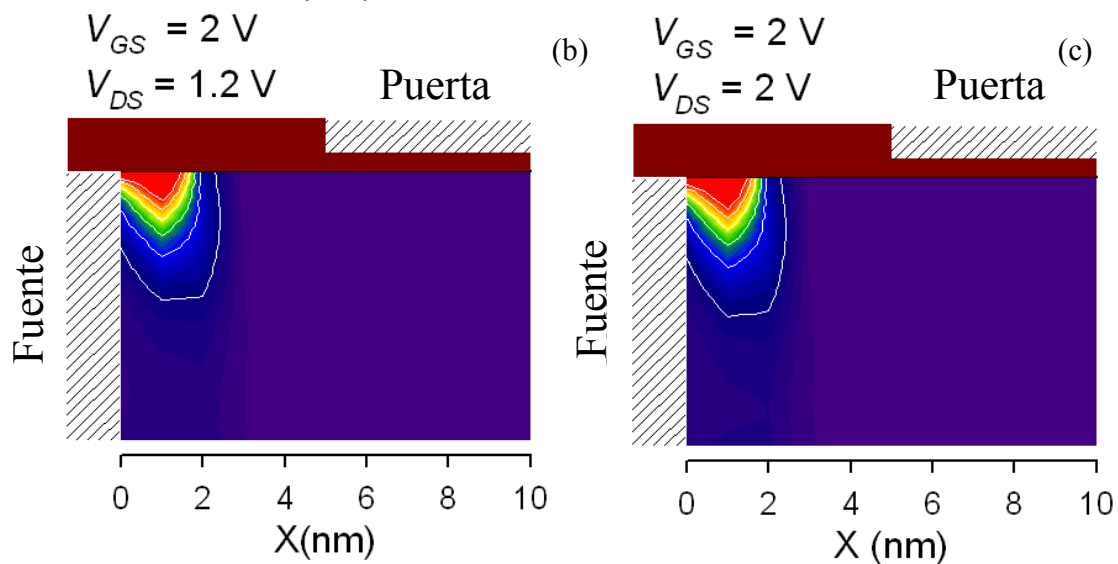

Figura V.18. Mapa de inyección total del contacto de fuente para $V_{G S}=2 \mathrm{Vy} . \boldsymbol{a} . V_{D S}=0.6 \mathrm{~V}$, .b. $1.2 \mathrm{Vy}$.c. $2 \mathrm{~V}$.

A medida que aumentamos el voltaje aplicado en drenador en el régimen de triodo, la cantidad de carga inyectada en la esquina superior de la fuente también es mayor. La zona donde la carga es inyectada por efecto túnel se extiende desde la fuente hasta unos $3 \mathrm{~nm}$ hacia el interior del canal en el eje longitudinal independientemente del $V_{D S}$ aplicado, mientras que en la dirección transversal la zona de inyección avanza hacia el óxido enterrado a medida que aumenta $V_{D S}$. Podemos observar este hecho si comparamos la Figura V.18.a. y .b., para $V_{D S}$ igual a $0.6 \mathrm{~V}$ y $1.2 \mathrm{~V}$ respectivamente. Por otro lado, una vez que el dispositivo entra en la región de saturación, el área de la zona de inyección de la carga permanece prácticamente constante con el voltaje aplicado en drenador, tal y como podemos comprobar al contrastar las Figura V.18.b. y .c. (correspondientes a $V_{D S}$ iguales a $1.2 \mathrm{~V}$ y $2 \mathrm{~V}$, respectivamente).

Con respecto al contacto de drenador, el área de inyección es muy reducida, limitada a una pequeña porción junto a la interfaz, ya que la corriente de inyección es prácticamente 
debida sólo a la emisión termoiónica, siendo la influencia de la emisión de campo muy débil en triodo y nula en saturación. Por este motivo, no mostramos los mapas de inyección correspondientes a este contacto nada más que para el caso de $V_{D S}$ nulo (Figura V.17).

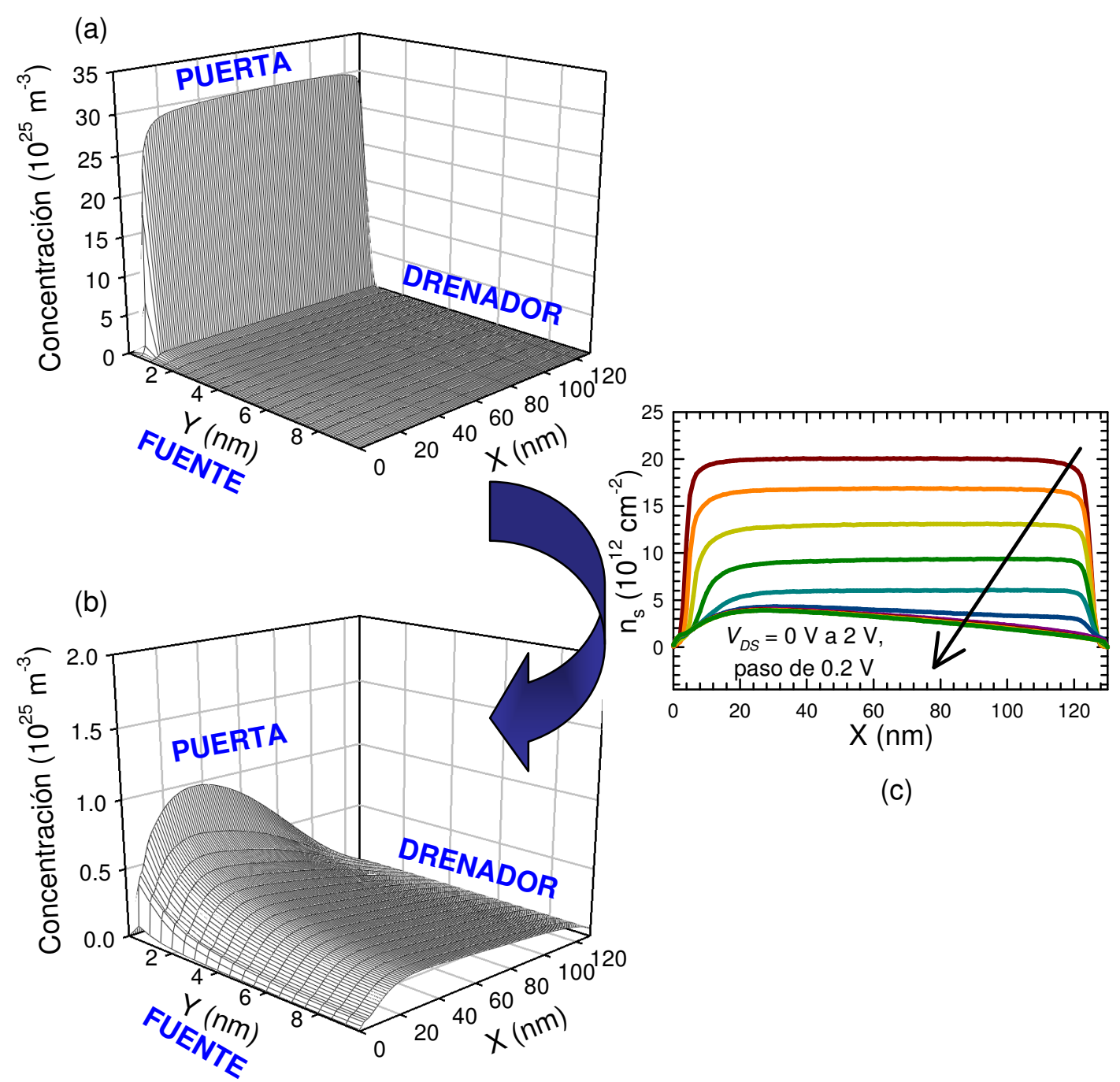

Figura V.19 Concentración de portadores en el canal para $V_{G S}=2$ V y dos valores de $V_{D S}:$.a. 0.2 V (triodo) y.b. $2 \mathrm{~V}$ (saturación) .c. Perfil de la concentración a lo largo del canal para varios $V_{D S}$.

Con objeto de examinar las diferencias del transporte entre el SB-MOSFET y el MOSFET convencional, nos interesa especialmente el estudio en volumen de la concentración de portadores tanto en triodo como en saturación, mostrada en la Figura V.19.a y .b. respectivamente para $V_{D S}=0.2 \mathrm{~V}$ (triodo) y $V_{D S}=2 \mathrm{~V}$ (saturación); en la Figura V.19.c aparece representado el perfil de la concentración de portadores a lo largo del canal para múltiples 
polarizaciones entre $02 \mathrm{~V}$ de $V_{D S}$ y $V_{G S}=2 \mathrm{~V}$, para de este modo observar de forma más gradual la transición del régimen de triodo a saturación. Posteriormente, en las Figuras V.20 y V.21 mostramos el campo eléctrico en el dispositivo en la dirección transversal y longitudinal para las tensiones de puerta anteriormente mencionadas.

En la región de triodo, los portadores se encuentran localizados muy próximos a la puerta, presentando valores muy elevados de concentración (Figura V.19.a). Como podemos ver, debido al elevado valor de $V_{G S}$ y bajo $V_{D S}$ aplicado, se origina un gran campo eléctrico transversal (1450 KV cm${ }^{-1}$ aproximadamente para $V_{D S}=0.2 \mathrm{~V}$, Figura V.20.a), que hace que la concentración de electrones en el SB-MOSFET en modo de acumulación sea muy similar a la que presenta un MOSFET convencional también en triodo. Sin embargo, en el régimen de saturación los portadores están situados en todo el volumen del canal (ya que el campo eléctrico transversal presenta un valor más reducido que en triodo - Figura V.20.b -), como ya comentamos en la Figura V.9.f. A diferencia del MOSFET convencional (en el cual el régimen de saturación viene determinado por el pinzamiento del canal), en este caso, al ser un dispositivo en acumulación la saturación aparece por un valor máximo de inyección de portadores a través del contacto de fuente, lo que origina que la concentración de electrones permanezca prácticamente invariante con $V_{D S}$ en este régimen de operación (véase la figura V.19.c).
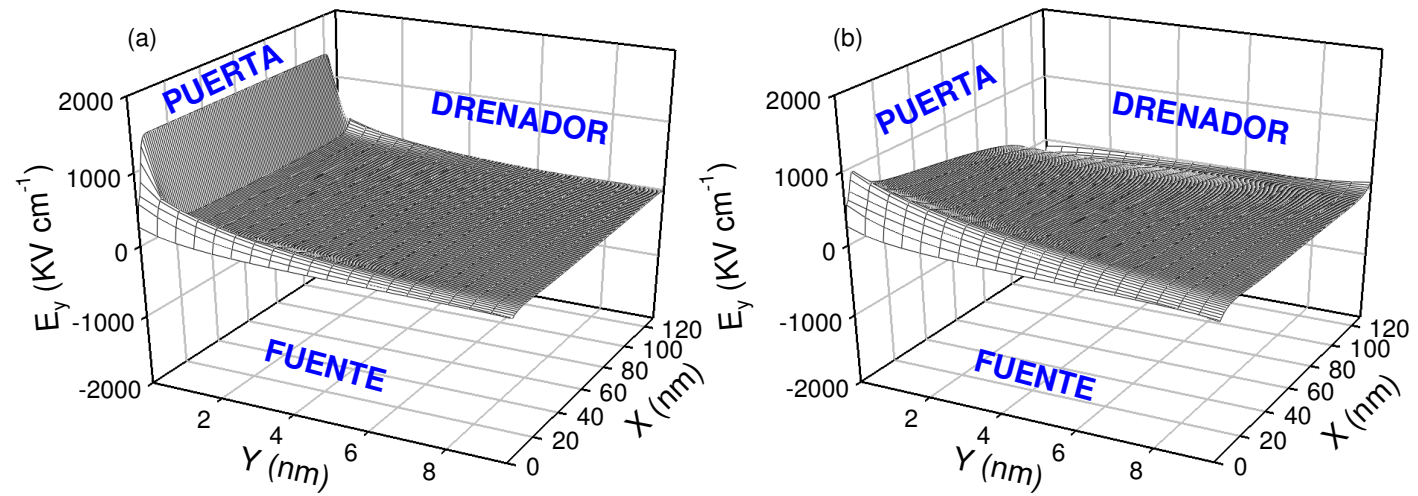

Figura V.20. Campo eléctrico en la dirección transversal para $V_{G S}=2 \mathrm{~V} y$ dos valores de $V_{D S}$ : a. $0.2 \mathrm{~V}$ (triodo) y .b. 2 V (saturación).

Con respecto al campo eléctrico longitudinal, como se aprecia en la Figura V.21, además de los elevados valores que presenta junto a los contactos de fuente y drenador, es 
importante destacar que la principal diferencia que aparece entre el régimen de triodo y saturación es que en las proximidades del drenador, el campo eléctrico longitudinal muestra signos contrarios. Por tanto, cuando el dispositivo está en triodo, el campo eléctrico longitudinal cerca del drenador es positivo, oponiéndose al paso de los portadores, dándose el fenómeno opuesto en saturación.
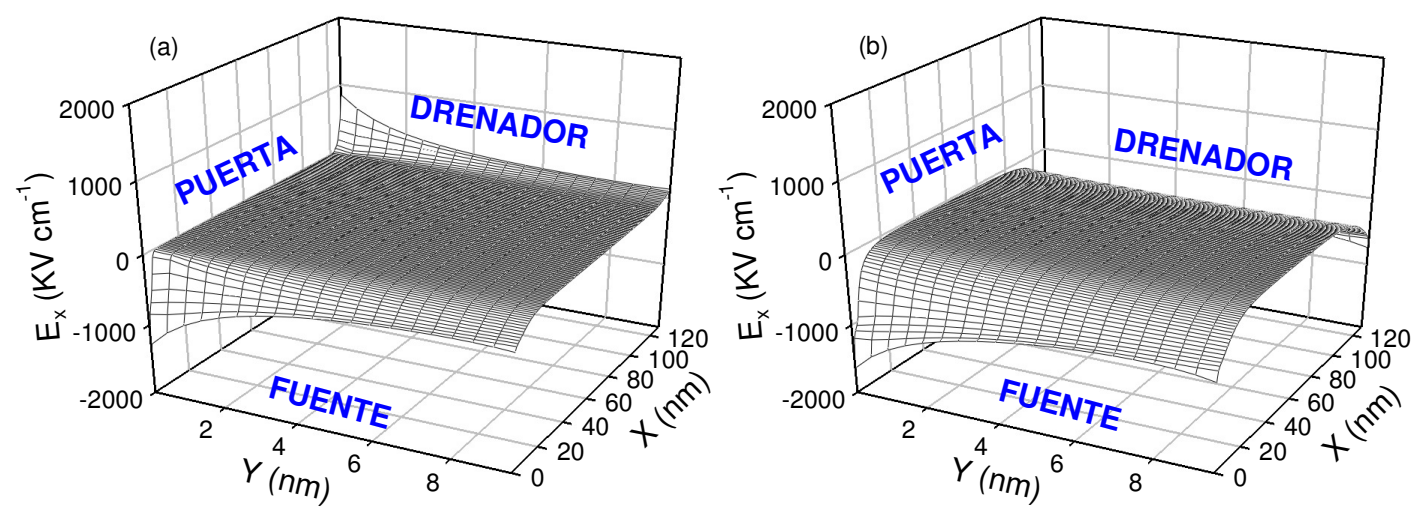

Figura V.21. Campo eléctrico en la dirección longitudinal para $V_{G S}=2 \mathrm{~V} y$ dos valores de $V_{D S}$ : a. $0.2 \mathrm{~V}$ (triodo) y.b. 2 V (saturación).

En la Figura V.22 mostramos los perfiles de velocidad y energía de los portadores para $V_{G S}$ igual a $2 \mathrm{~V}$ y diversos valores de $V_{D S}$. Como ya comentamos en la Figura V.13, la velocidad media de los portadores presenta un máximo local próximo a la región de fuente relacionado con el elevado campo longitudinal de arrastre que sufren los portadores que están siendo inyectados; dicho máximo varía dependiendo de la tensión de drenador aplicada desde $1.2 \cdot 10^{5} \mathrm{~ms}^{-1}\left(V_{D S}=0 \mathrm{~V}\right)$ a $1.8 \cdot 10^{5} \mathrm{~ms}^{-1}\left(V_{D S}=2 \mathrm{~V}\right)$.
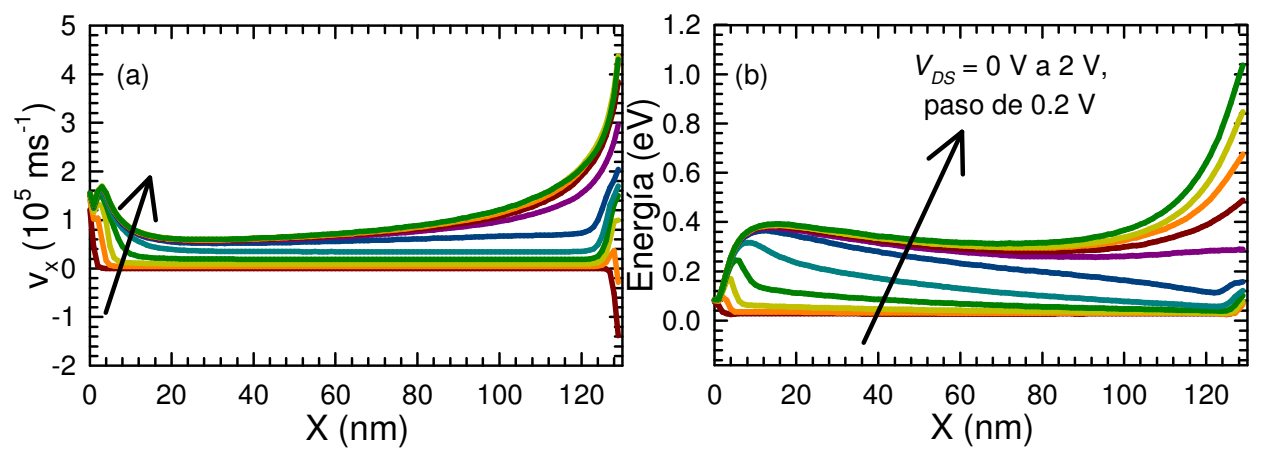

Figura V.22.a. Velocidad y b. energía de portadores en el canal para $V_{G S}=2$ Vy varios valores de $V_{D S}$. 
Sin embargo, vamos a centrarnos en lo que ocurre en las cercanías del drenador al variar $V_{D S}$. Para los valores de voltaje aplicado en drenador aplicados más débiles, para los que el campo eléctrico presenta valores positivos cerca del contacto de drenador, la velocidad junto a dicho contacto es negativa (lo cual está relacionado con los electrones que retroceden y con los inyectados en el propio contacto). Como hemos visto anteriormente (Figura V.20) cuando $V_{D S}$ aumenta, el campo eléctrico positivo en la región cercana al drenador disminuye, pasando a ser negativo en el régimen de saturación. De este modo, según aumenta $V_{D S}$, la velocidad media de los electrones es cada vez más elevada, presentando valores positivos asociados a los electrones que abandonan el dispositivo. Los valores alcanzados en las cercanías del drenador son muy altos (por ejemplo, $4.3 \cdot 10^{5} \mathrm{~ms}^{-1}$ para $V_{D S}$ igual a $2 \mathrm{~V}$ ). Además, en el régimen de saturación, la velocidad a lo largo del canal es cercana al valor de saturación en Silicio material $\left(10^{5} \mathrm{~ms}^{-1}\right)$.

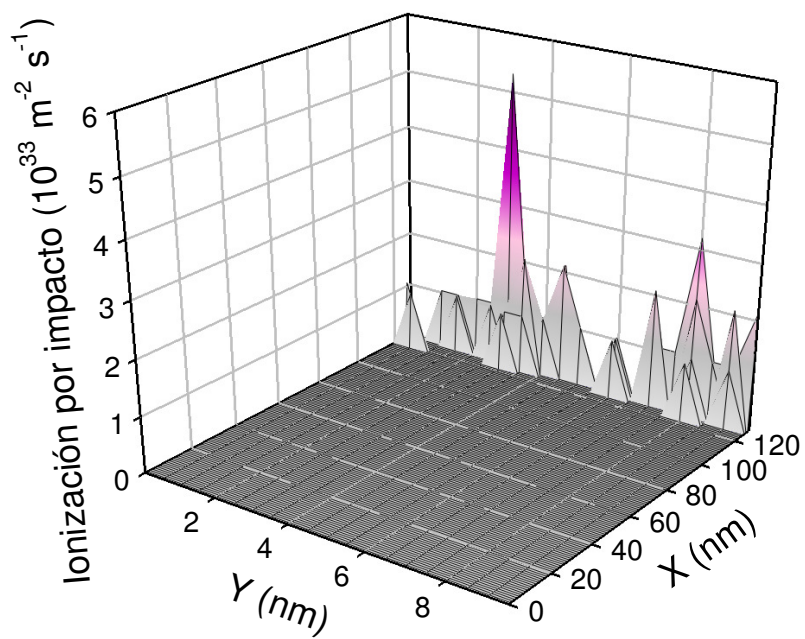

Figura V.23. Tasa de ionización por impacto para $V_{G S}=2$ Vy $V_{D S}=2 \mathrm{~V}$.

La energía aumenta con $V_{D S}$ de modo similar a la velocidad de los portadores: se ve incrementada con el voltaje aplicado en el drenador, presentando valores muy altos (para $V_{D S}=2 \mathrm{~V}$, la energía del máximo local junto a la fuente es de $0.39 \mathrm{eV}, 0.31 \mathrm{eV}$ en la mitad del canal y $1.03 \mathrm{eV}$ en el drenador). Se evidencia de este modo la existencia de portadores calientes en todo el dispositivo para valores elevados de $V_{D S}$, en particular en la región del canal más cercana al contacto de drenador. Por otro lado, las altas energías alcanzadas dan lugar a la aparición de fenómenos de ionización por impacto junto al drenador, como podemos verificar mediante la Figura V.23, en la que representamos la distribución espacial de los fenómenos de 
ionización por impacto ${ }^{5}$ de electrones en el dispositivo para la condición de polarización correspondiente a $V_{D S}=V_{G S}=2 \mathrm{~V}$. Como podemos ver, la multiplicación de portadores se da principalmente en la región del canal más cercana al contacto de drenador, la cual coincide con la zona en la que los portadores adquieren mayor energía (ver Figura V.22.b).

La función de ocupación (Figura V.24) muestra que en el régimen de triodo la probabilidad de ocupación de los valles X1 y X3 es superior a la del X2 (para $V_{D S}$ igual a 0.2 V). La discrepancia entre la ocupación de los valles X2 y X3 en este régimen de operación es debida al elevado campo eléctrico vertical (Figura V.20.a), para el cual la masa más elevada la presentan los portadores del valle X3. Por otro lado, en la región de saturación las diferencias se reducen, siendo el valle X1 el más ocupado, con un $38 \%$ de probabilidad de ocupación, sobre el $31 \%$ de los valles X2 y X3, como ya analizamos en la Figura V.14.

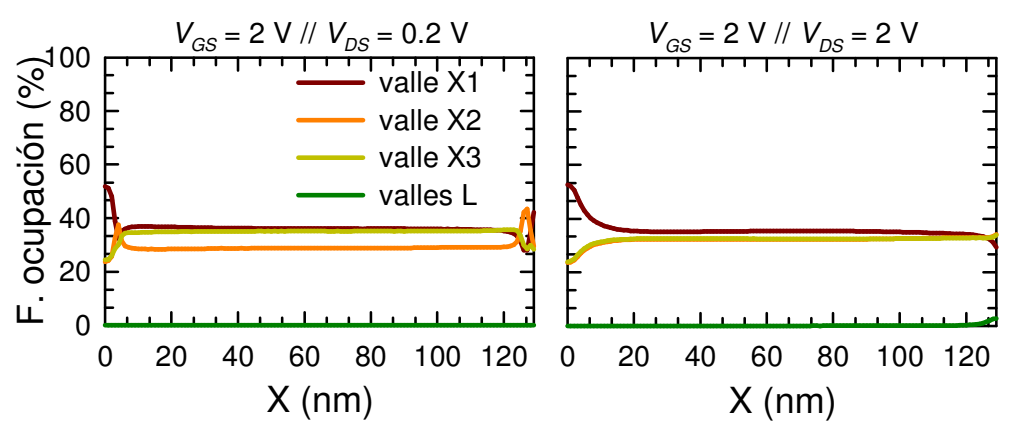

Figura V.24. Función de ocupación de los valles $X$ para $V_{G S}=2$ Vy varios valores de $V_{D S}$.

\section{V.5.d Estudio de las características microscópicas del transporte de portadores en el canal}

Como ya hemos explicado en el Capítulo II, Sección 3.f, nuestro simulador Monte Carlo nos permite llevar a cabo un análisis en profundidad de las propiedades microscópicas del transporte de portadores en una región de conteo dentro del dispositivo. Para ello, empleamos diversas magnitudes, como son el tiempo de tránsito medio (es decir, el tiempo real que tarda el portador en cruzar una determinada región), el número de mecanismos de scattering sufridos por los portadores al cruzar el canal desde la fuente al drenador, la distancia recorrida por los

\footnotetext{
${ }^{5}$ En el apéndice puede encontrarse la información acerca de nuestro modelo de ionización por impacto.
} 
mismos (total, longitudinal y transversal) o el recorrido libre medio. La región que se ha tenido en cuenta para el cálculo del valor de estas magnitudes en el transistor SB-MOSFET es la región justo debajo del contacto de puerta.
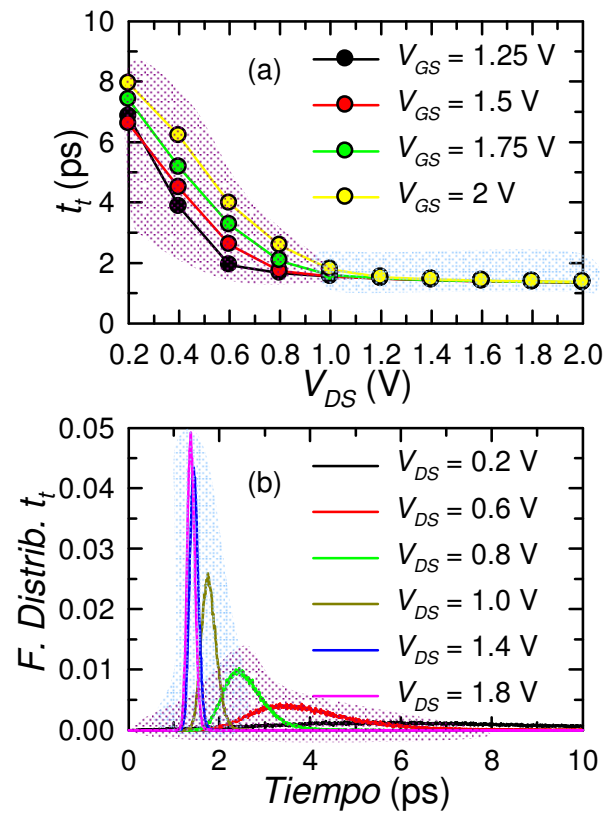

Figura V.25.a. Tiempo de tránsito medio en función de $V_{D S}$ para distintos valores de $V_{G S}$.b. Función de distribución del tiempo de tránsito para $V_{G S}=2 V y$ varios valores de $V_{D S}$.
En la Figura V.25.a mostramos el tiempo de tránsito medio de los portadores que atraviesan el canal $\left(t_{t}\right)$ para distintos valores de $V_{G S}$ (desde $1 \mathrm{~V}$ hasta $2 \mathrm{~V}$ ) en función de $V_{D S}$. Pueden observarse dos regiones muy diferentes: la primera (sombreada de violeta en la gráfica), en la que el dispositivo se encuentra en régimen de triodo, y una segunda región en saturación (sombreada de azul). Analizaremos a continuación ambos regímenes de polarización.

En la región de triodo se observa una notable reducción del tiempo de tránsito al aumentar el potencial de drenador, como respuesta al aumento homogéneo del campo

eléctrico longitudinal a lo largo del canal. En la Figura V.25.b mostramos la función de distribución del tiempo de tránsito para la tensión aplicada en la puerta de $2 \mathrm{~V}$ y diversos valores de tensión en drenador aplicada. Como podemos ver, la función de distribución en éste régimen presenta un máximo localizado en torno al valor medio (correspondiente al mostrado en la Figura V.25.a), sin embargo existe un elevado número de portadores que presentan un tiempo de tránsito tanto superior como inferior, abarcando así la función de distribución un ancho espectro.

Cuando el dispositivo está en saturación, el tiempo de tránsito presenta valores más bajos (alrededor de 1.35 ps) y permanece prácticamente constante (Figura V.25.a, región sombreada azul). La función de distribución en este régimen de operación es muy estrecha, lo cual es indicativo de que la mayoría de portadores presentan un valor del tiempo de tránsito muy similar (Figura V.25.b). 

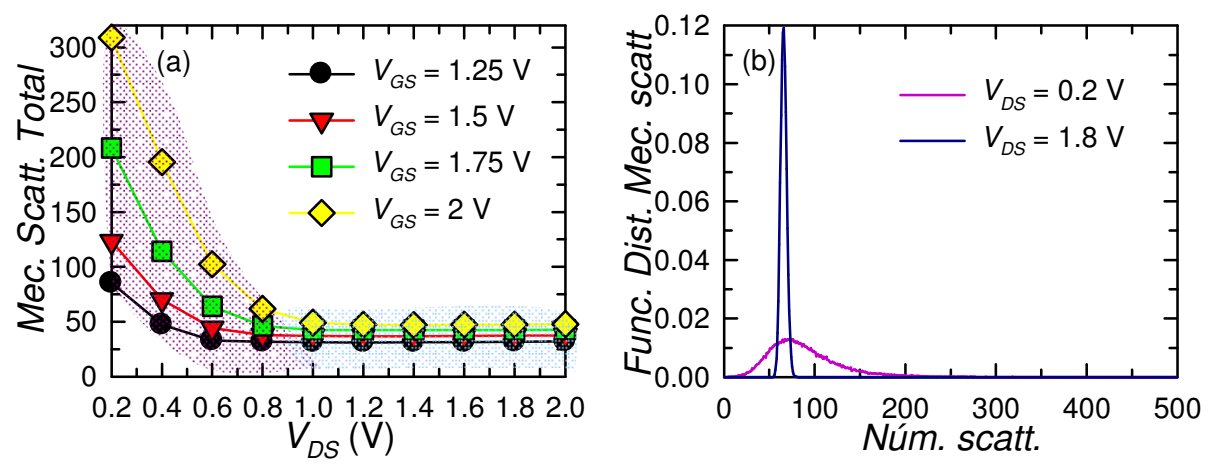

Figura V.26.a. Mecanismos de scattering totales en función de $V_{D S}$ para distintos valores de $V_{G S}$.b. Función de distribución de mecanismos de scattering totales para $V_{G S}=2 \mathrm{Vy}$ dos valores de $V_{D S}: 0.2 \mathrm{~V}$ (triodo) y $1.8 \mathrm{~V}$ (saturación).

El valor del tiempo de tránsito alcanzado por los portadores en el dispositivo está directamente relacionado con los mecanismos de scattering que sufren en su recorrido a través del canal; en la Figura V.26 mostramos la dependencia con $V_{D S}$ de los mecanismos de scattering totales sufridos por los portadores, así como su función de distribución para $V_{G S}=2 \mathrm{~V}$ y dos valores de $V_{D S}$. Como podemos observar, el número de mecanismos de scatterings sufridos por los portadores presenta un comportamiento similar al del tiempo de tránsito: en el régimen de triodo disminuye apreciablemente a medida que $V_{D S}$ aumenta, mientras que la función de distribución presenta un ancho espectro. Por otro lado, en el régimen de saturación el número de mecanismos es prácticamente constante y el ancho de la función de distribución es mucho menor.
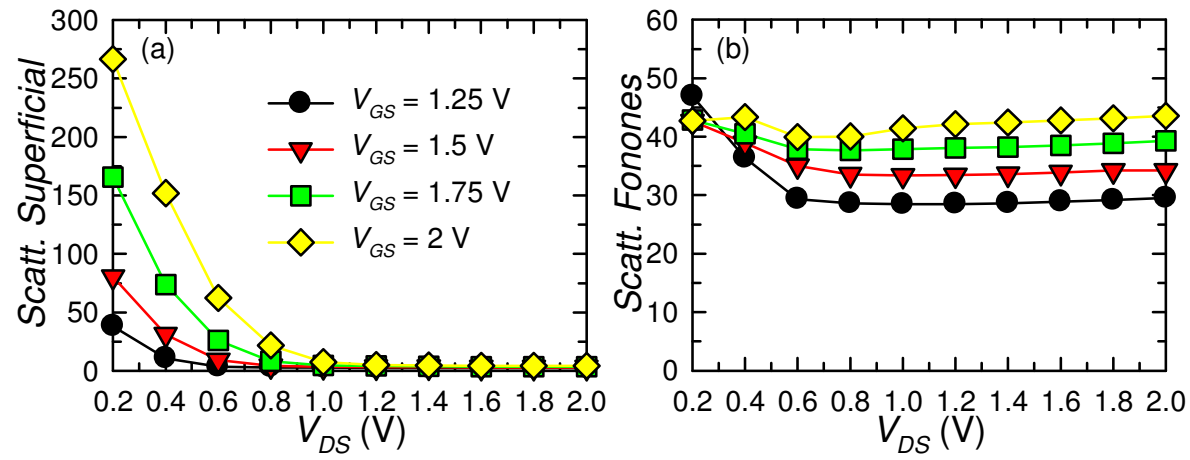

Figura V.27.a. Mecanismos de scattering con fonones y .b. superficiales en función de $V_{D S}$ para distintos valores de $V_{G S}$ 
Con el fin de explicar la variación del número de mecanismos de scattering con el voltaje aplicado en el contacto de drenador (promediados para todo el conjunto de portadores), y por tanto la variación del tiempo de tránsito, examinamos de manera independiente los distintos tipos de mecanismos de scattering considerados en nuestro simulador: las interacciones con la superficie $\mathrm{Si} / \mathrm{SiO}_{2}$ y los mecanismos con fonones (tanto acústicos como ópticos intervalle e intravalle), mostrados en la Figura V.27. Dado que el substrato se encuentra débilmente dopado y los valores de energía cinética de los portadores en el canal son relativamente elevados, el papel que juegan las impurezas sobre el transporte es despreciable.

El número de mecanismos de scattering superficial ${ }^{6}$ medio que sufren los portadores aumenta con la tensión aplicada en puerta en el régimen de triodo debido al campo eléctrico transversal que confina a los electrones junto a la puerta; recordemos que los portadores están concentrados prácticamente en su mayoría en una capa bajo el terminal de puerta (ver Figura V.19.a). Sin embargo, a medida que aumentamos el voltaje de drenador el valor del campo longitudinal también aumenta, reduciéndose la diferencia de potencial entre puerta y drenador; los portadores no están tan cercanos a la interfaz $\mathrm{Si} / \mathrm{SiO}_{2}$ y como consecuencia disminuye el número de mecanismos de scattering superficiales. Por tanto, podemos sostener que el scattering superficial es despreciable cuando el dispositivo opera en saturación, pero no en todo el rango del estado de conducción como afirman [Winstead, 2000], ya que como acabamos de ver, en el régimen de triodo estos mecanismos de scattering presentan un valor elevado.

Por otro lado, a medida que aumentan $V_{G S}$ y $V_{D S}$, aumenta también la energía de los portadores en el canal, y por tanto su probabilidad de interacción con fonones en la red. La influencia de los mismos frente a los mecanismos de scattering superficial en el régimen de triodo es muy débil; sin embargo, cuando el dispositivo entra en el régimen de saturación observamos que los mecanismos de scattering que predominan son los fonones (el scattering superficial toma valores despreciables frente a los anteriores -alrededor de 1 mecanismo de scattering-, ya que la carga ya no se encuentra tan localizada junto a la puerta, sino en todo el volumen del canal, como veíamos en la Figura V.19.b). Podemos así afirmar que, junto con una elección de dopaje bajo del canal, la arquitectura SB-MOS mejora la movilidad de los portadores en el interior de los dispositivos [Larson, 2004].

\footnotetext{
${ }^{6}$ Este mecanismo es suma de los mecanismos de scattering sufridos con la superficie $\mathrm{Si} / \mathrm{SiO}_{2}$ bajo la puerta y en la frontera con el óxido enterrado. Nótese que, contrariamente a lo que sucede en un MOSFET convencional el número de mecanismos en la superficie con el óxido enterrado no es nulo, aunque sí es muy pequeño en comparación con las que tienen lugar con el óxido de puerta.
} 

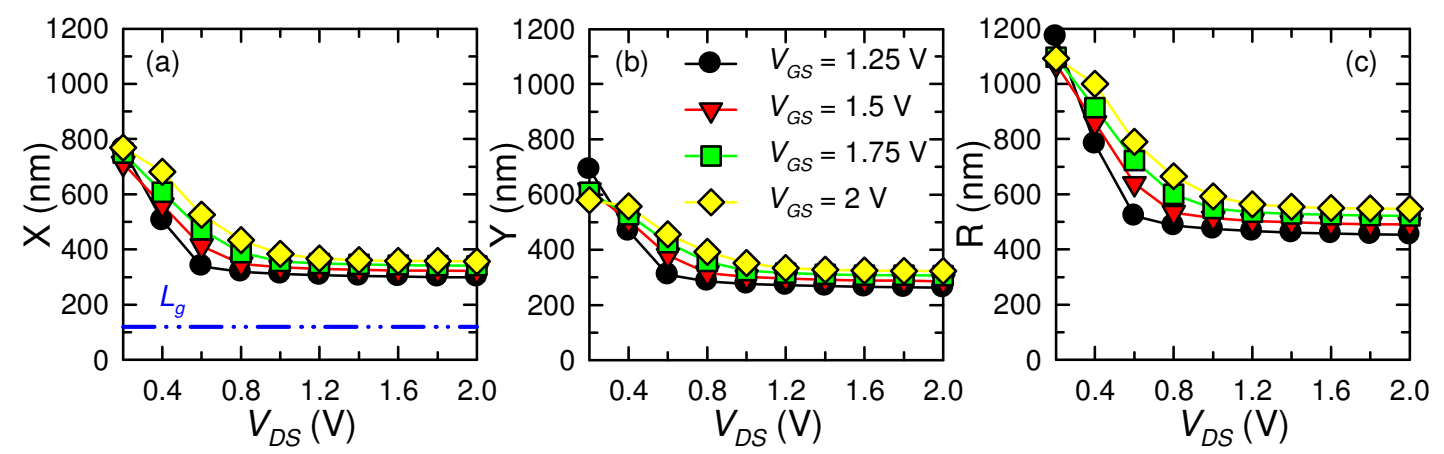

Figura V.28. Distancia recorrida en función de $V_{D S}$ para distintos valores de $V_{G S}$.

Nuestro modelo también nos permite determinar los valores medios del camino recorrido por los portadores a lo largo del eje longitudinal $(X)$ y transversal $(Y)$, así como la longitud recorrida total $(R)$. En la Figura V.28 mostramos la variación de estas magnitudes con la tensión en drenador aplicada para varios voltajes de puerta aplicados. Cuando el dispositivo está en triodo, la distancia recorrida por los electrones disminuye a medida que la tensión aplicada en drenador es mayor. Por otro lado, en saturación la longitud recorrida por los portadores, al igual que el valor de los mecanismos de scattering y el tiempo de tránsito, presenta valores prácticamente constantes con $V_{D S}$ y una ligera variación con $V_{G S}$ ya que estamos analizando el comportamiento de los portadores en la región de debajo de la puerta, en la que la banda de conducción y demás magnitudes internas presentan un valor prácticamente constante con $V_{D S}$. En el régimen de saturación, el valor de la longitud recorrida en media en la dirección $X$ no se aproxima al de la longitud de puerta, como sucede en el MOSFET convencional, presentando además un valor más elevado de la distancia media recorrida en $Y$ en comparación con dicho dispositivo, que nos corrobora que el movimiento es mucho más bidimensional en el SB-MOSFET al ser un dispositivo que trabaja en acumulación.

Hemos calculado también el tiempo medio entre mecanismos de scattering, $\tau$, así como el recorrido libre medio en la dirección longitudinal, $\lambda$, representados en la Figura V.29 para diferentes valores de $V_{G S}$ en función del voltaje aplicado en drenador. Tal y como podemos ver, en el régimen de triodo, el valor de estas magnitudes aumenta con $V_{D S}$ al reducirse el número de mecanismos de scattering totales. En el régimen de saturación permanecen prácticamente invariantes ( $\tau$ varía entre 25 fs y 40 fs y $\lambda$ entre $9 \mathrm{~nm}$ y $7.3 \mathrm{~nm}$ para los valores de $V_{G S}$ mostrados). 


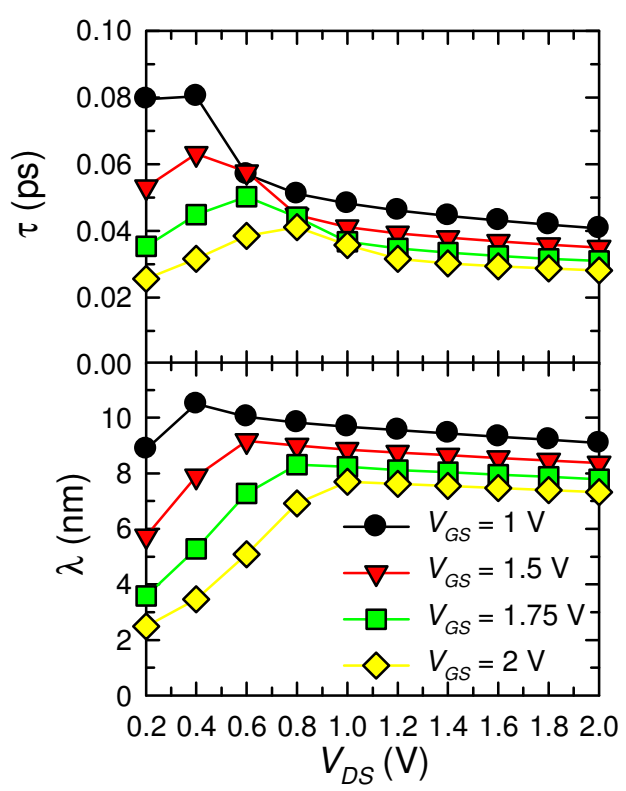

Figura V.29. Tiempo medio y recorrido libre medio entre mecanismos de scattering en función de $V_{D S}$ para distintos valores de $V_{G S}$.

En líneas generales, el comportamiento del tiempo de tránsito con el voltaje aplicado es análogo al de un MOSFET convencional, aunque con valores superiores en el régimen de triodo pero del mismo orden de magnitud en saturación, donde en el SB-MOSFET considerado encontramos un valor de 1.38 ps aproximadamente frente a $1.05 \mathrm{ps}$ del MOSFET convencional de longitud de puerta de $120 \mathrm{~nm}$ analizado en [Martín, 2007].

\section{V.6 Estudio de la variación de parámetros}

En esta sección nos centramos en investigar en detalle el efecto de la variación de diversos parámetros de la arquitectura del SB-MOSFET sobre el transporte de los portadores a través del canal. Este estudio es extremadamente útil en el estudio de dispositivos novedosos, pues permite conocer el efecto de dichos parámetros de manera individual, permitiéndonos optimizar la respuesta del dispositivo a través de la mejora de la corriente $I_{o n}$ a medida que modificamos tanto la altura de barrera Schottky, $q \Phi_{B n}$, como la longitud de underlap, $L_{u n}$. Además, en respuesta a la demanda tecnológica actual de reducir el tamaño de los dispositivos 
también analizamos la respuesta del transistor SB-MOSFET al reducir su longitud de puerta, $L_{g}$.

\section{V.6.a Altura de barrera Schottky}

Como ya hemos visto en los Capítulo III y IV, la variación de la altura de la barrera Schottky influye en gran medida sobre la corriente inyectada a través del contacto. Es por tanto el primer parámetro a reducir según la comunidad científica para que el SB-MOSFET alcance niveles de corriente análogos a los de los dispositivos MOSFET convencionales (véase Sección V.2).
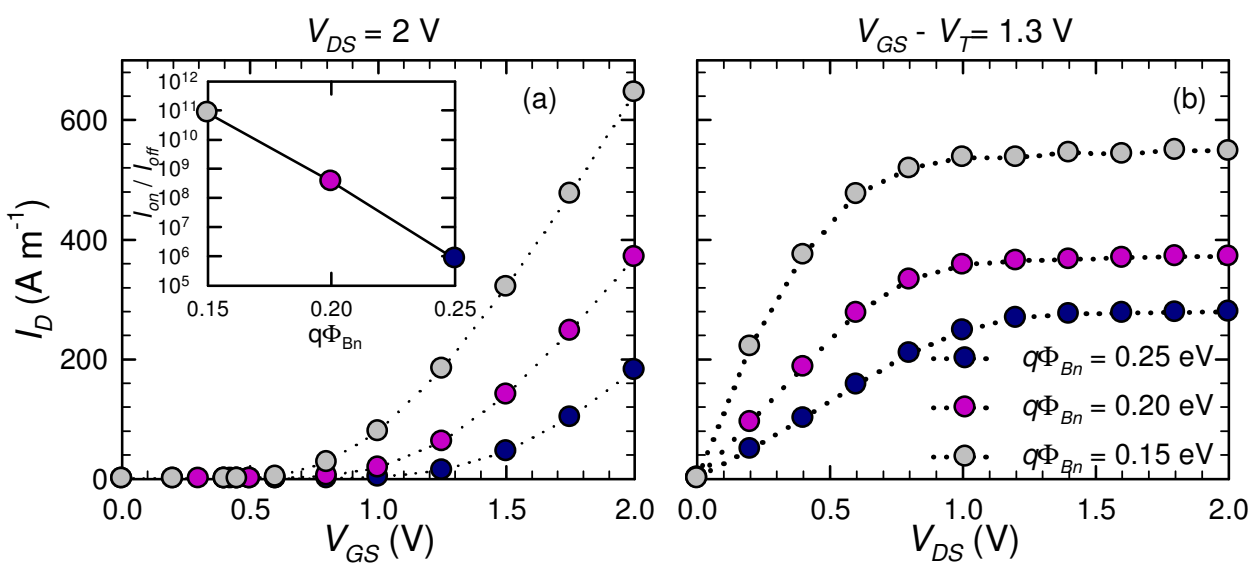

Figura V.30.a. Característica de transferencia para $V_{D S}=2 \mathrm{Vy}$.b. característica de salida para $V_{G S}-V_{T}=1.3 \mathrm{~V}$ para la estructura con $q \Phi_{B n}=0.25 \mathrm{eV}, 0.20 \mathrm{eV}$ y $0.15 \mathrm{eV}$. En el recuadro interior de a. representamos el ratio de corriente $I_{\text {on }} / I_{\text {off }}$, para el mismo valor de $V_{\text {overdrive: }}$ igual a $-0.6 \mathrm{~V}$ en corte y a $1.3 \mathrm{~V}$ en conducción.

Mostramos a continuación los principales resultados obtenidos del análisis de la variación de la altura de la barrera Schottky en el SB-MOSFET analizado. Para ello, hemos realizado simulaciones con la estructura bajo estudio considerando tres valores de $q \Phi_{B n}$ : $0.25 \mathrm{eV}, 0.20 \mathrm{eV}$ (estructura de partida) y $0.15 \mathrm{eV}$. En primer lugar presentamos en la Figura V.30.a la característica de transferencia $\left(V_{D S}=2 \mathrm{~V}\right)$ para las tres alturas de barrera. El valor del voltaje umbral, $V_{T}$, disminuye con la altura de la barrera, obteniéndose $0.95 \mathrm{~V}, 0.70 \mathrm{~V}$ y $0.55 \mathrm{~V}$ para $q \Phi_{B n}=0.25 \mathrm{eV}, 0.20 \mathrm{eV}$ y $0.15 \mathrm{eV}$ respectivamente. 
La Figura V.30.b representa las características de salida de la estructura simulada con los distintos valores de altura de barrera para el mismo voltaje de overdrive, que es igual a la diferencia entre el voltaje aplicado en la puerta y el voltaje umbral $\left(V_{G S}-V_{T}\right)$ (De ahora en adelante, los distintos resultados que mostramos están calculados en igual condición de $V_{\text {overdrive, }}$ con el fin de realizar una comparación más justa entre las distintas estructuras). Observamos el claro aumento de la corriente a medida que disminuimos el valor de la altura de la barrera Schottky, efecto que expondremos detalladamente más adelante. Los valores de corriente obtenidos son del mismo orden de magnitud que los presentados en [Vega, 2006] (con $q \Phi_{B p}=0.25 \mathrm{eV}$ para huecos) y [Su-Zhen, 2008] (con $q \Phi_{B n}$ variando entre $0.5 \mathrm{eV}$ y $0 \mathrm{eV}$ para electrones).

En el recuadro interior de la Figura V.31.a. hemos representado el ratio de corrientes $I_{o n} / I_{\text {off }}$ para los tres valores de $q \Phi_{B n}$. La determinación de este ratio ha sido llevada a cabo en las mismas condiciones de $V_{\text {overdrive, }}$, es decir, en el estado de corte se calcula para $V_{G S}-V_{T}=-0.6 \mathrm{~V}$ y $V_{D S}=2 \mathrm{~V}$ y en conducción para $V_{G S}-V_{T}=1.3 \mathrm{~V}$ y $V_{D S}=2 \mathrm{~V}$. Como podemos observar, obtenemos una mejora exponencial del ratio de corriente a medida que la altura de barrera disminuye. Sin embargo, en el caso de calcular este ratio para un valor de $V_{G S}$ constante, obtenemos el resultado contrario, es decir, el ratio disminuye para el caso de inferior altura de

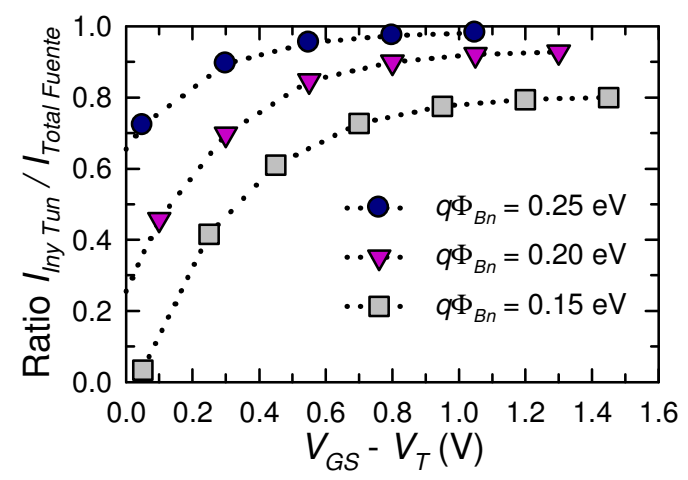

Figura V.31. Ratio entre la corriente de inyección túnel y la corriente total, en la fuente, para los distintos valores de altura de barrera. barrera [Sun, 2003], [Jan, 2004], [Pascual, 2009c].

Es importante estudiar la contribución de las distintas componentes a la corriente total cuando el dispositivo está en el estado de conducción, en especial la contribución de la corriente túnel. En la Figura V.31 mostramos el ratio entre la corriente de inyección túnel y la corriente total en la fuente; como podemos ver, el ratio aumenta con el valor de $V_{\text {overdrive }}$ debido al efecto de modulación de la barrera por la polarización de la puerta; además, presenta valores más cercanos a la unidad para el caso de mayor altura de barrera. Por tanto podemos afirmar que la altura de la barrera tiene un papel fundamental en la inyección de portadores en el canal debido al cambio de la curvatura de la banda junto al contacto de fuente. Esto puede ser corroborado si examinamos la banda de conducción para los tres valores de 
altura de barrera en condiciones de igual voltaje de overdrive $(1.3 \mathrm{~V})$ y $V_{D S}=2 \mathrm{~V}$, representado en la Figura V.32.a. Como podemos ver, al disminuir el valor de $\boldsymbol{q} \Phi_{B n}$, menor es la probabilidad de atravesar la barrera mediante emisión de campo.

Además, en la Figura V.32.b. y .c. mostramos la energía y la velocidad media de los portadores en el canal para $V_{D S}=2 \mathrm{~V}$ y $V_{G S}-V_{T}=1.3 \mathrm{~V}$. La variación de la banda de conducción en la región cercana al drenador con $q \Phi_{B n}$ (ver Figura V.32.a) implica que el valor del campo eléctrico disminuya con la altura de la barrera en la región cercana a la fuente (no mostrado en las gráficas). Este hecho está directamente relacionado con que el máximo de velocidad y energía de los portadores junto a la fuente también se vea reducido con $q \Phi_{B n}$.
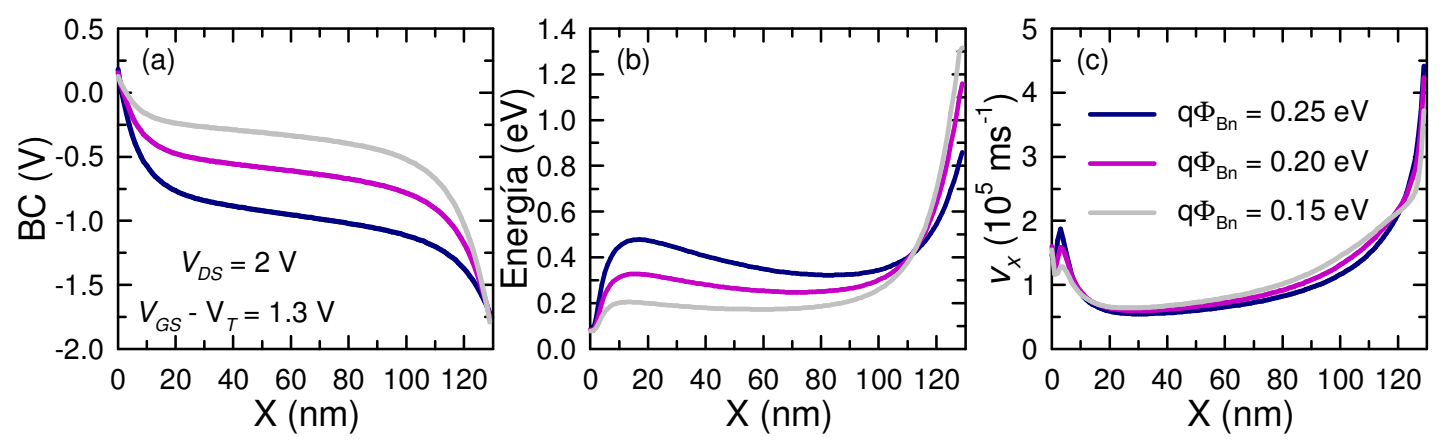

Figura V.32.a Banda de conducción, .b. energía media y .c. velocidad media de portadores en el canal para

$$
V_{D S}=2 \mathrm{Vy} V_{G S}-V_{T}=1.3 \mathrm{~V} \text {. }
$$

La mayor velocidad de los portadores junto al contacto de fuente para los dispositivos con mayor altura de barrera puede hacernos pensar que, aunque la cantidad de corriente es inferior, los portadores tardarán menos en atravesar el canal [Valentin, 2008]. Sin embargo, a medida que los portadores se alejan del contacto de fuente, la velocidad media de los mismos es menor en el caso del dispositivo de mayor altura de barrera Schottky (Figura V.32.c). Para explicar este hecho, en la Figura V.33 mostramos el tiempo de tránsito, $t_{t}$, y los mecanismos de scattering totales sufridos por los portadores en función de $V_{\text {overdrive }}$ en condiciones de saturación $\left(V_{D S}=2 \mathrm{~V}\right)$. Como podemos observar, el tiempo de tránsito disminuye ligeramente al reducir la altura de la barrera Schottky del contacto, lo cual está directamente relacionado con el menor número de mecanismos de scattering (ver Figura V.33.b). Los mecanismos de scattering que sufren los portadores al atravesar el canal son principalmente interacciones con las vibraciones de la red (fonones), que están ligados fundamentalmente a los valores de energía, por lo que, puesto que la energía de los portadores en la mayor parte del canal disminuye con la reducción de la altura de la barrera bajo iguales condiciones de $V_{\text {overdrive, }}$ este 
número es menor para el dispositivo de inferior $q \Phi_{B n}$ (lo que implica que la velocidad media de los portadores en el canal sea menor en el caso de mayor altura de barrera, como decíamos anteriormente). En comparación con el scattering con fonones, el scattering superficial es despreciable cuando el dispositivo está en saturación, como ya se ha explicado en la Sección V.5.d. La distancia total recorrida por los portadores también disminuye con la altura de la barrera, así como el recorrido libre medio y el tiempo entre mecanismos de scattering (no mostrados en las gráficas).
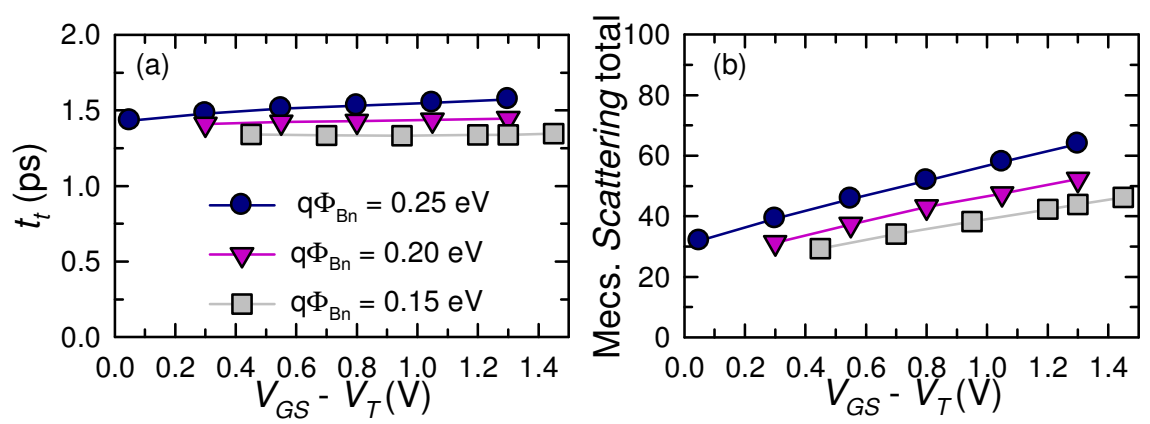

Figura V.33.a Tiempo de tránsito y .b. mecanismos de scattering totales sufridos por los portadores al atravesar el canal para las tres alturas de barrera bajo análisis para $V_{D S}=2 \mathrm{~V}$ y diversos valores de $V_{G S}$.

En la región cercana al contacto de drenador, de acuerdo con el perfil de la banda de conducción, el campo eléctrico es mayor a medida que $q \Phi_{B n}$ disminuye: de este modo, los portadores son más energéticos. Por otro lado, la velocidad de los portadores en esta región en el caso de la altura de barrera más baja se ve ligeramente disminuida. Esto es debido a que hay una mayor ocupación de los valles $\mathrm{L}$ en el caso de $q \Phi_{B n}=0.15 \mathrm{eV}$ que para el caso de alturas de barrera superiores (un $14 \%$ mayor si comparamos la ocupación junto al contacto del drenador para las alturas de $0.15 \mathrm{eV}$ y $0.25 \mathrm{eV}$ ).

Finalmente, podemos concluir que un elevado valor de la altura de barrera Schottky no es beneficioso para el correcto funcionamiento del transistor SB-MOSFET con respecto a las características estáticas debido al bajo nivel de corriente y el mayor tiempo de tránsito, lo cual está directamente relacionado con una peor respuesta dinámica del dispositivo, como veremos en el siguiente capítulo. Sin embargo, la reducción de la altura de la barrera presenta severas dificultades técnicas, debido a la elección de los Siliciuros de los contactos. Por este motivo se buscan también otras opciones para optimizar la respuesta del dispositivo, como es la variación de la longitud de underlap, que estudiamos a continuación. 


\section{V.6.b Longitud de underlap}

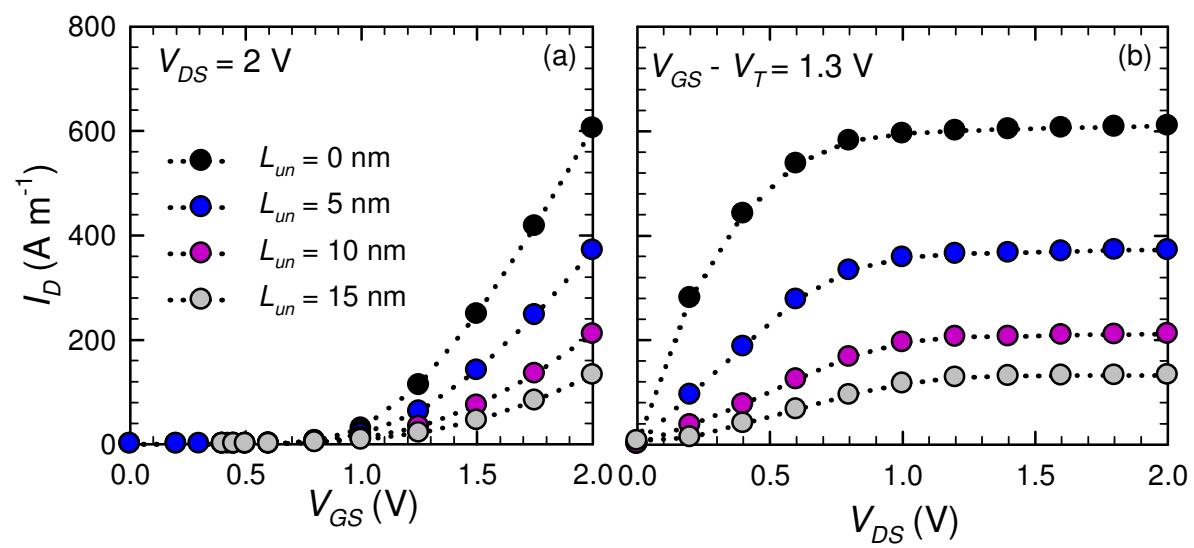

Figura V.34.a Característica de transferencia para $V_{D S}=2 \mathrm{Vy}$.b. característica de salida para $V_{G S}-V_{T}=1.3 \mathrm{~V}$ para la estructura con $L_{u n}=0 \mathrm{~nm}, 5 \mathrm{~nm}, 10 \mathrm{~nm}$ y $15 \mathrm{~nm}$.

La longitud de underlap, es decir, la separación de los contactos de fuente y drenador con el de la puerta es también un parámetro importante a modificar para lograr la optimización del rendimiento del transistor SB-MOSFET, como ya hemos comentado previamente en la Sección V.2. Hemos llevado a cabo el estudio del efecto de la variación de dicho parámetro, considerando cuatro valores de $L_{u n}$ : a $0 \mathrm{~nm}, 5 \mathrm{~nm}$ (estructura inicial), $10 \mathrm{~nm}$ y $15 \mathrm{~nm}$.
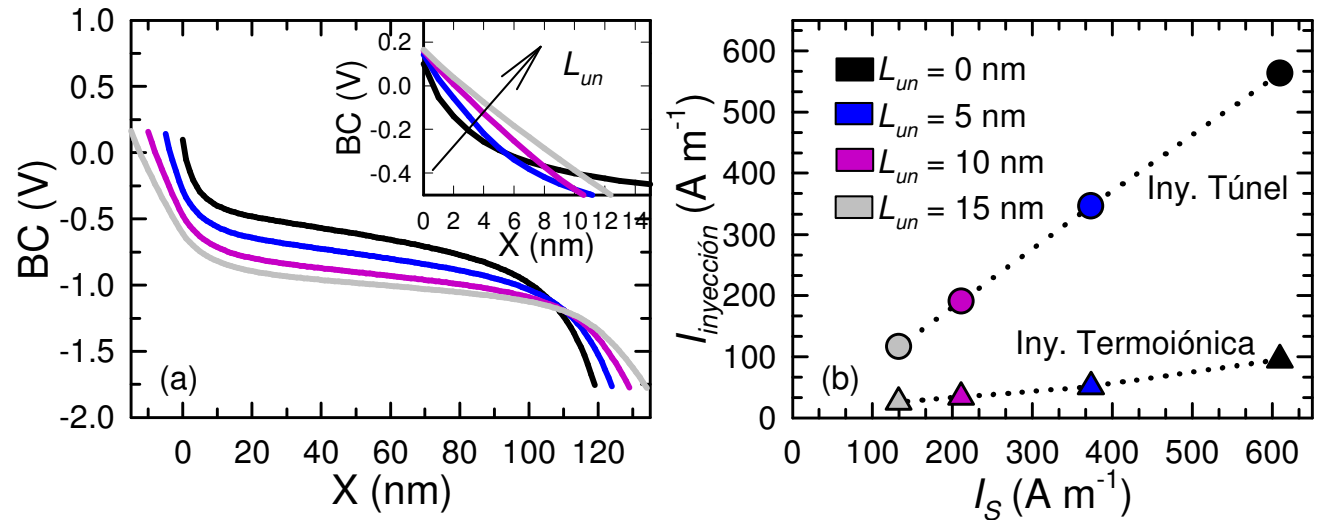

Figura V.35.a. Banda de conducción y .b. componentes de inyección túnel y termoiónica frente a la corriente de fuente para la estructura con $L_{u n}=0 \mathrm{~nm}, 5 \mathrm{~nm}, 10 \mathrm{~nm}$ y $15 \mathrm{~nm}$ bajo $V_{D S}=2 \mathrm{Vy} V_{G s}-V_{T}=1.3 \mathrm{~V}$. El recuadro interior de la Figura a. muestra una ampliación de la banda de conducción en la región cercana al contacto de fuente, desplazadas para tener el mismo origen. 
Las características de transferencia y las características de salida, mostradas en la Figura V.34, nos indican que a medida que la longitud de underlap disminuye, la corriente en el dispositivo aumenta. Además, a partir de la característica de transferencia podemos ver que el voltaje umbral se muestra invariante con $L_{u n}\left(V_{T}=0.7 \mathrm{~V}\right)$. De estos resultados podemos inferir que, incluso en el caso de contar con un dispositivo de altura de barrera Schottky ideal, es decir, inferior a $0.1 \mathrm{eV}$, si la longitud de underlap no se encontrara optimizada y presentase un valor elevado (por ejemplo superior a $15 \mathrm{~nm}$ ), la respuesta en corriente del dispositivo se vería seriamente limitada.

En la Figura V.35.a representamos el perfil de la banda de conducción a lo largo del canal para los diferentes valores de $L_{u n}$. Como podemos ver en el recuadro interior de dicha figura, la pendiente que presenta la barrera de potencial junto a la fuente es mayor cuando $L_{u n}$ es igual a $0 \mathrm{~nm}$, lo que implica un mayor valor de campo eléctrico (no mostrado en las gráficas). Por consiguiente, la inyección túnel y termoiónica se ven favorecidas cuanto menor es la región de underlap (debido a la menor distancia túnel y a una mayor reducción de la barrera por efecto Schottky, respectivamente), aumentando así la corriente total (la concentración de portadores es también mayor). Podemos comprobar este hecho examinando la Figura V.35.b, en la que representamos las componentes de corriente de inyección túnel y termoiónica frente a la corriente de fuente total, $I_{s}$; como podemos ver, ambas componentes aumentan de manera lineal al disminuir $L_{u n}$, siendo además el valor de la componente túnel muy próximo a la corriente total.
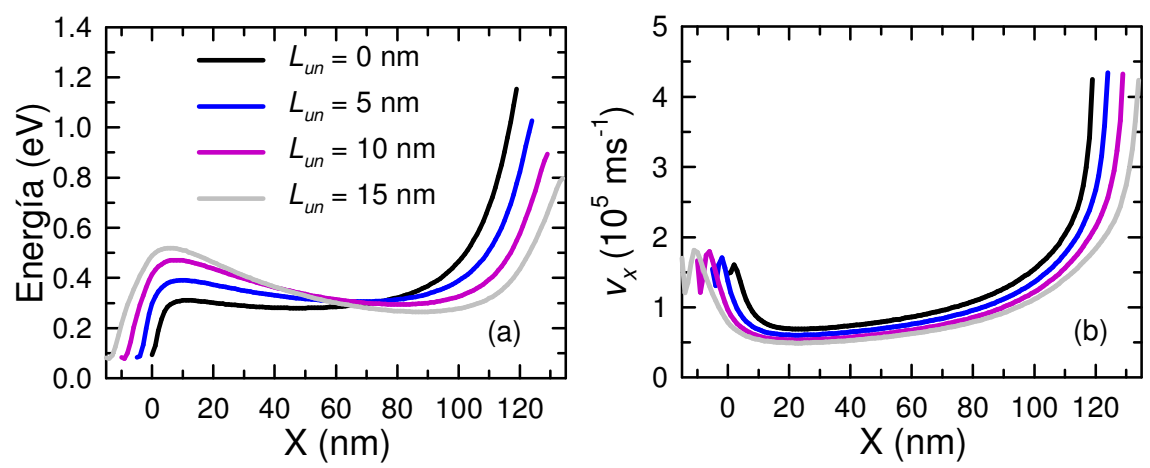

Figura V.36.a. Energía media y.b. velocidad media de portadores en el canal bajo $V_{D S}=2 \mathrm{Vy} V_{G S}-V_{T}=1.3 \mathrm{~V}$ para la estructura con $L_{u n}=0 \mathrm{~nm}, 5 \mathrm{~nm}, 10 \mathrm{~nm}$ y $15 \mathrm{~nm}$. 
La Figura V.36 muestra la variación de la energía y velocidad media de los portadores a lo largo del canal. Podemos ver que el máximo que presentan ambas magnitudes en la zona cercana a la fuente, es menor a medida que disminuye $L_{u n}$. Sin embargo, y como consecuencia de un campo eléctrico más elevado a lo largo del canal y en la zona cercana al drenador, la velocidad y la energía de los portadores aumentan en promedio cuando $L_{u n}$ disminuye.

En conclusión, la reducción de $\boldsymbol{L}_{u n}$ se traduce en un mejor control electrostático del potencial en la interfaz Schottky por parte de la tensión de puerta aplicada, lo que contribuye a aumentar el rendimiento del transistor. Sin embargo, esta alternativa de optimización es limitada, ya que la reducción de $L_{u n}$ dará lugar a un aumento de las capacidades parásitas entre la puerta y los contactos de fuente y drenador [Valentin, 2008b]. Por tanto, debe encontrarse un compromiso en la reducción de $\boldsymbol{L}_{u n}$ para mejorar el rendimiento del dispositivo, no sólo desde el punto de vista de la corriente, sino también considerando el comportamiento a alta frecuencia del dispositivo y los acoplamientos capacitivos.

\section{V.6.c Longitud de puerta}

Con el fin de mejorar el rendimiento de las tecnologías MOS de Silicio, el escalado de los dispositivos se convierte en uno de los principales requerimientos del ITRS [ITRS, 2007]. Por tanto, es primordial explorar las consecuencias de la reducción de la puerta de los dispositivos SOI SB-MOSFET, puesto que se plantean como posible solución a los problemas del escalado del MOSFET convencional.

\begin{tabular}{|c|c|}
\hline $\boldsymbol{L}_{\boldsymbol{g}}(\mathbf{n m})$ & $\boldsymbol{V}_{\boldsymbol{T}}(\mathbf{V})$ \\
\hline 120 & 0.7 \\
\hline 90 & 0.675 \\
\hline 65 & 0.65 \\
\hline 30 & 0.6 \\
\hline
\end{tabular}

Tabla V.2. Voltaje umbral correspondiente a cada valor de $L_{g}$.
A continuación mostramos los resultados más relevantes del análisis del efecto de la variación de la longitud de puerta sobre la respuesta estática del dispositivo. Las estructuras simuladas en este caso presentan longitudes de puerta igual a $120 \mathrm{~nm}$ (estructura inicial), $90 \mathrm{~nm}, 65 \mathrm{~nm}$ y $30 \mathrm{~nm}$. En primer lugar, en la Figura V.37 mostramos las características de salida y transferencia para las cuatro estructuras. Podemos observar que el voltaje umbral apenas se ve modificado por la variación de este parámetro hasta valores de $L_{g}$ de $30 \mathrm{~nm}$, tal y como reflejamos en la Tabla V.2. 

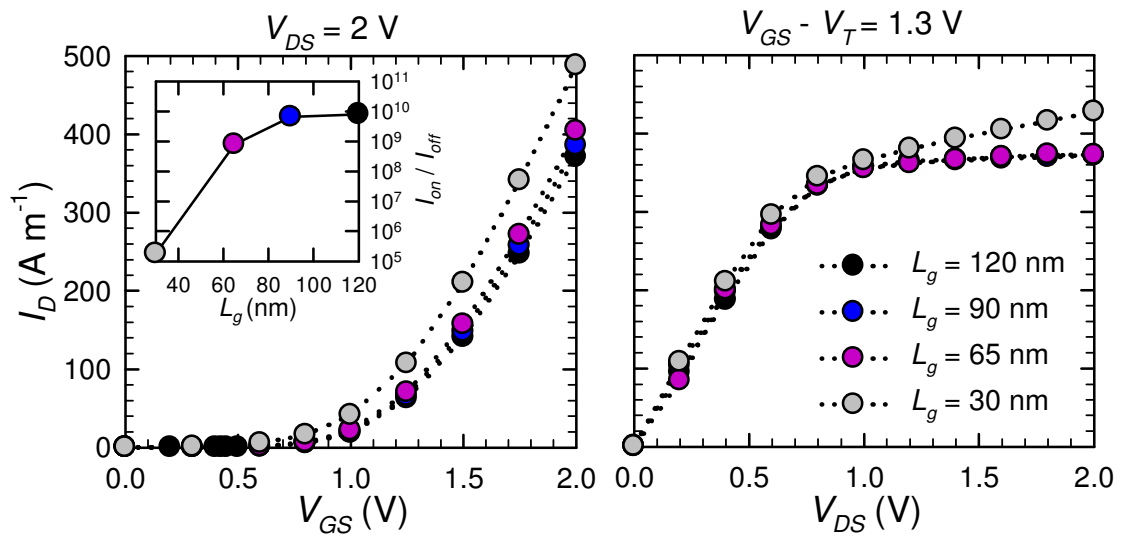

Figura V.37.a. Característica de transferencia para $V_{D S}=2 \mathrm{Vy}$.b. característica de salida para $V_{G S}-V_{T}=1.3 \mathrm{~V}$ para la estructura con $L_{g}=120 \mathrm{~nm}, 90 \mathrm{~nm}, 65 \mathrm{~nm}$ y $30 \mathrm{~nm}$. En el recuadro interior de.a. representamos el ratio de corriente $I_{\text {on }} / I_{\text {off }}$, para el mismo valor de $V_{\text {overdrive: }}$ igual a $-0.6 \mathrm{~V}$ en corte y a $1.3 \mathrm{~V}$ en conducción.

Al analizar el estado de corte de este dispositivo observamos que la corriente $I_{\text {off }}$ aumenta al disminuir la longitud de puerta. Este hecho se ve reflejado en la gráfica del recuadro interior de la Figura V.37.a, donde representamos el ratio $I_{o n} / I_{\text {off }}$ para los cuatro valores de $L_{g}$ simulados $\left(I_{o n}\right.$ calculado para $V_{\text {overdrive }}=1.3 \mathrm{~V}$ e $I_{\text {off }}$ para $\left.V_{\text {overdrive }}=-0.6 \mathrm{~V}\right)$. Como podemos ver, este ratio se mantiene constante hasta $90 \mathrm{~nm}$ aproximadamente y empeora al reducir la longitud de puerta, aunque de manera mucho más pronunciada al pasar de un valor de $65 \mathrm{~nm}$ a $30 \mathrm{~nm}$.
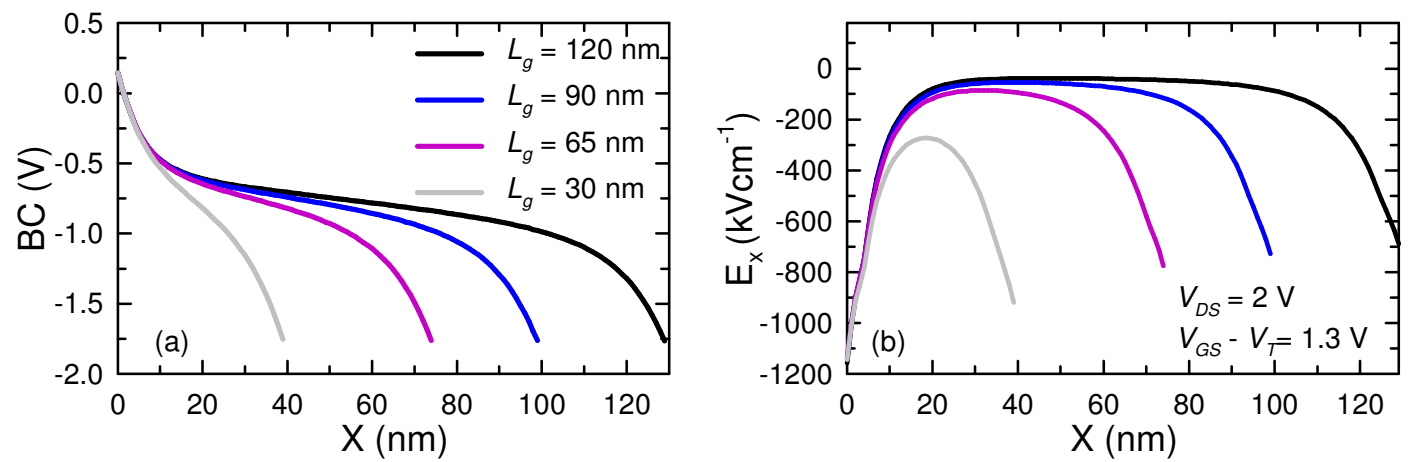

Figura V.38.a. Banda de conducción y .b. campo eléctrico a lo largo del canal para $V_{D S}=2 V y V_{G S}-V_{T}=1.3 \mathrm{~V}$

Por otro lado, la corriente de conducción del dispositivo permanece prácticamente constante al reducir la longitud de puerta, aumentando en mayor medida para la estructura de menor longitud de puerta simulada (los valores de corriente son del mismo orden de magnitud que los encontrados en la literatura para estructuras con diversas longitudes de puerta, por 
ejemplo, véase [Jang, 2004] para $L_{g}=50 \mathrm{~nm}$, [Saha, 2005] para $L_{g}=1 \mu \mathrm{m}$, [Vega, 2006] para $L_{g}<30 \mathrm{~nm}$ ). Este resultado sugiere una excelente inmunidad ante el efecto de canal corto, responsable de la caída del potencial umbral en los MOSFET convencionales al escalar el dispositivo. Debe tenerse en cuenta además que en los resultados presentados se han considerado condiciones de escalado no ideales, en las que el único parámetro modificado ha sido $L_{g}$, sin reducir la tensión aplicada en drenador ni otros parámetros de la simulación. La débil dependencia de la corriente de conducción del dispositivo con $L_{g}$ es fácil de entender si estudiamos las magnitudes internas para un mismo valor de $V_{\text {overdrive }}(=1.3 \mathrm{~V})$. En las Figuras V.38 y V.39 mostramos la banda de conducción junto con el campo eléctrico, la concentración y la velocidad media de los portadores, respectivamente, a lo largo del canal para las cuatro estructuras consideradas.
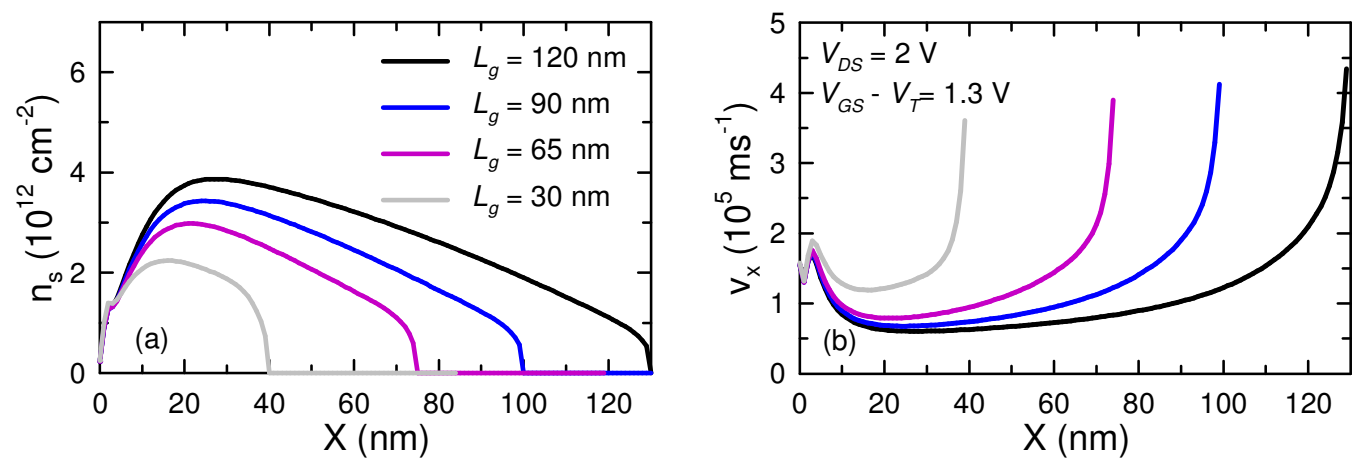

Figura V.39.a. Concentración y.b. velocidad media de portadores para $V_{D S}=2 \mathrm{Vy} V_{G S}-V_{T}=1.3 \mathrm{~V}$.

Tal y como vemos en la Figura V.38.a, la curvatura de la banda de conducción en la región cercana a la fuente se mantiene prácticamente invariante con la longitud de puerta dando lugar al mismo valor de campo eléctrico en esta zona (Figura V.38.b). En consecuencia los niveles de inyección túnel y termoiónicos son aproximadamente constantes y por tanto también la corriente total (la componente de inyección túnel constituye un $90 \%$ del total y la inyección termoiónica un 10\%, independientemente del valor de la longitud de puerta y en las mismas condiciones de polarización aplicada). Por otro lado, esto es congruente con los valores de concentración y velocidad media (Figura V.39), ya que la concentración disminuye con $L_{g}$ mientras que la velocidad de los portadores aumenta; el producto de ambas magnitudes, que es proporcional a la corriente, es prácticamente constante. Sin embargo, en el caso del transistor de $30 \mathrm{~nm}$ de longitud de puerta, la proximidad de los contactos de fuente y drenador da lugar a que la banda de conducción no presente la región plana en la región media del canal (entre ambos 
contactos) que sí se aprecia en los dispositivos de $L_{g}$ superior. En consonancia con este comportamiento, vemos que aunque el valor del campo eléctrico junto al contacto de fuente apenas varía con la longitud de puerta, el valor medio en el resto del canal sí se ve claramente incrementado en el caso de $L_{g}=30 \mathrm{~nm}$, presentando por tanto los portadores altas velocidades medias (superiores a $1.5 \cdot 10^{5} \mathrm{~ms}^{-1}$ en la zona media del canal).

Vamos a estudiar otra serie de factores que nos darán una idea del tipo de transporte en el canal. En la Figura V.40 mostramos la función de distribución de la distancia total recorrida por los portadores, $R$, es decir, la distancia real recorrida en nuestro simulador bidimensional por los portadores desde fuente a drenador bajo el terminal de puerta. Observamos como en general la función de distribución presenta un ancho espectro, siendo la distancia total recorrida mucho mayor que $L_{g}$. A medida que la longitud de puerta disminuye, la anchura de la función de distribución también es menor y

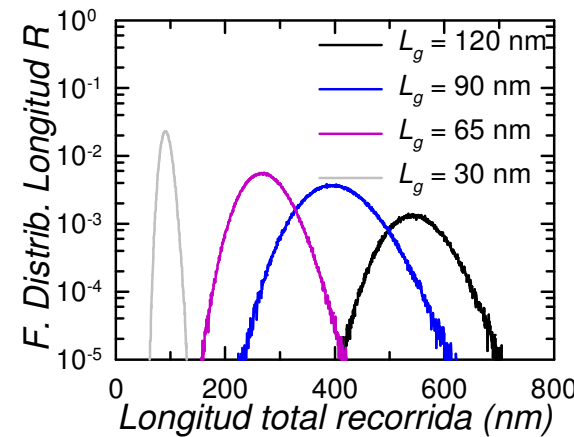

Figura V.40 Función de distribución de la distancia bidimensional recorrida, $R$, para distintos valores de $L_{g}$. su máximo se encuentra localizado en valores inferiores, aunque aún alejados del valor de $L_{g}$.
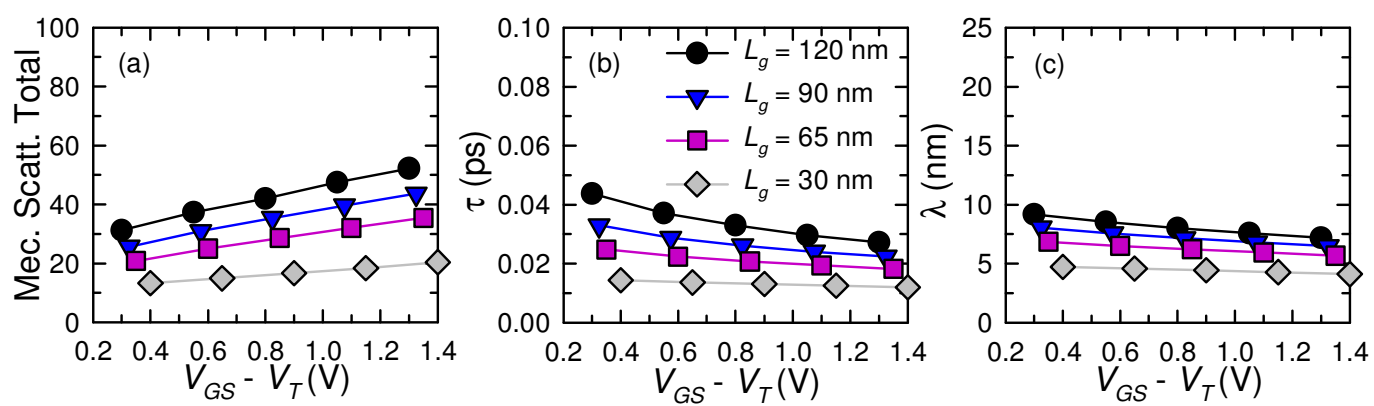

Figura V.41.a. Número de mecanismos de scattering .b. tiempo medio y.c. recorrido libre medio para

$$
V_{D S}=2 \mathrm{~V} \text {. }
$$

En la Figura V.41 mostramos el número de mecanismos de scattering, el tiempo medio entre dichos mecanismos, $\tau$, y el recorrido libre medio en la dirección longitudinal, $\lambda$, para las cuatro longitudes de puerta consideradas. Como es lógico, el número de mecanismos de scattering totales disminuye al reducirse la longitud de puerta (pasando, para las condiciones de polarización de $V_{D S}=2 \mathrm{~V}$ y $V_{G S}-V_{T}=1.3 \mathrm{~V}$, de 52 mecansimos de scattering en el caso de 
$L_{g}=120 \mathrm{~nm}$ a 19 mecanismos cuando $L_{g}=30 \mathrm{~nm}$ ). Sin embargo la influencia neta de los mecanismos de scattering sobre el transporte de portadores en el canal es mayor cuando $L_{g}$ se ve disminuida, lo que explica la reducción de $\lambda$ y $\tau$ con el menor valor de longitud de puerta, tal y como vemos en dicha figura. Esto indica que, a medida que se reduce la longitud del contacto de puerta, el canal conductor hace más difusivo el transporte de los portadores.

Para analizar las posibles propiedades del transporte balístico en el dispositivo a medida que disminuimos el valor de $L_{g}$, nuestro simulador nos permite desactivar artificialmente los mecanismos de scattering en el canal, asumiendo así una situación completamente balística (Capítulo II). De este modo, podemos obtener el valor de la corriente en condiciones idealmente balísticas, $I_{D, \text { bal }}$, y el tiempo de tránsito en ese contexto.

En la Figura V.42.a mostramos el valor de la corriente en drenador cuando se consideran los mecanismos de scattering, $I_{D}$, y la corriente en condiciones de balisticidad, $I_{D \text {, bal }}$, en función de $L_{g}$. Los cálculos han sido realizados para el mismo $V_{\text {overdrive, }}$ por lo que, como veíamos en la Figura V.37, el valor de $I_{D}$ permanece prácticamente constante para valores de $L_{g}$ superiores a $65 \mathrm{~nm}$, presentando un aumento en el caso de inferior longitud de puerta simulada. Como podemos observar, el valor de $I_{D \text {, bal }}$ disminuye al reducir $L_{g}$, siendo así más próximo al valor de $I_{D}$ en el caso de menor longitud de puerta simulada. Podemos comprobar este hecho en la Figura V.43.b, donde mostramos el ratio entre ambas corrientes, también denominado coeficiente de balisticidad [Eminente, 2005]. Como podemos ver, en el caso de $\boldsymbol{L}_{g}=\mathbf{3 0} \mathbf{~ n m}$ este ratio es del $75 \%$ aproximadamente, obteniéndose un valor muy similar al del caso del MOSFET convencional [Martín, 2007].
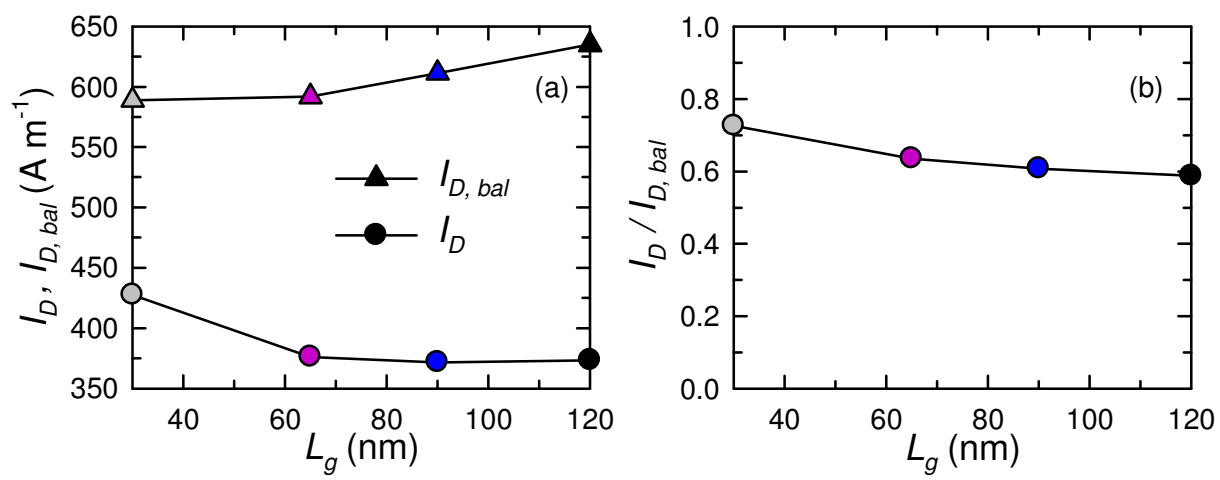

Figura V.42.a. Corriente en drenador con y sin mecanismos de scattering, $I_{D \text {, on }} y I_{D \text {, off }}$ respectivamente .b. Ratio entre ambas corrientes, también denominado coeficiente de balisticidad, en función de $L_{g}$. 
En conclusión, como acabamos de ver, podemos reducir el valor de la longitud puerta por cuestiones de empaquetamiento, aunque no se obtiene una mejora significativa en cuanto a la respuesta en corriente del dispositivo. 


\section{Bibliografía del Capítulo $V$}

Awano M. et al., "Advanced DSS MOSFET Technology for Ultrahigh Performance Applications", VLSI Symp. Tech. Dig., 24 (2008)

Brennan K. F. and Summers C. J., "Theory of resonant tunneling in a variably spaced multiquantum well structure: An Airy function approach," J. Appl. Phys., 61, 614 (1987)

Calvet L. E., Luebben H., et al., "Suppression of leakage current in Schottky barrier metaloxide-semiconductor field-effect transistors", Journal of Applied Physics, 91, 757 (2002)

Connelly D., Faulkner C., Grupp D. E., "Performance advantage of Schottky source/ drain in ultrathin body silicon on insulator and dual-gate CMOS", IEEE Trans. Electron Dev., 50, 1340 (2003)

Connelly D., Faulkner C., Grupp D. E., "Optimizing Schottky S/D Offset for 25-nm Dual-Gate CMOS Performance", IEEE Electron Device Lett., 24, 411 (2003b)

Datta S., "Nanoscale device modeling: the Green's function method" Superlattices and Microstructures, 28, 253 (2000)

Ding-Yu L. et al., "Schottky barrier MOSFET structure with silicide source/drain on buried metal", Chin. Phys. Soc., 16, 240 (2007)

Du G., Liu X. et al., "Characterizations of Double-gate SBTT studying by a 2-D full-band Monte Carlo device simulator", International workshop on junction technology, Shanghai, (IWJT '04), 325 (2004)

Dubois E. And Larrieu G., "Measurement of low Schottky barrier heights applied to metallic source/drain metal-oxide-semiconductor field effect transistors”, J. Appl. Phys., 96, 729 (2004)

Duboz J.Y., Badoz P. A., Arnaud F. and Chroboczek J. A., "Electronic transport properties of epitaxial erbium silicide/silicon heterostructures", Appl. Phys. Lett., 55, 84 (1989)

Eminente S. et al., "Understanding quasi-ballistic transport in nano-MOSFETs: part IITechnology scaling along the ITRS”, IEEE Trans. Electron Dev., 52, 2736 (2005) 
Fritze M., Chen C. L., et al. "High-speed Schottky-barrier pMOSFET with fT = $280 \mathrm{GHz}$," IEEE Electron Device Lett., 25, 220 (2004)

Guo J. and Lundstrom M. S., "A Computational Study of Thin-Body, Double-Gate, Schottky Barrier MOSFETs", IEEE Trans. on Electron Dev., TED.2002.804696 (2002)

Huang C. -K., Zhang W. E., and Yang C. H., "Two-Dimensional Numerical Simulation of Schottky Barrier MOSFET with Channel Length to $10 \mathrm{~nm}$ ", IEEE Trans. on Electron Dev., 45, 842, (1998)

Ieong M. et al., "Comparison of Raised and Schottky Source/Drain MOSFETs Using a Novel Tunneling Contact Model”, IEDM Tech. Dig., 98, 733 (1998)

ITRS "The International Technology Roadmap for Semiconductors (ITRS), Semiconductor Industry Association”, Edition, Austin, TX, International Sematech (2007) (disponible en http://public.ITRS.net)

Jang M. et al., "A 50-nm-gate-length erbium-silicided n-type Schottky barrier metal-oxidesemiconductor field-effect transistor", Applied Physics Letters, 84, 741 (2004)

Jiménez D. et al., "A drain current model for Schottky-barrier CNT-FETs", J. Comput Electron, 361 (2006)

Jiménez D., "A current-voltage model for Schottky-barrier graphene-based transistors", Nanotechnology, 19, 345204 (2008)

Kedzierski J., Xuan P., et al., “A $20 \mathrm{~nm}$ gate-length ultra-thin body p-MOSFET with silicide source/drain", Superlattices and Microstructures, 28, 445 (2000)

Kedzierski J., Xuan P., et al., "Complementary silicide source/drain thin-body MOSFETs for the $20 \mathrm{~nm}$ gate length regime", IEDM Tech. Dig., 57 (2000b)

Kinoshita A. et al., "Solution for High-Performance Schottky-Source/Drain MOSFETs: Schottky Barrier Height Engineering with Dopant Segregation Technique" VLSI Symp. Tech. Dig. 168 (2004)

Kinoshita T. et al., "Ultra Low Voltage Operations in Bulk CMOS Logic Circuits with Dopant Segregated Schottky Source/Drain Transistors", IEDM Tech. Dig., (2006)

Knoch J., Appenzeller J., "Impact of the channel thickness on the performance of Schottky barrier metal-oxide-semiconductor field-effect transistors", Applied Physics Letters, 81, 3082 (2002)

Knoch J., Zhang M. et al., "Effective Schottky barrier lowering in silicon-on-insulator Schottkybarrier metal-oxide-semiconductor field-effect transistors using dopant segregation" Applied Physics Letters, 87, 263505 [1-3] (2005) 
Koeneke C. J., Sze S. M., Levin R. M., and Kinsbron E., "Schottky MOSFET for VLSI," IEDM Tech. Dig., 367 (1981)

Koeneke C. J. and Lynch W. T., "Lightly doped Schottky MOSFET," IEDM Tech. Dig., 466 (1982)

Larrieu G., Dubois E., 'Schottky-Barrier Source/Drain MOSFETs on Ultra-thin Silicon-onInsulator body with a Tungsten Metallic Midgap Gate', IEEE Electron Device Lett., 25, 801 (2004)

Larrieu G. and Dubois E., "Integration of PtSi-based Schottky-Barrier p-MOSFETs with a midgap Tungsten gate" IEEE Trans. Electron Dev., 52, 2720 (2005)

Larrieu G. and Dubois E. et al., "Low Temperature Implementation of Dopant-Segregated Band-edge Metallic S/D junctions in Thin-Body SOI p-MOSFETs.” IEDM Tech. Dig., 147 (2007)

Larson J. M., "High-speed Schottky-barrier pMOSFET with $f_{T}=280 \mathrm{GHz}$ ", IEEE Electron Device Lett., 25, no. 4, 220 (2004)

Larson J. M., "Schottky Barrier CMOS. Scalable, Lower Cost, Radiation Tolerant, High Performance Technology for Sub-25nm Gate Lengths", Spinnaker Semiconductor Inc., Technology overview (2004b)

Larson J. M., Snyder J. P., "Overview and Status of Metal S/D Schottky-Barrier MOSFET Technology", IEEE Trans. Electron Dev., 53, 1048 (2006)

Laux S. E. y Fischetti M. V., "Monte-Carlo Simulation of Submicrometer Si n-MOSFETs at 77$\mathrm{K}$ and 300-K”, IEEE Electron Device Lett., 9, 467 (1988)

Lepselter M. P. and Sze S. M., "SB-IGFET: An insulated-gate field-effect transistor using Schottky barrier contacts for source and drain," Proc. IEEE, 56, 1400, (1968)

Lui W. and Fukuma M., "Exact solution of the Schrödinger equation across an arbitrary onedimensional piece-linear potential barrier”, J. Appl. Phys. 60, Num. 5 (1986)

Martín M. J., Rengel R., Pascual E. and González T., "Monte Carlo analysis of carrier transport from diffusive to ballistic regime in nanometer SOI MOSFETs", IEEE, 2007 Spanish Conference on Electron Devices, 340 (2007)

Matsuzawa K., Uchida K. and Nishiyama A., "Monte Carlo simulation of sub-0.1mm devices with Schottky contact model” IEICE Trans. Electron., E83-C, 1212 (2000)

Mochizuki T. and Wise K. D., “An n-channel MOSFET with Schottky source and drain," IEEE Electron Device Lett., 5, 108 (1984) 
Nishi Y., "Insulated gate field effect transistor and its manufacturing method," patente japonesa $\mathbf{5 8 7 , 5 2 7 ,} 162(1970)$

Norde H., de Sousa Pries J., d'Heurle F., Petersson S., Pesavento F. and Tove P. A., "The Schottky-barrier height of the contact between some rare-earth metals (and silicides) and P-type silicon", Appl. Phys. Lett., 38, 865 (1981)

Ong D. G., "Modern MOS Technology - Processes, Devices \& Design", McGraw-Hill: New York (1984)

Pascual E., Rengel R. y Martín M. J., "Current drive in n- type Schottky Barrier MOSFETs: a Monte Carlo study”, IEEE, 2009 Spanish Conference on Electron Devices, 108 (2009b)

Pascual E., Rengel R. y Martín M. J., "Intrinsic Noise Sources in a Schottky Barrier MOSFET: a Monte Carlo Analysis", Proceedings of the 20th International Conference on Noise and Fluctuations, AIP Conference Proceedings (2009c)

Pierret R. F., "Dispositivos de Efecto de Campo", Wilmington, DE: Addison-Wesley Iberoamericana (1994)

Rengel R., Mateos J., Pardo D., González T. y Martín M. J., "Monte Carlo análisis of dynamic and noise performance of submicron MOSFETs at RF and microwave frequencies", Semicond. Sci. Technol., 16, 939 (2001)

Rengel R., “Análisis de los Fenómenos de Trasporte y Ruido Electrónico en Transistores MOSFET y SOI Submicrométricos", Tesis Doctoral, Universidad de Salamanca (2002)

Saha A. R., Chattopadhyay S., Bose C., Maiti C. K., "Technology CAD of silicided Schottky barrier MOSFET for elevated source-drain engineering”, Materials Science and Engineering B, 124-125, 424 (2005)

Saitoh W., Itoh A., Yamagami S., Asada M., “Analysis of short-channel Schottky source/drain Metal-Oxide-Semiconductor field-effect transistor on Silicon-on-Insulator substrate and demonstration of sub-50 nm n-type devices with metal gate", Jpn. J. Appl. Phys., 38, $6226(1999)$

Snyder J. P., Helms C. R., "Experimental investigation of a PtSi source and drain field emission transistor", Appl. Phys. Lett. 67, 1420 (1995)

Snyder J. P., "The physics and technology of platinum silicide source and drain field effect transistors", Ph.D. dissertation, Stanford Univ., Stanford, CA (1996)

Sugino M., Akers L. A., Rebeschini M. E., "CMOS latch-up elimination using Schottky barrier p" IEEE Electron Device Meeting IEDM, 462 (1982) 
Sugino M., Akers L. A., Rebeschini M. E., "Latchup-free Schottky-barrier CMOS," IEEE Trans. Electron Dev., 30, 110 (1983)

Sun L., Lu D. Y., Du G. y Han R. Q., "Monte Carlo Simulation of Schottky Contact with Direct Tunnelling Model”, Semiconductor Science and Technology, 18, 576 (2003)

Sun L., Liu X. Y., Du G. y Han R. Q., “A planar asymmetric Schottky barrier source/drain structure for nano-scale MOSFETs", Semiconductor Science and Technology, 21, 608 (2006)

Su-Zhen L., Hong-Xia L., "Quantum compact model for thin-body double-gate Schottky barrier MOSFETs", Chinese Physics B, 17, 3077 (2008)

Swirhun S. E., Sangiorgi E., Weeks A. J., Swanson R. M., Saraswat K. C. and Dutton R. W., "A VLSI-suitable Schottky-barrier CMOS process," IEEE Trans. Electron Dev., 32, 194 (1985)

Sze S. M., Physics of Semiconductor Devices, $2^{\text {nd }}$ edn., Wiley, New York (1981)

Tsui B. -Y. and Lu C. -P., "Current Transport Mechanisms of Schottky Barrier and Modified Schottky Barrier MOSFETs", Essderc, 307 (2007)

Tu K. N., Thompson R. D. and Tsau B. Y., "Low Schottky barrier of rare-earth silicide on nSi”, Appl. Phys. Lett., 38, 626 (1981)

Tucker J. R., Wang C. and Carney P. S., "Silicon field-effect transistor based on quantum tunnelling" Appl. Phys. Lett., 65, 618 (1994)

Tucker J. R., Wang C., Lyding J. W., Shen T.-C. and Abeln G. C., "Nanometer scale MOSFETs and STM paterning on Si," Proc. Int. Conf. Solid State Devices Mater., 322 (1994b)

Valentin R., Dubois E. et al., "RF small-signal analysis of Schottky-barrier p-MOSFET" IEEE Trans. Electron Dev., 55, 1192 (2008)

Valentin R., "Investigation du Comportement et des Potentialités en Haute Fréquence de transistors MOSFET Avancés à Contacts Source/Drain Métalliques" Tesis Doctoral, (2008b)

Vega R. A., "On the modeling and design of Schottky field-effect transistors," IEEE Trans. Electron Dev., 53, 866 (2006)

Vega R. A., "Comparison study of tunneling models for Schottky field effect transistors and the effect of Schottky barrier lowering," IEEE Trans. Electron Dev., 53, 1593 (2006b)

Vega R. A., "A Comparative Study of Dopant-Segregated Schottky and Raised Source/Drain Double-Gate MOSFETs", IEEE Trans. Electron Dev., 55, 2665, (2008) 
Wang C., Tucker J. R., "Sub-40 nm PtSi Schottky source/drain metal-oxide-semiconductor field-effect transistor”, Appl. Phys. Lett., 74, 1174 (1999)

Winstead B., Ravaioli U., "Simulation of Schottky barrier MOSFETs with a coupled quantum injection/Monte Carlo technique”, IEEE Trans. on Electron Dev., 47, 1241 (2000)

Wong H. -S., Koh A. T. -Y., et al., "Source and Drain Series Resistance Reduction for NChannel Transistors Using Solid Antimony (Sb) Segregation (SSbS) During Silicidation” IEEE Electron Dev. Lett., 29, 756 (2008)

Xia Z., Du G. et al., "Investigation of RF performance of nano-scale ultra-thin-body Schottkybarrier MOSFETs using Monte Carlo simulation”, IEEE Conference on Electron devices and Solid-State circuits, 305 (2005)

Xiong S., King T. -J., and Bokor J., "A Comparison Study of Symmetric Ultrathin-Body Double-Gate Devices With Metal Source/Drain and Doped Source/Drain”, IEEE Trans. Electron Dev., 52, 1859 (2005)

Yeh S. -P, Shih C. -H., Gong J. and Lien C., "Latent noise in Schottky barrier MOSFETs", Journal of Statistical Mechanics, P01036 (2009)

Zeng L., Yan Liu X., et al., "A Monte Carlo Study of Ambipolar Schottky Barrier MOSFETs", IEEE International Workshop on Computational Electronics 13 (2009)

Zeng L., Yan Liu X., et al., "A Computational Study of Dopant-Segregated Schottky Barrier MOSFETs", IEEE Transtactions on nanotechnology, 9 (2010)

Zhang M. et al., "Impact of dopant segregation on fully depleted Schottky-barrier SOIMOSFETs", Solid-State Electronics, 50, 594 (2006) 


\section{Comportamiento dinámico y análisis del ruido del SB-MOSFET}

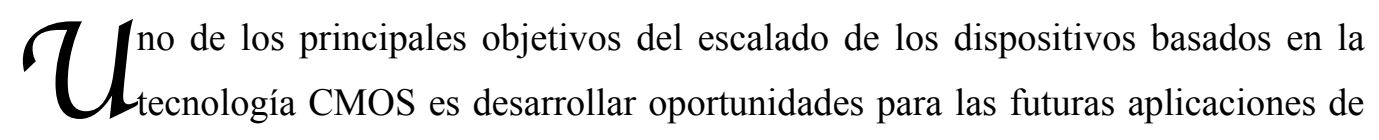
alta velocidad y baja potencia, ofreciendo así una mayor eficiencia para los dispositivos móviles. Por otro lado, a la hora de medir o amplificar señales de pequeña amplitud, el ruido electrónico (fluctuaciones en corriente, voltaje o temperatura) influye en las propiedades de los dispositivos, imponiendo un límite para el tratamiento de la señal. Un exceso de ruido puede limitar seriamente la funcionalidad del circuito tanto en aplicaciones analógicas como digitales [Deen, 2005], por lo que se hace imprescindible conocer las diferentes fuentes de ruido y su origen físico para así poder minimizar sus efectos. Por otra parte, a la hora de integrar los dispositivos SB-MOSFET en un circuito, es imprescindible poder predecir el comportamiento de los transistores en función de la polarización aplicada, la frecuencia, etc. De este modo se podrá optimizar el diseño de los dispositivos, y conocer tanto las ventajas como las desventajas de los mismos. En particular, en el caso que nos ocupa es fundamental cuantificar el impacto de considerar contactos de fuente y drenador metálicos en la respuesta dinámica del dispositivo, además de en el ruido electrónico del mismo. 
En el presente capítulo veremos en primer lugar el procedimiento de cálculo de los parámetros de pequeña señal de un dispositivo de tres terminales, centrándonos en el circuito equivalente que vamos a considerar para el estudio de los dispositivos. Posteriormente, en la Sección VI.2, pasaremos a presentar nuestros resultados. Inicialmente evaluaremos la respuesta dinámica del SB-MOSFET en el régimen de saturación frente a la variación del voltaje en puerta aplicado. A continuación, en la Sección VI.3 analizaremos además el comportamiento de los parámetros dinámicos con respecto a la variación de la altura de la barrera, la longitud de underlap y la longitud de puerta. Una vez analizados estos parámetros, y junto al estudio de las características estáticas llevado a cabo en el Capítulo V, procederemos al estudio del ruido en el SB-MOSFET en la Sección VI.4. En primer lugar presentamos las distintas etapas consideradas para llevar a cabo mediante el método Monte Carlo el análisis del ruido electrónico en transistores de tipo FET, además de describir los diversos parámetros que caracterizan este fenómeno. Llevaremos a cabo en esta sección el estudio de las fuentes internas de ruido, y finalmente analizaremos de las diversas figuras de mérito del ruido del dispositivo, estudiando el efecto de la variación de los parámetros geométricos anteriormente mencionados.

\section{VI.1. Caracterización de pequeña señal}

La respuesta dinámica de un SB-MOSFET puede ser caracterizada a través de la representación del dispositivo como una "caja negra" de tres terminales (véase Figura VI.1) caracterizada por una serie de parámetros que relacionen magnitudes como la corriente o el voltaje a la entrada con los obtenidos a la salida [van der Ziel, 1986], [Rengel, 2002]. De entre los distintos grupos de parámetros empleados usualmente para este estudio, en nuestros cálculos usaremos los parámetros admitancia $Y$, ya que pueden calcularse directamente en la simulación Monte Carlo [González G., 1997], [Rengel, 2002]. El método se basa en el análisis de Fourier de la respuesta transitoria de la corriente instantánea cuando aplicamos pequeños saltos de tensión en los terminales

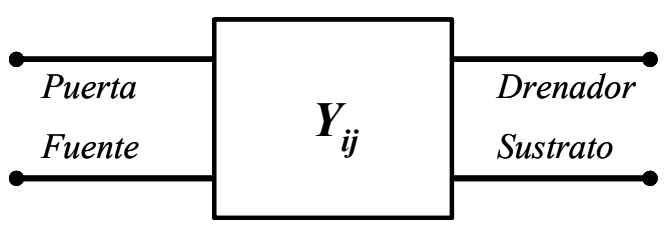

Figura VI.1. Representación del MOSFET como una "caja negra" de tres terminales (los terminales de fuente y sustrato suelen estar cortocircuitados en la práctica) que caracterizaremos por los parámetros admitancia $Y$, calculados directamente en la simulación Monte Carlo. 
( $\Delta V_{G S}$ en la puerta y $\Delta V_{D S}$ en el drenador, de manera independiente) partiendo de un punto de operación estacionario. Los valores de los saltos de tensión deben ser adecuadamente escogidos, es decir, lo suficientemente grandes como para imponerse al ruido numérico y físico, pero lo suficientemente pequeños como para evitar la generación de armónicos. La simulación tiene lugar hasta que se han alcanzado de nuevo condiciones estacionarias. Los parámetros $\mathrm{Y}$ se definen del siguiente modo [Vendelin, 1990]:

$$
\begin{aligned}
& i_{1}(f)=Y_{11}(f) v_{1}(f)+Y_{12}(f) v_{2}(f) \\
& i_{2}(f)=Y_{21}(f) v_{1}(f)+Y_{22}(f) v_{2}(f)
\end{aligned}
$$

Donde $i$ representa la variación con respecto al valor estacionario de las corrientes y $v$ respecto a los potenciales en terminales. De este modo, el parámetro $Y_{j k}$, representaría para cada frecuencia la variación de corriente en el electrodo $j$ cuando se aplica una variación de pequeña amplitud en el terminal $k$. Las partes real e imaginaria de este parámetro se pueden calcular como sigue [Laux, 1985], [Tomizawa, 1993], [Rengel, 2002]:

$$
\begin{aligned}
& \operatorname{Re}\left[Y_{j k}(f)\right]=\frac{I_{j}(\infty)-I_{j}(0)}{\Delta V_{k}}+\frac{\omega}{\Delta V_{k}} \int_{0}^{\infty}\left[I_{j}(t)-I_{j}(\infty)\right] \sin (2 \pi f t) d t \\
& \operatorname{Im}\left[Y_{j k}(f)\right]=\frac{\omega}{\Delta V_{k}} \int_{0}^{\infty}\left[I_{j}(t)-I_{j}(\infty)\right] \cos (2 \pi f t) d t
\end{aligned}
$$

Siendo $I_{j}(0)$ e $I_{j}(\infty)$ las corrientes estacionarias, respectivamente antes $\mathrm{y}$ después del salto en el electrodo $j$.

Con el fin de minimizar el ruido numérico y garantizar la reproducibilidad de los resultados, la simulación de cada variación en voltaje se lleva a cabo numerosas veces considerando en cada una diferentes semillas para la generación de números aleatorios en el simulador Monte Carlo; el cálculo de los parámetros $Y$ se realiza teniendo en cuenta la media las corrientes transitorias y estacionarias medias del conjunto de simulaciones. La validez y exactitud de este método en dispositivos

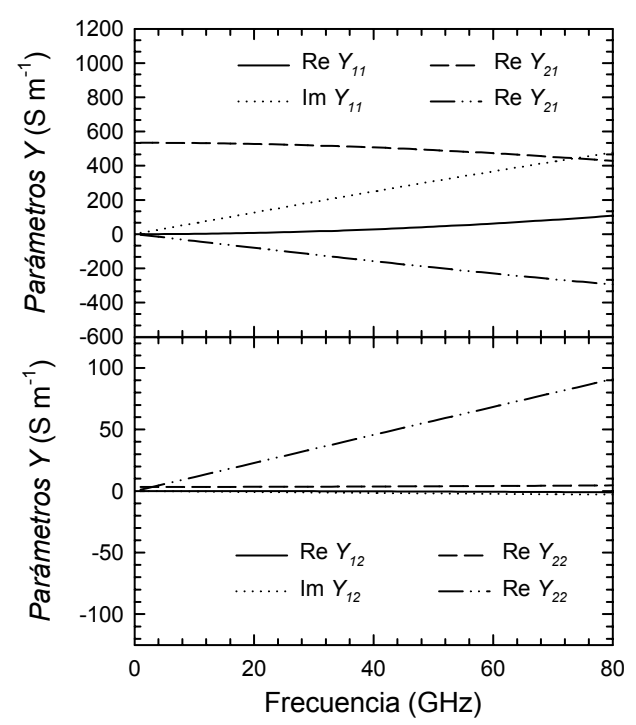

Figura VI.2. Parámetros $Y$ en función de la frecuencia para el SB-MOSFET con $V_{G S}=2 V y V_{D S}=2 \mathrm{~V}$. El salto de voltaje aplicado en cada uno de los terminales es: $\Delta V_{G S}=0.0625 \mathrm{~V}$ y $\Delta V_{D S}=0.15 \mathrm{~V}$. 
MOSFET convencionales y SOI MOSFET ha sido probada al comparar satisfactoriamente los resultados obtenidos de la simulación con medidas experimentales [Rengel, 2001], [Rengel, 2002b], [Rengel, 2006b].

Siguiendo este procedimiento descrito, hemos determinado los parámetros admitancia del transistor SB-MOSFET tipo $n$, con contactos metálicos en fuente y drenador caracterizados por una altura de barrera Schottky de $0.20 \mathrm{eV}$. La longitud de puerta de este transistor es de 120 $\mathrm{nm}$, y el transporte en el mismo bajo condiciones de tensiones constantes aplicadas en los electrodos fue examinado con detalle en el Capítulo V. A modo de ejemplo, en la Figura VI.2 mostramos la parte real e imaginaria de los parámetros admitancia para el punto de polarización de $V_{G S}=2 \mathrm{~V}$ y $V_{D S}=2 \mathrm{~V}$ y en función de la frecuencia hasta valores de $80 \mathrm{GHz}$. La dependencia de estos parámetros con la frecuencia es muy similar al caso del MOSFET convencional [Rengel, 2001].

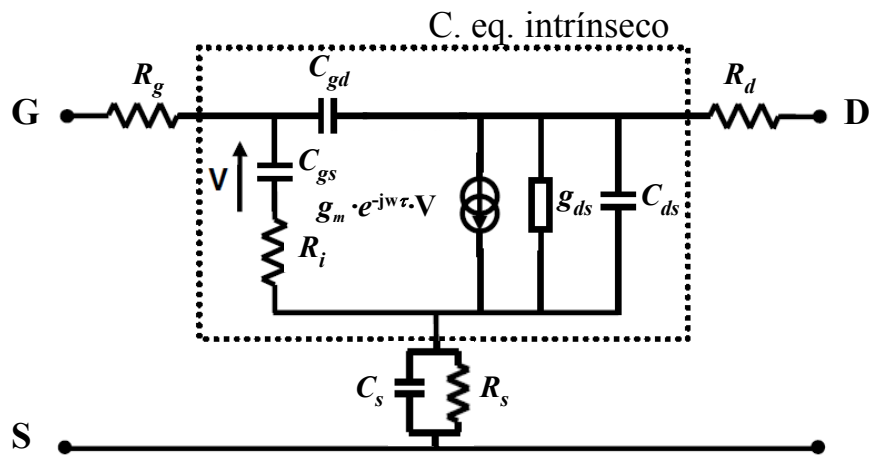

Figura VI.3. Circuito equivalente de pequeña señal de un transistor SB-MOSFET en saturación en el régimen de acumulación. La unión de fuente se encuentra polarizada en inversa mientras que la de drenador está en directa.

Para afrontar el análisis del comportamiento dinámico del dispositivo es altamente conveniente, desde un punto de vista físico, describir el dispositivo en términos de un circuito equivalente de pequeña señal, que puede obtenerse a partir de los parámetros $Y$. En la Figura VI.3 mostramos el esquema del circuito equivalente propuesto para el caso de transistores SB-MOSFET operando en régimen de saturación [Dawson, 1975], [Saha, 2005], [Xia, 2005], [Valentin, 2008]. Como podemos ver, la parte intrínseca (en el interior del recuadro de línea punteada) está basada en el modelo propuesto para transistores MOSFET convencionales [Dambrine, 1999], [Flandre, 2001], [Rengel, 2002]. Los elementos que constituyen el circuito equivalente intrínseco son la capacidad de puerta a fuente $C_{g s}$, la capacidad de puerta a drenador $C_{g d}$, la capacidad de drenador a fuente $C_{d s}$, la transconductancia $g_{m}$ y la conductancia de salida 
$g_{d s}$. Se tienen en cuenta además parámetros no-cuasiestáticos, como la resistencia de carga del canal $R_{i}$ o el retardo de la transconductancia $\tau$.

Como puede observarse en esta figura, la naturaleza de los contactos Schottky ha sido tenida en cuenta mediante una resistencia $R_{s}$ en paralelo con una capacidad $C_{s}$ para la unión de fuente en inversa [González, 1994] y una única resistencia $R_{d}$ asociada a la unión de drenador en directa. Junto con la resistencia de puerta $R_{g}$, estos parámetros son los elementos extrínsecos del circuito equivalente.

Una de las principales dificultades de la extracción de las resistencias $R_{s}$ y $R_{d}$ es que su valor depende de la polarización aplicada. Es por ello que se realizan diversas consideraciones para abordar el problema [Valentin, 2008]. En primer lugar, dado que en el régimen de saturación los portadores no deben superar ninguna barrera de potencial a la hora de abandonar el canal por el contacto de drenador, se puede considerar que $R_{d}$ (asociada a dicho contacto) es despreciable. La segunda hipótesis a tener en cuenta es que, puesto que el camino túnel que deben atravesar los portadores en este régimen de saturación es muy estrecho junto a la fuente, el valor de la capacidad $C_{s}$ es despreciable, correspondiendo la impedancia equivalente de este contacto a la contribución de $R_{s}$.

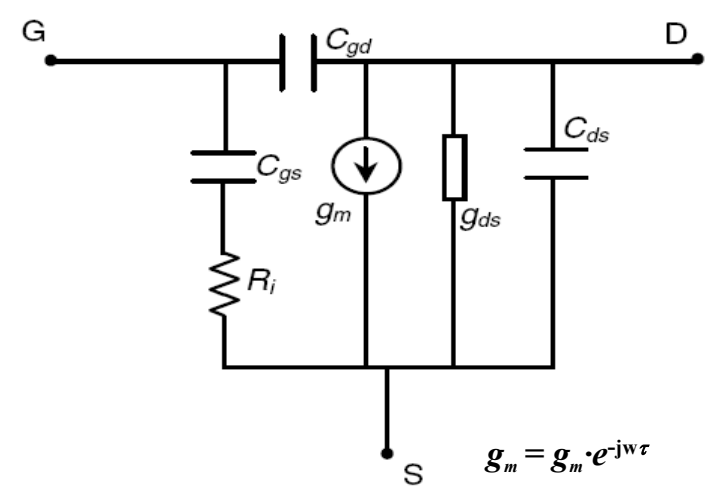

Figura VI.4. Topología del circuito equivalente de pequeña señal simplificado de un transistor SB-MOSFET.

Diversos autores realizan un tratamiento global de la resistencia $R_{s}$, considerando su contribución incluida en el resto de parámetros [Valentin, 2008], al tratarse de un parámetro no desligable del propio dispositivo en sí. En nuestro caso también hemos tenido en cuenta esta hipótesis, utilizando por tanto el circuito equivalente simplificado que mostramos en la Figura VI.4. El método Monte Carlo nos proporciona de manera directa los parámetros que constituyen dicho circuito, sin embargo, estos parámetros podrían no considerarse totalmente intrínsecos de 
acuerdo con la interpretación del circuito mostrado en la Figura VI.3. Una interpretación más amplia del concepto de dispositivo intrínseco, sin embargo, nos permite efectuar esta aproximación.

Este circuito equivalente simplificado (Figura VI.4) es empleado también en los dispositivos FET para representar su comportamiento dinámico en el rango de $\mathrm{GHz}$, ya que incorpora importantes parámetros no-cuasiestáticos (como $R_{i}$ o $\tau$ ) [Dambrine, 1999]. Cuando realizamos el estudio del comportamiento de dispositivos MOSFET a alta frecuencia, es importante tener en cuenta una representación no cuasiestática para conseguir así una descripción lo más exacta posible de la respuesta de pequeña señal [Raskin, 1998], [Manku, 1999], [Lee, 2001]. Es importante destacar que, para los dispositivos estudiados en este trabajo, los distintos elementos del circuito equivalente son independientes de la frecuencia al menos hasta la frecuencia de corte correspondiente, lo que asegura la validez del circuito equivalente [Raskin, 1998].

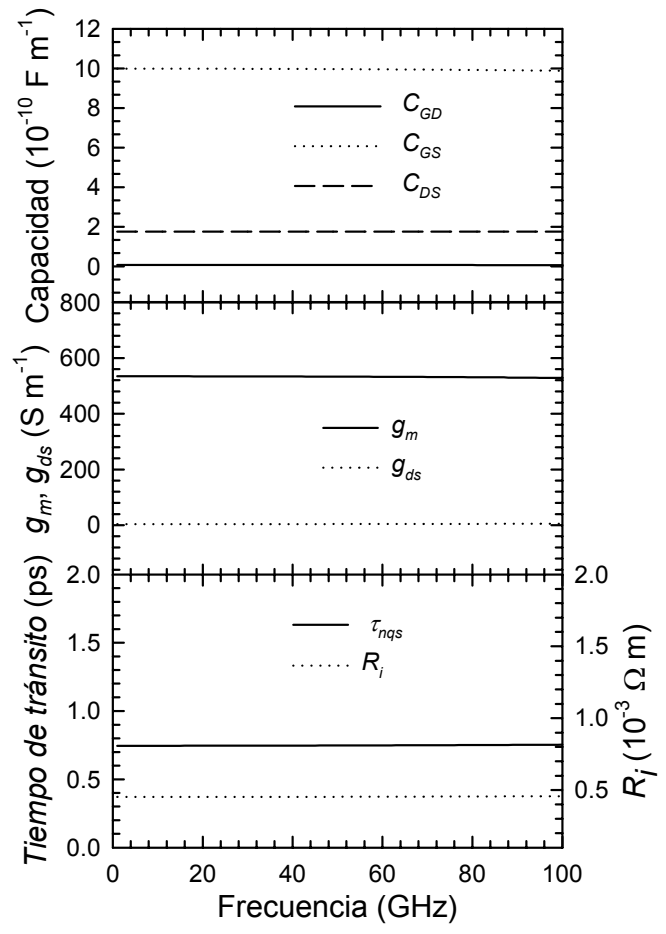

Figura VI.5 Parámetros del circuito equivalente en función de la frecuencia para el SB-MOSFET con $V_{G S}=2 V y V_{D S}=2 \mathrm{~V}$.
En la Figura VI.5 mostramos la dependencia de los parámetros del circuito equivalente con la frecuencia en el caso del transistor SB-MOSFET de $0.20 \mathrm{eV}$ de altura de barrera y $120 \mathrm{~nm}$ de longitud de puerta. Como podemos ver, permanecen constantes al menos hasta $100 \mathrm{GHz}$ en el caso de $V_{G S}=V_{D S}=2 \mathrm{~V}$. Esto sucede para todas las polarizaciones (no mostrado en las gráficas), lo que indica la validez del método de extracción.

A partir de los parámetros admitancia es también posible determinar el valor de otras importantes figuras de mérito, como la frecuencia máxima de oscilación $\left(f_{\max }\right.$, la frecuencia para la que la ganancia en potencia es la unidad) y de la frecuencia de corte $\left(f_{t}\right.$, la

frecuencia a la que la ganancia en corriente del dispositivo es la unidad). Ambos parámetros son excelentes indicadores del comportamiento a alta frecuencia del transistor; en nuestro caso, nos 
hemos centrado en el estudio de la frecuencia de corte de las distintas estructuras. Este parámetro $\left(f_{t}\right)$ representa en cierto modo la capacidad de amplificación de los dispositivos [Colinge, 1998].

En la Figura VI.6 mostramos el estado actual de las figuras de mérito $f_{t}$ y $f_{\max }$ en función de la longitud de puerta efectiva. Las líneas corresponden a la predicción para CMOS del ITRS, mientras que los símbolos representan el estado del arte de los resultados publicados para MOSFET convencionales de Silicio tipo $n$ y tipo $p$ (no se han tenido en cuenta los MOSFET multipuerta).

(a)

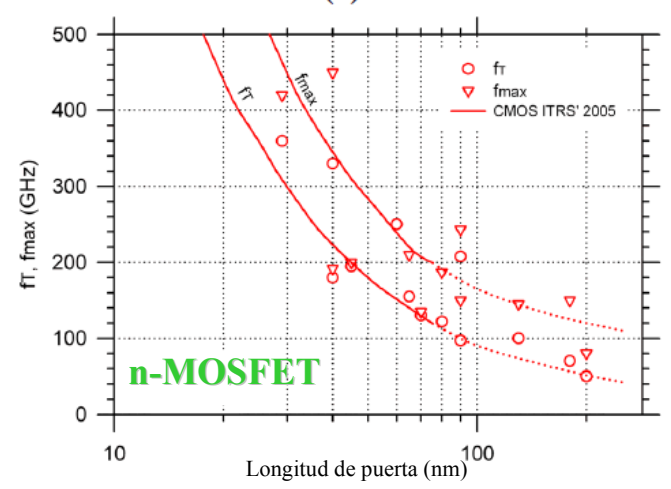

(b)

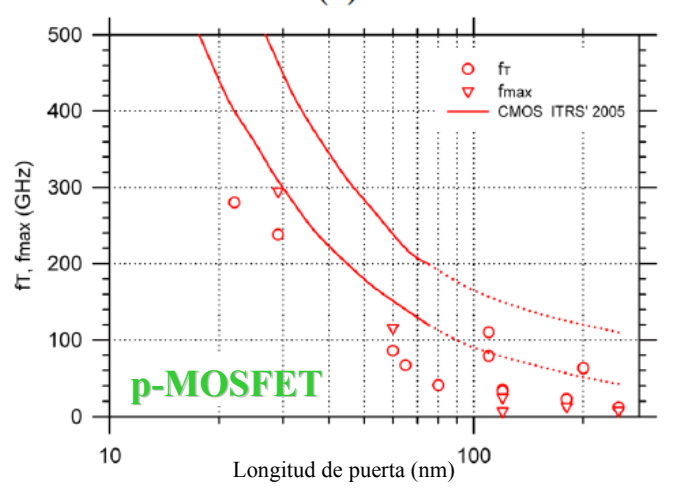

Figura VI.6. Estado del arte de $f_{t} y f_{\max }$ para transistores SOI MOSFETs: .a. tipo n [Matsumoto, 2001], [Momose, 2001], [Narashima, 2001], [Tiemeijer, 2001], [Zamdmer, 2001], [Chen, 2002], [CSM, 2002], [Vanmackelberg, 2002], [Zamdmer, 2002], [Zamdmer, 2004], [Dambrine, 2005], [Sungjae Lee, 2005] y .b. tipo p [Taur, 1993], [Ferlet-Cavrois, 1998], [Burghartz, 2000], [Barlage, 2001], [Chen, 2002] , [Hisayo, 2002], [Fritze, 2004], [Post, 2006]. En ambas figuras representamos la predicción para CMOS del ITRS [ITRS, 2005].

Como podemos en dicha figura, se ha registrado un valor record de $f_{t}$ de $330 \mathrm{GHz}$ en un MOSFET convencional tipo $n$ de $27 \mathrm{~nm}$ de longitud de puerta [Sungjae Lee, 2005]. Las figuras de mérito de los transistores de Silicio tipo $n$ presentan mejores valores en comparación con su homólogo tipo $p$, lo que supone una clara ventaja en las aplicaciones de los mismos. Esto se debe fundamentalmente a la menor movilidad de los huecos. En el caso de los transistores tipo $p$, se ha obtenido un valor de $240 \mathrm{GHz}$ para un MOSFET de tipo strained de $29 \mathrm{~nm}$ de longitud de puerta [Post, 2006]; sin embargo, el valor más elevado de $f_{t}$ del que se ha informado es $280 \mathrm{GHz}$, conseguido en un SB-MOSFET tipo $p$ de $22 \mathrm{~nm}$ de longitud de puerta [Fritze, 2004] (Nótese que en el caso de los dispositivos tipo $n$ aún no se encuentran valores de $f_{t}$ para longitudes de puerta tan pequeñas puesto que aún no se han fabricado de manera optimizada, tal y como comentamos en la Sección V.1 del capítulo anterior). 
En el caso de la estructura simulada que nos ocupa (el transistor SB-MOSFET de $120 \mathrm{~nm}$ de longitud de puerta y $0.20 \mathrm{eV}$ de altura de barrera), la frecuencia de corte $f_{t}$ obtenida mediante la simulación Monte Carlo alcanza un valor máximo de $84.5 \mathrm{GHz}$ para $V_{G S}=V_{D S}=2 \mathrm{~V}$; este valor se encuentra en completo acuerdo con la predicción del ITRS para un dispositivo de $120 \mathrm{~nm}$ de longitud de puerta (véase Figura VI.6). Los resultados obtenidos (a partir de la extrapolación de los valores de la ganancia en corriente $Y_{21} / Y_{11}$ tal y como vemos en la Figura VI.7) muestran un buen acuerdo con los que proporcionan expresiones simplificadas como la que sigue [Tvidisis, 1999]:

$$
f_{t}=\frac{g_{m}}{2 \pi\left(C_{g s}+C_{g d}\right)}
$$

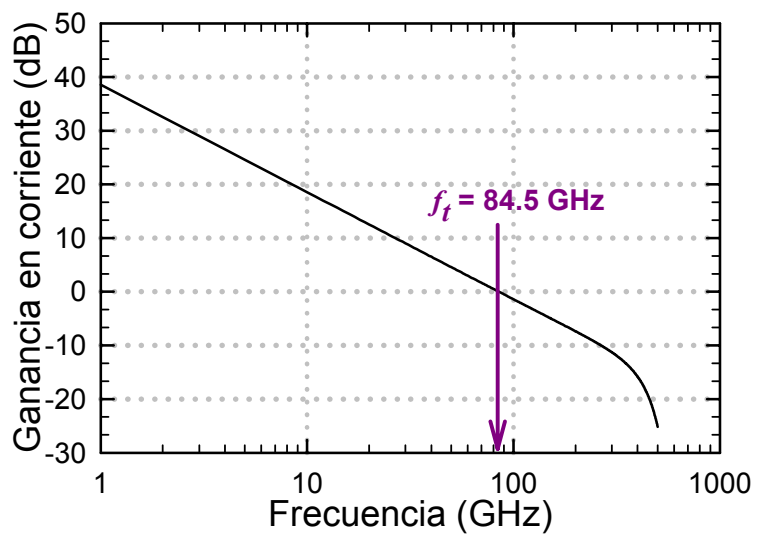

Figura VI.7. Ganancia en corriente del transistor SB-MOSFET bajo estudio en este trabajo, cuya longitud de puerta es de $120 \mathrm{~nm}$ sometido a la polarización $V_{G S}=V_{D S}=2 \mathrm{~V}$. A partir de los valores de $Y_{21} / Y_{11}$ obtenemos una frecuencia de corte de $84.5 \mathrm{GHz}$.

\section{VI.2. Sensibilidad de las características dinámicas del SB-MOSFET con la variación de parámetros geométricos}

En esta sección nos proponemos examinar las posibilidades existentes para mejorar el rendimiento del transistor SB-MOSFET en aplicaciones de alta frecuencia y optimizar su diseño. Para ello, investigaremos con detalle influencia de diversos parámetros sobre las características dinámicas y el ruido del dispositivo. Los parámetros a variar de la arquitectura del transistor son la altura de barrera Schottky, la longitud de underlap y la longitud de puerta, cuyo efecto sobre las características estáticas fue analizado en el Capítulo V. Con el fin de 
realizar comparaciones bajo iguales condiciones de polarización entre los diversos dispositivos, presentaremos el estudio de las distintas magnitudes en función del voltaje de overdrive $\left(V_{G S}-V_{T}\right)$, tal y como ya hicimos en el Capítulo V.

\section{VI.2.a Variación de la altura de barrera Schottky}

A continuación procedemos a analizar la influencia de la altura de barrera Schottky en las características dinámicas del dispositivo cuando se encuentra polarizado en régimen de saturación $\left(V_{D S}=2 \mathrm{~V}\right)$.

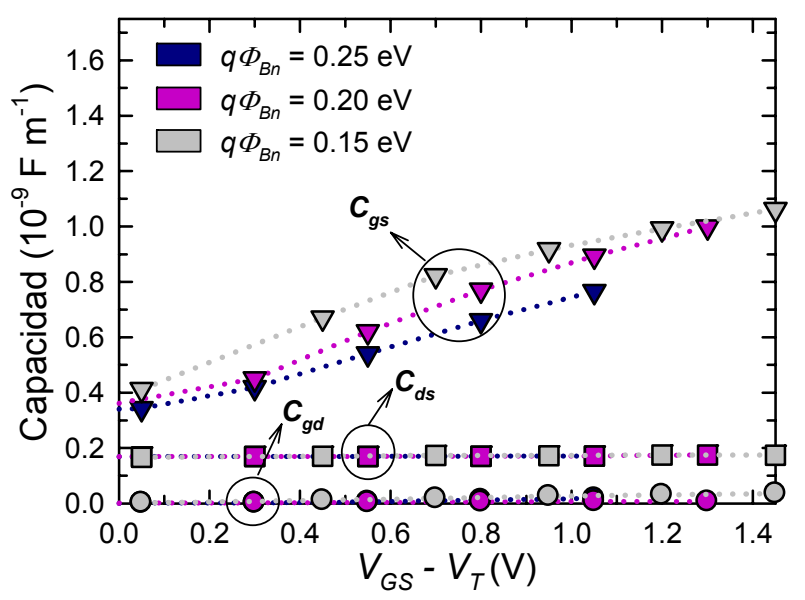

Figura VI.8. Resultados Monte Carlo de las capacidades $C_{g s}, C_{d s} y C_{g d}$ del circuito equivalente para el transistor SB-MOSFET considerando tres valores diferentes de altura de la barrera Schottky: $0.25 \mathrm{eV}, 0.20 \mathrm{eV}$ y $0.15 \mathrm{eV}$, en función de $V_{\text {overdrive }}$ con $V_{D S}=2 \mathrm{~V}$.

Comenzamos la investigación mostrando en la Figura VI.8 la dependencia de las capacidades con el voltaje de overdrive para el transistor SB-MOSFET bajo estudio, considerando tres valores distintos de altura de barrera Schottky $\left(q \Phi_{B n}=0.15 \mathrm{eV}, 0.20 \mathrm{eV}\right.$ y $0.25 \mathrm{eV})$. Como puede observarse, la capacidad de puerta a fuente $\left(C_{g s}\right)$ presenta los valores más altos, mientras que la de puerta a drenador $\left(C_{g d}\right)$ los valores más bajos; ambas capacidades aumentan con $\mathrm{V}_{\text {overdrive }}$ (aunque el aumento de $C_{g d}$ es poco apreciable en la gráfica). El comportamiento de $C_{g s}$ con el voltaje en puerta aplicado es análogo al que presenta el MOSFET convencional; sin embargo, en el dispositivo convencional la presencia de la capacidad $C_{g d}$ es más relevante [Rengel, 2002b] siendo prácticamente despreciable en el caso del SB-MOSFET. 
La explicación para el comportamiento de las capacidades de puerta podemos encontrarla al analizar la Figura VI.9, en la que mostramos el incremento de carga en inversión en el canal que se produce al aplicar un incremento de voltaje en puerta en el régimen de saturación. Como veíamos en el análisis de la concentración de portadores (Figura V.12.b del capítulo anterior), la mayor cantidad de carga de la capa de acumulación se sitúa en la región del canal más cercana a la fuente. Esta carga se ve incrementada en gran medida cuando aplicamos un incremento de voltaje $V_{G S}$ (mientras $V_{D S}$ permanece constante, ver Figura VI.9), lo cual explica el elevado valor de la capacidad $C_{g s}$.

El ligero aumento de $C_{g d}$ con $V_{G S}$ se debe a que para los valores más elevados de voltaje en puerta aplicado, aunque el dispositivo se encuentra aún en el régimen de operación de saturación, se encuentra más cercano al régimen de triodo que para los valores más bajos de $V_{G S}$, obteniéndose un incremento de la carga cercana al contacto de drenador, tal y como vemos en la Figura VI.9.

A continuación analizamos la dependencia de estas capacidades con la

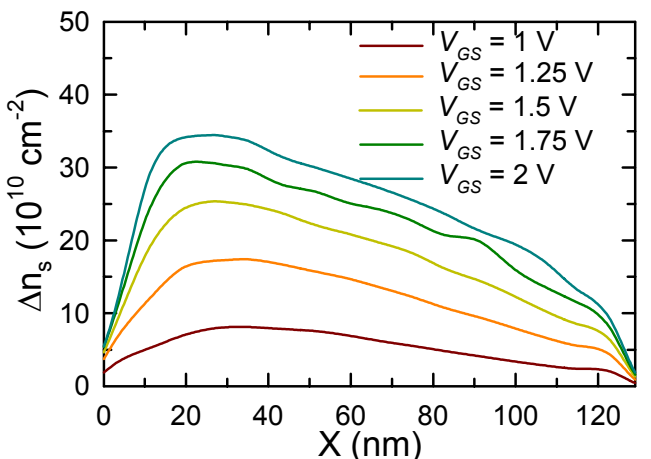

Figura VI.9. Resultados obtenidos mediante nuestro simulador MC2D que muestran el incremento de carga en el canal que se produce al aplicar un incremento de

$V_{G S}=0.625$ V sobre varios valores de $V_{G S}\left(V_{D S} s e\right.$ mantiene constante e igual a $2 \mathrm{~V}$. altura de barrera Schottky. La reducción del valor de dicha altura implica un aumento de la capacidad $C_{g s}$, lo que indica un mayor incremento de carga con el incremento de $V_{G S}$ en la región del canal más próxima a la de fuente cuanto menor es $q \Phi_{B n}$. Esta variación de carga no sólo es debida al efecto de la puerta, sino que también está directamente relacionada con el incremento de carga que está siendo inyectada en el canal a través de la barrera, que claramente es superior a medida que $q \Phi_{B n}$ disminuye. $C_{g d}$ posee valores muy reducidos en comparación con $C_{g s}$, presentando también un pequeño aumento al reducir la altura de la barrera, mientras que $C_{d s}$ permanece invariante con $q \Phi_{B n}$.

En la Figura VI.10 mostramos la transconductancia $g_{m}$ de la estructura simulada para los tres valores de altura de barrera. La transconductancia sigue el comportamiento de otros dispositivos MOSFET [Wong, 1996], [Rengel, 2002b], aumentando con el voltaje en puerta 
aplicado en el régimen de saturación $\left(V_{G S}<1.75 \mathrm{~V}\right)$. Sin embargo, cuando el dispositivo se aproxima a régimen de triodo (para los voltajes más elevados), el aumento de $g_{m}$ es cada vez menor. En cuanto a la conductancia de salida $g_{d s}$ (no representado en la figura), el SB-MOSFET presenta un comportamiento prácticamente ideal, con un valor muy próximo a cero, lo que se corresponde con la escasa pendiente de las características de salida en condiciones de saturación (véase Figura V.7).

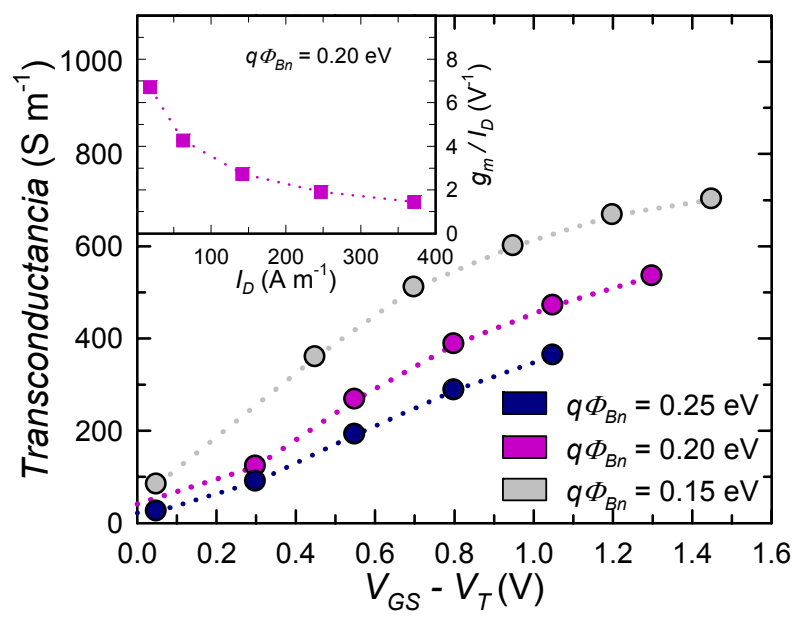

Figura VI.10. Transconductancia $g_{m}$ para tres valores de altura de barrera de $0.25 \mathrm{eV}, 0.20 \mathrm{eV}$ y $0.15 \mathrm{eV}$ en función de $V_{\text {overdrive }} y V_{D S}$ fijo $(2 \mathrm{~V})$. En el recuadro interior mostramos el ratio $g_{m} / I_{D}$ en función de $I_{D}$ para $V_{D S}=2 \mathrm{~V}$ en el caso de la estructura de $0.20 \mathrm{eV}$ de altura de barrera.

Con respecto a la dependencia de la transconductancia con la altura de la barrera, se observa un aumento significativo a medida que reducimos $q \Phi_{B n}$. El comportamiento de $g_{m}$ puede explicarse por la mayor variación de corriente de drenador cuando aplicamos un salto de voltaje en el terminal de puerta al reducir $q \Phi_{B n}$, como puede deducirse de la característica de transferencia (véase Figura V.30.a), indicando así que el electrodo de puerta ejerce un mayor control sobre la corriente de salida para el caso en que la altura de barrera es menor.

Por otro lado, la relación entre la transconductancia y la corriente de drenador es una figura de mérito importante en el diseño de aplicaciones analógicas [Silveira, 1996], [Colinge, 1998]. Mientras que la transconductancia del dispositivo representa la amplificación proporcionada por el mismo, la corriente de drenador está relacionada con la potencia disipada para obtener dicha amplificación. De este modo, el ratio $g_{m} / I_{D}$ nos indica la eficacia del uso de la corriente para conseguir un cierto valor de transconductancia [Silveira, 1996]. En el recuadro 
interior de la Figura VI.10 mostramos el valor de este ratio en función de la corriente de drenador para el dispositivo con altura de barrera de $0.20 \mathrm{eV}$.

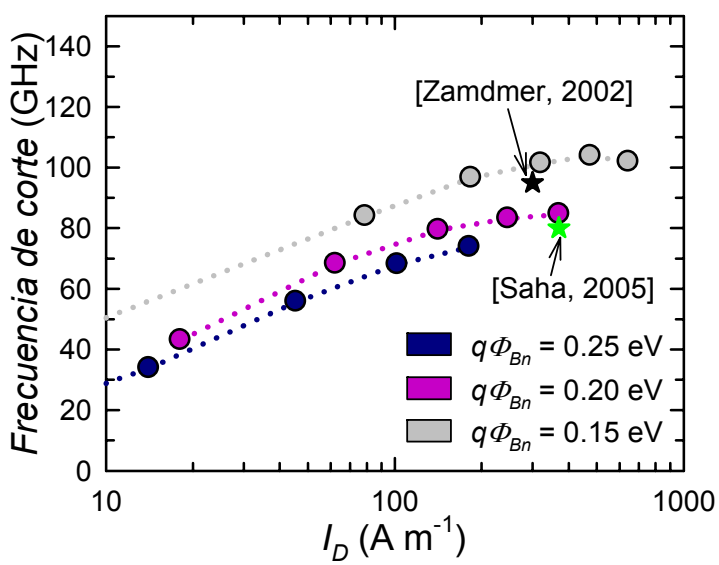

Figura VI.11. Frecuencia de corte, $f_{t}$, en función de $I_{D}$ para el transistor SB-MOSFET con tres valores de $q \Phi_{B n}$ : $0.25 \mathrm{eV}, 0.20 \mathrm{eV}$ y $0.15 \mathrm{eV}$. Mostramos además los resultados de $f_{t}$ mostrados en [Zamdmer, 2002](SOI-MOSFET, $\left.L_{g}=130 \mathrm{~nm}\right)$ y [Saha, 2005] (SB-MOSFET tipo n, $L_{g}=100 \mathrm{~nm}$, contactos Schottky de NiSi).

El aumento de la transconductancia observado tanto al disminuir el valor de la altura de barrera como al incrementar el valor de $V_{\text {overdrive }}$ está directamente relacionado con el valor creciente de la frecuencia de corte a través de la Ecuación VI.5 (en la Figura VI.11 presentamos $f_{t}$ representada en función de $V_{\text {overdrive }}$ para el transistor SB-MOSFET con tres valores distintos de $q \Phi_{B n}$ ). Sin embargo, debido a la contribución de la capacidad $C_{g s}$ (véase Figura VI.8) el incremento de $f_{t}$ no se produce en la misma medida que el de la transconductancia (Figura VI.10). Este resultado coincide con el obtenido para estructuras tipo p [Valentin, 2008b], sin embargo está en contraposición con el trabajo publicado por Xia et al. para transistores SB-MOSFET tipo $n$, donde concluyen que, mientras $g_{m}$ aumenta al disminuir $q \Phi_{B n}$, la frecuencia de corte no se vería afectada [Xia, 2005].

En la Figura VI.11 mostramos además el valor de $f_{t}$ para diversos transistores. En primer lugar, comparamos con el resultado obtenido de $f_{t}$ para un transistor SOI-MOSFET experimental de longitud de puerta igual a $130 \mathrm{~nm}$ (véase Figura VI.6.a, [Zamdmer, 2002]), aunque su valor efectivo es de $70 \mathrm{~nm}$ (en Figura VI.11 representada mediante el símbolo en forma de estrella negra). Como podemos ver es ligeramente inferior al valor que presenta el dispositivo de $0.15 \mathrm{eV}$ de altura de barrera Schottky. Finalmente, mostramos la frecuencia de corte obtenida de la simulación de un transistor SB-MOSFET tipo $n$, de $100 \mathrm{~nm}$ de longitud de puerta y contactos Schottky formados por NiSi (estrella verde) [Saha, 2005]; como podemos 
ver, el valor de $f_{t}$ es $80 \mathrm{GHz}$ muy similar al obtenido en nuestro trabajo para el caso de $q \Phi_{B n}=0.20 \mathrm{eV}$. Estos resultados nos indican que al reducir el valor de la altura de barrera mejoramos en gran medida la respuesta del dispositivo con respecto a la frecuencia de corte y a la transconductancia; sin embargo no es necesario alcanzar el valor de $\mathbf{0} \mathbf{e V}$ de altura de barrera para obtener excelentes valores de las figuras de mérito dinámicas, ya que con valores más realistas como $0.15 \mathrm{eV}$ obtenemos mejor respuesta que en el caso de transistores SOI-MOSFET convencionales; además debe tenerse en cuenta que es de momento inviable desde el punto de vista de la fabricación obtener alturas de barreras muy cercanas a $0 \mathrm{eV}$.
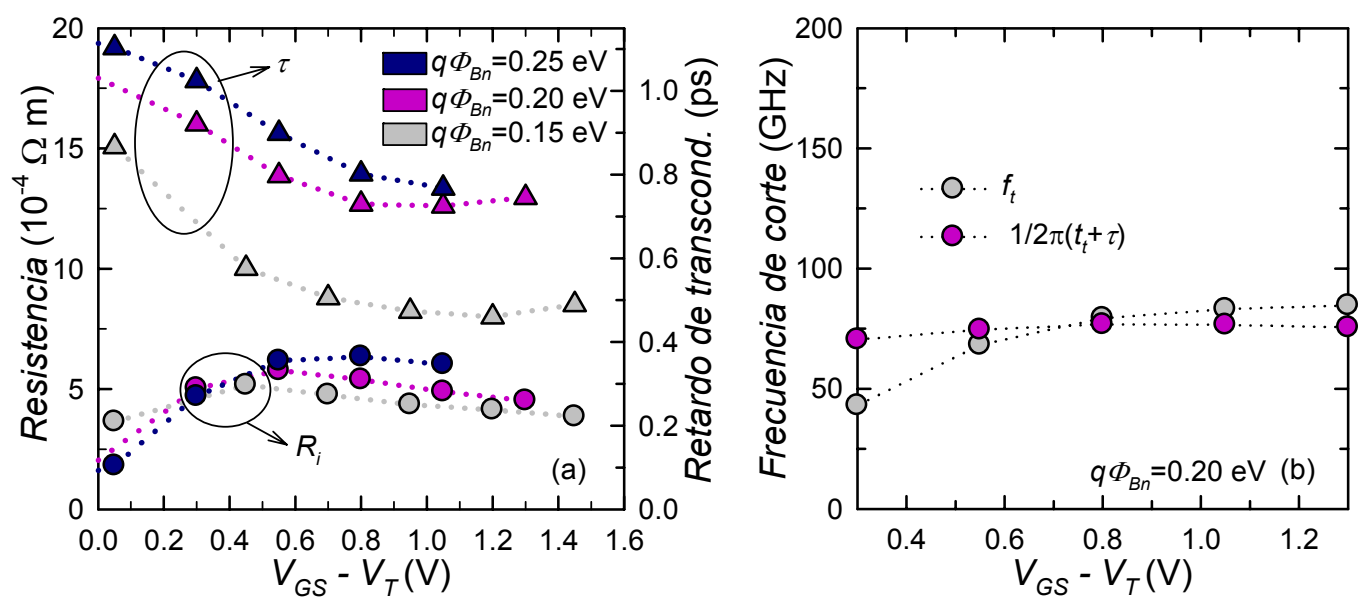

Figura VI.12.a. Resistencia de carga del canal $\left(R_{i}\right)$ y tiempo de retardo de transconductancia para el transistor $S B$-MOSFET con tres valores de $q \Phi_{B n}: 0.25 \mathrm{eV}, 0.20 \mathrm{eV}$ y $0.15 \mathrm{eV}(\tau)$.b. Frecuencia de corte y $1 / 2 \pi\left(t_{t}+\tau\right)$ para $q \Phi_{B n}=0.20 \mathrm{eV}$. Ambas figuras están expresadas en función de $V_{\text {overdrive }} y V_{D S}=2 \mathrm{~V}$.

En la Figura VI.12.a representamos la variación de la resistencia de carga $\left(R_{i}\right)$ y el tiempo de retardo de la transconductancia $(\tau)$ con $V_{\text {overdrive }}$ para $V_{D S}=2 \mathrm{~V}$ y diversos valores de altura de barrera. La consideración de estos parámetros está relacionada con el hecho de que la región activa no responde instantáneamente a las variaciones del voltaje en puerta aplicado. Como podemos observar, el valor de $R_{i}$ aumenta con $V_{\text {overdrive }}$ mientras el dispositivo se encuentra en la región subumbral, disminuyendo para valores de $V_{\text {overdrive }}$ elevados. Con respecto a la dependencia de esta magnitud con $q \Phi_{B n}, R_{i}$ aumenta ligeramente en el rango de bajos $V_{\text {overdrive }}$ aplicados y disminuye a voltajes elevados al reducir el valor de la altura de la barrera. Por otro lado, el tiempo de retardo de transconductancia puede también relacionarse con el tiempo requerido por la corriente de drenador para adaptarse a las variaciones de voltaje entre puerta y fuente. Como vemos en la Figura VI.12.a, $\tau$ disminuye con el voltaje en puerta, como se corresponde con el hecho de que para elevados $V_{\text {overdrive }}$ (en condiciones próximas al régimen 
de triodo) la capa de acumulación esté fuertemente ligada al contacto de puerta. Además, el valor de $\tau$ también es menor a medida que reducimos la altura de la barrera, consiguiéndose así un control más rápido de la puerta sobre la carga del canal. Los valores obtenidos para estas magnitudes ( $\tau$ y $R_{i}$ ) son similares a los obtenidos para un SOI-MOSFET convencional con capa activa de tamaño análogo [Rengel, 2004].

Teniendo en cuenta el valor del retardo de la transconductancia y el tiempo de tránsito del dispositivo (examinado en el Capítulo V, véase Figura V.25) podemos calcular el valor de la frecuencia de corte a partir de la siguiente expresión [Kuivalainen, 1997], [Sze, 2001]:

$f_{t}=\frac{1}{2 \pi\left(t_{t}+\tau\right)}$

Como podemos observar en la Figura VI.12.b, en la que comparamos el valor de la frecuencia de corte calculada a partir de la ganancia en corriente y la proporcionada por la ecuación anterior en el caso del dispositivo con $q \Phi_{B n}=0.20 \mathrm{eV}$, existe en general un buen acuerdo entre ambos métodos (presentando una mayor discrepancia en el régimen de bajos voltajes en puerta aplicados).

\section{VI.2.b Variación de la longitud de underlap}
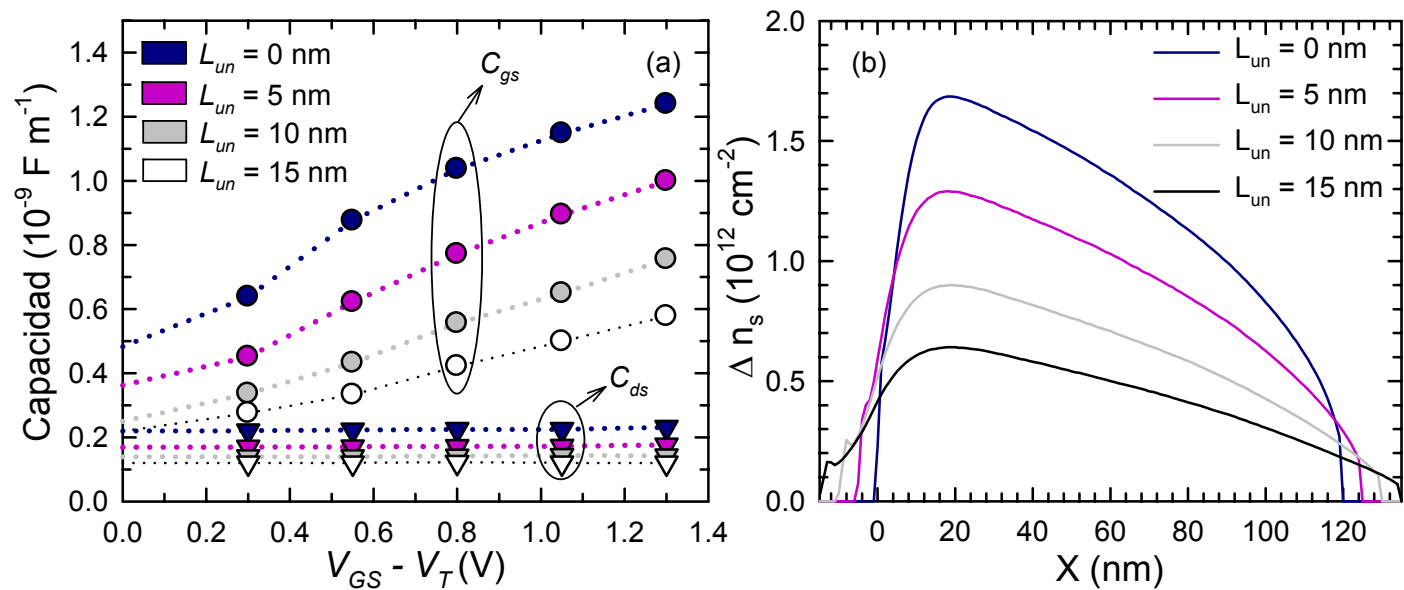

Figura VI.13.a. Capacidades del circuito equivalente para el SB-MOSFET con cuatro longitudes de underlap distintas: $0 \mathrm{~nm}, 5 \mathrm{~nm}, 10 \mathrm{~nm}$ y $15 \mathrm{~nm}$ en función de $V_{\text {overdrive }}$ con $V_{D S}=2 \mathrm{~V}$.b. Incremento de carga a lo largo del canal para los distintos valores de $L_{u n}\left(V_{D S}=2 \mathrm{~V}, V_{\text {overdrive }}=1.3 \mathrm{~V} \mathrm{y} \Delta V_{G S}=0.2 \mathrm{eV}\right)$.

A continuación estudiaremos el efecto de la longitud de underlap sobre las características dinámicas del dispositivo en régimen de saturación $\left(V_{D S}=2 \mathrm{~V}\right)$; como ya hicimos 
en la Sección V.6.b del anterior capítulo, consideraremos para este parámetro los valores de $0 \mathrm{~nm}, 5 \mathrm{~nm}, 10 \mathrm{~nm}$ y $15 \mathrm{~nm}$.

En la Figura VI.13 representamos las capacidades del circuito equivalente de pequeña señal en función del voltaje de overdrive así como el incremento de carga a lo largo del canal para las cuatro longitudes de underlap simuladas para $V_{\text {overdrive }}=1.3 \mathrm{~V}$. Observamos que $C_{g s}$ aumenta notablemente al disminuir $L_{u n}$. A medida que la distancia entre el contacto de fuente y de puerta se reduce, un incremento de voltaje aplicado en la puerta tiene un mayor efecto sobre el incremento de carga acumulada en la región cercana a la fuente. Además, también tiene lugar un mayor incremento de inyección de carga a través de la barrera Schottky del contacto de fuente cuando $L_{u n}$ es menor, como ya vimos en la Figura V.35. Por otro lado, $C_{d s}$ permanece constante con $V_{\text {overdrive, }}$ disminuyendo con el aumento de $L_{u n}$ como cabe esperar al aumentar la distancia que separa a ambos contactos.
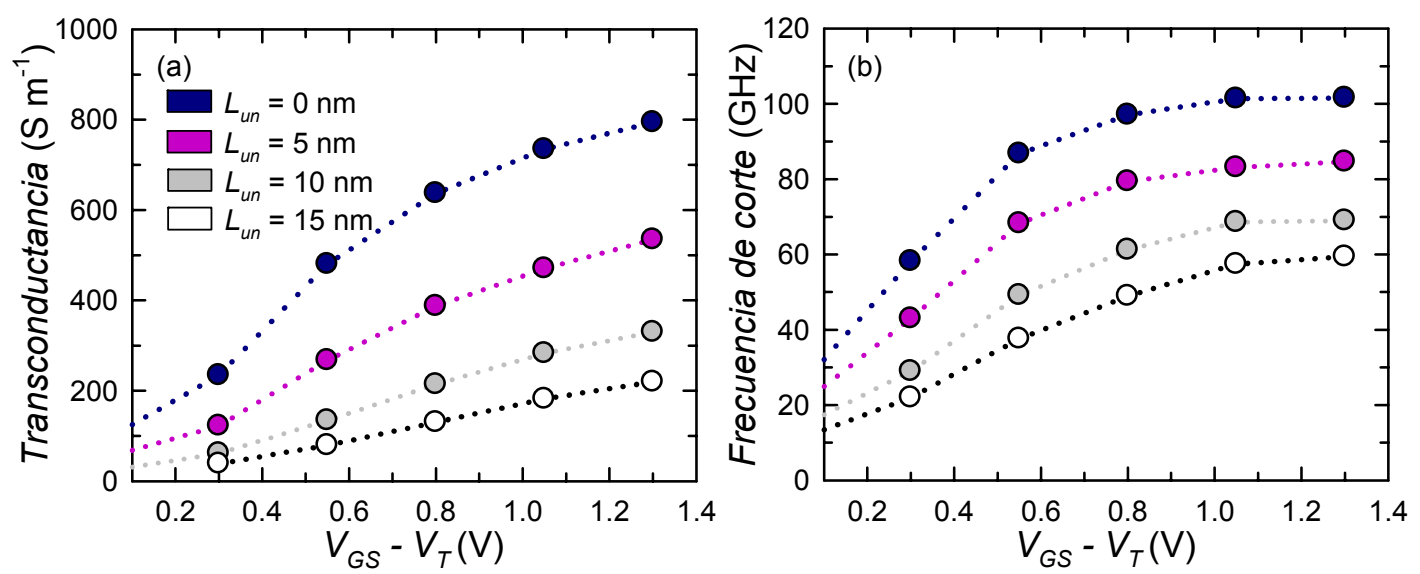

Figura VI.14.a. Transconductancia $g_{m} y$.b. frecuencia de corte $f_{t}$ para el SB-MOSFET con cuatro longitudes de underlap distintas: $0 \mathrm{~nm}, 5 \mathrm{~nm}, 10 \mathrm{~nm}$ y $15 \mathrm{~nm}$ en función de $V_{\text {overdrive }}$ con $V_{D S}=2 \mathrm{~V}$

Si analizamos la variación de la transconductancia y la frecuencia de corte con $L_{u n}$, representado en la Figura VI.14, vemos como ambas magnitudes se ven incrementadas al disminuir la longitud de underlap. Puesto que la transconductancia puede definirse como la relación entre la variación de corriente y la variación de tensión de puerta (a $V_{D S}$ constante) [Tsividis, 1999], podemos por tanto realizar el análisis de la dependencia de $g_{m}$ con el voltaje de overdrive en términos de la variación de la carga y velocidad a lo largo del canal. En la Figura VI.15.a. mostramos para el dispositivo con $L_{u n}=0 \mathrm{~nm}$ los diferentes términos que implican la variación de corriente, es decir, $\Delta n \cdot v_{x}, n \cdot \Delta v_{x} \mathrm{y} \Delta n \cdot \Delta v_{x}$; como podemos ver, $\Delta n \cdot v_{x}$ supone la mayor contribución a la variación de la corriente, siendo los otros dos productos prácticamente 
despreciables frente al anterior. En la Figura VI.15.b examinamos la dependencia de este producto (el incremento de carga por la velocidad total) con $L_{u n}$, que aumenta a medida que $L_{u n}$ es menor. El incremento de carga es el factor que más aumenta por esta variación, y explica por tanto la mejora de $g_{m}$.

Por otro lado, el aumento de la frecuencia de corte está relacionado no sólo con $g_{m}$, sino también con $C_{g s}$ tal y como veíamos en la Ecuación VI.5. Como vemos, aunque el valor de esta capacidad es mayor a medida que disminuye $L_{u n}$, el incremento de la transconductancia presenta una mayor contribución, dando lugar a valores superiores de $f_{t}$ (Figura VI.14.b). Además, teniendo en cuenta que el tiempo de tránsito se ve decrementado lógicamente con la longitud de underlap (puesto que la distancia que tienen que recorrer los portadores desde el contacto de fuente al de drenador es menor), este resultado es consistente con el comportamiento de la frecuencia de corte con $L_{u n}$ a través de la Ecuación VI.6. Estos resultados son similares a los encontrados para una estructura tipo $p$ en [Valentin, 2008b].
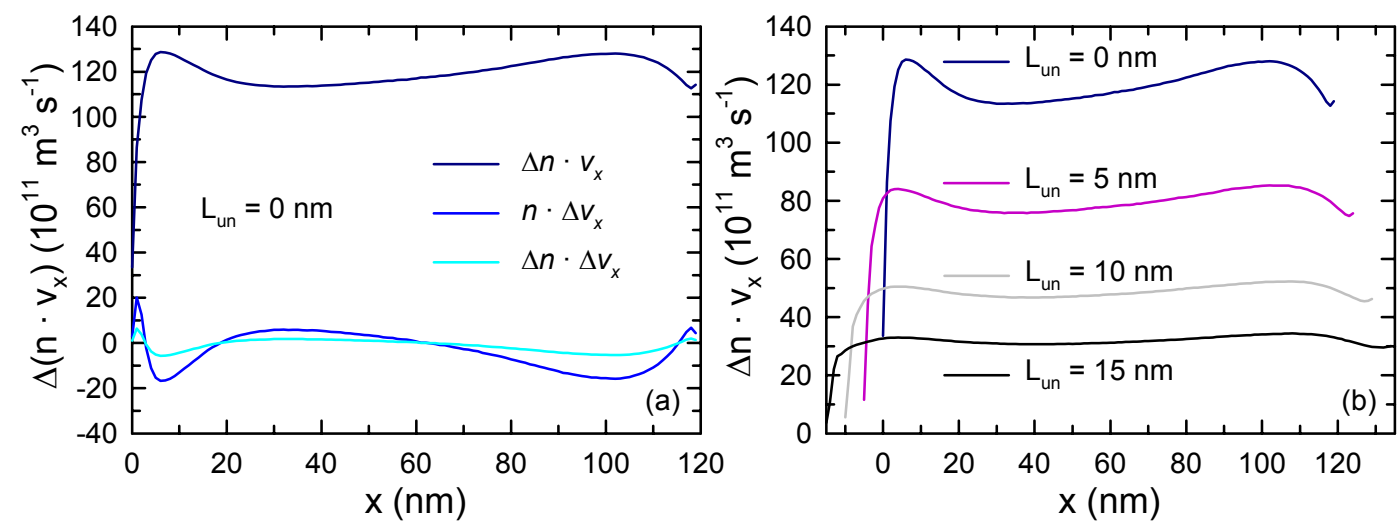

Figura VI.15.a. $\Delta n \cdot v, \Delta n \cdot v$ y $\Delta n \cdot v$ a lo largo del canal para el SB-MOSFET con longitudes de underlap de $0 \mathrm{~nm}$ .b. $\Delta n \cdot v$ para $L_{u n}=0 \mathrm{~nm}, 5 \mathrm{~nm}, 10 \mathrm{~nm}$ y $15 \mathrm{~nm}$.

A continuación, en la Figura VI.16 mostramos la resistencia de carga del canal $\left(R_{i}\right)$ y el tiempo de retardo de transconductancia $(\tau)$. Ambos parámetros no cuasiestáticos presentan valores inferiores a medida que $L_{u n}$ es menor, lo que indica que la variación de la carga en el canal y la transconductancia responden de manera más rápida a la variación del voltaje entre puerta y fuente en el caso de menor longitud de underlap. 


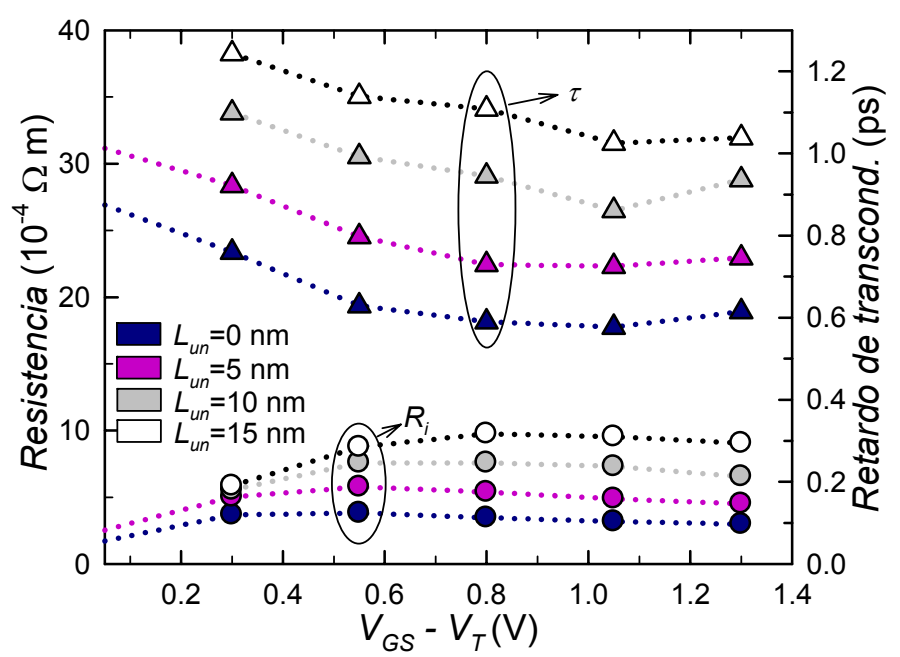

Figura VI.16. Resistencia de carga del canal $\left(R_{i}\right)$ y tiempo de retardo de transconductancia $(\tau)$ en función de de $V_{\text {overdrive }}$ con $V_{D S}=2 \mathrm{~V}$ para $L_{u n}=0 \mathrm{~nm}, 5 \mathrm{~nm}, 10 \mathrm{~nm}$ y $15 \mathrm{~nm}$.

Es por tanto importante reducir el valor de la longitud de underlap para obtener mayores valores de $\boldsymbol{g}_{\boldsymbol{m}} \mathbf{0} \boldsymbol{f}_{\boldsymbol{t}}$. Sin embargo, desde el punto de vista de fabricación, conseguir un dispositivo de underlap nulo es bastante difícil y nada recomendable, dado que los problemas de acoplamiento parásito son mayores. Podemos así afirmar que para la optimización del diseño del dispositivo debemos encontrar el equilibrio entre reducir los efectos de los elementos parásitos (aumentando la distancia entre fuente y drenador) y maximizar la conducción (reduciendo la separación de estos contactos), por lo que es recomendable una longitud de underlap no nula [Connelly, 2003].

\section{VI.2.c Variación de la longitud de puerta}

En este apartado analizamos la variación de las características dinámicas del SB-MOSFET con la longitud de puerta $\left(L_{g}\right)$ cuando el dispositivo opera en régimen de saturación $\left(V_{D S}=2 \mathrm{~V}\right)$; los valores que tendremos en cuenta para este parámetro son $120 \mathrm{~nm}$, $90 \mathrm{~nm}, 65 \mathrm{~nm}$ y $30 \mathrm{~nm}$. 


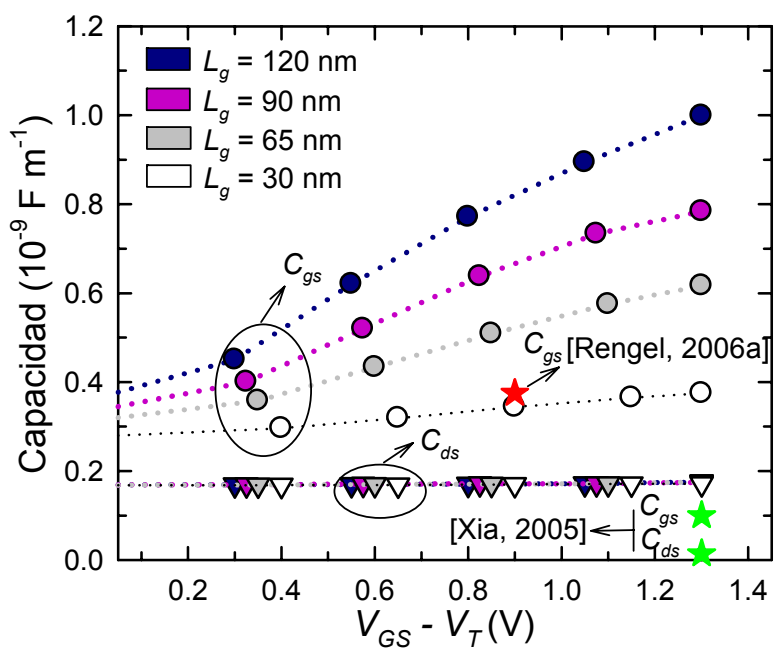

Figura VI.17. $C_{g s} y C_{d s}$ del SB-MOSFET con cuatro longitudes de puerta distintas: $120 \mathrm{~nm}, 90 \mathrm{~nm}, 65 \mathrm{~nm}$ y $30 \mathrm{~nm}$ en función de $V_{\text {overdrive }} c o n V_{D S}=2 \mathrm{~V}$. Representamos además los valores de dichas capacidades presentadas en [Xia, $2005]$ para un SB-MOSFET de $45 \mathrm{~nm}$ de longitud de puerta y $q \Phi_{B n}=0.20 \mathrm{eV}$ (estrellas verdes). La estrella roja se corresponde con el valor de $C_{g s}$ de un SOI-MOSFET convencional de $L_{g}=30 \mathrm{~nm}$ [Rengel, 2006]

En primer lugar analizamos la variación de las capacidades del circuito equivalente con la longitud de puerta, representadas en la Figura VI.17 en función del voltaje de overdrive para $V_{D S}=2 \mathrm{~V}$. Al igual que sucede en el MOSFET convencional [Rengel, 2002], al reducir la longitud de la puerta el valor de $C_{g s}$ es menor, lo que indica una menor variación de la carga en la zona del canal más próxima a la isla de fuente. Por otro lado, $C_{d s}$ permanece prácticamente constante con $L_{g}$ lo que nos indica que la presencia del óxido enterrado aísla la capa activa del mismo modo en este dispositivo al variar el tamaño de la puerta. Por otro lado, en esta figura mostramos además los resultados de la simulación Monte Carlo de un transistor SB-MOSFET de $45 \mathrm{~nm}$ de longitud de puerta, $t_{S i}=10 \mathrm{~nm}$ y q $\Phi_{\mathrm{Bn}}=0.20 \mathrm{eV}$ [Xia, 2005]. Como podemos observar, el valor presentado en dicha publicación para ambas capacidades es muy inferior al obtenido en nuestro trabajo para el dispositivo con $L_{g}=30 \mathrm{~nm}$; debe tenerse en cuenta, como ya dijimos en el Capítulo V, que los resultados se han obtenido a partir de simulaciones en condiciones estacionarias. Finalmente, al comparar con un dispositivo PDSOI-MOSFET convencional con $30 \mathrm{~nm}$ de longitud de puerta y $150 \mathrm{~nm}$ de espesor de canal [Rengel, 2006], vemos que el valor de la capacidad $C_{g s}$ se ve mejorado en el caso del transistor SB-MOSFET (téngase en cuenta que al disminuir el valor de $t_{S i}, C_{g s}$ es mayor [Rengel, 2004]). 

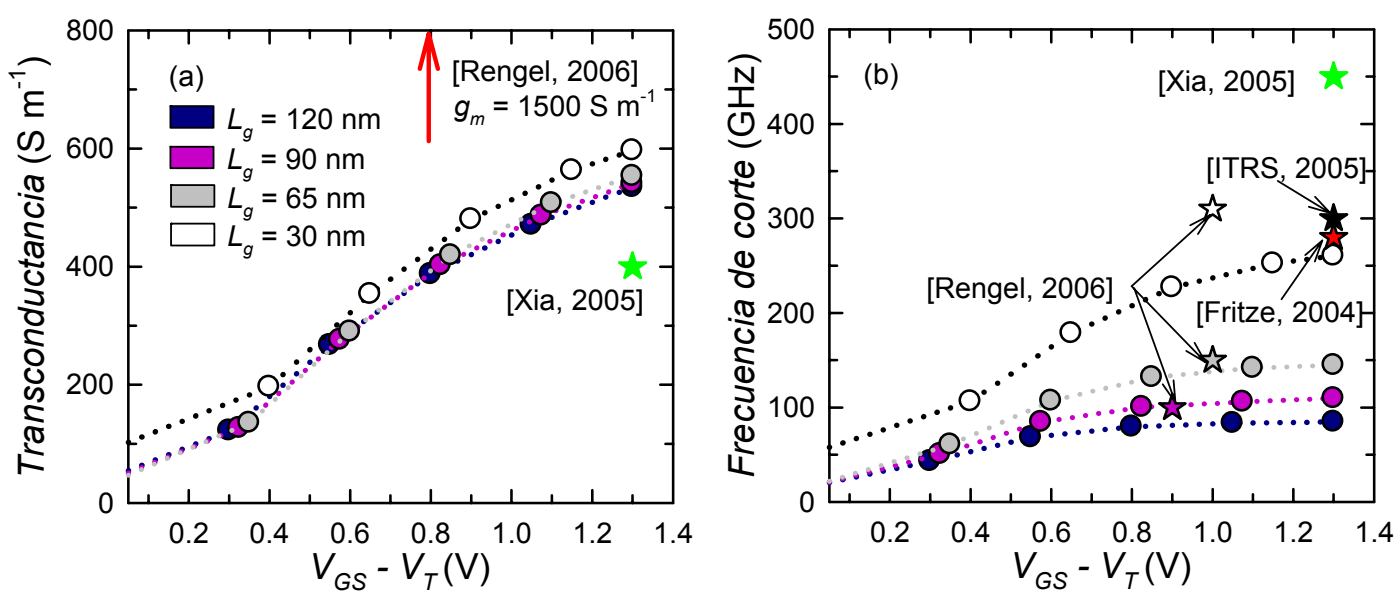

Figura VI.18.a. Transconductancia $g_{m} y$.b. frecuencia de corte $f_{t}$ para el SB-MOSFET con cuatro longitudes de puerta distintas: $120 \mathrm{~nm}, 90 \mathrm{~nm}, 65 \mathrm{~nm}$ y $30 \mathrm{~nm}$ en función de $V_{\text {overdrive }}$ con $V_{D S}=2 \mathrm{~V}$. También mostramos ambas magnitudes para los transistores estudiados en [Xia, 2005] (SB-MOSFET; estrella verde) y en [Rengel, 2006] (MOSFET convencional; en el caso de $f_{t}: L_{g}=90 \mathrm{~nm}$-estrella rosa-, $L_{g}=60 \mathrm{~nm}$-estrella gris- y $L_{g}=30 \mathrm{~nm}$ estrella blanca-, para $g_{m}$ : indicado valor para $\left.L_{g}=30 \mathrm{~nm}\right)$. Por último, también mostramos el valor de $f_{t}$ para el SB-MOSFET estudiado en [Frizte, 2004] (estrella roja) y la predicción teórica del [ITRS, 2005](estrella negra).

En la Figura VI.18.a mostramos la transconductancia. Como podemos ver, el valor de $\boldsymbol{g}_{\boldsymbol{m}}$ se ve ligeramente mejorado al reducir la longitud de puerta de $120 \mathrm{~nm}$ a $60 \mathrm{~nm}$, y más pronunciadamente al pasar de $60 \mathrm{~nm}$ a $30 \mathrm{~nm}$. Este comportamiento también se observa en el caso del MOSFET convencional analizado en [Rengel, 2006], donde se muestran resultados de $L_{g}=90 \mathrm{~nm}, 60 \mathrm{~nm}$ y $30 \mathrm{~nm}$. Sin embargo, tal y como vemos en esta figura para el caso del dispositivo convencional con $L_{g}=30 \mathrm{~nm}$, la transconductancia es muy superior en comparación con el SB-MOSFET de idéntica longitud de puerta $\left(g_{m}=1500 \mathrm{~S} \mathrm{~m}^{-1}\right.$, mientras que nuestros resultados indican que $g_{m}=600 \mathrm{~S} \mathrm{~m}^{-1}$ para el transistor SB-MOSFET). Esta gran diferencia viene explicada principalmente por el espesor del canal $\left(t_{S i}=150 \mathrm{~nm}\right.$ para el transistor convencional y $t_{S i}=10 \mathrm{~nm}$ para el SB-MOSFET). Finalmente, observamos que en el caso del SB-MOSFET de $45 \mathrm{~nm}$ simulado en [Xia, 2005] el valor de $g_{m}$ obtenido $\left(400 \mathrm{~S} \mathrm{~m}^{-1}\right)$ es inferior al presentado en este trabajo para $30 \mathrm{~nm}$.

Teniendo en cuenta los resultados de $g_{m}$ y de las capacidades del circuito equivalente, obtenemos el valor de la frecuencia de corte, representada en la Figura VI.18.b para los distintos valores de longitud de puerta. Como podemos ver, alcanza un valor máximo de $260 \mathrm{GHz}$ en el caso de $L_{g}=30 \mathrm{~nm}$, cuyo valor es muy similar al indicado en las predicciones de [ITRS, 2005] (estrella negra) y al que presenta el SB-MOSFET tipo $p$ estudiado por [Fritze, 2004]. Por otro lado, al compararlo con los resultados presentados en [Rengel, 2006] para el transistor SOIMOSFET convencional o en [Xia, 2005] para el SB-MOSFET, nuestros resultados son 
sensiblemente inferiores, consecuencia de las diferencias entre los valores de la transconductancia y las capacidades que ya hemos comentado anteriormente. En el caso de los transistores PDSOI-MOSFET convencional con longitudes de puerta iguales a $90 \mathrm{~nm}$ y $60 \mathrm{~nm}$, el valor de $f_{t}$ es prácticamente idéntico al obtenido en el dispositivo homólogo tipo Schottky.

Por último, mostramos a continuación en la Figura VI.19 la variación de la resistencia de carga del canal $\left(R_{i}\right)$ y el tiempo de retardo de transconductancia $(\tau)$ con la longitud de puerta. Como podemos observar, estas magnitudes se ven reducidas en gran medida al disminuir $L_{g}$, obteniendo por tanto una respuesta más rápida del dispositivo frente a variaciones del voltaje entre puerta y fuente.
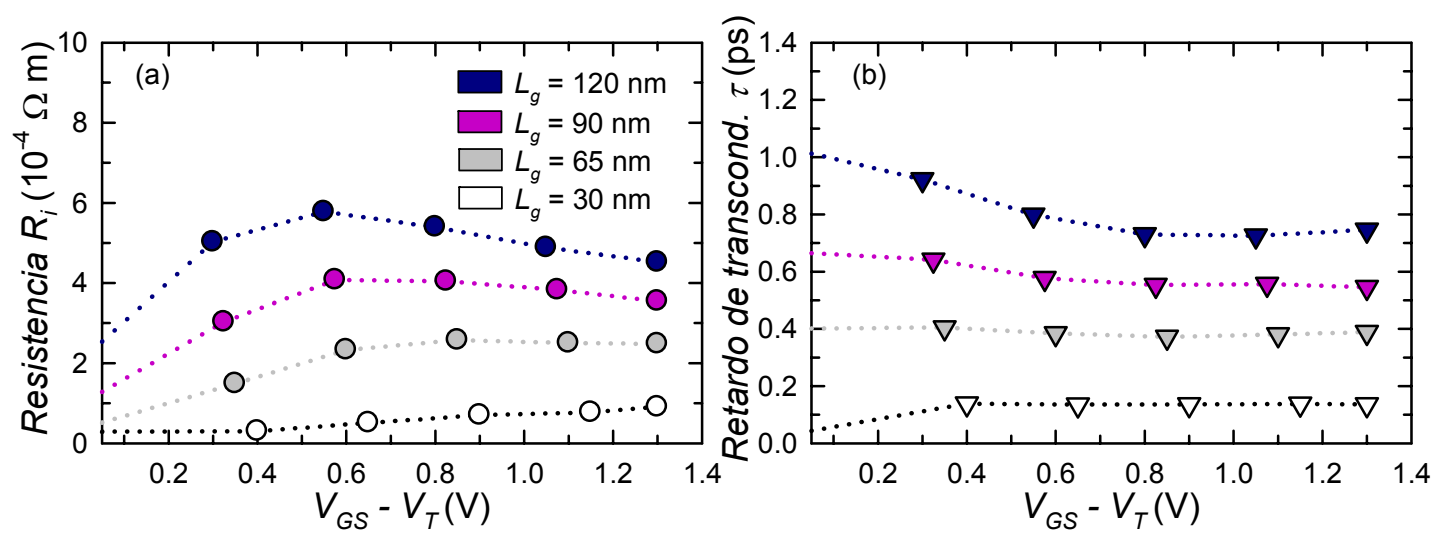

Figura VI.19.a. Resistencia de carga del canal $\left(R_{i}\right) y$. b. tiempo de retardo de transconductancia $(\tau)$ en función de de $V_{\text {overdrive }}$ con $V_{D S}=2 \mathrm{~V}$ para $L_{g}=120 \mathrm{~nm}, 90 \mathrm{~nm}, 65 \mathrm{~nm}$ y $30 \mathrm{~nm}$.

A partir del estudio de estas magnitudes podemos afirmar que la reducción de la longitud de puerta, aunque no proporciona un aumento significativo en la corriente del dispositivo (véase el Capítulo V, Sección 6.c) o la transconductancia, sí que resulta beneficiosa desde el punto de vista de la frecuencia de corte y de la capacidad de puerta a fuente. Sin embargo, es importante dejar claro que esta leve dependencia de $I_{D}$ o $g_{m}$ con $L_{g}$ no es debida a las propiedades del transistor SB-MOSFET, sino a un aumento de los efectos de canal corto justificado por las características de la simulación: es decir, el hecho de que no se ha tenido en cuenta la reducción adicional de los diversos parámetros del dispositivo (como $t_{S i}, t_{o x}$, etc) ni tampoco del voltaje de polarización, tal y como indican las reglas del escalado [Rengel, 2002]. 


\section{VI.3. Caracterización de ruido}

Llegados a este punto del trabajo, nos encontramos en condiciones de evaluar el fenómeno del ruido electrónico en este tipo de dispositivos. En primer lugar analizaremos las funciones de autocorrelación; a partir de las mismas podemos evaluar las densidades espectrales. Nos centraremos fundamentalmente en la dependencia con la frecuencia de las fuentes de ruido $S_{I D}$ y $S_{I G}$. En segundo lugar analizaremos la dependencia de $S_{I D}$ y $S_{I G}$ con el voltaje en puerta y en drenador aplicado. El efecto de la variación de los parámetros del dispositivo sobre el ruido y sus figuras de mérito será analizado en la siguiente sección.

\section{VI.3.a. Dependencia de las fuentes de ruido con la frecuencia}

El método Monte Carlo permite determinar de manera directa el valor de las fluctuaciones aleatorias de una magnitud dada, $A$, (ya sea corriente, voltaje u otra magnitud; téngase en cuenta que en el presente trabajo nos centraremos en analizar las fluctuaciones de corriente en terminales). Estas fluctuaciones, $\delta A$, vendrán definidas por la diferencia entre el valor de $A$ en cada instante de tiempo y su valor medio, $\bar{A}: \delta A=A(t)-\bar{A}$ [Van der Ziel, 1986]. En el presente trabajo nos centraremos en el estudio de las fluctuaciones de la corriente tanto de puerta como de drenador $\left(A=I_{G}, I_{D}\right)$.

Uno de los métodos más usados para estudiar la dependencia con la frecuencia, $f$, de las cantidades que fluctúan es el método de Fourier, con el que podemos expresar dichas cantidades en términos de su densidad espectral de fluctuaciones de corriente $S(f)$, con la ventaja de que esta magnitud puede ser comparada directamente con medidas experimentales del laboratorio. Como paso previo al cálculo de $S(f)$, debemos determinar la función de correlación correspondiente:

$$
C_{A B}(t)=\lim _{T \rightarrow \infty} \frac{1}{T} \int_{0}^{T} \delta A\left(t^{\prime}\right) \delta B\left(t^{\prime}+t\right) d t^{\prime}
$$

Cuando A es igual a $\mathrm{B}$, se denomina función de autocorrelación; a continuación enumeramos las funciones de correlación que analizaremos en esta memoria:

$$
C_{I G I D}(t)=\lim _{T \rightarrow \infty} \frac{1}{T} \int_{0}^{T} \delta I_{G}\left(t^{\prime}\right) \delta I_{D}\left(t^{\prime}+t\right) d t^{\prime}
$$




$$
\begin{aligned}
& C_{I G}(t)=\lim _{T \rightarrow \infty} \frac{1}{T} \int_{0}^{T} \delta I_{G}\left(t^{\prime}\right) \delta I_{G}\left(t^{\prime}+t\right) d t^{\prime} \\
& C_{I D}(t)=\lim _{T \rightarrow \infty} \frac{1}{T} \int_{0}^{T} \delta I_{D}\left(t^{\prime}\right) \delta I_{D}\left(t^{\prime}+t\right) d t^{\prime}
\end{aligned}
$$

Siendo $C_{I G I D}$ la función de correlación cruzada de las fluctuaciones de corriente de puerta y de drenador, $C_{I G}$ la función de autocorrelación de las fluctuaciones de la corriente de puerta y $C_{I D}$ la función de autocorrelación de las fluctuaciones de la corriente de drenador. A partir de estas funciones, podemos calcular las densidades espectrales correspondientes como su transformada de Fourier [McQuarrie, 1976]: $S_{A}(t)=2 \int_{-\infty}^{\infty} C_{A}(t) e^{j 2 \pi f t} d t$, que puede expresarse del siguiente modo:

$$
S_{A}(t)=2 \Delta t \sum_{m=-k}^{k} C_{A}(m \Delta t) \cos (2 \pi f m \Delta t)
$$

El factor 2 es debido a que integramos en $t$ únicamente en el rango de frecuencias positivas. En nuestro caso estudiaremos la densidad espectral de la correlación cruzada $\left(S_{I G I D}\right)$ y las densidades espectrales de las fluctuaciones de corriente de puerta $\left(S_{I G}\right)$ y drenador $\left(S_{I D}\right)$ :

$$
\begin{aligned}
& S_{I G I D}(t)=2 \Delta t \sum_{m=-k}^{k} C_{I G I D}(m \Delta t) \cos (2 \pi f m \Delta t) \\
& S_{I G}(t)=2 \Delta t \sum_{m=-k}^{k} C_{I G}(m \Delta t) \cos (2 \pi f m \Delta t) \\
& S_{I D}(t)=2 \Delta t \sum_{m=-k}^{k} C_{I D}(m \Delta t) \cos (2 \pi f m \Delta t)
\end{aligned}
$$

Téngase en cuenta que para la determinación adecuada de las densidades espectrales, es necesario asegurar que el valor de la correlación sea nulo en el tiempo de corte elegido para la transformada de Fourier. Aunque puede parecer que esta condición se alcanza para tiempos muy rápidos, en realidad existen "colas" de la función de correlación que hacen que el valor cero no se alcance verdaderamente hasta tiempos superiores a los $20 \mathrm{ps}$, dependiendo del dispositivo. Por este motivo es necesario realizar este corte de manera adecuada. 


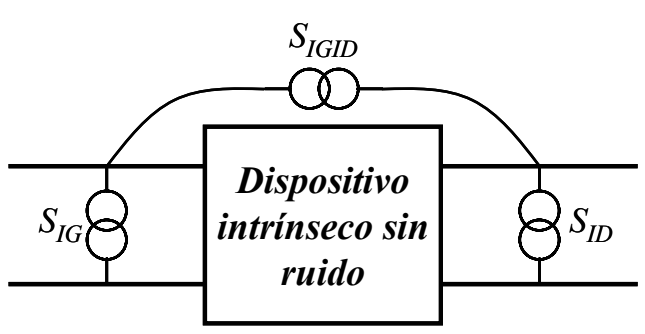

Figura VI.20. Representación circuital del ruido en un dispositivo de tres terminales.
Para facilitar el estudio del ruido del dispositivo, se representa al mismo (en configuración de fuente común) como un cuadripolo de dos puertos sin ruido ("noiseless") y representado por los parámetros de pequeña señal, al que se le añaden dos fuentes de ruido: una a la entrada (puerta) y otra a la salida (drenador) del dispositivo, ambas correlacionadas (ver Figura VI.20) [Van der Ziel, 1986]. A diferencia de otras metodologías, el simulador Monte Carlo nos permite calcular las fuentes de ruido de manera natural, sin realizar ninguna suposición acerca de su origen. Determinaremos, por tanto, los valores de las distintas densidades espectrales $\left(S_{I G}, S_{I D}, S_{I G I D}\right)$ mediante el análisis de las fluctuaciones de corriente instantánea en los terminales en simulaciones de larga duración [Rengel, 2001], pudiendo así describir el ruido en un dispositivo de tres terminales, más concretamente del SB-MOSFET, el caso que nos ocupa.

A continuación estudiamos las funciones de autocorrelación asociadas a las fluctuaciones de corriente en los terminales de puerta $\left(C_{I G}\right)$ y drenador $\left(C_{I D}\right)$ y la correlación cruzada $\left(C_{I G I D}\right)$, determinadas de la manera descrita por la Ecuación VI.7. Se muestran en la Figura VI.21.a en condiciones de polarización $V_{G S}=2 \mathrm{~V}$ y $V_{D S}=0.1 \mathrm{~V}$. Por otro lado, en la Figura VI.21.b representamos la variación de $C_{I D}$ para diferentes valores de voltaje en drenador.
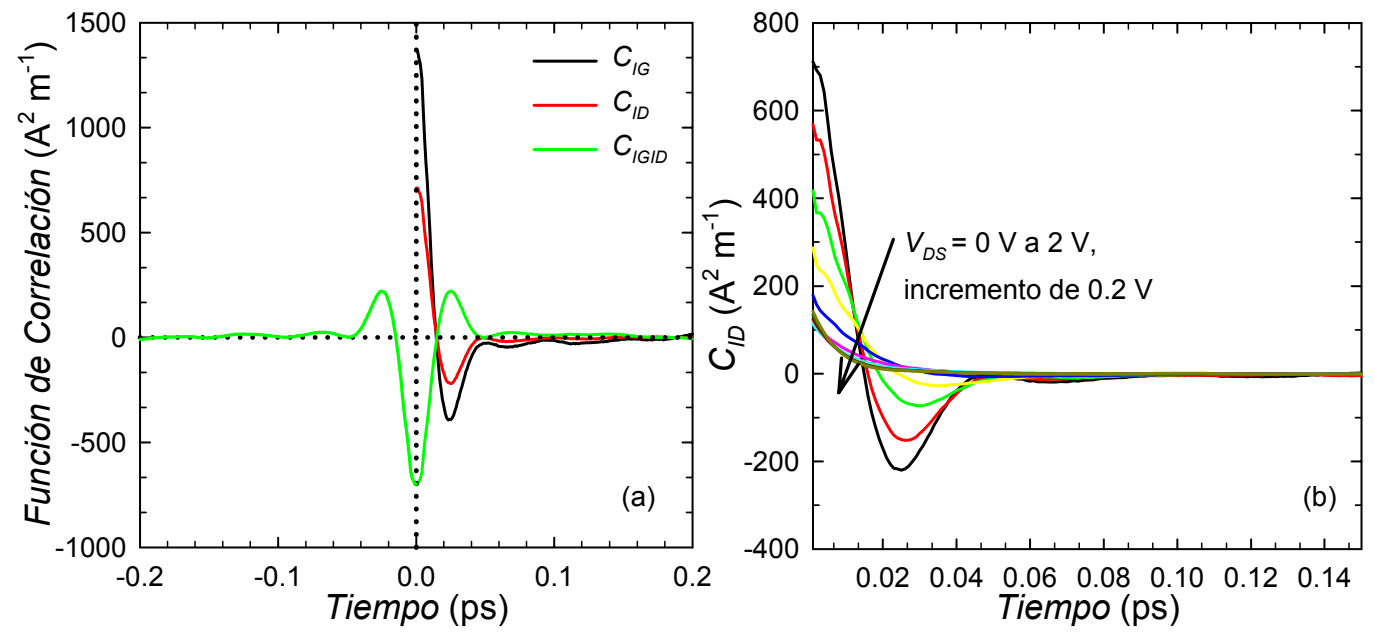

Figura VI.21.a. Funciones de autocorrelación de las fluctuaciones de corriente de drenador, de puerta y correlación cruzada para el SBMOSFET con $V_{G S}=2 \mathrm{~V}$ y $V_{D S}=0.1 \mathrm{~V}$.b. Función de autocorrelación de drenador para $V_{G S}=2 \mathrm{~V}$ 
Como podemos observar en la Figura VI.21.a, dado que las funciones de autocorrelación, $C_{I G}$ y $C_{I D}$, son funciones pares, han sido representadas únicamente para tiempos positivos, mientras que la asimetría que muestra la correlación cruzada con respecto al tiempo nos indica que el valor de $S_{I G I D}$ (magnitud que analizaremos posteriormente) ha de ser complejo [González, 1994]. Por otro lado, si examinamos la evolución de la función de autocorrelación de la corriente de drenador con $V_{D S}$ (Figura VI.21.b), observamos que su valor para tiempo cero disminuye a medida que el voltaje en drenador aumenta de $0 \mathrm{~V}$ a $1.2 \mathrm{~V}$, pasando a aumentar ligeramente para valores de $V_{D S}$ superiores, una vez que el dispositivo se encuentra en el régimen de saturación. Para valores débiles del campo eléctrico aplicado (bajo $V_{D S}$ ), la dependencia temporal de $C_{I D}$ se corresponde con la de una exponencial decreciente, cuya constante de evolución temporal coincide con el tiempo de relajación del momento $\tau_{m}$. A bajos voltajes aplicados $\left(0<V_{D S}<0.8 \mathrm{~V}\right), C_{I D}$ presenta un cambio de signo para tiempos en torno a 15 ns, alcanzando un mínimo que se desplaza a tiempos superiores a medida que $V_{D S}$ aumenta; el origen de este mínimo será analizado posteriormente.
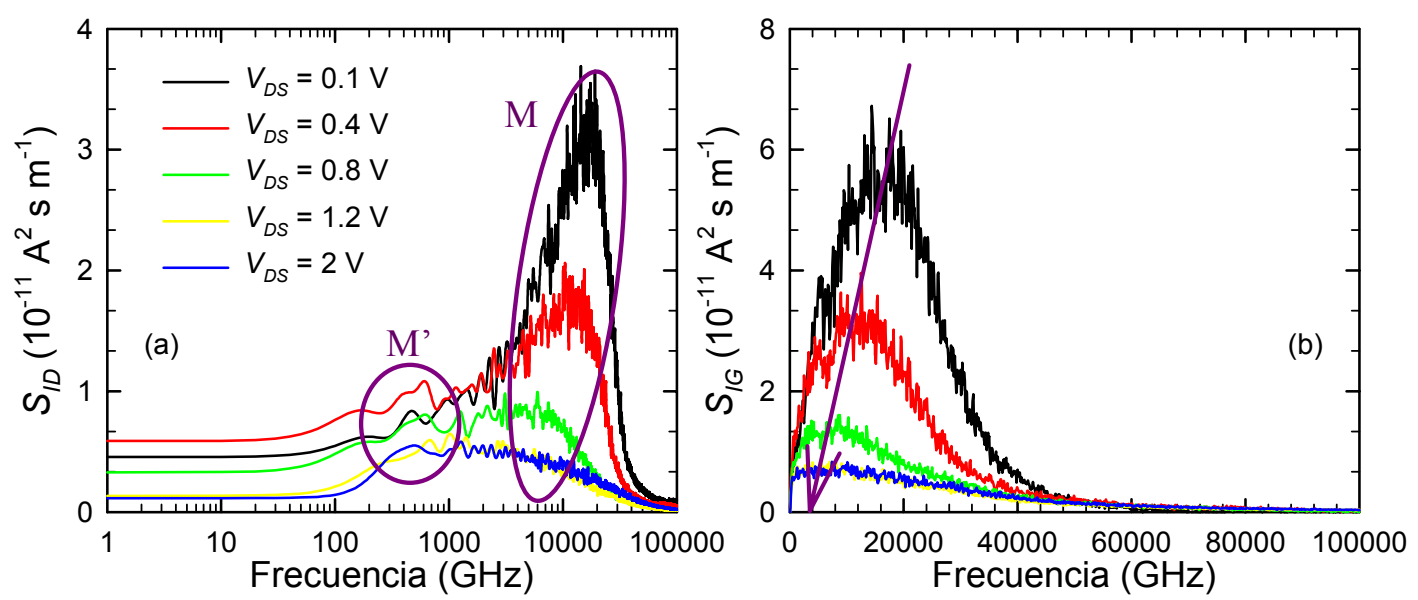

Figura VI.22.a. Densidades espectrales de las fluctuaciones de corriente en drenador .b. y en puerta para el SBMOSFET $\left(V_{G S}=2 V\right.$ y varios $\left.V_{D S}\right)$. Observamos la aparición de dos máximos en $S_{I D}\left(M y M^{\prime}\right)$ y de uno en $S_{I G}$.

En la Figura VI.22 representamos las densidades espectrales de las fluctuaciones de corriente de drenador y puerta, $S_{I D}$ y $S_{I G}$, en función de la frecuencia hasta $100 \mathrm{THz}$ para distintos valores de $V_{D S}\left(\right.$ con $\left.V_{G S}=2 \mathrm{~V}\right)$. Como puede observarse en la Figura VI.22.a, $S_{I D}$ presenta un máximo absoluto, $\mathbf{M}$ (en torno a $20 \mathrm{THz}$ para $V_{D S}=0.1 \mathrm{~V}$ ), que se reduce notablemente y se va desplazando a frecuencias menores a medida que aumenta el voltaje en 
drenador. Existe un segundo máximo local, M', situado a frecuencias en torno a $500 \mathrm{GHz}$ que no depende del valor del voltaje en drenador aplicado.

Finalmente, destacamos que el comportamiento de $S_{I D}$ en el rango inferior a $40 \mathrm{GHz}$ es el típico de ruido "blanco", es decir, independiente de la frecuencia. En el caso de $V_{D S}=2 \mathrm{~V}$ (régimen de saturación) es independiente hasta valores de $100 \mathrm{GHz}$ aproximadamente.

Por otro lado, si examinamos el comportamiento de la densidad espectral de las fluctuaciones de la corriente de puerta, $S_{I G}$, en función de la frecuencia (Figura VI.22.b) vemos que presenta un máximo cuyo valor disminuye a medida $V_{D S}$ que es mayor y su posición se corresponde a frecuencias cada vez menores.

Analizamos a continuación el comportamiento de los anteriormente comentados máximos (M y M') que se observan en la dependencia con la frecuencia de $S_{I D}$. Para esclarecer el origen de los mismos hemos realizado diversas pruebas con nuestro simulador Monte Carlo tomando como base los resultados en condiciones de polarización $V_{G S}=2 \mathrm{~V} \mathrm{y} V_{D S}=0.4 \mathrm{~V}$.

Hemos de comentar que en nuestro simulador hemos incorporado la posibilidad de realizar el cálculo de ruido en el dispositivo sin resolver la ecuación de Poisson cada paso temporal de manera autoconsistente, considerando un campo eléctrico "congelado" correspondiente a un perfil medio y estable para una determinada polarización. En la Figura VI.23.a mostramos para la polarización anteriormente mencionada $\left(V_{G S}=2 \mathrm{~V}\right.$ y $\left.V_{D S}=0.4 \mathrm{~V}\right)$ los resultados de la simulación habitual (resolviendo Poisson de manera autoconsistente, línea roja) y la simulación con campo congelado (línea gris).

Al igual que sucede en el MOSFET convencional, es lógico pensar el máximo $\mathbf{M}$ se encuentre asociado con el mínimo de la parte negativa de las funciones de autocorrelación (Figura VI.21.b). Por tanto, su origen sería el acoplamiento entre las fluctuaciones del campo eléctrico y las fluctuaciones de velocidad de los portadores que se produce al resolver de manera autoconsistente la ecuación de Poisson; estaría por tanto asociado a la frecuencia de oscilación del plasma [Martín, 1996]. Sin embargo, si éste fuera el único origen de M, al considerar el campo congelado en la simulación Monte Carlo (línea gris de Figura VI.23.a), este máximo debería desaparecer completamente, pero observamos que se desplaza a frecuencias inferiores en dicha simulación. Podemos afirmar por tanto que el máximo $\mathbf{M}$ tiene su origen en la superposición de dos efectos a distintas frecuencias $\left(\mathbf{M}_{\mathbf{1}}+\mathbf{M}_{\mathbf{2}}\right)$, siendo el primero de estos efectos $\left(\mathbf{M}_{1}\right)$ las oscilaciones del plasma, mientras que el origen de $\mathbf{M}_{2}$ será discutido posteriormente. Es interesante destacar que el máximo $\mathbf{M}_{1}$ no se presenta a para una frecuencia fija, sino que se desplaza a frecuencias inferiores a medida que aumenta la polarización $V_{D S}$, 
esto se debe a la dependencia de la frecuencia de plasma con la concentración de portadores (véase dicha dependencia en el Apéndice).
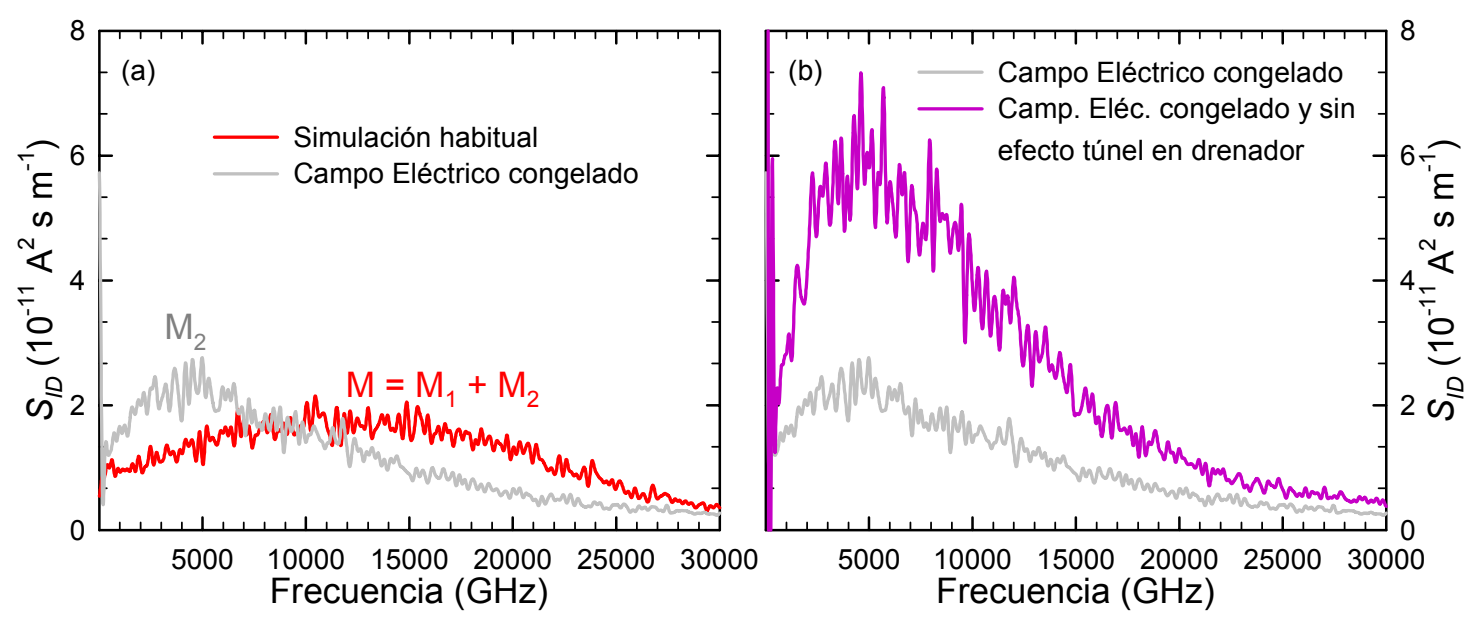

Figura VI.23. $S_{I D}$ en función de la frecuencia para el SBMOSFET bajo la polarización: $V_{G S}=2$ Vy $V_{D S}=0.4 \mathrm{~V}:$ la linea roja se corresponde con la simulación habitual (ver Figura VI.22.a), la línea gris representa el resultado de la simulación Monte Carlo con el campo congelado y la linea violeta representa el resultado con el campo congelado y sin considerar el efecto túnel en el contacto de drenador.

En la Figura VI.23.b mostramos la dependencia de $S_{I D}$ con la frecuencia para la simulación Monte Carlo con campo congelado (línea gris) junto con el resultado de desactivar el efecto túnel en el contacto de drenador (-además de considerar el campo congelado tambiénlínea malva). Podemos observar cómo el valor de $\mathbf{M}_{2}$ aumenta notablemente al anular el efecto túnel (principalmente absorción) en el contacto de drenador. Podemos deducir por tanto que este máximo está relacionado con los portadores que no tienen energía suficiente para superar la barrera y deben retroceder, denominados en inglés "returning carriers", ya que en el caso de desactivar el efecto túnel en el drenador estamos obligando a los portadores a darse la vuelta, de ahí el gran aumento en el valor de este máximo (Figura VI.23.b, línea malva). Debe tenerse en cuenta que las condiciones de polarización escogidas para estas simulaciones $\left(V_{G S}=2 \mathrm{~V}, V_{D S}=\right.$ $0.4 \mathrm{~V}$ ) coinciden con el máximo valor de absorción túnel en el drenador (véase Figura V.16). Por tanto, para valores de $V_{D S}$ superiores a $1 \mathrm{~V}$ el máximo $\mathbf{M}$ es únicamente contribución del máximo $\mathbf{M}_{1}$, puesto que no existe posibilidad de absorción túnel ni returning carriers debido a la forma de la banda de conducción (véase Figura V.15). 
La frecuencia (a) a la que aparece el máximo $\mathbf{M}_{2}$ (para este caso de polarización, $V_{G S}=2 \mathrm{~V} \mathrm{y} V_{D S}=0.4 \mathrm{~V}$, como vemos, el máximo $\mathbf{M}_{2}$ está en torno a $4-5 \mathrm{THz}^{1}$ ) está asociada al tiempo característico del proceso de reflexión de los portadores en la barrera:

$$
a=\sqrt{\frac{q \xi_{\max }}{m^{*} d}}
$$

donde $\xi_{\max }$ es el campo eléctrico máximo junto al contacto y $d$ es la anchura de la zona de carga espacial [Trippe, 1986].
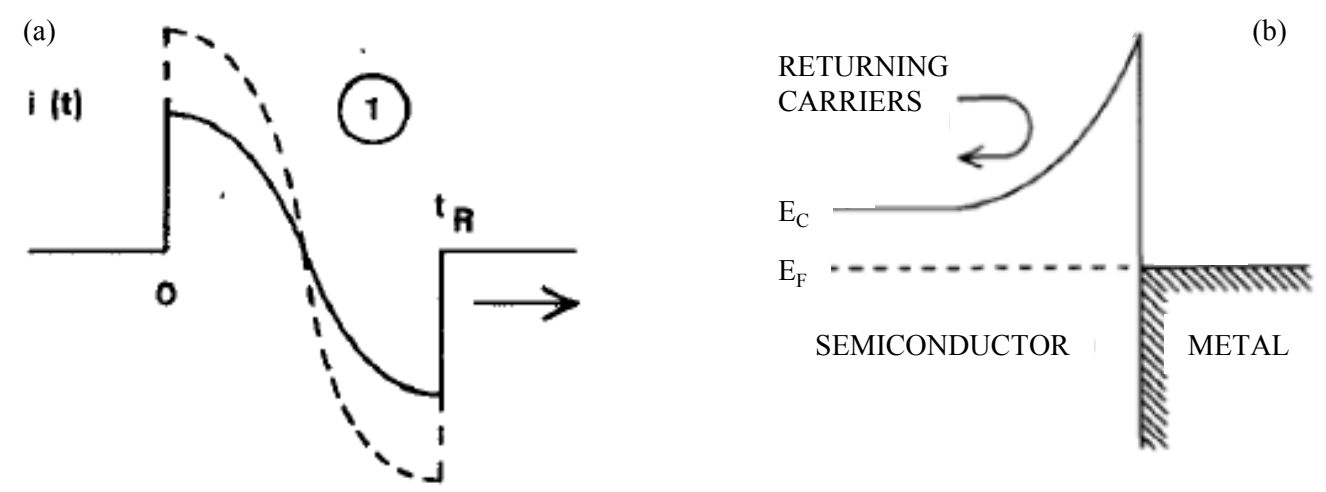

Figura VI.24.a. La línea continua en representa el pulso de corriente debido a un electrón que se da la vuelta (“returning carrier"). La línea discontinua se corresponde con un electrón con una velocidad inicial ligeramente superior. Esta figura muestra que todos los "returning carriers" tienen el mismo tiempo característico de vuelo. En .b. mostramos los "returning carriers" (tienen energía inferior a $q \Phi_{B n}$ y se dan la vuelta al semiconductor neutro).

[Trippe, 1986].

Estos electrones denominados "returning carriers" regresan a la región de vaciamiento, asociándoseles un tiempo de vuelo; durante este tiempo, la corriente asociada a dichos portadores presenta la dependencia temporal que mostramos en la Figura V.24 [Trippe, 1986]. En esta gráfica se muestra el caso para un único electrón, pero en la práctica, la amplitud debe ser multiplicada por el número de electrones inyectados con la misma velocidad inicial. Los portadores que se han dado la vuelta, independientemente de la velocidad con la que entraron en la zona de carga espacial, adquieren el mismo tiempo característico una vez alcanzada la zona neutra del semiconductor. La amplitud del pulso de corriente sí presenta dependencia con el valor inicial de velocidad del electrón, tal y como vemos en la Figura VI.24. El tiempo característico de un electrón para darse la vuelta es el siguiente:

\footnotetext{
${ }^{1}$ Para el valor correspondiente de $\xi_{\max }$-campo máximo- y de $d$-anchura de región de carga espacial-, los valores se corresponden con los valores observados en la figura.
} 
$\tau_{r c}=\pi / a$

Estos electrones no tienen asociada una corriente DC y no presentan una contribución importante a la admitancia del dispositivo a bajas frecuencias [Trippe, 1986].

Por último, para estudiar el origen del máximo local $\mathbf{M}$ ' a frecuencias de centenas de $\mathrm{GHz}$, presentamos en la Figura VI.25 la comparativa entre el resultado de $S_{I D}$ de la simulación habitual y la simulación desactivando el efecto túnel en ambos contactos (tanto en fuente como en drenador, con $V_{G S}=2 \mathrm{~V}$ y $V_{D S}=0.4 \mathrm{~V}$ ). Observamos que en ausencia de efecto túnel en ambos contactos el máximo M' desaparece mientras que el valor de $\mathbf{M}$ es mayor debido al aumento "returning carrriers" en el contacto de drenador. Podemos inferir de este análisis que el máximo M' está asociado en cierto modo al efecto túnel a través del contacto Schottky de fuente.

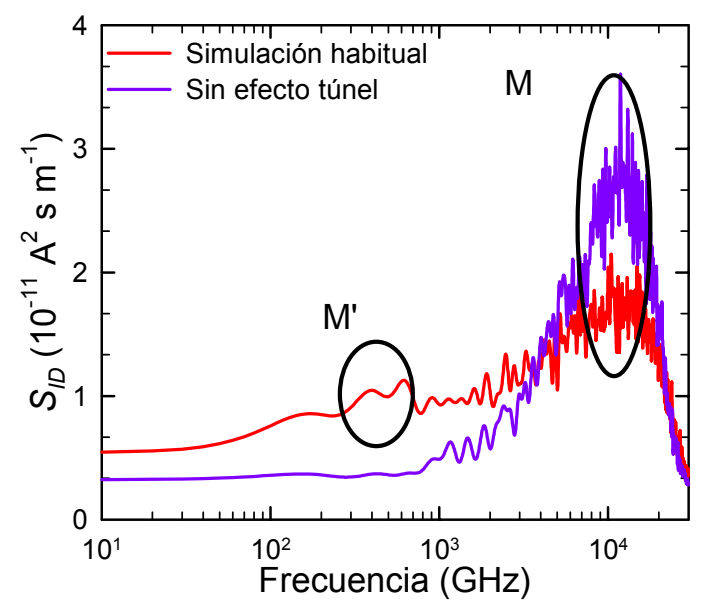

Figura VI.25. $S_{I D}$ en función de la frecuencia para el SBMOSFET bajo la polarización: $V_{G S}=2$ Vy $V_{G S}=0.4 \mathrm{~V}$ : simulación original, es decir, resolviendo Poisson y considerando el efecto túnel en fuente y drenador = línea roja y simulación sin considerar efecto túnel en los contactos de fuente y drenador.

La existencia del máximo $\mathrm{M}_{2}$ (asociado a los "returning carriers") en el caso del transistor SB-MOSFET bajo estudio concuerda los resultados observados en diodos Schottky [González, 1994]. Debe tenerse en cuenta que en dicho trabajo ([González, 1994]) el diodo simulado se encuentra polarizado en directa con lo que los fenómenos de inyección túnel son despreciables (además de que no están implementados en el simulador), lo que explica la ausencia del máximo M'. 

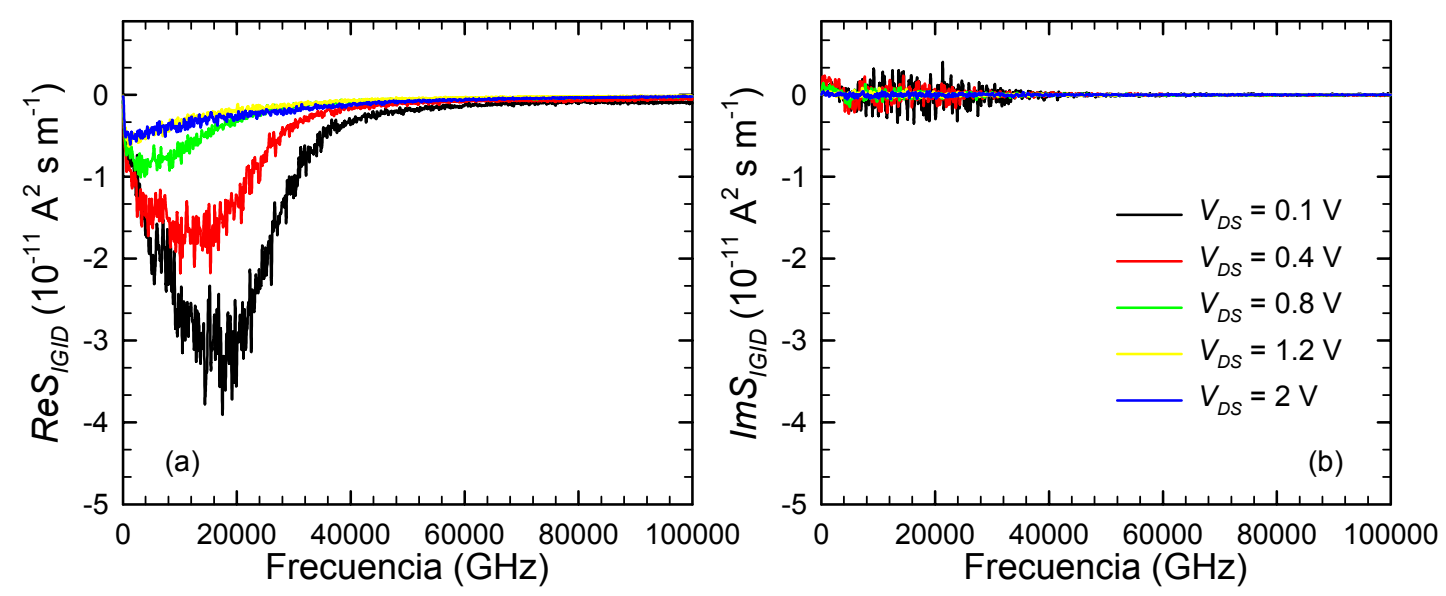

Figura VI.26.a. Parte real y .b. parte imaginaria de la densidad espectral de la correlación cruzada para el $\operatorname{SBMOSFET}\left(V_{G S}=2\right.$ Vy varios $\left.V_{D S}\right)$.

A continuación analizamos la dependencia con la frecuencia de la parte real e imaginaria de la densidad espectral de la correlación cruzada (véase Figura VI.26). Como podemos ver, la parte real presenta valores negativos y además, aparece un mínimo a las mismas frecuencias que el máximo de $S_{I G}$. Por otro lado, la parte imaginaria presenta valores inferiores en el rango de frecuencias analizado. Sin embargo, a frecuencias no tan elevadas (RF y microondas), es decir, dentro del rango de frecuencias para el comportamiento de ruido blanco de $S_{I D}$, como comentábamos con anterioridad, y centrándonos en el régimen de saturación (en el que desarrollaremos principalmente el estudio del ruido del dispositivo en las posteriores secciones), el valor de la parte real es despreciable frente al valor de la parte imaginaria. Por tanto, en el régimen de saturación podremos considerar que $\operatorname{Re} S_{I G I D}$ es nulo en el rango de radiofrecuencias.

A partir de este momento nos centraremos exclusivamente en el rango de frecuencias que acabamos de mencionar, por ser el que tiene mayor interés práctico desde el punto de vista de las aplicaciones de telecomunicaciones. En la Figura VI.27 mostramos la dependencia de $S_{I G}$, $S_{I D}$ e $\operatorname{Im} S_{I G I D}$ en función de la frecuencia para dos voltajes en puerta aplicados $\left(V_{G S}=1 \mathrm{~V}\right.$ and $1.5 \mathrm{~V})$ en el régimen de saturación $\left(V_{D S}=2 \mathrm{~V}\right)$. Como ya vimos en la Figura VI.22.a, la densidad espectral de las fluctuaciones de corriente en drenador, $S_{I D}$, presenta una dependencia constante con la frecuencia (ruido blanco). También podemos ver que $S_{I D}$ aumenta con $V_{G S}$, al igual que lo hace la corriente en drenador. El modo de operación básico de un transistor de efecto de campo consiste en la modulación de un canal conductor mediante el terminal de 
puerta. Por tanto, del mismo modo que en un MOSFET convencional, el dispositivo opera mediante la modulación de resistencia del canal; como consecuencia, es de esperar que aparezca ruido térmico en el mismo: $S_{I D}$ tiene de hecho su origen en el ruido térmico generado por los portadores en la región del canal.

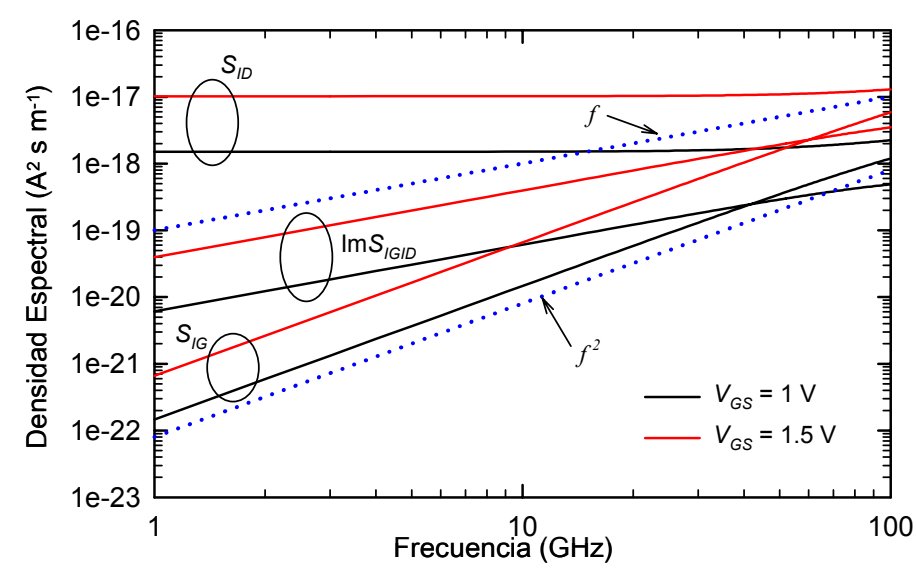

Figura VI.27. Densidades espectrales de fluctuaciones de corriente y su correlación cruzada en función de la frecuencia para dos valores de tensión en la puerta. También mostramos la dependencia con $f y f^{2}$.

Por otro lado, el ruido en puerta, $S_{I G}$, se asocia al acoplamiento capacitivo (con la puerta) de las fluctuaciones de carga en el canal. En el rango de frecuencias en que nos encontramos, podemos considerar al dispositivo como una red RC distribuida: la resistencia distribuida representa al canal conductor (que, como acabamos de ver, lleva asociada $S_{I D}$ ), mientras que la capacidad distribuida representa el acoplamiento capacitivo en puerta [Van der Ziel, 1986].

Como vemos en la Figura VI.27, $S_{I G}$ tiene una dependencia del tipo $f^{2}$ con la frecuencia, mientras que la parte imaginaria de $S_{I G I D}$ aumenta de manera lineal con la misma (la parte real es prácticamente despreciable en el rango de frecuencias de decenas de $\mathrm{GHz}$ ). Estas dependencias de las densidades espectrales con la frecuencia coinciden con el comportamiento predicho por la teoría para un MOSFET en general [Van der Ziel, 1986], además de con los resultados publicados en la literatura para otros dispositivos FET, como MESFETs [González, 1995] o HEMTS [Mateos, 1998], así como con SOI-MOSFETs [Rengel, 2006 y 2006b]. 


\section{VI.3.b. Dependencia de $S_{I D}$ con el voltaje aplicado}

En esta sección nos centraremos en el valor de la densidad espectral de ruido en corriente de drenador en el rango de RF y microondas, es decir, en la región donde $S_{I D}$ es una fuente de ruido blanco y presenta normalmente valores más elevados que $S_{I G}$ y $S_{I G I D}$, y suele por tanto ser considerada en la literatura como la fuente de ruido más representativa en un MOSFET.
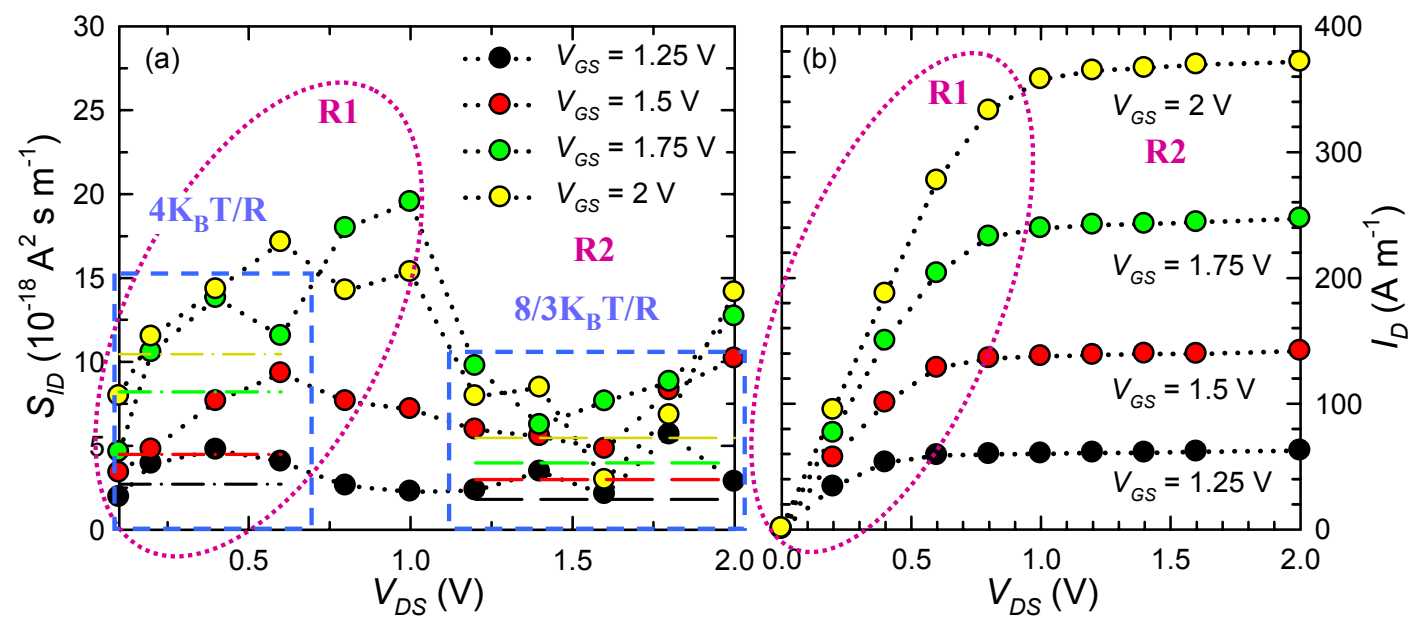

Figura VI.28.a. Densidad espectral de las fluctuaciones de corriente en drenador en función de $V_{D S}$ para $V_{G S}=1.25 \mathrm{~V}, 1.5 \mathrm{~V}, 1.75 \mathrm{Vy} 2 \mathrm{~V} . \mathrm{R} 1$ y R2 son las dos regiones de comportamiento .b. Características de salida correspondientes a los anteriores valores de $V_{G S}$.

En la Figura VI.28 mostramos la variación de $S_{I D}$ con $V_{D S}$ para diferentes valores de voltaje en puerta aplicados: $1.25 \mathrm{~V}, 1.5 \mathrm{~V}, 1.75 \mathrm{~V}$ y $2 \mathrm{~V}$. Hemos representado de nuevo la característica de salida (Figura VI.28.b) para facilitar la discusión.

Podemos distinguir claramente entre dos regiones de comportamiento: una primera región, para valores de $V_{D S}$ correspondientes a la región triodo (R1 en la figura, región englobada en línea púrpura) y una segunda región (R2, el resto) coincidente con el régimen de saturación del dispositivo.

En la región R1, el canal debería comportarse como un elemento fundamentalmente resistivo (sobretodo para los valores de $V_{D S}$ más pequeños), como se deduce del aumento lineal de $I_{D}$ con $V_{D S}$ en las características de salida correspondientes. Por este motivo, en la Figura VI.28.a también representamos la densidad espectral asociada al ruido térmico generado por un elemento resistivo $\left(4 K_{B} T / R\right)$, donde el valor de $R$ ha sido calculado a partir de las características 
$I-V$ para muy bajos valores de $V_{D S}$ [Nyquist, 1928]. Sin embargo, observamos que los resultados obtenidos de la simulación Monte Carlo no presentan un comportamiento constante en este régimen, sino que muestran un aumento con la tensión en drenador aplicada (aunque coinciden con la predicción teórica para los valores inferiores de $V_{D S}$ ). Este aumento de $\boldsymbol{S}_{I D}$ en la región triodo está relacionado con diferentes fenómenos; en primer lugar, en dicha región existe un rápido aumento de la velocidad y energía de los portadores con $V_{D S}$, como ya comentamos en el anterior capítulo (Figura V.22), lo que apunta a una importante contribución al ruido de los denominados portadores calientes (hot carriers) en ese rango. Por otra parte, existe también una importante influencia de los returning carriers en el contacto de drenador para valores bajos de $V_{D S}\left(V_{D S}<0.4 \mathrm{~V}\right.$ aproximadamente, ya que para estas polarizaciones la probabilidad de que un portador se de la vuelta es mayor debido a la forma que presenta la banda de conducción junto al drenador, como veíamos en la Figura V.15.b). Finalmente, el aumento en el número de portadores que son inyectados al interior del canal por emisión de campo a través del contacto de fuente también es otro factor a tener en cuenta para explicar este comportamiento.

Al aumentar más aún el voltaje aplicado en el drenador, a medida que nos acercamos al régimen de saturación $S_{I D}$ tiende a reducirse, presentando un valor prácticamente constante en la región R2, coincidiendo con la disminución del número de returning carriers en el drenador así como con la saturación del nivel de inyección por efecto túnel en la fuente.

Comparamos los resultados obtenidos en R2 con la expresión ideal en saturación para canal largo proporcionada por el modelo de van der Ziel [van der Ziel, 1986].

$$
S_{I D}=8 / 3 g_{m} \mathrm{~K}_{\mathrm{B}} \mathrm{T}
$$

Como podemos ver en la figura, los valores que se obtienen en la simulación Monte Carlo son muy cercanos a la expresión teórica en el caso de menor $V_{G S}$ simulado, incrementándose la diferencia a medida que el voltaje en puerta aumenta. Este resultado también ha sido observado con anterioridad en MOSFETs convencionales [Rengel, 2001] así como en SOI MOSFETs [Rengel, 2006b].

Dado que el comportamiento estático del SB-MOSFET está basado en gran medida en el fenómeno de emisión de campo, es necesario también comprobar la influencia del efecto túnel sobre $S_{I D}$ en este rango de frecuencias y particularmente en la región de polarización de triodo. En la Figura VI.29, mostramos la densidad espectral $S_{I D}$ para $V_{G S}=2 \mathrm{~V}$ resultante de la simulación Monte Carlo incluyendo la emisión de campo junto con el resultado de las simulaciones sin considerar el efecto túnel en los contactos de fuente y drenador y también desactivando dicho efecto únicamente en el contacto de drenador. 


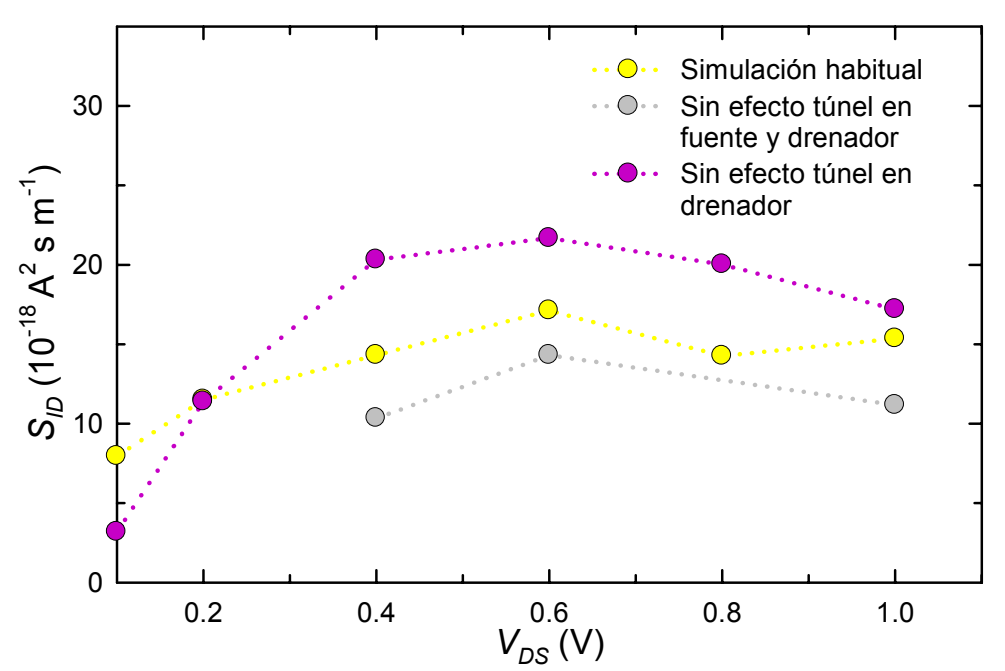

Figura VI.29 Densidad espectral de las fluctuaciones de corriente en drenador en función de $V_{D S}$ para $V_{G S}=2 \mathrm{~V}$ : simulación habitual (símbolos amarillos), simulación desactivando el túnel en los contactos de fuente y drenador (simbolos grises) y sin considerar dicho efecto en el contacto de drenador (símbolos malvas)

Como podemos observar, al desactivar la emisión de campo en los contactos de fuente y drenador - símbolos grises - el valor de $S_{I D}$ disminuye (aunque $S_{I D}$ se ve incrementado por el aumento relativo de los "returning carriers" en el contacto de drenador, es más importante la reducción de portadores por la no consideración de la inyección túnel en la fuente), mientras que $S_{I D}$ aumenta cuando desactivamos dicho fenómeno únicamente en el contacto de drenador símbolos violeta - (como consecuencia del aumento de portadores que se dan la vuelta en el drenador). Podemos afirmar por tanto que el valor de $S_{I D}$ en la región de triodo se ve notablemente influenciado por la cantidad de portadores inyectados por efecto túnel a través del contacto de fuente y por la absorción túnel y "returning carriers" en el contacto de drenador.

\section{VI.4. Efecto de la variación de parámetros geométricos sobre los resultados de ruido del SB-MOSFET}

En esta sección estudiaremos la influencia de la variación de los parámetros topológicos (altura de barrera Schottky, longitud de underlap y longitud de puerta) sobre el ruido del dispositivo en el régimen de operación de saturación; es decir, para un voltaje de drenador fijo, $V_{D S}=2 \mathrm{~V}$. Con este fin, analizaremos la dependencia de las densidades espectrales y de los parámetros de ruido normalizados $P, R$ y $C$ con el voltaje en puerta aplicado, además de estudiar 
los parámetros de ruido más interesantes desde el punto de vista circuital y experimental, como son la figura mínima de ruido, la resistencia de ruido, etc.

\section{VI.4.a Variación de la altura de barrera}

En la Figura VI.30 mostramos la dependencia con $V_{\text {overdrive }}$ de las densidades espectrales $S_{I G}($ a $4 \mathrm{GHz})$ y $S_{I D}$ para $V_{D S}=2 \mathrm{~V}$ para los tres valores de $q \Phi_{B n}$ analizados. Como podemos observar, ambas magnitudes aumentan a medida que $V_{\text {overdrive }}$ es mayor para todos los casos. En el caso del ruido en drenador, también mostramos el valor estimado de $S_{I D}$ mediante el modelo de van der Ziel para un MOSFET convencional de canal largo [van der Ziel, 1986].

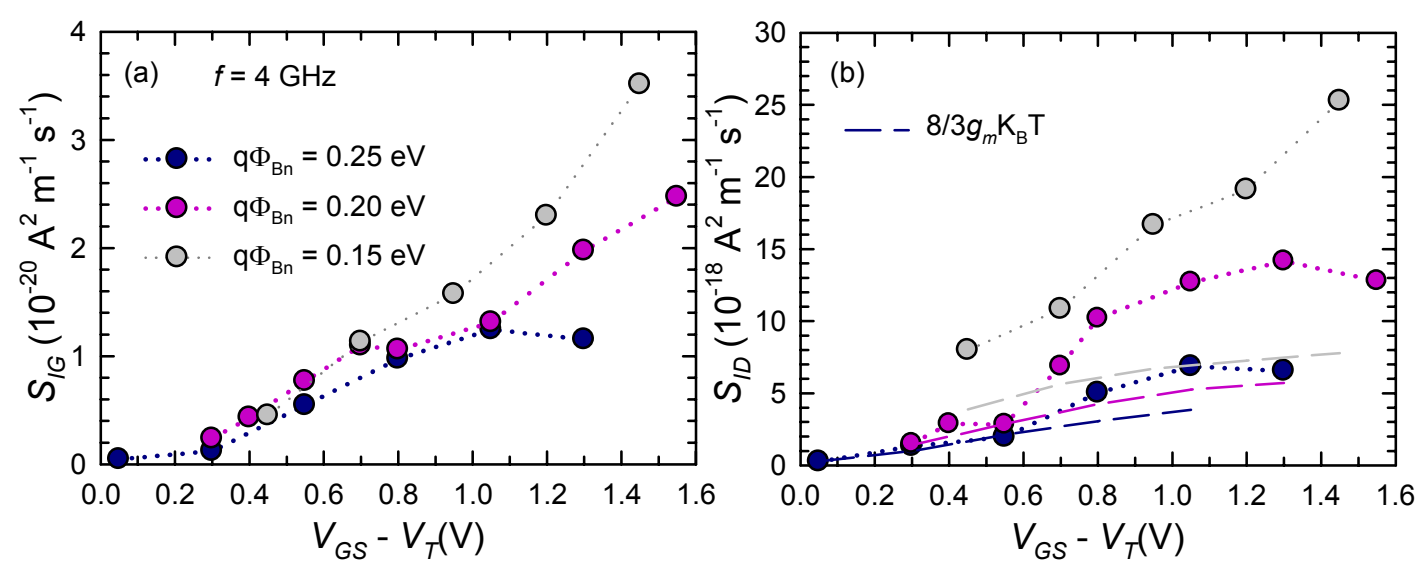

Figura VI.30.a. $S_{I G} y$.b. $S_{I D}$ para tres alturas de barrera de $0.25 \mathrm{eV}, 0.20 \mathrm{eV}$ y $0.15 \mathrm{eV}$ en función de $V_{\text {overdrive }}$ con $V_{D S}=2 \mathrm{~V}$.

Con respecto al efecto de la altura de la barrera Schottky, cuando $q \Phi_{B n}$ disminuye, $S_{I G}$ aumenta para valores de $V_{\text {overdrive }}$ superiores a $0.8 \mathrm{~V}$ (Figura VI.28.a), lo que indica que aparece un mayor ruido inducido en la puerta como consecuencia de las fluctuaciones de carga a lo largo del canal. Como veíamos en la Figura VI.8, $C_{g s}$ aumenta al disminuir la altura de la barrera, es decir, el acople capacitivo entre la puerta y la fuente es mayor, lo que explica que el ruido $S_{I G}$ sea también mayor. 
Como podemos ver en la Figura VI.30.b, $S_{I D}$ aumenta notablemente al disminuir la altura de la barrera (el valor de la corriente de drenador es también mayor al disminuir la

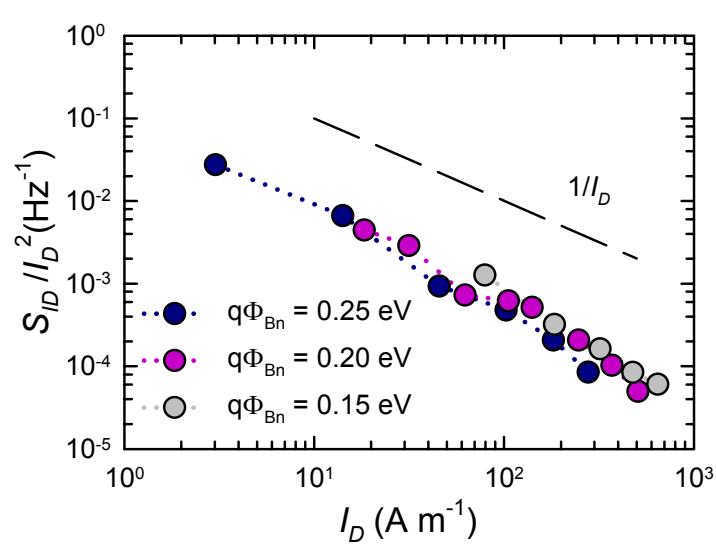

Figura VI.31. $S_{I D} / I_{D}^{2}$ (ruido en corriente normalizado) en función de $I_{D}$ para tres alturas de barrera de $0.25 \mathrm{eV}$, $0.20 \mathrm{eV}$ y $0.15 \mathrm{eV}$ para condición de polarización de $V_{D S}=2 \mathrm{~V}$. barrera), lo que implica un mayor ruido difusivo asociado al movimiento de los portadores en el canal. Para analizar la dependencia de $S_{I D}$ con la corriente de drenador, presentamos en la Figura VI.31 el ruido normalizado en corriente $\left(S_{I D} / I_{D}{ }^{2}\right)$ en función de $I_{D}$. La proporcionalidad con $1 / I_{D}$ de esta magnitud para los diferentes valores de altura de barrera consolida la idea de que el aumento de $S_{I D}$ con la reducción de $q \Phi_{B n} \quad$ se debe principalmente al aumento de la corriente de drenador.

Los resultados de la simulación para $S_{I D}$ muestran valores similares a la predicción teórica en el caso de bajo $V_{\text {overdrive; }}$ sin embargo, los resultados de la simulación Monte Carlo son más elevados a medida que aumenta $V_{\text {overdrive. }}$ Esta diferencia entre los valores MC de $S_{I D}$ y la predicción teórica de canal largo es también más evidente a medida que es menor $q \Phi_{B n}$.

A la vista de estos resultados podemos afirmar que, en principio, el valor de la principal fuente de ruido interno en un SBMOSFET depende fuertemente de la altura de la barrera Schottky, lo cual nos indica la importancia de la depuración de las técnicas de fabricación de los contactos Siliciuro/Silicio, intentando evitar en la medida de lo posible la aparición de estados interfaciales que puedan alterar la formación de la barrera Schottky; estos resultados son coincidentes con el estudio a baja frecuencia presentado por [Yeh, 2009]. Esta afirmación tiene sentido cuando realizamos el estudio en iguales condiciones de $V_{G S}-V_{T}$, sin embargo en idénticas condiciones de corriente no hay dependencia de la altura de la barrera.

A la hora de examinar los resultados de ruido del dispositivo es necesario usar parámetros que permitan una comparación directa con los obtenidos para otros transistores, como los parámetros $P, R$ y $C$ normalizados [Van der Ziel, 1986]. Estos parámetros pueden darnos información relevante acerca del origen físico del ruido. $P$ representa el ruido en la corriente de drenador producido por las fluctuaciones de la velocidad de portadores en el canal 
conductor, $R$ está asociado al ruido inducido en el terminal de puerta por las fluctuaciones de carga en el canal y $C$ a la correlación cruzada entre ambas fuentes de ruido. Para la determinación de estos parámetros, además de las fuentes de ruido es necesario el cálculo previo de los parámetros dinámicos [Pucel, 1974], [Danneville, 1994], en concreto, de los parámetros admitancia:

$$
\begin{aligned}
& P=\frac{S_{I D}}{4 k_{B} T\left|Y_{21}\right|} \\
& R=\frac{S_{I G}\left|Y_{21}\right|}{4 k_{B} T\left|Y_{11}\right|^{2}} \\
& C=\frac{\operatorname{Im}\left[S_{I G I D}\right]}{\sqrt{S_{I G} S_{I D}}}
\end{aligned}
$$

donde $Y$ son los parámetros admitancia (1 y 2 se corresponden con los terminales de puerta y drenador, respectivamente).

Aunque los parámetros $P, R$ y $C$ proporcionan información muy útil acerca del comportamiento en ruido del dispositivo, el parámetro más típico para una caracterización experimental del ruido a nivel circuital es la figura mínima de ruido, $N F_{\min }$. Esta figura de mérito nos indica cuánto incrementa el dispositivo el nivel de ruido de la señal que está siendo amplificada (definiéndose como el cociente del valor de la relación señal-ruido a la entrada y el valor a la salida del dispositivo). Para un análisis completo del ruido del dispositivo, además de la figura mínima de ruido es necesario determinar otros tres parámetros [Cappy, 1998], [Dambrine, 1999]: la resistencia de ruido $R_{n}$, el coeficiente de reflexión óptimo $\Gamma_{\text {opt }}$ y la ganancia asociada $G_{a s s}$, a partir de los parámetros admitancia y de las fuentes de ruido en corriente.

En nuestro caso, hemos seguido un procedimiento análogo al mostrado en [Pailloncy, 2004]. Para la determinación de los parámetros del ruido es necesario calcular $\mathrm{CH}_{s}$, la matriz cadena equivalente en configuración de fuente común y $\mathrm{Ca}_{s}$, la matriz de correlación de ruido asociada a $\mathrm{CH}_{s}^{\prime}$, considerando los elementos extrínsecos del circuito equivalente (véase [Pailloncy, 2004]).

Calculamos la matriz de parámetros $Z$ a partir de los parámetros admitancia:

$$
Z=\frac{1}{|Y|} \cdot\left(\begin{array}{cc}
Y_{22} & -Y_{12} \\
-Y_{21} & Y_{11}
\end{array}\right)
$$


A partir de la misma, calculamos la matriz $\mathrm{CH}_{s}$ :

$$
C H_{s}^{\prime}=\frac{1}{Z_{21}} \cdot\left(\begin{array}{cc}
Z_{11} & \operatorname{det}(Z) \\
1 & Z_{22}
\end{array}\right)
$$

Y posteriormente, la matriz de correlación de ruido, $\mathrm{Ca}_{s}{ }_{s}$ :

$$
C a_{s}^{\prime}=\left(\begin{array}{ll}
C a_{s 11}^{\prime} & C a_{s 12}^{\prime} \\
C a_{s 21}^{\prime} & C a_{s 22}^{\prime}
\end{array}\right)=\frac{4 K_{B} T}{|Y|^{2} \cdot \overline{Z_{21}}} \cdot\left(\begin{array}{ll}
A_{11} & A_{12} \\
A_{21} & A_{22}
\end{array}\right)
$$

donde

$$
\begin{aligned}
& A_{11}=\left[Y_{22}\left(S_{I G} \overline{Y_{22}}-S_{I G I D} \overline{Y_{12}}\right)-Y_{12}\left(S_{I D I G} \overline{Y_{22}}-S_{I D} \overline{Y_{12}}\right)+R_{S}+R_{G}\right]- \\
& {\left[Y_{22}\left(-S_{I G} \overline{Y_{21}}+S_{I G I D} \overline{Y_{11}}\right)-Y_{12}\left(-S_{I D I G} \overline{Y_{21}}-S_{I D} \overline{Y_{11}}\right)+R_{S}\right]\left(\overline{Z_{11}}+R_{S}+R_{G}\right)} \\
& A_{12}=\left[Y_{22}\left(-S_{I G} \overline{Y_{21}}+S_{I G I D} \overline{Y_{11}}\right)-Y_{12}\left(-S_{I D I G} \overline{Y_{21}}-S_{I D} \overline{Y_{11}}\right)+R_{S}\right]\left(1+R_{S}\right) \\
& A_{21}=\left[-Y_{21}\left(S_{I G} \overline{Y_{22}}-S_{I G I D} \overline{Y_{12}}\right)+Y_{11}\left(S_{I D I G} \overline{Y_{22}}-S_{I D} \overline{Y_{12}}\right)+R_{S}\right] \\
& -\left[-Y_{21}\left(-S_{I G} \overline{Y_{21}}+S_{I G I D} \overline{Y_{11}}\right)+Y_{11}\left(-S_{I D I G} \overline{Y_{21}}-S_{I D} \overline{Y_{11}}\right)+R_{S}+R_{D}\right]\left(\overline{Z_{11}}+R_{S}+R_{G}\right) \\
& A_{22}=\left[-Y_{21}\left(-S_{I G} \overline{Y_{21}}+S_{I G I D} \overline{Y_{11}}\right)+Y_{11}\left(-S_{I D I G} \overline{Y_{21}}-S_{I D} \overline{Y_{11}}\right)+R_{S}+R_{D}\right]\left(1+R_{S}\right)
\end{aligned}
$$

El coeficiente de reflexión óptimo, $\Gamma_{o p t}$, se calcula a partir de la admitancia óptima $Y_{o p t}$, que es la admitancia que habría que conectar en la entrada para tener la condición de ruido mínimo:

$$
\begin{aligned}
& y_{o p t}=\sqrt{\frac{C a_{s 22}^{\prime}}{C a_{s 11}^{\prime}},}, \quad y_{o p t}=\frac{C a_{s 21}^{\prime}}{C a_{s 11}^{\prime}} \\
& g_{c o r}=\operatorname{Re}\left(y_{c o r}\right), \quad b_{c o r}=\operatorname{Im}\left(y_{c o r}\right) \\
& g_{o p t}=\sqrt{y_{o p t}^{2}-b_{o p t}^{2}}, \quad b_{o p t}=-b_{c o r} \\
& Y_{o p t}=g_{o p t}+j \cdot b_{o p t} \\
& \Gamma_{o p t}=\frac{Y_{0}-Y_{o p t}}{Y_{0}+Y_{o p t}}
\end{aligned}
$$

Donde $Y_{0}$ es el inverso de la impedancia característica del sistema, usualmente $50 \Omega$. La resistencia de ruido $R_{n}$ da idea de cómo la figura de ruido aumenta cuando la admitancia de entrada es diferente de $Y_{\text {opt }}$. En condiciones intrínsecas su valor es tal que el ruido de difusión 
generado por ella correspondería al valor del ruido de la corriente de drenador. Se calcula a partir de la siguiente expresión:

$$
R_{n}=\frac{\left|C a_{s 11}^{\prime}\right|}{4 K_{B} T \Delta f}
$$

La ganancia asociada se define como la ganancia del dispositivo cuando la impedancia de entrada al mismo es $Y_{\text {opt, }}$ que indica la capacidad del dispositivo de amplificar una señal en condiciones de ruido mínimo

$$
G_{\text {ass }}=\frac{\operatorname{Re}\left(Y_{o p t}\right)}{\operatorname{Re}\left(Y_{22}-\frac{Y_{21} Y_{12}}{Y_{o p t}-Y_{11}}\right)}\left|\frac{Y_{21}}{Y_{o p t}+Y_{11}}\right|^{2}
$$

Finalmente, la figura mínima de ruido, expresada en $\mathrm{dB}$, se determina como sigue:

$$
N F_{\min }=10 \log _{10}\left(1+2 \cdot R_{n} \cdot\left(g_{\text {opt }}+g_{\text {cor }}\right)\right)
$$

A continuación mostramos la dependencia de algunos de estos parámetros con la altura de barrera Schottky. En primer lugar analizamos a través de la Figura VI.32 los resultados de $P$, $R$ y $C$ obtenidos para los transistores con distintas alturas de barrera en función de $V_{\text {overdrive }}$ para $V_{D S}=2 \mathrm{~V}$. Los resultados Monte Carlo para estos parámetros son prácticamente independientes con la frecuencia en el rango de frecuencias considerado.

El parámetro $P$ nos permite la comparación directa del ruido de drenador entre las distintas estructuras analizadas. Como podemos observar, $P$ aumenta con el voltaje de overdrive. Teniendo en cuenta la Ecuación VI.5 ( $g_{m}$ se corresponde prácticamente con la parte real de la magnitud $\mathrm{Y}_{21}$ en saturación), y que tanto $g_{m}$ como $S_{I D}$ aumentan con el voltaje (véanse Figuras VI.10 y VI.30.b), podemos confirmar que el valor de la transconductancia no compensa el incremento de $S_{I D}$ completamente, dando lugar al aumento de $P$ con $V_{\text {overdrive }}$. Observamos además que el valor de $P$ es mayor a medida que disminuye la altura de la barrera. Comparando las Ecuaciones VI.11 y VI.12, podemos admitir que el valor de $P$ para un dispositivo de canal largo ideal debe ser cercano a 2/3. En nuestras simulaciones de transistores SB-MOSFETs de con longitud del canal de $130 \mathrm{~nm}$ obtenemos valores más elevados debido al aumento del ruido en drenador anteriormente comentado.

Con respecto al parámetro $R$, su aumento con el voltaje en puerta está directamente relacionado con el aumento del ruido inducido en la puerta, $S_{I G}$ (ver Ecuación VI.13). Esto se debe a un mayor acoplamiento capacitivo; confirmamos este hecho con el aumento de la 
capacidad $C_{g s}$ con el $V_{\text {overdrive }}$ (Figura VI.8). Esta misma afirmación es válida para explicar el aumento de $R$ al disminuir $q \Phi_{B n}$. Por otro lado, también observamos que el valor de este

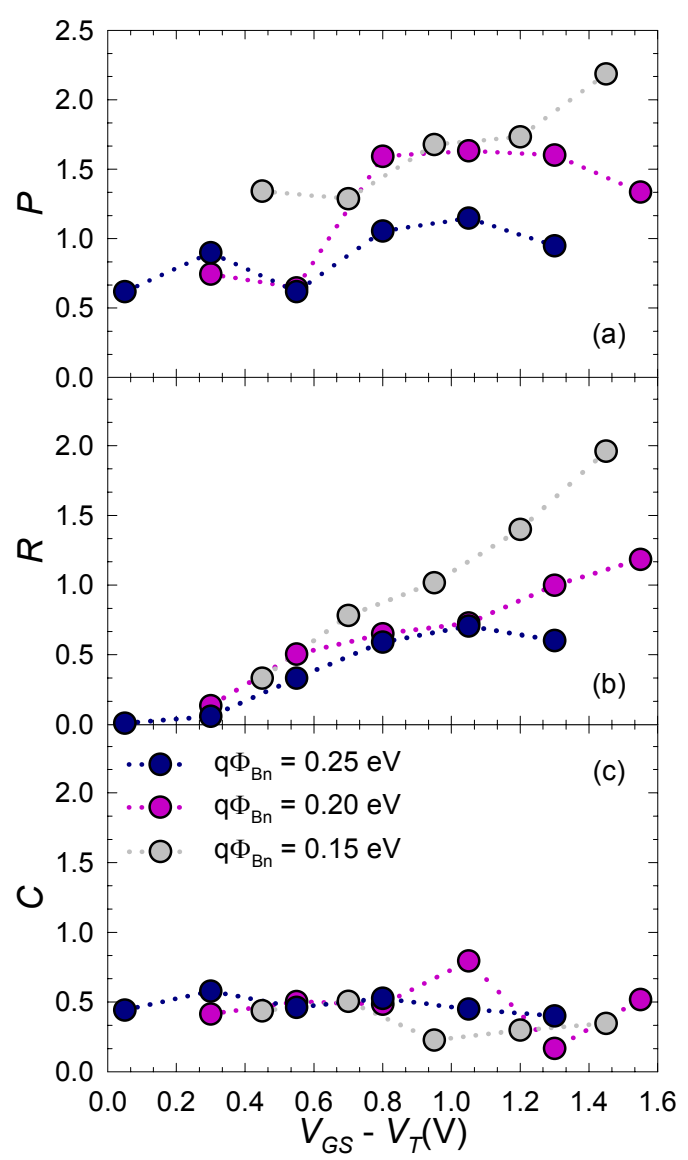

Figura VI.32. Parámetros $P, R$ y $C$ para tres alturas de barrera Schottky de $0.25 \mathrm{eV}, 0.20 \mathrm{eV}$ y $0.15 \mathrm{eV}$ en función de $V_{\text {overdrive }} \operatorname{con} V_{D S}=2 \mathrm{~V}$. parámetro se aleja de la predicción teórica para dispositivos de canal largo $(R \approx 0.3)$. El aumento de las fluctuaciones en el número de portadores por la mayor inyección a través de la fuente con $V_{\text {overdrive }}$ implica por tanto no sólo el aumento de $P$, también de $R$.

En cuanto al coeficiente de correlación entre drenador y puerta, $C$, está relacionado con $S_{I G I D}$ (Ecuación VI.14), cuya parte real es prácticamente nula en el rango de frecuencias analizado (Figura VI.13); de este modo $C$ puede ser considerado completamente imaginario, del mismo modo que se hace en los modelos teóricos para FETs en general. El valor teórico para dispositivos de canal largo es 0.395 [van der Ziel, 1986]. En nuestro caso, dentro de los amplios márgenes de error que posee este parámetro calculado mediante el método Monte Carlo, observamos que $C$ permanece prácticamente constante en el rango de voltajes analizados, viéndose además muy poco afectado por la variación de la altura de la barrera.

En la Figura VI.33 mostramos los resultados Monte Carlo obtenidos para $N F_{\min }$ y el módulo y la fase de $\Gamma_{\text {opt }}$ para $f=6 \mathrm{GHz}$ para nuestro SB-MOSFET con tres valores diferentes de altura de barrera.

Observamos que $\boldsymbol{N} \boldsymbol{F}_{\min }$, que aumenta con $\boldsymbol{V}_{\text {overdrive, }}$ presenta valores prácticamente independientes de la altura de la barrera en el rango de bajos voltajes aplicados. Sin embargo, para voltajes superiores a $1 \mathrm{~V}, N F_{\min }$ se ve ligeramente degradada con la disminución 
de la altura de la barrera. En este caso, es importante destacar que se obtienen excelentes valores de este parámetro (variando en el caso de $q \Phi_{B n}=0.15 \mathrm{eV}$ desde un valor mínimo de $0.3 \mathrm{~dB}$ a $V_{\text {overdrive }}=0.45 \mathrm{~V}$, cuyo valor de $f_{t}$ correspondiente es de $80 \mathrm{GHz}$, hasta $0.9 \mathrm{~dB}$ a $V_{\text {overdrive }}=1.45 \mathrm{~V}$, con $f_{t}=103 \mathrm{GHz}$ ). Los resultados Monte Carlo aquí mostrados son del orden de los presentados en [Saha, 2005] para un transistor SB-MOSFET tipo $n$ de $100 \mathrm{~nm}$ de longitud de puerta y contactos metálicos compuestos por $\mathrm{NiSi}$, que para un valor de voltaje en puerta aplicado de $1.2 \mathrm{~V}$ presenta un valor de $N F_{\min }=0.39 \mathrm{~dB}$.

El aumento de $N F_{\min }$ con $V_{G S}$ puede ser explicado mediante la siguiente expresión aproximada para la figura mínima de ruido [Dambrine, 1999]:

$$
N F_{\text {min }}=10 \log \left(1+2 \frac{f}{f_{t}} \sqrt{P R\left(1-C^{2}\right)}\right)
$$
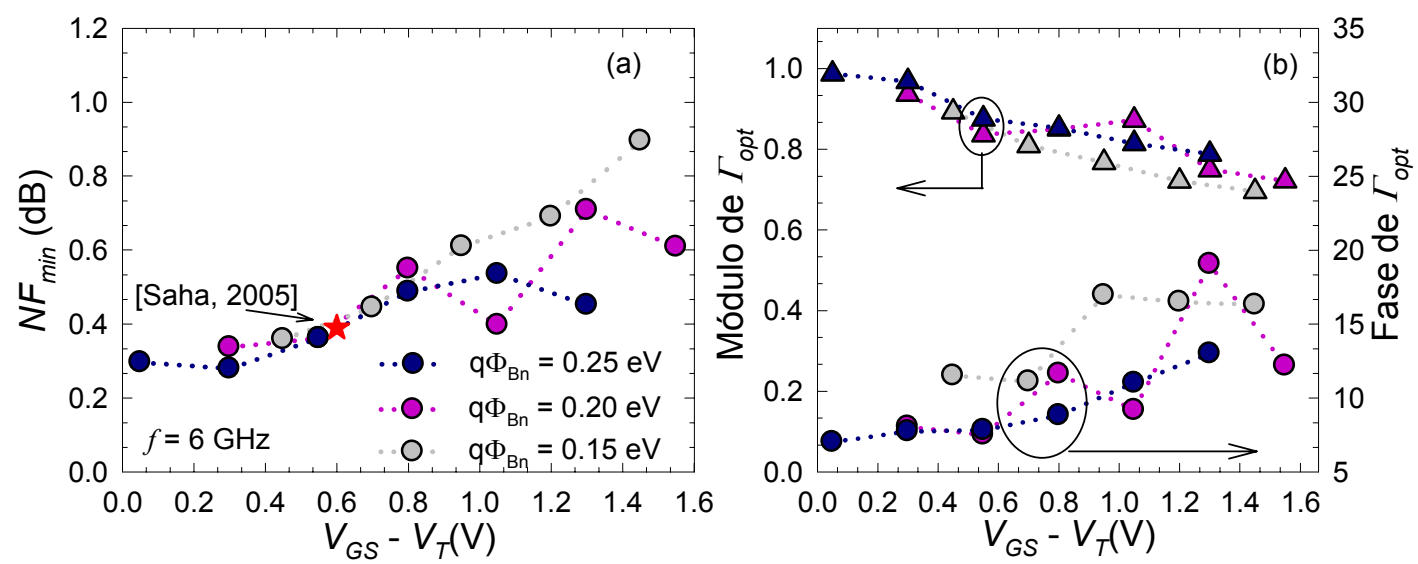

Figura VI.33.a. Figura mínima de Ruido y .b. Coeficiente de reflexión óptimo a 6 GHz para tres alturas de barrera Schottky de $0.25 \mathrm{eV}, 0.20 \mathrm{eV}$ y $0.15 \mathrm{eV}$ en función de $V_{\text {overdrive }}$ con $V_{D S}=2 \mathrm{~V}$. Mostramos también el resultado obtenido de la simulación de un SB-MOSFET de $100 \mathrm{~nm}$ de longitud de puerta (estrella roja) [Saha, 2005].

Debe tenerse en cuenta que aunque esta expresión subestima el valor de $N F_{\min }$ a baja corriente y frecuencia elevada en comparación con el cálculo exacto [Rengel, 2001], en general supone una buena aproximación al cálculo de este parámetro, permitiéndonos relacionar $N F_{\min }$ con los parámetros previamente analizados $P, R$ y $C$. Partiendo de esta expresión, por tanto, es posible deducir que el aumento de $N F_{\min }$ con $V_{G S}$ es debido tanto al incremento del ruido en puerta como en fuente (a través de los parámetros $P$ y $R$ ). El mismo argumento puede ser usado para explicar el aumento de $N F_{\min }$ al reducir $q \Phi_{B n}$ en el caso de altos valores de voltaje en puerta aplicados. Sin embargo, cabe destacar que aunque las diferencias observadas para $S_{I D}$ y $S_{I G}$ al variar la altura de la barrera son significativas, el efecto en el ruido desde el punto de vista 
circuital se ve suavizado al examinar $N F_{\min }$ debido a que el aumento intrínseco del ruido se compensa con el aumento de la frecuencia de corte del dispositivo.

Tanto la resistencia de ruido como la ganancia asociada (no mostradas en las gráficas) apenas se ven influenciadas por el cambio en la altura de la barrera. Esto nos indica, en el caso de $R_{n}$, que la sensibilidad de la figura mínima de ruido cuando la admitancia de entrada es distinta de $Y_{\text {opt }}$ no depende de $q \Phi_{B n}$. Por otro lado, desde el punto de vista de $G_{a s s}$, podemos afirmar que la capacidad de amplificar una señal en condiciones de mínimo ruido es similar para dispositivos con distinta altura de barrera.

En relación al módulo de $\Gamma_{o p t}$, es prácticamente independiente del valor de $q \Phi_{B n}$. Además, a medida que el voltaje en puerta aplicado aumenta, el valor de $\left|\Gamma_{\text {opt }}\right|$ se reduce (Figura VI.33.b). La fase de $\Gamma_{\text {opt }}$, que presenta una dependencia lineal con la frecuencia, aumenta con $V_{\text {overdrive, }}$ y además, su valor es mayor a medida que $q \Phi_{B n}$ disminuye. Esto implica una mayor facilidad de adaptación dentro de un circuito para el dispositivo de menor altura de barrera.

Finalmente, podemos concluir que la disminución de la altura de la barrera da lugar al realce en general de la corriente de conducción (en detrimento del ratio $I_{o n} / I_{o f f}$ ), así como de las principales figuras de mérito dinámicas como $g_{m}$ y $f_{t}$. Con respecto al ruido, las densidades espectrales de las fluctuaciones de corriente de drenador y puerta se ven aumentadas (como consecuencia fundamentalmente de un mayor nivel de corriente); sin embargo es importante destacar que la figura mínima de ruido apenas se ve afectada con la variación de $q \Phi_{B n}$.

\section{VI.4.b Variación de la longitud de underlap}

En esta sección analizaremos el efecto de la longitud de underlap, $L_{u n}$, sobre el ruido del dispositivo. En la Figura VI.34 presentamos los resultados de la simulación MC2D para las densidades espectrales $S_{I G}$ y $S_{I D}$ en función del $V_{\text {overdrive }}$ para los cuatro valores de $L_{u n}$ considerados.

Como podemos observar en la Figura VI.34.a, $S_{I G}$ apenas varía con $L_{u n}$, lo que significa que el efecto de las fluctuaciones de carga en el terminal de puerta es relativamente similar cuando este parámetro geométrico cambia (debe tenerse en cuenta que la longitud de puerta permanece constante). Por otro lado, $S_{I D}$ aumenta cuando disminuye $L_{u n}$ (Figura VI.34.b), al igual que le ocurre a la corriente de drenador (véase Figura V.33, del capítulo anterior). 
Como veíamos en el capítulo anterior, la inyección de portadores por emisión de campo aumenta a medida que la longitud de underlap es menor (Figura V.35, del capítulo anterior); este resultado explica el aumento de la corriente y consecuentemente el obtenido para $S_{I D}$.
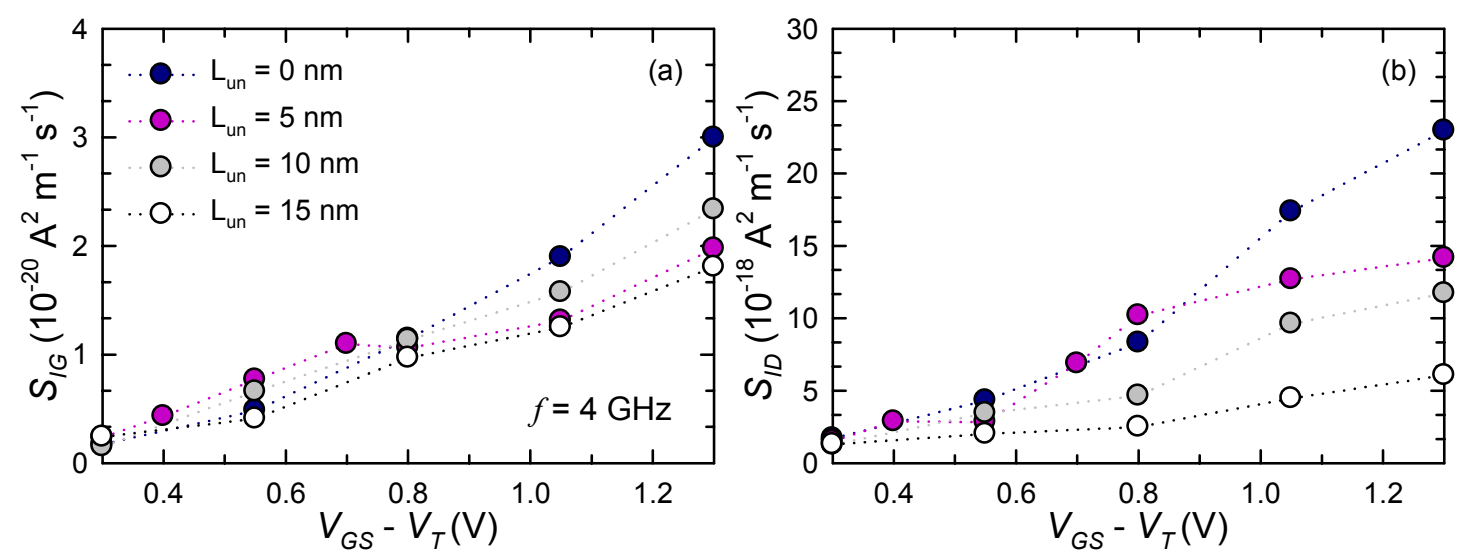

Figura VI.34.a. $S_{I G}$ y.b. $S_{I D}$ para los $S B$-MOSFETs simulados con cuatro longitudes de underlap $\left(L_{u n}=0 \mathrm{~nm}, 5 \mathrm{~nm}\right.$, $10 \mathrm{~nm}$ y $15 \mathrm{~nm}$ ) para $V_{D S}=2 \mathrm{~V}$ y distintos valores de $V_{\text {overdrive. }}$

Nuestro estudio de los parámetros $P, R$ y $C$ (no mostrados en las gráficas) nos indica que no hay diferencias significativas de $R$ y $C$ cuando $L_{u n}$ varía. Sin embargo, $P$ tiende a disminuir para los valores más pequeños de $L_{u n}$ en el rango de bajos voltajes en puerta aplicados; esto es debido a que el incremento de $g_{m}$ al disminuir $L_{u n}$ es más importante que el de $S_{I D}$ en las condiciones de polarización analizadas.
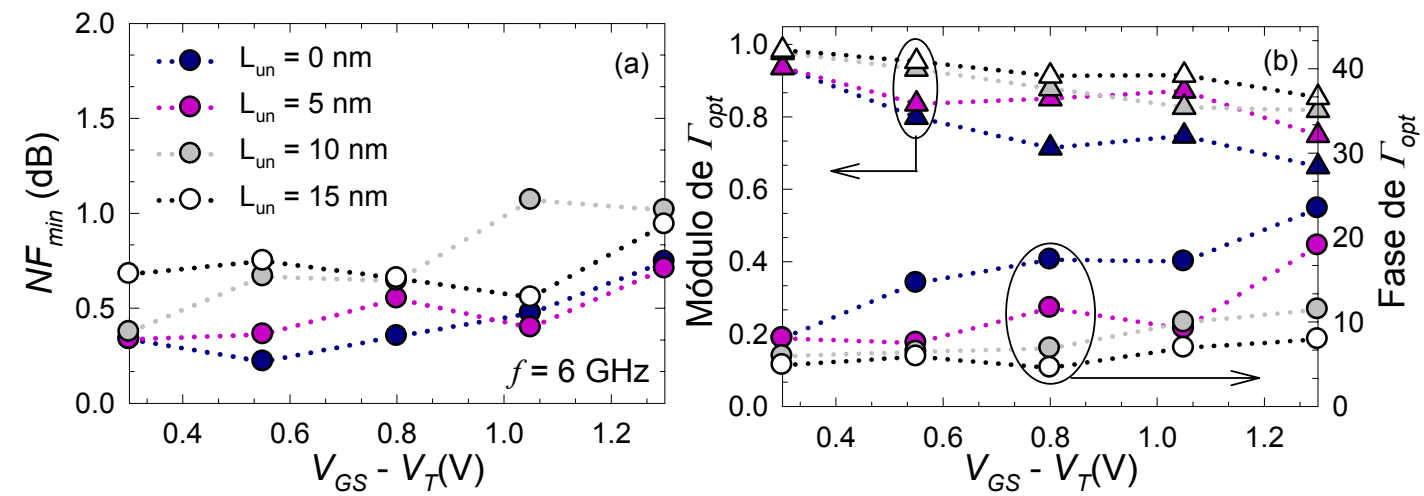

Figura VI.35.a. Figura minima de ruido $N F_{\min } y$.b. modulo y fase de $\Gamma_{\text {opt }}$ frente a $V_{G S}$ para $V_{D S}=2 \mathrm{~V}$. Mostramos los resultados para cuatro valores diferentes de longitud de underlap ( $L_{u n}=0 \mathrm{~nm}, 5 \mathrm{~nm}, 10 \mathrm{~nm}$ y $15 \mathrm{~nm}$ ). 
En la Figura VI.35 mostramos la figura mínima de ruido y el módulo y la fase de $\Gamma_{\text {opt }}$. Como podemos observar, $N F_{\min }$ disminuye a medida que reducimos $L_{u n}$. Evaluando la Ecuación VI.23 y teniendo en cuenta que las fuentes de ruido $S_{I G}$ y $S_{I D}$ aumentan al disminuir $L_{u n}$ (Figura VI.35), podemos afirmar que la contribución de los parámetros dinámicos da lugar a la mejora de la figura mínima de ruido cuando la longitud de underlap se reduce; recordemos que la frecuencia de corte es mayor a medida que $L_{u n}$ disminuye (Figura VI.14).

Como podemos observar en la Figura VI.35.b, el módulo de $\Gamma_{o p t}$ presenta una disminución y su fase un aumento a medida que la longitud de underlap es menor, indicando el mejor acoplamiento circuital de este dispositivo cuando reducimos $L_{u n}$.

A continuación, mediante la Figura VI.36, analizaremos la resistencia de ruido y la ganancia asociada del dispositivo. Respecto a la resistencia de ruido, sus valores son menores a medida que $L_{u n}$ disminuye. De este modo podemos afirmar que al disminuir la longitud de underlap el dispositivo presenta una menor sensibilidad de la figura mínima de ruido a cambios en la admitancia de entrada del dispositivo.
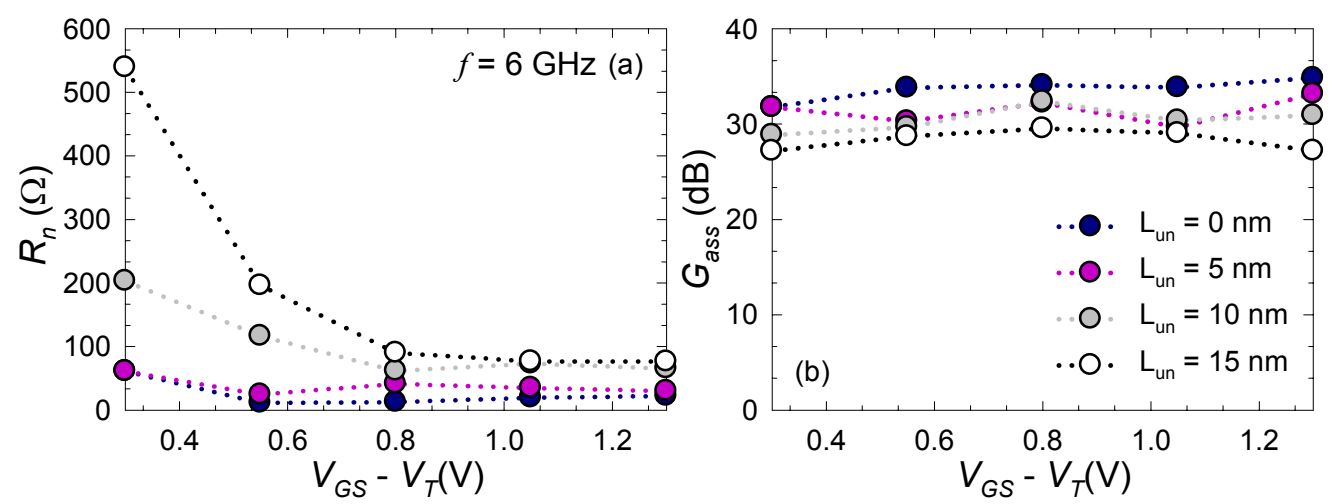

Figura VI.36.a. Resistencia de ruido y .b. ganancia asociada frente a $V_{G S}$ para $V_{D S}=2 \mathrm{~V}$. Mostramos los resultados para cuatro valores diferentes de longitud de underlap $\left(L_{u n}=0 \mathrm{~nm}, 5 \mathrm{~nm}, 10 \mathrm{~nm}\right.$ y $\left.15 \mathrm{~nm}\right)$.

En cuanto la ganancia asociada (Figura VI.36.b), ésta se ve aumentada ligeramente al disminuir $L_{u n}$, lo que denota una mayor capacidad de amplificación en condiciones de bajo ruido si reducimos este parámetro.

Podemos afirmar por tanto que la disminución de la longitud de underlap, además de dar lugar al aumento de la corriente de conducción, permite como ya comentamos la mejora de las figuras de mérito dinámicas. Por otro lado la figura mínima de ruido es mejor a medida que la longitud de underlap se reduce. Sin embargo, es importante tener en cuenta que la reducción 
de $L_{u n}$ es asumible hasta cierto punto (dependiendo de las demás características del dispositivo, como longitud de puerta, altura de la barrera, etc [Connelly, 2003]), ya que daría lugar al aumento de las capacidades parásitas entre los contactos, que no han sido consideradas en estos resultados, correspondientes al comportamiento intrínseco del dispositivo.

\section{VI.4.c Variación de la longitud de puerta}

Finalmente estudiamos la variación del ruido en el canal del transistor SB-MOSFET cuando reducimos la longitud del contacto de puerta, $L_{g}$. Nos centramos en primer lugar en los resultados de la simulación Monte Carlo para la densidad espectral de las fluctuaciones de la corriente de puerta y drenador, $S_{I G}$ y $S_{I D}$, en función del $V_{\text {overdrive }}$ para los cuatro valores de $L_{g}$, mostradas en la Figura VI.37. Como podemos observar, $S_{I G}$ presenta una notable reducción con el escalado del dispositivo, más importante a medida que $V_{\text {overdrive }}$ aumenta. Por otro lado, $S_{I D}$ presenta una disminución más suave, siendo prácticamente constante con $L_{g}$ a bajos voltajes aplicados.
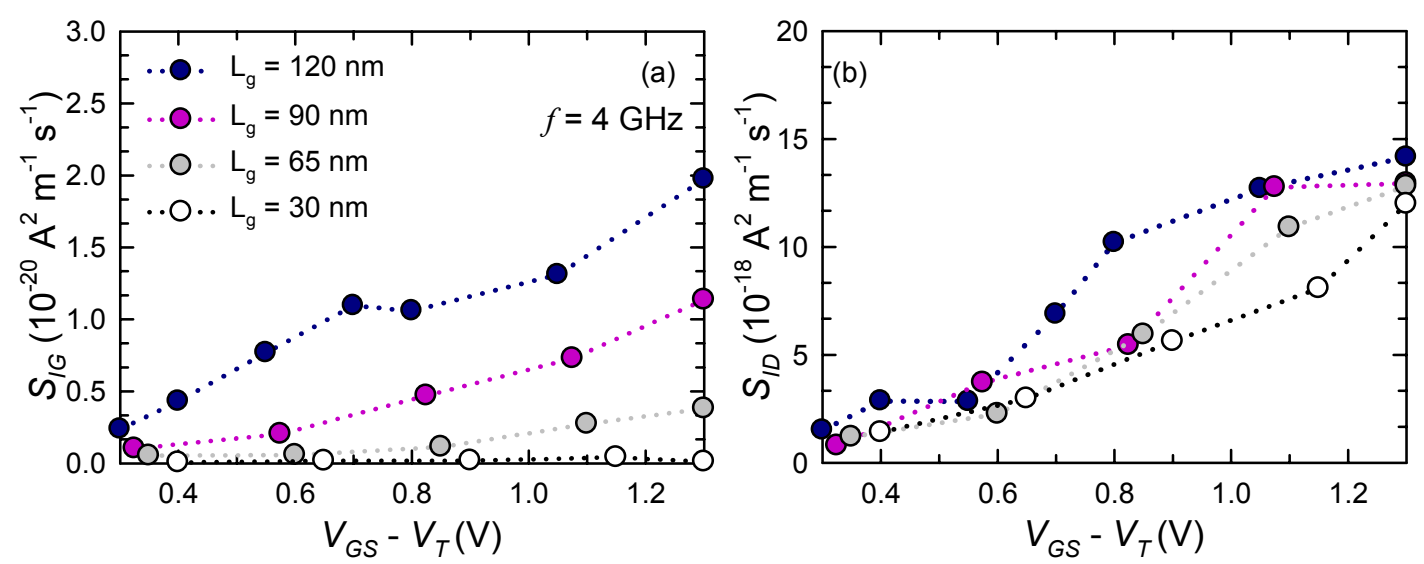

Figura VI.37.a. $S_{I G}$ y.b. $S_{I D}$ para los SB-MOSFETs simulados con cuatro longitudes de puerta $\left(L_{g}=120 \mathrm{~nm}, 90 \mathrm{~nm}\right.$, $65 \mathrm{~nm}$ y $30 \mathrm{~nm}$ ) para $V_{D S}=2 V$ y distintos valores de $V_{\text {overdrive. }}$

El descenso del valor de $S_{I G}$ con la longitud de puerta es debido a que hay un menor acoplamiento entre las fluctuaciones de corriente, lo cual es consistente con la disminución del valor de $C_{g s}$ previamente comentada (véase Figura VI.17) y con el hecho de que reducimos el tamaño de la puerta. Al evaluar el comportamiento de $S_{I D}$ con $L_{g}$ debemos tener en cuenta que el número de portadores inyectados se reduce muy significativamente al reducir la longitud de puerta (véase Figura V.39 en el Capítulo V). Como podemos observar, existe una reducción en 
el valor de $S_{I D}$ (si bien no es tan notable como la reducción del ruido en puerta) al disminuir el tamaño de los dispositivos.

En relación con la tecnología SOI-MOSFET convencional, el comportamiento de $S_{I G}$ al reducir el valor de $L_{g}$ es análogo al observado en [Martín, 2007]. Por otro lado, $S_{I D}$ aumenta al disminuir $L_{g}$ en el transistor convencional [Rengel, 2006], al contrario que en el SB-MOSFET, además de presentar valores muy superiores (por ejemplo, en el caso de $L_{g}=30 \mathrm{~nm}, S_{I D}=10^{-16}$ $\mathrm{A}^{2} \mathrm{~m}^{-1} \mathrm{~s}^{-1}$ para $\left.V_{\text {overdrive }}=1 \mathrm{~V}\right)$.

Los parámetros $P, R$ y $C$ (no mostrados en las gráficas) muestran una disminución a medida que $L_{g}$ es menor. En el caso de las longitudes de $90 \mathrm{~nm}, 65 \mathrm{~nm}$ y $30 \mathrm{~nm}, P$ alcanza valores máximos de $1.45,1.4$ y 1.21 respectivamente, mientras que en el transistor SOIMOSFET convencional su valor es de 2.8, 5.2 y 5.8 para $L_{g}=90 \mathrm{~nm}, 60 \mathrm{~nm}$ y $30 \mathrm{~nm}$ [Rengel, 2006], esto indica que el ruido en exceso aumenta progresivamente al reducir la longitud de puerta en un transistor convencional, mientras que disminuye en el caso del SB-MOSFET, fundamentalmente debido a la reducción de $S_{I D}$ previamente comentada.
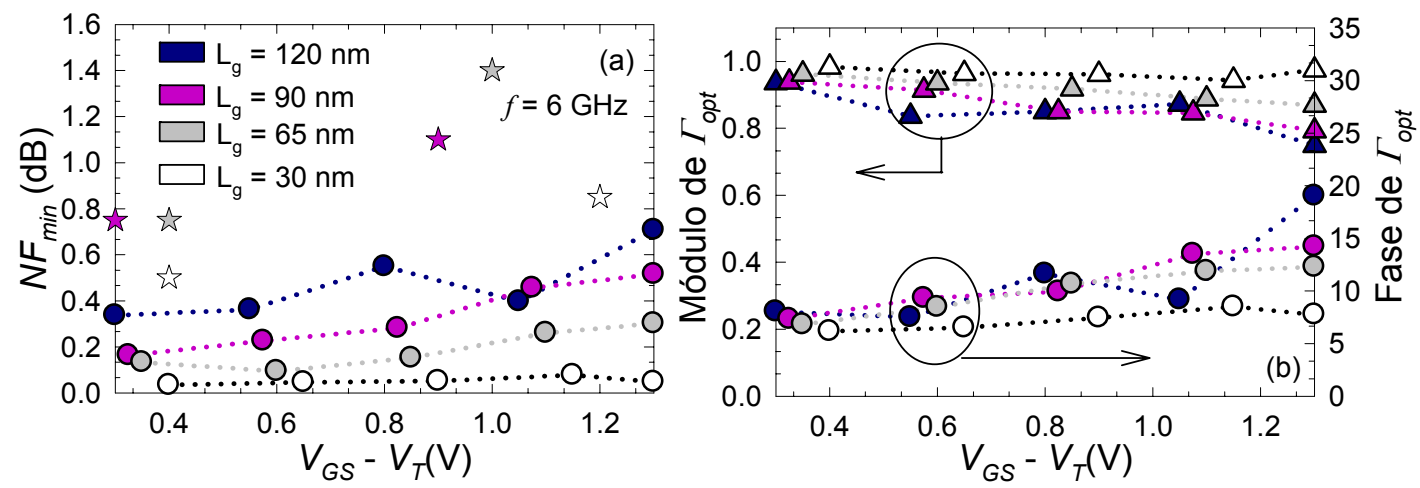

Figura VI.37.a. Figura mínima de ruido $N F_{\min } y$.b. modulo y fase de $\Gamma_{\text {opt }}$ frente a $V_{G S}$ para $V_{D S}=2$ V. Mostramos los resultados para cuatro valores diferentes de longitud puerta $\left(L_{g}=120 \mathrm{~nm}, 90 \mathrm{~nm}, 65 \mathrm{~nm}\right.$ y $\left.30 \mathrm{~nm}\right)$. Los simbolos en forma de estrella se corresponden con los resultados de $N F_{\text {min }}$ para un transistor SOI-MOSFET convencional de $90 \mathrm{~nm}$ (rosa), $60 \mathrm{~nm}$ (gris) y $30 \mathrm{~nm}$ (blanca) de longitud de puerta [Rengel, 2006].

Para concluir, vamos a evaluar la variación de la figura mínima de ruido y el módulo y la fase de $\Gamma_{\text {opt }}$ con la longitud de puerta (Figura VI.37). $N F_{\text {min }}$ presenta una clara reducción al escalar el dispositivo, del mismo modo que sucede en el transistor SOI-MOSFET convencional, cuyos valores máximo y mínimo mostramos en la Figura VI.37.a (símbolos en forma de estrella) para diversas longitudes de puerta ( $90 \mathrm{~nm}, 60 \mathrm{~nm}$ y $30 \mathrm{~nm}$ ) [Rengel, 2006]. Los valores de $N F_{\min }$ son significativamente inferiores a los obtenidos en el dispositivo convencional para la 
misma frecuencia, lo que confirma la idoneidad del transistor SB-MOSFET para ser usado en aplicaciones de alta frecuencia.

En la Figura VI.38.a mostramos los mejores valores de figura mínima de ruido publicados para MOSFETs convencionales de Si tipo $n$ en función de la frecuencia. De entre estos resultados destacamos los mínimos valores de $N F_{\text {min }}$ registrados son inferiores a $0.5 \mathrm{~dB}$ (para $f=12 \mathrm{GHz}$ ) en dispositivos de $90 \mathrm{~nm}$ de longitud de puerta [Chen, 2003], [Tiemeijer, 2004], [Schwierz, 2007] y menores a $1 \mathrm{~dB}$ (para $f=24 \mathrm{GHz}$ ) en dispositivos de longitud de puerta inferior de $46 \mathrm{~nm}$ [Lee, 2005], [Schwierz, 2007]. Como podemos observar, los valores de $N F_{\min }$ obtenidos mediante la simulación Monte Carlo para el SB-MOSFEt son coherentes con la tendencia mostrada en esta figura. Por otro lado, en la Figura VI.38.b representamos los objetivos marcados por el International Technology Roadmap for Semiconductors (ITRS); en el caso de los SB-MOSFETs estudiados en este trabajo, los resultados cumplirían esta predicción (por ejemplo, para el SB-MOSFET de $L_{g}=65 \mathrm{~nm}$ el valor de $N F_{\min }$ sería de $1.1 \mathrm{~dB}$ a $24 \mathrm{GHz}$ ).
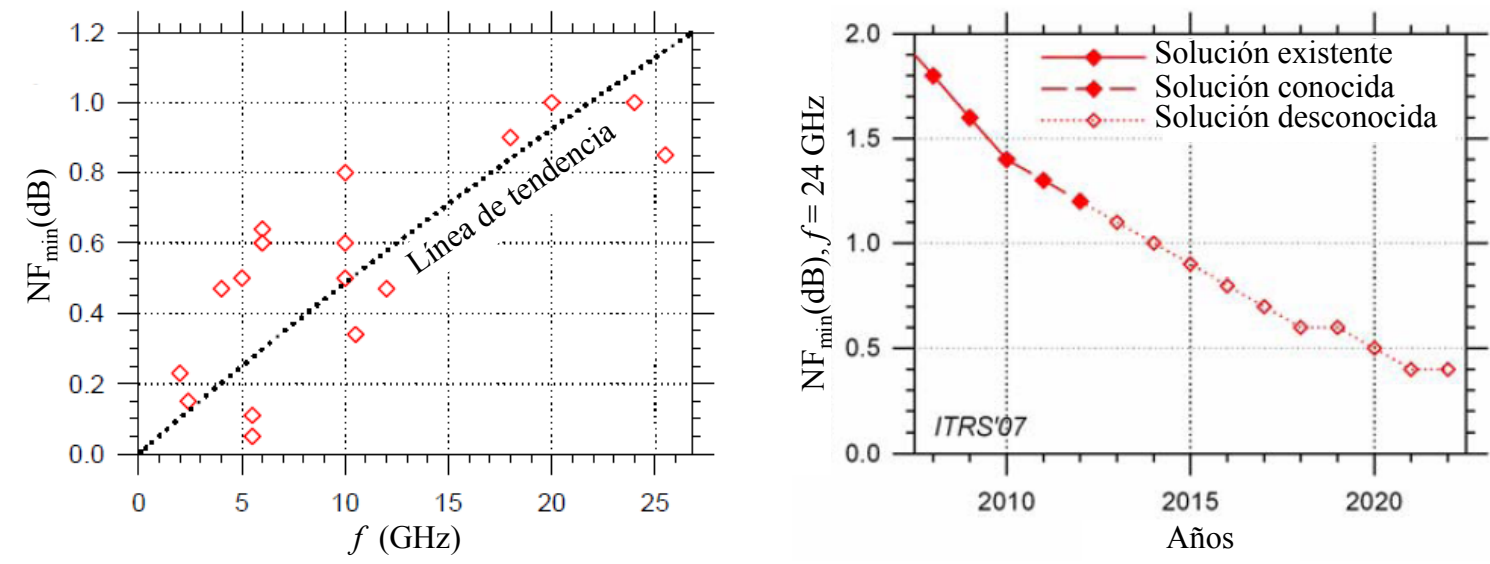

Figura VI.38.a. Figura mínima de ruido $N F_{\text {min }}$ de MOSFET de Si convencionales en función de la frecuencia (los resultados mostrados pertenecen a dispositivos con distintas longitudes de puerta: de $90 \mathrm{~nm}$ [Chen, 2003], [Tiemeijer, 2004], [Kao, 2007], de $65 \mathrm{~nm}$ [Dambrine, 2005], inferiores a $46 \mathrm{~nm}$ [Lee, 2005] y varios valores entre $30 \mathrm{~nm}$ y $90 \mathrm{~nm}$ [Schwierz, 2007] .b. $N F_{\min }$ para $f=24 \mathrm{GHz}$ en función de los años, tomado de la predicción del ITRS [ITRS, 2007]. La linea continua representa que la solución es conocida, mientras que la discontinua hace referencia a la situación contraria.

Con respecto al coeficiente de reflexión óptimo, $\Gamma_{\text {opt }}$, su módulo presenta un aumento y su fase una reducción a medida que la longitud de puerta es menor, del mismo modo que sucede en el transistor SOI-MOSFET convencional [Rengel, 2006], lo que indica que existiría una mayor dificultad para fabricar redes de adaptación a la entrada del transistor cuando reducimos la longitud de puerta. 
La ganancia asociada (no mostrada en las gráficas), no muestra una variación significativa con la longitud de puerta, indicando que la capacidad de amplificación del SB-MOSFET es prácticamente independiente de este parámetro.

Por último, en la Figura VI.39 representamos la resistencia de ruido del dispositivo para distintas longitudes de puerta. Como podemos ver, $R_{n}$ se ve reducida al escalar el dispositivo, pudiendo así afirmar que la reducción de $L_{g}$ tiene como consecuencia una menor sensibilidad de la figura mínima de ruido a cambios en la admitancia de entrada.

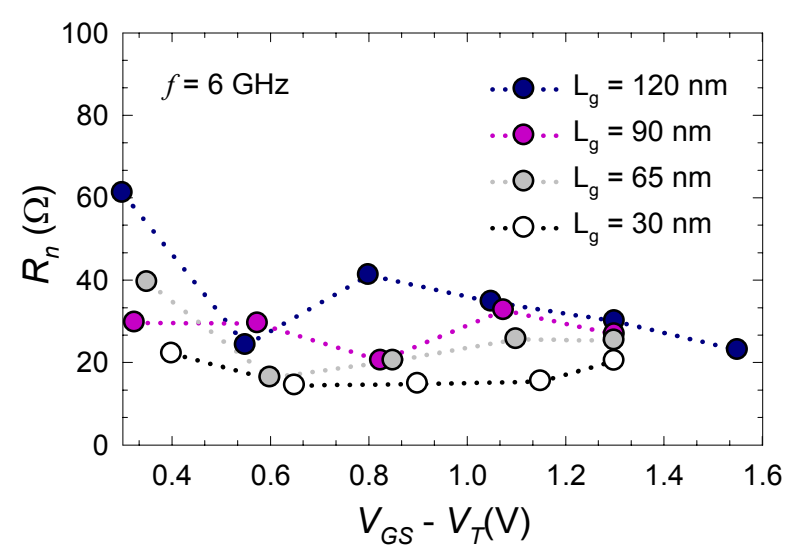

Figura VI.39.a. Resistencia de ruido frente a $V_{G S}$ para $V_{D S}=2$ V. Mostramos los resultados para cuatro valores diferentes de longitud de puerta $\left(L_{g}=120 \mathrm{~nm}, 90 \mathrm{~nm}, 65 \mathrm{~nm}\right.$ y $\left.30 \mathrm{~nm}\right)$.

En conclusión, aunque la disminución de la longitud de puerta no proporcione ventajas significativas desde el punto de vista de la corriente o de la transconductancia, encontramos numerosas ventajas desde el punto de vista del ruido electrónico a alta frecuencia, como es la disminución del valor de las fuentes de ruido $S_{I D}$ y $S_{I G}$ e inferiores valores de $N F_{\min }$. Si bien en los resultados mostrados debe tenerse en cuenta que no se ha efectuado un escalado riguroso, sino que únicamente se ha reducido el tamaño de la puerta, podemos concluir que los dispositivos SB-MOSFET presentan un notable comportamiento ante el escalado, con excelentes valores intrínsecos de los parámetros circuitales del ruido, que los convierten en una alternativa fiable para cumplir los requisitos a este respecto mencionados en el ITRS. 


\section{Bibliografía del Capítulo VI}

Barlage, D et al., "High-frequency response of $100 \mathrm{~nm}$ integrated CMOS transistors with highK gate dielectrics", Electron Devices Meeting, IEDM Technical Digest. International (2001)

Burghartz J. N. et al., "RF Potential of a $0.18 \mu \mathrm{m}$ CMOS Logic Device Technology", IEEE, (2000)

Chen C.L. et al., "High-performance fully-depleted SOI RF CMOS", IEEE Electron Device Letters, 23, 52 (2002)

Colinge J. P., "Fully-Depleted SOI CMOS for Analog Applications", IEEE Trans. Electron Dev. 451010 (1998)

Connelly D., Faulkner C., Grupp D. E., "Performance advantage of Schottky source/ drain in ultrathin-body silicon-oninsulator and dual-gate CMOS", IEEE Trans. Electron Dev., 50, $1340(2003)$

CSM, Compound Semiconductor Magazine, 31, January/February (2002)

Dambrine G., Raskin J.-P. et al. "High-Frequency Four Noise Parameters of Silicon-onInsulator-Based Technology MOSFET for the Design of Low-Noise RF Integrated Circuits", IEEE Trans. Electron Dev., 46, 1733 (1999)

Dambrine G. et al., "High frequency low noise potentialities of down to $65 \mathrm{~nm}$ technology nodes MOSFETs", Proc. of 13th European Gallium Arsenide \& Other Compound Semiconductors Application Symposium (GaAs), 03 (2005)

Danneville F., Happy H., Dambrine G., Belquin J. M. y Cappy A., "Microscopic noise modelling and macroscopic noise models: how good a connection?", IEEE Trans. Electron Dev., 41, 779 (1994)

Dawson R. H., "Equivalent Circuit of the Schottky-Barrier Field-Effect Transistor at Microwave Frequencies", IEEE Transactions on microwave theory and techniques, 499 (1975) 
Deen J. M. and Marinov O., "Noise in advanced electronic device and circuits", ICNF: Int. Conf. Noise and Fluctuations, 3 (2005)

Ferlet-Cavrois V. et al., "High-Frequency Performances of a Partially Depleted $0.18-\mu \mathrm{m}$ SOI/CMOS Technology at Low Supply Voltage-Influence of Parasitic Elements", IEEE Electron Device Letters, 19 (1998)

Flandre D., Adriaensen S. et al., "Fully depleted SOI CMOS technology for heterogeneous micropower, high-temperature of RF microsystems", Solid-State Electron., 45, 541 (2001)

Fritze M. et al., "High-Speed Schottky-Barrier pMOSFET With fT=280GHz", IEEE Electron Devices Letters, 25, 220 (2004)

González G., "Microwave Transistor Amplifiers Analysis and Design", 2nd edition Upper Saddle River: Prentice Hall (1997)

González T., "Análisis del Ruido Electrónico en Materiales y Dispositivos Semiconductores Unipolares mediante el Método de Monte Carlo", Tesis Doctoral, Universidad de Salamanca (1994)

González T., Pardo D., Varani L. y Reggiani L., "Monte Carlo Analysis of the Behaviour and Spatial Origin of Electronic Noise in GaAs MESFET's", IEEE Trans. Electron Dev. 42 991 (1995)

Hisayo Sasaki Momose, et al., "110 GHz cutoff frequency of ultra-thin gate oxide p-MOSFETs on (110) surface-oriented Si substrate", Toshiba Corporation, IEEE (2002)

ITRS "The International Technology Roadmap for Semiconductors (ITRS), Semiconductor Industry Association", Edition, Austin, TX, International Sematech (2007) (disponible en http://public.ITRS.net)

Kao H. et al., "Very Low Noise in 90nm Node RF MOSFETs using a New Layout", 2007 Topical Meeting on Silicon Monolithic Integrated Circuits in RF Systems, 44 (2007)

Kuivalainen P., Tarvainen E., Ronkainen H., Majamaa T. and Hovinen A., "Model for the Cutoff Frequency in RF MOSFETs", Physica Scripta. T69, 193 (1997)

Laux S. E., "Techniques for small-signal analysis of semiconductor devices", IEEE Trans. Electron Dev., 32, 2028 (1985)

Lee S. and $\mathrm{Yu} \mathrm{H.} \mathrm{K.,} \mathrm{"Determining} \mathrm{non-quasi-static} \mathrm{small-signal} \mathrm{equivalent} \mathrm{circuit} \mathrm{of} \mathrm{a} \mathrm{RF}$ silicon MOSFET" Solid-State Electron., 45, 359 (2001)

Lee $\mathrm{S}$, et al., "Record RF performance of sub-46 nm Lgate NFETs in microprocessor SOI CMOS technologies". IEDM Tech Dig., 10.7. (2005) 
Manku T., "Microwave CMOS - Device Physics and Design", IEEE J. Solid-State Circuits, 34, 277 (1999)

Martín M. J., “Análisis del Transporte de Carga y de los Fenómenos de Ruido Electrónico en Estructuras Si/Si1-xGex bipolares", Tesis Doctoral, Universidad de Salamanca (1996)

Mateos J. et al., "Noise analysis of $0.1 \mu \mathrm{m}$ gate MESFETs and HEMTs", Solid-State Electron., 42, 79 (1998)

Matsumoto T. et al., "70 nm SOI CMOS of $135 \mathrm{GHz}$ fmax with dual offset-implanted sourcedrain extension structure for RF/analog and logic applications", IEDM'01 Technical Digest, 219 (2001)

McQuarrie D. A., "Statistical Mechanics", New York: Harper and Row (1976)

Momose H. S. et al., "Cutoff frequency and propagation delay time of 1.5-nm gate oxide CMOS", IEEE Transaction on Electron Devices, 48, 6, 1165 (2001)

Narashima S. et al., "High performance sub-40 nm CMOS devices on SOI for the $70 \mathrm{~nm}$ technology node", IEDM'01 Technical Digest, 625 (2001)

Nyquist H., "Thermal agitation of electric charge in conductors", Phys. Rev., 32, 110 (1928)

Post I. et al., "A 65nm CMOS SOC Technology Featuring Strained Silicon Transistors for RF Applications", Proceeding of IEDM, (2006)

Pailloncy G. et al., "Noise modeling in fully depleted SOI MOSFETs", Solid-State Electron, 48, $813(2004)$

Pucel R. A., Haus H. A. y Statz H., "Signal and noise properties of gallium arsenide field effect transistors", Advances in Electronics and Electron Physics 38195 (1974)

Raskin J. -P., Guillon R., Chen J., Vanhoenacker-Janvier D. y Colinge J.-P., “Accurate SOI MOSFET Characterization at Microwave Frequencies for Device Performance Optimization and Analog Modeling”, IEEE Trans. Electron Dev. 451017 (1998)

Rengel R., Mateos J., Pardo D., González T. and Martín M. J., "Monte Carlo analysis of dynamic and noise performance of submicron MOSFETs at RF and microwave frequencies", Semicond. Sci. Technol., 16, 939 (2001)

Rengel R., "Análisis de los Fenómenos de Trasporte y Ruido Electrónico en Transistores MOSFET y SOI Submicrométricos”, Tesis Doctoral, Universidad de Salamanca (2002)

Rengel R. et al., "Numerical and experimental study of a $0.25 \mu \mathrm{m}$ fully-depleted silicononinsulator MOSFET: static and dynamic radio-frequency behaviour", Semicond. Sci. Technol., 17, 1149 (2002b) 
Rengel R., Pardo D., and Martín M. J., "A physically based investigation of the small-signal behaviour of bulk and fully-depleted silicon-on-insulator MOSFETs for microwave applications", Semicond. Sci. Technol., 19, 634 (2004)

Rengel R., Pardo D., and Martín M. J., "Towards the nanoscale: influence of scaling on the electronic transport and small-signal behaviour of MOSFETs", Nanotechnology 15, S276 (2004b)

Rengel R., Martín M. J., Dambrine G. and Danneville F., "A Monte Carlo investigation of the RF performance of partially-depleted SOI MOSFETs", Semicond. Sci. Technol., 21, 273, (2006)

Rengel R. et al., "A Microscopic Interpretation of the RF Noise Performance of Fabricated FDSOI MOSFETs", IEEE Trans. Electron Devices, 53, (2006b)

Saha A. R., Chattopadhyay S., Bose C., Maiti C. K., "Technology CAD of silicided Schottky barrier MOSFET for elevated source-drain engineering”, Materials Science and Engineering B, 124-125, 424 (2005)

Schwierz F. and Schippel C., "Performance trends of Si-based RF transistors", Microelectronics Reliability, 47, 384 (2007)

Sungjae Lee et al., "Record RF Performance of Sub-46 nm Lgate NFETs in Microprocessor SOI CMOS Technologies", Proceeding of IEDM, (2005)

Taur Y. et al., "High performance $0.1 \mu \mathrm{m}$ CMOS devices with $1.5 \mathrm{~V}$ power supply," IEDM Tech. Dig., 127 (1993)

Tiemeijer L. F. et al., "A record high $150 \mathrm{GHz}$ fmax realized at $0.18 \mu \mathrm{m}$ gate length in an industrial RFCMOS technology", IEDM'01 Technical Digest, 223 (2001)

Tomizawa K., Numerical Simulation of Submicron Semiconductor Devices (Boston: Artech House, 1993)

Trippe M., Bosman G. y van der Ziel A., "Transit-time effects in the noise of Schottky-barrier diodes", IEEE Trans. Microwave Theory Tech. 341183 (1986)

Tsividis Y.,"Operation and modelling of the MOS Transistor", $2^{\text {nd }}$ edition Boston: McGraw-Hill (1999)

Valentin R., Dubois E. et al., "RF small-signal analysis of Schottky-barrier p-MOSFET" IEEE Trans. Electron Dev., 55, 1192 (2008)

Valentin R., "Investigation du Comportement et des Potentialités en Haute Fréquence de transistors MOSFET Avancés à Contacts Source/Drain Métalliques" Tesis Doctoral, (2008b) 
van der Ziel A., "Noise in Solid State Devices and Circuits", New York: Wiley (1986)

Vanmackelberg M. et al., "90 nm SOI-CMOS of $150 \mathrm{GHz}$ fmax and 0.8 dB NFmin @ $6 \mathrm{GHz}$ for SOC", IEEE International SOI Conference, Wiliamsburg, VA, 7 (2002)

Vendelin G. D., Pavio A. M. Y Rohde U. L., "Microwave Circuit Design Using Linear and Nonlinear Techniques", New York: Wiley (1990)

Wong H., Man K. F. y Poon M. C., "Modeling of saturation transconductance for short-channel MOSFETs", Solid-State Electronics, 39, 1401 (1996)

Xia Z., Du G. et al., "Investigation of RF performance of nano-scale ultra-thin-body Schottkybarrier MOSFETs using Monte Carlo simulation”, IEEE Conference on Electron devices and Solid-State circuits, 305 (2005)

Yeh S. -P, Shih C. -H., Gong J. and Lien C., "Latent noise in Schottky barrier MOSFETs", Journal of Statistical Mechanics, P01036 (2009)

Zamdmer N. et al., "A $0.13-\mu \mathrm{m}$ SOI CMOS Technology for low-power Digital and RF Applications”, 2001 Symposium on VLSI Technology Digest, 85 (2001)

Zamdmer N. et al., "Suitability of scaled SOI CMOS for high-frequency analog circuits", ESSDERC, Proceedings of the $32^{\text {nd }}$ European Solid State Device Research Conference, $511(2002)$

Zamdmer, N. et al., "243-GHz $f_{t}$ and 208-GHz $f_{\max }$ 90-nm SOI CMOS SoC technology with lowpower millimeter-wave digital and RF circuit capability", Symposium on VLSI Technology, Digest of Technical Papers, 15, 98 (2004) 


\section{Conclusiones}

1 principal objetivo de este trabajo ha sido el estudio de los principios de
operación y del rendimiento para aplicaciones de alta frecuencia de los transistores MOSFET de barrera Schottky, una estructura alternativa al MOSFET de Silicio convencional. Esta tarea ha sido desarrollado a través de una herramienta de simulación Monte Carlo; para considerar las distintas componentes que contribuyen a la corriente total a través del contacto Schottky, hemos implementado un modelo de inyección/absorción que incorpora el transporte por túnel cuántico a través de barreras de potencial. Tal y como se estableció en la introducción, como paso previo al análisis del transporte en los transistores SB-MOSFET, hemos estudiado el transporte a través de la barrera Schottky en estructuras más sencillas, como son los diodos Schottky y los diodos back-to-back. A continuación desgranamos las principales conclusiones que se extraen de los resultados obtenidos. 


\section{Diodos de barrera Schottky}

Hemos llevado a cabo la simulación de diversas estructuras con contacto SilicioSiliciuro con alturas de barrera elevadas (de $0.50 \mathrm{eV}$ a $0.90 \mathrm{eV}$ para estructuras tipo $\mathrm{n}$ y $0.33 \mathrm{eV}$ para tipo p), lo que suponen las condiciones más exigentes desde el punto de vista de la simulación Monte Carlo. Para ello, empleamos inicialmente un simulador unidimensional que incluye un algoritmo de expansión-compresión para un correcto modelado de la zona de vaciamiento. Hemos comprobado que existe un excelente acuerdo de la característica corrientetensión obtenida mediante el simulador Monte Carlo y modelos teóricos sencillos evaluados en un primer momento, así como con las medidas experimentales disponibles. De esta manera verificamos que el modelo de inyección/absorción implementado es adecuado para el estudio de estos dispositivos. Del análisis de las magnitudes que nos proporciona el método de Monte Carlo extraemos las siguientes conclusiones:

Hemos analizado de manera breve el comportamiento de los diodos tipo $n$ con contacto de $\mathrm{ZrSi}_{2}$ (barrera de $0.55 \mathrm{eV}$ ) en el régimen de polarización directa. Destacamos que para voltajes superiores a $0.2 \mathrm{~V}$ la influencia de las componentes de corriente de inyección es despreciable frente a las de absorción. Además, para voltajes superiores al de banda plana las componentes de inyección y absorción túnel desaparecen al modificarse el perfil de la barrera. Comprobamos en esta región de polarización en directa que los resultados Monte Carlo concuerdan con el comportamiento predicho por los modelos teóricos.

El análisis del transporte de carga ha sido evaluado en mayor profundidad en el régimen de polarización inversa, ya que en esta región se favorece la inyección túnel. A medida que la tensión en inversa aumenta, la forma que adquiere la barrera de potencial favorece la inyección por efecto túnel, mientras que la corriente de inyección termoiónica permanece constante. Por otro lado, las componentes de absorción (termoiónica y túnel) disminuyen. Se ha comprobado además el carácter cuasibalístico de los portadores en la zona próxima al contacto.

De la comparación de la característica corriente-tensión considerando y sin considerar los fenómenos de túnel cuántico, se ha probado que dichos efectos son imprescindibles para reproducir las medidas experimentales, sobre todo a medida que disminuimos la altura de la barrera, ya que la corriente de inyección túnel se ve favorecida aportando la mayor contribución a la corriente total. 
Hemos considerado además el efecto de las cargas imagen, donde la reducción de la altura de barrera (mayor a medida que aumenta la tensión inversa aplicada) provoca el aumento de la componente de inyección termoiónica, que deja de ser constante con la tensión aplicada.

D Del análisis de los diodos con diferentes alturas de barrera podemos concluir que la densidad de corriente total aumenta para cada polarización aplicada a medida que la altura de la barrera es menor.

$>$ La corriente total también se ve favorecida por el aumento de la temperatura de operación. Sin embargo, examinando las distintas componentes, observamos que la inyección túnel aumenta al disminuir la temperatura proporcionalmente. La influencia de este parámetro sobre la densidad de corriente ha sido evaluada de manera más detallada a través del diagrama de Arrhenius. En el caso de los diodos tipo $n$ analizados, puesto que la altura de la barrera considerada es elevada, el modelo teórico presentado está en perfecto acuerdo con los resultados Monte Carlo. Sin embargo, para los diodos tipo $p$ estudiados, con barrera Schottky de altura inferior y más estrecha (por el mayor dopaje del substrato) que en los tipo $n$, aparecen grandes discrepancias en el diagrama de Arrhenius entre los valores Monte Carlo y el modelo teórico simple. Esto se debe a que en el modelo teórico no se tiene en cuenta la componente de inyección túnel, que presenta una mayor contribución en la región de bajas temperaturas. Sin embargo, para altas temperaturas observamos la aparición de un máximo asociado a la caída brusca de las componentes túnel (de inyección y absorción).

\section{Diodos back-to-back}

Este tipo de estructuras, constituidas por dos contactos Schottky situados sobre un sustrato de Silicio, presentan una configuración del transporte en corriente muy cercana a la del SB-MOSFET (con excepción de la ausencia del terminal de puerta). Su interés radica en la caracterización, desde el punto de vista experimental, de contactos con alturas de barrera reducidas. En particular dentro del Proyecto Europeo METAMOS, este tipo de estructuras han sido fundamentales para poder explorar nuevos materiales de contacto y técnicas de fabricación con el fin de realizar contactos de altura de barrera reducida en transistores MOSFET de barrera 
Schottky. Las principales conclusiones obtenidas acerca del transporte en los diodos back-toback son las siguientes.

El comportamiento global de estos dispositivos puede describirse de manera simplificada mediante la combinación del efecto de dos resistencias, en serie, una asociada al substrato de Silicio y otra asociada a la región de vaciamiento del contacto Schottky polarizado en inversa.

Cuando la altura de la barrera Schottky disminuye y aumenta la temperatura, la resistencia del contacto Schottky tiene una menor influencia en el comportamiento en corriente del dispositivo, imponiéndose así la resistencia del sustrato de Silicio.

$>\quad$ A partir del estudio de la función de distribución de velocidad hemos demostrado que el transporte de portadores es de tipo balístico junto a la interfaz Schottky. Este efecto es más pronunciado a medida que la temperatura disminuye, como consecuencia del mayor campo eléctrico y mayor velocidad de los portadores a bajas temperaturas, así como por el menor número de mecanismos de scattering.

Es importante destacar el excelente acuerdo de los resultados de la simulación Monte Carlo con medidas experimentales proporcionadas por nuestros colegas dentro del Proyecto Europeo METAMOS.

Hemos estudiado también el efecto de la inclusión de una capa de segregación de dopantes adyacente al contacto Schottky. El interés de esta técnica se debe a que según algunos autores supone un método adecuado para reducir el valor de la altura efectiva de la barrera Schottky y por tanto permitir la posibilidad de obtener contactos con muy bajo valor de barrera, imposibles de obtener mediante Siliciuros convencionales. Mediante nuestro estudio Monte Carlo podemos extraer las principales conclusiones de la inclusión de una capa de segregación de dopantes:

El efecto sobre la corriente total es equivalente a una reducción de la altura de barrera Schottky efectiva.

$>\quad$ La banda de conducción presenta una fuerte curvatura junto a la interfaz, haciéndose prácticamente transparente para la corriente túnel.

Aunque los portadores en contactos sin capa de dopantes presentan características balísticas en una región más amplia que en contactos con capa, los electrones en este segundo caso son más rápidos en las cercanías del contacto debido al elevado campo eléctrico originado en la región más dopada. 
En general, se puede concluir que la segregación de dopantes es una técnica adecuada para aumentar la corriente de inyección, promover el trasporte balístico y conseguir barreras efectivas más bajas en estructuras que así lo requieran, como es el caso del SB-MOSFET.

\section{Transistores SB-MOSFET}

El estudio de los diodos de barrera Schottky y de las estructuras back-to-back nos ha proporcionado las herramientas necesarias para proceder al estudio de transistores MOSFET de barrera Schottky en acumulación. En primer lugar, nos centramos en el análisis del comportamiento de los dispositivos, sus principios físicos de operación, sus características estáticas y las magnitudes internas proporcionadas por el simulador.

En el estado de corte $\left(V_{G S}<V_{T}\right)$, aparece una barrera de potencial adicional a la barrera Schottky, impidiendo que los portadores sean inyectados en el interior del dispositivo. Cuando $V_{G S}$ aumenta, dicha barrera disminuye hasta desaparecer, teniendo lugar así el paso de corte a conducción.

$>\quad$ En el régimen de triodo la concentración de portadores se encuentra fuertemente localizada justo debajo la puerta, mientras que en saturación la concentración en acumulación aún siendo mayor debajo de la puerta, se encuentra también distribuida de forma significativa en el resto del canal.

En las proximidades del contacto de drenador, la mayor diferencia con respecto a la banda de conducción entre los regímenes de triodo y saturación es la modificación en su curvatura, en triodo permitiendo la emisión de campo (siendo la absorción túnel la componente de corriente más importante) y prohibiéndolo en saturación (predominando la corriente de absorción termoiónica sobre las demás). Debe tenerse en cuenta que, tanto en triodo como en saturación, la corriente de inyección túnel es la mayor contribución a la corriente total en el contacto de fuente.

A partir del estudio de las propiedades del transporte microscópico (del tiempo de tránsito medio, número de mecanismos de scattering y recorrido libre medio) hemos comprobado que el transporte en este dispositivo es fundamentalmente de tipo difusivo. Además, podemos afirmar que, junto con una elección de dopaje bajo del canal, la arquitectura SB-MOS mejora la movilidad de los portadores en el interior de los dispositivos. 
Hemos llevado el estudio de la optimización el rendimiento del SB-MOSFET mediante la variación de diversos parámetros característicos de su arquitectura, como son la altura de la barrera Schottky y la longitud de underlap, además del estudio del efecto de la longitud del contacto de puerta. Podemos destacar las siguientes conclusiones:

○ Al disminuir la altura de barrera Schottky, $q \Phi_{B n}$, se obtiene un notable aumento de la corriente total del dispositivo, disminuyendo la importancia relativa de la inyección túnel. El número de mecanismos de scattering y el tiempo de tránsito también es menor, lo que indica que obtendremos una mejor respuesta dinámica. En general, ésta sería una buena opción para mejorar el rendimiento del dispositivo (idealmente debería tenderse a barreras de altura inferior a $0.1 \mathrm{eV}$, incluso de $0 \mathrm{eV}$ ), pero actualmente existen importantes dificultades técnicas para lograr una reducción tan drástica de la altura de la barrera.

○ Si se reduce la longitud de underlap, $L_{u n}$, la corriente total del dispositivo aumenta, viéndose favorecidas las componentes de corriente de inyección túnel y termoiónica. Se obtiene así un mejor control electrostático del potencial en la interfaz por parte de $V_{G S}$, aumentado el rendimiento del transistor. Sin embargo, una drástica reducción de $L_{u n}$ implica un aumento de las capacidades parásitas. Por tanto debe buscarse un compromiso entre la reducción de $L_{u n} \mathrm{y}$ $q \Phi_{B n}$ para mejorar el rendimiento del dispositivo, tanto desde el punto de vista de corriente como de la respuesta dinámica teniendo en cuenta las limitaciones tecnológicas existentes.

○ La reducción de la longitud de puerta, $L_{g}$, produce un efecto muy débil sobre la corriente total, permaneciendo los niveles de inyección túnel y termoiónicos aproximadamente constantes; sólo se aprecia un ligero aumento de la corriente total para el inferior valor de $L_{g}$ considerado en este estudio $(30 \mathrm{~nm})$. Observamos que el número de mecanismos de scattering y tiempo de tránsito es más elevado que en un MOSFET convencional. Sin embargo, el coeficiente de balisticidad para longitud de puerta de $30 \mathrm{~nm}$ es comparable al obtenido en un MOSFET convencional. Podemos concluir que en el caso del SB-MOSFET, la reducción de la longitud de puerta puede llevarse a cabo por motivos de empaquetamiento, aunque no aporta una gran mejora respecto a la corriente $I_{o n}$ del dispositivo.

En cuanto a los parámetros dinámicos, hemos evaluado la dependencia de los mismos con la altura de la barrera Schottky, la longitud de underlap y la longitud de puerta, pudiéndose extraer de este estudio las siguientes conclusiones: 
o Los transistores SB-MOSFET presentan en general excelentes valores tanto de transconductancia, $g_{m}$, como de frecuencia de corte de la ganancia en corriente, $f_{t}$, equiparables e incluso superiores a los de la tecnología MOSFET convencional.

- Al disminuir la altura de barrera Schottky, $q \Phi_{B n}$, se obtiene un importante incremento de la transconductancia y de la frecuencia de corte, así como de la capacidad $C_{g s}$. Esto indica que el electrodo de puerta ejerce un mayor control sobre la corriente de salida cuanto menor sea la altura de barrera del contacto Schottky. La disminución de la resistencia de carga del canal (en el rango de elevados voltajes en puerta aplicados) así como del retardo de la transconductancia nos indican la mayor rapidez de la puerta en controlar la carga al disminuir $q \Phi_{B n}$.

○ Reducir la longitud de underlap, $L_{u n}$, es otra posibilidad para mejorar notablemente las figuras de mérito dinámicas anteriormente mencionadas. La mejora en la transconductancia tiene lugar fundamentalmente por una mayor eficiencia en la inyección de carga en el canal al variar la tensión de puerta.

o Con la disminución de la longitud de puerta, $g_{m}$ presenta un ligero aumento, mientras que la frecuencia de corte aumenta de forma más apreciable. Estos valores de $f_{t}$ son similares a los obtenidos para un transistor SOI-MOSFET convencional. Al reducir la longitud de puerta la respuesta del dispositivo ante variaciones del voltaje puerta-fuente es más rapida, como evidencia la reducción de la resistencia de carga del canal y del retardo de transconductancia. La leve dependencia de $I_{D}$ y $g_{m}$ con este parámetro es debida a las características del escalado considerados en esta Memoria (reducción de $L_{g}$ únicamente, sin tener en cuenta otros parámetros como $t_{S i} \mathrm{o} t_{o x}$.).

Finalmente, llevamos a cabo el estudio del fenómeno del ruido electrónico a alta frecuencia. Del análisis de la densidad espectral de las fluctuaciones de corriente en drenador, $S_{I D}$, en función de la frecuencia hemos podido determinar la influencia de tres fenómenos, las oscilaciones plasmáticas, el efecto de los portadores que se dan la vuelta al aproximarse al contacto de drenador (returning carriers) y los fenómenos de inyección túnel a través del contacto de fuente. Destacamos que existe una elevada influencia de los returning carriers y de la inyección túnel en la dependencia de $S_{I D}$ con $V_{D S}$; por otro lado, es importante destacar que esta influencia está relacionada directamente con el aumento del nivel de corriente, que da lugar a un mayor valor de $S_{I D}$. 
○ La reducción de la altura de barrera Schottky, $q \Phi_{B n}$, produce un aumento de $S_{I G}$ (por un mayor acople capacitivo entre el contacto de puerta y fuente) y $S_{I D}$ (debido principalmente al aumento de la corriente de drenador y de la emisión de campo). En cuanto a los parámetros de ruido normalizados, $P$ y $R$ aumentan, mientras que $C$ permanece prácticamente invariante; estos parámetros presentan valores similares a los de un SOI-MOSFET convencional. La figura mínima de ruido, $N F_{\min }$, no varía a bajos voltajes, viéndose ligeramente incrementada con la reducción de $q \Phi_{B n}$ a elevados voltajes en puerta aplicados. Se obtienen excelentes valores de figura mínima de ruido (para $q \Phi_{B n}=0.15 \mathrm{eV}$ oscila entre $0.3 \mathrm{~dB}$ y $0.9 \mathrm{~dB}$ en el rango de voltajes estudiados), significativamente inferiores a los del dispositivo convencional. $R_{n}$ y $G_{a s s}$ apenas varían, lo que indica que la capacidad de amplificación de este dispositivo en condiciones de mínimo nivel de ruido no se ve afectada intrínsecamente por la variación de $q \Phi_{B n}$.

- Cuando disminuimos la longitud de underlap, $L_{u n}, S_{I G}$ varía ligeramente (influido por el valor constante de $L_{g}$ ) y $S_{I D}$ aumenta (por el aumento de $I_{D}$ y del mayor nivel de inyección túnel). Por otro lado, $N F_{\min }$ presenta una mejora debido fundamentalmente al mejor comportamiento dinámico al reducir $L_{u n}$, mientras que $R_{n}$ disminuye y $G_{a s s}$ aumenta.

○ El efecto de reducir la longitud de puerta, $L_{g}$, da lugar a una reducción de $S_{I G}$ (debido a la menor influencia de los mecanismos de scattering bajo la puerta: las fluctuaciones de corriente inducidas en la puerta por la carga en el canal son menos importantes) y $S_{I D}$ (el ruido asociado a la emisión de campo permanece prácticamente constante cuando se reduce $L_{g}$; por otro lado, el ruido difusivo inherente al movimiento de portadores en el canal es muy similar, incluso disminuye a voltajes elevados). Es importante destacar que el comportamiento de estas magnitudes es opuesto al del SOI-MOSFET convencional, presentando además valores inferiores en el caso del SB-MOSFET. Se obtiene una mejora de $N F_{\min }$, con valores muy inferiores a los presentados por el SOI-MOSFET convencional. También existe una disminución del valor de $R_{n}$. Todos estos indicadores nos demuestran las grandes posibilidades de este tipo de dispositivos para ser escalados en comparación con el transistor convencional debido a la disminución del nivel de ruido al escalar $L_{g}$.

Como conclusión final, podemos decir que el presente trabajo ha puesto de manifiesto la idoneidad del método de Monte Carlo para el estudio de uniones Siliciuro-semiconductor de tipo Schottky y dispositivos SB-MOSFET, permitiendo la mejor comprensión de la física del transporte de carga a través de la barrera Schottky y reproduciendo con exactitud las medidas 
experimentales disponibles. Además, en el caso del transistor SB-MOSFET se ha efectuado un análisis exhaustivo del comportamiento estático junto con una investigación de las propiedades dinámicas y de los fenómenos de ruido a alta frecuencia, pudiendo así facilitar las labores de optimización del rendimiento de los dispositivos. A la vista de los resultados presentados, podemos confirmar que la tecnología SB-MOSFET presenta un enorme potencial para reemplazar a los transistores MOSFET convencionales a corto y medio plazo. 



\section{Apéndice. Modelado del material de Silicio. Parámetros de la simulación}

$\mathcal{E}_{\text {física del Silicio material considerada en nuestra simulación. En la Sección A.1, }}^{\text {neste Apéndice, vamos a presentar los principales detalles de la descripción }}$ mostraremos las características de la estructura de bandas (tanto de conducción como de valencia) que ha sido tenida en cuenta. En la Sección A.2, detallaremos los mecanismos de scattering considerados en la simulación, junto con los principales parámetros asociados. Finalmente, en la Sección A.3, presentaremos los resultados obtenidos con el simulador Monte Carlo para algunas magnitudes de interés, tales como velocidad de los portadores, movilidad, coeficiente de difusión, etc... junto a su comparación con datos experimentales, que permiten confirmar la validez del simulador a este nivel.

\section{A.1. Estructura de bandas del Silicio}

A continuación vamos a analizar de manera separada las principales características de la banda de conducción y de la banda de valencia para este semiconductor, centrándonos en los valles y subbandas considerados en nuestra simulación. 


\section{A. 1. a. Banda de Conducción}

La banda de conducción del Silicio posee dos tipos de mínimos [Cohen, 1988]. El mínimo absoluto está formado por seis valles equivalentes situados en el espacio $\vec{k}$ a lo largo de las direcciones $<100>$ o direcciones $\Delta$ dentro de la $1^{\text {a }}$ zona de Brillouin, a los que denominamos valles X. Los siguientes mínimos relativos están situados en el borde de la $1^{\mathrm{a}}$ zona de Brillouin en la dirección $<111>$ y equivalentes (punto $\mathrm{L}$ ), en total $8 / 2$ valles equivalentes, a los que denominaremos valles L. Para energías cinéticas moderadas, todos estos valles se caracterizan por el hecho de que sus superficies de energía constante se pueden aproximar localmente por elipsoides de revolución. De este modo, cerca de los mínimos relativos X y L los electrones en cada valle muestran una masa efectiva longitudinal $m_{l}$ diferente y mayor que la masa efectiva transversal $m_{t}$. En la Figura A.1 hemos representado de manera simplificada las superficies de energía constante para los valles en las direcciones equivalentes $<100>$ (izquierda) y $<111>$ (derecha).
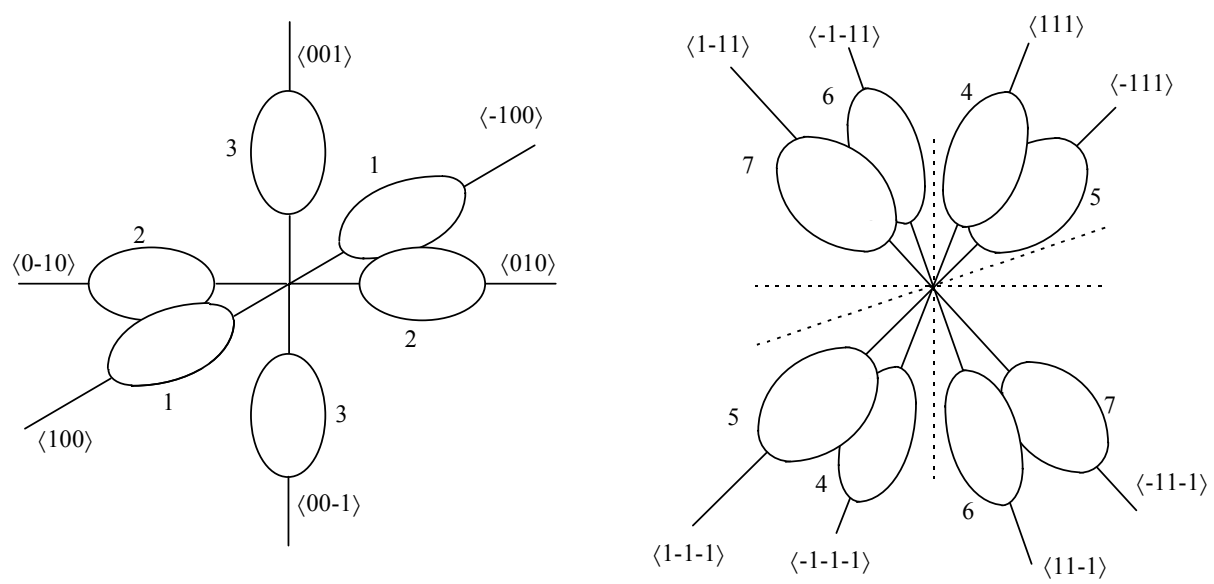

Figura A.1. Superficies de energía constante de los valles X (izquierda) y L (derecha) del Silicio.

La expresión analítica que relaciona la energía y el momento adoptada en nuestro modelo [Martín, 1996; Rengel, 2002] es la siguiente:

$$
E(1+\alpha E)=\frac{\hbar^{2}}{2}\left(\frac{k_{l}^{2}}{m_{l}}+\frac{k_{t}^{2}}{m_{t}}\right)
$$

siendo $\hbar$ la constante de Planck reducida, $\alpha$ el factor de no parabolicidad, $E$ la energía, $k_{l}$ y $k_{t}$ las componentes longitudinal y transversal del momento respectivamente, y $m_{l}$ y $m_{t}$ las componentes de la masa (longitudinal y transversal) del fondo de la banda de conducción. 
Los parámetros característicos de los valles X y L de electrones y para los valles de huecos pesados y ligeros (masas y coeficientes de no parabolicidad) utilizados en la simulación se muestran en la Tabla A.1.

\begin{tabular}{|c|c|c|c|c|c|c|c|}
\hline $\begin{array}{c}\mathrm{m}_{\mathrm{l}} \mathrm{X} \\
\left(\mathrm{m}_{\mathrm{o}}\right)^{*}\end{array}$ & $\begin{array}{c}\mathrm{m}_{\mathrm{t}} \mathrm{X} \\
\left(\mathrm{m}_{\mathrm{o}}\right)^{*}\end{array}$ & $\begin{array}{c}\mathrm{m}_{\mathrm{l}} \mathrm{L} \\
\left(\mathrm{m}_{\mathrm{o}}\right)^{*}\end{array}$ & $\begin{array}{c}\mathrm{m}_{\mathrm{t}} \mathrm{L} \\
\left(\mathrm{m}_{\mathrm{o}}\right)^{*}\end{array}$ & $\begin{array}{c}\alpha \mathrm{Xy} \mathrm{L} \\
\left(\mathrm{eV}^{-1}\right)\end{array}$ & $\begin{array}{c}\text { m pesados } \\
\left(\mathrm{m}_{\mathrm{o}}\right)^{*}\end{array}$ & $\begin{array}{c}\text { m ligeros } \\
\left(\mathrm{m}_{\mathrm{o}}\right)^{*}\end{array}$ & $\begin{array}{c}\alpha \text { pesados y ligeros } \\
\left(\mathrm{eV}^{-1}\right)\end{array}$ \\
\hline \hline 0.90 & 0.19 & 1.634 & 0.126 & 0.5 & 0.537 & 0.153 & 0.3 \\
\hline
\end{tabular}

Tabla A.1. Parámetros fisicos de las bandas de conducción y de valencia del Si ("Madelung 1991)

\section{A. 1. b. Banda de Valencia}

La banda de valencia del Silicio tiene su máximo en el punto $\Gamma$, y está formada por dos subbandas (huecos ligeros y pesados) degeneradas en este punto. También existe una tercera banda (split-off) separada de las anteriores [Dresselhaus, 1955], [Kane, 1965], [Madelung, 1991] cuya contribución al transporte de huecos es muy pequeña [Ottaviani,. 1975] y consecuentemente no ha sido incluida en nuestra simulación [Martín, 1996; Rengel, 2002]. Las dos subbandas más elevadas en energía potencial son consideradas en nuestro modelo como esféricas no parabólicas:

$$
E(1+\alpha E)=\frac{\hbar^{2} k^{2}}{2 m^{*}}
$$

donde $m^{*}$ es la masa efectiva de los huecos para cada sub-banda de valencia.

En la Tabla A.2 indicamos los valores para la permitividad relativa del Silicio y del $\mathrm{SiO}_{2}$, el valor de la densidad intrínseca $\left(n_{i}\right)$ a $300 \mathrm{~K}$ y los valores de la densidad equivalente de estados de la banda de conducción $\left(N_{C}\right)$ y de valencia $\left(N_{V}\right)$, así como el valor del gap considerado entre el mínimo absoluto de la banda de conducción y el máximo de la banda de valencia.

\begin{tabular}{|c|c|c|c|c|c|}
\hline$\varepsilon_{S i}$ & $\varepsilon_{S_{i O_{2}}}$ & $n_{i}$ & $N_{C}$ & $N_{V}$ & Gap \\
\hline \hline 11.7 & 3.9 & $1.45 \times 10^{16} \mathrm{~m}^{-3}$ & $2.24 \times 10^{25} \mathrm{~m}^{-3}$ & $8.44 \times 10^{24} \mathrm{~m}^{-3}$ & $1.05 \mathrm{eV}$ \\
\hline
\end{tabular}




\section{A.2. Mecanismos de scattering}

\section{A. 2. a. Electrones}

Los mecanismos de scattering considerados para electrones en nuestro modelo son: scattering con impurezas ionizadas (elástico y anisótropo), scattering intravalle acústico (elástico e isótropo) e interacciones intervalle equivalente y no equivalente (inelásticas e isótropas). Las expresiones para las diferentes probabilidades pueden encontrarse en [Martín, 1996; Rengel, 2002], así como en diferentes publicaciones fácilmente accesibles (como ejemplo especialmente significativo, véase la correspondiente a [Jacoboni y Lugli 1989]); a continuación comentaremos brevemente las principales características de cada mecanismo e indicaremos los diferentes parámetros considerados en nuestra simulación.

\section{Interacción con impurezas: [Brooks 1951, Jacoboni y Lugli 1989]}

En nuestra simulación hemos supuesto que todas las impurezas se encuentran ionizadas. Por tanto, debe aparecer un efecto de apantallamiento del campo coulombiano, que debe provocar que el potencial que experimenta el electrón en la banda tenga una fuerte dependencia espacial [Brooks, 1951]. En consecuencia, hemos optado por la descripción de Brooks-Herring para describir el scattering con impurezas. A causa de la naturaleza coulombiana de este mecanismo, su importancia es dominante cuando el electrón tiene una energía cinética débil, es decir, a bajas temperaturas o a bajos campos eléctricos aplicados. Las colisiones son elásticas, ya que la masa de la impureza excede enormemente a la del electrón. Además, este mecanismo es anisótropo, por lo que el estado del vector de onda después del mecanismo depende de su orientación anterior.

\section{Interacción acústica con potencial de deformación: [Jacoboni, 1989]}

En general, la energía transferida en este tipo de mecanismo es significativamente inferior a la energía cinética del electrón. Por esta razón, es considerado habitualmente como un proceso elástico y además isótropo (la orientación del vector de onda $\vec{k}$ tras la interacción es aleatoria). 


\begin{tabular}{|c|c|c|}
\hline & Valle X & Valle L \\
\hline Pot. deformación acústico (eV) & 9.0 & 9.0 \\
\hline
\end{tabular}

Tabla A.3. Potencial de deformación acústico de electrones

\section{Interacción intervalle: [Canali, 1975], [Chen, 1991]}

Para la descripción de la estructura de la banda de conducción en nuestro simulador hemos considerado los valles $\mathrm{X}$ (seis valles degenerados en las direcciones $<100>$ ) así como los valles $\mathrm{L}$ (ocho valles en las direcciones $<111>$. Es necesario tener en cuenta que las transiciones pueden darse entre valles equivalentes o no según los estados iniciales y finales del electrón correspondan a valles con el mínimo de energía en niveles iguales o distintos, respectivamente. Respecto a la orientación final del vector de onda, este tipo de colisiones son de carácter isótropo.

- Transiciones no equivalentes: consideraremos las X-L y las L-X. En ambos tipos de interacciones existen cuatro términos fonónicos. Los potenciales de deformación intervalle y energías de los correspondientes fonones se muestran en la Tabla A.4. Estos mecanismos pueden realizarse con emisión o absorción asistida de un fonón.

- Transiciones equivalentes: consideramos en primer lugar las transiciones X-X. Existen seis ramas fonónicas, de las cuales tres están asociadas a transiciones entre valles adyacentes (tipo $f$ ) y las otras tres corresponden a transiciones entre los valles opuestos (tipo $g$ ) (ver Figura A.1). Las transiciones tipo $f$ se producen con fonones de tipo longitudinal acústico y transversal óptico. Tras la colisión, el electrón va a un valle elegido de manera aleatoria entre los valles posibles. Las transiciones $g$ se producen por fonones ópticos longitudinales. El valle final al que va el electrón está determinado en este caso por el valle inicial. También se consideran transiciones equivalentes las transiciones L-L. Las constantes de acoplamiento, así como las energías del fonón intervalle para las seis ramas fonónicas de las transiciones $\mathrm{X}-\mathrm{X}$ y para las transiciones L-L, se muestran en la Tabla A.4. 


\begin{tabular}{|r|r|r|r|r|r||c||}
\hline Parámetros XX & $\mathrm{f} 1$ & $\mathrm{f} 2$ & $\mathrm{f3}$ & $\mathrm{g} 1$ & $\mathrm{~g} 2$ & $\mathrm{~g} 3$ \\
\hline \hline 1 & 0.15 & 3.4 & 4.0 & 0.5 & 0.8 & 3.0 \\
\hline \hline 2 & 18.1 & 43.1 & 54.3 & 12.1 & 18.1 & 60.3 \\
\hline \hline Parámetros X-L=L-X & & & & & & \\
\hline \hline 1 & 4.0 & 4.0 & 4.0 & 4.0 & - & - \\
\hline \hline 2 & 57.9 & 54.6 & 41.4 & 17.0 & - & - \\
\hline \hline Parámetros L-L & & & & & & \\
\hline \hline 1 & 2.63 & - & - & - & - & - \\
\hline \hline 2 & 38.87 & - & - & - & - & - \\
\hline
\end{tabular}

Tabla A.4 Parámetros utilizados en probabilidades de transición intervalle en la banda de conducción. 1: Constantes de acoplamiento intervalle $\left(10^{8} \mathrm{eV} / \mathrm{cm}\right), 2$ : Energía del fonón intervalle (meV).

Dada la dispersión de valores encontrada en la literatura para los valores de los potenciales de deformación intervalle [Canali, 1975], [Sano, 1990], estos han sido elegidos dentro del rango de valores encontrado en la bibliografía con el propósito de ajustar los resultados de la simulación a los datos experimentales disponibles.

Adicionalmente, a pesar de que no tratarse de un mecanismo de scattering propiamente dicho, hemos considerado como tal a la ionización por impacto, con una probabilidad asociada. Como ya comentamos en el Capítulo II, este es el tratamiento habitual de este fenómeno en las simulaciones Monte Carlo [Throngnumchai, 1986], [Canali, 1996], [Dunn, 1997], [Duncan, 1998], [Zandler, 1999], [Ong, 2000]. Para describir la probabilidad de ionización, hemos adoptado un modelo de Kane modificado [Kane, 1967]:

$P_{\text {IONIZA }}=\left\{\begin{array}{l}P\left(\frac{E-E_{t h}}{E_{t h}}\right)^{4} \text { si } E>E_{t h} \\ 0 \text { si } E<E_{t h}\end{array}\right.$

donde $P=9 \cdot 10^{12} \mathrm{~s}^{-1}$ y $E_{t h}=1.2 \mathrm{eV}$. Estos parámetros han sido ajustados para reproducir de la manera más exacta posible, por una parte la probabilidad de ionización asociada a este fenómeno obtenida por otros autores [Kane 1967], [Tang, 1983], [Fischetti y Laux, 1988], [Brunetti et al. 1989], [Vogelsang y Hänsch 1991], [Sano, 1992], [Bude, 1992], [Fiegna, 1993] 
y por otra parte el valor experimental del coeficiente de ionización, como veremos en la Sección A.3.

\section{A. 2. b. Huecos}

Respecto a los huecos, las dos subbandas de valencia consideradas (ligeros y pesados) están degeneradas, y en consecuencia todos los mecanismos de scattering para huecos deben ser tanto interbanda como intrabanda. Los mecanismos de scattering de huecos son básicamente los mismos que los de electrones: scattering con impurezas ionizadas (elástico y anisótropo), scattering con fonones acústicos (inelástico e isótropo) y ópticos (inelástico e isótropo).

\section{Interacción con impurezas:}

En el modelo de Brooks-Herring para las colisiones con impurezas ionizadas deben tenerse en cuenta las expresiones de solapamiento dadas por Wiley en 1971 para las transiciones intrabanda e interbanda. En consecuencia, existen dos tipos de transiciones diferentes según los procesos sean interbanda e intrabanda [Costato y Reggiani 1973].

\section{Interacción con fonones acústicos:}

Este tipo de mecanismo es isótropo y cuasi-elástico [Viso et al. 1979, Canali et al. 1975, Brudevoll et al. 1990]. El pequeño intercambio de energía entre los huecos y la red es necesario para poder alcanzar un estado estacionario adecuado en el caso de que la simulación se realice para bajos campos eléctricos y temperaturas.

\begin{tabular}{|c|c|c|c|}
\hline Pot. Def. Acústico(eV) & $\begin{array}{c}\text { Pot. Def. } \\
\text { Optico }(\mathrm{eV})\end{array}$ & $\begin{array}{c}\text { Energía. fonón } \\
\text { acústico }(\mathrm{meV})\end{array}$ & \begin{tabular}{c} 
Energía fonón óptico $(\mathrm{meV})$ \\
\hline $5.0^{* *}$
\end{tabular} \\
\hline $26.6^{* *}$ & 0.105 & $63.2^{*}$ \\
\hline
\end{tabular}

Tabla A.5. Parámetros de probabilidades de scattering de huecos (* Sze 1981, ** Reggiani 1980)

\section{Interacción con fonones ópticos:}

Este mecanismo de scattering es isótropo e inelástico. A causa de este carácter inelástico, debe considerarse tanto la absorción como la emisión de fonones. 


\section{A. 2. c. Probabilidades de scattering}

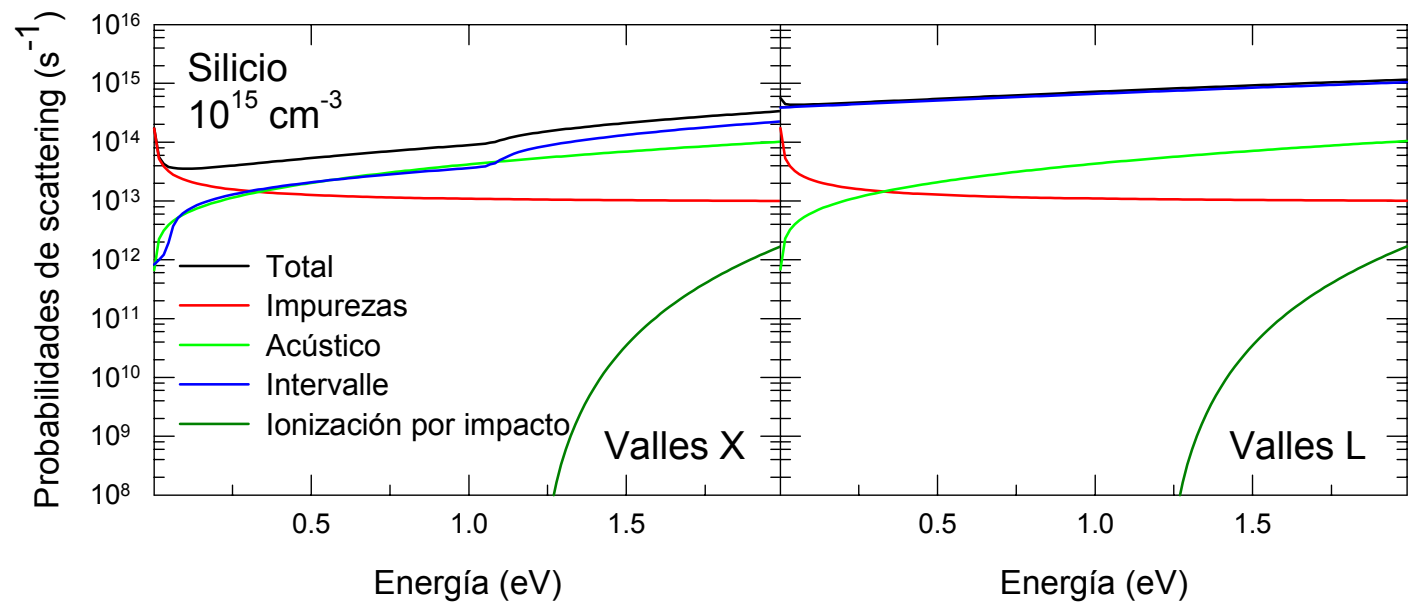

Figura A.2. Probabilidad de scattering de electrones en cada uno de los valles en Silicio

La Figura A.2. muestra la probabilidad total, la de scattering con impurezas ionizadas, la de ionización por impacto, la de acústicos intravalle y las suma de todas las probabilidades de interacciones intervalle correspondientes al valle X (izquierda) y al valle L (derecha) para Silicio con una densidad de impurezas de $10^{15} \mathrm{~cm}^{-3}$.

La Figura A.3 muestra la probabilidad total, scattering con impurezas ionizadas, acústica y ópticos del valle de huecos pesados y de huecos para Silicio con una densidad de impurezas de $10^{15} \mathrm{~cm}^{-3}$.

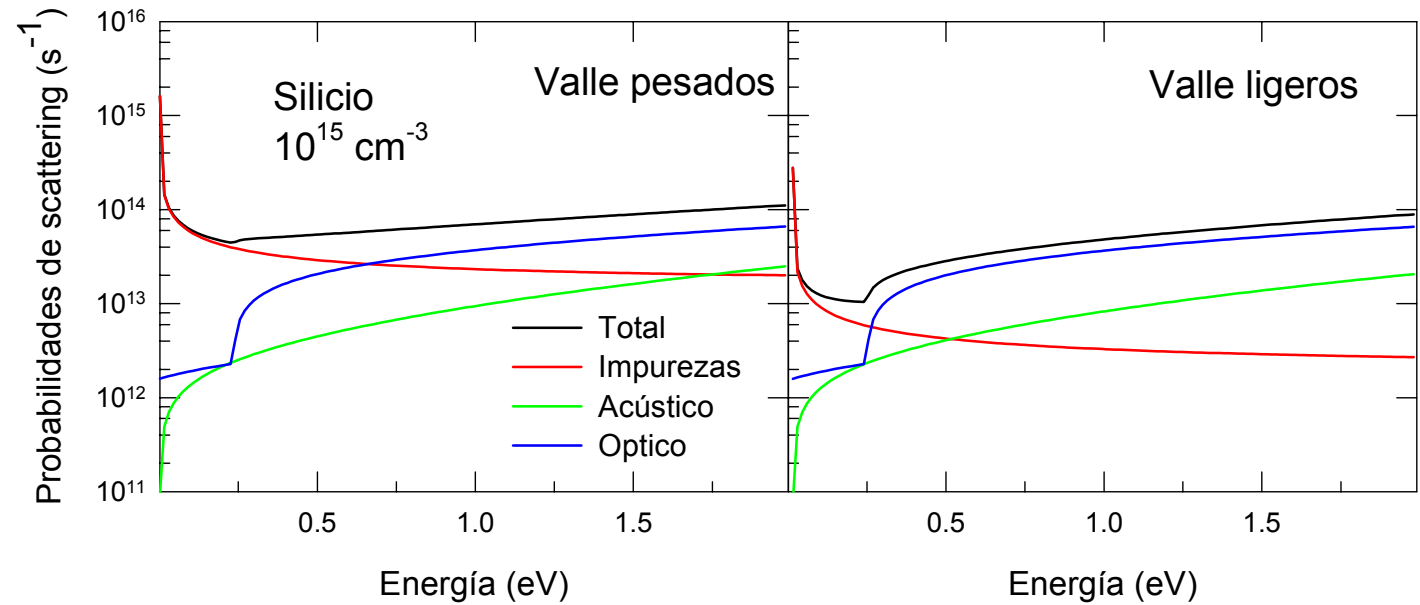

Figura A.3. Probabilidad de scattering de huecos en cada una de las subbandas en Silicio 


\section{A.3. Modelado de Silicio material}

En esta sección, vamos a exponer los principales resultados obtenidos en el estudio del transporte tanto de electrones como de huecos (con dopaje tipo $n$ o tipo $p$, respectivamente) en Silicio. Las simulaciones han sido efectuadas en condiciones de campo eléctrico constante y material homogéneo y sin límites. Los resultados Monte Carlo han sido calculados empleando un simulador de partícula única que considera los mismos mecanismos de scattering y estructura de bandas del simulador bidimensional de dispositivos utilizado en esta Tesis para el análisis de los transistores SB MOSFET considerados.

En nuestro estudio de las propiedades de transporte de los electrones consideraremos el campo eléctrico aplicado en dos direcciones cristalográficas diferentes, [100] y [111]. Todas las simulaciones se han realizado a temperatura de $300 \mathrm{~K}$. En algunos casos disponemos de resultados experimentales obtenidos por diferentes autores que han servido de referencia a la hora de comprobar la validez del modelo.

\section{3. a. Tiempo de permanencia en cada valle}

El tiempo de permanencia de los electrones en cada valle es mostrado en la Figura A.4 (izquierda) para valores del campo eléctrico aplicados en la dirección [100]. Podemos destacar, tal y como vemos en la gráfica, que para un campo eléctrico de aproximadamente $10 \mathrm{kV} \mathrm{cm}^{-1}$, la ocupación del valle $<100>$ alcanza su máximo.

En el caso de que el campo eléctrico se aplicase en la dirección [111], los tres valles X presentarían la misma ocupación.

La ocupación de los valles L ocurre para campos eléctricos aplicados superiores a $30 \mathrm{kV}$ $\mathrm{cm}^{-1}$, aunque inicialmente es muy reducida. La contribución al transporte de los valles $\mathrm{L}$ comienza ser significativa en Silicio material para campos eléctricos superiores a $150 \mathrm{kV} \mathrm{cm}^{-1}$ [Martín, 1992].

La ocupación de las subbandas de valencia en función del campo eléctrico aplicado es mostrada en la Figura A.4 (derecha). Como puede observarse, no existen grandes modificaciones incluso en el caso de campos elevados (la población de la subbanda de ligeros se mantiene cercana al 11-12\%). 


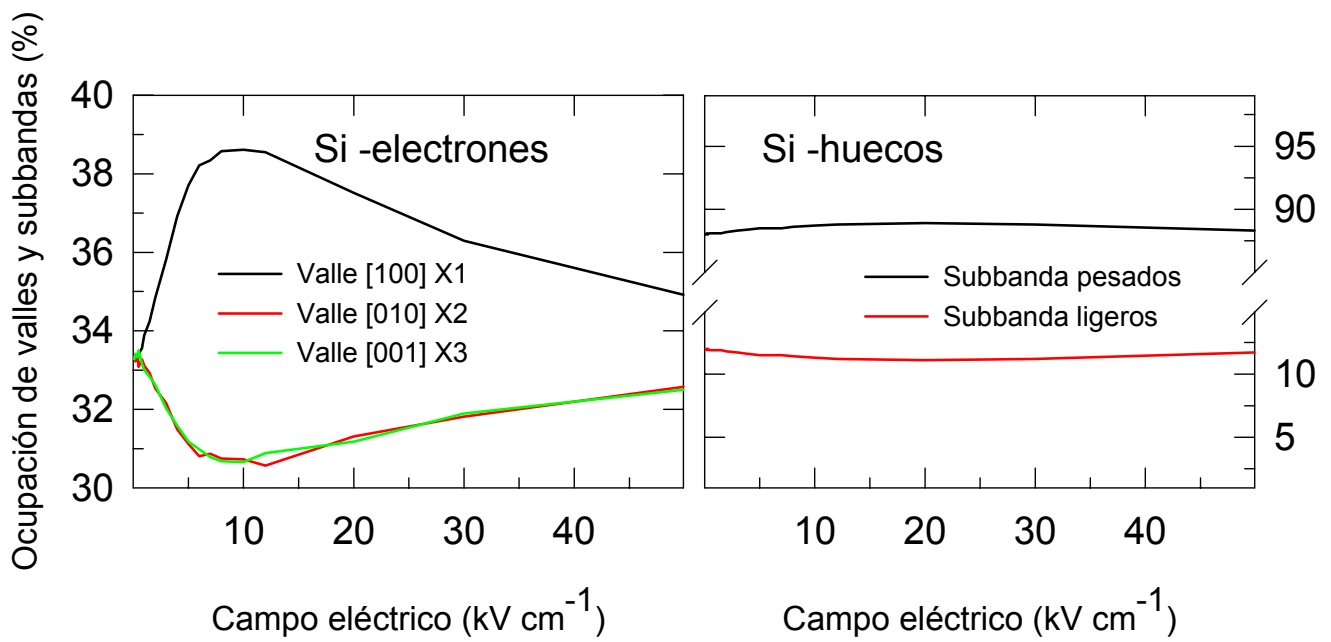

Figura A.4. Ocupación media de los valles X de la banda de conducción (izquierda) y de las subbandas de valencia (derecha) en Silicio material en función del campo eléctrico aplicado en la dirección [100].

\section{A. 3. b. Velocidad de arrastre. Movilidad}

En la Figura A.5 puede observarse la dependencia de la velocidad de arrastre con el campo eléctrico aplicado para electrones y huecos en Silicio material. Para el caso de material tipo $n$, se muestran los resultados obtenidos para campos eléctricos aplicados en las direcciones [100] y [111].

Junto a los resultados de la simulación representamos los valores experimentales obtenidos por Canali et al. en 1975 para electrones y por Ottaviani et al. en 1975 para huecos. Estos datos fueron obtenidos en muestras "altamente puras". La simulación Monte Carlo fue efectuada considerando un valor de densidad de impurezas ionizadas, tanto donadoras como aceptadoras, de $10^{13} \mathrm{~cm}^{-3}$. 


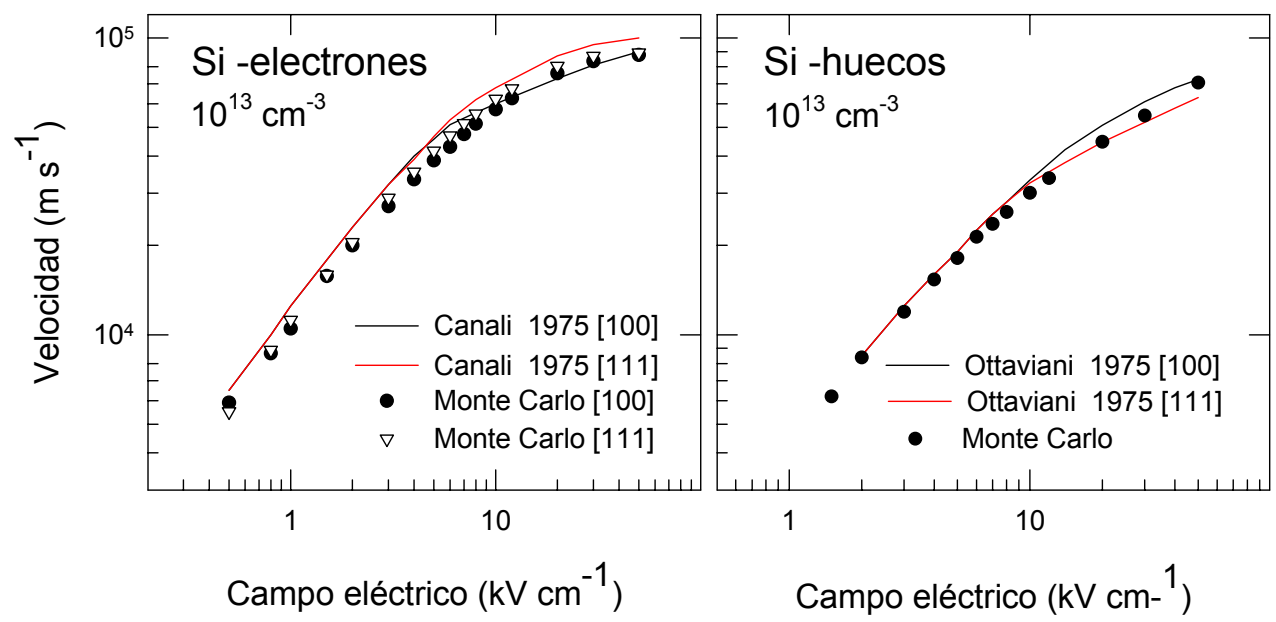

Figura A. 5. Velocidad de arrastre en función del campo eléctrico aplicado para electrones y huecos en Silicio. Junto a los resultados Monte Carlo se muestran distintos resultados experimentales.

Como puede observarse en la figura, el acuerdo entre los resultados Monte Carlo y los datos experimentales es notable, tanto en el caso de electrones como en el de huecos. Esto confirma la validez del simulador en cuanto a la estructura de bandas y probabilidades de scattering del Silicio material, que sirven de base para el desarrollo del simulador MC2D.

Para finalizar este apéndice, a continuación mostramos algunos de los parámetros físicos del Silicio considerados en las simulaciones (Tabla A.6), así como los valores de algunas magnitudes interesantes correspondientes a Silicio homogéneo dopado tipo $n$ (Tabla A.7) y tipo $p$ (Tabla A.8).

\begin{tabular}{||c|c|}
\hline Densidad & $3.329 \mathrm{gr} / \mathrm{cm}^{3}$ \\
\hline Velocidad del sonido Transversal & $9.04 \cdot 10^{5} \mathrm{~cm} / \mathrm{s}$ \\
\hline Velocidad del sonido Longitudinal & $5.30 \cdot 10^{5} \mathrm{~cm} / \mathrm{s}$ \\
\hline Constante Dieléctrica & $11.7 \mathrm{~F} / \mathrm{cm}$ \\
\hline
\end{tabular}




\begin{tabular}{||c|c|c|c|c|c|c||c||}
\hline \multicolumn{1}{|c|}{ Si-n } & & & $\mathrm{m}_{\mathrm{e}}=0.25$ & $\mathrm{~L}=1.0 \mu \mathrm{m}$ & & \\
$\mathrm{N}_{\mathrm{D}}\left(\mathrm{cm}^{-3}\right)$ & $\begin{array}{c}\tau\left(\mathrm{cm}^{2}\right. \\
\left.\mathrm{V}^{-1} \mathrm{~s}^{-1}\right)\end{array}$ & $\begin{array}{c}\mathrm{r}_{\mathrm{o}} \\
\left(\Omega \mathrm{m}^{2}\right)\end{array}$ & $\begin{array}{c}\tau_{\mathrm{p}} \\
(\mathrm{s})\end{array}$ & $\begin{array}{c}\mathrm{f}_{\mathrm{p}} \\
(\mathrm{GHz})\end{array}$ & $\begin{array}{c}\tau_{\mathrm{d}} \\
(\mathrm{s})\end{array}$ & $\begin{array}{c}\tau_{\mathrm{m}} \\
(\mathrm{s})\end{array}$ & $\begin{array}{c}\lambda_{\mathrm{D}} \\
(\mathrm{m})\end{array}$ \\
\hline $10^{16}$ & 1027 & $6.0710^{-9}$ & $3.0310^{-13}$ & 525 & $6.2910^{-13}$ & $1.4610^{-13}$ & $4.1010^{-8}$ \\
\hline \hline $10^{17}$ & 780 & $8.0010^{-10}$ & $9.5810^{-14}$ & 1660 & $8.2910^{-14}$ & $1.1110^{-13}$ & $1.3010^{-8}$ \\
\hline $10^{18}$ & 405 & $1.5410^{-10}$ & $3.0310^{-14}$ & 5246 & $5.7510^{-14}$ & $3.0310^{-14}$ & $4.0910^{-9}$ \\
\hline
\end{tabular}

Tabla A.7

\begin{tabular}{||c|c||c|c|c|c||c||c||}
\hline \multicolumn{1}{|c|}{ Si-p } & & & $\mathrm{m}_{\mathrm{h}}=0.49$ & $\mathrm{~m}_{\mathrm{hh}}=0.54$ & $\mathrm{~m}_{\mathrm{hl}}=0.15$ & $\mathrm{~L}=1.0 \mu \mathrm{m}$ & \\
$\mathrm{N}_{\mathrm{D}}\left(\mathrm{cm}^{-3}\right)$ & $\mu\left(\mathrm{cm}^{2}\right.$ & $\begin{array}{c}\mathrm{r}_{\mathrm{o}} \\
\left(\Omega \mathrm{m}^{2}\right)\end{array}$ & $\begin{array}{c}\tau_{\mathrm{p}} \\
(\mathrm{s})\end{array}$ & $\begin{array}{c}\mathrm{f}_{\mathrm{p}} \\
(\mathrm{GHz})\end{array}$ & $\begin{array}{c}\tau_{\mathrm{d}} \\
(\mathrm{s})\end{array}$ & $\begin{array}{c}\tau_{\mathrm{m}} \\
(\mathrm{s})\end{array}$ & $\begin{array}{c}\lambda_{\mathrm{D}} \\
(\mathrm{m})\end{array}$ \\
\hline \hline $10^{16}$ & 446 & $1.4010^{-8}$ & $4.2310^{-13}$ & 376 & $1.4510^{-12}$ & $1.2310^{-13}$ & $4.1010^{-8}$ \\
\hline \hline $10^{17}$ & 386 & $1.6110^{-9}$ & $1.3410^{-13}$ & 1190 & $1.6810^{-13}$ & $1.0710^{-13}$ & $1.3010^{-8}$ \\
\hline $10^{18}$ & 210 & $2.9710^{-10}$ & $4.2310^{-14}$ & 3762 & $3.0810^{-14}$ & $5.8010^{-14}$ & $4.1010^{-9}$ \\
\hline
\end{tabular}

Tabla A.8

Siendo

- movilidad: $\mu$

- resistencia (para una muestra de longitud igual a $1 \mu \mathrm{m}$ ), $r_{o} S=L / q \mu n_{o}$

- tiempo de relajación dieléctrico, $\tau_{d}=\varepsilon_{o} \varepsilon_{r} / q n \mu$

- tiempo de relajación del momento, $\tau_{m}=\mu \mathrm{m}^{*} / q$

D tiempo del plasma, $\tau_{p}=\sqrt{m^{*} \varepsilon_{o} \varepsilon_{r} / q^{2} n}$

- frecuencia del plasma, $f_{p}=1 / 2 \pi \tau_{p}$

- longitud de Debye (300 K), $\lambda_{D}=\sqrt{\varepsilon_{o} \varepsilon_{r} K_{B} T / q^{2} n}$ 


\section{Bibliografía del Apéndice}

Brooks H., "Scattering by ionised impurities in semiconductors", Phys. Rev., 83, 879 (1951)

Brudevoll T., Fjeldly T. A., Back J y Shur S., "Scattering rates for holes near the valence band edge in semiconductors", J. Appl. Phys., 67, 7373 (1990)

Brunett R., Jacoboni C., Nava F., Reggiani L., Bosman G. Y Zijlstra J. J., “Difussion coefficient of electrons in Silicon“, J. Appl. Phys., 52, 6713 (1996)

Brunetti R., Jacoboni C. et al., "A many-band silicon model for hot-electron transport at high temperature", Solid-State Electron., 32, 1663 (1989)

Bude J., Hess K. y Iafrate G., "Impact ionization in semiconductors - effects of high electricfields and hig sattering rates", Phys. Rev., B 45, 10958 (1992)

Canali C., Jacoboni C, Nava F., Ottaviani G. y Albegiri-Quaranta A., "Electrón drift velocity in silicon”, Phys. Rev., B 12, 2265 (1975)

Canali C., Pavan P. et al., "Experimental and Monte Carlo analysis of impact-ionization in AlGaAs HBT's", IEEE Trans. Electron Dev., 43, 1769 (1996)

Chen R. y Pan D.-S. J., “A Monte Carlo study of many-valley effects on the temporal electrón transient transport in silicon", J. Appl. Phys., 70, 4938 (1991)

Cohen M. L. y Chelikowsky J. R., "Electronic Structure and Optical Properties of Semiconductors”, Berlin, Springer-Verlag, (1988)

Costato M. y Reggiani L., "Scattering probabilities for holes”, Phys. Stat. Sol. B 58, 471 (1973)

Dewey J. y Osman M. A., "Monte Carlo Study of hole transport in Silicon”, J. Appl. Phys., 74, 3219 (1993)

Dresselhaus G., Kip A. F. y Kittel C., "Cyclotron resonance of electron and holes in Silicon and Germanium crystals", Phys. Rev., 98, 368 (1955)

Duncan A., Ravailoi U. y Jakumeit J., "Full-Band Monte Carlo investigation of hot carrier trends in the scaling of Metal-Oxide-Semiconductor Field-Effect-Transistors", IEEE Trans. Electron. Dev., 45, 867 (1998)

Dunn G. M., Rees G. J. y David J. P. R., "Monte Carlo simulation of impact ionization in MESFET's", Electron. Lett., 33, 639 (1997)

Fiegna C. y Sangiorgi E., "Modeling of high-energy electron in MOS devices at the microscopic level”, IEEE Trans. Electron Dev., 40, 619 (1993)

Fischetty M. y Laux. S., "Monte Carlo analysis of electron transport in small semiconductor devices including band-structure and space-charge effects", Phys. Rev. B. 38, 9721 (1988) 
Gómez M. y González P., "Effective mass dependence on the energy and the wavenumber in Diamond and Zinc-Blende Semiconductors", Semicond. Sci. Technol. (1996)

Jacoboni C. y Lugli P. "The Monte Carlo Method for Semiconductor Device Simulation", Viena: Springer (1989)

Kane O., "Energy Band Structure in p-type Germanium and Silicon", J. Phys. Chem. Solids, 1, $82(1965)$

Kane O., "Electron scattering by pair production in Silicon", Phys. Rev, 159, 624 (1967)

Klaasen D. B. M., "A unified mobility model for device simulation- I. Model equations and concentraton dependence", Solid State Electron., 35, 953 (1992)

Madelung O., “Group IV elements and III.V compounds”, Springer-Verlag, Berlin (1991)

Martín M. J., "Estudio del transporte de carga en Silicio mediante un método de Monte Carlo", Grado de Salamanca (Salamanca, 1992)

Martín M.J., “Análisis del Transporte de Carga y de los Fenómenos de Ruido Electrónico en Estructuras Si/Si1-xGex bipolares", Tesis Doctoral, Universidad de Salamanca (1996)

Ong D. G. et al., "Full band Monte Carlo modelling of impact ionization, avalanche multiplication and noise in submicron GaAs $\mathrm{p}^{+}-\mathrm{i}^{+} \mathrm{n}^{+}$diodes", J. Appl. Phys., 87, 7885 (2000)

Ottaviani G., Reggiani L., Canali C., Nava F. y Albegiri-Quaranta A., "Hole drift velocity in silicon”, Phys. Rev., B 12, 3318 (1975)

Reggiani L. y Brunetti R., "Difussion coefficient of holes in Silicon by Monte Carlo simulation”, J. Appl. Phys., 59, 1212 (1986)

Sano N. Aokiy T., Tomizawa M. y Yoshii A., "Electron transport and impact ionization in Si", Phys. Rev., B 41, 12122 (1990)

Sano N. y Yoshii A., "Impact ionization theory consistent with a realistic band structure of Silicon”, Phys. Rev., B 45, 4171 (1992)

Tang J. y Hess K., "Impact ionization of electrons in silicon (steady state)", J. Appl. Phys., 54, $5139(1983)$

Throngnumchai K., Asada K. y Sugano T., "Modeling of 0.1- m MOSFET on SOI structure using Monte Carlo simulation techinque", IEEE Trans. Electron. Dev., 33, 1005 (1986)

Viso S., Jacoboni C. y Reggiani L., J. Phys. C: Solid State Phys., 12, 1525 (1979)

Vogelsang T. y Hänsch W., "A novel-approach for including band-structure effects in a Monte Carlo simulation of electron-transport in Silicon”, J. Appl. Phys., 70. 1493 (1991)

Zandler G., Rossi L. et al., "Monte Carlo simulation of impact ionization and light emission in pseudomorphic HEMTs", Physica B 272, 558 (1999) 


\section{Bibliografía}

Albella J. M. Y Martínez-Duartz J. M., "Fundamentos de electrónica física y microelectrónica", Addison-Wesley / Universidad Autónoma de Madrid (1996)

Aldegunde M., García-Loureiro A. J., Kalna K., Asenov A., "Study of fluctuations in advanced MOSFETs using a 3D finite element parallel simulator" J. Comput. Electron., 5, 311, (2006)

Aldegunde M., García-Loureiro A. J., Martínez A., Kalna K., "3D Monte Carlo Simulation of Tri-Gate MOSFETs Using Tetrahedral Finite Elements" IEEE, International Conference on Simulation of Semiconductor Processes and Devices, 2008. SISPAD. (2008)

Andrews J. M. and Lepselter M. P, "Reverse current-voltage characteristics of metal-Silicide Schottky diodes", Solid State Electronics, 13, 1011 (1970)

ATLAS "New Thermionic Emission and Tunneling Models in ATLAS", The Simulation Standard, 10, no. 8, pp. 6-7 (1999).

Awano M. et al., "Advanced DSS MOSFET Technology for Ultrahigh Performance Applications", VLSI Symp. Tech. Dig., 24 (2008)

Bardeen J., "Surface States and Rectification at a Metal Semi-Conductor Contact", Phys. Rev. 71, 717 (1947)

Barlage, D et al., "High-frequency response of $100 \mathrm{~nm}$ integrated CMOS transistors with highK gate dielectrics", Electron Devices Meeting, IEDM Technical Digest. International (2001)

Bethe H. A., "Theory of the boundary layer of crystal rectifiers", Mass. Inst. Technol. Radiat. Lab. Rep. 43-12 (1942)

Boardman A. D., "Computer Simulations of Hot Electron Behaviour in Semiconductor Using Monte Carlo Methods", en Physics Programs, Boardman A. D., Wiley, New York, 315 (1980)

Bransden B. H. and Joachain C. J., "Introduction to quantum mechanics", Longman Scientific \& Technical (1986) 
Brattain W.H., Bardeen J., Bell Systems Tech. J., 32, 1 (1953)

Brennan K. F., Hess K., Tang J.Y. y Lafrate G. J., "Transient Electronic Transport in InP Ander the Condition of High-Energy Electron Injection", IEEE Trans. Electron. Devices, ED30, 1750, (1983)

Brennan K. F. and Summers C. J., "Theory of resonant tunneling in a variably spaced multiquantum well structure: An Airy function approach," J. Appl. Phys., 61, 614 (1987)

Brennan K. y Hess K., "High field transport in GaAs InP and InAs", Solid-State Electron. 27, 347 (1984)

Brillouin L., Comptes Rendus 183, 24 (1926)

Brooks H., "Scattering by ionised impurities in semiconductors", Phys. Rev., 83, 879 (1951)

Brudevoll T., Fjeldly T. A., Back J y Shur S., "Scattering rates for holes near the valence band edge in semiconductors", J. Appl. Phys., 67, 7373 (1990)

Brunett R., Jacoboni C., Nava F., Reggiani L., Bosman G. Y Zijlstra J. J., "Difussion coefficient of electrons in Silicon“, J. Appl. Phys., 52, 6713 (1996)

Brunetti R., Jacoboni C. et al., "A many-band silicon model for hot-electron transport at high temperature", Solid-State Electron., 32, 1663 (1989)

Bude J., Hess K. y Iafrate G., "Impact ionization in semiconductors - effects of high electricfields and hig sattering rates", Phys. Rev., B 45, 10958 (1992)

Burghartz J. N. et al., "RF Potential of a $0.18 \mu \mathrm{m}$ CMOS Logic Device Technology", IEEE, (2000)

Calvet L. E., Luebben H., et al., "Suppression of leakage current in Schottky barrier metaloxide-semiconductor field-effect transistors", Journal of Applied Physics, 91, 757 (2002)

Canali C., Jacoboni C, Nava F., Ottaviani G. y Albegiri-Quaranta A., "Electrón drift velocity in silicon”, Phys. Rev., B 12, 2265 (1975)

Canali C., Pavan P. et al., "Experimental and Monte Carlo analysis of impact-ionization in AlGaAs HBT's", IEEE Trans. Electron Dev., 43, 1769 (1996)

Celler G. K.and Cristoloveanu S., "Frontiers of silicon-on-insulator", J. Appl. Phys, 93, 4955 (2003)

Chang C. Y. and Sze S. M. "Carrier transport across Metal-semiconductor barriers", Solid-State Electron, 13, 727 (1970)

Chen B. S. and Chen M. C. "Formation of cobalt silicided shallow junction using implant into/through silicide technology and low temperature furnace annealing”, IEEE Trans. Electron Devices, 43, 258 (1996) 
Chen C. L. et al., "High-performance fully-depleted SOI RF CMOS", IEEE Electron Device Letters, 23, 52 (2002)

Chen R. y Pan D.-S. J., “A Monte Carlo study of many-valley effects on the temporal electrón transient transport in silicon", J. Appl. Phys., 70, 4938 (1991)

Cohen M. L. y Bergstresser T. K., "Band Structure and Pseudopotential Form Factors for Fourteen Semiconductors of the Diamond and Zinc-Blende Structures", Phys. Rev. 141, 789, (1966)

Cohen M. L. y Chelikowsky J. R., "Electronic Structure and Optical Properties of Semiconductors", Berlin, Springer-Verlag, (1988)

Colinge J. P., "Fully-Depleted SOI CMOS for Analog Applications", IEEE Trans. Electron Dev. 451010 (1998)

Connelly D., Faulkner C., Grupp D. E., "Performance advantage of Schottky source/ drain in ultrathin body silicon on insulator and dual-gate CMOS”, IEEE Trans. Electron Dev., 50 , 1340 (2003)

Connelly D., Faulkner C., Grupp D. E., "Optimizing Schottky S/D Offset for 25-nm Dual-Gate CMOS Performance", IEEE Electron Device Lett., 24, 411 (2003b)

Constant E., "Modelling of Sub-Micron Devices", Solid State Devices, Carroll J. E., IOP Conf. Series, 57, 141, (1980)

Costato M. y Reggiani L., “Scattering probabilities for holes”, Phys. Stat. Sol. B 58, 471 (1973)

Cowley A. M. and Sze S. M. "Surface States and Barrier Height of Metal-Semiconductor Systems", J. Appl. Phys., 36, 3212 (1965)

Crowell C. R., Sze S. M., Spitzer W. G., "Equality of the temperature dependence of the GoldSilicon surface barrier and the silicon energy gap in Au n-type Si diodes", Appl. Phys. Lett., 4, 91 (1964)

Crowell C. R., "The Richardson constant for thermionic emission in Schottky barrier diodes", Solid State Electronics, 8, 395 (1965)

Crowell C. R. and Sze S. M., "Electron-Optical-Phonon scattering in the emitter and collector barriers of semiconductor-metal-semiconductor structures", Solid-State Electron, 8, 979 (1965b)

Crowell C. R. and Sze S. M., "Current Transport in Metal-Semiconductor barriers", Solid-State Electron, 9, 1035 (1966)

Crowell C. R., "Richardson constant and tunneling effective mass for thermionic and thermionic-field emission in Schottky barrier diodes", Solid-State Electronics, 12, 55 (1969) 
Crowell C. R. and Rideout V. L., "Normalized Thermionic-fielg emission in metalsemiconductor (Schottky) barriers ”, Solid-State Electronics, 12, 89 (1969b)

CSM, Compound Semiconductor Magazine, 31, January/February (2002)

Dambrine G., Raskin J.-P. et al. "High-Frequency Four Noise Parameters of Silicon-onInsulator-Based Technology MOSFET for the Design of Low-Noise RF Integrated Circuits”, IEEE Trans. Electron Dev., 46, 1733 (1999)

Dambrine G. et al., "High frequency low noise potentialities of down to $65 \mathrm{~nm}$ technology nodes MOSFETs", Proc. of 13th European Gallium Arsenide \& Other Compound Semiconductors Application Symposium (GaAs), 03 (2005)

Danneville F., Happy H., Dambrine G., Belquin J. M. y Cappy A., "Microscopic noise modelling and macroscopic noise models: how good a connection?", IEEE Trans. Electron Dev., 41, 779 (1994)

Datta S., "Nanoscale device modeling: the Green's function method" Superlattices and Microstructures, 28, 253 (2000)

Dawson R. H., "Equivalent Circuit of the Schottky-Barrier Field-Effect Transistor at Microwave Frequencies", IEEE Transactions on microwave theory and techniques, 499 (1975)

Deen J. M. and Marinov O., "Noise in advanced electronic device and circuits", ICNF: Int. Conf. Noise and Fluctuations, 3 (2005)

Dennard R. H., Gaensslen F. H., Yu H. N., Rideout V. L., Bassous E., and LeBlanc A. R., "Design of Ion-Implanted MOSFETs with Very Small Physical Dimensions," IEEE J. Solid-State Circuits 9, 256 (1974)

Derdour A M, Nobili D and Solmi S, "High-Temperature Equilibrium Carrier Density of Arsenic-Doped Silicon"J. Electrochem. Soc. 138857 (1991)

Dewey J. y Osman M. A., "Monte Carlo Study of hole transport in Silicon", J. Appl. Phys., 74, $3219(1993)$

Ding-Yu L. et al., "Schottky barrier MOSFET structure with silicide source/drain on buried metal", Chin. Phys. Soc., 16, 240 (2007)

Dresselhaus G., Kip A. F. y Kittel C., "Cyclotron resonance of electron and holes in Silicon and Germanium crystals", Phys. Rev., 98, 368 (1955)

Du G., Liu X. et al., "Characterizations of Double-gate SBTT studying by a 2-D full-band Monte Carlo device simulator", International workshop on junction technology, Shanghai, (IWJT'04), 325 (2004) 
Dubois E. and Larrieu G., "Low Schottky barrier source/drain for advanced MOS architecture: device design and material considerations", Solid-State Electronics, 46, 997 (2002)

Dubois E. and Larrieu G., "Measurement of low Schottky barrier heights applied to metallic source-drain metal-oxide-semiconductor field effect transistors" J. Appl. Phys., 91, 729 (2004)

Duboz J. Y., Badoz P.A., Arnaud F. and Chroboczek J.A., "Electronic transport properties of epitaxial erbium silicide/silicon heterostructures". Appl. Phys. Lett. 55, 84 (1989)

Duncan A., Ravailoi U. y Jakumeit J., "Full-Band Monte Carlo investigation of hot carrier trends in the scaling of Metal-Oxide-Semiconductor Field-Effect-Transistors", IEEE Trans. Electron. Dev., 45, 867 (1998)

Dunn G. M., Rees G. J. y David J. P. R., "Monte Carlo simulation of impact ionization in MESFET's", Electron. Lett., 33, 639 (1997)

Eminente S. et al., "Understanding quasi-ballistic transport in nano-MOSFETs: part IITechnology scaling along the ITRS”, IEEE Trans. Electron Dev., 52, 2736 (2005)

Ferlet-Cavrois V. et al., "High-Frequency Performances of a Partially Depleted $0.18-\mu \mathrm{m}$ SOI/CMOS Technology at Low Supply Voltage_-Influence of Parasitic Elements”, IEEE Electron Device Letters, 19 (1998)

Fiegna C. y Sangiorgi E., "Modeling of high-energy electron in MOS devices at the microscopic level”, IEEE Trans. Electron Dev., 40, 619 (1993)

Fischetty M. y Laux. S., "Monte Carlo analysis of electron transport in small semiconductor devices including band-structure and space-charge effects", Phys. Rev. B. 38, 9721 (1988)

Flandre D., Adriaensen S. et al., "Fully depleted SOI CMOS technology for heterogeneous micropower, high-temperature of RF microsystems", Solid-State Electron., 45, 541 (2001)

Frank D. J., Taur Y.and Wong H. S., "Generalized scale length for two-dimensional effects in MOSFET's", IEEE Electron Device Lett. 19, 385 (1998)

Fritze M., Chen C. L., et al. "High-speed Schottky-barrier pMOSFET with fT = $280 \mathrm{GHz}$," IEEE Electron Device Lett., 25, 220 (2004)

Fuchs K. and Wills H. H., Proceedings of the Cambridge Philosophical Society, 100-108 (1937)

Gámiz F., Roldán J. B., Godoy A., Carceller J. E., Cartujo P., "Double gate silicon on insulator transistors. A Monte Carlo study", Solid-State Electronics, 48, 937 (2004) 
García-Loureiro A. J., Kalna K., Asenov A., "Intrinsic fluctuations induced by a high-kappa gate dielectric in sub-100 nm Si MOSFETs" ICNF: Int. Conf. Noise and Fluctuations, 3 (2005)

Ghandhi S. K., VLSI Fabrication Principles, Wiley, New York (1994)

Godoy A., Ruiz-Gallardo A., Sampedro C., Gámiz F., "Quantum-mechanical effects in multiple-gate MOSFETs", J. Comput. Electron., 6,145 (2007)

Gómez M. y González P., "Effective mass dependence on the energy and the wavenumber in Diamond and Zinc-Blende Semiconductors", Semicond. Sci. Technol. (1996)

González G., "Microwave Transistor Amplifiers Analysis and Design", 2nd edition Upper Saddle River: Prentice Hall (1997)

González T., "Análisis del Ruido Electrónico en Materiales y Dispositivos Semiconductores Unipolares mediante el Método de Monte Carlo", Tesis Doctoral, Universidad de Salamanca (1994)

González T. y Pardo D., "Physical Models of Ohmic Contacts for Monte Carlo Device Simulation", Solid-State Electron. 39, 555 (1996)

González T., Pardo D., Varani L. y Reggiani L., "Monte Carlo Analysis of the Behaviour and Spatial Origin of Electronic Noise in GaAs MESFET's", IEEE Trans. Electron Dev. 42 $991(1995)$

González T., Pardo D., Varani L. and Reggiani L., "Microscopic analysis of electron noise in GaAs Schottky-barrier diodes", J. Appl. Phys., 82, 2349 (1997)

González T., Vasallo B. G., Pardo D. and Mateos J., "Room temperature nonlinear transport in ballistic nanodevices", Semiconductor Science and Technology, 19, S125-S127 (2004)

Guo J. and Lundstrom M. S., "A Computational Study of Thin-Body, Double-Gate, Schottky Barrier MOSFETs", IEEE Trans. on Electron Dev., TED.2002.804696 (2002)

Havart R., Lheurette E., "Step-like heterostructure barrier varactor" IEEE Transactions on Electron Devices, 45, Num. 11 (1998)

Heine V. "Theory of Surface States", Phys. Rev., 138, 1689 (1965)

Hisayo Sasaki Momose, et al., "110 GHz cutoff frequency of ultra-thin gate oxide p-MOSFETs on (110) surface-oriented Si substrate", Toshiba Corporation, IEEE (2002)

Hockney R. W. y Eastwood J. W., "Semiconductor device simulation" en Computer simulation using particles, IOP, Bristol (1988)

Huang C. K., Zhang W. E. And Yang C. H. "Two - dimensional numerical simulation of Schottky Barrier MOSFET with channel length to $10 \mathrm{~nm}$ " IEEE Transactions on Electron Devices, 45, 4 (1998) 
Hutchby J. A., Bourianoff G. I., Zhirnov V. V. and Brewer J. E., "Extending the road beyond CMOS”, IEEE Circuits and Devices Magazine 18, 28 (2002)

Ieong M. et al., "Comparison of Raised and Schottky Source/Drain MOSFETs Using a Novel Tunneling Contact Model", IEDM Tech. Dig., 98, 733 (1998)

ITRS "The International Technology Roadmap for Semiconductors (ITRS), Semiconductor Industry Association", Edition, Austin, TX, International Sematech (2007) (disponible en http://public.ITRS.net)

Iwai H., "Outlook of MOS devices into the next century", Microelectronics Engineering, 48, 7 (1999)

Jacoboni C. y Reggiani L., "The Monte Carlo Method for the Solution of Charge Transport in Semiconductors with Applications to Covalent Materials", Rev. Mod. Phys., 55, 645 (1983)

Jacoboni C. y Lugli P. “The Monte Carlo Method for Semiconductor Device Simulation”, Viena: Springer (1989)

Jang M. et al., "A 50-nm-gate-length erbium-silicided n-type Schottky barrier metal-oxidesemiconductor field-effect transistor", Applied Physics Letters, 84, 741 (2004)

Jang M. et al., "Characterization of Erbium-Silicided Schottky Diode Junction" IEEE Elec. Dev. Letters, 26, 354 (2005)

Jian Y. L., "Schottky contact barrier height extraction by admittance measurement" J. Appl. Phys., 053705, 101 (2007)

Jiménez D. et al., "A drain current model for Schottky-barrier CNT-FETs", J. Comput Electron, $361(2006)$

Jiménez D., "A current-voltage model for Schottky-barrier graphene-based transistors", Nanotechnology, 19, 345204 (2008)

Jiménez J. R., Xiao X., Sturm J.C., Pellegreni P.W., "Tunable, long-wavelength PtSi/SiGe/Si Schottky diode infrared detector", Applied Physics Letters, 67, 506 (1995)

Kalna K., Asenov A., "Role of Multiple Delta Doping in PHEMTs scaled to sub-100 nm Dimensions" Solid-State Electronics, 48, 1223 (2004)

Kane O., "Energy Band Structure in p-type Germanium and Silicon", J. Phys. Chem. Solids, 1, $82(1965)$

Kane O., "Electron scattering by pair production in Silicon", Phys. Rev, 159, 624 (1967)

Kao H. et al., "Very Low Noise in 90nm Node RF MOSFETs using a New Layout", 2007 Topical Meeting on Silicon Monolithic Integrated Circuits in RF Systems, 44 (2007) 
Kedzierski J., Xuan P. et al., "A $20 \mathrm{~nm}$ gate-length ultra-thin body p-MOSFET with silicide source/drain", Superlattices and Microstructures, 28, 445 (2000)

Kedzierski J., Xuan P., et al., "Complementary silicide source/drain thin-body MOSFETs for the $20 \mathrm{~nm}$ gate length regime", IEDM Tech. Dig., 57 (2000b)

Khanna V. K., "Physics of carrier-transport mechanisms and ultra-small scale phenomena for theoretical modelling of nanometer MOS transistors from diffusive to ballistic regimes of operation”, Physics Reports, 67, 398 (2004)

Kim S. D., Park C. -M., and Woo J. C. S., "Advanced Model and Analysis for Series Resistance in Sub-100nm CMOS Including Poly Depletion and Overlap Doping Gradient Effect", IEDM Tech. Dig., (2000)

Kim H., Min H. S., Tang T. W. y Park Y. J., "An extended proof of the Ramo-Shockley theorem", Solid-State Electron. 34, 1251 (1991)

Kinoshita A. et al., "Solution for High-Performance Schottky-Source/Drain MOSFETs: Schottky Barrier Height Engineering with Dopant Segregation Technique”, VLSI Symp. Technical Digest, 168 (2004)

Kinoshita T. et al., "Ultra Low Voltage Operations in Bulk CMOS Logic Circuits with Dopant Segregated Schottky Source/Drain Transistors", IEDM Tech. Dig., (2006)

Klaasen D. B. M., “A unified mobility model for device simulation- I. Model equations and concentraton dependence", Solid State Electron., 35, 953 (1992)

Knoch J., Appenzeller J., "Impact of the channel thickness on the performance of Schottky barrier metal-oxide-semiconductor field-effect transistors", Applied Physics Letters, 81, $3082(2002)$

Knoch J., Zhang M. et al., "Effective Schottky barrier lowering in silicon-on-insulator Schottkybarrier metal-oxide-semiconductor field-effect transistors using dopant segregation" Applied Physics Letters, 87, 263505 [1-3] (2005)

Koeneke C. J., Sze S. M., Levin R. M., and Kinsbron E., "Schottky MOSFET for VLSI," IEDM Tech. Dig., 367 (1981)

Koeneke C. J. and Lynch W. T., "Lightly doped Schottky MOSFET," IEDM Tech. Dig., 466 (1982)

Kramers H. A., Zeits. F. Phys., 39, 828 (1926)

Kuivalainen P., Tarvainen E., Ronkainen H., Majamaa T. and Hovinen A., "Model for the Cutoff Frequency in RF MOSFETs", Physica Scripta. T69, 193 (1997)

Kurosawa T., "Monte Carlo calculation of hot electron problems", J. Phys. Soc. Japan Suppl. S 21, 424 (1966) 
Kurosawa T., "Proceedings of the International Conference on the Physics of Semiconductors", Kyoto, J. Phys. Soc. Japan Suppl. A 49 345, (1966b)

Larrieu G. and Dubois E., "Schottky-Barrier Source/Drain MOSFETs on Ultrathin SOI Body With a Tungsten Metallic Midgap Gate”, IEEE Trans. Electron Dev., 25, 801 (2004)

Larrieu G. "Elaboration et caractérisation de transistors MOS Schottky en regime nanométrique", Tesis Doctoral, Universidad de Ciencias y Tecnología de Lille (2004)

Larrieu G. and Dubois E., "Integration of PtSi-based Schottky-Barrier p-MOSFETs with a midgap Tungsten gate" IEEE Trans. Electron Dev., 52, 2720 (2005)

Larrieu G. et al., "Low Temperature Implementation of Dopant-Segregated Band-edge Metallic S/D junctions in Thin-Body SOI p-MOSFETs", International Electron Device Meeting, IEDM'07 (Washington), 147 (2007)

Larson J. M., "High-speed Schottky-barrier pMOSFET with $f_{T}=280 \mathrm{GHz}$ ", IEEE Electron Device Lett., 25, no. 4, 220 (2004)

Larson J. M., "Schottky Barrier CMOS. Scalable, Lower Cost, Radiation Tolerant, High Performance Technology for Sub-25nm Gate Length", Spinnaker Semiconductor Inc., Technology overview (2004b)

Larson J. M., Snyder J. P., "Overview and Status of Metal S/D Schottky-Barrier MOSFET Technology", IEEE Trans. Electron Dev., 53, 1048 (2006)

Laux S. E., "Techniques for small-signal analysis of semiconductor devices", IEEE Trans. Electron Dev., 32, 2028 (1985)

Laux S. E. y Fischetti M. V., "Monte-Carlo Simulation of Submicrometer Si n-MOSFETs at 77K and 300-K”, IEEE Electron Device Lett., 9, 467 (1988)

Lee S. and $\mathrm{Yu} \mathrm{H.} \mathrm{K.,} \mathrm{"Determining} \mathrm{non-quasi-static} \mathrm{small-signal} \mathrm{equivalent} \mathrm{circuit} \mathrm{of} \mathrm{a} \mathrm{RF}$ silicon MOSFET" Solid-State Electron., 45, 359 (2001)

Lee $\mathrm{S}$, et al., "Record RF performance of sub-46 nm Lgate NFETs in microprocessor SOI CMOS technologies". IEDM Tech Dig., 10.7. (2005)

Lenoble D., "Advanced junction fabrication challenges at the $45 \mathrm{~nm}$ node", Semiconductor Fabtech 30th edition, 114 (2006)

Lepselter M. P. and Sze S. M., "SB-IGFET: An insulated-gate field-effect transistor using Schottky barrier contacts for source and drain," Proc. IEEE, 56, 1400, (1968)

Leroux M., Grandjean N., Beaumont B., Nataf G., Semond F., Massies J., Gilbart P., "Temperature quenching of photoluminescence intensities in undoped and doped GaN" $J$. Appl. Phys. 86, 3721 (1999) 
Liou J. J., Ortiz-Conde A., García-Sánchez F., "Analysis and design of MOSFETs- Modelling, Simulation and Parameter extraction", Kluwer, Norwer MA, (1998)

Lugli P., "Monte Carlo Models and Simulations", Compound Semiconductor Device Modelling, Ed. C. M. Snowden y R. E. Miles, Londres: Springer-Verlag (1993)

Lui W. and Fukuma M., "Exact solution of the Schrödinger equation across an arbitrary onedimensional piece-linear potential barrier", J. Appl. Phys. 60, Num. 5 (1986)

Lundstrom M., "Is nanoelectronics the future of microelectronics?", Proceedings of the 2002 International Symposium on Low Power Electronics and Design, 172 (2002)

Lusakowski J., Martín M. J., Rengel R. et al, "Quasiballistic transport in nonometer Si metaloxide-semiconductor field-effect transistors: Experimental and Monte Carlo analysis", Journal of Applied Physics, 101, 114511(1-6) (2007)

Madelung O., "Introduction to Solid-State Theory", Springer-Verlag, Berlin (1981)

Madelung O., "Group IV elements and III.V compounds", Springer-Verlag, Berlin (1991)

Manku T., "Microwave CMOS - Device Physics and Design", IEEE J. Solid-State Circuits, 34, 277 (1999)

Martín M. J., "Estudio del transporte de carga en silicio mediante un método de Monte Carlo", Grado de Salamanca (1992)

Martín M. J., González T., Velázquez J. E. y Pardo D., "Simulation of electron transport in silicon: impact-ionization processes", Semicond. Sci. Technol. 8, 1291 (1993)

Martín M. J., González T., Pardo D. y Velázquez J. E., "Monte Carlo Analysis of a Schottky Diode with an Automatic Space-Variable Charge Algorithm", Semicond. Sci. Technol. 9, $1316(1994)$

Martín M. J., González T., Pardo D. y Velázquez J. E., "Monte Carlo Analysis of a Schottky Diode with an Automatic Space-Variable Charge Algorithm”, Semicond. Sci. Technol. 11, 380 (1996)

Martín M. J., "Análisis del transporte de carga y de los fenómenos de ruido electrónico en estructuras $\mathrm{Si} / \mathrm{Si}_{1-\mathrm{x}} \mathrm{Ge}_{\mathrm{x}}$ bipolares", Tesis Doctoral, Universidad de Salamanca (1996b)

Martín M. J., Rengel R., Pascual E. and González T., "Monte Carlo analysis of carrier transport from diffusive to ballistic regime in nanometer SOI MOSFETs", IEEE, 2007 Spanish Conference on Electron Devices, 340 (2007)

Martínez J. C. And Polatdemir E., "Semiclassical approach to tunneling throug double barrier structure" Quant-ph/0111103 (2001) 
Mateos J, González T., Pardo D., Hoel V. and Cappy A., "Effect of the T-gate on the performance of recessed HEMTs. A Monte Carlo analysis" Semicond. Sci. Technol. 14, 864 (1999)

Mateos J. et al., "Noise analysis of $0.1 \mu \mathrm{m}$ gate MESFETs and HEMTs", Solid-State Electron., 42, 79 (1998)

Mateos J., Vasallo B. G., Pardo D. and González T., "Operation and high-frequency performance of nanoscale unipolar rectifying diodes", Applied Physics Letters 86, 212103 [1-3] (2005)

Matsumoto T. et al., "70 nm SOI CMOS of $135 \mathrm{GHz}$ fmax with dual offset-implanted sourcedrain extension structure for RF/analog and logic applications", IEDM'01 Technical Digest, 219 (2001)

Matsuzawa, K. Uchida, K. Nishiyama, A. "Monte Carlo simulation of $50 \mathrm{~nm}$ devices with Schottky contact model" International Conference on Simulation of Semiconductor Processes and Devices, SISPAD '99, 35 (1999)

Matsuzawa K., Uchida K. and Nishiyama A., "Monte Carlo simulation of sub-0.1mm devices with Schottky contact model" IEICE Trans. Electron., E83-C, 1212 (2000)

McQuarrie D. A., "Statistical Mechanics”, New York: Harper and Row (1976)

Mochizuki T. and Wise K. D., "An n-channel MOSFET with Schottky source and drain," IEEE Electron Device Lett., 5, 108 (1984)

Moglestue C., "A Self-Consistent Monte Carlo Particle Model to Analyze Semiconductor Microcomponents of Any Geometry", IEEE Trans. Computer-Aided Design, 5, 326 (1986)

Moglestue C., "Monte Carlo Simulation of Semiconductor Devices", Boston: Artech House (1993)

Momose H. S. et al., "Cutoff frequency and propagation delay time of 1.5 -nm gate oxide CMOS", IEEE Transaction on Electron Devices, 48, 6, 1165 (2001)

Mönch W., "Electronic properties of semiconductor interfaces", Springer, Germany (2004)

Moore G., "Progress in Digital Integrated Electronics," IEDM Tech. Digest, 11 (1975)

Morrison S. R., J. Phys. Chem. 57, 860 (1953)

Mott N. F., "Note on the contact between a metal and an insulator or semiconductor", Proc. Cambr. Phil. Soc. 34, 568 (1938)

Nag B. R., "Electron Transport in Compound Semiconductors", Springer-Verlag, Berlin (1980)

Narashima S. et al., "High performance sub-40 nm CMOS devices on SOI for the $70 \mathrm{~nm}$ technology node", IEDM'01 Technical Digest, 625 (2001) 
Neamen D. A., "Semiconductor Physics and devices", Richard Irwin (1992)

Neudeck G. W., "El diodo PN de Unión”, $2^{a}$ Edn. Addison-Wesley Iberoamericana, S. A., Delaware, USA (1993)

$\mathrm{Ng} \mathrm{K}$. K. and Lynch W. T., 'Analysis of the gate-voltage-dependent series resistance of MOSFET's', IEEE Trans. Electron Devices, 33, 965 (1986)

Nishi Y., "Insulated gate field effect transistor and its manufacturing method," patente japonesa $\mathbf{5 8 7 , 5 2 7 ,} 162(1970)$

Norde H., de Sousa Pries J., d'Heurle F., Petersson S., Pesavento F. and Tove P. A., "The Schottky-barrier height of the contact between some rare-earth metals (and silicides) and P-type silicon", Appl. Phys. Lett., 38, 865 (1981)

Nyquist H., "Thermal agitation of electric charge in conductors", Phys. Rev., 32, 110 (1928)

Ong D. G., "Modern MOS Technology - Processes, Devices \& Design", McGraw-Hill: New York (1984)

Ong D. G. et al., "Full band Monte Carlo modelling of impact ionization, avalanche multiplication and noise in submicron GaAs $\mathrm{p}^{+}-\mathrm{i}-\mathrm{n}^{+}$diodes", J. Appl. Phys., 87, 7885 (2000)

Ottaviani G., Reggiani L., Canali C., Nava F. y Albegiri-Quaranta A., "Hole drift velocity in silicon", Phys. Rev., B 12, 3318 (1975)

Pailloncy G. et al., "Noise modeling in fully depleted SOI MOSFETs", Solid-State Electron, 48, 813 (2004)

Pardo D. y Bailón L. A., "Elementos de electrónica", Secretariado de Publicaciones e Intercambio Editorial, Universidad de Valladolid (2007)

Pascual E., Rengel R., Martín M. J., "Microscopic modelling of reverse biased Schottky diodes: influence of non-equilibrium transport phenomena", Semicond. Sci. Technol., 22, 1003 (2007)

Pascual E., Rengel R., Martín M. J., "Monte Carlo analysis of quantum tunnelling and thermionic transport in a reverse biased Schottky diode", 2007 Spanish Conference on Electron Devices, IEEE Catalog 07EX1644, 108 (2007b)

Pascual E., Rengel R., Reckinger N., Tang X., Bayot V., Dubois E., Martín M. J., “A Monte Carlo investigation of carrier transport in fabricated back-to-back Schottky diodes: Influence of direct quantum tunnelling and temperature" Physica Status Solidi (c), 5, 119 (2008) 
Pascual E., Martín M. J., Rengel R., Larrieu G. and Dubois E., "Enhanced carrier injection in Schottky contacts using dopant segregation: a Monte Carlo research", Semicond. Sci. Technol., 24, 025022 [1-6] (2009)

Pascual E., Rengel R. y Martín M. J., "Current drive in n- type Schottky Barrier MOSFETs: a Monte Carlo study", IEEE, 2009 Spanish Conference on Electron Devices, 108 (2009b)

Pascual E., Rengel R. y Martín M. J., "Intrinsic Noise Sources in a Schottky Barrier MOSFET: a Monte Carlo Analysis", Proceedings of the 20th International Conference on Noise and Fluctuations, AIP Conference Proceedings (2009c)

Pérez S. and González T., "Current noise spectra of Schottky barrier diodes with electron traps in the active layer", J. Appl. Phys., 073708, 97 (2005)

Pierret R. F., "Dispositivos de efecto de campo", Wilmington, DE: Addison-Wesley Iberoamericana, (1994)

Pikus F. G., K. K. Likharev, "Nanoscale field effect transistors: an ultimate size analysis", Appl. Phys. Lett., 71, 3661 (1997)

Post I. et al., "A 65nm CMOS SOC Technology Featuring Strained Silicon Transistors for RF Applications", Proceeding of IEDM, (2006)

Pucel R. A., Haus H. A. y Statz H., "Signal and noise properties of gallium arsenide field effect transistors", Advances in Electronics and Electron Physics 38195 (1974)

Ramo S., "Currents Induced by Electron Motion", Proc. IRE 27, 584 (1939)

Raskin J. -P., Guillon R., Chen J., Vanhoenacker-Janvier D. y Colinge J.-P., “Accurate SOI MOSFET Characterization at Microwave Frequencies for Device Performance Optimization and Analog Modeling”, IEEE Trans. Electron Dev. 451017 (1998)

Reggiani L. y Brunetti R., "Difussion coefficient of holes in Silicon by Monte Carlo simulation”, J. Appl. Phys., 59, 1212 (1986)

Rengel R., Mateos J., Pardo D., González T. and Martín M. J., "Monte Carlo analysis of dynamic and noise performance of submicron MOSFETs at RF and microwave frequencies” Semicond. Sci. Technol., 16939 (2001)

Rengel R., "Análisis de los Fenómenos de Trasporte y Ruido Electrónico en Transistores MOSFET y SOI Submicrométricos”, Tesis Doctoral, Universidad de Salamanca (2002)

Rengel R. et al., "Numerical and experimental study of a $0.25 \mu \mathrm{m}$ fully-depleted silicononinsulator MOSFET: static and dynamic radio-frequency behaviour", Semicond. Sci. Technol., 17, 1149 (2002b) 
Rengel R., Pardo D., and Martín M. J., "A physically based investigation of the small-signal behaviour of bulk and fully-depleted silicon-on-insulator MOSFETs for microwave applications", Semicond. Sci. Technol., 19, 634 (2004)

Rengel R., Pardo D., and Martín M. J., "Towards the nanoscale: influence of scaling on the electronic transport and small-signal behaviour of MOSFETs", Nanotechnology 15, S276 (2004b)

Rengel R., Martín M. J., Dambrine G. and Danneville F., "A Monte Carlo investigation of the RF performance of partially-depleted SOI MOSFETs", Semicond. Sci. Technol., 21, 273, (2006)

Rengel R. et al., "A Microscopic Interpretation of the RF Noise Performance of Fabricated FDSOI MOSFETs", IEEE Trans. Electron Devices, 53, (2006b)

Rengel R., Pascual E., Martín M. J., "Injected Current and Quantum Transmission Coefficient in Low Schottky Barriers: WKB and Airy Approaches", IEEE Elec. Dev. Letters, 28, 171 (2007)

Richardson O. W., "The Emission of Electricity from Hot Bodies", Longmans Green, Harlow, Essex (1921)

Rideout V. L., "A Review of the Theory, Technology and Applications of Metal-Semiconductor Rectifiers", Thin Solid Films, 48, 261 (1978)

Ridley K. K., "Quantum Processes in Semiconductors”, Oxford: Clarendon Press (1983)

Roberts G. I. and Crowell C. R. "Capacitive effects of $\mathrm{Au}$ and $\mathrm{Cu}$ impurity levels in Pt n-type Schottky barriers", Solid State Electronic., 16, 29 (1973)

Saha A. R., Chattopadhyay S., Bose C., Maiti C. K., "Technology CAD of silicided Schottky barrier MOSFET for elevated source-drain engineering", Materials Science and Engineering B, 124-125, 424 (2005)

Saitoh W., Itoh A., Yamagami S., Asada M., “Analysis of short-channel Schottky source/drain Metal-Oxide-Semiconductor field-effect transistor on Silicon-on-Insulator substrate and demonstration of sub-50 nm n-type devices with metal gate", Jpn. J. Appl. Phys., 38, $6226(1999)$

Sano N. Aokiy T., Tomizawa M. y Yoshii A., "Electron transport and impact ionization in Si", Phys. Rev., B 41, 12122 (1990)

Sano N. y Yoshii A., "Impact ionization theory consistent with a realistic band structure of Silicon", Phys. Rev., B 45, 4171 (1992)

Schottky W., Phys. Z., 15, 872 (1914)

Schottky W., "Halbleirtertheorie der Sperrschicht”, Naturwissenschaften, 26,843 (1938) 
Schottky W. and Spenke E., Wiss. Veroff. Siemens-Werken, 18, 225 (1939)

Schottky, W., Physik. Zeitschr. 30, 839 (1940)

Schrödinger E., "Quantization as a problem of proper values. Part I", Annalen der Physik, Leipzig. 79, 361 (1926)

Schrödinger E., "An undulatory theory of the mechanics of atoms and molecules", Phys. Rev. 28, 1049 (1926b)

Schwierz F. and Schippel C., "Performance trends of Si-based RF transistors", Microelectronics Reliability, 47, 384 (2007)

Selberherr, S., "Analysis and Simulation of Semiconductor Devices", Springer-Verlag, Wien (1984)

Shalish I., Kronik L., Segal G., Shapira Y., "Yellow luminescence and Fermi level pinning in GaN layers", Applied Physics Letters, 77, 987 (2000)

Shen M., Saikin S, Cheng M., "Monte Carlo Modelling of Spin Injection Through a Schottky Barrier and Spin Transport in a Semiconductor Quantum Well", Journal of Applied Physics, 96, 4319 (2004)

Shenai K. et al., "Modeling and Characterization of Dopant Redistlributions in Metal and Silicide Contact" IEEE Trans. on Elec. Devices, ED-32, 793 (1985)

Shenai K. et al., "Current Transport Mechanisms in Atomically Abrupt Metal-Semiconductor Interfaces" IEEE Trans. on Elec. Devices, 35, 468 (1988)

Shiktorov P., Starikov E., Gruzinskis V., Perez S., González T., Reggiani L., Varani L. and Vaissiere J. C., "Monte Carlo simulation of Schottky diodes operating under TeraHertz cyclostationary conditions", IEEE Electron Dev. Lett., 25, 1, (2004)

Shockley, W. And Pearson G. L., Physic Rev. 74, 232 (1948)

Shockley W., "Current to conductors induced by a moving point charge", J. Appl. Phys., 9, 635 (1938)

Shockley W., Copeland J. A. y James P., "Quantum Theory of Atoms, Molecules and the Solid State", ed. P. O. Löwdin, New York: Academic Press, (1966)

Skotnicki T., Pouydebasque A. and Villanueva D.; USJ Conf. Proceedings, (2003)

Skotnicki T. et al. http://www.itrs.net/Common/2005ITRS/Linked\%20Files/2005Files/PIDS/

MASTAR_Download.htm (2005)

Snowden C. M., "Introduction to Semiconductor Device Modelling", Singapur: World Scientific (1986)

Snyder J. P., Helms C. R., "Experimental investigation of a PtSi source and drain field emission transistor", Appl. Phys. Lett. 67, 1420 (1995) 
Snyder J. P., "The physics and technology of platinum silicide source and drain field effect transistors", Ph.D. dissertation, Stanford Univ., Stanford, CA (1996)

Stratton R. "Difussion of hot and cold electrons in semiconductor barriers", Phys. Rev., 2002, (1962)

Sugino M., Akers L. A., Rebeschini M. E., "CMOS latch-up elimination using Schottky barrier p" IEEE Electron Device Meeting IEDM, 462 (1982)

Sugino M., Akers L. A., Rebeschini M. E., "Latchup-free Schottky-barrier CMOS," IEEE Trans. Electron Dev., 30, 110 (1983)

Sun L., Liu X. Y., Du G. y Han R. Q., "Monte Carlo Simulation of Schottky Contact with Direct Tunnelling Model”, Semiconductor Science and Technology, 18, 576 (2003)

Sun L., Liu X. Y., Du G. y Han R. Q., "A planar asymmetric Schottky barrier source/drain structure for nano-scale MOSFETs", Semiconductor Science and Technology, 21, 608 (2006)

Sungjae Lee et al., "Record RF Performance of Sub-46 nm Lgate NFETs in Microprocessor SOI CMOS Technologies", Proceeding of IEDM, (2005)

Su-Zhen L., Hong-Xia L., "Quantum compact model for thin-body double-gate Schottky barrier MOSFETs", Chinese Physics B, 17, 3077 (2008)

Swirhun S. E., Sangiorgi E., Weeks A. J., Swanson R. M., Saraswat K. C. and Dutton R. W., "A VLSI-suitable Schottky-barrier CMOS process," IEEE Trans. Electron Dev., 32, 194 (1985)

Sze S. M., Crowell C. R. and Kahng D., "Photoelectric Determination of the Image Force Dielectric Constant for Hot Electrons in Schottky Barriers", J. Appl. Phys., 35, 2534 (1964)

Sze S. M., "Physics of Semiconductor Devices", $2^{\text {nd }}$ Edn. Wiley, New York (1981)

Sze S. M., VLSI Technology, New York, McGraw-Hill (1983)

Sze S. M., "Semiconductor Devices. Physics and Technology", $2^{\text {nd }}$ Edn. John Wiley and Sons Inc., New York (2001)

Tang J. y Hess K., "Impact ionization of electrons in silicon (steady state)", J. Appl. Phys., 54, $5139(1983)$

Taur Y. et al., "High performance $0.1 \mu \mathrm{m}$ CMOS devices with $1.5 \mathrm{~V}$ power supply," IEDM Tech. Dig., 127 (1993)

Taur Y., "CMOS design near the limit of scaling", IBM Journal of Research and Development 46, $213(2002)$ 
Throngnumchai K., Asada K. y Sugano T., "Modeling of 0.1- m MOSFET on SOI structure using Monte Carlo simulation techinque”, IEEE Trans. Electron. Dev., 33, 1005 (1986)

Tiemeijer L. F. et al., "A record high $150 \mathrm{GHz}$ fmax realized at $0.18 \mu \mathrm{m}$ gate length in an industrial RFCMOS technology", IEDM'01 Technical Digest, 223 (2001)

Tomizawa K., "Numerical Simulation of Submicron Semiconductor Devices", Artech House, Boston (1993)

Trippe M., Bosman G. y van der Ziel A., "Transit-time effects in the noise of Schottky-barrier diodes", IEEE Trans. Microwave Theory Tech. 341183 (1986)

Tsividis Y., "Operation and modelling of the MOS transistor" $2^{\text {nd }}$ Edition, McGraw-Hill Boston (1999)

Tsui B-Y and Lu C-P, "Current Transport Mechanisms of Schottky Barrier and Modified Schottky Barrier MOSFETs" Conf. Proc. ESSDERC 07, 307 (2007)

Tu K. N., Thompson R. D. and Tsau B. Y., "Low Schottky barrier of rare-earth silicide on nSi”, Appl. Phys. Lett., 38, 626 (1981)

Tucker J. R., Wang C. and Carney P. S., "Silicon field-effect transistor based on quantum tunnelling" Appl. Phys. Lett., 65, 618 (1994)

Tucker J. R., Wang C., Lyding J. W., Shen T.-C. and Abeln G. C., "Nanometer scale MOSFETs and STM paterning on Si," Proc. Int. Conf. Solid State Devices Mater., 322 (1994b)

Tung R. T., "Recent advances in Schottky barrier concepts", Material Science and Engineering $R, 35,1$ (2001)

Valentin R., Dubois E. et al., "RF small-signal analysis of Schottky-barrier p-MOSFET" IEEE Trans. Electron Dev., 55, 1192 (2008)

Valentin R., "Investigation du Comportement et des Potentialités en Haute Fréquence de transistors MOSFET Avancés à Contacts Source/Drain Métalliques" Tesis Doctoral, (2008b)

Van de Roer T. G., "Microwave electronic devices", Chapman and Hall, London (1994)

van der Ziel A., "Noise in Solid State Devices and Circuits", New York: Wiley (1986)

Vanmackelberg M. et al., "90 nm SOI-CMOS of $150 \mathrm{GHz}$ fmax and 0.8 dB NFmin@ 6 GHz for SOC", IEEE International SOI Conference, Wiliamsburg, VA, 7 (2002)

Van Vliet K., Friedmann A. et al., "Noise in Single Injections Diodes. I. Survey of Methods", J. Appl. Phys., 46, 1804 (1975)

Vasallo B. G., González T., Pardo D. and Mateos J., "Monte Carlo analysis of four-terminal ballistic rectifiers", Nanotechnology, 15, S250-S253 (2004) 
Vatannia S. and Gildenblat G., "Airy's functions implementation of the transfer-matrix method for resonant tunneling in variably spaced finite superlattices," IEEE J. Quantum Electron., 32, 1093 (1996)

Vega R. A., "On the modeling and design of Schottky field-effect transistors," IEEE Trans. Electron Devices, 53, 4, 866 (2006)

Vega R. A., "Comparison study of tunneling models for Schottky field effect transistors and the effect of Schottky barrier lowering," IEEE Trans. Electron Devices, 53, 1593 (2006b)

Vega R. A., "A Comparative Study of Dopant-Segregated Schottky and Raised Source/Drain Double-Gate MOSFETs", IEEE Trans. Electron Dev., 55, 2665, (2008)

Vendelin G. D., Pavio A. M. Y Rohde U. L., "Microwave Circuit Design Using Linear and Nonlinear Techniques", New York: Wiley (1990)

Viso S., Jacoboni C. y Reggiani L., J. Phys. C: Solid State Phys., 12, 1525 (1979)

Vogelsang T. y Hänsch W., "A novel-approach for including band-structure effects in a Monte Carlo simulation of electron-transport in Silicon”, J. Appl. Phys., 70. 1493 (1991)

Wang C., Tucker J. R., "Sub-40 nm PtSi Schottky source/drain metal-oxide-semiconductor field-effect transistor", Appl. Phys. Lett., 74, 1174 (1999)

Wentzel G., "Una generailzacione delle condizioni quantiche al fini della meccanica ondulatoria”, Zeitschr. F. Phys. 38, 518 (1926)

Winstead B., Ravaioli U., “Simulation of Schottky Barrier MOSFET’s with a Coupled Quantum Injection/Monte Carlo Technique”, IEEE Trans. on Elect. Dev., 47, 1241 (2000)

Wong H., Man K. F. y Poon M. C., "Modeling of saturation transconductance for short-channel MOSFETs", Solid-State Electronics, 39, 1401 (1996)

Wong H.-S. P., "Beyond the conventional transistor", IBM Journal of Research and Development 46, 133 (2002)

Wong H. -S., Koh A. T. -Y., et al., "Source and Drain Series Resistance Reduction for NChannel Transistors Using Solid Antimony (Sb) Segregation (SSbS) During Silicidation" IEEE Electron Dev. Lett., 29, 756 (2008)

Xia Z., Du G. et al., "Investigation of RF performance of nano-scale ultra-thin-body Schottkybarrier MOSFETs using Monte Carlo simulation”, IEEE Conference on Electron devices and Solid-State circuits, 305 (2005)

Xiong S., King T. -J., and Bokor J., "A Comparison Study of Symmetric Ultrathin-Body Double-Gate Devices With Metal Source/Drain and Doped Source/Drain”, IEEE Trans. Electron Dev., 52, 1859 (2005)

Yang E. S., "Microelectronic Devices", McGraw-Hill, Singapore (1988) 
Yeh S. -P, Shih C. -H., Gong J. and Lien C., "Latent noise in Schottky barrier MOSFETs", Journal of Statistical Mechanics, P01036 (2009)

Zamdmer N. et al., "A $0.13-\mu \mathrm{m}$ SOI CMOS Technology for low-power Digital and RF Applications", 2001 Symposium on VLSI Technology Digest, 85 (2001)

Zamdmer N. et al., "Suitability of scaled SOI CMOS for high-frequency analog circuits", ESSDERC, Proceedings of the $32^{\text {nd }}$ European Solid State Device Research Conference, $511(2002)$

Zamdmer, N. et al., "243-GHz Ft and 208-GHz Fmax 90-nm SOI CMOS SoC technology with lowpower millimeter-wave digital and RF circuit capability", Symposium on VLSI Technology, Digest of Technical Papers, 15, 98 (2004)

Zandler G., Rossi L. et al., "Monte Carlo simulation of impact ionization and light emission in pseudomorphic HEMTs", Physica B 272, 558 (1999)

Zeng L., Yan Liu X., et al., "A Monte Carlo Study of Ambipolar Schottky Barrier MOSFETs", IEEE International Workshop on Computational Electronics 13 (2009)

Zeng L., Yan Liu X., et al., "A Computational Study of Dopant-Segregated Schottky Barrier MOSFETs", IEEE Transtactions on nanotechnology, 9 (2010)

Zhang M. et al., "Impact of dopant segregation on fully depleted Schottky-barrier SOIMOSFETs", Solid-State Electronics, 50, 594 (2006)

Zhang Z., Qiu Z., Liu R., Östling M. and Zhang S. L. "Schottky-barrier height tuning by means of ion implantation into preformed silicide films followed by drive-in anneal", Solid State Electronics., 28, 565 (2007)

Zhu S. et al., "N-Type Schottky Barrier Source/Drain MOSFET Using Ytterbium Silicide" IEEE Elec. Dev. Letters, 25, 565 (2004)

Zubrilov A. S., Nikishin S. A., Kipshidze G. D., Kuryatkov V. V., Temkin H., Prokofyeva T. I., Holtz M. "Optical properties of GaN grown on Si (111) by gas source molecular beam epitaxy with ammonia", J. Appl. Phys. 91, 1209 (2002) 
